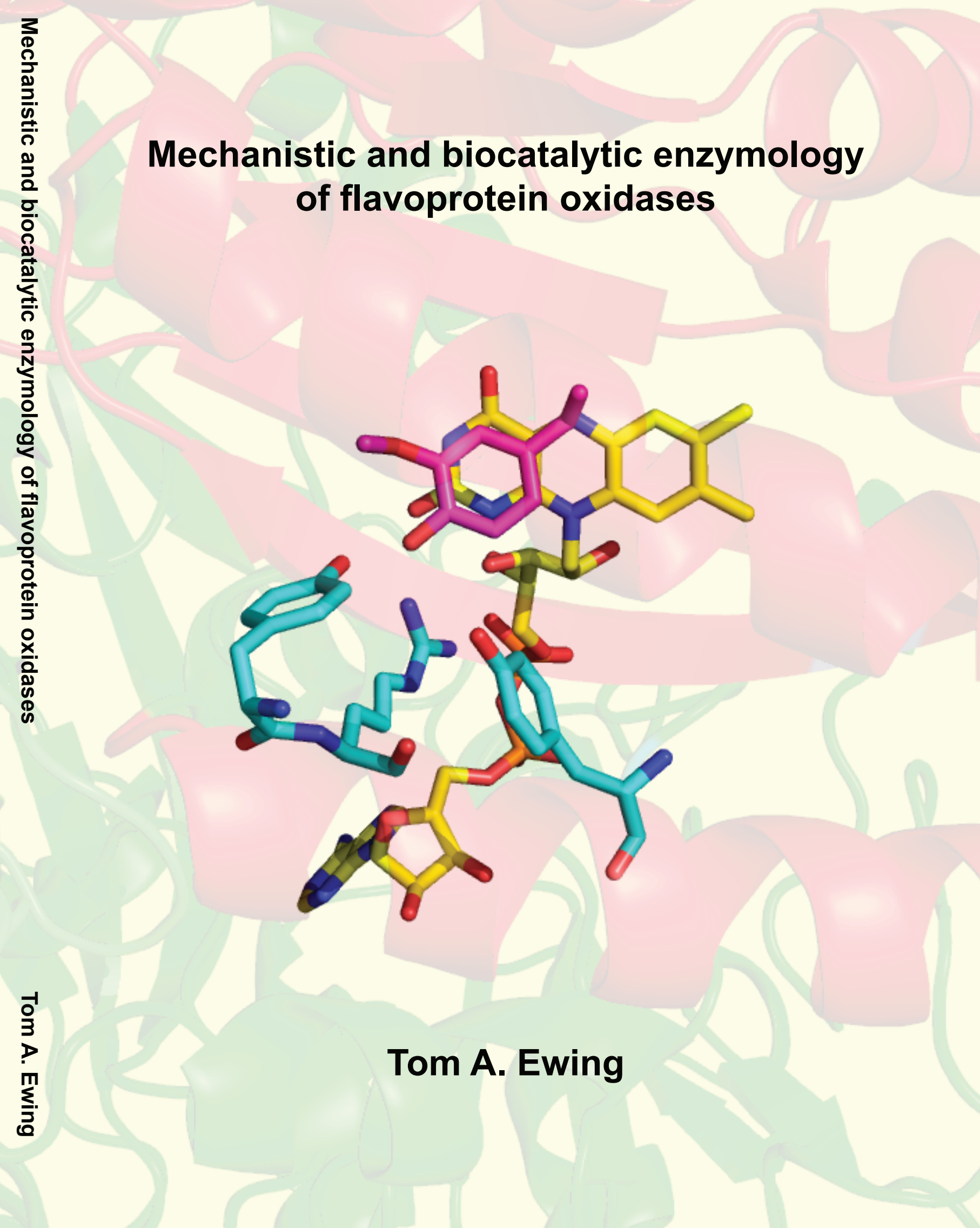




\section{Propositions}

1. Vanillyl alcohol oxidase reacts with both deprotonated and protonated phenolic substrates.

(this thesis)

2. Flavin-dependent alcohol oxidases catalyse the oxidation of thiols to thiocarbonyls. (this thesis)

3. Catalytic efficiency $\left(k_{\mathrm{cat}} / K_{\mathrm{m}}\right)$ is a poor measure by which to compare enzymes.

4. General conclusions regarding the mechanism of an enzyme should not be drawn from experiments involving only a single substrate.

5. The era of biocatalysis requires the exploration of the interdisciplinary field of biocatalytic enzymology.

6. Big data necessitates big legislation.

7. The greatest threat to a productive working day is waking up to find that one has run out of coffee.

Propositions belonging to the thesis, entitled

"Mechanistic and biocatalytic enzymology of flavoprotein oxidases"

Tom A. Ewing

Wageningen, 3 July 2018 


\section{Mechanistic and biocatalytic enzymology of flavoprotein oxidases}

Tom A. Ewing 


\section{Thesis committee}

\section{Promotor}

Prof. Dr W. J. H. van Berkel

Personal chair at the Laboratory of Biochemistry

Wageningen University \& Research

\section{Other members}

Prof. Dr F. L. van Delft, Wageningen University \& Research

Prof. Dr G. J. Poelarends, University of Groningen

Prof. Dr N. S. Scrutton, The University of Manchester, United Kingdom

Dr R. Ludwig, University of Natural Resources and Life Sciences, Vienna, Austria

This research was conducted under the auspices of the Graduate School VLAG (Advanced studies in Food Technology, Agrobiotechnology, Nutrition and Health Sciences). 


\title{
Mechanistic and biocatalytic enzymology of flavoprotein oxidases
}

\author{
Tom A. Ewing
}

Thesis

submitted in fulfilment of the requirements for the degree of doctor at Wageningen University

by the authority of the Rector Magnificus

Prof. Dr A. P. J. Mol,

in the presence of the

Thesis Committee appointed by the Academic Board

to be defended in public

on Tuesday 3 July 2018

at 4 p.m. in the Aula. 
Tom A. Ewing

Mechanistic and biocatalytic enzymology of flavoprotein oxidases 236 pages

PhD thesis, Wageningen University, Wageningen, the Netherlands (2018)

With references, with summaries in English and Dutch

ISBN 978-94-6343-865-0

DOI https://doi.org/10.18174/447033 


\section{Table of contents}

$\begin{array}{ll}\text { Summary } & 7\end{array}$

Nederlandse samenvatting $\quad 9$

Chapter 1: General introduction 13

Chapter 2: The VAO/PCMH flavoprotein family 31

Chapter 3: A single loop is essential for the octamerisation of vanillyl alcohol oxidase 63

Chapter 4: Two tyrosine residues, Tyr-108 and Tyr-503, are responsible for the 85 deprotonation of phenolic substrates in vanillyl alcohol oxidase

Chapter 5: Biocatalysis in organic synthesis: Oxidations using alcohol oxidases 109

Chapter 6: A xylenol orange-based screening assay for the substrate specificity of 141 flavin-dependent para-phenol oxidases

Chapter 7: Multigram scale enzymatic synthesis of (R)-1-(4'-hydroxyphenyl)ethanol using vanillyl alcohol oxidase

Chapter 8: The oxidation of thiols by flavoprotein oxidases: A biocatalytic route to reactive thiocarbonyls

Chapter 9: Discussion 209

$\begin{array}{ll}\text { Acknowledgements } & 225\end{array}$

$\begin{array}{ll}\text { About the author } & 231\end{array}$ 



\section{Summary}

Flavoprotein oxidases catalyse the two-electron oxidation of their substrates using molecular oxygen as an electron acceptor. This makes them interesting candidates for biocatalytic applications. Many flavin-dependent oxidases belong to one of a number of families of structurally related proteins. One such family is the VAO/PCMH family, which contains enzymes that share a common FAD-binding fold. Within this family, a subfamily of enzymes is found that catalyse the oxidation of para-substituted phenols, the 4-phenol oxidising (4PO) subfamily. Enzymes from this subfamily include the oxidases vanillyl alcohol oxidase (VAO) and eugenol oxidase (EUGO) and the dehydrogenase para-cresol methylhydroxylase (PCMH). This thesis focusses on understanding the mode of action of these enzymes and exploring their potential for biocatalytic applications. Although the main focus is on the $4 \mathrm{PO}$ subfamily, other flavin-dependent enzymes are also discussed at some points.

Broadly speaking, the thesis consists of two parts. In the first, the emphasis is on understanding the mode of action of $4 \mathrm{PO}$ subfamily members at the molecular level. Chapter 2 provides an overview of the current state of our knowledge on members of the VAO/PCMH family. The family was divided into eleven subfamilies based on a phylogenetic analysis of 115 members. These subfamilies are discussed in terms of the reactions they catalyse and our mechanistic understanding thereof. Chapter 3 explores the differing oligomerisation states of VAO and EUGO. Despite their highly similar secondary and tertiary structures, VAO forms octamers, which can be described as tetramers of stable dimers, whereas EUGO is exclusively dimeric. A single loop, which is located at the dimer-dimer interface of a VAO octamer, was shown to be essential for the octamerisation of VAO. Its deletion led to a VAO variant that is exclusively dimeric in solution and displays similar catalytic properties to the wild-type enzyme. Chapter 4 focusses on understanding the mechanism of substrate activation in VAO. To enable catalysis, the substrate must first be bound in the active site in its phenolate form. The role of two tyrosine residues, Tyr- 108 and Tyr-503, in facilitating substrate deprotonation was studied by site-directed mutagenesis. These tyrosine residues play a crucial role in enabling substrate activation by $\mathrm{VAO}$, both by stimulating preferential binding of the phenolate form of the substrate and by deprotonating the substrate when it is bound in its protonated form.

The second part of the thesis focusses on the biocatalytic applications of flavin-dependent oxidases. Chapter 5 provides an overview of how flavin-dependent oxidases can be used in synthetic organic chemistry. Chapter 6 describes the development of a high-throughput screening assay for the substrate specificity of variants of VAO and EUGO based on the detection of hydrogen peroxide formed by the enzymes using a xylenol orange assay. This assay was used to screen the substrate specificity of the wild-type enzymes and a small library of variants, leading to the identification of novel substrates of the enzymes and two variants with improved activity towards at least one substrate. Chapter 7 describes the scale-up of the synthesis of a chiral secondary alcohol, $(R)-1-\left(4^{\prime}\right.$-hydroxyphenyl)ethanol, using VAO. Under optimised reaction conditions, we obtained $4.1 \mathrm{~g}$ of pure $(R)-1-\left(4^{\prime}\right.$-hydroxyphenyl)ethanol. In Chapter 8, the focus moves away from the $4 \mathrm{PO}$ subfamily. It describes the oxidation of thiols 
by a number of flavin-dependent alcohol oxidases. Alditol oxidase (AldO) and 5(hydroxymethyl)furfural oxidase (HMFO) were shown to oxidise thiol analogues of their alcohol substrates to the corresponding thiocarbonyls, which have a number of potential applications in organic synthesis.

These results provide advances in our understanding of the mode of action and potential applications of flavoprotein oxidases and will facilitate their future application in industrial biocatalytic processes. 


\section{Nederlandse samenvatting}

Flavine-afhankelijke oxidases katalyseren redoxreacties waarbij twee elektronen van hun substraat worden overgedragen aan een zuurstof molecuul. Hierdoor zijn ze uitstekend geschikt voor gebruik in een verscheidenheid aan toepassingen binnen de biokatalyse. Een groot deel van de flavine-afhankelijke oxidases behoort tot een van een aantal eiwitfamilies bestaande uit eiwitten met een soortgelijke driedimensionale structuur. Een van deze families is de $\mathrm{VAO} / \mathrm{PCMH}$ familie. De leden van deze familie zijn flavine-afhankelijke enzymen die allen een geconserveerd FAD-bindend domein bevatten. De familie kan opgedeeld worden in een aantal subfamilies bestaande uit enzymen die structureel sterk op elkaar lijken en (meestal) hetzelfde type reactie katalyseren. Een voorbeeld van een dergelijke subfamilie is de 4-fenol oxiderende (4PO) subfamilie. Deze subfamilie bestaat uit enzymen die allen de oxidatie van para-gesubstitueerde fenolen katalyseren. Lid van deze subfamilie zijn onder andere de oxidases vanillyl alcohol oxidase (VAO) en eugenol oxidase (EUGO) en de dehydrogenase para-cresol methylhydroxylase (PCMH). Het onderzoek dat in deze thesis omschreven wordt betreft het moleculaire werkingsmechanisme van deze enzymen en hun mogelijke toepassingen binnen de biokatalyse. Het grootste deel van het omschreven werk betreft enzymen uit de $4 \mathrm{PO}$ subfamilie. Flavine-afhankelijke enzymen uit andere (sub)families zullen op bepaalde plekken ook aan bod komen.

De thesis kan beschouwd worden als bestaande uit twee thematisch verschillende delen. In het eerste deel ligt de nadruk op de moleculaire werkingsmechanismen van de leden van de $4 \mathrm{PO}$ subfamilie. In hoofdstuk 2 wordt een overzicht gegeven van onze huidige kennis van de leden van de VAO/PCMH familie. Op basis van een fylogenetische analyse wordt de familie opgedeeld in elf subfamilies. Vervolgens wordt per subfamilie besproken welke reacties door haar leden gekatalyseerd worden en wat de huidige stand van zaken is betreffende ons begrip van het moleculaire mechanisme van deze reacties. In hoofdstuk 3 worden de verschillende oligomerisatie toestanden van de oxidases VAO en EUGO onder het licht gehouden. Ondanks het feit dat VAO en EUGO qua secundaire en tertiaire structuur erg op elkaar lijken, verschillen ze qua quaternaire structuur van elkaar. VAO moleculen vormen vooral octamerische structuren bestaande uit vier stabiele dimeren die door middel van relatief losse contacten de octameer vormen. EUGO moleculen vormen uitsluitend dimerische structuren die erg lijken op een VAO dimeer. In hoofdstuk 3 bewijzen we dat een enkel lusvormig structuurelement, dat zich in een VAO octameer op het raakvlak tussen twee stabiele dimeren bevindt, van essentieel belang is voor het vormen van VAO octameren. Een mutant van VAO waaruit deze lus verwijderd was vormde uitsluitend dimerische structuren, maar behield wel dezelfde katalytische eigenschappen als het ongemuteerde enzym. Hoofdstuk 4 betreft het mechanisme waarmee VAO haar substraten voor de oxidatie reactie activeert. Om geoxideerd te kunnen worden, moeten deze substraten eerst als een gedeprotoneerde fenolaat-anion binden in het actieve centrum van het enzym. In hoofdstuk 4 laten we met behulp van plaatsgerichte mutagenese zien dat twee tyrosine residuen, Tyr-108 en Tyr-503, een cruciale rol spelen bij het activeren van de substraten van VAO door het binden van het fenolaat-anion in het actieve centrum te faciliteren. 
In het tweede deel van de thesis ligt de nadruk op de mogelijke toepassingen van flavineafhankelijke oxidases in de biokatalyse. Hoofdstuk 5 geeft een overzicht van hoe alcohol oxidases toegepast kunnen worden in de synthetische chemie. Hoofdstuk 6 omschrijft de ontwikkeling van een screening methode voor de substraatspecificiteit van VAO en EUGO. Deze methode is gebaseerd op het meten van de hoeveelheid waterstofperoxide die in een bepaalde tijd door de enzymen gevormd wordt door middel van een reactie tussen waterstofperoxide, ijzer ionen en xylenol orange. Hierbij ontstaat een gekleurd complex, waarbij de intensiteit van de kleur afhangt van de hoeveelheid waterstofperoxide dat gevormd is. Deze methode werd toegepast om nieuwe substraten van VAO en EUGO te identificeren en om mutanten van de enzymen te vinden die verhoogde activiteit hadden met een of meerdere substraten. In hoofdstuk 7 omschrijven we de multigram schaal synthese van een chiraal secundair alcohol met behulp van VAO. Na optimalisatie van de reactieomstandigheden waren we in staat om $4.1 \mathrm{~g}$ zuiver $(R)-1-\left(4^{\prime}\right.$-hydroxyfenyl)ethanol in handen te krijgen. In hoofdstuk 8 beschrijven we de vinding dat een aantal flavineafhankelijke alcohol oxidases, bijvoorbeeld alditol oxidase (AldO) of 5(hydroxymethyl)furfural oxidase (HMFO), in staat zijn thiolen om te zetten in thiocarbonyl verbindingen. Zulke thiocarbonylen hebben een aantal mogelijke toepassingen in de synthetische chemie.

De hier gepresenteerde resultaten vergroten ons begrip van de moleculaire werkingsmechanismen van flavine-afhankelijke oxidases. Bovendien geven ze nieuwe inzichten in de mogelijke toepassingen van deze enzymen binnen de biokatalyse. Deze kennis zal bruikbaar zijn bij het ontwikkelen van toekomstige industriële processen waarbij gebruik wordt gemaakt van dit type enzymen. 


General introduction 



\section{Flavins and flavoproteins}

Flavins are organic molecules that contain a tricyclic isoalloxazine ring structure. They are well known for their biologically important role as protein cofactors, with flavin-dependent proteins being present in all kingdoms of life (1). In proteins, flavin is usually present as flavin mononucleotide (FMN) or flavin adenine dinucleotide (FAD) (Scheme 1). Both are biosynthetically derived from riboflavin (vitamin $B_{2}$ ), which is an essential vitamin in humans (2). Most flavin-dependent proteins function as oxidoreductase enzymes. Classes of flavindependent oxidoreductases include monooxygenases, which catalyse the introduction of an oxygen atom originating from molecular oxygen into their substrates, reductases, which catalyse the two-electron reduction of their substrates, and oxidases and dehydrogenesases, which catalyse the two-electron oxidation of their substrates using either molecular oxygen (oxidases) or other molecules (dehydrogenases) as an electron acceptor (3-7). Although flavoproteins are best-known for their role as oxidoreductases, they may also act as electrontransfer proteins (e.g. flavodoxins or electron-transfer flavoproteins), photo- or magnetoreceptors (e.g. cryptochromes) or as non-oxidoreductase enzymes such as transferases or lyases (e.g. alkyl-dihydroxyacetone phosphate synthase or DNA photolyase) (8-13).

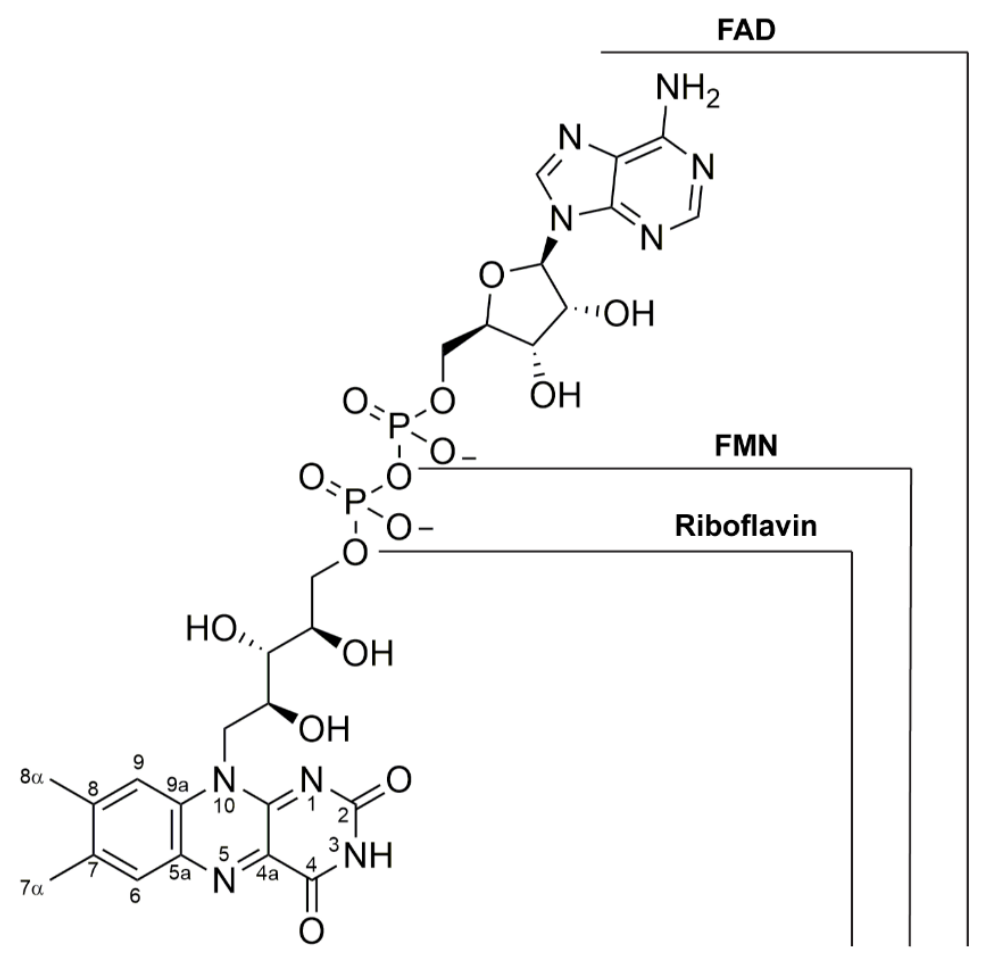

Scheme 1: Structures of riboflavin, FMN and FAD. Scheme adapted from (14).

Flavoproteins achieve their diverse range of functions by using the redox properties of the isoalloxazine ring system, which can be present in one of three redox states: fully oxidised, one-electron reduced, or two-electron reduced (Scheme 2). These redox states can readily be distinguished from each other by their distinct spectral properties and thus colour. In their oxidised form, flavins are typically yellow [hence flavin, from the latin flavus (yellow)]. The one-electron reduced form, a radical that is referred to as the flavin semiquinone, can exist as 
Oxidised (quinone)<smiles>[R]N1c2cc(C)c(C)cc2N=C2NC(=O)NC(=O)C21</smiles><smiles>[18F]C1CCC1[PH3+]</smiles>

One-electron reduced (semiquinone)<smiles>[R]N1C2=NC(=O)NC(=O)C2Nc2cc(C)c(C)cc21</smiles><smiles>[R]n1c2nc(=O)[nH]c(=O)c-2nc2cc(C)c([BH])cc21</smiles>

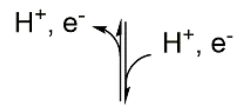

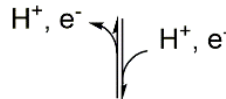

Two-electron reduced (hydroquinone)<smiles>[R]N1c2cc(C)c(C)cc2Nc2c1[nH]c(=O)[nH]c2=O</smiles><smiles></smiles>

Scheme 2: Redox states of the isoalloxazine ring system. Under physiological conditions, flavins can be present in the fully oxidised (quinone), one-electron reduced (semiquinone) or fully reduced (hydroquinone) form. The semiquinone and hydroquinone may be present in their neutral protonated or anionic deprotonated form. Scheme adapted from (15).

a red anionic form or a blue neutral form. The fully reduced hydroquinone form is colourless.

The catalytic mechanism of flavin-dependent oxidoreductases varies depending on the reaction they catalyse. In reductases and monooxygenases, the flavin cofactor is first reduced to the hydroquinone form by accepting two electrons from an electron donor, typically nicotinamide adenine dinucleotide (phosphate) $(\mathrm{NAD}[\mathrm{P}] \mathrm{H})$. In reductases, the electrons are subsequently transferred to the substrate, achieving its two-electron reduction and regenerating the oxidised flavin. In monooxygenases, the reduced flavin reacts with molecular oxygen, yielding a (hydro)peroxyflavin intermediate. This intermediate subsequently attacks the substrate, yielding the oxygenated product and a hydroxyflavin intermediate. Release of water from the hydroxyflavin regenerates the oxidised flavin cofactor. Oxidases and dehydrogenases catalyse the two-electron oxidation of their substrates. After formation of the reduced flavin cofactor by abstraction of two electrons from the substrate, the flavin is reoxidised by its reaction with molecular oxygen (oxidases) or other electron acceptors (dehydrogenases). In recent years, a number of flavin-dependent enzymes have been identified that do not follow one of these canonical flavin-dependent oxidoreductase mechanisms. Examples include EncM from Streptomyces maritimus, which uses an unusual flavin oxidation state, the flavin-N5-oxide, to catalyse a sequential monooxygenationoxidation of its substrate and fungal Fdc1, which uses a prenylated flavin cofactor to catalyse the decarboxylation of its substrate (16-18). 


\section{Reaction mechanism of flavin-dependent oxidases and dehydrogenases}

The catalytic cycle of flavin-dependent oxidases and dehydrogenases can be divided into two distinct half-reactions (Scheme 3). During the reductive half-reaction, a hydride equivalent is transferred from the site of oxidation on the substrate to the N5 atom of the flavin cofactor. Although the mechanism by which this transfer occurs has historically been a major point of debate in the field of flavoenzymology, the current consensus is that the direct transfer of a hydrogen anion is the most probable mechanism for the majority of oxidases and dehydrogenases (19). After completion of the reductive half-reaction, the flavin cofactor must be reoxidised to enable further rounds of catalysis. This occurs during the oxidative halfreaction. In dehydrogenases, the electrons are transferred to other proteins or to small organic molecules. In oxidases, the electrons are transferred to molecular oxygen. The reaction of reduced flavin with molecular oxygen occurs through two sequential single-electron transfer steps (20). The first electron transfer yields a short-lived flavin semiquinone and superoxide anion radical pair. Subsequently, the second electron transfer yields hydrogen peroxide and oxidised flavin. The second electron transfer may proceed either through the direct transfer of an electron from the flavin semiquinone to superoxide anion (concomitant with its protonation) or

through the formation of a covalent (hydro)peroxyflavin intermediate, as is found in monooxygenases. Such an intermediate has been observed in pyranose-2-oxidase, but it is unclear whether it is also formed in other oxidases (21). Detailed studies on the oxidative halfreaction of choline oxidase revealed that it does not involve the formation of a (hydro)peroxyflavin intermediate (22). In recent years, much progress has been made in understanding the molecular determinants of the oxygen reactivity of flavoenzymes. Important factors include the presence of a hydrophobic oxygen binding pocket close to the C4a locus of the isoalloxazine ring and the presence of a positive charge in the vicinity of this binding site, which helps activate oxygen for the electron transfer reaction (23-29).

\section{4-Phenol oxidising flavoenzymes}

Many flavoenzymes that catalyse different reactions share common structural elements and as such can be classified as belonging to structurally related protein families $(5,30)$. One such family of structurally related flavoenzymes is the VAO/PCMH family. This family contains FAD-dependent oxidases, dehydrogenases, reductases and transferases that catalyse a wide range of reactions (31, Chapter 2). Family members are characterised by a two-domain architecture consisting of a conserved FAD-binding domain and a more variable substratebinding or cap domain (32). The FAD-binding domain is responsible for forming the majority of interactions with the FAD cofactor. The ribityl and adenosine diphosphate moieties of FAD are deeply embedded within it, while the isoalloxazine ring is positioned at the interface between the FAD-binding and substrate-binding domains. The shape of the substrate binding pocket is predominantly defined by the substrate-binding domain. This two-domain architecture allows for a wide variety in the type of substrates that are converted by 


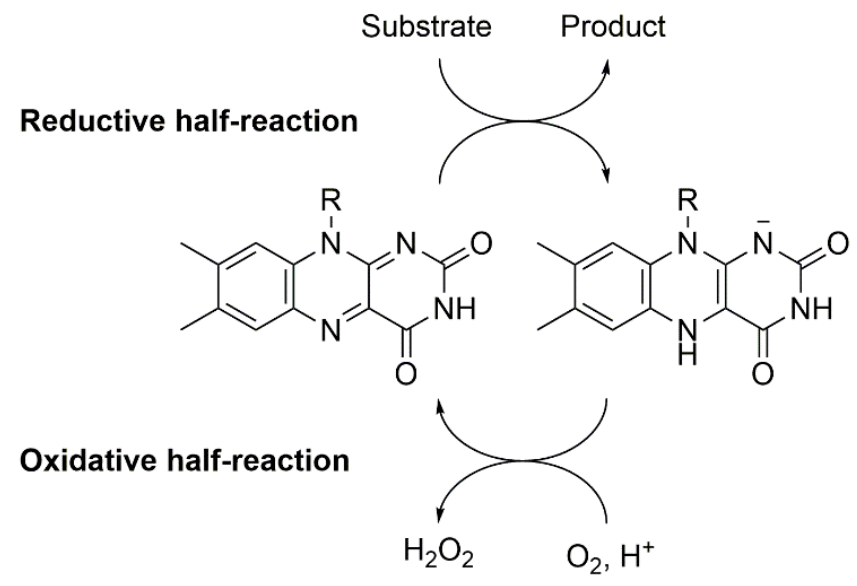

Scheme 3: Catalytic cycle of flavin-dependent oxidases. The catalytic cycle of oxidases consists of two half-reactions. In the reductive half-reaction, the substrate undergoes a two-electron oxidation by transferring a hydride equivalent to the flavin cofactor. During the oxidative half-reaction, the flavin cofactor is oxidised by its reaction with molecular oxygen, which is converted to hydrogen peroxide. Flavin-dependent dehydrogenases follow a similar catalytic cycle, but they are poorly reactive with molecular oxygen, instead transferring the electrons to a different electron acceptor in the oxidative half-reaction.

$\mathrm{VAO} / \mathrm{PCMH}$ family members while maintaining the conserved interactions with the FAD cofactor. Various subfamilies of structurally and functionally related enzymes can be defined within the VAO/PCMH family. One of them, the 4-phenol oxidising (4PO) subfamily, which contains a number of enzymes that catalyse the oxidation of para-substituted phenols, will be the main focus of this thesis.

The three enzymes from the $4 \mathrm{PO}$ subfamily that have been most extensively characterised are vanillyl alcohol oxidase (VAO, EC 1.1.3.38) from the fungus Penicillium simplicissimum, eugenol oxidase (EUGO) from the actinobacterium Rhodococcus jostii RHA1 and para-cresol methylhydroxylase (PCMH, EC 1.17.99.1) from the proteobacterium Pseudomonas putida (3335). These three enzymes catalyse the same basic reaction, the two-electron oxidation of parasubstituted phenols at the $\mathrm{C} \alpha$ atom of their side chain. However, they differ in terms of their substrate specificity, preferred electron acceptor and oligomerisation state. VAO displays the broadest substrate scope of the three, catalysing the oxidation of benzyl alcohols, the oxidative deamination of benzylic amines, the oxidative demethylation of 4-(methoxymethyl)phenol, the hydroxylation of 4-allylphenols and the hydroxylation or dehydrogenation of 4alkylphenols (Scheme 4) $(36,37)$. EUGO catalyses many of the same reactions as VAO, but is poorly active with 4-alkylphenols and 4-(methoxymethyl)phenol $(35,38)$. PCMH is primarily active on short-chain 4-alkylphenols and benzyl alcohols $(33,39)$. The oxidases VAO and EUGO use molecular oxygen as their electron acceptor, whereas PCMH does not react with molecular oxygen and relies on the presence of alternative electron acceptors for its activity. In terms of their structure, all three enzymes share a highly similar core structural unit consisting of two flavoprotein subunits that form a closely packed homodimer $(38,40,41)$. In $\mathrm{VAO}$, four of these dimers can associate to form a homo-octamer (Fig. 1). In solution, VAO is present as an equilibrium of dimers and octamers, with the octameric form predominating 
(42). PCMH forms heterotetramers, with two cytochrome $c$ subunits bound to the dimeric flavoprotein core. In vivo, the heme cofactor of the cytochrome $c$ subunit acts as an electron shuttle, accepting electrons from reduced FAD and transferring them to an azurin electron acceptor protein (43). EUGO forms homodimers consisting simply of the flavoprotein dimer core. In all three enzymes, the FAD cofactor is covalently bound to the protein. In VAO and EUGO, the covalent bond is to a histidine residue ( $8 \alpha-N 3$-histidyl-FAD bond), wherease in $\mathrm{PCMH}$ it is to a tyrosine residue ( $8 \alpha$-O-tyrosyl-FAD bond).<smiles>COc1cc(CO)ccc1O</smiles>

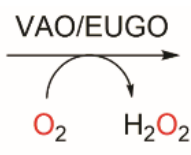<smiles>COc1cc(C=O)ccc1O</smiles><smiles>CCCc1ccc(O)c(OC)c1</smiles>

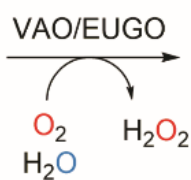<smiles>COc1cc(/C=C/CO)ccc1O</smiles><smiles>Cc1ccc(O)cc1</smiles>

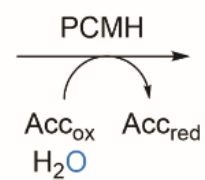<smiles>OCc1ccc(O)cc1</smiles><smiles>OCc1ccc(O)cc1</smiles>

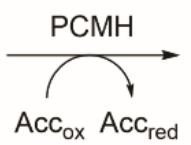<smiles>O=Cc1ccc(O)cc1</smiles><smiles>CCc1ccc(O)cc1</smiles>

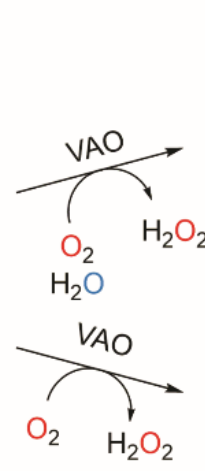<smiles>CC(O)c1ccc(O)cc1</smiles>

Scheme 4: Reactions catalysed by $4 \mathrm{PO}$ subfamily members. From top to bottom: the oxidation of vanillyl alcohol (4-hydroxy-3-methoxybenzyl alcohol) to vanillin (4-hydroxy-3-methoxybenzaldehyde) as catalysed by VAO or EUGO; the oxidation of eugenol (4-allyl-2-methoxyphenol) to coniferyl alcohol [4-(3-hydroxyprop-1-en-1-yl)-2-methoxyphenol] as catalysed by VAO or EUGO; the oxidation of paracresol (4-methylphenol) to 4-hydroxybenzyl alcohol as catalysed by $\mathrm{PCMH}$; the oxidation of 4hydroxybenzyl alcohol to 4-hydroxybenzaldehyde as catalysed by $\mathrm{PCMH}$; the oxidation of 4ethylphenol to a mixture of (R)-1-(4'-hydroxyphenyl)ethanol and 4-vinylphenol as catalysed by VAO.

In addition to these well-characterised enzymes, the $4 \mathrm{PO}$ subfamily contains a number of enzymes about which less is known. Two Pseudomonas sp. strains (OPS1 and HR199) that are able to use eugenol (4-allyl-2-methoxyphenol) as a carbon source have been shown to contain eugenol hydroxylases, flavocytochromes that catalyse the oxidation of eugenol to coniferyl 


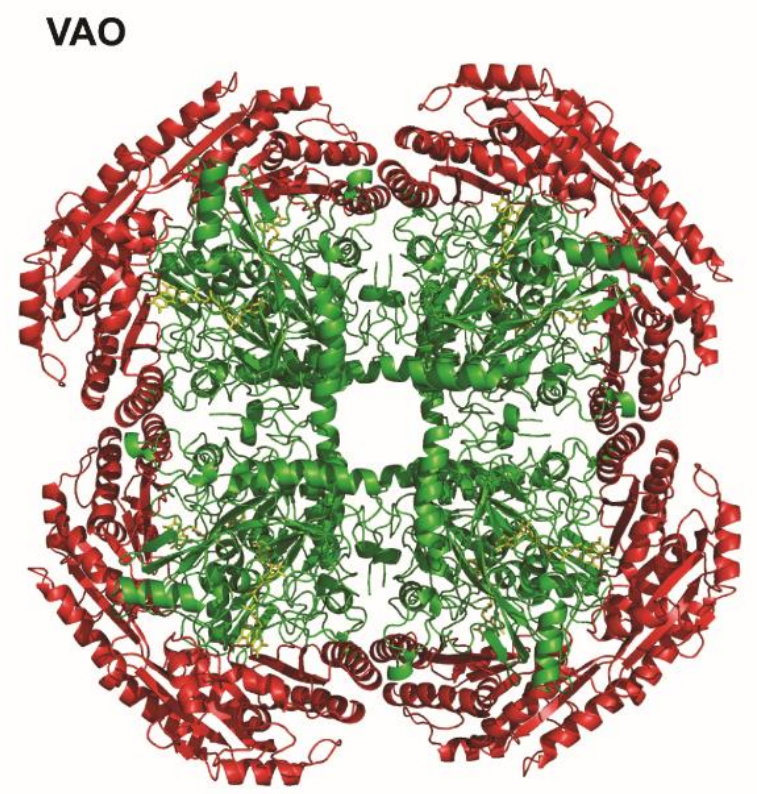

\section{EUGO}

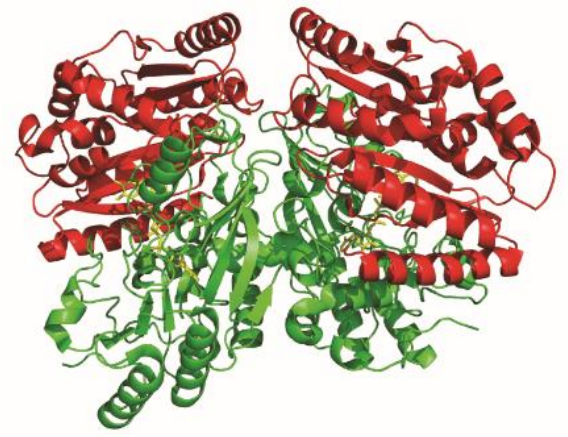

PCMH

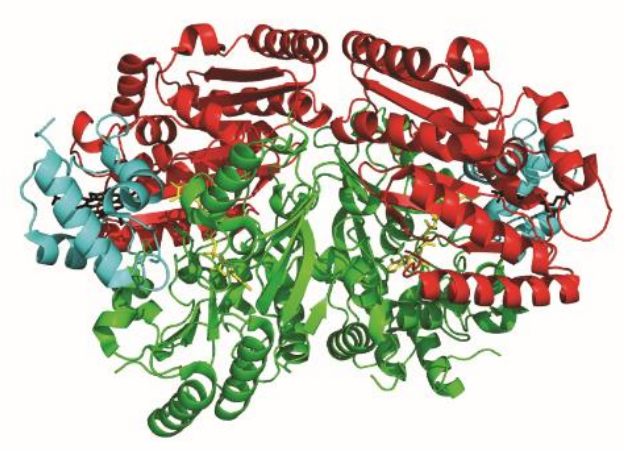

Figure 1: Crystal structures of VAO, EUGO and PCMH. Structures are shown in cartoon representation with the FAD-binding domain of the flavoprotein subunits shown in green, the substrate-binding domain of the flavoprotein subunits shown in red and the cytochrome $c$ subunit of PCMH shown in cyan. The FAD cofactor is shown as yellow sticks and the heme cofactor of PCMH is shown as black sticks. VAO forms homo-octamers that can be described as tetramers of dimers. Each quarter of the ring-like octameric structure corresponds to a tightly packed dimer, with the inter-dimer packing being more loose. EUGO forms homodimers that structurally closely resemble a VAO dimer. PCMH forms heterotetramers consisting of two flavoprotein subunits and two cytochrome $c$ subunits. The flavoprotein subunits form a dimer that closely resembles a VAO or EUGO dimer. One cytochrome $c$ subunit binds to each flavoprotein subunit at a binding site located far from the dimer interface. Figures were prepared from PDB files downloaded from the Protein Data Bank website [PDB IDs: 1VAO for VAO, 1WVE for PCMH and 5FXD for EUGO] using PyMOL (Schrödinger LLC, New York, NY, USA).

alcohol [4-(3-hydroxyprop-1-en-1-yl)-2-methoxyphenol], the first step in its degradation (44, 45). The sequences of these proteins reveal that they belong to the $4 \mathrm{PO}$ subfamily, showing highest similarity to PCMH, and they are predicted to contain $8 \alpha$-O--tyrosyl-FAD as a cofactor. Although the purified enzymes from these strains have not been characterised, a flavocytochrome that converts eugenol to coniferyl alcohol has been purified from the eugenol-degrading bacterium Pseudomonas fluorescens E118 and named eugenol 
dehydrogenase (46). N-terminal sequencing revealed that it displays significant similarity to eugenol hydroxylase from Pseudomonas sp. HR199. In addition to eugenol, 4-alkylphenols and 4-hydroxybenzyl alcohols were converted by the enzyme. Another flavocytochrome that likely belongs to the $4 \mathrm{PO}$ subfamily, but for which no sequence information is available, is 4ethlyphenol methylenehydroxylase from Pseudomonas putida JD1. This enzyme catalyses the oxidation of 4-alkylphenols and 4-allylphenols, with linear 4-alkylphenols with side chains of two to five carbon atoms being most efficiently converted $(47,48)$. In addition to in Penicillium simplicissimum, enzymes that display VAO activity have been identified in the fungi Byssochlamys fulva V107 and Fusarium moniliforme MRC $6155(49,50)$. The 4 PO subfamily also contains two enzymes that catalyse the oxidative decarboxylation of $\mathrm{N}$-substituted tyrosine derivatives: CndG from Chondromyces crocatus and FeeG from an unidentified soil microbe (51, $52)$.

Despite their different substrate scopes, all 4PO subfamily members share a strict requirement for a para-substituted phenol as their substrate. This can be understood by taking their catalytic mechanism into account (Scheme 5) $(36,53-55)$. In VAO, the substrate must first be bound in the active site in its phenolate form. Deprotonation to the phenolate activates the substrate for oxidation by allowing the formation of a para-quinone methide intermediate upon transfer of a hydride to the enzyme's FAD cofactor. Following hydride transfer, the paraquinone methide intermediate either rearranges to form a dehydrogenated product or reacts with water to yield a hydroxylated product. In VAO, the reaction of the reduced flavin with molecular oxygen takes place either with the para-quinone methide intermediate still bound in the active site (ternary complex mechanism) or after the release of the final product (pingpong mechanism). The mechanism that is followed depends on the substrate: with 4(methoxymethyl)phenol the enzyme exclusively follows a ternary complex mechanism, whereas with vanillyl alcohol a ping-pong mechanism is also possible (54).

Although the catalytic mechanisms of other $4 \mathrm{PO}$ subfamily members have not been studied in as much detail, they presumably act through a similar mechanism involving the formation and breakdown of a para-quinone methide intermediate.

\section{Biocatalytic applications of 4-phenol oxidising flavoenzymes}

In recent decades, biocatalysis, the use of enzymes to achieve organic chemical transformations, has been gathering attention as a potential alternative to traditional methods of chemical synthesis. Biocatalytic processes have a number of advantages as compared to conventional chemical ones. Enzymes are often highly regioselective, making them ideally suited to converting one of multiple identical functional groups on a molecule without the need for protection chemistry. For example, many enzymes are known to catalyse the oxidation of a specific hydroxyl group of carbohydrate molecules (56). In addition, the asymmetric nature of an enzyme's active site enables them to perform highly enantioselective reactions. This is of particular interest to the pharmaceutical industry, as it makes enzymes highly suitable for the challenging task of generating the chiral centres that are often required in active pharmaceutical ingredients (57). Biocatalytic processes may also have benefits in 


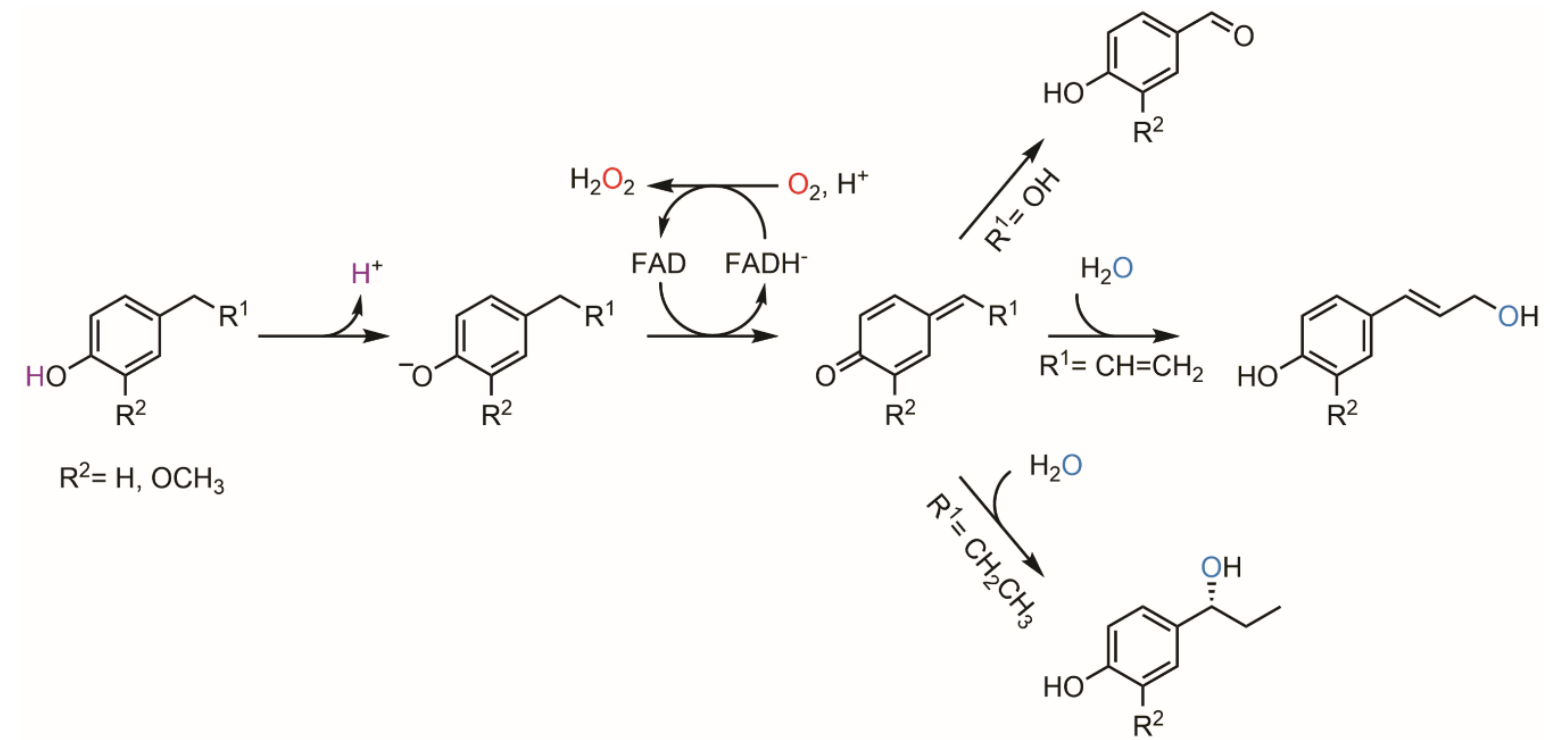

Scheme 5: Catalytic mechanism of VAO. First, the substrate must be bound in the enzyme's active site in its phenolate form. Subsequently, transfer of a hydride to the FAD cofactor yields a para-quinone methide intermediate that is stabilised in the enzyme's active site. Depending on the substrate, this intermediate either rearranges or is hydroxylated at the $\mathrm{C} \alpha$ or $\mathrm{C} \gamma$ position to give the reaction product. Meanwhile, the FAD cofactor is reoxidised by its reaction with molecular oxygen, which is converted to hydrogen peroxide. Depending on the substrate, this reoxidation may occur with the para-quinone methide intermediate still bound in the active site (ternary complex mechanism) or after product release (ping-pong mechanism). Other members of the $4 \mathrm{PO}$ subfamily are thought to operate via a similar mechanism.

terms of their environmental impact, as enzymes can be sustainably sourced from biological materials and are active under mild process conditions.

A number of the reactions that are catalysed by $4 \mathrm{PO}$ subfamily members are of interest from the point of view of biocatalytic applications. One interesting target compound is vanillin (4hydroxy-3-methoxybenzaldehyde), the main flavour and fragrance compound in vanilla. Vanillin is one of the most-used flavouring compounds in the world, with an annual market of approximately 16,000 tonnes (58). Most of this is currently produced synthetically from the petrochemical guaiacol (2-methoxyphenol). VAO could potentially be used to synthesise vanillin from precursors found in natural sources such as para-creosol (4-methyl-2methoxyphenol), which is found in wood tar, or capsaicin, which is found in chilli peppers (Scheme 6) (59). para-Creosol can be converted to vanillin by VAO in a two-step reaction involving its hydroxylation to vanillyl alcohol (4-hydroxy-3-methoxybenzyl alcohol) followed by oxidation of the formed vanillyl alcohol to vanillin. Hydrolysis of capsaicin, by e.g. penicillin G acylase, yields vanillyl amine [4-(aminomethyl)-2-methoxyphenol], which can subsequently be converted to vanillin via oxidative deamination by VAO. The conversion of eugenol to coniferyl alcohol by VAO has also gathered interest as a potential starting point for the synthesis of vanillin by microbial fermentation (60-65). Eugenol is the major constituent of the essential oil from cloves and is therefore readily available from natural sources. The coniferyl alcohol formed by its conversion can be converted to vanillin in four biosynthetic steps. 
In addition to the synthesis of vanillin, members of the 4PO subfamily can be employed for the synthesis of chiral secondary alcohols. Creating chiral centres in achiral compounds is one of the most challenging reactions in synthetic organic chemistry, yet it is of great importance for the synthesis of active pharmaceutical ingredients $(66,67)$. A number of $4 \mathrm{PO}$ subfamily members are capable of catalysing the synthesis of chiral 1-(4'-hydroxyphenyl)alcohols from linear 4-alkylphenols. The enantiomer that is formed depends on the enzyme, e.g. VAO catalyses the formation of the $(R)$-enantiomers (Scheme 4 , bottom panel), whereas PCMH catalyses the formation of the $(S)$-enantiomers $(68,69)$. In VAO, this enantioselectivity can be explained by the presence of an active site base (Asp-170) that activates water for attack on one side of the planar para-quinone methide intermediate. Replacing this base with one that is positioned on the opposite side of the para-quinone methide intermediate was sufficient to reverse the enantioselectivity of the reaction, with the resulting D170S/T457E variant preferentially forming the (S)-enantiomers of the alcohol products (70).

Another potential biocatalytic application of $\mathrm{VAO}$ is the synthesis of the plant lignan pinoresinol, which is of interest due to its potential health benefits (71). Pinoresinol was synthesised via a cascade reaction combining the oxidation of eugenol to coniferyl alcohol by $\mathrm{VAO}$ with the dimerisation of coniferyl alcohol catalysed by a laccase (Scheme 7) (72).

Subsequent reduction of the formed pinoresinol by enantioselective reductases was used to form both pure enantiomers by kinetic resolution (73). The oxidation of eugenol to coniferyl alcohol by EUGO has been used in a cascade reaction for the synthesis of lignin model

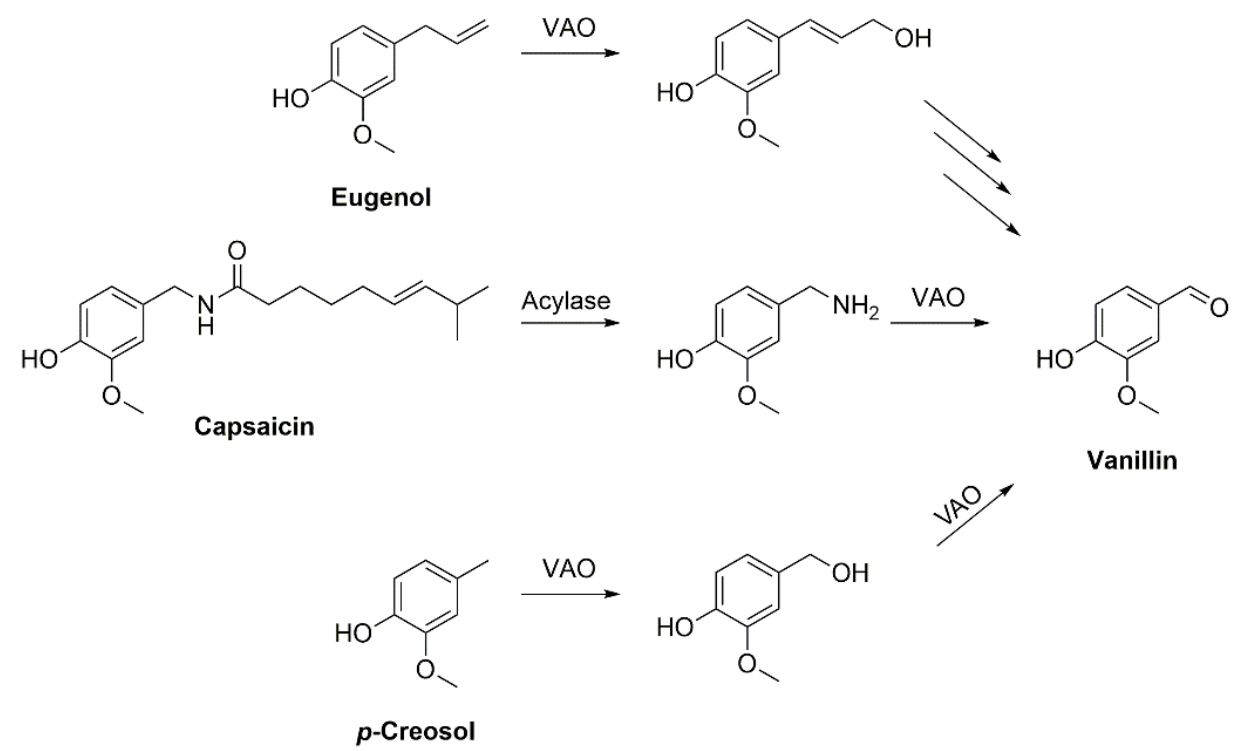

Scheme 6: Biocatalytic pathways for the synthesis of vanillin from naturally-sourced precursors involving VAO. VAO can convert eugenol, which is found in clove oil, to coniferyl alcohol as the first step in a fermentation process that yields vanillin as the final product. Capsaicin, which is found in chilli peppers, can be deacylated to vanillyl amine by penicillin $\mathrm{G}$ acylase. The formed vanillyl amine can be converted to vanillin by VAO. para-Creosol, which is found in wood tar, can be converted to vanillin in a two-step reaction involving its hydroxylation to vanillyl alcohol followed by the oxidation of the alcohol to vanillin. Both steps of the reaction are catalysed by VAO. 
compounds (Scheme 7). The heterogeneous aromatic polymer lignin is one of the major constituents of lignocellulosic biomass. It is formed by polymerisation of the monolignols para-coumaryl alcohol, coniferyl alcohol and sinapyl alcohol through a radical reaction catalysed by laccases and peroxidases (74). This yields a complex polymer in which the monolignols are linked through a variety of different bond types. In order to synthesise lignin model compounds, the oxidation of eugenol to coniferyl alcohol by EUGO was followed by the radical polymerisation of coniferyl alcohol catalysed by a peroxidase (75). Many of the bond types commonly found in natural lignin could be identified in the resulting lignin model compounds. As EUGO can also oxidise chavicol (4-allylphenol) and 4-allyl-2,6dimethoxyphenol to para-coumaryl alcohol and sinapyl alcohol respectively, the cascade could be employed to synthesise lignin model compounds reflecting the heterogeneity of natural lignin both in terms of the presence of all three monolignols and the various bond types between them.

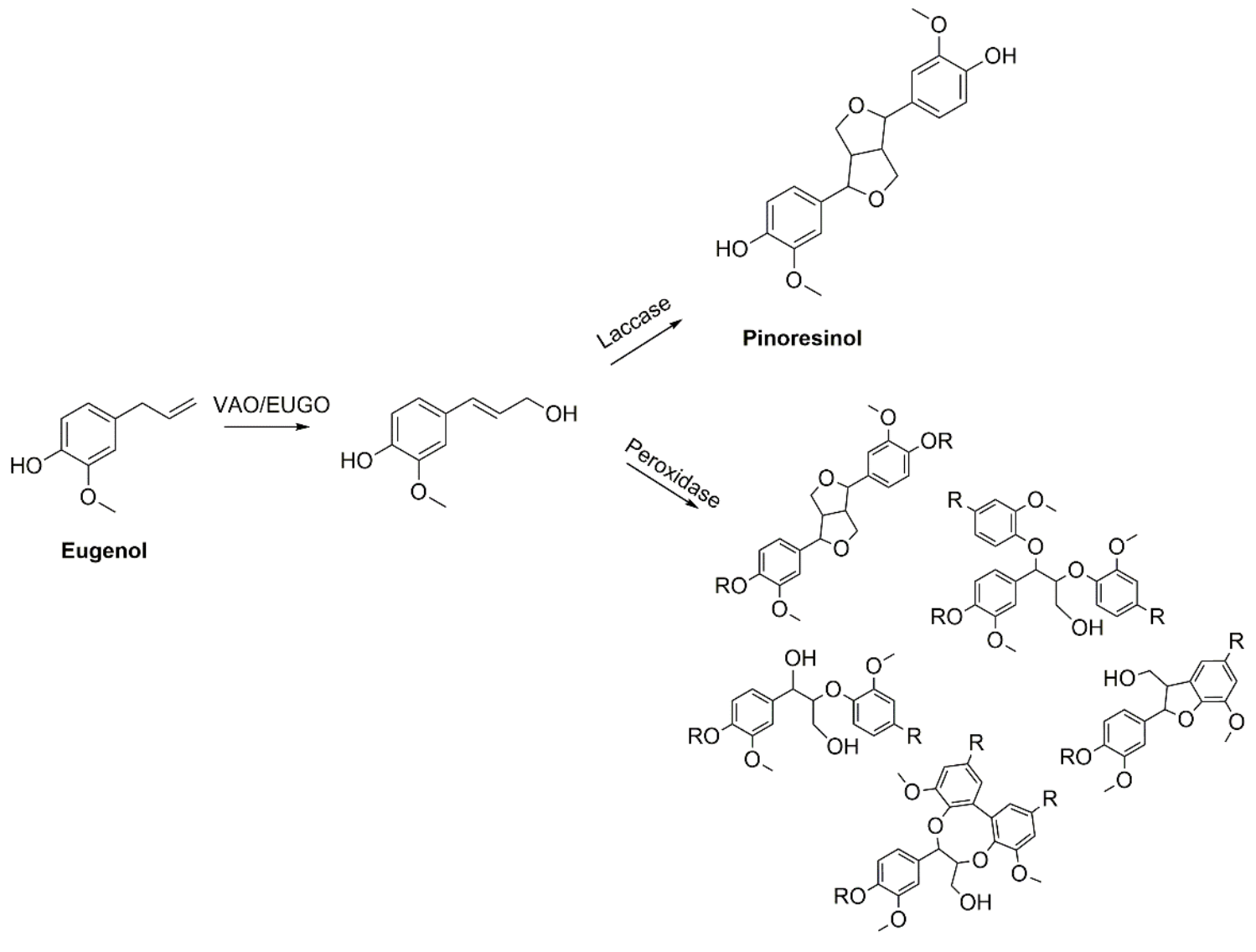

Lignin Model Compounds

Scheme 7: Biocatalytic pathways for the synthesis of pinoresinol or lignin model compounds involving $\mathrm{VAO}$ or EUGO. VAO/EUGO catalyses the conversion of eugenol to coniferyl alcohol. Subsequently, radical polymerisation of coniferyl alcohol is induced by a laccase or peroxidase. One of the main products from the laccase-catalysed reaction is pinoresinol, a coniferyl alcohol dimer that is of interest due to its potential health benefits. The peroxidase-catalysed reaction yields heterogenous lignin model compounds, containing most of the bond types commonly found in natural lignin. 


\section{Outline of this thesis}

The research described in this thesis primarily concerns the 4PO subfamily members VAO and EUGO. Two main themes can be distinguished within the thesis. Chapters two through four consist of fundamental enzymological studies. The goal here is to understand how the enzymes function at a molecular level: mechanistic enzymology. The focus of chapters five through eight lies more towards the biocatalytic applications of these enzymes. Here, the goal is to study how they can be used to produce industrially relevant compounds and how they may be modified in order to achieve this goal. This field of research, which focussess on studying enzymes in the context of their biocatalytic applications can be referred to as biocatalytic enzymology. Although the main focus of the thesis is on $4 \mathrm{PO}$ subfamily members, other members of the $\mathrm{VAO} / \mathrm{PCMH}$ flavoprotein family and other, structurally unrelated, alcohol oxidases are also discussed at certain points.

Chapter 2 provides an overview of the members of the VAO/PCMH flavoprotein family characterised to date. The family is divided into eleven subgroups based on a phylogenetic analysis and the subgroups are discussed in terms of their enzymological properties.

Chapter 3 concerns the differing oligomerisation states of $4 \mathrm{PO}$ subfamily members. The molecular determinants and function of the octamerisation of VAO are studied by protein engineering.

In Chapter 4, the role played by two conserved tyrosine residues in activating the substrates of $4 \mathrm{PO}$ subfamily members for oxidation is studied by site-directed mutagenesis.

Chapter 5 marks the start of the biocatalytically oriented part of the thesis. This chapter provides an overview of the use of alcohol oxidases in organic chemical synthesis.

Chapter 6 describes the development of an assay for efficient screening of VAO and EUGO variants for changes in their substrate specificity and its use to discover novel substrates for the wild-type enzymes.

Chapter 7 concerns the use of VAO to synthesise the chiral secondary alcohol (R)-1-(4'hydroxyphenyl)ethanol at multigram scale.

In Chapter 8, the focus shifts away from the $4 \mathrm{PO}$ subfamily. It describes the oxidation of thiols to thioaldehydes by a number of different flavin-dependent oxidases.

Finally, Chapter 9 provides a general discussion of the most important findings of this thesis.

\section{References}

1. Macheroux, P., Kappes, B., and Ealick, S. E. (2011) Flavogenomics: A genomic and structural view of flavin-dependent proteins. FEBS J. 278, 2625-2634

2. Northrop-Clewes, C. A., and Thurnham, D. I. (2012) The discovery and characterization of riboflavin. Ann. Nutr. Metab. 61, 224-230

3. Joosten, V., and van Berkel, W. J. H. (2007) Flavoenzymes. Curr. Opin. Chem. Biol. 11, 195-202

4. Dijkman, W. P., de Gonzalo, G., Mattevi, A., and Fraaije, M. W. (2013) Flavoprotein oxidases: Classification and applications. Appl. Microbiol. Biotechnol. 97, 5177-5188 
5. Huijbers, M. M. E., Montersino, S., Westphal, A. H., Tischler, D., and van Berkel, W. J. H. (2014) Flavin dependent monooxygenases. Arch. Biochem. Biophys. 544, 2-17

6. Scholtissek, A., Tischler, D., Westphal, A. H., van Berkel, W. J. H., and Paul, C. E. (2017) Old yellow enzyme-catalysed asymmetric hydrogenation: Linking family roots with improved catalysis. Catalysts. 7, 130

7. Romero, E., Gómez Castellanos, J. R., Gadda, G., Fraaije, M. W., and Mattevi, A. (2018) Same substrate, many reactions: Oxygen activation in flavoenzymes. Chem. Rev. 118, 1742-1769

8. Sancho, J. (2006) Flavodoxins: Sequence, folding, binding, function and beyond. Cell. Mol. Life Sci. 63, 855-864

9. Toogood, H. S., Leys, D., and Scrutton, N. S. (2007) Dynamics driving function: New insights from electron transferring flavoproteins and partner complexes. FEBS J. 274, 5481-5504

10. Dodson, C. A., Hore, P. J., and Wallace, M. I. (2013) A radical sense of direction: Signalling and mechanism in cryptochrome magnetoreception. Trends Biochem. Sci. 38, 435-446

11. Fortunato, A. E., Annunziata, R., Jaubert, M., Bouly, J.-P., and Falciatore, A. (2015) Dealing with light: The widespread and multitasking cryptochrome/photolyase family in photosynthetic organisms. J. Plant Physiol. 172, 42-54

12. Kavakli, I. H., Baris, I., Tardu, M., Gül, Ş., Öner, H., Çal, S., Bulut, S., Yarparvar, D., Berkel, Ç., Ustaoğlu, P., and Aydın, C. (2017) The photolyase/cryptochrome family of proteins as DNA repair enzymes and transcriptional repressors. Photochem. Photobiol. 93, 93-103

13. Piano, V., Palfey, B. A., and Mattevi, A. (2017) Flavins as covalent catalysts: New mechanisms emerge. Trends Biochem. Sci. 42, 457-469

14. Huijbers, M. M. E., Martínez-Júlvez, M., Westphal, A. H., Delgado-Arciniega, E., Medina, M., and van Berkel, W. J. H. (2017) Proline dehydrogenase from Thermus thermophilus does not discriminate between FAD and FMN as cofactor. Sci. Rep. 7, 43880

15. Müller, F. (1991) Free flavins: Syntheses, chemical and physical properties. In: Chemistry and Biochemistry of Flavoenzymes, Volume I (Müller, F. ed.), pp. 1-71

16. Teufel, R., Miyanaga, A., Michaudel, Q., Stull, F., Louie, G., Noel, J. P., Baran, P. S., Palfey, B., and Moore, B. S. (2013) Flavin-mediated dual oxidation controls an enzymatic Favorskii-type rearrangement. Nature. 503, 552-556

17. Payne, K. A. P., White, M. D., Fisher, K., Khara, B., Bailey, S. S., Parker, D., Rattray, N. J. W., Trivedi, D. K., Goodacre, R., Beveridge, R., Barran, P., Rigby, S. E. J., Scrutton, N. S., Hay, S., and Leys, D. (2015) New cofactor supports $\alpha, \beta$-unsaturated acid decarboxylation via 1,3-dipolar cycloaddition. Nature. 522, 497-501

18. Leys, D., and Scrutton, N. S. (2016) Sweating the assets of flavin cofactors: New insight of chemical versatility from knowledge of structure and mechanism. Curr. Opin. Struct. Biol. 41, 19-26

19. Fitzpatrick, P. F. (2015) Combining solvent isotope effects with substrate isotope effects in mechanistic studies of alcohol and amine oxidation by enzymes. Biochim. Biophys. Acta - Proteins Proteomics. 1854, 1746-1755

20. Chaiyen, P., Fraaije, M. W., and Mattevi, A. (2012) The enigmatic reaction of flavins with oxygen. Trends Biochem. Sci. 37, 373-380

21. Sucharitakul, J., Prongjit, M., Haltrich, D., and Chaiyen, P. (2008) Detection of a C4ahydroperoxyflavin intermediate in the reaction of a flavoprotein oxidase. Biochemistry. 47, 84858490

22. Gannavaram, S., and Gadda, G. (2013) Relative timing of hydrogen and proton transfers in the reaction of flavin oxidation catalyzed by choline oxidase. Biochemistry. 52, 1221-1226

23. Roth, J. P., and Klinman, J. P. (2003) Catalysis of electron transfer during activation of $\mathrm{O}_{2}$ by the flavoprotein glucose oxidase. Proc. Natl. Acad. Sci. U. S. A. 100, 62-67

24. Zhao, G., Bruckner, R. C., and Jorns, M. S. (2008) Identification of the oxygen activation site in monomeric sarcosine oxidase: Role of Lys265 in catalysis. Biochemistry. 47, 9124-9135

25. Leferink, N. G. H., Fraaije, M. W., Joosten, H.-J., Schaap, P. J., Mattevi, A., and van Berkel, W. J. 
H. (2009) Identification of a gatekeeper residue that prevents dehydrogenases from acting as oxidases. J. Biol. Chem. 284, 4392-4397

26. Baron, R., Riley, C., Chenprakhon, P., Thotsaporn, K., Winter, R. T., Alfieri, A., Forneris, F., van Berkel, W. J. H., Chaiyen, P., Fraaije, M. W., Mattevi, A., and McCammon, J. A. (2009) Multiple pathways guide oxygen diffusion into flavoenzyme active sites. Proc. Natl. Acad. Sci. U. S. A. 106, 10603-10608

27. Hernández-Ortega, A., Lucas, F., Ferreira, P., Medina, M., Guallar, V., and Martínez, A. T. (2011) Modulating $\mathrm{O}_{2}$ reactivity in a fungal flavoenzyme: Involvement of aryl-alcohol oxidase Phe-501 contiguous to catalytic histidine. J. Biol. Chem. 286, 41105-41114

28. Gadda, G. (2012) Oxygen activation in flavoprotein oxidases: The importance of being positive. Biochemistry. 51, 2662-2669

29. Zafred, D., Steiner, B., Teufelberger, A. R., Hromic, A., Karplus, P. A., Schofield, C. J., Wallner, S., and Macheroux, P. (2015) Rationally engineered flavin-dependent oxidase reveals steric control of dioxygen reduction. FEBS J. 282, 3060-3074

30. Wongnate, T., and Chaiyen, P. (2013) The substrate oxidation mechanism of pyranose 2-oxidase and other related enzymes in the glucose-methanol-choline superfamily. FEBS J. 280, 3009-3027

31. Leferink, N. G. H., Heuts, D. P. H. M., Fraaije, M. W., and van Berkel, W. J. H. (2008) The growing VAO flavoprotein family. Arch. Biochem. Biophys. 474, 292-301

32. Fraaije, M. W., van Berkel, W. J. H., Benen, J. A. E., Visser, J., and Mattevi, A. (1998) A novel oxidoreductase family sharing a conserved FAD-binding domain. Trends Biochem. Sci. 23, 206207

33. Keat, M. J., and Hopper, D. J. (1978) $p$-Cresol and 3,5-xylenol methylhydroxylases in Pseudomonas putida N.C.I.B. 9869. Biochem. J. 175, 649-658

34. de Jong, E., van Berkel, W. J. H., van der Zwan, R. P., and de Bont, J. A. M. (1992) Purification and characterization of vanillyl-alcohol oxidase from Penicillium simplicissimum: A novel aromatic alcohol oxidase containing covalently bound FAD. Eur. J. Biochem. 208, 651-657

35. Jin, J., Mazon, H., van den Heuvel, R. H. H., Janssen, D. B., and Fraaije, M. W. (2007) Discovery of a eugenol oxidase from Rhodococcus sp. strain RHA1. FEBS J. 274, 2311-2321

36. Fraaije, M. W., Veeger, C., and van Berkel, W. J. H. (1995) Substrate specificity of flavindependent vanillyl-alcohol oxidase from Penicillium simplicissimum: Evidence for the production of 4-hydroxycinnamyl alcohols from 4-allylphenols. Eur. J. Biochem. 234, 271-277

37. van den Heuvel, R. H. H., Fraaije, M. W., Laane, C., and van Berkel, W. J. H. (1998) Regio- and stereospecific conversion of 4-alkylphenols by the covalent flavoprotein vanillyl-alcohol oxidase. J. Bacteriol. 180, 5646-5651

38. Nguyen, Q.-T., de Gonzalo, G., Binda, C., Rioz-Martínez, A., Mattevi, A., and Fraaije, M. W. (2016) Biocatalytic properties and structural analysis of eugenol oxidase from Rhodococcus jostii RHA1: A versatile oxidative biocatalyst. ChemBioChem. 17, 1359-1366

39. McIntire, W., Hopper, D. J., and Singer, T. P. (1985) $p$-Cresol methylhydroxylase: Assay and general properties. Biochem. J. 228, 325-335

40. Mattevi, A., Fraaije, M. W., Mozzarelli, A., Olivi, L., Coda, A., and van Berkel, W. J. H. (1997) Crystal structures and inhibitor binding in the octameric flavoenzyme vanillyl-alcohol oxidase: The shape of the active-site cavity controls substrate specificity. Structure. 5, 907-920

41. Cunane, L. M., Chen, Z.-W., Shamala, N., Mathews, F. S., Cronin, C. N., and McIntire, W. S. (2000) Structures of the flavocytochrome $p$-cresol methylhydroxylase and its enzyme-substrate complex: Gated substrate entry and proton relays support the proposed catalytic mechanism. J. Mol. Biol. 295, 357-374

42. Fraaije, M. W., Mattevi, A., and van Berkel, W. J. H. (1997) Mercuration of vanillyl-alcohol oxidase from Penicillium simplicissimum generates inactive dimers. FEBS Lett. 402, 33-35

43. Causer, M. J., Hopper, D. J., Mcintire, W. S., and Singer, T. P. (1984) Azurin from Pseudomonas putida: An electron acceptor for $p$-cresol methylhydroxylase. Biochem. Soc. Trans. 12, 1131-1132 
44. Priefert, H., Overhage, J., and Steinbüchel, A. (1999) Identification and molecular characterization of the eugenol hydroxylase genes (ehyA/ehyB) of Pseudomonas sp. strain HR199. Arch. Microbiol. 172, 354-363

45. Brandt, K., Thewes, S., Overhage, J., Priefert, H., and Steinbüchel, A. (2001) Characterization of the eugenol hydroxylase genes (ehyA/ehyB) from the new eugenol-degrading Pseudomonas sp. strain OPS1. Appl. Microbiol. Biotechnol. 56, 724-730

46. Furukawa, H., Wieser, M., Morita, H., Sugio, T., and Nagasawa, T. (1998) Purification and characterization of eugenol dehydrogenase from Pseudomonas fluorescens E118. Arch. Microbiol. $171,37-43$

47. Reeve, C. D., Carver, M. A., and Hopper, D. J. (1989) The purification and characterization of 4ethylphenol methylenehydroxylase, a flavocytochrome from Pseudomonas putida JD1. Biochem. J. 263, 431-437

48. Hopper, D. J., and Cottrell, L. (2003) Alkylphenol biotransformations catalyzed by 4-ethylphenol methylenehydroxylase. Appl. Environ. Microbiol. 69, 3650-3652

49. Furukawa, H., Wieser, M., Morita, H., Sugio, T., and Nagasawa, T. (1999) Purification and characterization of vanillyl-alcohol oxidase from Byssochlamys fulva V107. J. Biosci. Bioeng. 87, 285-290

50. van Rooyen, N. (2012) Identification, cloning and heterologous expression of fungal vanillylalcohol oxidases. Ph.D. thesis. Available online at: http://scholar.ufs.ac.za:8080/xmlui/handle/11660/2055

51. Brady, S. F., Chao, C. J., and Clardy, J. (2002) New natural product families from an environmental DNA (eDNA) gene cluster. J. Am. Chem. Soc. 124, 9968-9969

52. Rachid, S., Revermann, O., Dauth, C., Kazmaier, U., and Müller, R. (2010) Characterization of a novel type of oxidative decarboxylase involved in the biosynthesis of the styryl moiety of chondrochloren from an acylated tyrosine. J. Biol. Chem. 285, 12482-12489

53. Hopper, D. J. (1976) The hydroxylation of $p$-cresol and its conversion to $p$-hydroxybenzaldehyde in Pseudomonas putida. Biochem. Biophys. Res. Commun. 69, 462-468

54. Fraaije, M. W., and van Berkel, W. J. H. (1997) Catalytic mechanism of the oxidative demethylation of 4-(methoxymethyl)phenol by vanillyl-alcohol oxidase: Evidence for formation of a $p$-quinone methide intermediate. J. Biol. Chem. 272, 18111-18116

55. Fraaije, M. W., van den Heuvel, R. H. H., Roelofs, J. C. A. A., and van Berkel, W. J. H. (1998) Kinetic mechanism of vanillyl-alcohol oxidase with short-chain 4-alkylphenols. Eur. J. Biochem. 253, 712-719

56. van Hellemond, E. W., Leferink, N. G. H., Heuts, D. P. H. M., Fraaije, M. W., and van Berkel, W. J. H. (2006) Occurrence and biocatalytic potential of carbohydrate oxidases. Adv. Appl. Microbiol. $60,17-54$

57. Huisman, G. W., and Collier, S. J. (2013) On the development of new biocatalytic processes for practical pharmaceutical synthesis. Curr. Opin. Chem. Biol. 17, 284-292

58. Waltz, E. (2015) Engineers of scent. Nat. Biotechnol. 33, 329-332

59. van den Heuvel, R. H. H., Fraaije, M. W., Laane, C., and van Berkel, W. J. H. (2001) Enzymatic synthesis of vanillin. J. Agric. Food Chem. 49, 2954-2958

60. Overhage, J., Steinbüchel, A., and Priefert, H. (2003) Highly efficient biotransformation of eugenol to ferulic acid and further conversion to vanillin in recombinant strains of Escherichia coli. Appl. Environ. Microbiol. 69, 6569-6576

61. Plaggenborg, R., Overhage, J., Loos, A., Archer, J. A. C., Lessard, P., Sinskey, A. J., Steinbüchel, A., and Priefert, H. (2006) Potential of Rhodococcus strains for biotechnological vanillin production from ferulic acid and eugenol. Appl. Microbiol. Biotechnol. 72, 745-755

62. Overhage, J., Steinbüchel, A., and Priefert, H. (2006) Harnessing eugenol as a substrate for production of aromatic compounds with recombinant strains of Amycolatopsis sp. HR167. J. Biotechnol. 125, 369-376

63. Zucca, J., Lambert, F., Mane, J., Ness, F., and Aigle, M. (2013) System for producing aromatic 
molecules by bioconversion. United States Patent. 8,404,472

64. Lambert, F., Zucca, J., Ness, F., and Aigle, M. (2014) Production of ferulic acid and coniferyl alcohol by conversion of eugenol using a recombinant strain of Saccharomyces cerevisiae. Flavour Fragr. J. 29, 14-21

65. Zhou, R., and Xiaodan, Y. (2015) Methods of making vanillin via the microbial fermentation of ferulic acid from eugenol using a plant dehydrogenase. Patent application. WO/2015/066722

66. Karl, U., and Simon, A. (2009) BASF's ChiPros ${ }^{\circledR}$ chiral building blocks: The cornerstones of your API syntheses! Chim. Oggi. 27, 66-69

67. Patel, R. N. (2013) Biocatalytic synthesis of chiral alcohols and amino acids for development of pharmaceuticals. Biomolecules. 3, 741-777

68. McIntire, W., Hopper, D. J., Craig, J. C., Everhart, E. T., Webster, R. V., Causer, M. J., and Singer, T. P. (1984) Stereochemistry of 1-(4'-hydroxyphenyl)ethanol produced by hydroxylation of 4ethylphenol by $p$-cresol methylhydroxylase. Biochem. J. 224, 617-621

69. Drijfhout, F. P., Fraaije, M. W., Jongejan, H., van Berkel, W. J. H., and Franssen, M. C. R. (1998) Enantioselective hydroxylation of 4-alkylphenols by vanillyl alcohol oxidase. Biotechnol. Bioeng. 59, 171-177

70. van den Heuvel, R. H. H., Fraaije, M. W., Ferrer, M., Mattevi, A., and van Berkel, W. J. H. (2000) Inversion of stereospecificity of vanillyl-alcohol oxidase. Proc. Natl. Acad. Sci. U. S. A. 97, 94559460

71. López-Biedma, A., Sánchez-Quesada, C., Delgado-Rodríguez, M., and Gaforio, J. J. (2016) The biological activities of natural lignans from olives and virgin olive oils: A review. J. Funct. Foods. 26, 36-47

72. Ricklefs, E., Girhard, M., Koschorreck, K., Smit, M. S., and Urlacher, V. B. (2015) Two-step onepot synthesis of pinoresinol from eugenol in an enzymatic cascade. ChemCatChem. 7, 1857-1864

73. Ricklefs, E., Girhard, M., and Urlacher, V. B. (2016) Three-steps in one-pot: Whole-cell biocatalytic synthesis of enantiopure (+)- and (-)-pinoresinol via kinetic resolution. Microb. Cell Fact. 15, 78

74. Rinaldi, R., Jastrzebski, R., Clough, M. T., Ralph, J., Kennema, M., Bruijnincx, P. C. A., and Weckhuysen, B. M. (2016) Paving the way for lignin valorisation: Recent advances in bioengineering, biorefining and catalysis. Angew. Chem. Int. Ed. 55, 8164-8215

75. Habib, M. H. M., Deuss, P. J., Lončar, N., Trajkovic, M., and Fraaije, M. W. (2017) A biocatalytic one-pot approach for the preparation of lignin oligomers using an oxidase/peroxidase cascade enzyme system. Adv. Synth. Catal. 359, 3354-3361

\section{Abbreviations}

4PO: 4-phenol oxidising, EUGO: eugenol oxidase, FAD: flavin adenine dincucleotide, FMN: flavin mononucleotide, $\mathrm{NAD}(\mathrm{P}) \mathrm{H}$ : nicotinamide adenine dinucleotide (phosphate), $\mathrm{PCMH}$ : para-cresol methylhydroxylase, VAO: vanillyl alcohol oxidase 



\section{The VAO/PCMH flavoprotein family}

Tom A. Ewing ${ }^{1}$, Marco W. Fraaije ${ }^{2}$, Andrea Mattevi ${ }^{3}$ and Willem J. H. van Berkel ${ }^{1}$

${ }^{1}$ Laboratory of Biochemistry, Wageningen University \& Research, Wageningen, The Netherlands

${ }^{2}$ Molecular Enzymology Group, University of Groningen, Groningen, The Netherlands

${ }^{3}$ Department of Biology and Biotechnology, University of Pavia, Pavia, Italy

Based on Archives of Biochemistry and Biophysics (2017), 632, 104-117

\section{Summary}

The VAO/PCMH flavoprotein family consists of structurally homologous flavin-dependent enzymes that catalyse a wide range of chemical reactions. Family members share an architecture consisting of a conserved FAD-binding domain and a more variable substratebinding domain, which enables varying interactions with a range of substrates while maintaining the same cofactor binding fold. Here, we provide an overview of the current state of our knowledge on the members of the $\mathrm{VAO} / \mathrm{PCMH}$ family. Based on a phylogenetic analysis, we divide the family into eleven subfamilies. We discuss the properties of these subfamilies, focusing on recent developments in terms of the discovery of new family members and mechanistic advances. We also highlight open questions that will provide challenges for future research. 



\section{Introduction}

Flavoenzymes, which contain one of the vitamin $\mathrm{B}_{2}$-derived cofactors flavin adenine dinucleotide (FAD) or flavin mononucleotide (FMN), are found in all domains of life and fulfill important roles in a variety of biological processes $(1,2)$. They utilise the reactive isoalloxazine ring of their cofactor to catalyse a diverse range of chemical reactions. Most flavoenzymes are oxidoreductases, using the electron transferring properties of the isoalloxazine ring system to catalyse the two electron oxidation or reduction of their substrates $(3,4)$. However, in recent years, a number of new chemical reactivities of the isoalloxazine ring have been unearthed, including some where it is used to catalyse non-redox reactions (5, 6). The VAO/PCMH (vanillyl alcohol oxidase/para-cresol methylhydroxylase) flavoprotein family contains flavoproteins that are characterised by the presence of a shared FAD-binding domain (pfam_FAD_binding_4). In addition to this conserved FAD-binding domain, which is responsible for binding the adenosine diphosphate and ribityl moieties of FAD, members of the $\mathrm{VAO} / \mathrm{PCMH}$ family contain a more variable substrate-binding domain, which is positioned over the isoalloxazine ring of the cofactor (Fig. 1A). This architecture allows for great variation in the structure of the active sites and therefore the catalytic activity of these enzymes, while maintaining the conserved cofactor binding fold. The VAO/PCMH family was originally identified in 1998 and at that time was found to encompass thirteen enzymes of known function (7). Almost a decade ago, the family was reviewed and found to encompass around 50 characterised enzymes (8). Here, we provide an update on the state of our knowledge on the members of this flavoprotein family.

\section{Phylogenetic analysis of the VAO/PCMH family}

In order to identify members of the VAO/PCMH family, we performed a search in the structure and sequence databases. The Swissprot sequence database and Protein Data Bank were searched for annotated sequences and deposited structures respectively that contain a domain belonging to the pfam_FAD_binding_4 family. In addition, we searched the primary literature for VAO/PCMH family members of known sequence and enzymatic function. This led to the identification of 115 sequences of VAO/PCMH family members with known enzymatic activity, as determined either by characterisation of the purified protein or by genetic analysis. Of the 115 sequences, 35 encode proteins of which the three-dimensional structure has been determined. Most of the identified enzymes are classic flavin-dependent oxidoreductases that catalyse two-electron reductions (known as reductases) or oxidations (known as oxidases if molecular oxygen is used as an electron acceptor and as dehydrogenases if another molecule is used as an electron acceptor). In addition, a small number of the identified enzymes display oxygenase or non-redox transferase activity (see below for details).

Almost all of the enzymes for which the presence of a cofactor has been studied contain FAD, as would be expected based on the presence of the conserved FAD-binding domain. The only exceptions are D-arabinono-1,4-lactone oxidase from Trypanosoma brucei and L-galactono-1,4lactone dehydrogenase from Brassica oleracea (cauliflower), which have been reported to contain FMN, and L-gulono-1,4-lactone dehydrogenase from Mycobacterium tuberculosis, in which no flavin could be identified (9-11). However, two other VAO/PCMH-type enzymes 

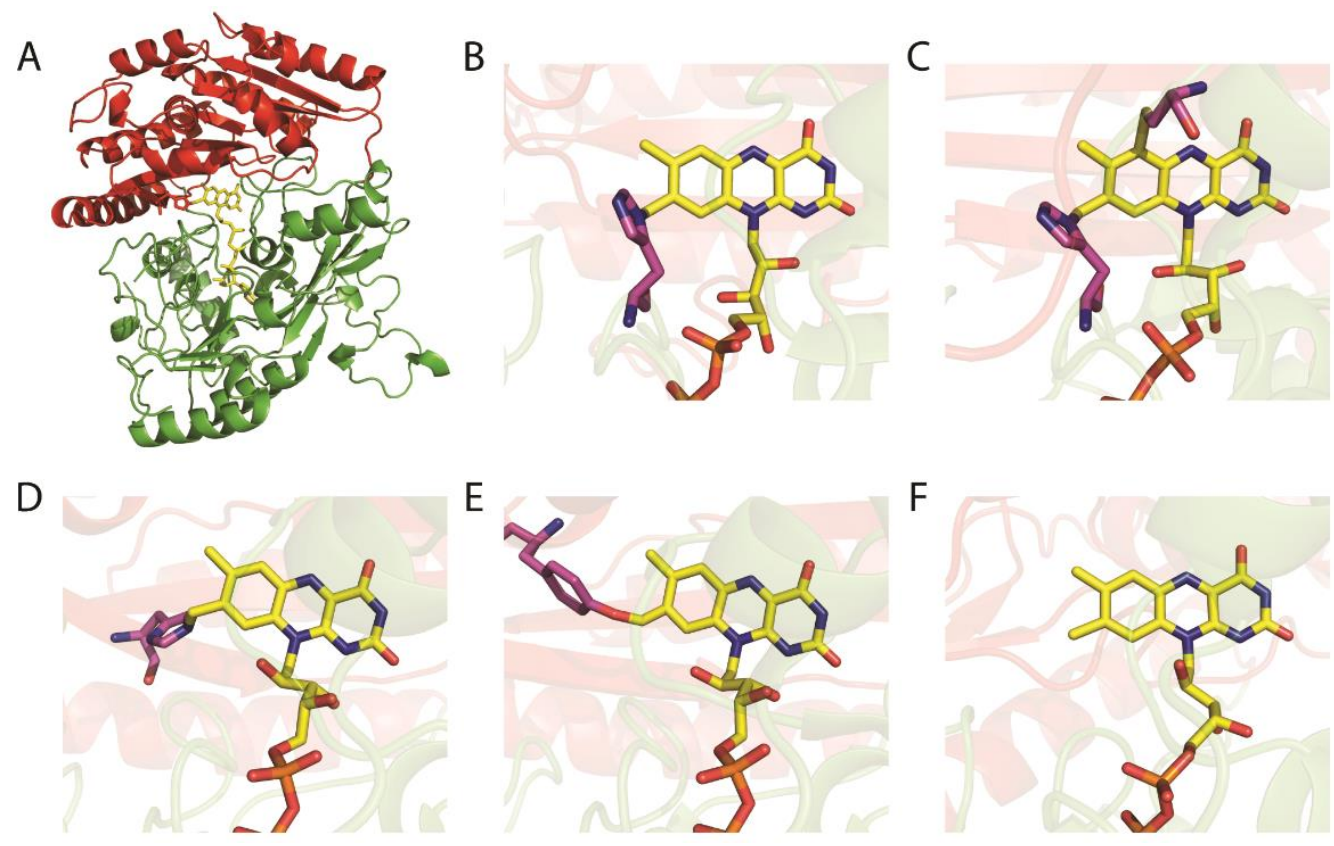

Figure 1: Structural architecture and cofactor binding modes of VAO/PCMH family members. (A) Cartoon representation of the three-dimensional structure of vanillyl alcohol oxidase (VAO) from Penicillium simplicissimum (PDB ID: 1VAO) showing the two-domain topology that is characteristic of members of the VAO/PCMH family. The FAD-binding domain, which is conserved throughout the family, is shown in green. The substrate-binding domain, which is more variable, is shown in red. The FAD cofactor, which is covalently bound to the enzyme via an $8 \alpha-N 3$-histidyl-FAD bond, is shown as yellow sticks. (B-F) Stick representations of flavin binding modes found in members of the VAO/PCMH family. (B) Monocovalent $8 \alpha$-N1-histidyl-FAD of cholesterol oxidase (ChoX) from Brevibacterium sterolicum (PDB ID: 1I19). (C) Bicovalent 6-S-cysteinyl-8 $\alpha$-N1-histidyl-FAD of glucooligosaccharide oxidase (GOOX) from Sarocladium strictum (PDB ID: 1ZR6). (D) Monocovalent $8 \alpha-N 3$-histidyl-FAD of VAO. (E) 8 $\alpha$-O-tyrosyl-FAD of para-cresol methylhydroxylase (PCMH) from Pseudomonas putida (PDB ID: 1WVF). (F) Non-covalent FAD of MurB from Escherichia coli (PDB ID: 2MBR).

that were originally reported to contain FMN, were later found to contain FAD (Dbv29 from Nonomuraea sp. ATCC 39727 and L-galactono-1,4-lactone oxidoreductase from Trypanosoma cruzi) with the original misassignment being attributed to the hydrolysis of FAD during analysis $(12,13)$. Therefore, further research is required to confirm the identity of the flavin cofactor in the above-mentioned enzymes.

A large number of $\mathrm{VAO} / \mathrm{PCMH}$ family members contain a covalently bound flavin cofactor. In total, five different types of flavin binding mode can be distinguished within the family (Fig. 1B-F): non-covalent, monocovalent $8 \alpha-N 1$-histidyl-FAD, monocovalent $8 \alpha-N 3$-histidylFAD, monocovalent $8 \alpha$-O-tyrosyl-FAD and bicovalent 6-S-cysteinyl-8 $\alpha$-N1-histidyl-FAD. Covalent flavinylation increases the redox potential of the cofactor and thus enhances the oxidative power of the enzymes (14). A structure-based alignment of the identified sequences was performed using the PROMALS3D webserver and subsequently used to generate a phylogenetic tree using the phylogeny.fr webserver's BioNJ function. This phylogenetic tree is shown in Figure 2. Using this tree, we divided the VAO/PCMH family into eleven subfamilies of related enzymes based on sequence similarity (and in most cases) function. One sequence, glycolate oxidoreductase subunit E from Escherichia coli, did not belong to any of 
the identified subfamilies. Below, we will discuss these subfamilies in terms of their shared properties and functions and highlight newly discovered members and recent developments in our mechanistic understanding of the enzymes.

\section{Subfamily 1: BBE-like}

The subfamily with most characterised members is the berberine bridge enzyme (BBE)-like subfamily. This subfamily is named after berberine bridge enzyme, or reticuline oxidase (EC 1.21.3.3), which catalyses the oxidative ring closure of (S)-reticuline to $(S)$-scoulerine, a key intermediate in the biosynthesis of protoberberine and benzophenanthridine alkaloids in plants (15). The BBE-like subfamily is the most functionally diverse of the identified subfamilies. Many of the members are involved in the biosynthesis of secondary metabolites in plants (16-18) or microorganisms $(12,19-30)$. The subfamily also contains a number of enzymes that catalyse the oxidation of the $\mathrm{C} 1$ hydroxyl groups of the aldose moieties of (oligo)saccharides (31-36). Examples include glucooligosaccharide oxidase from Sariocladum strictum (previously known as Acremonium strictum), chitooligosaccharide oxidase from Fusarium graminearum and the recently identified xylooligosaccharide oxidase from Myceliophthora thermophila C1. Many members of the BBE-like subfamily contain a bicovalently-linked 6-S-cysteinyl-8 $\alpha$-N1-histidyl-FAD cofactor. The bicovalent linkage increases the redox potential of FAD and may be of importance in maintaining the correct position of the flavin cofactor in the active site, which is typically quite open in bicovalent flavoproteins.

An interesting recent development is the characterisation of EncM, a BBE-like enzyme that utilises a hitherto unknown flavin redox state, the flavin-N5-oxide, to catalyse the oxidative ring closure required to form the tricyclic core of the antibiotic enterocin in Streptomyces maritimus $(27,37)$. This occurs through a mechanism where the oxygen atom from the flavinN5-oxide is first incorporated into the substrate at the C4 position, yielding hydroxylated substrate and oxidised FAD. Subsequent oxidation of the formed hydroxyl group, yielding a ketone and reduced flavin, triggers a Favorskii-type rearrangement reaction that leads to lactone formation, yielding the tricyclic enterocin core (Scheme 1). Subsequently, reduced flavin reacts with molecular oxygen, regenerating the flavin-N5-oxide. This has been proposed to occur via the initial formation of an N5-peroxide intermediate, which forms the flavin-N5-oxide after expelling water. However, a mechanism consisting of the formation and subsequent rearrangement of a C4a-peroxide intermediate, as typically found in flavindependent monooxygenases, is also possible. Elucidating the mechanism by which EncM enables the formation and stabilisation of the flavin-N5-oxide will be an interesting challenge for future research. In addition to EncM, a flavin-N5-oxide was also found to be an intermediate in the catalytic cycle of the non-VAO/PCMH-type flavoenzyme DszA (38). It will be interesting to see whether more flavoenzymes that utilise this redox state will be identified in future. For a more comprehensive overview of the BBE-like subgroup, see (39). 


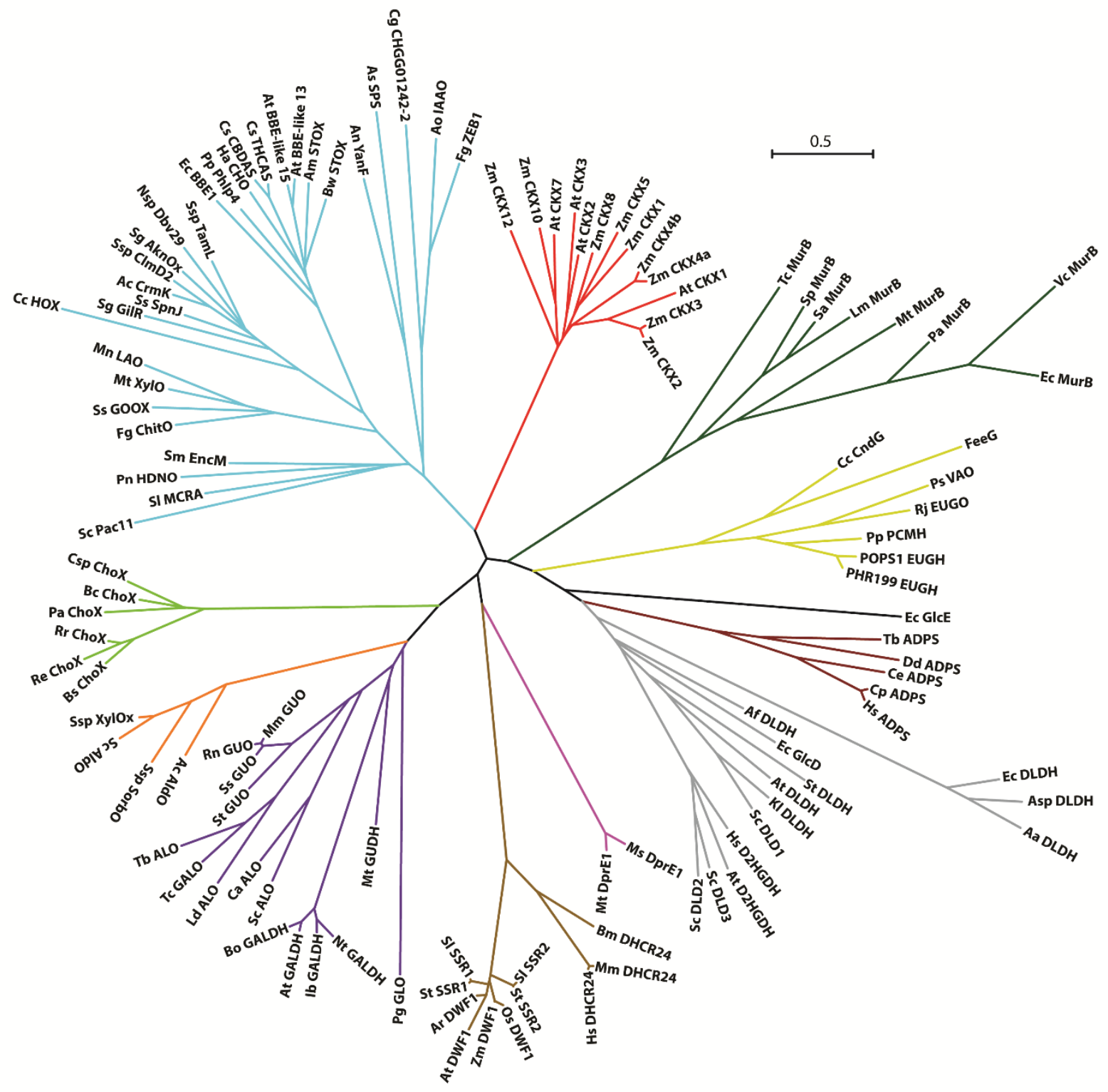

Figure 2: Phylogenetic tree of the VAO/PCMH family showing the division into eleven subfamilies. The subfamilies are indicated by the colours of the branches. They are: berberine bridge enzyme-like (blue), cytokinin dehydrogenases (light red), MurBs (dark green), 4-phenol oxidising (yellow), alkyldihydroxyacetone phosphate synthases (dark red), $\alpha$-hydroxy acid dehydrogenases (grey) DprE1s (pink) sterol reductases (brown), aldonolactone oxidoreductases (purple), alditol oxidases (orange) and cholesterol oxidases (light green). Glycolate oxidoreductase subunit E from Escherichia coli (black) was not deemed to belong to any of the identified subfamilies. The sequences in the tree are: Sc Pac11: Pac11 from Streptomyces coeruleorubidus, Sl MCRA: mitomycin radical oxidase from Streptomyces lavendulae, Pn HDNO: 6-hydroxy-D-nicotine oxidase from Paenarthrobacter nicotinovorans, Sm EncM: EncM from Streptomyces maritimus, Fg ChitO: chitooligosaccharide oxidase from Fusarium graminearum, Ss GOOX: glucooligosaccharide oxidase from Sarocladium strictum, Mt XylO: xylooligosaccharide oxidase from Myceliophthora thermophila C1, Mn LAO: lactose oxidase from Microdochium nivale, Cc HOX: hexose oxidase from Chondrus crispus, Sg GilR: GilR from Streptomyces griseoflavus, Ss SpnJ: SpnJ from Saccharopolyspora spinosa, Ac CrmK: CrmK from Actinoalloteichus cyanogriseus WH1-2216-6, Ssp ClmD2: ClmD2 from Streptomyces sp. CS40, Sg AknOx, aclacinomycin oxidoreductase from Streptomyces galilaeus, Nsp Dbv29: Dbv29 from Nonomuraea sp. ATCC 39727, Ssp TamL: TamL from Streptomyces sp. 
307-9, Ec BBE1: berberine bridge enzyme from Eschscholzia californica (california poppy), Pp Phlp4: Phl p 4 from Phleum pratense (timothy-grass), Ha CHO: carbohydrate oxidase from Helianthus annuus (sunflower), Cs CBDAS: cannabidiolic acid synthase from Cannabis sativa, Cs THCAS: tetrahydrocannabinolic acid synthase from Cannabis sativa, At BBE-like 15: BBE-like protein 15 from Arabidopsis thaliana, At BBE-like 13: BBE-like protein 13 from Arabidopsis thaliana, Am STOX: (S)tetrahydroprotoberberine oxidase from Argemone mexicana, Bw STOX: (S)-tetrahydroprotoberberine oxidase from Berberis wilsoniae, An YanF: YanF from Aspergillus niger, As SPS: solanapyrone synthase from Alternaria solani, Cg CHGG01242-2: CHGG_01242-2 from Chaetomium globosum, Ao IAAO: isoamyl alcohol oxidase from Aspergillus oryzae, Fg ZEB1: ZEB1 from Fusarium graminearum, Zm CKX: cytokinin dehydrogenase from Zea mays (maize, numbered by isoform), At CKX: cytokinin dehydrogenase from Arabidopsis thaliana (numbered by isoform), Tc MurB: MurB from Thermus caldophilus, Sp MurB: MurB from Streptococcus pneumoniae, Sa MurB: MurB from Staphylococcus aureus, Lm MurB: MurB from Listeria monocytogenes, Mt MurB: MurB from Mycobacterium tuberculosis, Pa MurB: MurB from Pseudomonas aeruginosa, Vc MurB: MurB from Vibrio cholerae, Ec MurB: MurB from Escherichia coli, Cc CndG: CndG from Chondromyces crocatus $\mathrm{Cm}$ c5, FeeG: FeeG from an unidentified soil microbe, Ps VAO: vanillyl alcohol oxidase from Penicillium simplicissimum, Rj EUGO: eugenol oxidase from Rhodococcus jostii RHA1, Pp PCMH: para-cresol methylhydroxylase from Pseudomonas putida, POPS1 EUGH: eugenol hydroxylase from Pseudomonas sp. OPS1, PHR199 EUGH: eugenol hydroxylase from Pseudomonas sp. HR199, Ec GlcE: glycolate oxidoreductase subunit E from Escherichia coli, Tb ADPS: alkyldihydroxyacetone phosphate synthase (ADPS) from Trypanosoma brucei, Dd ADPS: ADPS from Dictyostelium discoideum, Ce ADPS: ADPS from Caenorhabditis elegans, Cp ADPS: ADPS from Cavia porcellus (guinea pig), Hs ADPS, human ADPS, Ec DLDH: D-lactate dehydrogenase (DLDH) from Escherichia coli, Asp DLDH: DLDH from Acidocella sp. MX-AZ02, Aa DLDH: DLDH from Acetobacter aceti, Af DLDH: DLDH from Archaeoglobus fulgidus, Ec GlcD: glycolate oxidoreductase subunit D from Escherichia coli, St DLDH: DLDH from Sulfolobus tokodaii 7, At DLDH: DLDH from Arabidopsis thaliana, K1 DLDH: DLDH from Kluyveromyces lactis, Sc DLD1: DLD1 from Saccharomyces cerevisiae, Hs D2HGDH: human D-2-hydroxyglutarate dehydrogenase, At D2HGDH: D-2-hydroxyglutarate dehydrogenase from Arabidopsis thaliana, Sc DLD3: DLD3 from Saccharomyces cerevisiae, Sc DLD2: DLD2 from Saccharomyces cerevisiae, Ms DprE1: DprE1 from Mycobacterium smegmatis, Mt DprE1: DprE1 from Mycobacterium tuberculosis, Bm DHCR24: 24-dehydrocholesterol reductase (DHCR24) from Bombyx mori (silkworm), Mm DHCR24: DHCR24 from Mus musculus (mouse), Hs DHCR24: human DHCR24, S1 SSR2: sterol side chain reductase (SSR) 2 from Solanum lycopersicum (tomato), St SSR2: SSR2 from Solanum tuberosum (potato), Os DWF1: DWARF1 from Oryza sativa (rice), Zm DWF1: DWARF1 from Zea mays (maize), At DWF1: DWARF1 from Arabidopsis thaliana, Ar DWF1: DWARF1 from Ajuga reptans, St SSR1: SSR1 from Solanum tuberosum (potato), SI SSR1: SSR1 from Solanum lycopersicum (tomato), Ac AldO: alditol oxidase from Acidothermus cellulolyticus 11B, Ssp SorbO: sorbitol oxidase from Streptomyces sp. H-7775, Sc AldO: alditol oxidase from Streptomyces coelicolor A3(2), Ssp XylOx: xylitol oxidase from Streptomyces sp. IKD472, Pg GLO: D-glucono-1,4-lactone oxidase from Penicillium griseoroseum, Mt GUDH: L-gulono-1,4-lactone dehydrogenase from Mycobacterium tuberculosis, Nt GALDH: L-galactono-1,4-lactone dehydrogenase (GALDH) from Nicotiana tabacum (tobacco), Ib GALDH: GALDH from Ipomoea batatas (sweet potato), At GALDH: GALDH from Arabidopsis thaliana, Bo GALDH: GALDH from Brassica oleracea (cauliflower), Sc ALO: D-arabinono-1,4-lactone oxidase (ALO) from Saccharomyces cerevisiae, Ca ALO: ALO from Candida albicans, Ld ALO: ALO from Leishmania donovani, Tc GALO: L-galactono-1,4-lactone oxidoreductase from Trypanosoma cruzi, Tb ALO: ALO from Trypanosoma brucei, St GUO: L-gulono-1,4-lactone oxidase (GUO) from Scyliorhinus torazame (cloudy catshark), Ss GUO: GUO from Sus scrofa (pig), Rn GUO: GUO from Rattus norvegicus (rat), Mm GUO: GUO from Mus musculus (mouse), Bs ChoX: cholesterol oxidase (ChoX) from Brevibacterium sterolicum, Re ChoX: ChoX from Rhodococcus erythropolis CECT3014, Rr ChoX: ChoX from Rhodococcus 
ruber Chol-4, Pa ChoX: ChoX from Pseudomonas aeruginosa PA157, Bc ChoX: ChoX from Burkholderia cepacia ST-200, Csp ChoX: ChoX from Chromobacterium sp. DS-1. The scale bar indicates the branch length corresponding to 0.5 changes per position.

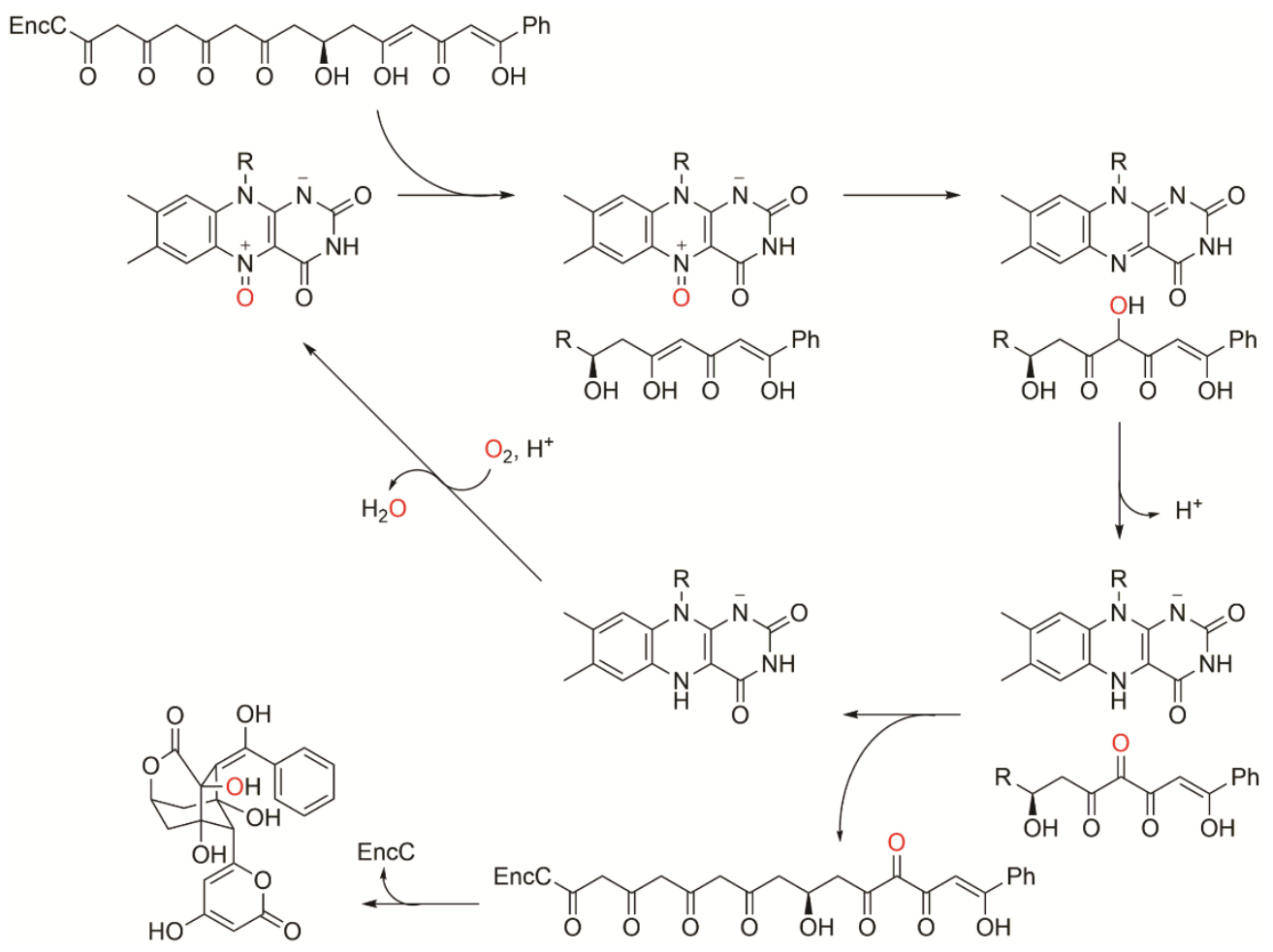

Scheme 1: Synthesis of the tricyclic core of the antibiotic enterocin catalysed by EncM. EncM utilises a novel flavin oxidation state, the flavin-N5-oxide, to catalyse a double oxidation of its substrate. The proposed physiological substrate, a dihydrooctaketide intermediate bound to the carrier protein EncC, is bound in the enzyme's active site with EncM's FAD cofactor in the N5-oxide form. Subsequently, the flavin-N5-oxide oxygenates the substrate at the C4 position, yielding an alcohol and oxidised FAD. Following this, oxidation of the formed alcohol to a ketone takes place, yielding fully reduced FAD. The product is then released from the enzyme and undergoes a Favorskii-type rearrangement to yield the tricyclic enterocin core. Meanwhile, the reduced flavin reacts with molecular oxygen, regenerating the flavin-N5-oxide.

\section{Subfamily 2: Cytokinin dehydrogenases}

Cytokinin dehydrogenases (CKX, EC 1.5.99.12) are found in a wide variety of plants, where they are responsible for the breakdown of cytokinins, plant hormones that are involved in cell division and differentiation. Cytokinins are adenine derivatives with a substituent bound to the N6 atom, which can be an isoprenoid or aromatic functionality. CKX oxidises the side chain amine of cytokinins to an imine, which is subsequently hydrolysed, yielding adenine and an aldehyde (40) (Scheme 2). Following this, the FAD cofactor of CKX, which is covalently linked to the enzyme through an $8 \alpha-N 1$-histidyl-FAD bond, is reoxidised. Various electron acceptors, such as quinones or 2,6-dichlorophenolindophenol (DCPIP) can be used by the enzyme in vitro (41). For the Zea mays (maize) CKX1 ( $Z m C K X 1)$ isoform, evidence exists that the physiological electron acceptor is a free radical formed by the oxidation of 2,4-dihydroxy7-methoxy-1,4-benzoxazin-3-one, a compound found in maize phloem sap, by laccases or peroxidases (42). Higher plants typically contain multiple genes encoding CKXs, with the best 
studied CKX families being those from Arabidopsis thaliana (seven isoforms) and Z. mays (thirteen isoforms). The various members of these families differ in terms of their tissuespecific expression patterns, (predicted) subcellular localisation and substrate specificity, suggesting they have distinct functions in vivo (43-47). The crystal structure of ZmCKX1 revealed that the substrate binding site consists of an internal cavity, where the isoprenoid or aromatic side chain of the substrate binds, linked to the surface through a narrow pore (48). The $\mathrm{C}-\mathrm{N}$ bond that is cleaved occupies the pore, with the adenine ring structure bound to a funnel-shaped pocket on the surface of the protein. Based on the crystal structure, a mechanism for substrate oxidation was proposed where a conserved aspartate residue (Asp169 in ZmCKX1) plays a crucial role in catalysis. By interacting with the N10 atom of the cytokinin, Asp-169 induces polarisation of the substrate, facilitating the transfer of a hydride from the $\mathrm{C} 11$ atom to FAD. The importance of Asp-169 for catalysis is supported by a recent site-directed mutagenesis study, which showed that the ZmCKX1 D169N variant displays significantly lowered activity towards all tested substrates, while $K_{\mathrm{m}}$ values were not affected (49).<smiles>[R]C=Nc1ncnc2[nH]cnc12</smiles>

Scheme 2: Oxidation of cytokinins as catalysed by cytokinin dehydrogenase (CKX). The cytokinin is first oxidised at its side chain amine group, yielding an imine. The imine is subsequently hydrolysed, yielding adenine and an aldehyde.

\section{Subfamily 3: MurBs}

UDP- $N$-acetylglucosamine enolpyruvate reductase, also known as MurB (EC 1.3.1.98), is involved in bacterial cell wall synthesis, where it catalyses the second step in the biosynthesis of peptidoglycan, the reduction of UDP- $N$-acetylglucosamine enolpyruvate (UDP-GlcNAcEP) to UDP-N-acetylmuramic acid (UDP-MurNAc) (Scheme 3). The enzyme achieves this by utilising a non-covalently bound FAD cofactor to transfer a hydride equivalent from nicotinamide adenine dinucleotide phosphate (NADPH) to UDP-GlcNAc-EP (50).

Based on their three-dimensional structures, two different types of MurB can be distinguished. The two types share a similar overall fold, but differ in terms of their mechanism of substrate binding. Type I MurB, which is found in Gram-negative bacteria such as Escherichia coli and Pseudomonas aeruginosa, is characterised by the presence of a loop region that is involved in substrate binding, closing the active site after binding $(51,52)$. The loop contains a tyrosine residue (Tyr-190 in E. coli MurB) that forms an interaction with one of the phosphate groups of UDP-GlcNAc-EP or NADPH, which bind at a similar position. Type II MurBs are found in bacteria such as Staphylococcus aureus and Thermus caldophilus and lack this loop region as well as part of the substrate-binding domain that forms interactions with the uridine moiety of UDP-GlcNAc-EP in Type I MurBs $(53,54)$. Type II MurBs can be further divided into two 
groups based on the identity of an active site acid that protonates the carbanion intermediate formed after hydride transfer to UDP-GlcNAc-EP. In S. aureus MurB, this role is performed by a serine residue (Type II-a), while in T. caldophilus MurB, it is performed by a cysteine residue (Type II-b). All type I MurBs contain a serine residue that acts as the proton donor (55).

Recently, it was found that Verrucomicrobium spinosum contains a novel form of MurB, where it is fused to MurC, the enzyme that catalyses the following step in peptidoglycan synthesis, the addition of L-alanine to the carboxylic acid group of UDP-MurNAc (56). Expression of the fusion protein was found to complement the phenotype of E. coli murB and murC mutants. However, although MurC activity could be detected using the purified enzyme in vitro, MurB activity could not. Further characterisation of this novel MurB will be an interesting future research line. MurB's role in bacterial cell wall synthesis makes it an interesting target for the development of novel antibiotics and various inhibitors have been identified and shown to possess antibacterial activity [see (57) for a review].

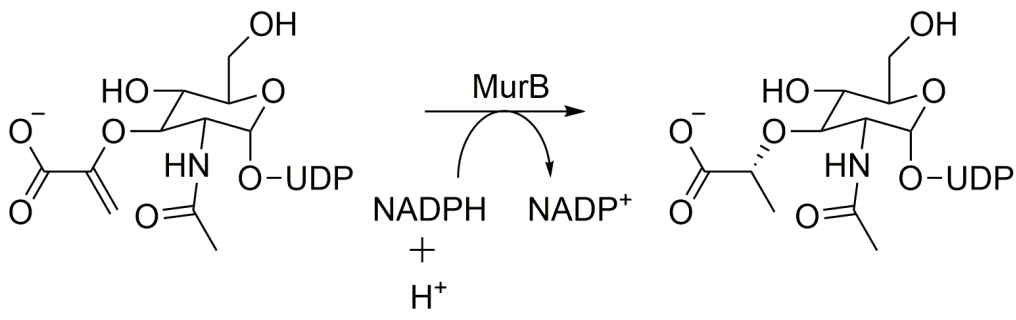

Scheme 3: Reduction of UDP- $N$-acetylglucosamine enolpyruvate to UDP- $N$-acetylmuramic acid catalysed by MurB.

\section{Subfamily 4: 4-Phenol oxidising}

The 4-phenol oxidising (4PO) subfamily contains enzymes that catalyse the oxidation of parasubstituted phenols at the $\mathrm{C} \alpha$ atom of the para-substituent. The subfamily contains both oxidases, such as vanillyl alcohol oxidase (VAO, EC 1.1.3.38) from Penicillium simplicissimum, and eugenol oxidase (EUGO) from Rhodococcus jostii RHA1, and dehydrogenases, such as para-cresol methylhydroxylase (PCMH, EC 1.17.99.1) from Pseudomonas putida and eugenol hydroxylase (EUGH) from Pseudomonas sp., that utilise a cytochrome $c$ subunit as an electron acceptor (58-62). Depending on the enzyme and type of substrate, these 4PO subfamily members can catalyse the oxidation of benzylic alcohols to aldehydes, the oxidative deamination of benzylic amines, the oxidative demethylation of 4-(methoxymethyl)phenol, the hydroxylation of 4-allylphenols, and the hydroxylation or dehydrogenation of 4alkylphenols (Scheme 4A-C) (63-65). Catalysis proceeds through the abstraction of a hydride from the $\mathrm{C} \alpha$ atom of the side chain of an enzyme-bound phenolate form of the substrate, yielding a para-quinone methide intermediate that subsequently rearranges or reacts with water to give the reaction products $(66,67)$. In vivo, these enzymes are presumably involved in the metabolism of para-substituted phenolic compounds, possibly derived from the degradation of lignin (68).

These $4 \mathrm{PO}$ subfamily members are unique among the VAO/PCMH family members in that their FAD cofactor is covalently bound to a loop that is close to the C-terminus of the protein, 40 
rather than the more commonly found covalent linkages to a loop close to the $\mathrm{N}$-terminus of the protein. This occurs through an $8 \alpha-N 3$-histidyl-FAD bond in the case of VAO and EUGO and an $8 \alpha-O$-tyrosyl-FAD bond in the case of PCMH and (as predicted from its sequence) EUGH (69-71). Recent progress in studying this group of enzymes includes the publication of the crystal structure of EUGO and a mutagenesis study that focused on understanding the molecular determinants and function of the differing oligomerisation states of the oxidases VAO and EUGO $(71,72)$. Although the secondary and tertiary structures of these proteins are rather similar, they differ in terms of their quaternary structure. EUGO is exclusively dimeric in solution, whereas VAO forms both dimers and octamers, which can be described as tetramers of dimers. A loop that is positioned at the dimer-dimer interface of $\mathrm{VAO}$, but is not present in EUGO, was shown to be responsible for this difference, with its deletion leading to the formation of a VAO variant that exclusively forms dimers without significantly affecting catalytic function. However, a recent report described the expression of a number of VAO homologues that displayed an octameric quaternary structure, despite not containing such a loop region (73). Further research is required to understand the mechanism by which these novel VAO-like enzymes obtain their octameric oligomerisation state.

In addition to these well characterised enzymes, the $4 \mathrm{PO}$ subfamily contains two enzymes that catalyse the oxidative decarboxylation of $\mathrm{N}$-substituted tyrosine derivatives: CndG from Chondromyces crocatus and FeeG from an unidentified soil microbe (Scheme 4D) $(74,75)$. These enzymes both contain three residues that are thought to be crucial for enabling the formation of the para-quinone methide intermediate in other $4 \mathrm{PO}$ subfamily members (Tyr-108, Tyr-503 and Arg-504 in VAO). This suggests that the decarboxylation reaction proceeds through the initial formation of a para-quinone methide intermediate, which subsequently expels carbon dioxide. Unlike the other $4 \mathrm{PO}$ subfamily members, CndG contains a non-covalently bound FAD cofactor, in agreement with the fact that neither the histidine residue involved in covalent flavin binding in VAO nor the tyrosine residue involved in covalent flavin binding in PCMH are conserved in this enzyme.

A<smiles>COc1cc(CO)ccc1O</smiles>

B<smiles>Cc1ccc(O)cc1</smiles>

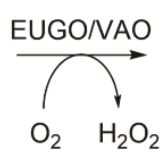

$\mathrm{HO}$<smiles>CCOc1cc(C=O)ccc1O</smiles><smiles>OCc1ccc(O)cc1</smiles>

C<smiles>C=CCc1ccc(O)c(OC)c1</smiles>

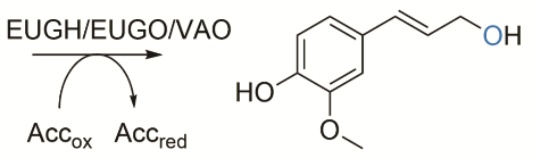
$\mathrm{H}_{2} \mathrm{O}$<smiles>[R]C(=O)N[C@@H](Cc1ccc(O)c(Cl)c1)C(=O)O</smiles><smiles>[R]C(=O)N/C=C/c1ccc(O)c(Cl)c1</smiles>

Scheme 4: Examples of reactions catalysed by members of the 4-phenol oxidising subfamily. (A) The oxidation of vanillyl alcohol to vanillin by eugenol oxidase (EUGO) or vanillyl alcohol oxidase (VAO).

(B) The oxidation of para-cresol to 4-hydroxybenzyl alcohol by para-cresol methylhydroxylase (PCMH).

(C) The oxidation of eugenol to coniferyl alcohol by eugenol hydroxylase (EUGH), EUGO or VAO. (D) The oxidative decarboxylation of $N$-substituted tyrosine derivatives by CndG. 


\section{Subfamily 5: Alkyl-dihydroxyacetone phosphate synthases}

Alkyl-dihydroxyacetone phosphate synthase (ADPS, EC 2.5.1.26) is involved in the biosynthesis of ether phospholipids, lipids which contain an ether-bond linked alkyl chain instead of one of the ester-bond linked acyl chains found in most phospholipids. Ether lipids form a major constituent of mammalian membranes and defects in their biosynthesis, in some cases caused by mutations in the gene encoding ADPS, are linked to the genetic disorder rhizomelic chondrodysplasia punctata in humans (76). Ether phospholipid biosynthesis starts in the peroxisomes with the synthesis of acyl-dihydroxyacetone phosphate (acyl-DHAP) from DHAP and acyl-CoA, catalysed by an acyltransferase. Subsequently, ADPS catalyses the conversion of the acyl-DHAP to an alkyl-DHAP by the incorporation of a long-chain fatty alcohol, thus yielding the ether bond that is characteristic of ether phospholipids. ADPS is unique among $\mathrm{VAO} / \mathrm{PCMH}$ family members in that it is not an oxidoreductase, but a transferase that uses a non-covalently bound FAD cofactor to catalyse a reaction that does not involve any change in the redox states of the substrates (77). The reaction occurs through the formation of a covalent intermediate between acyl-DHAP and FAD and is proposed to proceed as follows (Scheme 5) $(78,79)$. First, acyl-DHAP is bound to the enzyme's active site in its enolate form. Tautomerisation to the enolate may be stimulated by Tyr-578 (numbering according to Cavia porcellus [guinea pig] ADPS), which could act as a proton acceptor, and His-616 and His-617, which could stabilise a negative charge on the DHAP O2 atom. These residues are conserved among all ADPS sequences included in our alignment and have been shown to be essential for activity by site-directed mutagenesis (77-79). This tautomerisation enables the subsequent nucleophilic attack of the C1 atom of DHAP on the N5 atom of FAD, yielding a covalent N5-acyl-DHAP adduct. Next, a fatty acid is expelled from the intermediate, yielding an N5-DHAP iminium intermediate. This intermediate breaks down through the attack of a fatty alcohol, yielding the alkyl-DHAP product and the oxidised FAD cofactor. In addition to the two domains typically found in VAO/PCMH family members, ADPS contains a third domain at its N-terminus $(78,79)$. This domain contains a gating helix that closes off the substrate-binding cavity. Recently, ADPS has gained interest as a potential anti-cancer drug target, as its inhibition leads to significant changes in the lipid composition of tumour cells and impairs their pathogenicity $(80,81)$.

\section{Subfamily 6: $\alpha$-Hydroxy acid dehydrogenases}

$\alpha$-Hydroxy acid dehydrogenases catalyse the oxidation of the hydroxyl group of $\alpha$-hydroxy acids to a carbonyl group, using electron acceptors other than molecular oxygen (Scheme 6). Depending on their substrate specificity and physiological function they are known either as D-lactate dehydrogenase (DLDH, EC 1.1.2.4), D-2-hydroxyglutarate dehydrogenase (D2HGDH, EC 1.1.99.39) or glycolate oxidoreductase. DLDH is found in bacteria, archaea, yeasts and plants, where it catalyses the oxidation of D-lactate to pyruvate, the first step in its metabolism (82-86). The crystal structure of Escherichia coli DLDH revealed that it contains a non-covalently bound FAD cofactor and that, in addition to the FAD-binding and substratebinding domains typically found in VAO/PCMH family members, it contains a third domain that is responsible for association of the protein with the cytoplasmic membrane (87). Similar to DLDH, D2HGDH catalyses the oxidation of the hydroxyl groups of $\alpha$-hydroxy acids, but it shows highest activity for the oxidation of D-2-hydroxyglutarate to $\alpha$-ketoglutarate (88). 


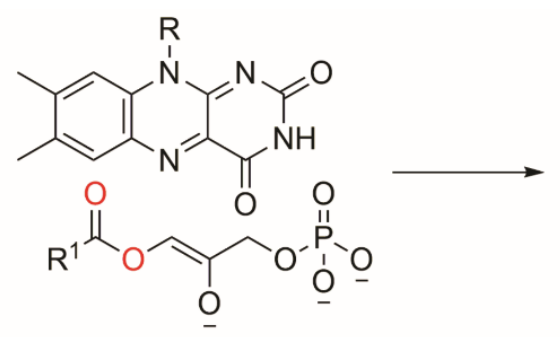<smiles></smiles>

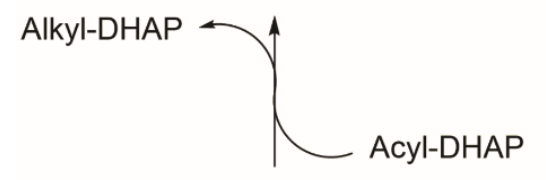<smiles>CC1CC1[R]C(=O)O</smiles><smiles>[R2]OC=C(O)COP(=O)([O-])OCc1cc2nc3c(=O)[nH]c(=O)nc-3n([R])c2cc1C</smiles><smiles></smiles>

Scheme 5: Mechanism of the synthesis of alkyl-dihydroxyacetone phosphate (alkyl-DHAP) from acylDHAP by alkyl-dihydroxyacetone phosphate synthase (ADPS). Upon binding to the enzyme, acylDHAP is tautomerised to its enolate form. This enables the formation of a covalent adduct between the enzyme's FAD cofactor and acyl-DHAP. Subsequently, a fatty acid is released from this intermediate, yielding a covalent iminium intermediate. This subsequently reacts with a fatty alcohol, yielding the alkyl-DHAP product.

Mutations in the human D2HGDH gene cause the genetic disorder D-2-hydroxyglutaric aciduria, which is characterised by heightened levels of D-2-hydroxyglutarate in body fluids, with patients displaying severe developmental defects (89).

A recent study revealed that Saccharomyces cerevisiae contains three $\alpha$-hydroxy acid dehydrogenases (named DLD1, DLD2 and DLD3). DLD1 functions as a conventional DLDH. However, DLD2 and DLD3 do not only catalyse the oxidation of $\alpha$-hydroxy acids using electron acceptors such as DCPIP, but can also transfer the electrons to $\alpha$-keto acids, yielding new $\alpha$-hydroxy acids (90). Based on the fact that a dld3 $\Delta$ mutant displayed increased D-2hydroxyglutarate levels and decreased D-lactate levels, it was suggested that the enzyme may function as a transhydrogenase in vivo, converting D-2-hydroxyglutarate and pyruvate to $\alpha$ ketoglutarate and D-lactate respectively.

Glycolate oxidoreductase from E. coli catalyses the first step of glycolate metabolism, its oxidation to glyoxylate (91). Two genes encoding the putative VAO/PCMH-type oxidoreductases glcD and glcE have been shown to be required for glycolate oxidoreductase activity (92). However, the function of the two gene products has not been studied in detail. It should be noted that the sequence of glcE is significantly shorter than that of the other $\alpha$ hydroxy acid dehydrogenases and it is not closely related to the other sequences according to the phylogenetic tree. As such, we do not deem it to belong to the $\alpha$-hydroxy acid dehydrogenase subfamily. 


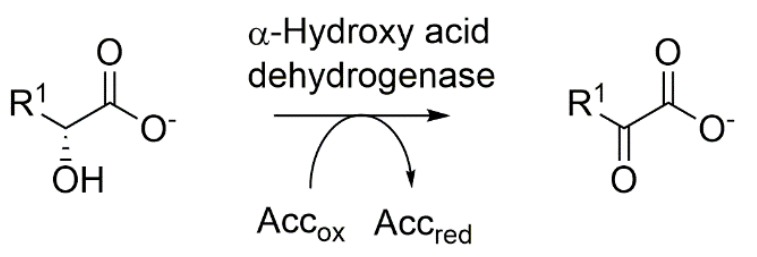

$$
\begin{aligned}
\mathrm{R}^{1}= & \mathrm{H} \text { (gylcolate oxidoreductase) } \\
& \mathrm{CH}_{3}(\mathrm{DLDH}) \\
& \mathrm{CH}_{2} \mathrm{CH}_{2} \mathrm{COOH}(\mathrm{D} 2 \mathrm{HGDH})
\end{aligned}
$$

Scheme 6: Oxidation of $\alpha$-hydroxy acids catalysed by the $\alpha$-hydroxy acid dehydrogenases glycolate oxidoreductase, D-lactate dehydrogenase (DLDH) and D-2-hydroxyglutarate dehydrogenase (D2HGDH).

\section{Subfamily 7: Sterol reductases}

Members of the sterol reductase subfamily are responsible for the reduction of a carboncarbon double bond during sterol biosynthesis. Their exact physiological function differs depending on the source organism. Sterol reductases from mammals are known as 24dehydrocholesterol reductase (DHCR24, also known as seladin-1, EC 1.3.1.72) and catalyse the final step of cholesterol biosynthesis, the reduction of 24-dehydrocholesterol (desmosterol) to cholesterol (Scheme 7A) (93). The reaction is NADPH dependent and stimulated by the presence of FAD, suggesting DHCR24 contains a non-covalently bound FAD cofactor and uses NADPH as an electron donor. Mutations in the human DHCR24 gene cause the congenital disorder desmosterolosis, with patients displaying heightened plasma desmosterol levels and various congenital and developmental abnormalities (93). In addition to mammals, DHCR24 has been identified in Bombyx mori (silkworm) (94). This invertebrate cannot synthesise cholesterol de novo, but rather converts plant sterols to cholesterol via a process where they are first dealkylated to desmosterol, which is subsequently reduced by DHCR24.

Sterol reductases have also been identified in plants such as Arabidopsis thaliana and Oryza sativa (rice), where they are involved in the biosynthesis of C24-alkylated sterols such as campesterol or sitosterol $(95,96)$. These sterols are synthesised from their $\Delta 24-28$ unsaturated derivatives, which are reduced by the sterol reductase DWARF1 (Scheme 7B). Typically, this reaction is believed to take place in two steps, with the $\Delta 24-28$ unsaturated sterols first being isomerised to the $\Delta 24-25$ unsaturated derivatives, which are subsequently reduced to the saturated products. Thus, like DHCR24, DWARF1 catalyses the reduction of $\Delta 24-25$ double bonds, with the main difference in the reactions lying in the prior isomerisation step. Interestingly, Ajuga reptans DWARF1 was recently reported to catalyse direct reduction of $\Delta 24-28$ double bonds without the prior isomerisation step (97).

Cholesterol is generally only found in trace amounts in plants. However, plants from the genus Solanum, such as Solanum tuberosum (potato) and Solanum lycopersicum (tomato) rely on cholesterol as a precursor to steroidal glycoalkyloid toxins. Both $S$. tuberosum and $S$. lycopersicum contain two sterol reductase-encoding genes. One encodes a typical DWARF1type reductase that catalyses the $\Delta 24-28$ double bond reduction required for the synthesis of 
plant sterols (SSR1), while the other encodes a reductase that only catalyses the reduction of $\triangle 24-25$ double bonds enabling it to convert desmosterol to cholesterol (SSR2) (98).

A thorough understanding of the molecular determinants of the differences in the reactions catalysed by sterol reductases is hampered by a lack of structural data. Homology modelling of human DHCR24 did lead to a hypothesis regarding the cause of desmosterolosis (99). Mutations found in patients may affect interactions with the FAD cofactor, the desmosterol substrate and the endoplasmic reticulum membrane, with which DHCR24 is associated (100, 101). Further structural and biochemical studies are required to confirm these hypotheses.
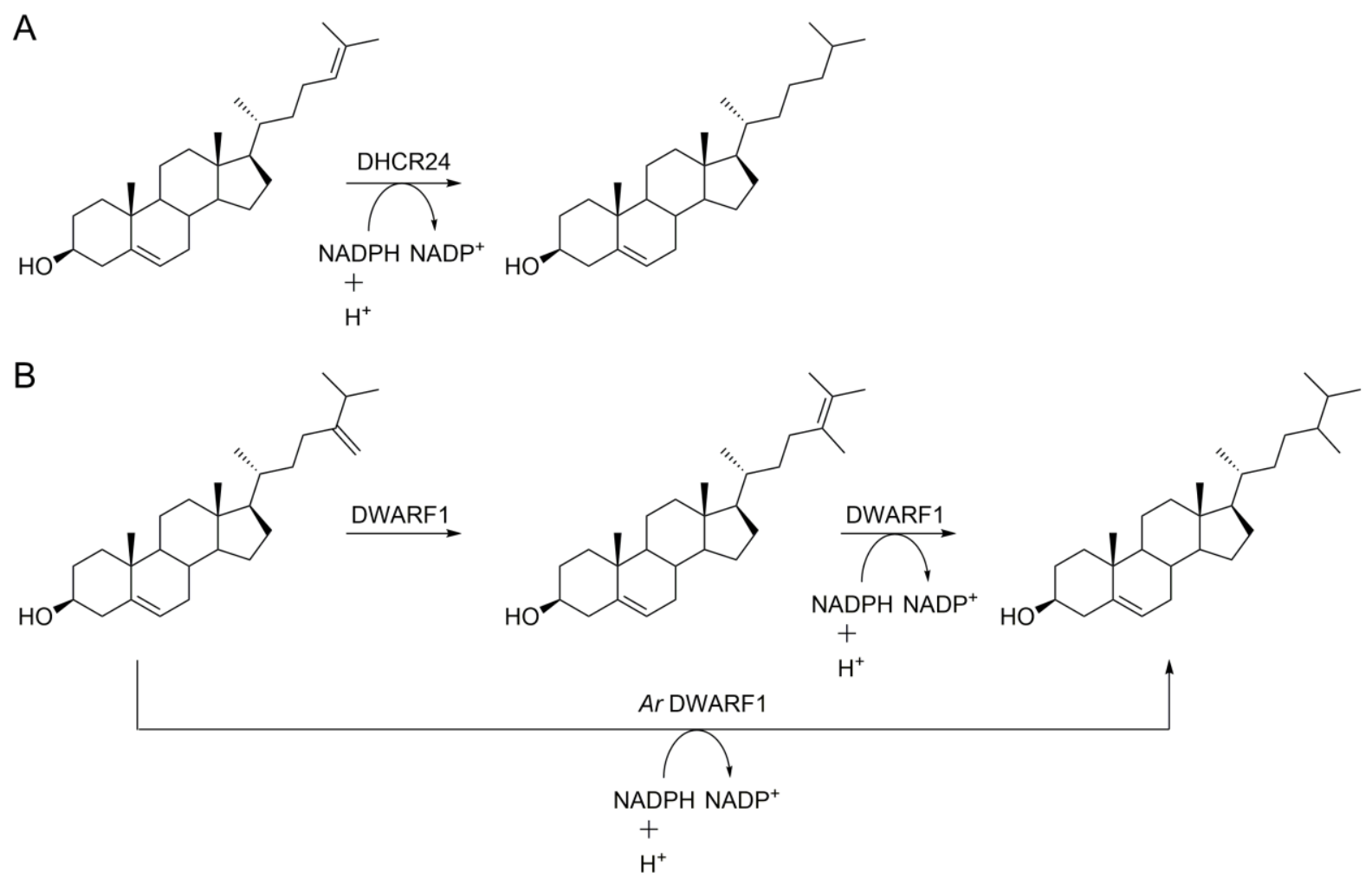

Scheme 7: Reductions of sterols catalysed by sterol reductases. (A) The mammalian enzyme DHCR24 reduces the $\triangle 24-25$ double bond of desmosterol, yielding cholesterol. (B) The plant enzyme DWARF1 catalyses the isomerisation of the $\Delta 24-28$ double bond of sterols such as 24-methylenecholesterol to a $\Delta 24-25$ double bond, followed by the reduction of this double bond, in this case yielding campesterol. Ajuga reptans DWARF1 ( $A r$ DWARF1) can catalyse the direct reduction of $\Delta 24-28$ double bonds without the prior isomerisation step.

\section{Subfamily 8: DprE1s}

The most recently identified subfamily of the VAO/PCMH family consists of DprE1 (EC 1.1.98.3). Together with a second enzyme, DprE2, DprE1 is responsible for the epimerisation of decaprenylphosphoryl- $\beta$-D-ribofuranose (DPR) to decaprenylphosphoryl- $\beta$-Darabinofuranose (DPA), the precursor of the arabinan moiety of the cell wall of mycobacteria such as Mycobacterium tuberculosis, the pathogen that causes tuberculosis (102). The importance of this reaction is highlighted by the fact that the dprE1 gene has been shown to be essential for growth in Mycobacterium smegmatis (103). Epimerisation of DPR to DPA occurs 
in a two-step process, where DPR is first oxidised to decaprenylphosphoryl-2-keto- $\beta$-Derythro-pentofuranose (DPX) by DprE1, after which DPX is reduced to DPA by DprE2 (Scheme 8A) (104). To achieve substrate oxidation, DprE1 uses a non-covalently bound FAD cofactor. Though DprE1 can use oxygen as an electron acceptor, artificial electron acceptors such as DCPIP lead to more efficient catalysis, suggesting the enzyme is a dehydrogenase rather than an oxidase. It has been suggested that membrane-embedded menaquinone may be the physiological electron acceptor (105).

Due to its crucial role in mycobacterial cell wall synthesis, DprE1 has gathered major interest as a potential antitubercular drug target in recent years. The DprE1 inhibitors identified so far can be classed into two groups: nitro-group containing suicide inhibitors and non-covalent competitive inhibitors (106). The covalent inhibitors are first activated by reduction of their nitro group to a nitroso group, which only occurs after reduction of the enzyme's FAD cofactor by its substrate (Scheme $8 \mathrm{~B})(104,105,107,108)$. The nitroso group subsequently reacts with the thiol group of an active site cysteine (Cys-387 in M. tuberculosis DprE1), leading to the bulky compounds permanently blocking the active site and preventing catalysis. The identification of further DprE1 inhibitors and their application as antitubercular drugs will likely be an important line of research in the coming years.

A<smiles>[R]OP(=O)(O)OC1OC(CO)C(O)C1O</smiles>

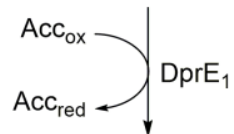<smiles>[R]OP(=O)(O)OC1OC(CO)C(=O)C1O</smiles>

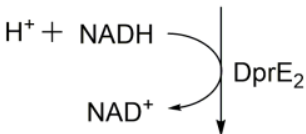<smiles>O=P(O)(O)OC1OC(CO)C(O)C1O</smiles>

B<smiles>CC1COC2(CCN(c3nc(=O)c4cc(C(F)(F)F)cc([N+](=O)[O-])c4s3)CC2)O1</smiles><smiles>CC1COC2(CCN(c3nc(=O)c4cc(C(F)(F)F)cc(NO)c4s3)CC2)O1</smiles><smiles>CC1COC2(CCN(c3nc(=O)c4cc(C(F)(F)F)cc(NO)c4s3)CC2)O1</smiles>

$\mathrm{R}=$ decaprenol

Scheme 8: (A) Epimerisation of decaprenylphosphoryl- $\beta$-D-ribofuranose (DPR) to decaprenylphosphoryl- $\beta$-D-arabinofuranose (DPA) as catalysed by DprE1 and DprE2. DPR is first oxidised to decaprenylphosphoryl-2-keto- $\beta$-D-erythro-pentofuranose (DPX) by DprE1. DPX is subsequently reduced to DPA by the NADH-dependent reductase DprE2. (B) Mechanism of inhibition of DprE1 by the benzothiazone inhibitor BTZ043. In order for inhibition to occur, the enzyme's FAD cofactor must first be reduced by its substrate. Subsequently, the nitro group of BTZ043 is reduced to a nitroso group by reaction with the FAD cofactor. This nitroso group reacts with the thiol group of an active site cysteine residue (Cys-387 in Mycobacterium tuberculosis DprE1), covalently modifying the enzyme and blocking the active site. 


\section{Subfamily 9: Aldonolactone oxidoreductases}

Aldonolactone oxidoreductases are found in a wide variety of organisms where they catalyse the final step in the synthesis of L-ascorbic acid (vitamin C) or related antioxidant compounds. The exact reaction that is catalysed depends on the host organism (Scheme 9) $(109,110)$. In animals, L-ascorbic acid is synthesised from L-gulono-1,4-lactone by L-gulono-1,4-lactone oxidase (GUO, EC 1.1.3.8) (111, 112). In plants, L-ascorbic acid is mainly synthesised from Lgalactono-1,4-lactone by L-galactono-1,4-lactone dehydrogenase (GALDH, EC 1.3.2.3) (113116). Another enzyme that catalyses the formation of L-ascorbic acid is L-gulono-1,4-lactone dehydrogenase (GUDH) from Mycobacterium tuberculosis (10). However, it is unclear what the physiological function of this enzyme is. Fungal aldonolactone oxidoreductases do not synthesise L-ascorbic acid, but rather the similar compounds D-erythorbic acid, which is synthesised from D-glucono-1,4-lactone by D-glucono-1,4-lactone oxidase (GLO) in Penicillium griseoroseum (also known as Penicillium cyaneo-fulvum), or dehydro-D-arabinono-1,4-lactone (also known as D-erythroascorbic acid), which is synthesised from D-arabinono-1,4-lactone by D-arabinono-1,4-lactone oxidase (ALO, EC 1.1.3.37) in yeasts such as Saccharomyces cerevisiae and Candida albicans (117-120). Aldonolactone oxidoreductases have also been identified in the protozoans Trypanosoma brucei (ALO), Trypanosoma cruzi (L-galactono-1,4-lactone oxidoreductase, GALO), and Leishmania donovani (ALO) $(11,121,122)$. These enzymes are named after the best substrate identified in in vitro experiments. However, the identity of their physiological substrate is unclear.

Despite the differences in substrate specificity, the basic reaction catalysed by all aldonolactone oxidoreductases is similar. The $\mathrm{C} 2-\mathrm{C} 3$ bond of the lactone substrate is oxidised to a double bond by transfer of two electrons to the enzyme's flavin cofactor. The subsequent reoxidation of the flavin cofactor can occur either by its reaction with molecular oxygen (GUO, yeast ALO, GLO) or electron acceptors such as cytochrome $c$ (GUDH, GALDH). Arabidopsis thaliana GALDH can be converted to an oxidase by a single alanine to glycine mutation (123). A glycine or proline is found at this position in the oxidases GUO, yeast ALO and GLO and it is predicted to be located close to the $\mathrm{C} 4 \mathrm{a}$ atom of $\mathrm{FAD}$, the site of the reaction with oxygen. This suggests that in GALDH, this alanine residue acts as a gatekeeper, blocking access of oxygen to the flavin cofactor. The binding mode of the flavin cofactor also differs depending on the enzyme, GUO, yeast ALO and GLO contain a covalently bound FAD cofactor, while GALDH from A. thaliana and GALO from T. cruzi contain non-covalently bound FAD $(13,124-$ 126). A number of arabinonolactone oxidoreductases have been reported to contain noncovalently bound FMN (ALO from T. brucei and GALDH from B. oleracea) and no flavin was detected in GUDH (9-11). However, as hydrolysis of FAD during analysis may lead to misassignment of the cofactor of flavoproteins as FMN instead of FAD, further investigations are required to confirm the identity of the flavin in these enzymes.

\section{Subfamily 10: Alditol oxidases}

Members of the alditol oxidase subfamily [referred to as alditol oxidase (AldO), sorbitol oxidase or xylitol oxidase depending on their substrate specificity, EC 1.1.3.41] catalyse the oxidation of the primary hydroxyl groups of alditols (sugar alcohols), yielding the corresponding aldoses, with molecular oxygen acting as the electron acceptor (Scheme 10A). 


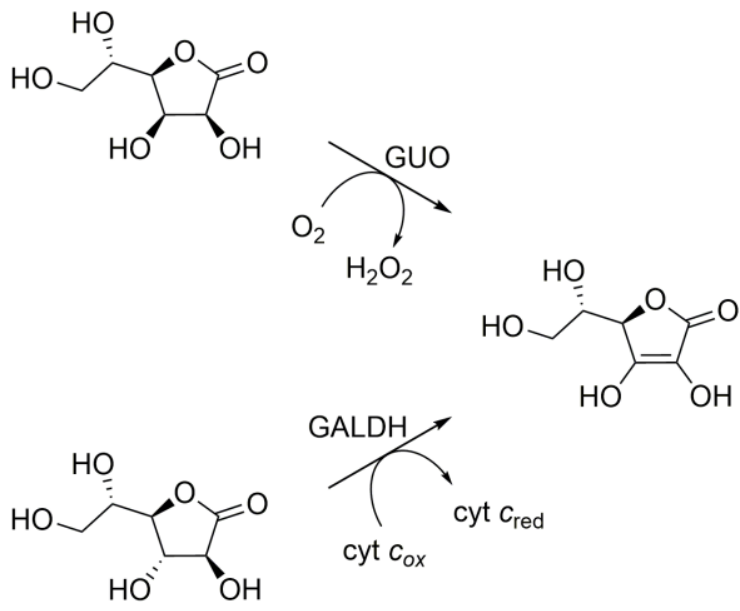<smiles>O=C1OC(CO)C(O)C1O</smiles>

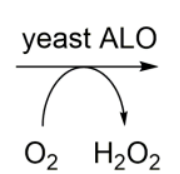<smiles>O=C1OC(CO)C(O)=C1O</smiles><smiles>O=C1OC([C@H](O)CO)C(O)[C@H]1O</smiles><smiles>O=C1OC(C(O)CO)C(O)=C1O</smiles>

Scheme 9: Oxidations catalysed by aldonolactone oxidoreductases. L-gulono-1,4-lactone oxidase (GUO) and L-galactono-1,4-lactone dehydrogenase (GALDH) catalyse the formation of L-ascorbic acid from Lgulono-1,4-lactone and L-galactono-1,4-lactone respectively. D-arabinono-1,4-lactone oxidase (ALO) from yeast catalyses the oxidation of D-arabinono-1,4-lactone to dehydro-D-arabinono-1,4-lactone. DGlucono-1,4-lactone oxidase (GLO) catalyses the oxidation of D-glucono-1,4-lactone to D-erythorbic acid.

They have been identified in various Streptomyces species and in the thermophilic bacterium Acidothermus cellulolyticus 11B (127-130). All characterised alditol oxidases contain a covalently bound FAD cofactor, which the crystal structure of the enzyme from Streptomyces coelicolor A3(2) revealed to be covalently bound to the enzyme via an $8 \alpha-N 1$-histidyl-FAD bond (131). The diffusion of oxygen into the active site of $S$. coelicolor AldO was studied by a combination of molecular dynamics simulations and site-directed mutagenesis (132). This revealed that there are multiple defined diffusion pathways through which oxygen reaches the active site of the enzyme. The majority of these pathways converge at a single point of entrance to the active site. The residue located at this site, Ala-105, is homologous to the gatekeeper alanine residue identified in GALDH, suggesting this may be a crucial site in determining the oxygen reactivity of $\mathrm{VAO} / \mathrm{PCMH}$-type flavoenzymes.

In addition to the oxidation of alcohols, AldO from S. coelicolor, can catalyse the oxidation of thiol groups (133). For example, L-dithiothreitol, which is identical to the sugar alcohol Lthreitol except for the presence of thiol groups instead of hydroxyl groups at the terminal carbon atoms, is oxidised to the corresponding thioaldehyde, which subsequently cyclises to 
a dithiohemiacetal (Scheme 10B). A number of other flavin-dependent alcohol oxidases from the structurally unrelated glucose-methanol-choline (GMC) oxidoreductase family were also shown to oxidise thiol analogues of their alcohol substrates, suggesting the ability to do so may be a general property of flavin-dependent alcohol oxidases.

A
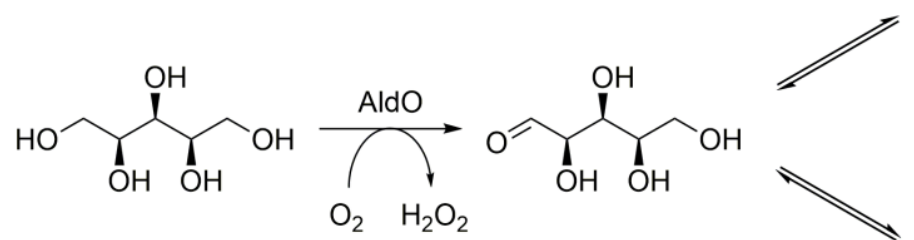<smiles>OC1[C@@H](O)CO[C@@H](O)[C@@H]1O</smiles>

B

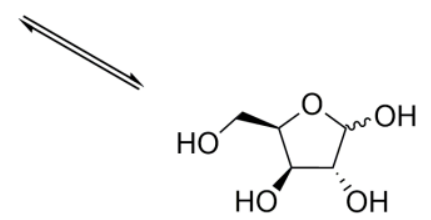<smiles>OC(CS)C(O)CS</smiles>

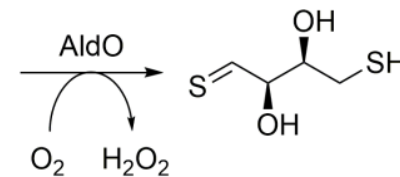<smiles>C=C</smiles><smiles>OC1CSC(S)C1O</smiles>

Scheme 10: (A) Oxidation of xylitol to D-xylose catalysed by alditol oxidase (AldO). (B) Oxidation of Ldithiothreitol to the corresponding thioaldehyde, which rearranges to a cyclic dithiohemiacetal, catalysed by AldO.

\section{Subfamily 11: Cholesterol oxidases}

Cholesterol oxidase (ChoX, EC 1.1.3.6) is found in bacteria that are capable of metabolising cholesterol, where it catalyses the first two steps in its metabolism. The first is the oxidation of the $3 \beta$-hydroxyl group to a carbonyl, yielding cholest-5-en-3-one (Scheme 11). In most cases, this is subsequently isomerised to cholest-4-en-3-one (134). Molecular oxygen is utilised as the electron acceptor for the first step of the reaction, being converted to hydrogen peroxide. Two types of ChoX exist, a VAO/PCMH-type that contains a covalently bound FAD cofactor linked to the protein through an $8 \alpha-N 1$-histidyl-FAD bond and another type that belongs to the GMC oxidoreductase family and contains non-covalently bound FAD. VAO/PCMH-type ChoXs are found in Gram-positive bacteria such as Brevibacterium sterolicum and various species of Rhodococcus and Gram-negative bacteria such as Chromobacterium sp. DS-1, Pseudomonas aeruginosa and Burkholderia cepacia (135-140). Unlike most ChoXs, the VAO/PCMH-type enzymes from Gram-negative bacteria do not catalyse the isomerization of cholest-5-en-3-one to cholest-4-en-3-one and the main product of the reaction is 6-hydroperoxycholest-4-en-3one (Scheme 11) (141). The exact mechanism by which 6-hydroperoxycholest-4-en-3-one is formed is unclear. It has been proposed that it may be the result of dioxygenase activity of the enzyme, but a mechanism where the oxidation proceeds through enzyme-initiated radical reactions is also a possibility $(141,142)$. The molecular determinants of which product is formed are unclear and would be an interesting topic for future studies, particularly considering that a crystal structure is available of both the cholest-4-en-3-one-forming 

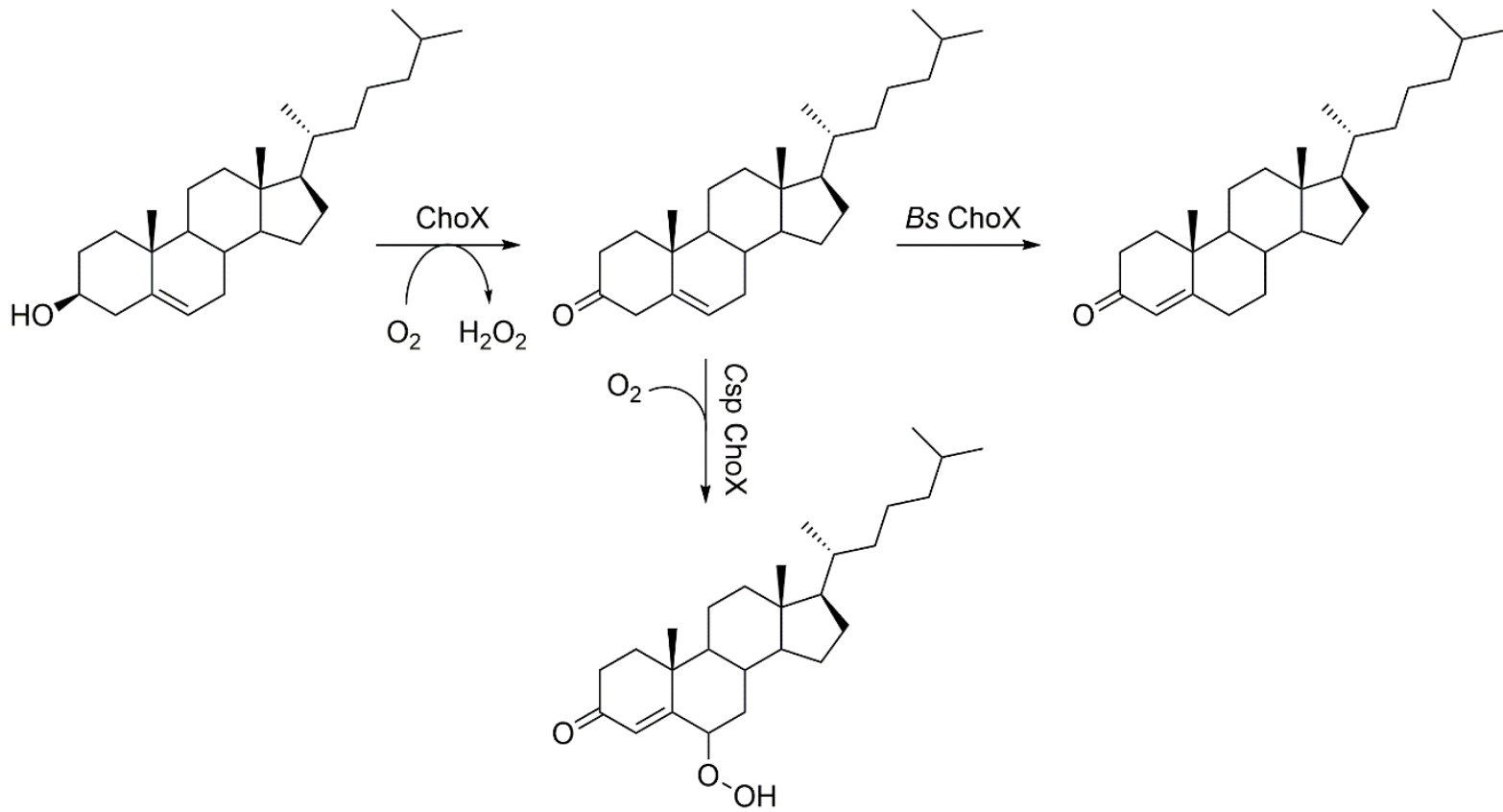

Scheme 11: Oxidation of cholesterol as catalysed by cholesterol oxidase (ChoX). Cholesterol is first oxidised to cholest-5-en-3-one. VAO/PCMH-type ChoX from Gram-positive bacteria, such as Brevibacterium sterolicum (Bs ChoX), subsequently catalyses its isomerisation to cholest-4-en-3-one. VAO/PCMH-type ChoX from Gram-negative bacteria, such as Chromobacterium sp. DS-1 (Csp ChoX), does not catalyse this isomerisation step. Instead, oxygen is incorporated into the formed cholest-5-en3-one, yielding 6-hydroperoxycholest-4-en-3-one as the product.

VAO/PCMH-type ChoX from Brevibacterium sterolicum and the 6-hydroperoxycholest-4-en-3one-forming VAO/PCMH-type ChoX from Chromobacterium sp. DS-1 $(143,144)$.

\section{Concluding remarks}

The past ten years have seen significant and surprising advances in our knowledge of enzymes of the $\mathrm{VAO} / \mathrm{PCMH}$ family, including the characterisation of the flavin-N5-oxide, a novel flavin redox state, and DprE1, a promising potential antitubercular drug target. As highlighted above, open questions regarding the biochemical and mechanistic characterisation of these enzymes remain and will provide interesting challenges for future research. A number of interesting advances have already been reported since the publication of our analysis in June 2017. Jiang et al. reported the characterization of a DLDH from Pseudomonas putida KT2440 that contains an Fe-S cluster-binding domain in addition to its $\mathrm{VAO} / \mathrm{PCMH}$-type flavoprotein domains (145). After oxidation of D-lactate, electrons are transferred from the FAD cofactor to a quinone electron acceptor via the Fe-S clusters. Other advances include the characterisation of the novel VAO/PCMH family member daurichromenic acid synthase from Rhododendron dauricum, which catalyses the oxidative cyclisation of grifolic acid to daurichromenic acid and the determination of the crystal structure of Mycobacterium tuberculosis MurB, which could facilitate the design of antitubercular drugs targeting this enzyme $(146,147)$. 


\section{Methods}

To perform the phylogenetic analysis of the VAO/PCMH family, a list of family members with known sequence and enzymatic function was compiled by searching the Uniprot and Protein Data Bank databases and the primary literature. A structure-based multiple sequence alignment of 112 obtained sequences was performed using the PROMALS3D webserver (148). PDB files with the following PDB IDs were used for the alignment: 1F0X (Escherichia coli DLDH), 1HSK (Staphylococcus aureus MurB), $1 \mathrm{I19}$ (Brevibacterium sterolicum ChoX), 1VAO (Penicillium simplicissimum VAO), 1W1O (Zea mays CKX1), 1WVF (Pseudomonas putida PCMH), 1ZR6 (Sarocladium strictum glucooligosaccharide oxidase), 2BVF (Paenarthrobacter nicotinovorans 6-hydroxy-D-nicotine oxidase), 2EXR (Arabidopsis thaliana CKX7), 2GQT (Thermus caldophilus MurB), 2IPI (Streptomyces galilaeus aclacinomycin oxidoreductase), 2MBR (Escherichia coli MurB), 2UUU (Dictyostelium discoideum ADPS), 2VFR (Streptomyces coelicolor A3(2) AldO), 2Y08 (Streptomyces sp. 307-9 TamL), 3D2H (Eschscholzia californica BBE), 3JS8 (Chromobacterium sp. DS-1 ChoX), 3RJ8 (Microdochium nivale lactose oxidase) and 3TSH (Phleum pratense Phl p 4). Subsequently, three sequences, GilR from Streptomyces griseoflavus, CndG from Chondromyces crocatus and FeeG from an unkown source organism, were added to the alignment using the MAFFT version 7 webserver (149). Subsequently, a phylogenetic tree was constructed using the phylogeny.fr webserver's BioNJ function (150). The iTOL webserver was used for tree visualisation (151).

\section{Acknowledgements}

This work was supported by the European Union through the INDOX project (FP7-KBBE2013-7-613549).

\section{References}

1. Macheroux, P., Kappes, B., and Ealick, S. E. (2011) Flavogenomics: A genomic and structural view of flavin-dependent proteins. FEBS J. 278, 2625-2634

2. Lienhart, W.-D., Gudipati, V., and Macheroux, P. (2013) The human flavoproteome. Arch. Biochem. Biophys. 535, 150-162

3. Fraaije, M. W., and Mattevi, A. (2000) Flavoenzymes: Diverse catalysts with recurrent features. Trends Biochem. Sci. 25, 126-132

4. Joosten, V., and van Berkel, W. J. H. (2007) Flavoenzymes. Curr. Opin. Chem. Biol. 11, 195-202

5. Leys, D., and Scrutton, N. S. (2016) Sweating the assets of flavin cofactors: New insight of chemical versatility from knowledge of structure and mechanism. Curr. Opin. Struct. Biol. 41, 19-26

6. Piano, V., Palfey, B. A., and Mattevi, A. (2017) Flavins as covalent catalysts: New mechanisms emerge. Trends Biochem. Sci. 42, 457-469

7. Fraaije, M. W., van Berkel, W. J. H., Benen, J. A. E., Visser, J., and Mattevi, A. (1998) A novel oxidoreductase family sharing a conserved FAD-binding domain. Trends Biochem. Sci. 23, 206207

8. Leferink, N. G. H., Heuts, D. P. H. M., Fraaije, M. W., and van Berkel, W. J. H. (2008) The growing VAO flavoprotein family. Arch. Biochem. Biophys. 474, 292-301

9. Mapson, L. W., and Breslow, E. (1958) Biological synthesis of ascorbic acid: L-Galactono- $\gamma-$ lactone dehydrogenase. Biochem. J. 68, 395-406

10. Wolucka, B. A., and Communi, D. (2006) Mycobacterium tuberculosis possesses a functional 
enzyme for the synthesis of vitamin C, L-gulono-1,4-lactone dehydrogenase. FEBS J. 273, 44354445

11. Logan, F. J., Taylor, M. C., Wilkinson, S. R., Kaur, H., and Kelly, J. M. (2007) The terminal step in vitamin $\mathrm{C}$ biosynthesis in Trypanosoma cruzi is mediated by a FMN-dependent galactonolactone oxidase. Biochem. J. 407, 419-426

12. Liu, Y.-C., Li, Y.-S., Lyu, S.-Y., Hsu, L.-J., Chen, Y.-H., Huang, Y.-T., Chan, H.-C., Huang, C.-J., Chen, G.-H., Chou, C.-C., Tsai, M.-D., and Li, T.-L. (2011) Interception of teicoplanin oxidation intermediates yields new antimicrobial scaffolds. Nat. Chem. Biol. 7, 304-309

13. Kudryashova, E. V., Leferink, N. G. H., Slot, I. G. M., and van Berkel, W. J. H. (2011) Galactonolactone oxidoreductase from Trypanosoma cruzi employs a FAD cofactor for the synthesis of vitamin C. Biochim. Biophys. Acta - Proteins Proteomics. 1814, 545-552

14. Heuts, D. P. H. M., Scrutton, N. S., McIntire, W. S., and Fraaije, M. W. (2009) What's in a covalent bond?: On the role and formation of covalently bound flavin cofactors. FEBS J. 276, 3405-3427

15. Winkler, A., Łyskowski, A., Riedl, S., Puhl, M., Kutchan, T. M., Macheroux, P., and Gruber, K. (2008) A concerted mechanism for berberine bridge enzyme. Nat. Chem. Biol. 4, 739-741

16. Taura, F., Sirikantaramas, S., Shoyama, Y., Yoshikai, K., Shoyama, Y., and Morimoto, S. (2007) Cannabidiolic-acid synthase, the chemotype-determining enzyme in the fiber-type Cannabis sativa. FEBS Lett. 581, 2929-2934

17. Gesell, A., Chávez, M. L. D., Kramell, R., Piotrowski, M., Macheroux, P., and Kutchan, T. M. (2011) Heterologous expression of two FAD-dependent oxidases with (S)tetrahydroprotoberberine oxidase activity from Argemone mexicana and Berberis wilsoniae in insect cells. Planta. 233, 1185-1197

18. Shoyama, Y., Tamada, T., Kurihara, K., Takeuchi, A., Taura, F., Arai, S., Blaber, M., Shoyama, Y., Morimoto, S., and Kuroki, R. (2012) Structure and function of $\Delta 1$-tetrahydrocannabinolic acid (THCA) synthase, the enzyme controlling the psychoactivity of Cannabis sativa. J. Mol. Biol. 423, 96-105

19. Kim, Y.-T., Lee, Y.-R., Jin, J., Han, K.-H., Kim, H., Kim, J.-C., Lee, T., Yun, S.-H., and Lee, Y.-W. (2005) Two different polyketide synthase genes are required for synthesis of zearalenone in Gibberella zeae. Mol. Microbiol. 58, 1102-1113

20. Kim, H. J., Pongdee, R., Wu, Q., Hong, L., and Liu, H.-W. (2007) The biosynthesis of spinosyn in Saccharopolyspora spinosa: Synthesis of the cross-bridging precursor and identification of the function of SpnJ. J. Am. Chem. Soc. 129, 14582-14584

21. Alexeev, I., Sultana, A., Mäntsälä, P., Niemi, J., and Schneider, G. (2007) Aclacinomycin oxidoreductase (AknOx) from the biosynthetic pathway of the antibiotic aclacinomycin is an unusual flavoenzyme with a dual active site. Proc. Natl. Acad. Sci. U. S. A. 104, 6170-6175

22. Kasahara, K., Miyamoto, T., Fujimoto, T., Oguri, H., Tokiwano, T., Oikawa, H., Ebizuka, Y., and Fujii, I. (2010) Solanapyrone synthase, a possible Diels-Alderase and iterative type I polyketide synthase encoded in a biosynthetic gene cluster from Alternaria solani. ChemBioChem. 11, 12451252

23. Carlson, J. C., Li, S., Gunatilleke, S. S., Anzai, Y., Burr, D. A., Podust, L. M., and Sherman, D. H. (2011) Tirandamycin biosynthesis is mediated by co-dependent oxidative enzymes. Nat. Chem. 3, 628-633

24. Noinaj, N., Bosserman, M. A., Schickli, M. A., Piszczek, G., Kharel, M. K., Pahari, P., Buchanan, S. K., and Rohr, J. (2011) The crystal structure and mechanism of an unusual oxidoreductase, GilR, involved in gilvocarcin V biosynthesis. J. Biol. Chem. 286, 23533-23543

25. Ragab, A. E., Grüschow, S., Tromans, D. R., and Goss, R. J. M. (2011) Biogenesis of the unique 4',5'-dehydronucleoside of the uridyl peptide antibiotic pacidamycin. J. Am. Chem. Soc. 133, 15288-15291

26. Ishiuchi, K., Nakazawa, T., Yagishita, F., Mino, T., Noguchi, H., Hotta, K., and Watanabe, K. (2013) Combinatorial generation of complexity by redox enzymes in the chaetoglobosin A biosynthesis. J. Am. Chem. Soc. 135, 7371-7377 
27. Teufel, R., Miyanaga, A., Michaudel, Q., Stull, F., Louie, G., Noel, J. P., Baran, P. S., Palfey, B., and Moore, B. S. (2013) Flavin-mediated dual oxidation controls an enzymatic Favorskii-type rearrangement. Nature. 503, 552-556

28. Garcia, I., Vior, N. M., González-Sabín, J., Braña, A. F., Rohr, J., Moris, F., Méndez, C., and Salas, J. A. (2013) Engineering the biosynthesis of the polyketide-nonribosomal peptide collismycin A for generation of analogs with neuroprotective activity. Chem. Biol. 20, 1022-1032

29. Holm, D. K., Petersen, L. M., Klitgaard, A., Knudsen, P. B., Jarczynska, Z. D., Nielsen, K. F., Gotfredsen, C. H., Larsen, T. O., and Mortensen, U. H. (2014) Molecular and chemical characterization of the biosynthesis of the 6-MSA-derived meroterpenoid yanuthone D in Aspergillus niger. Chem. Biol. 21, 519-529

30. Zhu, Y., Picard, M.-È., Zhang, Q., Barma, J., Després, X. M., Mei, X., Zhang, L., Duvignaud, J.-B., Couture, M., Zhu, W., Shi, R., and Zhang, C. (2016) Flavoenzyme CrmK-mediated substrate recycling in caerulomycin biosynthesis. Chem. Sci. 7, 4867-4874

31. Xu, F., Golightly, E. J., Fuglsang, C. C., Schneider, P., Duke, K. R., Lam, L., Christensen, S., Brown, K. M., Jørgensen, C. T., and Brown, S. H. (2001) A novel carbohydrate:acceptor oxidoreductase from Microdochium nivale. Eur. J. Biochem. 268, 1136-1142

32. Custers, J. H. H. V., Harrison, S. J., Sela-Buurlage, M. B., van Deventer, E., Lageweg, W., Howe, P. W., van der Meijs, P. J., Ponstein, A. S., Simons, B. H., Melchers, L. S., and Stuiver, M. H. (2004) Isolation and characterisation of a class of carbohydrate oxidases from higher plants, with a role in active defence. Plant J. 39, 147-160

33. Huang, C.-H., Lai, W.-L., Lee, M.-H., Chen, C.-J., Vasella, A., Tsai, Y.-C., and Liaw, S.-H. (2005) Crystal structure of glucooligosaccharide oxidase from Acremonium strictum: A novel

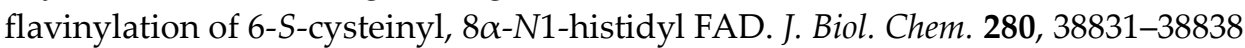

34. Rand, T., Qvist, K. B., Walter, C. P., and Poulsen, C. H. (2006) Characterization of the flavin association in hexose oxidase from Chondrus crispus. FEBS J. 273, 2693-2703

35. Heuts, D. P. H. M., Janssen, D. B., and Fraaije, M. W. (2007) Changing the substrate specificity of a chitooligosaccharide oxidase from Fusarium graminearum by model-inspired site-directed mutagenesis. FEBS Lett. 581, 4905-4909

36. Ferrari, A. R., Rozeboom, H. J., Dobruchowska, J. M., van Leeuwen, S. S., Vugts, A. S. C., Koetsier, M. J., Visser, J., and Fraaije, M. W. (2016) Discovery of a xylooligosaccharide oxidase from Myceliophthora thermophila C1. J. Biol. Chem. 291, 23709-23718

37. Teufel, R., Stull, F., Meehan, M. J., Michaudel, Q., Dorrestein, P. C., Palfey, B., and Moore, B. S. (2015) Biochemical establishment and characterization of EncM's flavin-N5-oxide cofactor. J. Am. Chem. Soc. 137, 8078-8085

38. Adak, S., and Begley, T. P. (2016) Dibenzothiophene catabolism proceeds via a flavin-N5-oxide intermediate. J. Am. Chem. Soc. 138, 6424-6426

39. Daniel, B., Konrad, B., Toplak, M., Lahham, M., Messenlehner, J., Winkler, A., and Macheroux, P. (2017) The family of berberine bridge enzyme-like enzymes: A treasure-trove of oxidative reactions. Arch. Biochem. Biophys. 632, 88-103

40. Popelková, H., Fraaije, M. W., Novák, O., Frébortová, J., Bilyeu, K. D., and Frébort, I. (2006) Kinetic and chemical analyses of the cytokinin dehydrogenase-catalysed reaction: Correlations with the crystal structure. Biochem. J. 398, 113-124

41. Frébortová, J., Fraaije, M. W., Galuszka, P., Šebela, M., Peč, P., Hrbáč, J., Novák, O., Bilyeu, K. D., English, J. T., and Frébort, I. (2004) Catalytic reaction of cytokinin dehydrogenase: Preference for quinones as electron acceptors. Biochem. J. 380, 121-130

42. Frébortová, J., Novák, O., Frébort, I., and Jorda, R. (2010) Degradation of cytokinins by maize cytokinin dehydrogenase is mediated by free radicals generated by enzymatic oxidation of natural benzoxazinones. Plant J. 61, 467-481

43. Galuszka, P., Popelková, H., Werner, T., Frébortová, J., Pospíšilová, H., Mik, V., Köllmer, I., Schmülling, T., and Frébort, I. (2007) Biochemical characterization of cytokinin oxidases/dehydrogenases from Arabidopsis thaliana expressed in Nicotiana tabacum L. J. Plant 
Growth Regul. 26, 255-267

44. Šmehilová, M., Galuszka, P., Bilyeu, K. D., Jaworek, P., Kowalska, M., Šebela, M., Sedlářová, M., English, J. T., and Frébort, I. (2009) Subcellular localization and biochemical comparison of cytosolic and secreted cytokinin dehydrogenase enzymes from maize. J. Exp. Bot. 60, 2701-2712

45. Gu, R., Fu, J., Guo, S., Duan, F., Wang, Z., Mi, G., and Yuan, L. (2010) Comparative expression and phylogenetic analysis of maize cytokinin dehydrogenase/oxidase (CKX) gene family. J. Plant Growth Regul. 29, 428-440

46. Kowalska, M., Galuszka, P., Frébortová, J., Šebela, M., Béres, T., Hluska, T., Šmehilová, M., Bilyeu, K. D., and Frébort, I. (2010) Vacuolar and cytosolic cytokinin dehydrogenases of Arabidopsis thaliana: Heterologous expression, purification and properties. Phytochemistry. 71, 1970-1978

47. Zalabák, D., Galuszka, P., Mrízová, K., Podlešáková, K., Gu, R., and Frébortová, J. (2014) Biochemical characterization of the maize cytokinin dehydrogenase family and cytokinin profiling in developing maize plantlets in relation to the expression of cytokinin dehydrogenase genes. Plant Physiol. Biochem. 74, 283-293

48. Malito, E., Coda, A., Bilyeu, K. D., Fraaije, M. W., and Mattevi, A. (2004) Structures of Michaelis and product complexes of plant cytokinin dehydrogenase: Implications for flavoenzyme catalysis. J. Mol. Biol. 341, 1237-1249

49. Kopečný, D., Končitíková, R., Popelka, H., Briozzo, P., Vigouroux, A., Kopečná, M., Zalabák, D., Šebela, M., Skopalová, J., Frébort, I., and Moréra, S. (2016) Kinetic and structural investigation of the cytokinin oxidase/dehydrogenase active site. FEBS J. 283, 361-377

50. Benson, T. E., Marquardt, J. L., Marquardt, A. C., Etzkorn, F. A., and Walsh, C. T. (1993) Overexpression, purification, and mechanistic study of UDP-N-acetylenolpyruvylglucosamine reductase. Biochemistry. 32, 2024-2030

51. Benson, T. E., Walsh, C. T., and Hogle, J. M. (1996) The structure of the substrate-free form of MurB, an essential enzyme for the synthesis of bacterial cell walls. Structure. 4, 47-54

52. Chen, M. W., Lohkamp, B., Schnell, R., Lescar, J., and Schneider, G. (2013) Substrate channel flexibility in Pseudomonas aeruginosa MurB accommodates two distinct substrates. PLoS One. 8, e66936

53. Benson, T. E., Harris, M. S., Choi, G. H., Cialdella, J. I., Herberg, J. T., Martin, Jr., J. P., and Baldwin, E. T. (2001) A structural variation for MurB: X-ray crystal structure of Staphylococcus aureus UDP-N-acetylenolpyruvylglucosamine reductase (MurB). Biochemistry. 40, 2340-2350

54. Kim, M.-K., Cho, M. K., Song, H.-E., Kim, D., Park, B.-H., Lee, J. H., Kang, G. B., Kim, S. H., Im, Y. J., Lee, D.-S., and Eom, S. H. (2007) Crystal structure of UDP-Nacetylenolpyruvylglucosamine reductase (MurB) from Thermus caldophilus. Proteins Struct. Funct. Bioinforma. 66, 751-754

55. Benson, T. E., Walsh, C. T., and Massey, V. (1997) Kinetic characterization of wild-type and S229A mutant MurB: Evidence for the role of Ser 229 as a general acid. Biochemistry. 36, 796-805

56. Naqvi, K. F., Patin, D., Wheatley, M. S., Savka, M. A., Dobson, R. C. J., Gan, H. M., Barreteau, H., Blanot, D., Mengin-Lecreulx, D., and Hudson, A. O. (2016) Identification and partial characterization of a novel UDP-N-acetylenolpyruvoylglucosamine reductase/UDP- $N$ acetylmuramate:L-alanine ligase fusion enzyme from Verrucomicrobium spinosum DSM $4136^{\mathrm{T}}$. Front. Microbiol. 7, 362

57. Hrast, M., Sosič, I., Šink, R., and Gobec, S. (2014) Inhibitors of the peptidoglycan biosynthesis enzymes MurA-F. Bioorg. Chem. 55, 2-15

58. Keat, M. J., and Hopper, D. J. (1978) p-Cresol and 3,5-xylenol methylhydroxylases in Pseudomonas putida N.C.I.B. 9869. Biochem. J. 175, 649-658

59. de Jong, E., van Berkel, W. J. H., van der Zwan, R. P., and de Bont, J. A. M. (1992) Purification and characterization of vanillyl-alcohol oxidase from Penicillium simplicissimum: A novel aromatic alcohol oxidase containing covalently bound FAD. Eur. J. Biochem. 208, 651-657

60. Priefert, H., Overhage, J., and Steinbüchel, A. (1999) Identification and molecular 
characterization of the eugenol hydroxylase genes (ehyA/ehyB) of Pseudomonas sp. strain HR199. Arch. Microbiol. 172, 354-363

61. Brandt, K., Thewes, S., Overhage, J., Priefert, H., and Steinbüchel, A. (2001) Characterization of the eugenol hydroxylase genes (ehyA/ehyB) from the new eugenol-degrading Pseudomonas sp. strain OPS1. Appl. Microbiol. Biotechnol. 56, 724-730

62. Jin, J., Mazon, H., van den Heuvel, R. H. H., Janssen, D. B., and Fraaije, M. W. (2007) Discovery of a eugenol oxidase from Rhodococcus sp. strain RHA1. FEBS J. 274, 2311-2321

63. McIntire, W., Hopper, D. J., and Singer, T. P. (1985) p-Cresol methylhydroxylase: Assay and general properties. Biochem. J. 228, 325-335

64. Fraaije, M. W., Veeger, C., and van Berkel, W. J. H. (1995) Substrate specificity of flavindependent vanillyl-alcohol oxidase from Penicillium simplicissimum: Evidence for the production of 4-hydroxycinnamyl alcohols from 4-allylphenols. Eur. J. Biochem. 234, 271-277

65. van den Heuvel, R. H. H., Fraaije, M. W., Laane, C., and van Berkel, W. J. H. (1998) Regio- and stereospecific conversion of 4-alkylphenols by the covalent flavoprotein vanillyl-alcohol oxidase. J. Bacteriol. 180, 5646-5651

66. Fraaije, M. W., and van Berkel, W. J. H. (1997) Catalytic mechanism of the oxidative demethylation of 4-(methoxymethyl)phenol by vanillyl-alcohol oxidase: Evidence for formation of a $p$-quinone methide intermediate. J. Biol. Chem. 272, 18111-18116

67. Fraaije, M. W., van den Heuvel, R. H. H., Roelofs, J. C. A. A., and van Berkel, W. J. H. (1998) Kinetic mechanism of vanillyl-alcohol oxidase with short-chain 4-alkylphenols. Eur. J. Biochem. 253, 712-719

68. Fraaije, M. W., Pikkemaat, M., and van Berkel, W. J. H. (1997) Enigmatic gratuitous induction of the covalent flavoprotein vanillyl-alcohol oxidase in Penicillium simplicissimum. Appl. Environ. Microbiol. 63, 435-439

69. Mattevi, A., Fraaije, M. W., Mozzarelli, A., Olivi, L., Coda, A., and van Berkel, W. J. H. (1997) Crystal structures and inhibitor binding in the octameric flavoenzyme vanillyl-alcohol oxidase: The shape of the active-site cavity controls substrate specificity. Structure. 5, 907-920

70. Cunane, L. M., Chen, Z.-W., Shamala, N., Mathews, F. S., Cronin, C. N., and McIntire, W. S. (2000) Structures of the flavocytochrome $p$-cresol methylhydroxylase and its enzyme-substrate complex: Gated substrate entry and proton relays support the proposed catalytic mechanism. J. Mol. Biol. 295, 357-374

71. Nguyen, Q.-T., de Gonzalo, G., Binda, C., Rioz-Martínez, A., Mattevi, A., and Fraaije, M. W. (2016) Biocatalytic properties and structural analysis of eugenol oxidase from Rhodococcus jostii RHA1: A versatile oxidative biocatalyst. ChemBioChem. 17, 1359-1366

72. Ewing, T. A., Gygli, G., and van Berkel, W. J. H. (2016) A single loop is essential for the octamerization of vanillyl alcohol oxidase. FEBS J. 283, 2546-2559

73. Gygli, G., and van Berkel, W. J. H. (2017) Vanillyl alcohol oxidases produced in Komagataella phaffii contain a highly stable noncovalently bound anionic FAD semiquinone. Biocatalysis. 3, 1726

74. Brady, S. F., Chao, C. J., and Clardy, J. (2002) New natural product families from an environmental DNA (eDNA) gene cluster. J. Am. Chem. Soc. 124, 9968-9969

75. Rachid, S., Revermann, O., Dauth, C., Kazmaier, U., and Müller, R. (2010) Characterization of a novel type of oxidative decarboxylase involved in the biosynthesis of the styryl moiety of chondrochloren from an acylated tyrosine. J. Biol. Chem. 285, 12482-12489

76. Braverman, N. E., and Moser, A. B. (2012) Functions of plasmalogen lipids in health and disease. Biochim. Biophys. Acta - Mol. Basis Dis. 1822, 1442-1452

77. de Vet, E. C. J. M., Hilkes, Y. H. A., Fraaije, M. W., and van den Bosch, H. (2000) Alkyldihydroxyacetonephosphate synthase: Presence and role of flavin adenine dinucleotide. J. Biol. Chem. 275, 6276-6283

78. Razeto, A., Mattiroli, F., Carpanelli, E., Aliverti, A., Pandini, V., Coda, A., and Mattevi, A. (2007) The crucial step in ether phospholipid biosynthesis: Structural basis of a noncanonical reaction 
associated with a peroxisomal disorder. Structure. 15, 683-692

79. Nenci, S., Piano, V., Rosati, S., Aliverti, A., Pandini, V., Fraaije, M. W., Heck, A. J. R., Edmondson, D. E., and Mattevi, A. (2012) Precursor of ether phospholipids is synthesized by a flavoenzyme through covalent catalysis. Proc. Natl. Acad. Sci. U. S. A. 109, 18791-18796

80. Benjamin, D. I., Cozzo, A., Ji, X., Roberts, L. S., Louie, S. M., Mulvihill, M. M., Luo, K., and Nomura, D. K. (2013) Ether lipid generating enzyme AGPS alters the balance of structural and signaling lipids to fuel cancer pathogenicity. Proc. Natl. Acad. Sci. U. S. A. 110, 14912-14917

81. Piano, V., Benjamin, D. I., Valente, S., Nenci, S., Marrocco, B., Mai, A., Aliverti, A., Nomura, D. K., and Mattevi, A. (2015) Discovery of inhibitors for the ether lipid-generating enzyme AGPS as anti-cancer agents. ACS Chem. Biol. 10, 2589-2597

82. Futai, M. (1973) Membrane D-lactate dehydrogenase from Escherichia coli: Purification and properties. Biochemistry. 12, 2468-2474

83. Lodi, T., and Ferrero, I. (1993) Isolation of the DLD gene of Saccharomyces cerevisiae encoding the mitochondrial enzyme D-lactate ferricytochrome $c$ oxidoreductase. Mol. Gen. Genet. 238, 315-324

84. Lodi, T., O'Connor, D., Goffrini, P., and Ferrero, I. (1994) Carbon catabolite repression in Kluyveromyces lactis: Isolation and characterization of the KIDLD gene encoding the mitochondrial enzyme D-lactate ferricytochrome $c$ oxidoreductase. Mol. Gen. Genet. 244, 622-629

85. Reed, D. W., and Hartzell, P. L. (1999) The Archaeoglobus fulgidus D-lactate dehydrogenase is a $\mathrm{Zn}^{2+}$ flavoprotein. J. Bacteriol. 181, 7580-7587

86. Engqvist, M., Drincovich, M. F., Flügge, U.-I., and Maurino, V. G. (2009) Two D-2-hydroxy-acid dehydrogenases in Arabidopsis thaliana with catalytic capacities to participate in the last reactions of the methylglyoxal and $\beta$-oxidation pathways. J. Biol. Chem. 284, 25026-25037

87. Dym, O., Pratt, E. A., Ho, C., and Eisenberg, D. (2000) The crystal structure of D-lactate dehydrogenase, a peripheral membrane respiratory enzyme. Proc. Natl. Acad. Sci. U. S. A. 97, 9413-9418

88. Achouri, Y., Noël, G., Vertommen, D., Rider, M. H., Veiga-da-Cunha, M., and van Schaftingen, E. (2004) Identification of a dehydrogenase acting on D-2-hydroxyglutarate. Biochem. J. 381, 3542

89. Struys, E. A., Salomons, G. S., Achouri, Y., van Schaftingen, E., Grosso, S., Craigen, W. J., Verhoeven, N. M., and Jakobs, C. (2005) Mutations in the D-2-hydroxyglutarate dehydrogenase gene cause D-2-hydroxyglutaric aciduria. Am. J. Hum. Genet. 76, 358-360

90. Becker-Kettern, J., Paczia, N., Conrotte, J.-F., Kay, D. P., Guignard, C., Jung, P. P., and Linster, C. L. (2016) Saccharomyces cerevisiae forms D-2-hydroxyglutarate and couples its degradation to D-lactate formation via a cytosolic transhydrogenase. J. Biol. Chem. 291, 6036-6058

91. Lord, J. M. (1972) Glycolate oxidoreductase in Escherichia coli. Biochim. Biophys. Acta - Bioenerg. 267, 227-237

92. Pellicer, M.-T., Badía, J., Aguilar, J., and Baldomà, L. (1996) glc Locus of Escherichia coli: Characterization of genes encoding the subunits of glycolate oxidase and the glc regulator protein. J. Bacteriol. 178, 2051-2059

93. Waterham, H. R., Koster, J., Romeijn, G. J., Hennekam, R. C. M., Vreken, P., Andersson, H. C., Fitzpatrick, D. R., Kelley, R. I., and Wanders, R. J. A. (2001) Mutations in the 3 $\beta$-hydroxysterol $\Delta^{24}$-reductase gene cause desmosterolosis, an autosomal recessive disorder of cholesterol biosynthesis. Am. J. Hum. Genet. 69, 685-694

94. Ciufo, L. F., Murray, P. A., Thompson, A., Rigden, D. J., and Rees, H. H. (2011) Characterisation of a desmosterol reductase involved in phytosterol dealkylation in the silkworm, Bombyx mori. PLoS One. 6, e21316

95. Klahre, U., Noguchi, T., Fujioka, S., Takatsuto, S., Yokota, T., Nomura, T., Yoshida, S., and Chua, N.-H. (1998) The Arabidopsis DIMINUTO/DWARF1 gene encodes a protein involved in steroid synthesis. Plant Cell. 10, 1677-1690

96. Hong, Z., Ueguchi-Tanaka, M., Fujioka, S., Takatsuto, S., Yoshida, S., Hasegawa, Y., Ashikari, M., Kitano, H., and Matsuoka, M. (2005) The rice brassinosteroid-deficient dwarf2 mutant, defective in 
the rice homolog of Arabidopsis DIMINUTO/DWARF1, is rescued by the endogenously accumulated alternative bioactive brassinosteroid, dolichosterone. Plant Cell. 17, 2243-2254

97. Tsukagoshi, Y., Suzuki, H., Seki, H., Muranaka, T., Ohyama, K., and Fujimoto, Y. (2016) Ajuga $\Delta 24$-sterol reductase catalyzes the direct reductive conversion of 24-methylenecholesterol to campesterol. J. Biol. Chem. 291, 8189-8198

98. Sawai, S., Ohyama, K., Yasumoto, S., Seki, H., Sakuma, T., Yamamoto, T., Takebayashi, Y., Kojima, M., Sakakibara, H., Aoki, T., Muranaka, T., Saito, K., and Umemoto, N. (2014) Sterol side chain reductase 2 is a key enzyme in the biosynthesis of cholesterol, the common precursor of toxic steroidal glycoalkaloids in potato. Plant Cell. 26, 3763-3774

99. Pedretti, A., Bocci, E., Maggi, R., and Vistoli, G. (2008) Homology modelling of human DHCR24 (seladin-1) and analysis of its binding properties through molecular docking and dynamics simulations. Steroids. 73, 708-719

100. Lu, X., Li, Y., Liu, J., Cao, X., Wang, X., Wang, D., Seo, H., and Gao, B. (2012) The membrane topological analysis of $3 \beta$-hydroxysteroid- $\Delta 24$ reductase (DHCR24) on endoplasmic reticulum. J. Mol. Endocrinol. 48, 1-9

101. Zerenturk, E. J., Sharpe, L. J., and Brown, A. J. (2014) DHCR24 associates strongly with the endoplasmic reticulum beyond predicted membrane domains: Implications for the activities of this multi-functional enzyme. Biosci. Rep. 34, 107-117

102. Mikušová, K., Huang, H., Yagi, T., Holsters, M., Vereecke, D., D’Haeze, W., Scherman, M. S., Brennan, P. J., McNeil, M. R., and Crick, D. C. (2005) Decaprenylphosphoryl arabinofuranose, the donor of the D-arabinofuranosyl residues of mycobacterial arabinan, is formed via a twostep epimerization of decaprenylphosphoryl ribose. J. Bacteriol. 187, 8020-8025

103. Crellin, P. K., Brammananth, R., and Coppel, R. L. (2011) Decaprenylphosphoryl- $\beta$-D-ribose 2'epimerase, the target of benzothiazinones and dinitrobenzamides, is an essential enzyme in Mycobacterium smegmatis. PLoS One. 6, e16869

104. Trefzer, C., Škovierová, H., Buroni, S., Bobovská, A., Nenci, S., Molteni, E., Pojer, F., Pasca, M. R., Makarov, V., Cole, S. T., Riccardi, G., Mikušová, K., and Johnsson, K. (2012) Benzothiazinones are suicide inhibitors of mycobacterial decaprenylphosphoryl- $\beta$-D-ribofuranose 2'-oxidase DprE1. J. Am. Chem. Soc. 134, 912-915

105. Neres, J., Pojer, F., Molteni, E., Chiarelli, L. R., Dhar, N., Boy-Röttger, S., Buroni, S., Fullam, E., Degiacomi, G., Lucarelli, A. P., Read, R. J., Zanoni, G., Edmondson, D. E., De Rossi, E., Pasca, M. R., McKinney, J. D., Dyson, P. J., Riccardi, G., Mattevi, A., Cole, S. T., and Binda, C. (2012) Structural basis for benzothiazinone-mediated killing of Mycobacterium tuberculosis. Sci. Transl. Med. 4, 150ra121

106. Piton, J., Foo, C. S.-Y., and Cole, S. T. (2017) Structural studies of Mycobacterium tuberculosis DprE1 interacting with its inhibitors. Drug Discov. Today. 22, 526-533

107. Trefzer, C., Rengifo-Gonzalez, M., Hinner, M. J., Schneider, P., Makarov, V., Cole, S. T., and Johnsson, K. (2010) Benzothiazinones: Prodrugs that covalently modify the decaprenylphosphoryl- $\beta$-D-ribose 2'-epimerase DprE1 of Mycobacterium tuberculosis. J. Am. Chem. Soc. 132, 13663-13665

108. Batt, S. M., Jabeen, T., Bhowruth, V., Quill, L., Lund, P. A., Eggeling, L., Alderwick, L. J., Fütterer, K., and Besra, G. S. (2012) Structural basis of inhibition of Mycobacterium tuberculosis DprE1 by benzothiazinone inhibitors. Proc. Natl. Acad. Sci. U. S. A. 109, 11354-11359

109. Leferink, N. G. H., and van Berkel, W. J. H. (2014) Aldonolactone oxidoreductases. In: Flavins and Flavoproteins: Methods and Protocols (Weber, S., and Schleicher, E. eds.), Methods Mol. Biol. vol. 1146, pp. 95-111

110. Aboobucker, S. I., and Lorence, A. (2016) Recent progress on the characterization of aldonolactone oxidoreductases. Plant Physiol. Biochem. 98, 171-185

111. Nishikimi, M., Tolbert, B. M., and Udenfriend, S. (1976) Purification and characterization of Lgulono- $\gamma$-lactone oxidase from rat and goat liver. Arch. Biochem. Biophys. 175, 427-435

112. Kiuchi, K., Nishikimi, M., and Yagi, K. (1982) Purification and characterization of L- 
gulonolactone oxidase from chicken kidney microsomes. Biochemistry. 21, 5076-5082

113. Østergaard, J., Persiau, G., Davey, M. W., Bauw, G., and van Montagu, M. (1997) Isolation of a cDNA coding for L-galactono- $\gamma$-lactone dehydrogenase, an enzyme involved in the biosynthesis of ascorbic acid in plants: Purification, characterization, cDNA cloning, and expression in yeast. J. Biol. Chem. 272, 30009-30016

114. Imai, T., Karita, S., Shiratori, G., Hattori, M., Nunome, T., Ôba, K., and Hirai, M. (1998) LGalactono- $\gamma$-lactone dehydrogenase from sweet potato: Purification and cDNA sequence analysis. Plant Cell Physiol. 39, 1350-1358

115. Yabuta, Y., Yoshimura, K., Takeda, T., and Shigeoka, S. (2000) Molecular characterization of tobacco mitochondrial L-galactono- $\gamma$-lactone dehydrogenase and its expression in Escherichia coli. Plant Cell Physiol. 41, 666-675

116. Leferink, N. G. H., van den Berg, W. A. M., and van Berkel, W. J. H. (2008) L-Galactono- $\gamma$-lactone dehydrogenase from Arabidopsis thaliana, a flavoprotein involved in vitamin $C$ biosynthesis. FEBS J. 275, 713-726

117. Takahashi, T., Yamashita, H., Kato, E., Mitsumoto, M., and Murakawa, S. (1976) Purification and some properties of D-glucono- $\gamma$-lactone dehydrogenase: D-Erythorbic acid producing enzyme of Penicillium cyaneo-fulvum. Agric. Biol. Chem. 40, 121-129

118. Huh, W.-K., Kim, S.-T., Yang, K.-S., Seok, Y.-J., Hah, Y. C., and Kang, S.-O. (1994) Characterisation of D-arabinono-1,4-lactone oxidase from Candida albicans ATCC 10231. Eur. J. Biochem. 225, 1073-1079

119. Huh, W.-K., Lee, B.-H., Kim, S.-T., Kim, Y.-R., Rhie, G.-E., Baek, Y.-W., Hwang, C.-S., Lee, J.-S., and Kang, S.-O. (1998) D-Erythroascorbic acid is an important antioxidant molecule in Saccharomyces cerevisiae. Mol. Microbiol. 30, 895-903

120. Salusjärvi, T., Kalkkinen, N., and Miasnikov, A. N. (2004) Cloning and characterization of gluconolactone oxidase of Penicillium cyaneo-fulvum ATCC 10431 and evaluation of its use for production of D-erythorbic acid in recombinant Pichia pastoris. Appl. Environ. Microbiol. 70, 55035510

121. Wilkinson, S. R., Prathalingam, S. R., Taylor, M. C., Horn, D., and Kelly, J. M. (2005) Vitamin C biosynthesis in trypanosomes: A role for the glycosome. Proc. Natl. Acad. Sci. U. S. A. 102, 1164511650

122. Biyani, N., and Madhubala, R. (2011) Leishmania donovani encodes a functional enzyme involved in vitamin c biosynthesis: Arabino-1,4-lactone oxidase. Mol. Biochem. Parasitol. 180, 76-85

123. Leferink, N. G. H., Fraaije, M. W., Joosten, H.-J., Schaap, P. J., Mattevi, A., and van Berkel, W. J. H. (2009) Identification of a gatekeeper residue that prevents dehydrogenases from acting as oxidases. J. Biol. Chem. 284, 4392-4397

124. Kenney, W. C., Edmondson, D. E., Singer, T. P., Nakagawa, H., Asano, A., and Sato, R. (1976) Identification of the covalently bound flavin of L-gulono- $\gamma$-lactone oxidase. Biochem. Biophys. Res. Commun. 71, 1194-1200

125. Kenney, W. C., Edmondson, D. E., Singer, T. P., Nishikimi, M., Noguchi, E., and Yagi, K. (1979) Identification of the covalently-bound flavin of L-galactonolactone oxidase from yeast. FEBS Lett. 97, 40-42

126. Harada, Y., Shimizu, M., Murakawa, S., and Takahashi, T. (1979) Identification of FAD of Dgluconolactone dehydrogenase: D-Erythorbic acid producing enzyme of Penicillium cyaneofulvum. Agric. Biol. Chem. 43, 2635-2636

127. Hiraga, K., Eto, T., Yoshioka, I., and Oda, K. (1998) Molecular cloning and expression of a gene encoding a novel sorbitol oxidase from Streptomyces sp. H-7775. Biosci. Biotechnol. Biochem. 62, 347-353

128. Yamashita, M., Omura, H., Okamoto, E., Furuya, Y., Yabuuchi, M., Fukahi, K., and Murooka, Y. (2000) Isolation, characterization, and molecular cloning of a thermostable xylitol oxidase from Streptomyces sp. IKD472. J. Biosci. Bioeng. 89, 350-360

129. Heuts, D. P. H. M., van Hellemond, E. W., Janssen, D. B., and Fraaije, M. W. (2007) Discovery, 
characterization, and kinetic analysis of an alditol oxidase from Streptomyces coelicolor. J. Biol. Chem. 282, 20283-20291

130. Winter, R. T., Heuts, D. P. H. M., Rijpkema, E. M. A., van Bloois, E., Wijma, H. J., and Fraaije, M. W. (2012) Hot or not? Discovery and characterization of a thermostable alditol oxidase from Acidothermus cellulolyticus 11B. Appl. Microbiol. Biotechnol. 95, 389-403

131. Forneris, F., Heuts, D. P. H. M., Delvecchio, M., Rovida, S., Fraaije, M. W., and Mattevi, A. (2008) Structural analysis of the catalytic mechanism and stereoselectivity in Streptomyces coelicolor alditol oxidase. Biochemistry. 47, 978-985

132. Baron, R., Riley, C., Chenprakhon, P., Thotsaporn, K., Winter, R. T., Alfieri, A., Forneris, F., van Berkel, W. J. H., Chaiyen, P., Fraaije, M. W., Mattevi, A., and McCammon, J. A. (2009) Multiple pathways guide oxygen diffusion into flavoenzyme active sites. Proc. Natl. Acad. Sci. U. S. A. 106, 10603-10608

133. Ewing, T. A., Dijkman, W. P., Vervoort, J. M., Fraaije, M. W., and van Berkel, W. J. H. (2014) The oxidation of thiols by flavoprotein oxidases: A biocatalytic route to reactive thiocarbonyls. Angew. Chem. Int. Ed. 53, 13206-13209

134. Doukyu, N. (2009) Characteristics and biotechnological applications of microbial cholesterol oxidases. Appl. Microbiol. Biotechnol. 83, 825-837

135. Gadda, G., Wels, G., Pollegioni, L., Zucchelli, S., Ambrosius, D., Pilone, M. S., and Ghisla, S. (1997) Characterization of cholesterol oxidase from Streptomyces hygroscopicus and Brevibacterium sterolicum. Eur. J. Biochem. 250, 369-376

136. Doukyu, N., and Aono, R. (2001) Cloning, sequence analysis and expression of a gene encoding an organic solvent- and detergent-tolerant cholesterol oxidase of Burkholderia cepacia strain ST200. Appl. Microbiol. Biotechnol. 57, 146-152

137. Doukyu, N., Shibata, K., Ogino, H., and Sagermann, M. (2008) Purification and characterization of Chromobacterium sp. DS-1 cholesterol oxidase with thermal, organic solvent, and detergent tolerance. Appl. Microbiol. Biotechnol. 80, 59-70

138. Fernández de las Heras, L., Mascaraque, V., García Fernández, E., Navarro-Llorens, J. M., Perera, J., and Drzyzga, O. (2011) ChoG is the main inducible extracellular cholesterol oxidase of Rhodococcus sp. strain CECT3014. Microbiol. Res. 166, 403-418

139. Fernández de las Heras, L., Perera, J., and Navarro Llorens, J. M. (2014) Cholesterol to cholestenone oxidation by ChoG, the main extracellular cholesterol oxidase of Rhodococcus ruber strain Chol-4. J. Steroid Biochem. Mol. Biol. 139, 33-34

140. Doukyu, N., and Nihei, S. (2015) Cholesterol oxidase with high catalytic activity from Pseudomonas aeruginosa: Screening, molecular genetic analysis, expression and characterization. J. Biosci. Bioeng. 120, 24-30

141. Doukyu, N., and Aono, R. (1999) Two moles of $\mathrm{O}_{2}$ consumption and one mole of $\mathrm{H}_{2} \mathrm{O}_{2}$ formation during cholesterol peroxidation with cholesterol oxidase from Pseudomonas sp. strain ST-200. Biochem. J. 341, 621-627

142. Sampson, N. S., and Kass, I. J. (1997) Isomerization, but not oxidation, is suppressed by a single point mutation, E361Q, in the reaction catalyzed by cholesterol oxidase. J. Am. Chem. Soc. 119, $855-862$

143. Coulombe, R., Yue, K. Q., Ghisla, S., and Vrielink, A. (2001) Oxygen access to the active site of cholesterol oxidase through a narrow channel is gated by an Arg-Glu pair. J. Biol. Chem. 276, 30435-30441

144. Sagermann, M., Ohtaki, A., Newton, K., and Doukyu, N. (2010) Structural characterization of the organic solvent-stable cholesterol oxidase from Chromobacterium sp. DS-1. J. Struct. Biol. 170, $32-40$

145. Jiang, T., Guo, X., Yan, J., Zhang, Y., Wang, Y., Zhang, M., Sheng, B., Ma, C., Xu, P., and Gao, C. (2017) A bacterial multidomain NAD-independent D-lactate dehydrogenase utilizes flavin adenine dinucleotide and Fe-S clusters as cofactors and quinone as an electron acceptor for Dlactate oxidization. J. Bacteriol. 199, e00342-17 
146. Iijima, M., Munakata, R., Takahashi, H., Kenmoku, H., Nakagawa, R., Kodama, T., Asakawa, Y., Abe, I., Yazaki, K., Kurosaki, F., and Taura, F. (2017) Identification and characterization of daurichromenic acid synthase active in anti-HIV biosynthesis. Plant Physiol. 174, 2213-2230

147. Eniyan, K., Dharavath, S., Vijayan, R., Bajpai, U., and Gourinath, S. (2018) Crystal structure of UDP-N-acetylglucosamine-enolpyruvate reductase (MurB) from Mycobacterium tuberculosis. Biochim. Biophys. Acta - Proteins Proteomics. 1866, 397-406

148. Pei, J., Kim, B.-H., and Grishin, N. V. (2008) PROMALS3D: A tool for multiple protein sequence and structure alignments. Nucleic Acids Res. 36, 2295-2300

149. Katoh, K., and Frith, M. C. (2012) Adding unaligned sequences into an existing alignment using MAFFT and LAST. Bioinformatics. 28, 3144-3146

150. Dereeper, A., Guignon, V., Blanc, G., Audic, S., Buffet, S., Chevenet, F., Dufayard, J.-F., Guindon, S., Lefort, V., Lescot, M., Claverie, J. M., and Gascuel, O. (2008) Phylogeny.fr: Robust phylogenetic analysis for the non-specialist. Nucleic Acids Res. 36, W465-469

151. Letunic, I., and Bork, P. (2011) Interactive Tree Of Life v2: Online annotation and display of phylogenetic trees made easy. Nucleic Acids Res. 39, W475-478

\section{Abbreviations}

4PO: 4-phenol oxidising, ADPS: alkyl-dihydroxyacetone phosphate synthase, AldO: alditol oxidase, ALO: D-arabinono-1,4-lactone oxidase, BBE: berberine bridge enzyme, ChoX: cholesterol oxidase, CKX: cytokinin dehydrogenase, D2HGDH: D-2-hydroxyglutarate dehydrogenase, DCPIP: 2,6dichlorophenolindophenol, DHAP: dihydroxyacetone phosphate, DHCR24: 24-dehydrocholesterol reductase, DLDH: D-lactate dehydrogenase, DPA: decaprenylphosphoryl- $\beta$-D-arabinofuranose, DPR: decaprenylphosphoryl- $\beta$-D-ribofuranose, $\quad$ DPX: decaprenylphosphoryl-2-keto- $\beta$-D-erythropentofuranose, EUGH: eugenol hydroxylase, EUGO: eugenol oxidase, FAD: flavin adenine dinucleotide, FMN: flavin mononucleotide, GALDH: L-galactono-1,4-lactone dehydrogenase, GALO: L-galactono-1,4-lactone oxidoreductase, GLO: D-glucono-1,4-lactone oxidase, GMC: glucose-methanolcholine, GUDH: L-gulono-1,4-lactone dehydrogenase, GUO: L-gulono-1,4-lactone oxidase, NAD $(\mathrm{P}) \mathrm{H}$ : nicotinamide adenine dinucleotide (phosphate), PCMH: para-cresol methylhydroxylase, SSR: sterol side chain reductase, UDP-GlcNAc-EP: uridine diphosphate- $N$-acetylglucosamine enolpyruvate, UDPMurNAc: uridine diphosphate- $N$-acetylmuramic acid, VAO: vanillyl alcohol oxidase 




\title{
3
}

\section{A single loop is essential for the octamerisation of vanillyl alcohol oxidase}

\author{
Tom A. Ewing, Gudrun Gygli and Willem J. H. van Berkel \\ Laboratory of Biochemistry, Wageningen University \& Research, Wageningen, The Netherlands
}

Based on FEBS Journal (2016), 283, 2546-2559

\section{Summary}

The VAO/PCMH family of flavoenzymes is a family of structurally related proteins that catalyse a wide range of reactions. It contains a subfamily of enzymes that catalyse the oxidation of para-substituted phenols (the 4PO subfamily). Among others, this subfamily contains the oxidases vanillyl alcohol oxidase (VAO) and eugenol oxidase (EUGO) and the flavocytochrome dehydrogenases para-cresol methylhydroxylase (PCMH) and eugenol hydroxylase (EUGH). Although they catalyse similar reactions, these enzymes differ in terms of their electron acceptor preference and oligomerisation state. For example, VAO forms homo-octamers that can be described as tetramers of stable dimers, whereas EUGO is exclusively dimeric in solution. A possible explanation for this difference is the presence of a loop at the dimer-dimer interface in VAO that is not present in EUGO. Here, the role played by this loop in determining the quaternary structure of these enzymes is investigated. A VAO variant where the loop was deleted, loopless VAO, exclusively formed dimers. However, introduction of the loop into EUGO was not sufficient to induce its octamerisation. Neither variant displayed major changes in its catalytic properties as compared to the wild-type enzyme. Bioinformatic analysis revealed that the presence of the loop is conserved within putative fungal oxidases of the $4 \mathrm{PO}$ subfamily, but it is never found in putative bacterial oxidases or dehydrogenases. Our results shed light on the molecular mechanism of homooligomerisation of VAO and the importance of oligomerisation for its enzymatic function. 



\section{Introduction}

Homo-oligomerisation of proteins is often of great importance for their function, e.g. by allowing the formation of novel binding sites at the oligomerisation interface, enabling or preventing interactions with other proteins, allosterically modulating enzymatic activity or promoting structural stability $(1,2)$. Evolutionary mechanisms by which homooligomerisation can be achieved include domain swapping, the introduction of structural oligomerisation motifs (e.g. coiled-coils) and amino acid substitutions, insertions or deletions at the interface region. Bioinformatic analyses have revealed that the deletion or insertion of loops at the oligomerisation interface is a common mechanism by which homologous proteins can obtain different oligomerisation states $(3,4)$. Here, we investigate the role of such a loop region in determining the oligomeric state of two flavin-dependent oxidases from the VAO/PCMH flavoprotein family: vanillyl alcohol oxidase (VAO, EC 1.1.3.38) from Penicillium simplicissimum and eugenol oxidase (EUGO) from Rhodococcus jostii RHA1.

The VAO/PCMH flavoprotein family is a group of structurally related flavoenzymes that catalyse a wide range of reactions $(5,6$, Chapter 2$)$. Members of the family share a two-domain structure composed of a conserved flavin-binding domain and a variable substrate-binding domain. Phylogenetic analysis of the VAO/PCMH family revealed that it contains a subfamily of related enzymes that all catalyse the oxidation of para-substituted phenolic compounds at the $\mathrm{C} \alpha$ position of their sidechain, the 4-phenol oxidising (4PO) subfamily (6, Chapter 2). Characterised members of the $4 \mathrm{PO}$ subfamily include vanillyl alcohol oxidase (VAO) from Penicillium simplicissimum, eugenol oxidase (EUGO) from Rhodococcus jostii RHA1, para-cresol methylhydroxylase (PCMH) from Pseudomonas putida and eugenol hydroxylase (EUGH) from Pseudomonas sp. OPS1 and HR199. Although these enzymes all catalyse the oxidation of parasubstituted phenols, they differ significantly in terms of their substrate specificity and quaternary structures.

VAO is a 560 amino acid (64 kDa) protein from the fungus Penicillium simplicissimum (7). It displays the broadest substrate scope of the $4 \mathrm{PO}$ subfamily members, catalysing the oxidation of benzylic alcohols and amines, the demethylation of 4-(methoxymethyl)phenol, the hydroxylation of 4-allylphenols and the hydroxylation or dehydrogenation of 4-alkylphenols $(8,9)$. Reoxidation of the flavin cofactor occurs through its reaction with molecular oxygen, which is converted to hydrogen peroxide. The crystal structure of VAO reveals that it forms homo-octameric structures that can be described as tetramers of stable dimers (10). In solution, VAO constitutes an equilibrium of octamers and dimers, with the octameric form predominating and both forms being catalytically active (11). VAO contains an FAD cofactor that is covalently linked to the protein through an $8 \alpha-N 3$-histidyl-FAD bond. Binding of FAD or ADP stimulates the octamerisation of the enzyme, suggesting that cofactor binding conveys subtle conformational changes to the protein's surface that stabilise the octameric form of the protein $(12,13)$.

EUGO is a 526 amino acid ( $59 \mathrm{kDa}$ ) enzyme from Rhodococcus jostii RHA1 that shares $45 \%$ sequence identity with VAO (14). Like VAO, EUGO contains an $8 \alpha-N 3$-histidyl-bound FAD cofactor and oxidises a range of para-substituted phenolic compounds with molecular oxygen acting as the final electron acceptor (15). However, it displays a number of interesting differences with VAO in terms of its substrate specificity. For example, the catalytic efficiency 
A

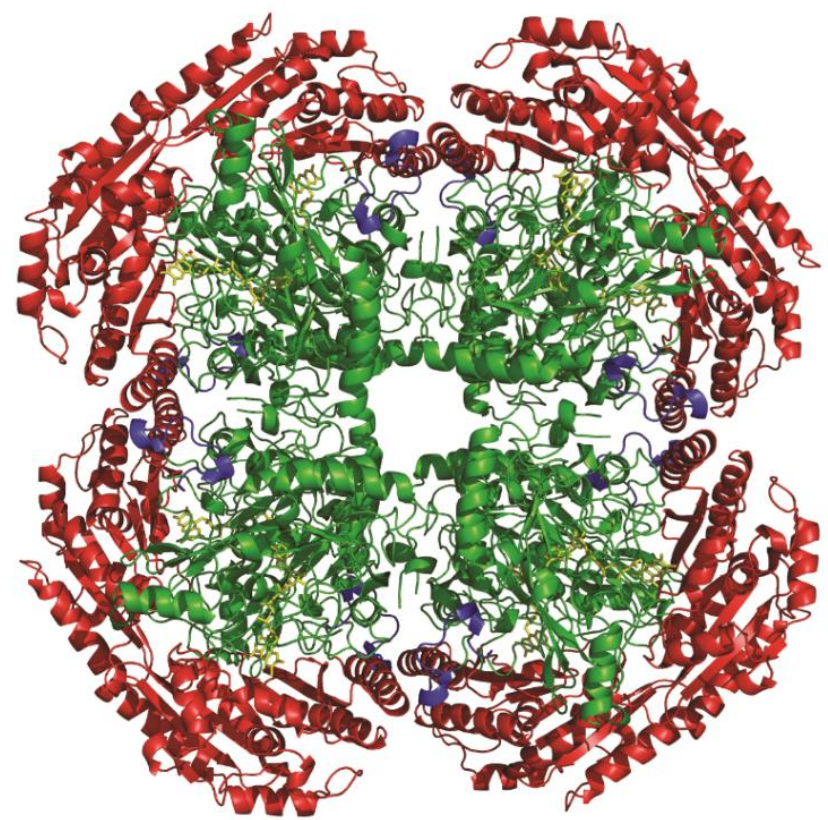

B
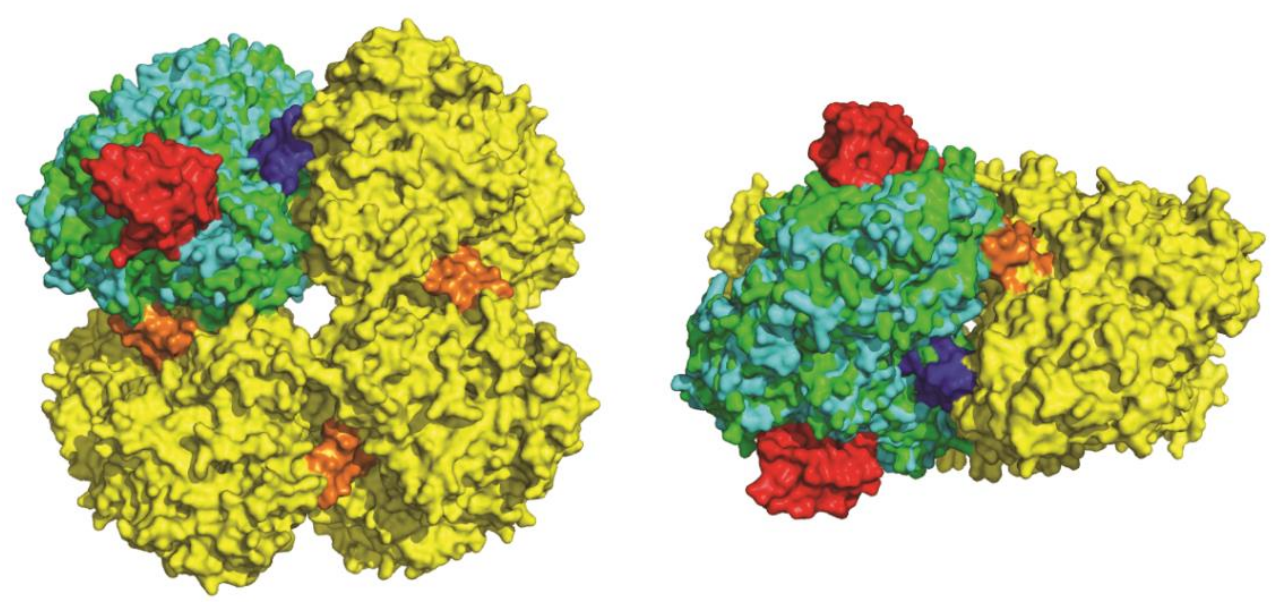

Figure 1: Structures of VAO and PCMH. (A) Cartoon representation of the structure of a VAO octamer showing the prominent role played by the dimer-dimer interacting loop in forming the dimer-dimer interface. The FAD-binding domain is shown in green, the substrate-binding domain in red, the dimerdimer interacting loop in blue and the FAD cofactor as yellow sticks. (B) Surface representation of the structure of a VAO homo-octamer with a PCMH hetero-tetramer (two flavoprotein and two cytochrome subunits) aligned to one dimer of the VAO octamer. This displays the prominent role played by the dimer-dimer interacting loop at the dimer-dimer interface of VAO and the position that the cytochrome subunit of PCMH would occupy in a VAO octamer. The flavoprotein subunits of PCMH are shown in cyan and the cytochrome subunits are shown in red. The VAO dimer to which the flavoprotein subunits of PCMH are aligned is shown in green with the dimer-dimer interacting loop shown in blue. The other VAO dimers are shown in yellow with the dimer-dimer interacting loops in orange. In the left panel, the octamer is positioned as in A, in the right panel it is rotated $90^{\circ}$ out of the plane. The VAO and PCMH structures were downloaded from the PDB website (PDB IDs: 1VAO and 1WVE respectively). The figure was prepared using PyMOL (Schrödinger LLC, New York, NY, USA). 
of the oxidation of vanillyl alcohol (4-hydroxy-3-methoxybenzyl alcohol) by EUGO is significantly higher than that of $\mathrm{VAO}$, while 4-(methoxymethyl)phenol is hardly accepted as a substrate by EUGO. Another remarkable difference between the two enzymes lies in their oligomerisation state. In contrast to the octameric VAO, EUGO is exclusively dimeric in solution (14). The crystal structure of EUGO revealed that it forms dimers that, in terms of their global fold, are highly similar to VAO dimers (15).

PCMH from Pseudomonas putida is a flavocytochrome that catalyses the hydroxylation of short-chain 4-alkylphenols and the oxidation of 4-hydroxybenzyl alcohols $(16,17)$. PCMH is a heterotetramer composed of two $59 \mathrm{kDa}$ flavoprotein subunits and two $9 \mathrm{kDa}$ cytochrome $c$ subunits. The flavoprotein subunits each contain an FAD cofactor, which is covalently bound to the protein through an $8 \alpha$-O-tyrosyl-FAD bond, and form dimers which are structurally similar to VAO dimers $(19,20)$. Unlike VAO and EUGO, PCMH cannot utilise molecular oxygen as an electron acceptor, instead requiring the addition of external electron acceptors.

EUGH is not as well-studied as the enzymes described above. EUGH genes have been identified in Pseudomonas sp. HR199 and OPS1 (21, 22). Based on genetic evidence, both enzymes were proposed to be flavocytochromes that catalyse the oxidation of eugenol to coniferyl alcohol, but neither has been characterised further. However, a eugenol dehydrogenase has been purified from Pseudomonas fluorescens E118 and N-terminal sequencing of the protein revealed that it displays sequence similarity to the eugenol hydroxylases mentioned above (23). The purified enzyme was reported to form heterodimers composed of a $58 \mathrm{kDa}$ flavoprotein subunit and a $10 \mathrm{kDa}$ cytochrome subunit. It oxidises eugenol, 4-alkylphenols and 4-hydroxybenzyl alcohols, relying on the addition of an external electron acceptor for activity. Like PCMH, the flavoprotein subunit of EUGH is predicted to

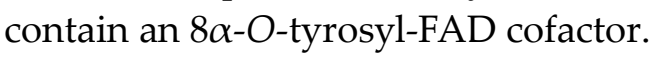

One of the striking differences between the members of the $4 \mathrm{PO}$ subfamily is the oligomerisation state of their flavoprotein subunits. Comparison of the crystal structures of $\mathrm{VAO}, \mathrm{EUGO}$ and PCMH provides a potential explanation for these differences. In octameric $\mathrm{VAO}$, a large proportion of the dimer-dimer interface is formed by a loop consisting of residues 220-235 (Fig. 1). Contacts involving this dimer-dimer interacting loop are responsible for $42 \%$ of the solvent accessible surface area that is buried at the dimer-dimer interface, as calculated using the PDBePISA webserver (24). Such a loop is not present in EUGO or the flavoprotein subunit of PCMH. Thus, the presence of the dimer-dimer interacting loop may explain why VAO forms octamers, whereas EUGO and the flavoprotein subunits of PCMH form dimers.

Here, we investigate the role of this loop in determining the oligomerisation state of VAO and the importance of said oligomerisation state for enzymatic function. To this end, we created a VAO variant where the loop was deleted, loopless VAO (ll-VAO), and a EUGO variant where the loop from VAO was introduced at the corresponding position in EUGO, loop-EUGO (loEUGO). These variants were expressed, purified and characterised in terms of their hydrodynamic and catalytic properties. In addition, a bioinformatic analysis of the $4 \mathrm{PO}$ subfamily was performed to provide information on the conservation of the loop. Our results 
provide insights into the role of the loop region in determining the oligomerisation state of $\mathrm{VAO}$ and the importance of this oligomerisation state for enzymatic function.

\section{Results}

Expression and purification of $l l-V A O$ and lo-EUGO

The 1l-VAO variant was created by deleting residues 220-235 from the sequence of wild-typeVAO (wt-VAO). Additionally, Asp-219 was mutated to glycine, which is found at this position in EUGO. The lo-EUGO variant was created by introducing the sequence of residues 220-235 from VAO (PKRPETMGLKPEDQPW) between Gly-200 and Ser-201 in the sequence of wtEUGO, which corresponds to Asp-219 and Ser-236 respectively in VAO. 1l-VAO and lo-EUGO were both successfully expressed in E. coli and purified. During the purification procedure, 11VAO displayed a tendency to aggregate, forming yellow precipitates when concentrated to concentrations of more than approximately $10 \mu \mathrm{M}$. wt-VAO could be concentrated to concentrations of $>150 \mu \mathrm{M}$ without displaying any such aggregation, suggesting the solubility of the enzyme has been significantly reduced by removal of the loop. SDS-PAGE analysis of the purified variants and wild-type enzymes is shown in Figure 2A. 11-VAO runs as a single band with a relative subunit molecular mass of $59 \mathrm{kDa}$ (theoretical: $62 \mathrm{kDa}$ ), as compared to wt-VAO which runs at a relative molecular mass of $61 \mathrm{kDa}$ (theoretical: $64 \mathrm{kDa}$ ). lo-EUGO runs as a single band with a relative molecular mass of $61 \mathrm{kDa}$ (theoretical: $61 \mathrm{kDa}$ ), as compared to wt-EUGO, which runs at a relative molecular mass of $60 \mathrm{kDa}$ (theoretical: 59 $\mathrm{kDa}$ ). Upon irradiation of the gel with UV light, the bands from all four proteins were fluorescent, demonstrating that all variants contain a covalently bound flavin cofactor.

\section{Hydrodynamic properties}

To evaluate the effect of the introduced mutations on the oligomerisation state of VAO and EUGO, the loop variants and wild-type enzymes were analysed by analytical gel filtration (Fig. $2 \mathrm{~B}+\mathrm{C}$ ). wt-VAO elutes as a single peak with an elution volume of $10.3 \mathrm{~mL}$. This corresponds to an apparent molecular mass of $485 \mathrm{kDa}$, in good agreement with the theoretical molecular mass of a wt-VAO octamer of $510 \mathrm{kDa}$. No peak corresponding to wtVAO dimers was observed under the utilised conditions. For ll-VAO, a single peak was observed with an elution volume of $13.3 \mathrm{~mL}$, yielding an apparent molecular mass of $114 \mathrm{kDa}$. This corresponds well with the theoretical mass of an ll-VAO dimer (123 kDa). No peak was observed at the elution volume expected for octameric ll-VAO. Thus, deletion of the dimerdimer interacting loop leads to the formation of a VAO variant that exclusively forms dimers in solution. wt-EUGO elutes as a single peak with an elution volume of $13.3 \mathrm{~mL}$, yielding an apparent molecular mass of $114 \mathrm{kDa}$, in good agreement with the expected molecular mass of a wt-EUGO dimer (119 kDa). lo-EUGO elutes as a single peak at $13.2 \mathrm{~mL}$, corresponding to an apparent molecular mass of $120 \mathrm{kDa}$. This is close to the theoretical mass of a lo-EUGO dimer $(123 \mathrm{kDa})$. Thus, although the deletion of the loop from VAO is sufficient to prevent its octamerisation, introduction of the loop into EUGO is not sufficient to cause this dimeric enzyme to octamerise. 

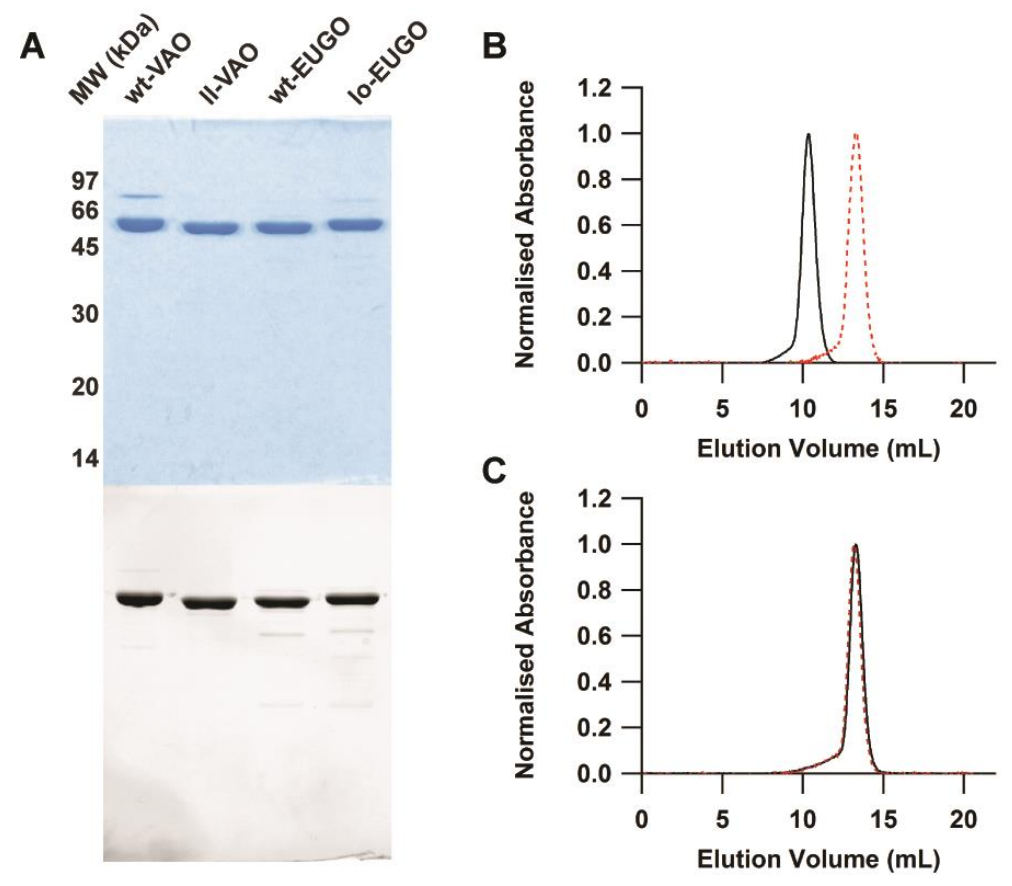

Figure 2: SDS-PAGE and analytical gel filtration analysis of purified VAO and EUGO variants. (A) The top panel shows an SDS-PAGE gel stained with Coomassie Brilliant Blue, the bottom panel shows the same gel with the fluorescence of the covalently bound flavin cofactor visualised by irradiating the gel with UV light after soaking it in an aqueous solution of $7.5 \%$ acetic acid and $5 \%$ ethanol. $(B+C)$ Analytical gel filtration analysis of VAO and EUGO variants. (B) wt-VAO (black solid line) and ll-VAO (red dashed line), (C) wt-EUGO (black solid line) and lo-EUGO (red dotted line). Samples were run on a Superdex $200 \mathrm{HR}$ 10/30 column in $50 \mathrm{mM}$ potassium phosphate buffer, $\mathrm{pH}$ 7.5, $150 \mathrm{mM} \mathrm{KCl}$. Traces show the absorbance at $280 \mathrm{~nm}$ normalised to 1 at the elution maximum.

\section{Spectral properties}

To determine whether the introduced mutations affect the electronic environment of the enzymes' flavin cofactor, absorption spectra were recorded. Figure 3A shows that the visible absorption spectrum of ll-VAO is highly similar to that of wt-VAO, displaying maxima at 437 $\mathrm{nm}$ and $354 \mathrm{~nm}$ as compared to $438 \mathrm{~nm}$ and $354 \mathrm{~nm}$ for wt-VAO. The absorption spectra of wt-EUGO and lo-EUGO (Fig. 3B) are almost indistinguishable from each other, displaying maxima at $366 \mathrm{~nm}$ and 442 and $440 \mathrm{~nm}$ respectively. Thus, neither mutation leads to major changes in the electronic environment of the flavin cofactor. To evaluate whether the introduced mutations affect the secondary structure content of the proteins, far-UV CD spectra were recorded. The CD spectra of wt-VAO and ll-VAO are highly similar (Fig. 3C), as are those of wt-EUGO and lo-EUGO recorded at $20^{\circ} \mathrm{C}$ (Fig. 3D), demonstrating that no major changes in secondary structure content occur due to the introduced mutations.

\section{Catalytic properties}

To evaluate the effect of the introduced mutations on the catalytic properties of VAO and EUGO, the enzyme variants were tested for activity towards a selection of VAO substrates: vanillyl alcohol, which is oxidised to the aldehyde vanillin (4-hydroxy-3methoxybenzaldehyde), eugenol (4-allyl-2-methoxyphenol), which is hydroxylated to 
A

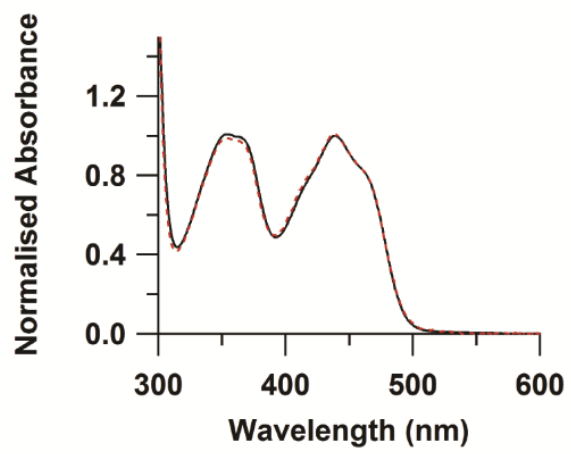

C

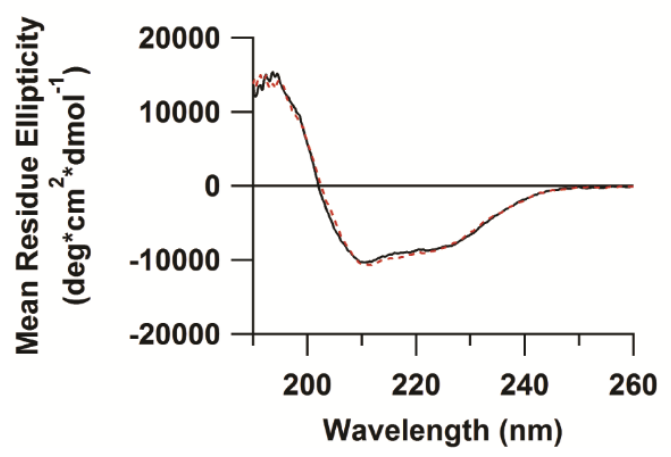

B

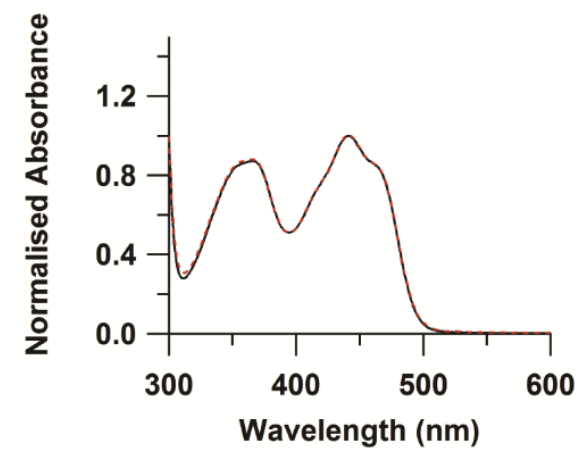

D

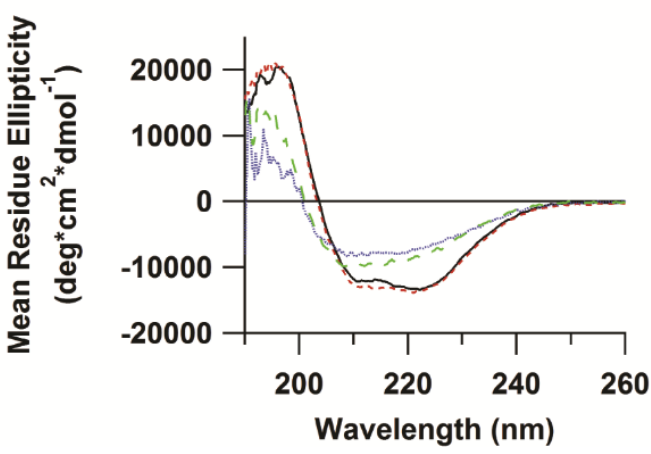

Figure 3: Spectral properties of VAO and EUGO variants. (A) Visible absorption spectra of wt-VAO (solid black line) and ll-VAO (dashed red line) normalised to an absorbance of 1.0 at $439 \mathrm{~nm}$. (B) Visible absorption spectra of wt-EUGO (solid black line) and lo-EUGO (dashed red line) normalised to an absorbance of 1.0 at $441 \mathrm{~nm}$. (C) CD spectra of wt-VAO (solid black line) and ll-VAO (dashed red line). (D) CD spectra of wt-EUGO at $20{ }^{\circ} \mathrm{C}$ (solid black line), at $80{ }^{\circ} \mathrm{C}$ (dotted blue line) and at $20{ }^{\circ} \mathrm{C}$ after 20 min incubation at $80{ }^{\circ} \mathrm{C}$ (long green dashes) and of lo-EUGO at $20{ }^{\circ} \mathrm{C}$ (short red dashes). Spectra were recorded in $50 \mathrm{mM}$ potassium phosphate buffer, $\mathrm{pH} 7.5$, at $25{ }^{\circ} \mathrm{C}(\mathrm{A}+\mathrm{B}), 20{ }^{\circ} \mathrm{C}(\mathrm{C})$, or 20 or $80{ }^{\circ} \mathrm{C}$ as indicated (D).

coniferyl alcohol [4-(3-hydroxyprop-1-en-1-yl)-2-methoxyphenol], and 4-ethylphenol, which is either hydroxylated or dehydrogenated, yielding a mixture of 1-(4'-hydroxyphenyl)ethanol and 4-vinylphenol. The steady-state kinetic parameters for these enzymatic oxidation reactions are given in Table 1 . The catalytic efficiencies for the reactionswith 1l-VAO are somewhat lower than those with wt-VAO. This is primarily due to an increase in the $K_{\mathrm{m}}$ values, which are 2-5 times higher than those of the wild-type enzyme. The $k_{\text {cat }}$ values are almost identical, never differing by more than a factor two. lo-EUGO displays similar catalytic properties to wt-EUGO. No activity was found with 4-ethylphenol, while the catalytic efficiencies with vanillyl alcohol and eugenol were slightly changed.

\section{Thermostability}

To determine whether the introduced mutations affect the stability of EUGO and VAO, thermal unfolding of the proteins was monitored by following changes in secondary structure using CD spectroscopy. For each variant, a single transition was observed (Fig. 4). With wt$\mathrm{VAO}$ and ll-VAO, the $\mathrm{CD}$ signal went to zero upon heating and insoluble aggregates were 
Table 1: Steady-state kinetic parameters for the oxidation of a set of VAO substrates by VAO and EUGO variants measured at $25^{\circ} \mathrm{C}$ in $50 \mathrm{mM}$ potassium phosphate buffer, $\mathrm{pH}$ 7.5. ND: not determined due to low reaction rate (no significant decrease in oxygen concentration was observed in reaction mixtures containing $2.3 \mu \mathrm{M}$ enzyme and $200 \mu \mathrm{M}$ 4-ethylphenol).

\begin{tabular}{|c|c|c|c|c|c|}
\hline & & wt-VAO & 11-VAO & wt-EUGO & lo-EUGO \\
\hline \multirow{3}{*}{ Vanillyl Alcohol } & $k_{\text {cat }}\left(\mathrm{s}^{-1}\right)$ & $2.6 \pm 0.1$ & $3.0 \pm 0.1$ & $7.1 \pm 0.2$ & $4.7 \pm 0.1$ \\
\hline & $K_{\mathrm{m}}(\mu \mathrm{M})$ & $149 \pm 6$ & $676 \pm 54$ & $79 \pm 8$ & $42 \pm 4$ \\
\hline & $k_{\text {cat }} / K_{\mathrm{m}}\left(\mathrm{s}^{-1} \mathrm{mM}^{-1}\right)$ & 17 & 4 & 90 & 112 \\
\hline \multirow[t]{3}{*}{ Eugenol } & $k_{\text {cat }}\left(\mathrm{s}^{-1}\right)$ & $11 \pm 1$ & $15 \pm 1$ & $2.9 \pm 0.1$ & $1.8 \pm 0.1$ \\
\hline & $K_{\mathrm{m}}(\mu \mathrm{M})$ & $3.9 \pm 0.2$ & $9.8 \pm 1$ & $2.2 \pm 0.2$ & $4.7 \pm 0.4$ \\
\hline & $k_{\text {cat }} / K_{\mathrm{m}}\left(\mathrm{s}^{-1} \mathrm{mM}^{-1}\right)$ & 2821 & 1531 & 1318 & 383 \\
\hline \multirow{3}{*}{ 4-Ethylphenol } & $k_{\text {cat }}\left(\mathrm{s}^{-1}\right)$ & $2.6 \pm 0.1$ & $1.5 \pm 0.1$ & ND & ND \\
\hline & $K_{\mathrm{m}}(\mu \mathrm{M})$ & $12 \pm 1$ & $31 \pm 1$ & ND & ND \\
\hline & $k_{\text {cat }} / K_{\mathrm{m}}\left(\mathrm{s}^{-1} \mathrm{mM}^{-1}\right)$ & 217 & 48 & ND & ND \\
\hline
\end{tabular}

observed in the sample after returning it to room temperature. For wt-EUGO and lo-EUGO, however, a significant $\mathrm{CD}$ signal was still observed even after the transition region, indicating that the proteins retain secondary structure at this point, and no insoluble aggregates were observed. The CD spectrum of wt-EUGO at $80{ }^{\circ} \mathrm{C}$ confirms that the protein retains secondary structure at this temperature, with a clear negative $C D$ signal being observed in the wavelength range from 200 to $240 \mathrm{~nm}$ (Fig. 3D). However, the shape of the spectrum is significantly different from that recorded at $20^{\circ} \mathrm{C}$. In particular, the peak that is observed at $222 \mathrm{~nm}$ in the spectrum of wt-EUGO at $20{ }^{\circ} \mathrm{C}$ is no longer present when the spectrum is recorded at $80^{\circ} \mathrm{C}$. After a $20 \mathrm{~min}$ treatment at $80^{\circ} \mathrm{C}$, wt-EUGO did not display any enzymatic activity and ran in the void volume of a Superdex 200 size exclusion column. A CD spectrum recorded at $20{ }^{\circ} \mathrm{C}$ after incubating wt-EUGO at $80{ }^{\circ} \mathrm{C}$ for $20 \mathrm{~min}$ displays a similar shape to the wt-EUGO spectrum recorded at $80{ }^{\circ} \mathrm{C}$ (Fig. 3D). Thus, it seems that wt-EUGO forms inactive, soluble aggregates upon temperature-induced unfolding and that this temperatureinduced aggregation is irreversible. Similar results were obtained with lo-EUGO. To evaluate whether the introduced mutations affect the thermostability of the enzymes, thermal midpoints of unfolding were determined by fitting a sigmoidal curve to the recorded thermal denaturation data (Table 2). 1l-VAO displays slightly impaired thermostability compared to wt-VAO, with its $T_{\mathrm{m}}$ lying $1.9^{\circ} \mathrm{C}$ lower. lo-EUGO is significantly less stable than wt-EUGO, with its $T_{\mathrm{m}}$ lying $9.3^{\circ} \mathrm{C}$ lower. Apparently, the introduction of the loop at the surface of the enzyme leads to a significant drop in its conformational stability.

\section{Bioinformatics}

In order to investigate the conservation of the dimer-dimer interacting loop within the 4PO subfamily, a bioinformatic search for homologues of VAO, EUGO, PCMH and EUGH was performed. This led to the identification of 46 unique sequences of putative $4 \mathrm{PO}$ subfamily members (including query sequences). A phylogenetic tree of these sequences is shown in Figure 5. This tree reveals that the $4 \mathrm{PO}$ subfamily can be divided into four main groups of related enzymes. The first two groups, which are putative dehydrogenases (PCMH-like and 
wt-VAO

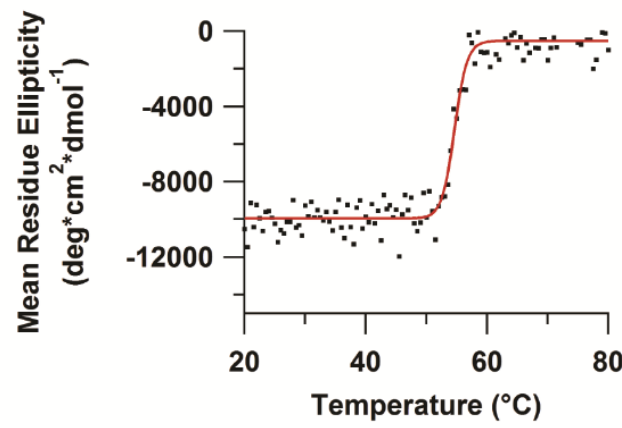

wt-EUGO

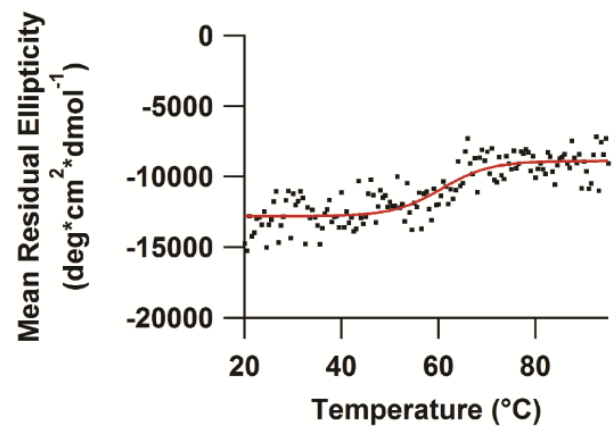

II-VAO

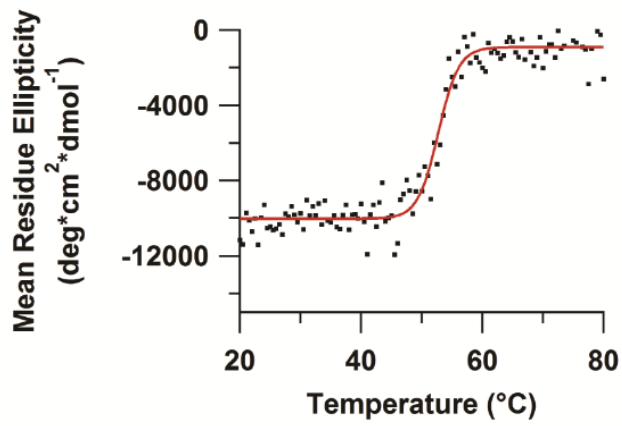

IO-EUGO

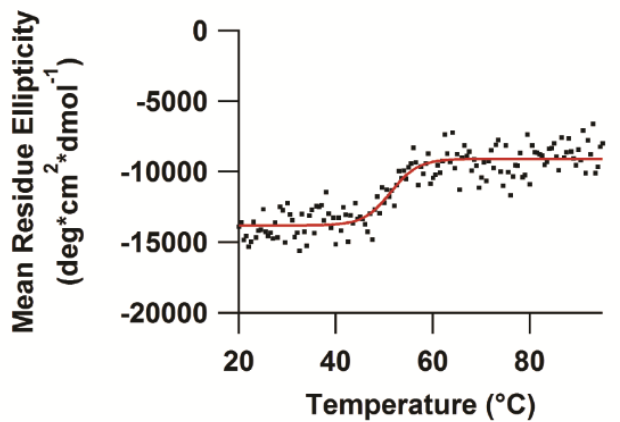

Figure 4: Thermal denaturation curves of VAO and EUGO variants. $1 \mu \mathrm{M}$ enzyme was subjected to a temperature gradient in $50 \mathrm{mM}$ potassium phosphate buffer, $\mathrm{pH}$ 7.5, and unfolding was followed by monitoring the CD signal at $220 \mathrm{~nm}$. Data points are shown in black (average of two measurements) and the sigmoidal fits used to determine $T_{\mathrm{m}}$ in red.

Table 2: Thermal midpoints of unfolding of VAO and EUGO variants determined by CD spectroscopy.

\begin{tabular}{|ll|}
\hline Enzyme & $\boldsymbol{T}_{\mathrm{m}}\left({ }^{\circ} \mathrm{C}\right)$ \\
\hline wt-VAO & $54.6 \pm 0.1$ \\
ll-VAO & $52.7 \pm 0.1$ \\
wt-EUGO & $60.6 \pm 1.4$ \\
lo-EUGO & $51.3 \pm 0.7$ \\
\hline
\end{tabular}

EUGH-like), almost exclusively contain sequences from proteobacteria, the one exception being a putative PCMH from Nitrospira defluvii (WP_013246640.1), a Gram-negative bacterium from the phylum Nitrospirae. The other two groups are putative oxidases, one group that is most closely related to EUGO and exclusively contains sequences from actinobacteria, and one group that is most closely related to $\mathrm{VAO}$, all of which are from ascomycetous fungi. When the search was performed with EUGO as a query sequence, a single sequence was found from the proteobacterium Cyclocasticus sp. (YP_006837036.1) that did not belong to any of the identified groups.

Analysis of the sequence region containing the dimer-dimer interacting loop revealed that none of the identified bacterial sequences carry the loop, whereas all of the fungal sequences 


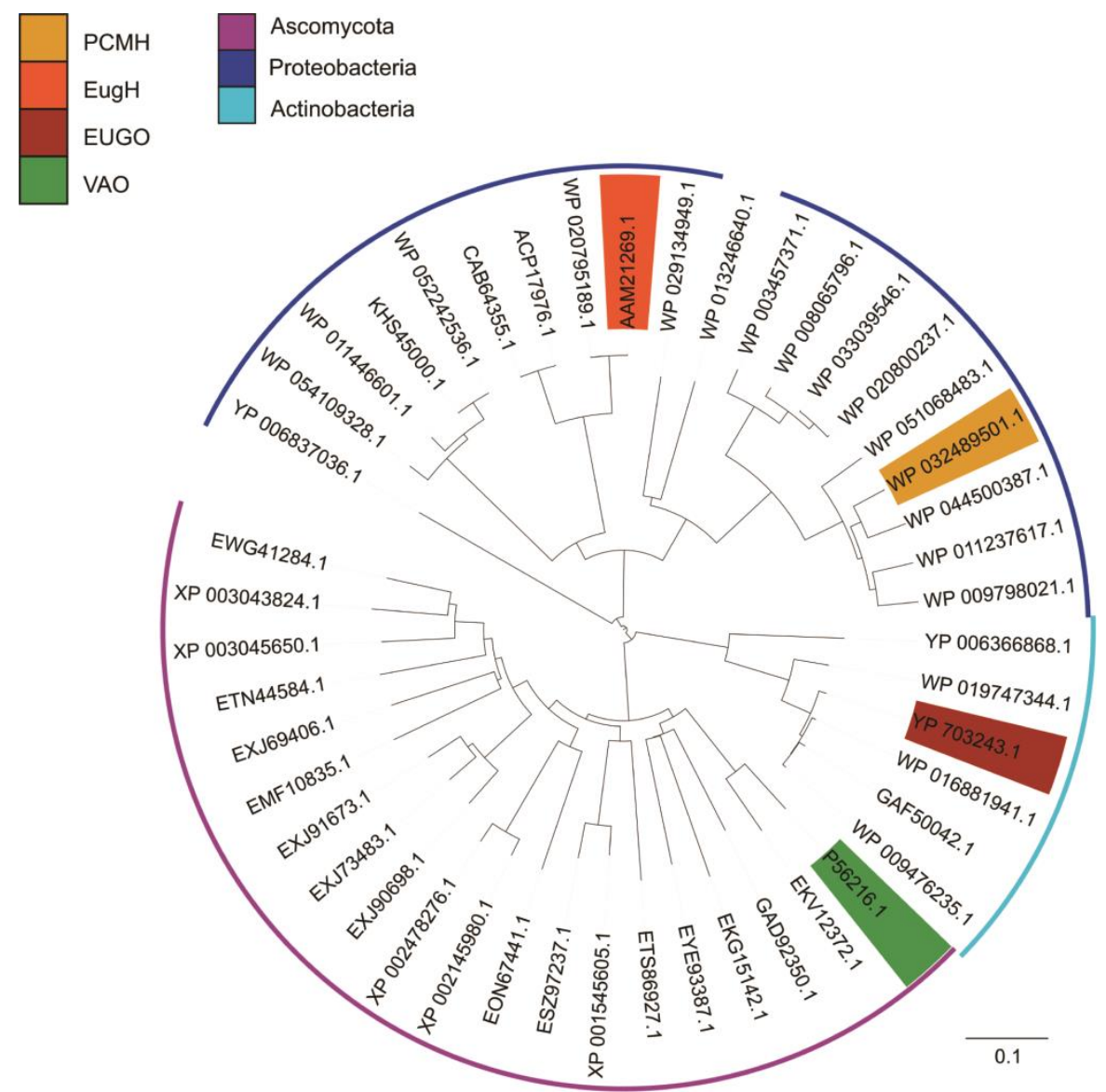

Figure 5: Phylogenetic tree of protein sequences of homologues of VAO from Penicillium simplicissimum (P56216.1, highlighted in green), EUGO from Rhodococcus jostii RHA1 (YP_703243.1, highlighted in red), PCMH from Pseudomonas putida (WP_032489501.1, highlighted in yellow) and EUGH from Pseudomonas sp. OPS1 (AAM21269.1, highlighted in orange). Accession codes for the sequences identified from several sources (GenBank, RefSeq and UniProt databases) are shown. The blue, cyan and purple lines indicate sequences from proteobacteria, actinobacteria and ascomycetous fungi respectively. YP_006837036.1 was used as an outgroup. Branch length is proportional to the number of substitutions, as indicated by the bar ( 0.1 substitutions per sequence position).

do contain a loop region (Fig. 6). No conserved motif is present in the sequences of the loop itself and the loops vary slightly in length. However, at least two proline residues are always present in the loop as well as a group of polar residues. The regions flanking the loop are highly conserved. The region opposite the loop in the crystal structure of VAO from $P$. simplicissimum was also analysed (Fig. 6). The loop is in direct contact with an $\alpha$-helix formed by residues 478-498. The inter-subunit loop-helix contact is primarily formed by seven residues: Lys-221, Pro-223 and Met-226 from the loop and Arg-482, Gln-485, Trp-486 and Thr490 from the helix (Fig. 7). The $\varepsilon$-amino group of Lys-221 is positioned within hydrogen bonding distance of the backbone carbonyl atoms of Gln-485 and Trp-486 and the hydroxyl group of Thr-490, providing a potential driving force for octamerisation. In addition, the hydrophobic side chains of Pro-223, Met-226, and Trp-486 and the aliphatic part of the side chain of Arg-482 are (partially) buried within the dimer-dimer interface. Analysis of these interacting residues in the multiple sequence alignment reveals that they are generally not strongly conserved. In the context of the fact that incorporation of the loop into EUGO does 


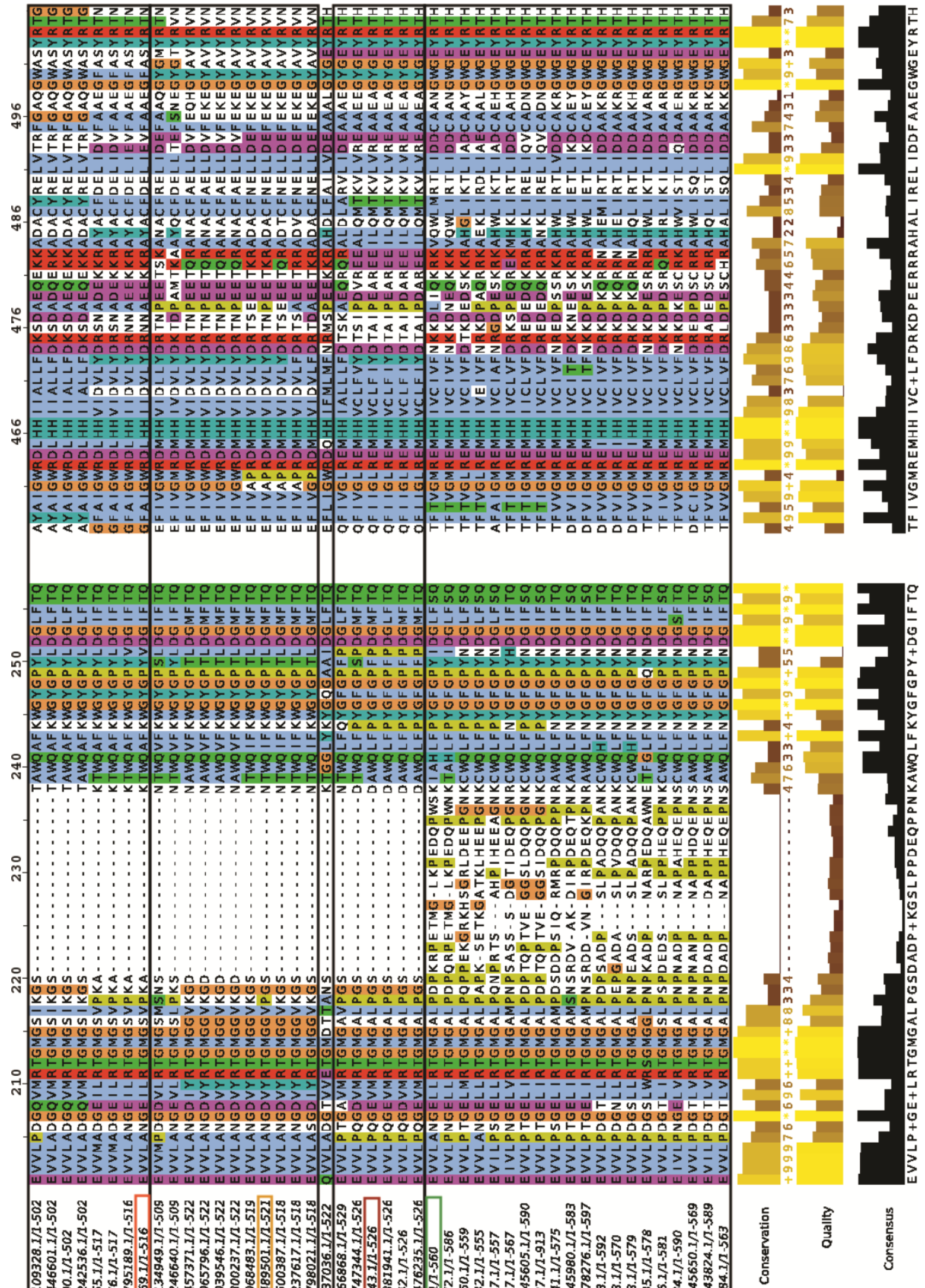

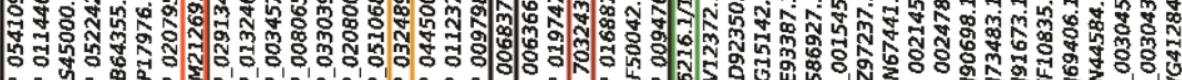

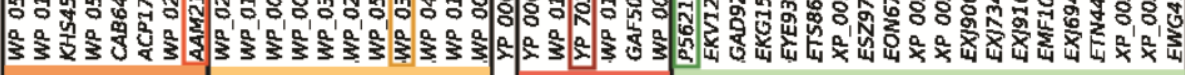


Figure 6: Excerpts from the multiple sequence alignment of 4PO enzymes. Coloured boxes indicate the sequences of $P$. simplicissimum VAO (green), R. jostii RHA1 EUGO (red), P. putida PCMH (yellow) and Pseudomonas sp. OPS1 EUGH (orange). The coloured bars at the bottom of the figure indicate the division into enzyme groups (VAO-like: green, EUGO-like: red, PCMH-like: yellow, EUGH-like: orange) based on the phylogenetic tree shown in Figure 5. The numbers behind the sequence IDs show the lengths of the aligned sequences. The bar charts below the alignment show the conservation and quality scores at each position, following the "Alignment Conservation Annotation" used by Jalview (25). Figure was prepared with Jalview, residue numbering was changed to match VAO. Residues are coloured according to the ClustalX colour scheme.

not lead to its octamerisation, it is interesting to note that Arg-482, Gln-485, Trp-486 and Thr490 are not conserved in EUGO, being replaced by glutamic acid, leucine, glutamine and valine respectively.

\section{Discussion}

Our results demonstrate that the loop formed by residues 220-235 in VAO plays a crucial role in determining the oligomerisation state of this enzyme. Deletion of the loop leads to a VAO variant that is exclusively dimeric in solution, even under conditions where wt-VAO only forms octamers. The ll-VAO variant does not differ much from wt-VAO in terms of its in vitro enzymatic properties, displaying similar thermostability and spectral and catalytic properties. The major difference between the two enzymes, other than their oligomerisation state, is their solubility. Unlike wt-VAO, ll-VAO displays a strong tendency to aggregate. The decreased solubility of ll-VAO may be due to hydrophobic residues that are normally buried in the dimer-dimer interface becoming solvent exposed in the dimeric variant, e.g. Trp-486, which is in contact with the loop from an opposite dimer in the octameric structure of VAO.

Although deletion of the loop was sufficient to cause VAO to dimerise, its introduction into EUGO was not sufficient to induce its octamerisation. Introduction of the loop from VAO at the surface of EUGO did not significantly alter the enzyme's catalytic properties. It did, however, lead to a significant decrease in thermostability, with the $T_{\mathrm{m}}$ of lo-EUGO lying $9.3^{\circ} \mathrm{C}$ lower than that of the wild-type enzyme.

As the introduction of the loop was not sufficient to cause EUGO to octamerise, other factors must also be important in enabling the octamersiation of VAO. These may include specific contacts between residues in the dimer-dimer interacting loop and residues in the region opposite it. Although the loop and the face of the helix formed by residues 478-498 that interacts with it primarily contain polar residues, three relatively hydrophobic residues, Pro223, Met-226 and Trp-486 are positioned at the point where the loop and helix make direct contact (Fig. 7). Burial of these hydrophobic residues at the dimer-dimer interface may provide an important driving force for octamerisation and is consistent with the fact that VAO dimerises at low ionic strength (11). Hydrogen bonds between the $\varepsilon$-amino group of Lys-221 and the main chain carbonyl groups of Gln-485 and Trp-486 and the hydroxyl group of Thr490 may also contribute to promoting octamerisation. Another factor that may be involved in promoting the octamerisation of $\mathrm{VAO}$ is interactions involving the extreme C-terminus of the enzyme (residues 554-560). This region of the protein is in contact with an $\alpha$-helix formed by residues 519-535 from one monomer of the opposite dimer and a turn formed by residues 203- 
209 from the other monomer of the opposite dimer. In this context it is interesting to note that the C-termini of VAO (residues 550-560) and EUGO (residues 518-526) are not conserved.

Bioinformatic analysis of the $4 \mathrm{PO}$ subfamily demonstrates that it can be divided into four groups of enzymes, fungal VAO-like enzymes and bacterial EUGO-like, PCMH-like and EUGH-like enzymes. All of the VAO-like enzymes contain a dimer-dimer interacting loop, whereas none of the bacterial enzymes contain it. Incorporation of the loop may have been an evolutionary mechanism by which these enzymes obtained differing oligomerisation states. Studies into the oligomeric nature of other members of the VAO-like group are necessary to evaluate this further. In this context, it should be noted that an enzyme has been isolated from the ascomycetous fungus Byssochlamys fulva V107 that displayed VAO activity, yet was reported to be exclusively dimeric in solution (26). However, no sequence or structural information is available on this protein.

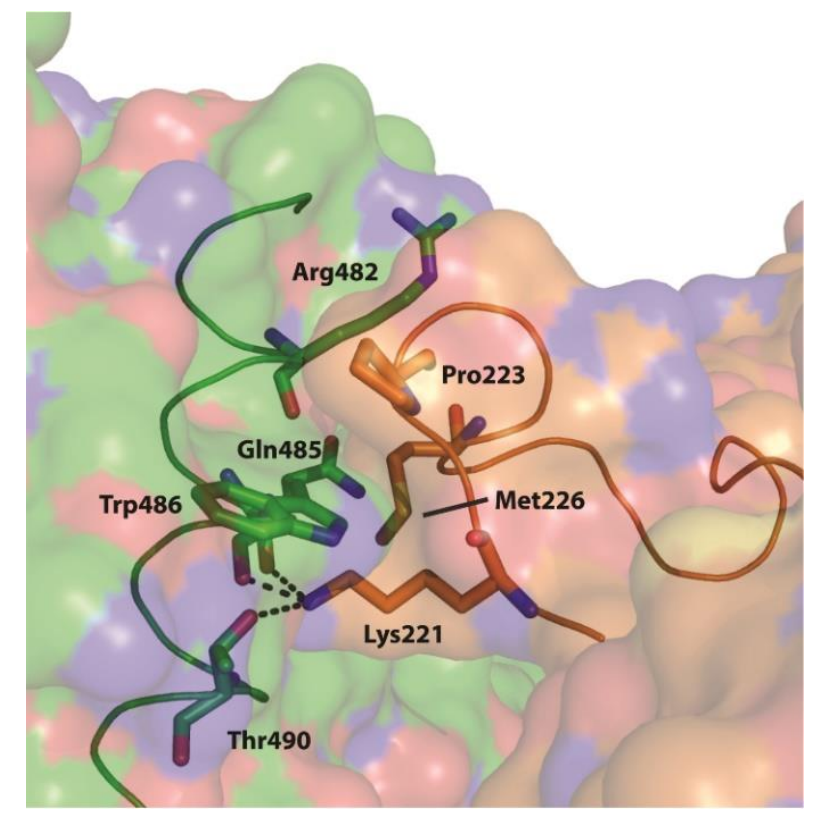

Figure 7: Inter-subunit contacts between the dimer-dimer interacting loop and the helix opposite it in a VAO octamer. The dimer in which the helix is shown has green carbon atoms and the dimer in which the loop is shown has orange carbon atoms. Nitrogen atoms are shown in blue and oxygen atoms in red. Lys-221, Pro-223, Met-226, Arg-482, Gln-485, Trp-486 and Thr-490, the residues that are primarily involved in inter-dimer contacts, are shown in stick representation. The potential hydrogen bonds between the $\varepsilon$-amino group of Lys-221 and the backbone carbonyl atoms of Gln-485 and Trp-486 and the hydroxyl group of Thr-490 are shown as black dashed lines. The VAO structure was downloaded from the PDB website (PDB ID: 1VAO). The figure was prepared using PyMOL (Schrödinger LLC, New York, NY, USA).

The exact role of the differing oligomerisation states of the $4 \mathrm{PO}$ enzymes is yet to be elucidated. The dimeric ll-VAO displays only slightly impaired catalytic efficiencies and thermostability as compared to the wild-type enzyme. The fact that ll-VAO still readily oxidises 4-ethylphenol, which is a very poor substrate for EUGO, demonstrates that the observed differences in substrate specificity between VAO and EUGO are not caused by their different oligomerisation states. It seems unlikely, therefore, that VAO evolved so as to be able to form octamers to directly modulate its enzymatic properties. In vivo, octamerisation may be of 
importance in modulating the interactions of $\mathrm{VAO}$ with other proteins. A possible function of octamerisation could be to prevent the binding of a cytochrome subunit such as is found in PCMH and EUGH. However, comparison of the structures of a PCMH heterotetramer and a $\mathrm{VAO}$ octamer demonstrates that the cytochrome binding site in PCMH lies far from the area that corresponds to the dimer-dimer interface of VAO, suggesting that preventing cytochrome binding is not the function of octamerisation of VAO (Fig. 1B). Another possibility is that octamerisation is simply an efficient way to cluster the active VAO dimers so as to achieve a high concentration of VAO molecules in a limited space. Alternatively, the oligomerisation state of VAO may be of importance in maintaining its subcellular localisation. VAO is not strictly compartmentalised in vivo, being localised to both the peroxisomes and cytosol of $P$. simplicissimum (27). Transport of proteins into peroxisomes typically takes place posttranslationally and is initiated by the recognition of a C-terminal peroxisomal targeting sequence. The sequence of the extreme C-terminus of VAO (WKL) is a putative variant of the canonical PTS1 peroxisomal targeting sequence (SKL). As in the octameric structure of VAO its C-terminus is buried at the dimer-dimer interface, it can be expected that VAO could only be transported into peroxisomes in its dimeric form, raising the intriguing possibility that the ability of VAO to form both dimers and octamers could allow it to maintain its dual localisation. More detailed studies on the physiological function of VAO are required in order to fully understand the role of octamer formation in vivo.

In conclusion, our results demonstrate that the dimer-dimer interacting loop determines the oligomerisation state of $\mathrm{VAO}$, with deletion of the loop changing the oligomerisation state of $\mathrm{VAO}$ from predominantly octameric to exclusively dimeric without significantly affecting the catalytic properties of the enzyme. All identified fungal 4PO subfamily members contain the loop, while all bacterial $4 \mathrm{PO}$ subfamily members do not. Octamerisation is not crucial for the enzymatic activity of $\mathrm{VAO}$, but may have other roles in vivo such as promoting enzyme solubility, enabling or preventing interactions with other proteins or modulating the subcellular localisation of the enzyme.

\section{Materials and methods}

\section{Materials}

Vanillyl alcohol, eugenol, and 4-ethylphenol were from Sigma-Aldrich (St Louis, MO, USA). All other chemicals were from commercial sources and of the purest grade available. The pBAD-EUGO plasmid was a kind gift from Prof. Dr. Marco Fraaije (University of Groningen, The Netherlands).

\section{Creation of the ll-VAO and lo-EUGO constructs}

A construct encoding for 1l-VAO was created by PCR amplification from the pBC11 plasmid (28), a pEMBL19 derivative containing the gene encoding for wt-VAO. First, two constructs were PCR amplified. The first consisted of the $105 \mathrm{bp}$ upstream from the VAO gene, the first $657 \mathrm{bp}$ of the VAO gene and a 3' extension consisting of bp 706 to 728 . The construct also contained a single point mutation in order to mutate Asp-219 to glycine. This construct was amplified using the following primers: forward primer: CAATTTCACACAGGAAACAGC 
TATGACC, reverse primer: GGAAACAGATGAGCGATTTTGCTACCAGGTAGAGCCCCC ATGCCAG. The second construct consisted of bp 635-657 of the VAO gene at the 5' end, again with a point mutation to change Asp-219 into glycine, followed by bp 706-1683 of the VAO gene and finally $147 \mathrm{bp}$ from the plasmid sequence downstream of the VAO gene. This construct was PCR amplified using the following primers: forward primer: CTGGCATGGGGGCTCTACCTGGTAGCAAAATCGCTCATC TGTTTCC, reverse primer: CCAGTCACGACGTTGTAAAACGACGG. Subsequently, the construct encoding for 11-VAO was PCR amplified from the two overlapping constructs using the forward primer for the first construct and the reverse primer for the second construct. This construct was digested with HindIII and SacI and ligated into HindIII-SacI digested pBC11, yielding pBC11-ll-VAO. The pBAD-lo-EUGO plasmid was created using a similar strategy. Two overlapping constructs were PCR amplified from the pBAD-EUGO plasmid (14). The first construct consisted of the $128 \mathrm{bp}$ directly upstream of the EUGO gene, the first $600 \mathrm{bp}$ of the EUGO gene and a $3^{\prime}$ extension consisting of bp 658 to 689 from the VAO gene. This construct was amplified using the following primers: forward primer: CGGCGTCACACTTTGCTATGCCATAGC, reverse primer: GGCTTTAGCCCCATCGTTTCGGGACGTTTAGGGCCGGGCAGTGCGCCCATCC CGGTG. The second construct consisted of bp 673-705 from the VAO gene, followed by bp 601-1581 of the EUGO gene and $100 \mathrm{bp}$ from the pBAD-EUGO plasmid downstream of the EUGO gene. This construct was PCR amplified using the following primers: forward primer: CGATGGGGCTAAAGCCAGAAGACCAGCCATGGAGCGACGCGTGGCAATTGTTCCCC T, reverse primer: GCCAAGCTGGAGAC CGTTTAAACTCAATG. The construct encoding loEUGO was subsequently PCR amplified from the two overlapping constructs using the forward primer for the first construct and the reverse primer for the second construct. The formed construct was digested with HindIII and NdeI and ligated into HindIII-NdeI digested pBAD-EUGO, yielding pBAD-lo-EUGO.

\section{Protein expression and purification}

wt-VAO was expressed from pBC11 in BL21 E. coli. Cells were grown at $37^{\circ} \mathrm{C}$ in LB medium (Duchefa Biochemie, Haarlem, The Netherlands) supplemented with $100 \mu \mathrm{g} / \mathrm{mL}$ ampicillin to an $\mathrm{OD}_{600}$ of 0.6 , after which protein expression was induced by adding $0.25 \mathrm{mM}$ IPTG and cells were grown overnight at $37^{\circ} \mathrm{C}$. Subsequently, wt-VAO was purified essentially as described previously (29) and transferred into $50 \mathrm{mM}$ potassium phosphate buffer, $\mathrm{pH} 7.5$, for storage. ll-VAO was expressed and purified according to a modified version of this protocol. BL21 E. coli containing the pBC11-11-VAO plasmid was grown in TB medium (Difco, Becton Dickinson, Franklin Lake, NJ, USA) supplemented with $100 \mu \mathrm{g} / \mathrm{mL}$ ampicillin at $37{ }^{\circ} \mathrm{C}$ until the $\mathrm{OD}_{600}$ of the culture was 0.5 . Subsequently, protein expression was induced by the addition of $0.25 \mathrm{mM}$ IPTG and the cultures were grown overnight at $30{ }^{\circ} \mathrm{C}$. The cells were then harvested by centrifugation $\left(2800 \mathrm{~g}, 20 \mathrm{~min}, 4{ }^{\circ} \mathrm{C}\right.$ ) and resuspended in $50 \mathrm{mM}$ potassium phosphate buffer, $\mathrm{pH} 7.0$, supplemented with $0.5 \mathrm{mM} \mathrm{MgSO}_{4}, 0.5 \mathrm{mM}$ dithiothreitol and two cOmplete $^{\mathrm{TM}}$ protease inhibitor pills (Roche, Basel, Switzerland) and $1 \mathrm{mg}$ DnaseI (Roche) per $50 \mathrm{~mL}$. The resuspended cells were lysed by passing them three times through a pre-cooled SLM Aminco French pressure cell. Cell debris was removed by centrifugation $(39,000 g, 45$ min, $4{ }^{\circ} \mathrm{C}$ ). Subsequently, protamine sulphate was added to the supernatant to a final concentration of $0.5 \%(\mathrm{w} / \mathrm{v})$, following which the precipitate was removed by centrifugation $\left(39,000 \mathrm{~g}, 20 \mathrm{~min}, 4{ }^{\circ} \mathrm{C}\right)$. $\left(\mathrm{NH}_{4}\right)_{2} \mathrm{SO}_{4}$ was added to the supernatant to $35 \%$ saturation and the 
precipitate was pelleted by centrifugation $\left(39,000 \mathrm{~g}, 20 \mathrm{~min}, 4^{\circ} \mathrm{C}\right)$. The pellet was dissolved in

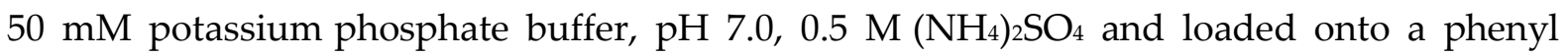
sepharose column equilibrated with the same buffer. After elution with a gradient from 0.5 to $0 \mathrm{M}\left(\mathrm{NH}_{4}\right)_{2} \mathrm{SO}_{4}$ in $50 \mathrm{mM}$ potassium phosphate buffer, $\mathrm{pH} 7.0$, 1l-VAO was still bound to the column. ll-VAO was then eluted with water which was subsequently adjusted to $20 \mathrm{mM}$ potassium phosphate buffer, $\mathrm{pH}$ 7.0. This sample was loaded onto a hydroxyapatite column equilibrated with the same buffer. After loading, bound proteins were eluted using a gradient from 20 to $1000 \mathrm{mM}$ potassium phosphate buffer, $\mathrm{pH}$ 7.0. Fractions containing ll-VAO were pooled and the buffer was exchanged to $50 \mathrm{mM}$ potassium phosphate buffer, $\mathrm{pH} 7.5$, by dialysis. The pooled fractions were run over a Q-sepharose column in $50 \mathrm{mM}$ potassium phosphate buffer, $\mathrm{pH}$ 7.5. The flow-through from this column was collected and contained pure ll-VAO.

wt-EUGO and lo-EUGO were expressed in TOP10 E. coli containing the appropriate plasmid. Cells were grown at $37^{\circ} \mathrm{C}$ in TB medium supplemented with $100 \mu \mathrm{g} / \mathrm{mL}$ ampicillin until the OD 600 of the culture was 0.6 . Then, protein expression was induced by adding $0.02 \%(\mathrm{w} / \mathrm{v}) \mathrm{L}-$ arabinose and cells were grown overnight at $30{ }^{\circ} \mathrm{C}$. Subsequently, cells were harvested by centrifugation $\left(2800 \mathrm{~g}, 20 \mathrm{~min}, 4^{\circ} \mathrm{C}\right.$ ) and resuspended in $20 \mathrm{mM}$ potassium phosphate buffer, $\mathrm{pH}$ 7.1, supplemented with $0.5 \mathrm{mM} \mathrm{MgSO}_{4}$ and two cOmplete ${ }^{\mathrm{TM}}$ protease inhibitor pills (Roche) and $1 \mathrm{mg}$ DnaseI (Roche) per $50 \mathrm{~mL}$. The resuspended cells were lysed by passing them three times through a pre-cooled SLM Aminco French pressure cell and cell debris was removed by centrifugation $\left(39,000 \mathrm{~g}, 45 \mathrm{~min}, 4^{\circ} \mathrm{C}\right)$. The obtained supernatant was loaded onto a Q-sepharose column equilibrated with $20 \mathrm{mM}$ potassium phosphate buffer, $\mathrm{pH}$ 7.1. Bound proteins were subsequently eluted using a linear gradient from 0 to $1 \mathrm{M} \mathrm{KCl}$. Fractions containing the protein of interest were pooled and run over a Superdex 200 column in $20 \mathrm{mM}$ potassium phosphate buffer, $\mathrm{pH} 7.1,150 \mathrm{mM} \mathrm{KCl}$. Subsequently, $\mathrm{KCl}$ was removed by dialysis and the samples were loaded onto a hydroxyapatite column in $20 \mathrm{mM}$ potassium phosphate buffer, $\mathrm{pH}$ 7.1. Bound proteins were eluted using a linear gradient from 20 to $1000 \mathrm{mM}$ potassium phosphate buffer, $\mathrm{pH}$ 7.1. Fractions containing the proteins of interest were pooled and dialysed to $20 \mathrm{mM}$ potassium phosphate buffer, $\mathrm{pH}$ 7.1. Then, $\left(\mathrm{NH}_{4}\right)_{2} \mathrm{SO}_{4}$ was added to $0.5 \mathrm{M}$ and the samples were loaded onto a phenyl sepharose column. Bound proteins were eluted using a gradient from 0.5 to $0 \mathrm{M}\left(\mathrm{NH}_{4}\right)_{2} \mathrm{SO}_{4}$ in $20 \mathrm{mM}$ potassium phosphate buffer, $\mathrm{pH}$ 7.1. Fractions containing pure wt-EUGO or lo-EUGO were pooled, concentrated using an Amicon spin filter (Merck, Kenilworth, NJ, USA) and dialysed to $50 \mathrm{mM}$ potassium phosphate buffer, $\mathrm{pH}$ 7.5. Purified wt-EUGO was not fully saturated with its FAD cofactor. To obtain fully flavinylated EUGO, $10 \mu \mathrm{M}$ wt-EUGO was incubated with $200 \mu \mathrm{M}$ FAD in $50 \mathrm{mM}$ potassium phosphate buffer, $\mathrm{pH} 7.5$ for $1 \mathrm{~h}$ at room temperature. Subsequently, remaining free FAD was removed by passing the sample over an Econo-Pac 10DG desalting column (BioRad, Hercules, CA, USA).

\section{Analytical methods}

Unless specified otherwise, all experiments were performed in $50 \mathrm{mM}$ potassium phosphate buffer, $\mathrm{pH}$ 7.5. Absorption spectra were recorded at $25^{\circ} \mathrm{C}$ on a Hewlett Packard 8453 diode array spectrophotometer (Agilent Technologies, Santa Clara, CA, USA). The concentration of $\mathrm{wt}-\mathrm{VAO}$ and ll-VAO solutions was determined from their absorption at $439 \mathrm{~nm}$, using the 
previously determined extinction coefficient of the wild-type enzyme $\left[\varepsilon=12,500 \mathrm{M}^{-1} \mathrm{~cm}^{-1}(7)\right]$. The concentration of wt-EUGO and lo-EUGO solutions was determined from their absorption at $441 \mathrm{~nm}\left[\varepsilon=14,200 \mathrm{M}^{-1} \mathrm{~cm}^{-1},(14)\right]$. CD spectra were recorded using $1 \mu \mathrm{M}$ enzyme solutions at $20{ }^{\circ} \mathrm{C}$ or $80{ }^{\circ} \mathrm{C}$ on a Jasco J-715 spectropolarimeter (Jasco Inc., Easton, MD, USA). Thermal denaturation curves were recorded by monitoring the $\mathrm{CD}$ signal at $220 \mathrm{~nm}$ as the temperature was increased from 20 to $80{ }^{\circ} \mathrm{C}$ for wt-VAO and $11-\mathrm{VAO}$ or from 20 to $95^{\circ} \mathrm{C}$ for wt-EUGO and lo-EUGO. The temperature was increased in a linear gradient at a rate of $35^{\circ} \mathrm{C} / \mathrm{h}$. Thermal midpoints of unfolding were determined by fitting a sigmoidal curve to the data using Igor Pro v. 6.10A (Wavemetrics, Lake Oswego, OR, USA). Analytical gel filtration was performed using a Superdex 200 HR 10/30 column on a Pharmacia Biotech Äkta system (GE Healthcare, Chicago, IL, USA). After loading, samples were eluted in $50 \mathrm{mM}$ potassium phosphate buffer, $\mathrm{pH} 7.5,150 \mathrm{mM} \mathrm{KCl}$. Apparent molecular masses were determined from a calibration curve based on proteins of known molecular mass.

\section{Enzyme activity measurements}

All activity measurements were performed in $50 \mathrm{mM}$ potassium phosphate buffer, $\mathrm{pH} 7.5$, at $25^{\circ} \mathrm{C}$. Enzymatic oxidation of vanillyl alcohol was followed by monitoring the absorption of the formed vanillin at $340 \mathrm{~nm}\left(\varepsilon=14,000 \mathrm{M}^{-1} \mathrm{~cm}^{-1}\right)$. Enzymatic oxidation of eugenol or 4ethylphenol was followed by monitoring oxygen consumption using a Hansatech Oxytherm system (Hansatech Instruments, King's Lynn, UK). Steady-state kinetic parameters were determined by fitting observed initial rates to the Michaelis-Menten equation using Igor Pro v. 6.10A.

\section{Bioinformatics}

YP_703243.1 (EUGO) from Rhodococcus jostii RHA1 and P56216.1 (VAO) from Penicillium simplicissimum were blasted and the results were filtered to contain 15 unique sequences sharing a high percentage of sequence identity and with high alignment scores. AAM21269.1 (EUGH) from Pseudomonas sp. OPS1 and WP_032489501.1 (PCMH) from Pseudomonas putida, were blasted, limiting the number of hits to 10. EXJ91673.1, EXJ73483.1, ETN44584.1 and XP_001545605.1 were identified via the blast with VAO as well as with EUGO. Therefore, a total of 46 unique sequences remained. These sequences were aligned and a phylogenetic tree was created using the Clustal Omega webserver (30). Manual verification of the alignment was performed and no changes were made to the alignment. The phylogenetic tree created by Clustal Omega was manually re-rooted to YP_006837036.1 and visualised using the iTOL webserver (31).

\section{Acknowledgements}

We thank Prof. Dr. Marco Fraaije (University of Groningen, The Netherlands) for kindly providing the pBAD-EUGO plasmid. This work was supported by the European Union through the INDOX project (FP7-KBBE-2013-7-613549). 


\section{References}

1. Hashimoto, K., Nishi, H., Bryant, S., and Panchenko, A. R. (2011) Caught in self-interaction: Evolutionary and functional mechanisms of protein homooligomerization. Phys. Biol. 8, 035007

2. Nishi, H., Hashimoto, K., Madej, T., and Panchenko, A. R. (2013) Evolutionary, physicochemical, and functional mechanisms of protein homooligomerization. Prog. Mol. Biol. Transl. Sci. 117, 324

3. Akiva, E., Itzhaki, Z., and Margalit, H. (2008) Built-in loops allow versatility in domain-domain interactions: Lessons from self-interacting domains. Proc. Natl. Acad. Sci. U. S. A. 105, 1329213297

4. Hashimoto, K., and Panchenko, A. R. (2010) Mechanisms of protein oligomerization, the critical role of insertions and deletions in maintaining different oligomeric states. Proc. Natl. Acad. Sci. U. S. A. 107, 20352-20357

5. Fraaije, M. W., van Berkel, W. J. H., Benen, J. A. E., Visser, J., and Mattevi, A. (1998) A novel oxidoreductase family sharing a conserved FAD-binding domain. Trends Biochem. Sci. 23, 206207

6. Leferink, N. G. H., Heuts, D. P. H. M., Fraaije, M. W., and van Berkel, W. J. H. (2008) The growing VAO flavoprotein family. Arch. Biochem. Biophys. 474, 292-301

7. de Jong, E., van Berkel, W. J. H., van der Zwan, R. P., and de Bont, J. A. M. (1992) Purification and characterization of vanillyl-alcohol oxidase from Penicillium simplicissimum: A novel aromatic alcohol oxidase containing covalently bound FAD. Eur. J. Biochem. 208, 651-657

8. Fraaije, M. W., Veeger, C., and van Berkel, W. J. H. (1995) Substrate specificity of flavindependent vanillyl-alcohol oxidase from Penicillium simplicissimum: Evidence for the production of 4-hydroxycinnamyl alcohols from 4-allylphenols. Eur. J. Biochem. 234, 271-277

9. van den Heuvel, R. H. H., Fraaije, M. W., Laane, C., and van Berkel, W. J. H. (1998) Regio- and stereospecific conversion of 4-alkylphenols by the covalent flavoprotein vanillyl-alcohol oxidase. J. Bacteriol. 180, 5646-5651

10. Mattevi, A., Fraaije, M. W., Mozzarelli, A., Olivi, L., Coda, A., and van Berkel, W. J. H. (1997) Crystal structures and inhibitor binding in the octameric flavoenzyme vanillyl-alcohol oxidase: The shape of the active-site cavity controls substrate specificity. Structure. 5, 907-920

11. Fraaije, M. W., Mattevi, A., and van Berkel, W. J. H. (1997) Mercuration of vanillyl-alcohol oxidase from Penicillium simplicissimum generates inactive dimers. FEBS Lett. 402, 33-35

12. Tahallah, N., van den Heuvel, R. H. H., van den Berg, W. A. M., Maier, C. S., van Berkel, W. J. H., and Heck, A. J. R. (2002) Cofactor-dependent assembly of the flavoenzyme vanillyl-alcohol oxidase. J. Biol. Chem. 277, 36425-36432

13. Jin, J., Mazon, H., van den Heuvel, R. H. H., Heck, A. J., Janssen, D. B., and Fraaije, M. W. (2008) Covalent flavinylation of vanillyl-alcohol oxidase is an autocatalytic process. FEBS J. 275, 51915200

14. Jin, J., Mazon, H., van den Heuvel, R. H. H., Janssen, D. B., and Fraaije, M. W. (2007) Discovery of a eugenol oxidase from Rhodococcus sp. strain RHA1. FEBS J. 274, 2311-2321

15. Nguyen, Q.-T., de Gonzalo, G., Binda, C., Rioz-Martínez, A., Mattevi, A., and Fraaije, M. W. (2016) Biocatalytic properties and structural analysis of eugenol oxidase from Rhodococcus jostii RHA1: A versatile oxidative biocatalyst. ChemBioChem. 17, 1359-1366

16. Keat, M. J., and Hopper, D. J. (1978) p-Cresol and 3,5-xylenol methylhydroxylases in Pseudomonas putida N.C.I.B. 9869. Biochem. J. 175, 649-658

17. McIntire, W., Hopper, D. J., and Singer, T. P. (1985) $p$-Cresol methylhydroxylase: Assay and general properties. Biochem. J. 228, 325-335

18. Cunane, L. M., Chen, Z.-W., McIntire, W. S., and Mathews, F. S. (2005) p-Cresol methylhydroxylase: Alteration of the structure of the flavoprotein subunit upon its binding to the cytochrome subunit. Biochemistry. 44, 2963-2973

19. McIntire, W., Edmondson, D. E., Hopper, D. J., and Singer, T. P. (1981) $8 \alpha-(O$-tyrosyl)flavin 
adenine dinucleotide, the prosthetic group of bacterial $p$-cresol methylhydroxylase. Biochemistry. 20, 3068-3075

20. Cunane, L. M., Chen, Z.-W., Shamala, N., Mathews, F. S., Cronin, C. N., and McIntire, W. S. (2000) Structures of the flavocytochrome $p$-cresol methylhydroxylase and its enzyme-substrate complex: Gated substrate entry and proton relays support the proposed catalytic mechanism. J. Mol. Biol. 295, 357-374

21. Priefert, H., Overhage, J., and Steinbüchel, A. (1999) Identification and molecular characterization of the eugenol hydroxylase genes (ehyA/ehyB) of Pseudomonas sp. strain HR199. Arch. Microbiol. 172, 354-363

22. Brandt, K., Thewes, S., Overhage, J., Priefert, H., and Steinbüchel, A. (2001) Characterization of the eugenol hydroxylase genes (ehyA/ehyB) from the new eugenol-degrading Pseudomonas sp. strain OPS1. Appl. Microbiol. Biotechnol. 56, 724-730

23. Furukawa, H., Wieser, M., Morita, H., Sugio, T., and Nagasawa, T. (1998) Purification and characterization of eugenol dehydrogenase from Pseudomonas fluorescens E118. Arch. Microbiol. 171, 37-43

24. Krissinel, E., and Henrick, K. (2007) Inference of macromolecular assemblies from crystalline state. J. Mol. Biol. 372, 774-797

25. Waterhouse, A. M., Procter, J. B., Martin, D. M. A., Clamp, M., and Barton, G. J. (2009) Jalview Version 2: A multiple sequence alignment editor and analysis workbench. Bioinformatics. 25, 1189-1191

26. Furukawa, H., Wieser, M., Morita, H., Sugio, T., and Nagasawa, T. (1999) Purification and characterization of vanillyl-alcohol oxidase from Byssochlamys fulva V107. J. Biosci. Bioeng. 87, 285-290

27. Fraaije, M. W., Sjollema, K. A., Veenhuis, M., and van Berkel, W. J. H. (1998) Subcellular localization of vanillyl-alcohol oxidase in Penicillium simplicissimum. FEBS Lett. 422, 65-68

28. van den Heuvel, R. H. H., Fraaije, M. W., Mattevi, A., and van Berkel, W. J. H. (2000) Asp-170 is crucial for the redox properties of vanillyl-alcohol oxidase. J. Biol. Chem. 275, 14799-14808

29. Benen, J. A. E., Sánchez-Torres, P., Wagemaker, M. J. M., Fraaije, M. W., van Berkel, W. J. H., and Visser, J. (1998) Molecular cloning, sequencing, and heterologous expression of the vaoA gene from Penicillium simplicissimum CBS 170.90 encoding vanillyl-alcohol oxidase. J. Biol. Chem. 273, 7865-7872

30. Sievers, F., Wilm, A., Dineen, D., Gibson, T. J., Karplus, K., Li, W., Lopez, R., McWilliam, H., Remmert, M., Söding, J., Thompson, J. D., and Higgins, D. G. (2011) Fast, scalable generation of high-quality protein multiple sequence alignments using Clustal Omega. Mol. Syst. Biol. 7, 539

31. Letunic, I., and Bork, P. (2011) Interactive Tree Of Life v2: Online annotation and display of phylogenetic trees made easy. Nucleic Acids Res. 39, W475-478

\section{Abbreviations}

4PO: 4-phenol oxidising, ADP: adenosine 5'-diphosphate, EUGO: eugenol oxidase, EUGH: eugenol hydroxylase, FAD: flavin adenine dinucleotide, PCMH: $p$-cresol methylhydroxylase, VAO: vanillyl alcohol oxidase 




\title{
Two tyrosine residues, Tyr-108 and Tyr-503, are responsible for the deprotonation of phenolic substrates in vanillyl alcohol oxidase
}

\author{
Tom A. Ewing ${ }^{1}$, Quoc-Thai Nguyen 2,3,4, Robert C. Allan ${ }^{1}$, Gudrun Gygli1 ${ }^{1}$ Elvira Romero ${ }^{3}$, \\ Claudia Binda ${ }^{2}$, Marco W. Fraaije ${ }^{3}$, Andrea Mattevi' ${ }^{2}$ and Willem J. H. van Berkel ${ }^{1}$ \\ ${ }^{1}$ Laboratory of Biochemistry, Wageningen University \& Research, Wageningen, The Netherlands \\ ${ }^{2}$ Department of Biology and Biotechnnology, University of Pavia, Pavia, Italy \\ 3Molecular Enzymology Group, University of Groningen, Groningen, The Netherlands \\ ${ }^{4}$ Faculty of Pharmacy, University of Medicine and Pharmacy, Ho Chi Minh City, Vietnam
}

Based on Journal of Biological Chemistry (2017), 292, 14668-14679

\begin{abstract}
Summary
A number of oxidoreductases from the VAO/PCMH flavoprotein family catalyse the oxidation of para-substituted phenols. One of the best-studied is vanillyl alcohol oxidase (VAO) from the fungus Penicillium simplicissimum. For oxidation of phenols by VAO to occur, they must first be bound in the enzyme's active site in their phenolate form. The crystal structure of VAO reveals that two tyrosine residues, Tyr-108 and Tyr-503, are positioned so as to facilitate deprotonation to the phenolate. To investigate their role in catalysis, we created three VAO variants, Y108F, Y503F, and Y108F/Y503F, and studied their biochemical properties. Steadystate kinetics indicated that the presence of at least one of the tyrosine residues is essential for efficient catalysis by VAO. Stopped-flow kinetics revealed that the reduction of VAO by

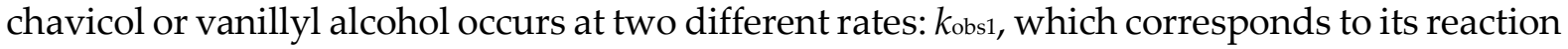
with the deprotonated form of the substrate, and $k_{\mathrm{obs} 2}$, which corresponds to its reaction with the protonated form of the substrate. In Y108F, Y503F, and Y108F/Y503F, the relative contribution of $k_{\text {obs2 }}$ to the reduction is larger than in wild-type VAO, suggesting deprotonation is impaired in these variants. Binding studies disclosed that the competitive inhibitor isoeugenol is predominantly in its deprotonated form when bound to wild-type $\mathrm{VAO}$, but predominantly in its protonated form when bound to the variants. These results indicate that Tyr-108 and Tyr-503 are responsible for the activation of substrates in VAO, providing new insights into the catalytic mechanism of VAO and related enzymes that oxidise para-substituted phenols.
\end{abstract}





\section{Introduction}

Vanillyl alcohol oxidase (VAO, EC 1.1.3.38) from Penicillium simplicissimum is a flavindependent oxidase that catalyses the oxidation of para-substituted phenolic compounds using molecular oxygen as an electron acceptor (1-3). It is a $64 \mathrm{kDa}$ (560 amino acid) protein that forms octameric structures with each subunit containing an FAD cofactor that is covalently linked to the protein via an $8 \alpha-N 3$-histidyl-FAD bond (4). VAO is a member of the $\mathrm{VAO} / \mathrm{PCMH}$ flavoprotein family, which consists of homologous oxidoreductases that catalyse a wide range of reactions, but share a conserved FAD-binding domain (5, Chapter 2). Although VAO catalyses a range of reactions, such as the oxidation of benzylic alcohols to aldehydes, the oxidative deamination of benzylic amines, the hydroxylation of 4-allylphenols, and the hydroxylation or dehydrogenation of 4 -alkylphenols, it displays a strict selectivity towards the oxidation of para-substituted phenols at the $\mathrm{C} \alpha$ atom of their side chain. The proposed catalytic mechanism of VAO provides an explanation for this selectivity (Scheme 1) $(2,6,7)$. First, VAO's substrates are bound in the active site in their deprotonated phenolate form. Subsequently, a hydride is transferred from the $\mathrm{C} \alpha$ atom of the substrate's side chain to the N5 atom of FAD, yielding the reduced enzyme and a para-quinone methide intermediate. Finally, this intermediate either reacts with water or rearranges to yield the product of the reaction and FAD is reoxidised by molecular oxygen, which is converted to hydrogen peroxide. The initial deprotonation of the substrate's phenolic hydroxyl group is likely a crucial step in this mechanism, as it promotes the hydride transfer reaction by enabling the formation of the para-quinone methide intermediate. Studies using the competitive inhibitor isoeugenol [2-methoxy-4-(1-propen-1-yl)phenol, Scheme 1] revealed that its $\mathrm{p} K_{\mathrm{a}}$ is lowered from 10 to 5 upon binding to $\mathrm{VAO}$ (2). A potential explanation for this decrease is provided by examining the crystal structure of VAO in complex with isoeugenol (4). Three polar residues, Tyr-108, Tyr-503 and Arg-504, form a phenolate binding pocket, with their side chains positioned within hydrogen bonding distance of isoeugenol's phenolic hydroxyl group (Fig. 1). These residues may facilitate deprotonation of the substrate either by promoting preferential binding of the deprotonated form or by one of the tyrosine residues acting as a base and actively deprotonating the substrate in the active site. All three residues are

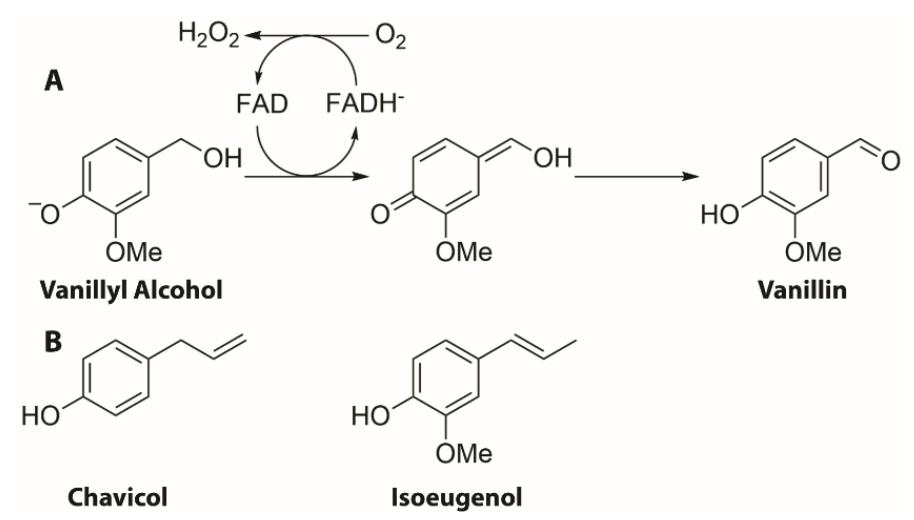

Scheme 1: (A) Oxidation of vanillyl alcohol (4-hydroxy-3-methoxybenzyl alcohol) as catalysed by VAO. First, the substrate is bound in the active site in its deprotonated phenolate form. This facilitates the transfer of a hydride from the $\mathrm{C} \alpha$ of the substrate to $\mathrm{FAD}$, yielding a para-quinone methide intermediate and the reduced enzyme. The para-quinone methide subsequently rearranges to give the product vanillin (4-hydroxy-3-methoxybenzaldehyde) and FAD is reoxidised by molecular oxygen, which is converted to hydrogen peroxide. (B) Structures of chavicol (4-allylphenol) and isoeugenol [2-methoxy4-(1-propen-1-yl)phenol]. 


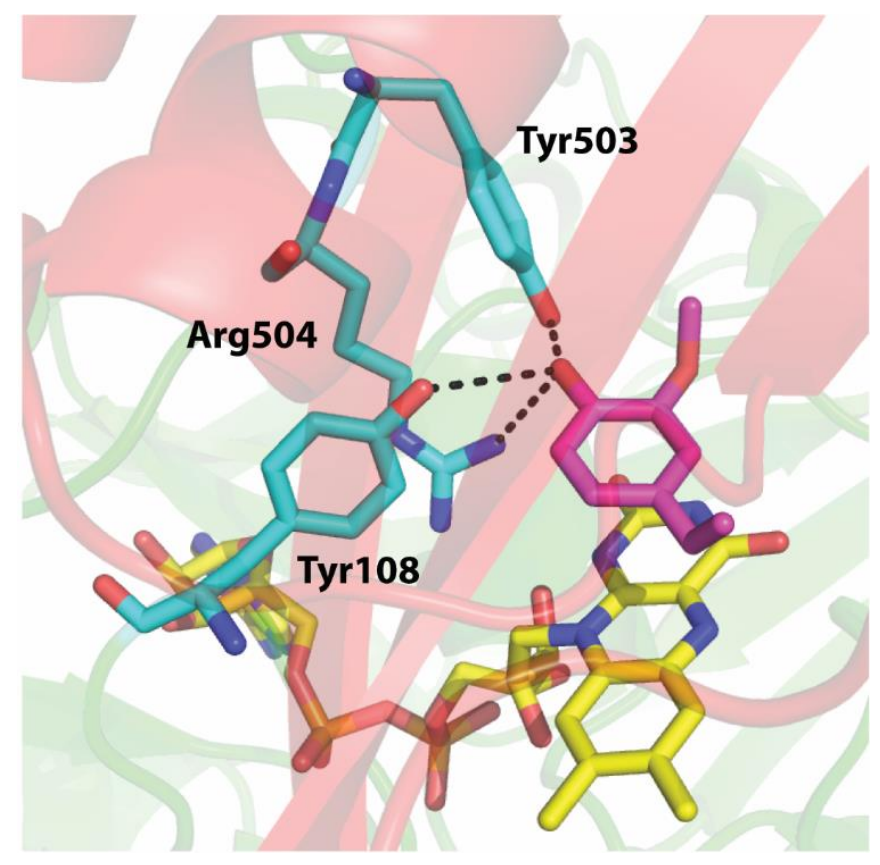

Figure 1: Structure of the phenolate binding pocket of VAO. Tyr-108, Tyr-503 and Arg-504 are shown in stick representation with cyan carbon atoms, the competitive inhibitor isoeugenol with pink carbon atoms and the FAD cofactor with yellow carbon atoms. Nitrogen atoms are shown in blue, oxygen atoms in red and phosphorus atoms in orange. Potential hydrogen bonds between the amino acids of the phenolate binding pocket and the phenolic hydroxyl group of isoeugenol are shown as black dashes. The protein backbone is shown in cartoon representation, with the FAD-binding domain in green and the substrate-binding domain in red. The structure of isoeugenol-bound VAO was downloaded from the PDB website (PDB ID: 2VAO). The figure was prepared using PyMOL (Schrödinger LLC, New York, NY, USA).

conserved in the homologous flavoenzymes eugenol oxidase (EUGO) and para-cresol methylhydroxylase (PCMH, EC 1.17.99.1), which also catalyse the oxidation of parasubstituted phenols $(8,9)$. The role of Arg-474 in PCMH, which corresponds to Arg-504 in $\mathrm{VAO}$, has been studied by site-directed mutagenesis and it was found to be of importance in achieving covalent incorporation of the enzyme's cofactor and in enabling efficient catalysis by modulating the redox properties of both the cofactor and enzyme-bound substrates (10). Here, we investigated the role played by Tyr-108 and Tyr-503 in VAO using site-directed mutagenesis. Three VAO variants, Y108F, Y503F and Y108F/Y503F, were created, expressed and purified and their catalytic properties were studied. Our results reveal that the presence of at least one of these tyrosine residues is essential for efficient catalysis.

\section{Results}

The Y108F, Y503F and Y108F/Y503F variants were successfully expressed in Escherichia coli and purified. Like wild-type (wt)-VAO, all three variants contained a covalently bound FAD cofactor, as judged from the yellow pellet and colourless supernatant obtained after precipitation of the proteins with trichloroacetic acid. In their native form, all variants displayed an octameric quaternary structure, as determined using analytical size exclusion chromatography. The flavin absorption spectra of the variants were highly similar to that of $\mathrm{wt}-\mathrm{VAO}$ (see spectral studies presented below), demonstrating that the introduced mutations do not lead to any major changes in the electronic environment of their flavin cofactor. To determine whether the introduced mutations affect the stability of VAO, thermal midpoints 
of unfolding of the variants were determined using the ThermoFAD method (11). This yielded thermal midpoints of unfolding of $55{ }^{\circ} \mathrm{C}$ for wt-VAO, $53{ }^{\circ} \mathrm{C}$ for $\mathrm{Y} 108 \mathrm{~F}$ and $\mathrm{Y} 503 \mathrm{~F}$, and $52{ }^{\circ} \mathrm{C}$ for $\mathrm{Y} 108 \mathrm{~F} / \mathrm{Y} 503 \mathrm{~F}$, suggesting the introduced mutations have a minor deleterious effect on the thermostability of VAO.

\section{Steady-state catalytic properties}

To determine the effect of the introduced mutations on the catalytic efficiency of VAO, we measured the steady-state kinetic parameters for the oxidation of two substrates: vanillyl alcohol (4-hydroxy-3-methoxybenzyl alcohol), which is oxidised to the aldehyde vanillin (4hydroxy-3-methoxybenzaldehyde), and chavicol (4-allylphenol, Scheme 1), which is hydroxylated to para-coumaryl alcohol [4-(3-hydroxyprop-1-en-1-yl)phenol]. The kinetic parameters for the oxidation of these substrates are listed in Table 1. Interestingly, the effect of the mutations varied depending on the substrate. With vanillyl alcohol, the $k_{\text {cat }}$ drops to approximately $2 \%$ of that of wt-VAO for both the Y108F and Y503F variants. With chavicol, a more minor drop in the $k_{\text {cat }}$ value to approximately $45 \%$ of that of the wild-type enzyme was observed for the Y108F and Y503F variants. With chavicol, the Y108F/Y503F variant displayed a $k_{\text {cat }}$ value of less than $2 \%$ of that of wt-VAO and with vanillyl alcohol, the activity was too low to accurately determine kinetic parameters. This indicates that the presence of at least one of the two tyrosine residues is essential for efficient catalysis. For each variant, the $K_{\mathrm{m}}$ increased for each substrate. The magnitude of the increase ranged from three- to twelvefold.

\section{Stopped-flow kinetics of the reductive half-reaction}

To determine whether the decreased catalytic efficiencies observed for the VAO variants were due to impaired reduction of their FAD cofactor, spectral changes during the anaerobic reduction of the enzymes by vanillyl alcohol or chavicol were followed by stopped-flow spectroscopy. Where possible, photodiode array detection was used to monitor changes over a spectral range from 254 to $725 \mathrm{~nm}$. However, with long measurement times $(>10 \mathrm{~s})$, the highintensity illumination required for this led to photoreduction of FAD. Therefore, in cases where the reduction reaction was slow, it was monitored using single wavelength detection at $439 \mathrm{~nm}$, the absorption maximum of the oxidised FAD in VAO. This was the case for the reduction of the $\mathrm{Y} 108 \mathrm{~F}$ and $\mathrm{Y} 503 \mathrm{~F}$ variants by $2 \mathrm{mM}$ vanillyl alcohol and for all reactions involving the $\mathrm{Y} 108 \mathrm{~F} / \mathrm{Y} 503 \mathrm{~F}$ variant.

Observed rates for the anaerobic reduction of wt-VAO and the $\mathrm{Y} 108 \mathrm{~F}$ and $\mathrm{Y} 503 \mathrm{~F}$ variants by $2 \mathrm{mM}$ vanillyl alcohol at $\mathrm{pH} 6.2$ and 7.5 are given in Table 2. The anaerobic reduction of wtVAO by vanillyl alcohol at $\mathrm{pH} 7.5$ has been studied previously and was found to be a biphasic process with rate constants at saturating substrate concentration of $k_{\mathrm{red} 1}=24 \mathrm{~s}^{-1}$ and $k_{\mathrm{red} 2}=3.5$ $\mathrm{s}^{-1}(6)$. The two different rates of reduction were interpreted to be caused by the presence of two conformations of the enzyme. The rate of the slower phase, $k_{\text {red2, }}$ lies close to $k_{\text {cat }}$ and is rate-limiting for catalysis. Our results with wt-VAO are in good agreement with these data. The effect of the Y108F and Y503F mutations on the rate of reduction by vanillyl alcohol was similar. In each variant, the reduction was a biphasic process. Each of the rates of reduction has dropped significantly as compared with wt-VAO. The rates of the two phases are in the same range and $k_{\text {obs2 }}$ is similar to the rate of steady-state catalysis, suggesting that flavin reduction is rate-limiting in these variants. In addition to the decrease in both reduction rates, the relative contribution of the two kinetic events to the drop in absorption at $439 \mathrm{~nm}$ (amplitude $a$ ) has changed. In wt-VAO, $k_{\mathrm{obs} 1}$ has a greater contribution than $k_{\mathrm{obs} 2}\left(a_{1}: a_{2} \approx 2: 1\right)$, 
Table 1: Steady-state kinetic parameters for the oxidation of vanillyl alcohol and chavicol by VAO variants. Kinetic parameters were determined at $25^{\circ} \mathrm{C}$ in $50 \mathrm{mM}$ potassium phosphate buffer, pH 7.5. $K_{\mathrm{i}}$ is the inhibition constant for substrate inhibition, which was observed for all variants when using chavicol as a substrate. ND: not determined due to low reaction rate (observed rate $<0.01 \mathrm{~s}^{-1}$ at $2 \mathrm{mM}$ vanillyl alcohol).

\begin{tabular}{|lllll|}
\hline Vanillyl alcohol & & & \\
\hline VAO variant & $k_{\text {cat }}\left(\mathrm{s}^{-1}\right)$ & $K_{\mathrm{m}}(\mu \mathrm{M})$ & $k_{\text {cat }} / K_{\mathrm{m}}\left(\mathrm{s}^{-1} \mathrm{mM}^{-1}\right)$ & \\
wild-type & $2.6^{\mathrm{a}}$ & $149^{\mathrm{a}}$ & 17 & \\
Y108F & $0.042 \pm 0.006$ & $1750 \pm 420$ & 0.024 & \\
Y503F & $0.055 \pm 0.002$ & $990 \pm 60$ & 0.056 & \\
Y108F/Y503F & $\mathrm{ND}$ & $\mathrm{ND}$ & $\mathrm{ND}$ & \\
\hline Chavicol & & & & $K_{\mathrm{i}}(\mathrm{mM})$ \\
& & & $k_{\text {cat }} / K_{\mathrm{m}}\left(\mathrm{s}^{-1} \mathrm{mM}^{-1}\right)$ & $7.0 \pm 1.1$ \\
\hline VAO variant & $k_{\text {cat }}\left(\mathrm{s}^{-1}\right)$ & $K_{\mathrm{m}}(\mu \mathrm{M})$ & 1138 & $2.9 \pm 0.4$ \\
wild-type & $7.4 \pm 0.1$ & $6.5 \pm 0.3$ & 51 & $0.45 \pm 0.04$ \\
Y108F & $3.4 \pm 0.1$ & $67 \pm 7$ & 157 & $1.4 \pm 0.2$ \\
Y503F & $3.3 \pm 0.1$ & $21 \pm 2$ & 1.8 & \\
Y108F/Y503F & $0.13 \pm 0.01$ & $74 \pm 6$ & & \\
\hline
\end{tabular}

aData taken from (12).

Table 2: Observed rates of reduction of VAO variants by $2 \mathrm{mM}$ vanillyl alcohol. Rates were determined by fitting appropriate exponential functions to single wavelength traces of the absorption at $439 \mathrm{~nm}$ obtained after mixing $10 \mu \mathrm{M}$ enzyme with $2 \mathrm{mM}$ vanillyl alcohol in $50 \mathrm{mM}$ potassium phosphate buffer, $\mathrm{pH} 6.2$ or 7.5 , under anaerobic conditions at $25{ }^{\circ} \mathrm{C}$ using a stopped-flow apparatus. Values in parentheses give the relative contribution of $k_{\mathrm{obs}}$ and $k_{\mathrm{obs} 2}$, the two phases associated with flavin reduction, to the drop in absorption. The observed rates for the oxidation of $2 \mathrm{mM}$ vanillyl alcohol under steady-state conditions $\left(k_{\mathrm{ss}}\right)$ at $\mathrm{pH} 7.5$ are given for comparison. $\mathrm{ND}=$ not determined due to low reaction rate.

\begin{tabular}{|llll|}
\hline pH 7.5 & & & \\
\hline VAO variant & $k_{\text {obs1 }}\left(\mathrm{s}^{-1}\right)$ & $k_{\text {obs2 }}\left(\mathrm{s}^{-1}\right)$ & $k_{\mathrm{ss}}\left(\mathrm{s}^{-1}\right)$ \\
wild-type & $27 \pm 1(0.67)$ & $3.5 \pm 0.1(0.33)$ & 2.4 \\
Y108F & $0.061 \pm 0.002(0.17)$ & $0.023 \pm 0.001(0.83)$ & 0.024 \\
Y503F & $0.075 \pm 0.001(0.26)$ & $0.025 \pm 0.001(0.74)$ & 0.037 \\
Y108F/Y503F & $\mathrm{ND}$ & $\mathrm{ND}$ & $<0.01$ \\
\hline pH 6.2 & & & \\
& & $k_{\text {obs2 }}\left(\mathrm{s}^{-1}\right)$ & \\
VAO variant & $k_{\text {obs1 }}\left(\mathrm{s}^{-1}\right)$ & $2.9 \pm 0.1(0.77)$ & \\
wild-type & $35 \pm 1(0.23)$ & $0.0085 \pm 0.0001(0.97)$ & \\
Y108F & $0.27 \pm 0.01(0.03)$ & $0.018 \pm 0.001(0.97)$ & \\
Y503F & $0.33 \pm 0.01(0.03)$ & $\mathrm{ND}$ & \\
Y108F/Y503F & $\mathrm{ND}$ & \multicolumn{2}{l}{} \\
\hline
\end{tabular}

whereas in the $\mathrm{Y} 108 \mathrm{~F}$ and $\mathrm{Y} 503 \mathrm{~F}$ variants, $k_{\mathrm{obs} 2}$ has a greater contribution than $k_{\mathrm{obs}}\left(a_{1}: a_{2} \approx 1: 5\right.$ for $\mathrm{Y} 108 \mathrm{~F}$ and 1:3 for Y503F). At $\mathrm{pH} 6.2$, reduction by vanillyl alcohol was also biphasic for all variants. For wt-VAO, the rates of the two phases are very similar to those at $\mathrm{pH}$ 7.5. However, their contributions to the reduction have changed, with $k_{\mathrm{obs} 2}$ having a greater contribution than $k_{\text {obs1 }}\left(a_{1}: a_{2} \approx 1: 3\right)$. In the $\mathrm{Y} 108 \mathrm{~F}$ and $\mathrm{Y} 503 \mathrm{~F}$ variants, $k_{\mathrm{obs}}$ is somewhat higher than observed at $\mathrm{pH} 7.5$ and $k_{\mathrm{obs} 2}$ is slightly lower in $\mathrm{Y} 108 \mathrm{~F}$ and does not change significantly in Y503F. As 
observed for wt-VAO, the contribution of the two rates to the change in absorption shifts, with $k_{\text {obs2 }}$ becoming more predominant ( $a_{1}: a_{2} \approx 1: 32$ for each variant).

Spectra obtained during the anaerobic reduction of wt-VAO by $100 \mu \mathrm{M}$ chavicol are shown in Figure 2. After mixing wt-VAO with chavicol, the characteristic peak of the enzyme's oxidised flavin cofactor (maximum at $439 \mathrm{~nm}$ ) rapidly disappears, indicating that FAD is reduced by the substrate. At the same time, the absorption between 320 and $430 \mathrm{~nm}$ increases, yielding an intense peak with a maximum at $373 \mathrm{~nm}$. This peak reaches its maximum intensity after approximately $600 \mathrm{~ms}$, after which it decreases in intensity. The formation of a species with similar spectral characteristics has been observed during the anaerobic reduction of wt-VAO by vanillyl alcohol $\left(\lambda_{\max }=362 \mathrm{~nm}\right)$ or 4 -(methoxymethyl)phenol $\left(\lambda_{\max }=364 \mathrm{~nm}\right)$ and was attributed to a complex between the para-quinone methide intermediate and the reduced enzyme formed after the electron transfer reaction has taken place (6). Intermediate spectra obtained upon spectral deconvolution of the stopped-flow data are shown in Figure 2. The data were fit to a three-phase consecutive model, yielding four distinct spectral intermediates. Species 1 represents the oxidised enzyme. Species 3 displays the highest absorption at $373 \mathrm{~nm}$ and complete loss of the characteristic oxidised flavin spectrum. It likely represents the paraquinone methide-reduced enzyme complex. Species 2 displays incomplete formation of the para-quinone methide intermediate as indicated by its lower absorption at $373 \mathrm{~nm}$. In addition, part of the FAD cofactor is still in its oxidised form in this species, as evidenced by the absorption in the range $440-500 \mathrm{~nm}$. The spectra of species 1, 2, and 3 display an isosbestic point at $423 \mathrm{~nm}$ and a spectrum calculated by assuming that the spectrum of species 2 is a simple composite of those of species $1(20 \%)$ and $3(80 \%)$ was virtually indistinguishable from the actual spectrum of species 2. This is consistent with species 2 not representing a real reaction intermediate, but rather reflecting the fact that there are two separate rates of conversion of species 1 to species 3 . Species 4 displays a spectrum that is typical of a reduced flavoprotein, suggesting that this species is formed by the breakdown of the para-quinone methide intermediate by its reaction with water, yielding the product para-coumaryl alcohol. Thus, the anaerobic reduction of wt-VAO by chavicol proceeds as follows. First, the substrate binds to the enzyme. Subsequently, the FAD cofactor is reduced by the substrate leading to the formation of a para-quinone methide-reduced enzyme complex with two kinetic phases that both contribute to this reduction being distinguished. Following reduction, the paraquinone methide intermediate reacts with water to yield para-coumaryl alcohol. Rate constants for these processes were obtained by fitting curves of the absorption at $440 \mathrm{~nm}$ to a triphasic exponential function. Rate constants for the two reduction rates ( $k_{\text {obs } 1}$ and $k_{\text {obs } 2}$ ) are given in Table 3. $k_{\text {obs2 }}$ is similar to the steady-state reaction rate, suggesting it is rate-limiting for catalysis. The observed rate of decay of the para-quinone methide intermediate, $k_{\text {obs3, was }}$ similar for all variants $\left(0.1-0.2 \mathrm{~s}^{-1}\right)$ and is too slow to be catalytically relevant. Presumably, breakdown of this intermediate is faster in the presence of oxygen, as has been observed previously for the para-quinone methide intermediate formed upon reduction of wt-VAO by 4-(methoxymethyl)phenol (6).

Upon reduction of the $\mathrm{Y} 108 \mathrm{~F}$ and $\mathrm{Y} 503 \mathrm{~F}$ variants by chavicol, the spectral changes observed were similar to those seen with wt-VAO, with reduction of FAD occurring concomitantly with the appearance of a putative para-quinone methide intermediate (Fig. 2). The absorption maximum of this intermediate is shifted to lower wavelengths in the variants (363 nm for Y108F and $368 \mathrm{~nm}$ for Y503F) compared with wt-VAO (373 nm). Maximum abundance of the 

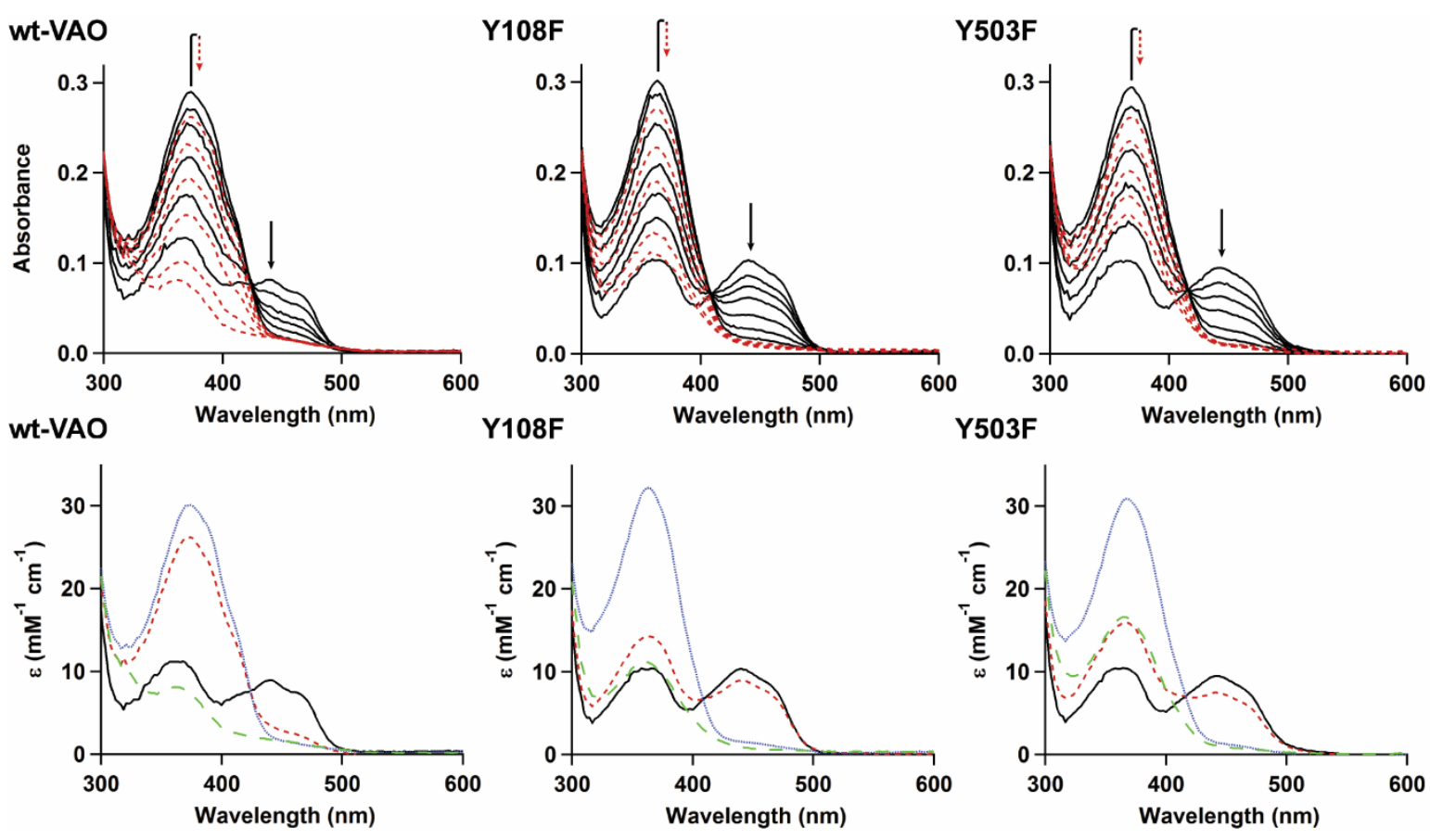

Figure 2: Spectral changes observed during the anaerobic reduction of VAO variants by chavicol. Top panels show spectra obtained after mixing $10 \mu \mathrm{M}$ wt-VAO, Y108F, or Y503F with $100 \mu \mathrm{M}$ chavicol in a stopped-flow apparatus under anaerobic conditions in $50 \mathrm{mM}$ potassium phosphate buffer, $\mathrm{pH} 7.5$, at $25{ }^{\circ} \mathrm{C}$. Initially, FAD is reduced and a complex between the reduced enzyme and a para-quinone methide intermediate is formed (black solid lines). Subsequently, the para-quinone methide intermediate decays, likely due to it reacting with water to yield the product (para-coumaryl alcohol) and the reduced enzyme (red dashed lines). For wt-VAO, spectra were obtained 0.001, 0.004, 0.009, $0.019,0.079,0.60,2.3,4.3,7.2,12,25$, and $75 \mathrm{~s}$ after mixing. For $\mathrm{Y} 108 \mathrm{~F}$, spectra were obtained $0.001,0.071$, $0.17,0.31,0.64,1.1,2.2,6.1,12,20,45$, and $120 \mathrm{~s}$ after mixing. For Y503F, spectra were obtained 0.001, $0.071,0.17,0.31,0.64,1.1,5.0,8.7,17,44$, and $120 \mathrm{~s}$ after mixing. Bottom panels show spectra of reaction intermediates obtained after spectral deconvolution of the stopped-flow data for wt-VAO, Y108F, and Y503F. Data was fit to a three-phase model $(1 \rightarrow 2 \rightarrow 3 \rightarrow 4)$, yielding four spectrally distinct species: 1 (black solid line), 2 (red short dashes), 3 (blue dots) and 4 (green long dashes). One- or two-phase models were not able to satisfactorily explain the data. The rates obtained for the three phases (average of at least 3 measurements) are: $k_{1}=134 \mathrm{~s}^{-1}, k_{2}=3.7 \mathrm{~s}^{-1}$, and $k_{3}=0.093 \mathrm{~s}^{-1}$ for wt-VAO, $k_{1}=29 \mathrm{~s}^{-1}, k_{2}=1.7 \mathrm{~s}^{-}$ 1 , and $k_{3}=0.051 \mathrm{~s}^{-1}$ for $\mathrm{Y} 108 \mathrm{~F}$, and $k_{1}=13 \mathrm{~s}^{-1}, k_{2}=2.7 \mathrm{~s}^{-1}$, and $k_{3}=0.083 \mathrm{~s}^{-1}$ for Y503F. These rates are in good agreement with those obtained by fitting to single wavelength traces at $440 \mathrm{~nm}$ (see Table 3 ).

intermediate was observed after $2.2 \mathrm{~s}$ for the Y108F variant and after $1.5 \mathrm{~s}$ for the Y503F variant,after which the intermediate decays. Spectral deconvolution again revealed a threephase process (Fig. 2). As with wt-VAO, species 1 and 3 represent the oxidised enzyme and the para-quinone methide-reduced enzyme complex respectively. Species 2 again displays an absorption spectrum that is a composite of that of species 1 and 3 and is likely not a real reaction intermediate. Species 4 appears to represent a mixture of para-quinone methidereduced enzyme complex and free reduced enzyme. This is likely due to the decay of the paraquinone methide not being complete at the end of the measurement (120 s). Fitting the data at $440 \mathrm{~nm}$ to triple exponential functions revealed that $k_{\mathrm{obs} 1}$ and $k_{\mathrm{obs} 2}$ are both decreased in each variant as compared with wt-VAO (Table 3 ). However, neither rate in either variant decreased more than tenfold. Thus, the effect of the $\mathrm{Y} 108 \mathrm{~F}$ and $\mathrm{Y} 503 \mathrm{~F}$ mutations on the rate of reduction of VAO by chavicol is less severe than that on the rate of reduction by vanillyl alcohol ( $k_{\mathrm{obs}}$ and $k_{\mathrm{obs} 2}$ decreased $>100$-fold in each variant). Interestingly, a similar effect on the relative 
Table 3: Observed rates of reduction of VAO variants by $100 \mu \mathrm{M}$ chavicol. Rates were determined by fitting appropriate exponential functions to traces of the absorption at $440 \mathrm{~nm}$ [from photodiode array detection (wt-VAO, Y108F, and Y503F)] or $439 \mathrm{~nm}$ [from single wavelength detection (Y108F/Y503F)] obtained after mixing $10 \mu \mathrm{M}$ enzyme with $100 \mu \mathrm{M}$ chavicol in $50 \mathrm{mM}$ potassium phosphate buffer, $\mathrm{pH}$ 6.2 or 7.5 , under anaerobic conditions at $25^{\circ} \mathrm{C}$ using a stopped-flow apparatus. Values in parentheses give the relative contribution of $k_{\mathrm{obs} 1}$ and $k_{\mathrm{obs} 2}$, the two phases associated with flavin reduction, to the drop in absorption. The observed rates for the oxidation of $100 \mu \mathrm{M}$ chavicol under steady-state conditions $\left(k_{\mathrm{ss}}\right)$ at $\mathrm{pH} 7.5$ are given for comparison. $\mathrm{NO}=$ not observed.

\begin{tabular}{|llll|}
\hline $\mathbf{p H} 7.5$ & & & \\
\hline VAO variant & $k_{\text {obs1 }}\left(\mathrm{s}^{-1}\right)$ & $k_{\text {obs2 }}\left(\mathrm{s}^{-1}\right)$ & $k_{\mathrm{ss}}\left(\mathrm{s}^{-1}\right)$ \\
wild-type & $139 \pm 1(0.76)$ & $4.5 \pm 0.1(0.24)$ & 6.8 \\
Y108F & $33 \pm 1(0.13)$ & $1.6 \pm 0.1(0.87)$ & 2.0 \\
Y503F & $14 \pm 1(0.07)$ & $2.6 \pm 0.1(0.93)$ & 2.3 \\
Y108F/Y503F & $19 \pm 1(0.05)$ & $0.11 \pm 0.01(0.95)$ & 0.069 \\
\hline pH 6.2 & & & \\
& & $k_{\text {obs2 }}\left(\mathrm{s}^{-1}\right)$ & \\
VAO variant & $k_{\text {obs1 }}\left(\mathrm{s}^{-1}\right)$ & $3.7 \pm 0.1(0.70)$ & \\
wild-type & $132 \pm 1(0.30)$ & $0.79 \pm 0.01(1.00)$ & \\
Y108F & $\mathrm{NO}$ & $1.7 \pm 0.1(1.00)$ & \\
Y503F & $\mathrm{NO}$ & $0.027 \pm 0.001(0.94)$ & \\
Y108F/Y503F & $21 \pm 1(0.06)$ & & \\
\hline
\end{tabular}

contributions of $k_{\mathrm{obs} 1}$ and $k_{\mathrm{obs} 2}$ to reduction was observed with chavicol as with vanillyl alcohol,

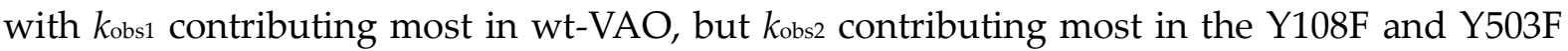
variants. Reduction of the $\mathrm{Y} 108 \mathrm{~F} / \mathrm{Y} 503 \mathrm{~F}$ variant by chavicol was monitored using single wavelength detection at $439 \mathrm{~nm}$. In this case, the data fit best to a double exponential function. Interestingly, $k_{\mathrm{obs}}$ is in the same range as for the $\mathrm{Y} 108 \mathrm{~F}$ and $\mathrm{Y} 503 \mathrm{~F}$ variants, whereas $k_{\mathrm{obs}}$ is an order of magnitude smaller. This brings it into the same range as $k_{\mathrm{obs} 3}$ observed for wt-VAO and the $\mathrm{Y} 108 \mathrm{~F}$ and $\mathrm{Y} 503 \mathrm{~F}$ variants. This provides an explanation for the absence of a third phase, as the decay of the para-quinone methide intermediate is now likely no longer distinguishable from the second flavin reduction phase. As with Y108F and Y503F, $k_{\text {obs } 2}$ makes the largest contribution to flavin reduction. For all variants, $k_{\text {obs } 2}$ lies close to the observed rate of steady-state catalysis, suggesting it is rate-limiting.

Upon reduction of wt-VAO and the $\mathrm{Y} 108 \mathrm{~F}$ and $\mathrm{Y} 503 \mathrm{~F}$ variants by $100 \mu \mathrm{M}$ chavicol at $\mathrm{pH} 6.2$, similar spectral changes were observed as at $\mathrm{pH}$ 7.5. Formation of a para-quinone methide intermediate and concomitant reduction of FAD were followed by the decay of the paraquinone methide intermediate, which was too slow to be catalytically relevant. The observed reduction rates are given in Table 3. For wt-VAO, the reduction is a biphasic process. The two rates are similar to those observed at $\mathrm{pH} \mathrm{7.5,} \mathrm{however,} \mathrm{as} \mathrm{with} \mathrm{vanillyl} \mathrm{alcohol,} \mathrm{the}$ contribution of the rates to the drop in absorbance changes, with $k_{\text {obs } 2}$ becoming more predominant ( $a_{1}: a_{2} \approx 1: 2$ at $\mathrm{pH} 6.2$ compared with $a_{1}: a_{2} \approx 3: 1$ at $\left.\mathrm{pH} 7.5\right)$. For the Y108F and Y503F variants, only a single reductive phase was observed, with rate constants that are similar to those of $k_{\mathrm{obs} 2}$ at $\mathrm{pH} 7.5$, suggesting that the observed rate can be attributed to the same process as $k_{\text {obs } 2}$ and that the process associated with $k_{\text {obs } 1}$ does not make an observable contribution to the reduction at $\mathrm{pH}$ 6.2. For the $\mathrm{Y} 108 \mathrm{~F} / \mathrm{Y} 503 \mathrm{~F}$ variant, the reduction was observed to be biphasic with the rates of the two phases and their relative contributions being similar to those 
observed at $\mathrm{pH}$ 7.5. However, the contribution of the slower phase to the reduction may be underestimated. After reduction of $\mathrm{Y} 108 \mathrm{~F} / \mathrm{Y} 503 \mathrm{~F}$ a residual absorbance of 0.03 was measured (usually approximately 0.015 ). With the $\mathrm{Y} 108 \mathrm{~F}$ and $\mathrm{Y} 503 \mathrm{~F}$ variants it was observed that after the reduction was complete, the absorption at $440 \mathrm{~nm}$ increased, likely due to the formation of a charge transfer interaction between the reduced enzyme and a bound substrate molecule. Due to the slow rate of reduction of the $\mathrm{Y} 108 \mathrm{~F} / \mathrm{Y} 503 \mathrm{~F}$ variant, formation of such a charge transfer complex could occur on a similar timescale as the reduction, which would cause part of the absorbance drop associated with the reduction to be masked.

To study the $\mathrm{pH}$-dependence of the reaction in more detail, the anaerobic reduction of wtVAO by $50 \mu \mathrm{M}$ chavicol was studied in the $\mathrm{pH}$ range 6.1-9.5. The overall spectral changes, as monitored using photodiode array detection, were similar to those observed during the experiments presented in Figure 2 at all $\mathrm{pH}$ values. Reduction of FAD and concomitant formation of a para-quinone methide-reduced enzyme complex was followed by the decay of the para-quinone methide intermediate to yield the fully reduced enzyme spectrum. As before, the absorption at $440 \mathrm{~nm}$ was fit to a triphasic exponential function, yielding two rates that are associated with flavin reduction: $k_{\mathrm{obs}}$ and $k_{\mathrm{obs} 2}$. The values of $k_{\mathrm{obs} 1}$ and $k_{\mathrm{obs} 2}$ remained similar in this $\mathrm{pH}$ range, with the lowest and highest values never differing more than a factor two (Fig. 3 inset). The $\mathrm{pH}$-dependence of the fractional contribution of $k_{\mathrm{obs} 1}$ to the reduction is shown in Figure 3. The contribution of $k_{\mathrm{obs} 1}$ increases with $\mathrm{pH}$. Attempts to fit the data to the Henderson-Hasselbach equation, which allows the determination of the $\mathrm{pK}$ a of an ionisable group were unsuccessful. Although the data display a sigmoidal form, the slope of the

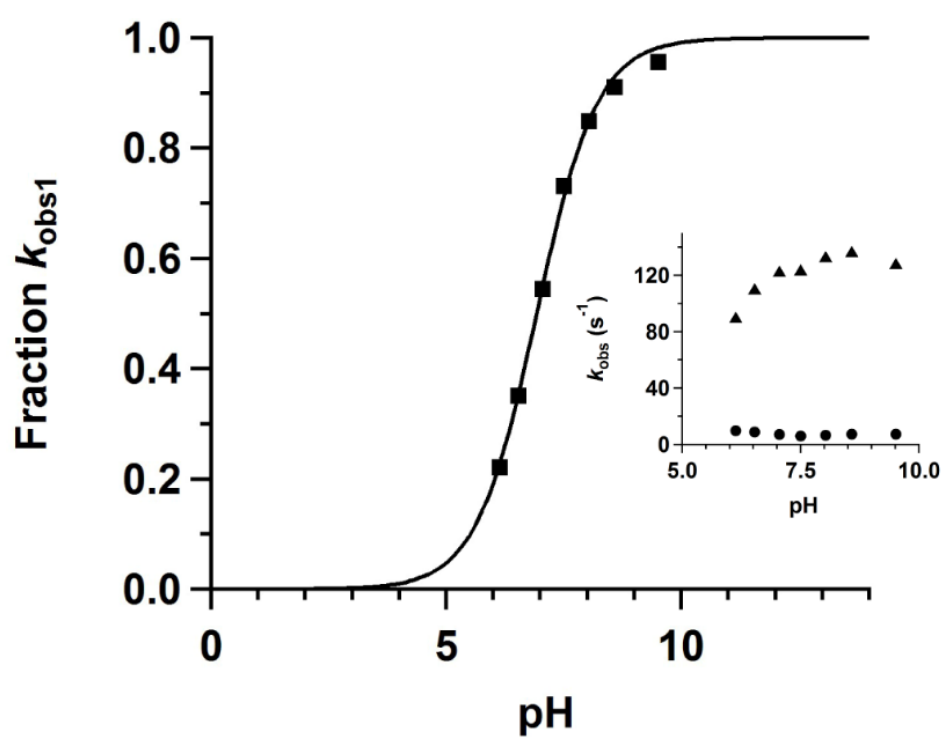

Figure 3: $\mathrm{pH}$-dependence of the contribution of $k_{\mathrm{obs}}$ to the reduction of $10 \mu \mathrm{M} w \mathrm{wt}-\mathrm{VAO}$ by $50 \mu \mathrm{M}$ chavicol. Anaerobic substrate and enzyme solutions in Britt-Robinson buffers of appropriate $\mathrm{pH}$ values were mixed in a stopped-flow apparatus and absorbance changes were monitored using a photodiode array detector. Fitting curves to the absorbance at $440 \mathrm{~nm}$ yielded two reduction rates: $k_{\text {obs }}$ (fast) and $k_{\mathrm{obs} 2}$ (slow). The graph shows the fraction of the change in absorbance associated with reduction that is contributed by $k_{\mathrm{obs} 1}$ [fraction $k_{\mathrm{obs} 1}=a_{\mathrm{obs} 1} /\left(a_{\mathrm{obs}} 1+a_{\mathrm{obs} 2}\right)$, where $a$ is the amplitude]. The solid line is a fit of a sigmoidal function to the data, from which a midpoint $\mathrm{pH}$ value (fraction $k_{\mathrm{obs} 1}=$ fraction $k_{\mathrm{obs} 2}=0.5$ ) of 6.9 was estimated. The inset shows the values of $k_{\text {obs } 1}(\boldsymbol{\Lambda})$ and $k_{\text {obs2 }}(\bullet)$. The error on each data point is less than $5 \%$. 
sigmoid is lower than described by the Henderson-Hasselbach equation. To estimate the midpoint at which the contribution of $k_{\text {obs } 1}$ and $k_{\text {obs2 }}$ to reduction is equal, the data was fit to a sigmoidal curve with the slope as a variable, yielding a midpoint of $\mathrm{pH}$ 6.9.

Isoeugenol binding studies

To evaluate whether the introduced mutations affect the protonation state of phenolic compounds bound to VAO, we studied the binding of the competitive inhibitor isoeugenol to the VAO variants. In solution, the phenolic hydroxyl group of isoeugenol has a $p K_{a}$ of approximately 10 and its absorption spectrum is dependent on its protonation state (2). In particular, deprotonated isoeugenol displays significantly higher absorption at $320 \mathrm{~nm}$ than protonated isoeugenol $\left(\varepsilon_{320}=0.21 \mathrm{mM}^{-1} \mathrm{~cm}^{-1}\right.$ at $\mathrm{pH} 6$ and $8.8 \mathrm{mM}^{-1} \mathrm{~cm}^{-1}$ at $\left.\mathrm{pH} 12\right)$. Therefore, the absorption of isoeugenol at $320 \mathrm{~nm}$ upon binding to VAO can act as a reporter on its protonation state. The $\mathrm{p} K_{\mathrm{a}}$ of isoeugenol bound to $\mathrm{wt}-\mathrm{VAO}$ is approximately 5 (2). To evaluate whether this has been perturbed by the introduced mutations, the VAO variants were titrated with isoeugenol at $\mathrm{pH}$ 7.5. At this $\mathrm{pH}$, isoeugenol is almost entirely protonated in solution, but almost entirely deprotonated when bound to wt-VAO.

Spectral changes observed upon titration of wt-VAO with isoeugenol are shown in Figure 4. Upon addition of isoeugenol, the intensity of the absorption peak of oxidised FAD at $439 \mathrm{~nm}$ decreases, while the absorption at wavelengths below $335 \mathrm{~nm}$ increases, which is due to the absorption of the added isoeugenol. Plots of the absorption at $439 \mathrm{~nm}$ and $320 \mathrm{~nm}$ against the concentration of isoeugenol displayed a curvature indicative of a binding event (Fig. 4 inset). The data at each of these wavelengths could be satisfactorily fit to a model assuming a simple 1:1 complex formation between isoeugenol and VAO. This yielded a dissociation constant of $6.8 \pm 0.5 \mu \mathrm{M}$ from the data at $439 \mathrm{~nm}$ and $6.8 \pm 1.0 \mu \mathrm{M}$ from the data at $320 \mathrm{~nm}$. The increase in the absorption at $320 \mathrm{~nm}$ associated with binding of isoeugenol to VAO is indicative of the fact that, as expected, it is primarily in its deprotonated form upon binding to the enzyme.

Somewhat different spectral changes were observed upon titration of the Y108F variant with isoeugenol (Fig. 4). As with wt-VAO, the intensity of the peak at $439 \mathrm{~nm}$ decreases and absorption below $335 \mathrm{~nm}$ increases. In addition, a broad absorption band appears at wavelengths between 500 and $680 \mathrm{~nm}$. Such a broad absorption band at high wavelengths is indicative of a charge transfer interaction between isoeugenol and FAD, likely caused by $\pi$ stacking of the aromatic rings of isoeugenol and isoalloxazine. Similar charge transfer bands have been observed upon binding of various aromatic compounds to the flavoprotein subunit of PCMH (13). Fitting of the data at $439 \mathrm{~nm}$ yielded a dissociation constant of $18 \pm 2 \mu \mathrm{M}$, suggesting binding of isoeugenol is slightly impaired in the $\mathrm{Y} 108 \mathrm{~F}$ variant as compared with $\mathrm{wt}-\mathrm{VAO}$. The absorption at $550 \mathrm{~nm}$ also displayed curvature indicative of a single binding event and fitting yielded a dissociation constant of $20 \pm 1 \mu \mathrm{M}$. This suggests that the drop in absorption at $439 \mathrm{~nm}$ and charge transfer band at wavelengths above $500 \mathrm{~nm}$ are both caused by the same binding event, the formation of a 1:1 complex between isoeugenol and Y108F. The absorption at $320 \mathrm{~nm}$, however, did not display the major increase associated with binding to $w t-V A O$. This suggests that isoeugenol is primarily in its protonated form when bound to $\mathrm{Y} 108 \mathrm{~F}$ and that the protonated form of isoeugenol forms a charge transfer complex with the FAD cofactor of $\mathrm{Y} 108 \mathrm{~F}$. 


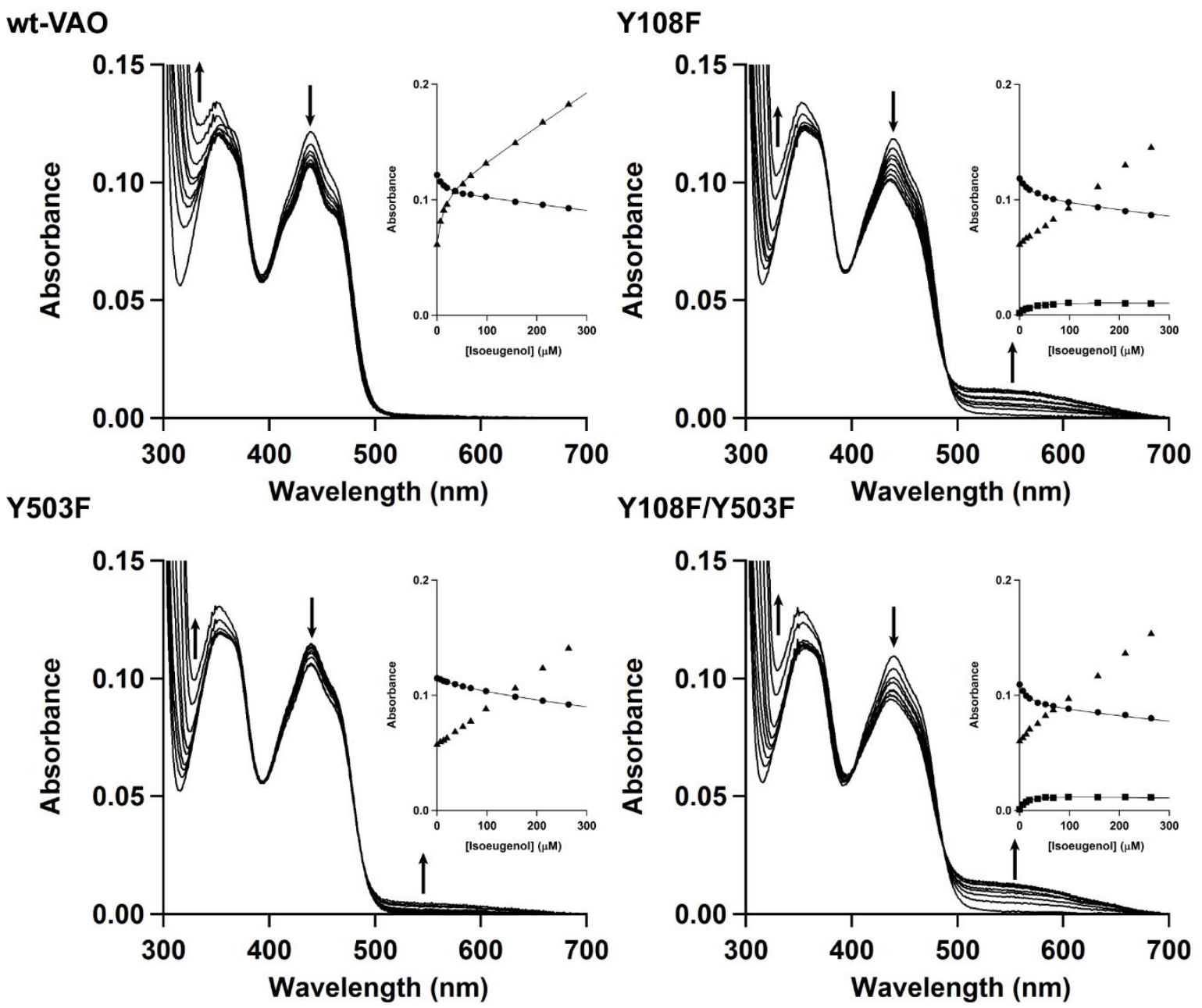

Figure 4: Spectral changes observed upon titration of wt-VAO, Y108F, Y503F, or Y108F/Y503F with the competitive inhibitor isoeugenol. Isoeugenol was added to a $10 \mu \mathrm{M}$ enzyme solution in $50 \mathrm{mM}$ potassium phosphate buffer, $\mathrm{pH}$ 7.5. After each addition, an absorption spectrum was recorded. Spectra recorded at isoeugenol concentrations of $6.6,13.2,19.8,36.0,51.9,98.3,211$, and $312 \mu \mathrm{M}$ are shown. The displayed spectra are corrected for dilution due to the addition of isoeugenol. Arrows indicate the direction of the observed changes in absorption upon increasing isoeugenol concentration. Insets show the absorption measured at $320(\boldsymbol{\Delta}), 439(\bullet)$, and $550(\boldsymbol{\bullet}) \mathrm{nm}$, with fitted lines used to determine dissociation constants. Absorbance values in these insets are not corrected for dilution.

For $\mathrm{Y} 503 \mathrm{~F}$, the absorption peak at $439 \mathrm{~nm}$ also decreased in intensity upon addition of isoeugenol (Fig. 4). Fitting to the data at $439 \mathrm{~nm}$ yielded a dissociation constant of $91 \pm 7 \mu \mathrm{M}$, indicating that binding of isoeugenol is significantly weaker than for wt-VAO. Similar to $\mathrm{Y} 108 \mathrm{~F}$, the appearance of a charge transfer band at wavelengths above $500 \mathrm{~nm}$ was observed. However, the measured absorption of this band was too low to reliably determine the dissociation constant. The absorption at $320 \mathrm{~nm}$ essentially increased linearly with the isoeugenol concentration, suggesting it is due to the presence of deprotonated isoeugenol in solution and that isoeugenol is predominantly in its protonated form upon binding to Y503F.

With Y108F/Y503F, the results of the titration strongly resemble those with Y108F (Fig. 4). Dissociation constants of $11 \pm 1$ and $7.0 \pm 0.4 \mu \mathrm{M}$ were obtained by fitting to the data at 439 and $550 \mathrm{~nm}$ respectively. The appearance of a charge transfer band is observed and the absorption at $320 \mathrm{~nm}$ does not show the large increase associated with binding to wt-VAO. 
This suggests that, like for the $\mathrm{Y} 108 \mathrm{~F}$ and $\mathrm{Y} 503 \mathrm{~F}$ variants, isoeugenol is predominantly in its protonated form when in complex with $\mathrm{Y} 108 \mathrm{~F} / \mathrm{Y} 503 \mathrm{~F}$.

\section{Crystal structures}

To evaluate the effects of the mutations on the architecture of VAO's active site, the crystal structures of the $\mathrm{Y} 108 \mathrm{~F}$ and $\mathrm{Y} 503 \mathrm{~F}$ variants were solved at $2.8 \AA$ resolution by molecular replacement using the VAO monomer [PDB ID: 2VAO (4)] as a search model. Attempts to solve the structure of the $\mathrm{Y} 108 \mathrm{~F} / \mathrm{Y} 503 \mathrm{~F}$ variant were hampered by fragility of the crystals. The overall structures of both $\mathrm{Y} 108 \mathrm{~F}$ and $\mathrm{Y} 503 \mathrm{~F}$ are essentially identical to that of wt-VAO, as indicated by an RMSD of $0.5 \AA$ for 1105 and 1108 pairs of $\mathrm{C} \alpha$ atoms, respectively. The asymmetric unit contains two monomers, which form the functional octamer through crystallographic symmetry, as in wt-VAO.

The geometry of the active site in the mutated enzymes is largely conserved, with the aromatic ring of Phe- 108 or Phe-503 located at the same position as the corresponding tyrosine residue in wt-VAO, where they each form a hydrogen bond with the phenolic hydroxyl group of isoeugenol (Fig. 5) (4). Additionally, Tyr-108 interacts with Ser-426 via a hydrogen bond. This interaction is disrupted in the $\mathrm{Y} 108 \mathrm{~F}$ variant, causing a shift of the Phe-108 side chain towards the FAD cofactor (Fig. 5B). In the Y503F variant, the plane of the aromatic ring of Phe-503 is slightly tilted compared to Tyr-503 in wt-VAO (Fig. 5C). This may partly be due to the lack of the hydrogen bond between Tyr-503 and Arg-504 that is present in wt-VAO.

In summary, both the overall structure of the Y108F and Y503F variants and the geometry of their active sites are extremely similar to wt-VAO. Therefore, changes in reactivity are not due to major structural perturbations, but can be attributed to the disruption of the hydrogen bonding capacity conferred by the two tyrosine residues.

A

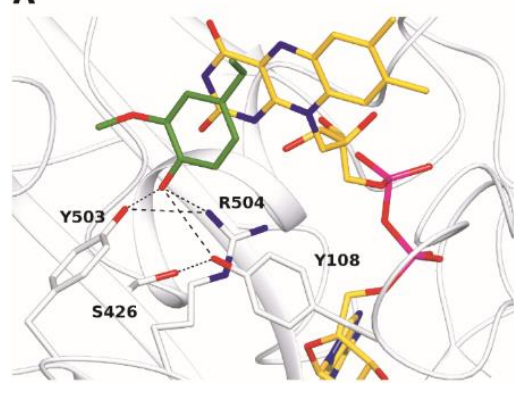

B

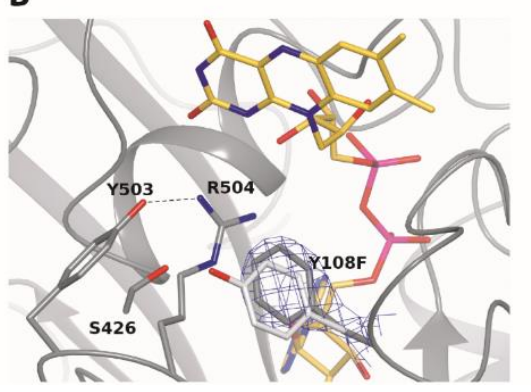

C

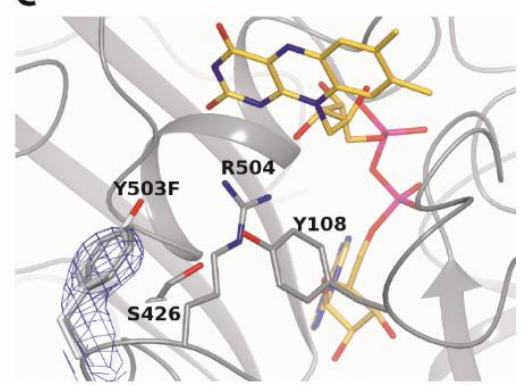

Figure 5: Structure of the phenolate binding pocket of VAO in the wild-type enzyme [A, carbon atoms in white (PDB ID: 2VAO)] and in the Y108F and Y503F variants (B and C respectively, carbon atoms in grey). The refined $2 F_{\mathrm{o}}-F_{\mathrm{c}}$ electron density map (contoured at $1.2 \sigma$ ) is shown for the mutated residues Phe-108 and Phe-503 as blue chicken-wire. The wt-VAO crystal structure in complex with the competitive inhibitor isoeugenol (A, carbon in green) is taken as a reference to analyse the mutant proteins. Phe/Tyr-108, Phe/Tyr-503, Arg-504, Ser-426, isoeugenol, and the FAD cofactor (carbon in yellow) are displayed in stick representation with the surrounding protein backbone shown in cartoon representation. Potential hydrogen bonds are depicted as black dashed lines. Nitrogen atoms are shown in blue, oxygen atoms in red and phosphorus atoms in pink. As reported in (4), binding of isoeugenol does not significantly influence the geometry of the active site. In B and C, the side chains of Tyr-108 and Tyr-503 respectively from wt-VAO are shown for comparison. As described in the text, in the Y503F variant Phe-503 is slightly tilted with respect to the corresponding Tyr-503 in wt-VAO. The figure was prepared using CCP4mg (14). 


\section{Conservation of the phenolate binding pocket in VAO homologues}

To determine whether the residues that form the phenolate binding pocket in VAO are also present in homologous proteins, we evaluated their conservation in a group of homologues of VAO, EUGO, PCMH and the related flavocytochrome eugenol hydroxylase from Pseudomonas sp. OPS1 (EUGH) (15) that we identified in a previous study and named the 4phenol oxidising (4PO) subfamily (12, Chapter 3). Additionally, we determined whether they are present in the decarboxylases $\mathrm{CndG}$ and FeeG, which catalyse the oxidative decarboxylation of $\mathrm{N}$-substituted tyrosine derivatives $(16,17)$. These also belong to the $4 \mathrm{PO}$ subfamily and their catalytic mechanism is believed to involve the initial formation of a paraquinone methide intermediate followed by the expulsion of carbon dioxide. This analysis revealed that Tyr-108, Tyr-503 and Arg-504 are strictly conserved suggesting they are of importance for catalysis in these related enzymes.

\section{Discussion}

Our results demonstrate that the presence of at least one of the active site tyrosine residues Tyr-108 and Tyr-503 is essential for efficient catalysis by VAO, with the $k_{\text {cat }}$ values for $\mathrm{Y} 108 \mathrm{~F} / \mathrm{Y} 503 \mathrm{~F}$ being less than $2 \%$ of those for wt-VAO for each tested substrate. The effect of the single tyrosine to phenylalanine mutations on catalytic efficiency was dependent on the substrate. The oxidation of vanillyl alcohol was severely impaired in both the Y108F and Y503F variants, whereas the oxidation of chavicol was only slightly impaired as compared with wt-VAO.

Stopped-flow kinetics revealed that reduction of the FAD cofactor of VAO by vanillyl alcohol or chavicol proceeds at two distinct rates: $k_{\text {obs } 1}$ (fast) and $k_{\text {obs2 }}$ (slow). $k_{\text {obs2 }}$ lies close to the rate of steady-state catalysis and, therefore, is probably rate-limiting. The observed reduction in the rate of steady-state catalysis for the tyrosine to phenylalanine variants corresponds with the observed reduction in $k_{\text {obs2, }}$ demonstrating that impaired flavin reduction is the cause of the reduced rate of catalysis. Titration with the competitive inhibitor isoeugenol indicated that, at $\mathrm{pH} 7.5$, this molecule is predominantly in its deprotonated phenolate form when bound to wt-VAO, but predominantly in its protonated form when bound to $\mathrm{Y} 108 \mathrm{~F}, \mathrm{Y} 503 \mathrm{~F}$, or $\mathrm{Y} 108 \mathrm{~F} / \mathrm{Y} 503 \mathrm{~F}$. In combination, these results suggest that Tyr-108 and Tyr-503 play a role in activating VAO's substrates for their reaction with its FAD cofactor by enabling their deprotonation in the active site of the enzyme. Interestingly, the relative contributions of the two reduction rates, $k_{\mathrm{obs}}$ and $k_{\mathrm{obs} 2}$, to the change in absorption associated with flavin reduction changes upon the introduction of the tyrosine to phenylalanine mutations. At $\mathrm{pH} 7.5, k_{\mathrm{obs}}$ contributes more to the drop in absorption in wt-VAO, whereas in the Y108F, Y503F, and $\mathrm{Y} 108 \mathrm{~F} / \mathrm{Y} 503 \mathrm{~F}$ variants, $k_{\mathrm{obs}}$ contributes more to the drop in absorption. The relative contributions of the two rates shift when the reduction is performed at $\mathrm{pH} 6.2$, with the relative contribution of $k_{\mathrm{obs}}$ becoming greater. The reduction of the $\mathrm{Y} 108 \mathrm{~F} / \mathrm{Y} 503 \mathrm{~F}$ variant by chavicol was an exception to this observation. However, this may be due to the contribution of $k_{\text {obs } 2}$ to the reduction at $\mathrm{pH} 6.2$ being underestimated due to the concomitant formation of a charge transfer interaction between the reduced enzyme and substrate. For the reduction of wt-VAO by chavicol, the contribution of $k_{\text {obs } 1}$ increases with $\mathrm{pH}$ in the $\mathrm{pH}$ range 6.1-9.5. The data was fit to a sigmoidal curve with a midpoint $\mathrm{pH}$, at which $k_{\mathrm{obs} 1}$ and $k_{\mathrm{obs} 2}$ contribute equally to the reduction, of 6.9 . 
Based on these results, we propose a somewhat modified model for the reaction mechanism of VAO (Scheme 2). Substrates can bind in the active site of VAO with their phenolic hydroxyl group either in its protonated or deprotonated form. The affinity of the enzyme for the substrate depends on the protonation state of the substrate, with wt-VAO displaying a higher affinity for deprotonated substrates. Subsequently, the substrate reacts with VAO, a reaction that occurs at different rates depending on the protonation state of the substrate. With the deprotonated substrate, the reaction simply involves the transfer of a hydride to the FAD cofactor to yield the para-quinone methide intermediate. Reaction with the protonated substrate requires the deprotonation of the substrate's phenolic hydroxyl group either prior to or at the same time as the hydride transfer reaction. The proton is transferred to either Tyr108 or Tyr-503, which may be transiently deprotonated in the enzyme's active site. This is stimulated by Arg-504, which is positioned so as to form a cation- $\pi$ interaction with Tyr-108, lowering its $\mathrm{pK}$. This model explains the two rates of flavin reduction observed for each substrate, with $k_{\text {obs1 } 1}$ representing the reaction with deprotonated substrate and $k_{\text {obs } 2}$ representing the reaction with protonated substrate. This is supported by the fact that the contribution of $k_{\text {obsi } 1}$ to flavin reduction increases with $\mathrm{pH}$, as at higher $\mathrm{pH}$ values a larger percentage of the substrate is in its deprotonated form. The results of the isoeugenol titrations support the idea that substrates bind to $\mathrm{Y} 108 \mathrm{~F}, \mathrm{Y} 503 \mathrm{~F}$, and $\mathrm{Y} 108 \mathrm{~F} / \mathrm{Y} 503 \mathrm{~F}$ predominantly in their protonated form at $\mathrm{pH} 7.5$, as opposed to wt-VAO where it predominantly binds in its deprotonated form. This explains the change in the relative contributions of $k_{\mathrm{obs} 1}$ and $k_{\mathrm{obs} 2}$ to flavin reduction upon introduction of the tyrosine to phenylalanine mutations. The mutations prevent the preferential binding of the deprotonated form of the substrates observed with wtVAO due to the loss of hydrogen bonding interactions that stabilise the negative charge on the phenolate oxygen. In support of this, the crystallographic analysis revealed that, though the mutations do not produce any major structural perturbation of the active site architecture, they modify the hydrogen bonding network that is essential to activate the substrate for the reaction with the flavin cofactor.

The fact that the curve of the fractional contribution of $k_{\mathrm{obs} 1}$ to the reduction versus $\mathrm{pH}$ does not obey the Henderson-Hasselbach equation may be attributable to the effects of functional groups near the phenolic hydroxyl group on its protonation state, which can have a distorting effect on the titration behaviour of ionisable groups in proteins (18). Additionally, interconversion of the enzyme-protonated substrate (Eox-SH) and enzyme-deprotonated substrate (Eox-S-) complexes may involve more significant changes than simply the deprotonation of the substrate's phenolic hydroxyl group. The fact that a charge transfer interaction is observed between the enzyme variants and protonated isoeugenol during titrations with this molecule, but not between the wild-type enzyme and deprotonated isoeugenol, suggests that the ligand may bind to the enzyme in a slightly different orientation depending on its protonation state. Thus, conversion of the Eox-SH complex to the Eox-Scomplex may require a reorientation of the substrate within the active site, and possibly minor changes in the conformation of active site residues. This could explain the reasonably low rates observed for $k_{\text {obs2 }}$. Although in our view this model provides the most satisfactory explanation of our observations, an alternative mechanism where the two observed reduction rates reflect the presence of two forms of the enzyme, one in which both Tyr-108 and Tyr-503 are protonated and one in which one of the two tyrosine residues is deprotonated, cannot be fully ruled out. 


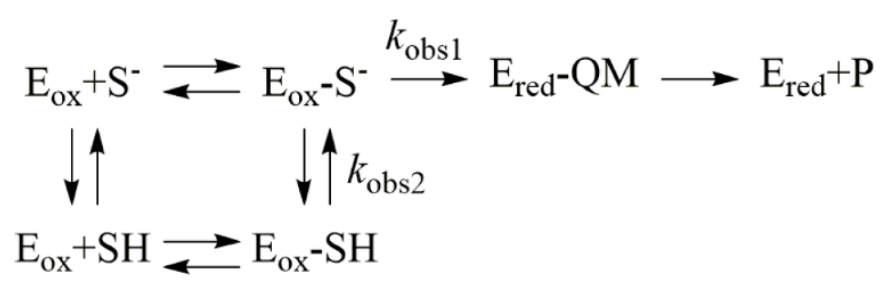

Scheme 2: Proposed model for the reductive half-reaction of VAO. Substrates can bind to the enzyme with their phenolic hydroxyl group in its protonated $(\mathrm{SH})$ or deprotonated ( $\left.\mathrm{S}^{-}\right)$form. Upon binding of the deprotonated form, the enzyme is reduced, yielding the para-quinone methide-reduced enzyme

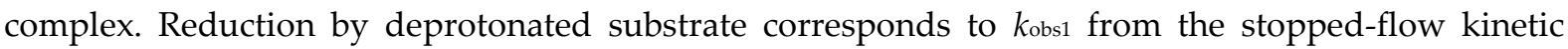
experiments presented here. Upon binding of the protonated form, the substrate must be deprotonated in order to allow flavin reduction to occur, i.e. the Eox-SH complex must be converted to the Eox-Scomplex. This corresponds to $k_{\text {obs2 }}$ from the stopped-flow kinetic experiments. After the reduction, the product is released from the reduced enzyme. This step is too slow to be catalytically relevant, suggesting product release is faster in the presence of molecular oxygen.

The effect of the single Y108F and Y503F mutations on the rate of reduction of VAO was dependent on the substrate used. With the benzylic alcohol vanillyl alcohol, a far more severe drop in both $k_{\text {obs1 }}$ and $k_{\text {obs2 }}$ was observed than with the 4-allyphenol chavicol. However, the change in the relative contributions of $k_{\mathrm{obs} 1}$ and $k_{\mathrm{obs} 2}$ to flavin reduction is similar. Possibly, the large effect on the reduction rate seen with vanillyl alcohol is due to incorrect positioning of the substrate in the active site of Y108F and Y503F. Hydrogen bonds between the substrate's phenolic hydroxyl group and the two tyrosine residues may be of importance in maintaining it in the correct orientation for the hydride transfer reaction to take place. This effect may be less important for chavicol than for vanillyl alcohol, as interactions involving its longer side chain may lock it into position in the active site. In this context, it is interesting to note that the $K_{\mathrm{m}}$ for the oxidation of chavicol by wt-VAO is significantly lower than that for the oxidation of vanillyl alcohol, indicating tighter binding of chavicol.

In addition to the $4 \mathrm{PO}$ subfamily members mentioned above, a catalytic motif composed of two tyrosine residues has been proposed to be involved in substrate activation in other enzymes in which catalysis proceeds through a para-quinone methide intermediate, such as the cofactor-independent hydroxycinnamoyl-CoA hydratase-lyase from Pseudomonas fluorescens AN103 (19) and bacterial phenolic acid decarboxylases [(20) [although an alternative mechanism has also been proposed $(21,22)])]$. This suggests that these structurally unrelated proteins have obtained similar mechanisms for the activation of the phenolic hydroxyl groups of their substrates through convergent evolution.

Catalytic motifs composed of two tyrosine residues have also been implicated in substrate activation in a number of enzymes that catalyse oxidation reactions that do not involve a parasubstituted phenol. They have been proposed to facilitate the activation of hydroxyl groups for their oxidation to carbonyls in other oxidoreductases from the VAO/PCMH family, such as aclacinomycin oxidoreductase from Streptomyces galilaeus ATCC 31615 (23), Dbv29 from Nonomuraea sp. ATCC 39727 (24), GilR from Streptomyces griseoflavus (25), and the monolignol oxidoreductase BBE-like protein 15 from Arabidopsis thaliana (26). These enzymes all belong to the same clade of the VAO/PCMH family, which primarily contains enzymes with a bicovalently bound FAD cofactor (27, Chapter 2). Despite their homology with VAO, a sequence and structural alignment of the five proteins demonstrated that the tyrosine pair 
found in these enzymes is not equivalent to that found in VAO, suggesting the motifs evolved separately.

In summary, the results presented here indicate that two tyrosine residues, Tyr-108 and Tyr503 , are responsible for activating the phenolic substrates of VAO for oxidation by facilitating the binding of their deprotonated phenolate form in the active site, shedding light on the catalytic mechanism of VAO and other homologous flavoenzymes that oxidise parasubstituted phenols.

\section{Materials and methods}

\section{Materials}

Vanillyl alcohol and isoeugenol were from Sigma-Aldrich (St. Louis, MO, USA). Chavicol was from Quest International (Naarden, NL). All other chemicals were from commercial sources and of the purest grade available.

\section{Cloning, protein expression and purification}

Constructs encoding for the $\mathrm{Y} 108 \mathrm{~F}$ and $\mathrm{Y} 503 \mathrm{~F}$ variants were created by linear whole-plasmid amplification from the pBC11 (28) plasmid using the mutagenic primers CATCTCTATTGGAAGGAATTCCGGATTTGGCGGTGCTGCGCC (Y108F) and ATGGATGGGGC GAATTTCGAACCCATCTGGC (Y503F) (nucleotides in italics are the sites of mutation for introduction of the tyrosine to phenylalanine mutations, the underlined nucleotide indicates the site of a silent mutation that introduces an EcoRI site). After amplification and concomitant ligation, template DNA was digested using DpnI and the mutagenised plasmids were transformed into DH5 $\alpha$ E. coli. Correct plasmids, as confirmed by sequencing, were transformed into BL21 E. coli for protein expression. The construct encoding for the $\mathrm{Y} 108 \mathrm{~F} / \mathrm{Y} 503 \mathrm{~F}$ variant was created in a similar manner, using the plasmid encoding for the $\mathrm{Y} 108 \mathrm{~F}$ variant as the template for amplification and the primer for the Y503F variant. All variants were expressed and purified as described previously for wt-VAO (12).

\section{Analytical methods}

Unless specified otherwise, all experiments were performed in $50 \mathrm{mM}$ potassium phosphate buffer, $\mathrm{pH}$ 7.5. Absorption spectra of purified proteins were recorded using a Hewlett Packard 8453 diode array spectrophotometer (Agilent Technologies, Santa Clara, CA, USA) and enzyme concentrations were determined using the extinction coefficient of wt-VAO at $439 \mathrm{~nm}$ $\left[\varepsilon=12,500 \mathrm{M}^{-1} \mathrm{~cm}^{-1}(1)\right]$. For trichloroacetic acid precipitations, $5 \%(\mathrm{w} / \mathrm{v})$ trichloroacetic acid was mixed with $10 \mu \mathrm{M}$ enzyme and samples were incubated on ice for $30 \mathrm{~min}$. Subsequently, the precipitated protein was pelleted by centrifugation $\left(21,000 \mathrm{~g}, 15 \mathrm{~min}, 4{ }^{\circ} \mathrm{C}\right)$, and the presence of FAD in the pellet or supernatant was judged from its colour. The absence of FAD from the supernatants was confirmed by measuring their absorption spectra. Analytical gel filtration was performed using a Superdex $200 \mathrm{HR} 10 / 30$ column on an Äkta Pure chromatography system (GE Healthcare, Chicago, IL, USA). Samples of enzyme at a concentration of $10 \mu \mathrm{M}$ were loaded onto the column and subsequently eluted in $50 \mathrm{mM}$ potassium phosphate buffer, $\mathrm{pH} 7.5$, containing $150 \mathrm{mM} \mathrm{KCl}$. Molecular masses were determined based on a calibration curve of proteins of known mass. Thermal unfolding of the VAO variants was monitored by the ThermoFAD method (11) using a MiniOpticon real-time PCR system (Bio-Rad, Hercules, CA, USA). Protein solutions at a concentration of $7 \mu \mathrm{M}$ were subjected to a temperature gradient from $25-70{ }^{\circ} \mathrm{C}$ and the fluorescence of the FAD cofactor 
was measured every $0.5^{\circ} \mathrm{C}$ with excitation and emission wavelengths of $485 \pm 30$ and $625 \pm 30$ $\mathrm{nm}$ respectively. Thermal midpoints of unfolding were determined by finding the maximum of the derivative of the unfolding curves.

\section{Steady-state kinetics}

All experiments were performed at $25^{\circ} \mathrm{C}$ in $50 \mathrm{mM}$ potassium phosphate buffer, $\mathrm{pH}$ 7.5. The oxidation of vanillyl alcohol to vanillin was followed by monitoring the absorption of the product at $340 \mathrm{~nm}\left(\varepsilon=14,000 \mathrm{M}^{-1} \mathrm{~cm}^{-1}\right)$. The oxidation of chavicol was followed by monitoring oxygen consumption using a Hansatech Oxytherm system (Hansatech Instruments, King's Lynn, UK). Steady-state kinetic parameters were determined by fitting the obtained data to the Michaelis-Menten equation (vanillyl alcohol) using Igor Pro v. 6.10A (Wavemetrics, Lake Oswego, OR, USA) or to a modified version of the Michaelis-Menten equation that includes a term to take substrate inhibition into account (chavicol):

$$
k_{o b s}=\frac{k_{c a t} *[S]}{K_{m}+[S]+\frac{[S]^{2}}{K_{i}}}
$$

Where $k_{\mathrm{obs}}$ is the measured initial rate, $k_{\mathrm{cat}}$ is the turnover number, $K_{\mathrm{m}}$ is the Michaelis constant, $K_{\mathrm{i}}$ is the inhibition constant for substrate inhibition and [S] is the substrate concentration.

\section{Stopped-flow kinetics}

The reduction of VAO variants by vanillyl alcohol or chavicol was followed using an SX20 stopped-flow system (Applied Photophysics, Leatherhead, UK) equipped with a photodiode array detector or a photomultiplier detector as applicable. Enzyme and substrate solutions in $50 \mathrm{mM}$ potassium phosphate buffer, $\mathrm{pH} 6.2$ or 7.5 as appropriate, containing $5 \mathrm{mM}$ glucose were made anaerobic by first flushing them with $\mathrm{N}_{2}$ gas for $10 \mathrm{~min}$ and then adding $0.3 \mu \mathrm{M}$ glucose oxidase. Subsequently, the enzyme and substrate solutions were mixed to final concentrations of $10 \mu \mathrm{M}$ enzyme and $2 \mathrm{mM}$ (vanillyl alcohol) or $100 \mu \mathrm{M}$ (chavicol) substrate and spectral changes were monitored at $25{ }^{\circ} \mathrm{C}$. Rate constants were obtained by global fitting of multiple single wavelength traces to appropriate exponential functions using Igor Pro. Spectral deconvolution of multiwavelength data was performed using Pro-Kineticist (Applied Photophysics). To study the $\mathrm{pH}$-dependence of the reduction of wt-VAO by chavicol in more detail, the reaction was performed at various $\mathrm{pH}$ values in the $\mathrm{pH}$ range 6.1-9.5. Experiments were performed in Britt-Robinson buffers consisting of $40 \mathrm{mM}$ acetic acid, $40 \mathrm{mM} \mathrm{H}_{3} \mathrm{PO}_{4}$, and $40 \mathrm{mM} \mathrm{H}_{3} \mathrm{BO}_{3}$ set to the correct $\mathrm{pH}$ using $\mathrm{KOH}$. The ionic strength of the buffers was set to 0.2 $\mathrm{M}$ by the addition of $\mathrm{K}_{2} \mathrm{SO}_{4}$. Subsequently, enzyme and substrate solutions were made anaerobic as described above, mixed to final concentrations of $10 \mu \mathrm{M}$ enzyme and $50 \mu \mathrm{M}$ chavicol, and the reduction was monitored at $25^{\circ} \mathrm{C}$ and data was analysed as described above.

\section{Isoeugenol binding studies}

Changes in the spectral properties of the VAO variants upon binding of the competitive inhibitor isoeugenol were monitored using a Cary 4000 spectrophotometer (Agilent Technologies). Isoeugenol was titrated to $10 \mu \mathrm{M}$ enzyme in $50 \mathrm{mM}$ potassium phosphate buffer, $\mathrm{pH} 7.5$, and an absorption spectrum was recorded after every addition. Dissociation constants were determined by fitting the data at a specific wavelength to a model assuming formation of a 1:1 enzyme-isoeugenol complex using the following formula with Igor Pro: 


$$
A=\varepsilon_{E} *[E]_{f}+\varepsilon_{E I} *[E I]+\varepsilon_{I} *[I]_{f}
$$

Where:

$$
[E I]=\frac{[E]_{t}+[I]_{t}+K_{d}-\sqrt{\left([E]_{t}+[I]_{t}+K_{d}\right)^{2}-4 *[I]_{t} *[E]_{t}}}{2}
$$

And:

$$
[E]_{f}=[E]_{t}-[E I]
$$

And:

$$
[I]_{f}=[I]_{t}-[E I]
$$

With $\varepsilon_{\mathrm{E}}, \varepsilon_{\mathrm{EI}}$, and $\varepsilon_{\mathrm{I}}$ being the molar extinction coefficients of the free enzyme, the enzymeinhibitor complex, and the free inhibitor respectively, $[\mathrm{E}]_{\mathrm{f}}$ being the concentration of free enzyme, $[\mathrm{EI}]$ the concentration of enzyme-inhibitor complex, $[\mathrm{I}]_{\mathrm{f}}$ the concentration of free inhibitor, $[E]_{\mathrm{t}}$ the total enzyme concentration, $[\mathrm{I}]_{\mathrm{t}}$ the total inhibitor concentration and $K_{\mathrm{d}}$ the dissociation constant.

\section{Crystallisation and structure determination}

VAO variants were crystallised by the hanging drop vapour diffusion method. Protein solutions at a concentration of $180-220 \mu \mathrm{M}$ in $50 \mathrm{mM}$ potassium phosphate buffer, $\mathrm{pH} 7.5$, were mixed 1:1 (v/v) with a mother liquor containing 6\% (w/v) PEG4000 in $100 \mathrm{mM}$ sodium acetate buffer, $\mathrm{pH} 4.6$, and equilibrated against the mother liquor at $20{ }^{\circ} \mathrm{C}$. Prior to data collection, crystals were transferred to a cryoprotectant solution containing $10 \%(\mathrm{w} / \mathrm{v})$ PEG4000 and 20\% (w/v) glycerol in $100 \mathrm{mM}$ sodium acetate buffer, $\mathrm{pH} 4.6$, and cryo-cooled in liquid nitrogen. X-ray diffraction data was collected at the X06DA beamline of the Swiss Light Source synchrotron in Villigen, Switzerland (SLS) and at the ID30B beamline of the European Synchrotron Radiation Facility in Grenoble, France (ESRF). Images were integrated using MOSFLM (29) and data was scaled using programs of the CCP4 suite (30). Detailed data processing statistics are shown in Table 4. Structures of VAO variants were solved by molecular replacement using PHASER (31) with the structure of wt-VAO (PDB ID: 2VAO) devoid of all ligands and water molecules as a search model. Subsequently, structures were refined by alternating refinement using REFMAC5 (32) and manual model building using COOT (33). Atomic coordinates and structure factors were deposited in the Protein Data Bank under the PDB ID codes 5MXJ (Y108F-VAO) and 5MXU (Y503F-VAO).

\section{Acknowledgements}

This work was financed by the European Union through the INDOX project (FP7-KBBE-20137-613549). We thank the European Synchrotron Radiation Facility (ESRF) and the Swiss Light Source (SLS) for providing beamtime and assistance and the European Community's Seventh Framework Programme (FP7/2007-2013) under BioStruct-X (Grants 7551 and 10205) for funding synchrotron trips. 
Table 4: Data collection and refinement statistics.

\begin{tabular}{|c|c|c|}
\hline & Y108F & Y503F \\
\hline PDB ID Code & $5 \mathrm{MXJ}$ & $5 \mathrm{MXU}$ \\
\hline Space group & $I 4$ & I4 \\
\hline Resolution $(\AA)$ & 2.8 & 2.8 \\
\hline$a, b, c(\AA)$ & $137.9,137.9,132.9$ & $138.0,138.0,132.9$ \\
\hline$R_{\text {sym }}$ a,b,c $(\%)$ & $8.7(67.9)$ & $10.1(63.8)$ \\
\hline $\mathrm{CC}_{1 / 2} \mathrm{a}^{\mathrm{a}, \mathrm{c}}$ & $0.99(0.54)$ & $0.99(0.54)$ \\
\hline Completeness ${ }^{\mathrm{b}}(\%)$ & 89.7 (91.9) & $91.8(94.3)$ \\
\hline Unique reflections & 27138 & 27846 \\
\hline Multiplicity & $2.3(2.1)$ & $2.4(2.4)$ \\
\hline $\mathrm{I} / \sigma^{\mathrm{c}}$ & $9.0(1.3)$ & $7.0(1.3)$ \\
\hline \multicolumn{3}{|l|}{ Number of atoms: } \\
\hline protein & 8697 & 8706 \\
\hline FAD/glycerol/water & $2 \times 53 / 3 \times 6 / 260$ & $2 \times 53 / 2 \times 6 / 232$ \\
\hline Average $B$ value for all atoms $\left(\AA^{2}\right)$ & 52.1 & 52.6 \\
\hline$R_{\text {cryst }^{\mathrm{c}, \mathrm{d}}(\%)}$ & $18.0(34.2)$ & $17.5(30.2)$ \\
\hline$R_{\text {free }}{ }^{c, d}(\%)$ & $25.9(33.7)$ & $24.9(37.4)$ \\
\hline Rms bond length $(\AA)$ & 0.01 & 0.01 \\
\hline Rms bond angles $\left(^{\circ}\right)$ & 1.56 & 1.48 \\
\hline Ramachandran outliers (\%) & 0 & 0 \\
\hline
\end{tabular}

a Data processing statistics were based on $\mathrm{CC}_{1 / 2}$ as described in $(34,35)$.

b $R_{\text {sym }}=\sum\left|\mathrm{I}_{i}-<\mathrm{I}>\right| / \sum \mathrm{I}_{i}$, where $\mathrm{I}_{i}$ is the intensity of $i^{\text {th }}$ observation and $<\mathrm{I}>$ is the mean intensity of the reflection.

c Values in parentheses are for reflections in the highest resolution shell.

d $R_{\text {cryst }}=\sum\left|F_{\text {obs }}-F_{\text {calc }}\right| / \sum\left|F_{\text {obs }}\right|$ where $F_{\text {obs }}$ and $F_{\text {calc }}$ are the observed and calculated structure factor amplitudes, respectively. $R_{\text {cryst }}$ and $R_{\text {free }}$ were calculated using the working and test sets, respectively

\section{References}

1. de Jong, E., van Berkel, W. J. H., van der Zwan, R. P., and de Bont, J. A. M. (1992) Purification and characterization of vanillyl-alcohol oxidase from Penicillium simplicissimum: A novel aromatic alcohol oxidase containing covalently bound FAD. Eur. J. Biochem. 208, 651-657

2. Fraaije, M. W., Veeger, C., and van Berkel, W. J. H. (1995) Substrate specificity of flavindependent vanillyl-alcohol oxidase from Penicillium simplicissimum: Evidence for the production of 4-hydroxycinnamyl alcohols from 4-allylphenols. Eur. J. Biochem. 234, 271-277

3. van den Heuvel, R. H. H., Fraaije, M. W., Laane, C., and van Berkel, W. J. H. (1998) Regio- and stereospecific conversion of 4-alkylphenols by the covalent flavoprotein vanillyl-alcohol oxidase. J. Bacteriol. 180, 5646-5651

4. Mattevi, A., Fraaije, M. W., Mozzarelli, A., Olivi, L., Coda, A., and van Berkel, W. J. H. (1997) Crystal structures and inhibitor binding in the octameric flavoenzyme vanillyl-alcohol oxidase: The shape of the active-site cavity controls substrate specificity. Structure. 5, 907-920

5. Fraaije, M. W., van Berkel, W. J. H., Benen, J. A. E., Visser, J., and Mattevi, A. (1998) A novel oxidoreductase family sharing a conserved FAD-binding domain. Trends Biochem. Sci. 23, 206207

6. Fraaije, M. W., and van Berkel, W. J. H. (1997) Catalytic mechanism of the oxidative demethylation of 4-(methoxymethyl)phenol by vanillyl-alcohol oxidase: Evidence for formation of a $p$-quinone methide intermediate. J. Biol. Chem. 272, 18111-18116

7. Fraaije, M. W., van den Heuvel, R. H. H., Roelofs, J. C. A. A., and van Berkel, W. J. H. (1998) 
Kinetic mechanism of vanillyl-alcohol oxidase with short-chain 4-alkylphenols. Eur. J. Biochem. 253, 712-719

8. Nguyen, Q.-T., de Gonzalo, G., Binda, C., Rioz-Martínez, A., Mattevi, A., and Fraaije, M. W. (2016) Biocatalytic properties and structural analysis of eugenol oxidase from Rhodococcus jostii RHA1: A versatile oxidative biocatalyst. ChemBioChem. 17, 1359-1366

9. Cunane, L. M., Chen, Z.-W., Shamala, N., Mathews, F. S., Cronin, C. N., and McIntire, W. S. (2000) Structures of the flavocytochrome $p$-cresol methylhydroxylase and its enzyme-substrate complex: Gated substrate entry and proton relays support the proposed catalytic mechanism. J. Mol. Biol. 295, 357-374

10. Efimov, I., Cronin, C. N., Bergmann, D. J., Kuusk, V., and McIntire, W. S. (2004) Insight into covalent flavinylation and catalysis from redox, spectral, and kinetic analyses of the R474K mutant of the flavoprotein subunit of $p$-cresol methylhydroxylase. Biochemistry. 43, 6138-6148

11. Forneris, F., Orru, R., Bonivento, D., Chiarelli, L. R., and Mattevi, A. (2009) ThermoFAD, a Thermofluor ${ }^{\circledR}$-adapted flavin ad hoc detection system for protein folding and ligand binding. FEBS J. 276, 2833-2840

12. Ewing, T. A., Gygli, G., and van Berkel, W. J. H. (2016) A single loop is essential for the octamerization of vanillyl alcohol oxidase. FEBS J. 283, 2546-2559

13. Engst, S., Kuusk, V., Efimov, I., Cronin, C. N., and McIntire, W. S. (1999) Properties of $p$-cresol methylhydroxylase flavoprotein overproduced by Escherichia coli. Biochemistry. 38, 16620-16628

14. McNicholas, S., Potterton, E., Wilson, K. S., and Noble, M. E. M. (2011) Presenting your structures: The CCP4mg molecular-graphics software. Acta Crystallogr. Sect. D Biol. Crystallogr. 67, 386-394

15. Brandt, K., Thewes, S., Overhage, J., Priefert, H., and Steinbüchel, A. (2001) Characterization of the eugenol hydroxylase genes (ehyA/ehyB) from the new eugenol-degrading Pseudomonas sp. strain OPS1. Appl. Microbiol. Biotechnol. 56, 724-730

16. Brady, S. F., Chao, C. J., and Clardy, J. (2002) New natural product families from an environmental DNA (eDNA) gene cluster. J. Am. Chem. Soc. 124, 9968-9969

17. Rachid, S., Revermann, O., Dauth, C., Kazmaier, U., and Müller, R. (2010) Characterization of a novel type of oxidative decarboxylase involved in the biosynthesis of the styryl moiety of chondrochloren from an acylated tyrosine. J. Biol. Chem. 285, 12482-12489

18. Ullmann, G. M., and Bombarda, E. (2013) $\mathrm{pK}$ a values and redox potentials of proteins. What do they mean? Biol. Chem. 394, 611-619

19. Bennett, J. P., Bertin, L., Moulton, B., Fairlamb, I. J. S., Brzozowski, A. M., Walton, N. J., and Grogan, G. (2008) A ternary complex of hydroxycinnamoyl-CoA hydratase-lyase (HCHL) with acetyl-CoA and vanillin gives insights into substrate specificity and mechanism. Biochem. J. 414, $281-289$

20. Sheng, X., Lind, M. E. S., and Himo, F. (2015) Theoretical study of the reaction mechanism of phenolic acid decarboxylase. FEBS J. 282, 4703-4713

21. Rodríguez, H., Angulo, I., de las Rivas, B., Campillo, N., Páez, J. A., Muñoz, R., and Mancheño, J. M. (2010) $p$-Coumaric acid decarboxylase from Lactobacillus plantarum: Structural insights into the active site and decarboxylaction catalytic mechanism. Proteins Struct. Funct. Bioinforma. 78, 1662-1676

22. Frank, A., Eborall, W., Hyde, R., Hart, S., Turkenburg, J. P., and Grogan, G. (2012) Mutational analysis of phenolic acid decarboxylase from Bacillus subtilis (BsPAD), which converts bioderived phenolic acids to styrene derivatives. Catal. Sci. Technol. 2, 1568-1574

23. Alexeev, I., Sultana, A., Mäntsälä, P., Niemi, J., and Schneider, G. (2007) Aclacinomycin oxidoreductase (AknOx) from the biosynthetic pathway of the antibiotic aclacinomycin is an unusual flavoenzyme with a dual active site. Proc. Natl. Acad. Sci. U. S. A. 104, 6170-6175

24. Liu, Y.-C., Li, Y.-S., Lyu, S.-Y., Hsu, L.-J., Chen, Y.-H., Huang, Y.-T., Chan, H.-C., Huang, C.-J., Chen, G.-H., Chou, C.-C., Tsai, M.-D., and Li, T.-L. (2011) Interception of teicoplanin oxidation intermediates yields new antimicrobial scaffolds. Nat. Chem. Biol. 7, 304-309 
25. Noinaj, N., Bosserman, M. A., Schickli, M. A., Piszczek, G., Kharel, M. K., Pahari, P., Buchanan, S. K., and Rohr, J. (2011) The crystal structure and mechanism of an unusual oxidoreductase, GilR, involved in gilvocarcin V biosynthesis. J. Biol. Chem. 286, 23533-23543

26. Daniel, B., Pavkov-Keller, T., Steiner, B., Dordic, A., Gutmann, A., Nidetzky, B., Sensen, C. W., van der Graaff, E., Wallner, S., Gruber, K., and Macheroux, P. (2015) Oxidation of monolignols by members of the berberine bridge enzyme family suggests a role in plant cell wall metabolism. J. Biol. Chem. 290, 18770-18781

27. Leferink, N. G. H., Heuts, D. P. H. M., Fraaije, M. W., and van Berkel, W. J. H. (2008) The growing VAO flavoprotein family. Arch. Biochem. Biophys. 474, 292-301

28. van den Heuvel, R. H. H., Fraaije, M. W., Mattevi, A., and van Berkel, W. J. H. (2000) Asp-170 is crucial for the redox properties of vanillyl-alcohol oxidase. J. Biol. Chem. 275, 14799-14808

29. Battye, T. G. G., Kontogiannis, L., Johnson, O., Powell, H. R., and Leslie, A. G. W. (2011) iMOSFLM: A new graphical interface for diffraction-image processing with MOSFLM. Acta Crystallogr. Sect. D Biol. Crystallogr. 67, 271-281

30. Winn, M. D., Ballard, C. C., Cowtan, K. D., Dodson, E. J., Emsley, P., Evans, P. R., Keegan, R. M., Krissinel, E. B., Leslie, A. G. W., McCoy, A., McNicholas, S. J., Murshudov, G. N., Pannu, N. S., Potterton, E. A., Powell, H. R., Read, R. J., Vagin, A., and Wilson, K. S. (2011) Overview of the CCP4 suite and current developments. Acta Crystallogr. Sect. D Biol. Crystallogr. 67, 235-242

31. McCoy, A. J., Grosse-Kunstleve, R. W., Adams, P. D., Winn, M. D., Storoni, L. C., and Read, R. J. (2007) Phaser crystallographic software. J. Appl. Crystallogr. 40, 658-674

32. Murshudov, G. N., Vagin, A. A., and Dodson, E. J. (1997) Refinement of macromolecular structures by the maximum-likelihood method. Acta Crystallogr. Sect. D Biol. Crystallogr. 53, 240255

33. Emsley, P., and Cowtan, K. (2004) Coot: Model-building tools for molecular graphics. Acta Crystallogr. Sect. D Biol. Crystallogr. 60, 2126-2132

34. Karplus, P. A., and Diederichs, K. (2012) Linking crystallographic model and data quality. Science. 336, 1030-1033

35. Karplus, P. A., and Diederichs, K. (2015) Assessing and maximizing data quality in macromolecular crystallography. Curr. Opin. Struct. Biol. 34, 60-68

\section{Abbreviations}

4PO: 4-phenol oxidising, EUGH: eugenol hydroxylase, EUGO: eugenol oxidase, FAD: flavin adenine dinucleotide, PCMH: para-cresol methylhydroxylase, VAO: vanillyl alcohol oxidase 




\title{
Biocatalysis in organic synthesis: Oxidations using alcohol oxidases
}

\author{
Tom A. Ewing ${ }^{1}$, Marco W. Fraaije ${ }^{2}$ and Willem J. H. van Berkel ${ }^{1}$ \\ ${ }^{1}$ Laboratory of Biochemistry, Wageningen University \& Research, Wageningen, The Netherlands \\ 2Molecular Enzymology Group, University of Groningen, Groningen, The Netherlands
}

Based on Science of Synthesis: Biocatalysis in Organic Synthesis 3 (2015), Chapter 3.3.3, pp. 157-185

\section{Summary}

The oxidation of alcohols to carbonyl compounds is one of the most important reactions in organic chemistry. Biocatalysis provides an attractive alternative to traditional methods of alcohol oxidation. Enzyme-catalysed oxidations are often highly regioselective, enabling the oxidation of polyols without the need for complex protection schemes. Many oxidative enzymes also display exquisite enantioselectivity and thus can be used for the preparation of enantiopure secondary alcohols by kinetic resolution or deracemisation. The use of biocatalysts also has advantages from the point of view of sustainability. This is particularly true for oxidases, which catalyse the oxidation of their substrates using molecular oxygen as the final electron acceptor. This chapter provides an overview of the known alcohol oxidases, the reactions they catalyse and, where available, examples of their use for synthetic purposes. 



\section{Introduction}

The oxidation of alcohols is one of the most important reactions in synthetic organic chemistry, as the products that are formed can be used as precursors for a wide variety of compounds. Primary alcohols can be oxidised to either aldehydes or carboxylic acids, while the oxidation of secondary alcohols usually results in the formation of ketones. Conventionally, alcohol oxidation is achieved using stoichiometric amounts of chemical oxidants. Well-known examples include metal-based oxidants (e.g. chromium, ruthenium or manganese compounds), various forms of activated dimethyl sulfoxide and hypervalent iodine compounds (1). These traditional oxidants suffer from a number of drawbacks. Their lack of specificity can lead to undesired side reactions and they display poor regio- and stereoselectivity, necessitating the use of complex protection schemes if multiple reactive functional groups are present in a compound. Additionally, their use in stoichiometric amounts is undesirable from an environmental perspective, especially for industrial-scale applications. In recent years, progress has been made in the development of compounds that can catalyse the oxidation of alcohols with cheap oxidants, e.g. molecular oxygen, acting as the final electron acceptor. These include various transition metal-based catalysts (e.g. copper, ruthenium or palladium compounds) and organocatalysts such as the nitroxyl radical 2,2,6,6tetramethylpiperidine- $N$-oxyl (TEMPO) $(2,3)$.

Another promising alternative to the use of traditional chemical oxidants is biocatalysis $(4,5)$. In contrast to chemical oxidation methods, enzymatic oxidation reactions are often highly regioselective. The asymmetric environment of an enzyme's active site also allows many enzymatic reactions to proceed stereoselectively, a feat that is hard to accomplish by chemical means. Additionally, enzymatic catalysis has advantages from the point of view of sustainability, as enzymes can be produced in a biological system and are active under mild reaction conditions and in aqueous environments. Enzymatic reactions also do not require the addition of toxic compounds (such as transition metals) and thus are ideally suited for use in the pharmaceutical and food industries. A large number of enzymes are known to be capable of oxidising alcohols to carbonyl compounds. These enzymes are either dehydrogenases or oxidases, depending on the identity of their final electron acceptor. Dehydrogenases do not react with oxygen and instead depend on the presence of alternative electron acceptors, such as $\mathrm{NAD}(\mathrm{P})^{+}$, quinones or heme for their activity. Therefore, the use of dehydrogenases in organic synthesis requires the addition of stoichiometric amounts of these cofactors or of efficient systems for their regeneration. Oxidases on the other hand, use cheap and ubiquitous oxygen as a final electron acceptor, making them attractive candidates for biocatalytic applications. This chapter provides an overview of the known classes of alcohol oxidase enzymes, the reactions they catalyse and examples of their application in organic chemical synthesis.

\section{Flavin-dependent alcohol oxidases}

Most alcohol oxidases are flavoenzymes, which contain a flavin cofactor (FAD or FMN). Flavoenzymes usually bind this cofactor non-covalently, but a number of oxidases have been identified where the flavin is anchored to the enzyme through either one or two covalent bonds (6). The flavin acts as a redox cofactor, shuttling electrons from the alcohol substrate to 
molecular oxygen. Although flavin-dependent oxidases convert a large variety of different alcohols, a number of general mechanistic principles can be defined that appear to hold true for the majority of the enzymes. The reactions can be divided into a reductive half-reaction, during which two electrons are transferred from the alcohol substrate to the oxidised flavin cofactor, and an oxidative half-reaction, during which the flavin is re-oxidised by the transfer of two electrons to molecular oxygen. At the start of the reductive half-reaction, the alcohol is bound in the active site of the enzyme, with its $\mathrm{C} \alpha$ atom positioned at a distance of approximately $3.5 \AA$ from the flavin N5 atom (7). In many cases, the enzyme stimulates the deprotonation of the hydroxyl group of the alcohol, which facilitates oxidation by promoting the formation of a $\mathrm{C}=\mathrm{O}$ bond $(7,8)$. Subsequently, two electrons are transferred from the alcohol $\mathrm{C} \alpha$ atom to the flavin N5 atom, yielding reduced flavin and the carbonyl product. The electron transfer is generally believed to proceed via the direct transfer of a hydrogen anion $(9,10)$. During the oxidative half-reaction the reduced flavin cofactor is reoxidised by molecular oxygen $(11,12)$.

Many flavin-dependent alcohol oxidases belong to one of two protein families: the glucosemethanol-choline oxidase (GMC) family or the vanillyl alcohol oxidase/para-cresol methylhydroxylase (VAO/PCMH) family (13). Members of each family can be recognised by the presence of a characteristic FAD-binding domain that is conserved within the family (14, 15). The presence of a more variable substrate-binding domain, which determines the architecture of the active site, allows great variety in the type of substrates that are oxidised by members of each family. The different types of flavin-dependent alcohol oxidases and the reactions they catalyse are discussed below.

\section{Methanol oxidase}

Methanol oxidase (MO, also known as alcohol oxidase, EC 1.1.3.13) is a member of the GMCtype oxidase family. $\mathrm{MO}$ is found in a large number of fungi and methylotrophic yeasts. A number of MOs are commercially available, including the enzymes from Pichia pastoris, Candida boidinii and Hansenula polymorpha (16-19). The subunit molecular masses of MOs range from 65 to $85 \mathrm{kDa}$ and most of the characterised enzymes form octamers, though tetrameric variants have also been reported $(20,21)$. Each MO subunit contains a noncovalently bound FAD cofactor, which is sometimes post-translationally modified to arabino$\operatorname{FAD}(22,23)$.

MOs catalyse the oxidation of short-chain unbranched primary alcohols with chain lengths of up to 5 carbon atoms (Scheme 1). Usually, methanol is the best substrate, with the catalytic efficiency decreasing as the chain length of the substrate increases. Many MOs readily oxidise unsaturated alcohols and alcohols with substituents such as chloro, bromo, thiol or cyano groups. Secondary and branched chain alcohols are generally poor MO substrates. Many MOs can also oxidise formaldehyde to formic acid, though at a far lower rate than they oxidise methanol.

A potential application of $\mathrm{MO}$ in organic synthesis is the in situ formation of reactive aldehydes. Siebum et al. utilised MO from $P$. pastoris in the one-pot synthesis of the aldol 1 from 4-penten-1-ol and acetone (Scheme 2) (24). This was achieved via a cascade reaction, 


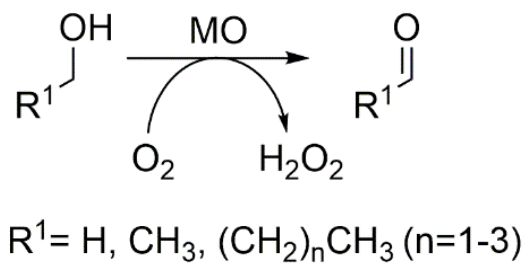

Scheme 1: Oxidation of primary alcohols catalysed by methanol oxidase (MO).

where conversion of the alcohol to the corresponding aldehyde by $\mathrm{MO}$ was followed by enantioselective aldol addition catalysed by 2-deoxyribo-5-phosphate aldolase. This procedure yielded 1 in a yield of $30 \%$ and an enantiomeric excess of $70 \%$.

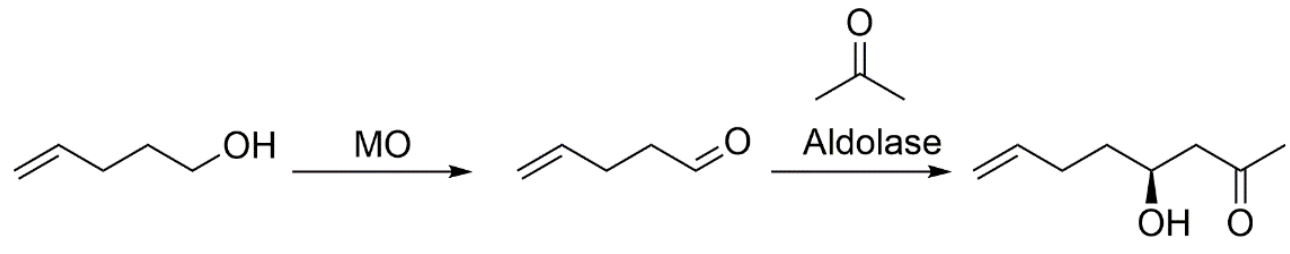

1

Scheme 2: Synthesis of (S)-4-hydroxyoct-7-en-2-one (1) using methanol oxidase (MO) and an aldolase (24).

MO has also been applied in the synthesis of enantiomerically pure 2-hydroxyketones 2 from benzaldehyde and various aliphatic alcohols (Scheme 3) (25). This was achieved using a onepot cascade reaction, where oxidation of the alcohols to aldehydes by $\mathrm{MO}$ was combined with enantioselective carboligation by benzaldehyde lyase. For short unbranched saturated alcohols this procedure yielded the $(R)$-enantiomers of 2, generally in good yields and excellent enantiomeric excess. The procedure with methanol was extended by the enantioselective reduction of the formed 2-hydroxy-1-phenylethan-1-one by an alcohol dehydrogenase, yielding enantiomerically pure (S)-1-phenylethane-1,2-diol in quantitative yield and an enantiomeric excess of $>99 \%$ (26).

Recently, a similar cascade was employed for the synthesis of chiral benzoins from benzyl alcohols (27). The oxidation of benzyl alcohols by MO from Pichia pastoris was combined with the enantioselective carboligation of the formed aldehydes catalysed by benzaldehyde lyase. With benzyl alcohol as a substrate, the cascade was employed for the synthesis of $4.6 \mathrm{~g}$ enantiomerically pure $(R)$-benzoin 3 (Scheme 4, 82\% isolated yield). Various substituted benzyl alcohols, particularly those bearing substituents at the para-position, were also successfully converted.

\section{Isoamyl alcohol oxidase}

Isoamyl alcohol oxidase (IAAO) from the fungus Aspergillus oryzae oxidises branched chain alkanols. IAAO is a $73 \mathrm{kDa}$ glycoprotein that is predicted to contain covalently-bound FAD (28). IAAO has a relatively narrow substrate range. Its best substrate is 3-methylbutan-1-ol (isoamyl alcohol), which is converted to 3-methylbutanal (isovaleraldehyde). IAAO is also 


\begin{tabular}{|c|c|c|}
\hline $\mathrm{MO}$ & benzaldehyde & \\
\hline $\mathbf{R}^{1}$ & Conversion to $2(\%)$ & $e e(\%)$ \\
\hline $\mathrm{H}$ & $>99$ & - \\
\hline $\mathrm{Me}$ & $>99$ & 99 \\
\hline Et & 15 & 98 \\
\hline $\operatorname{Pr}$ & 91 & 98 \\
\hline Pentyl & 0 & - \\
\hline $\mathrm{iPr}$ & 0 & - \\
\hline$s-\mathrm{Bu}$ & 0 & - \\
\hline Vinyl & 0 & - \\
\hline
\end{tabular}

Scheme 3: Synthesis of 2-hydroxyketones (2) from alcohols and benzaldehyde using methanol oxidase $(\mathrm{MO})$ and benzaldehyde lyase (25).

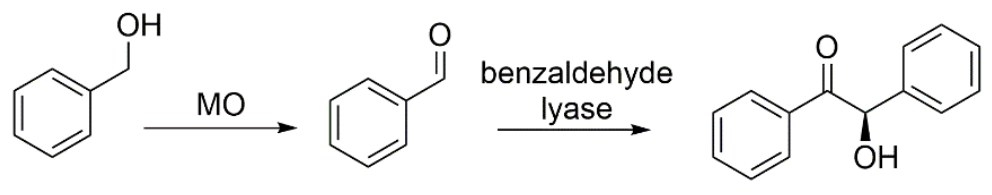

3

Scheme 4: Synthesis of (R)-benzoin (3) from benzyl alcohol using methanol oxidase (MO) and benzaldehyde lyase (27).

active with 1-pentanol, 1-hexanol and 4-methylpentan-1-ol. Alcohols with less than 5 or more than 6 carbon atoms are poor substrates for IAAO.

Long-chain alcohol oxidase

Long-chain alcohol oxidase (LCAO, EC 1.1.3.20, also known as fatty alcohol oxidase) oxidises various primary and secondary long-chain alcohols to the corresponding aldehydes or ketones. LCAO has been identified in various fungi and in a number of plants, e.g. Arabidopsis thaliana (29-31). LCAOs are membrane-bound proteins with subunit molecular masses of approximately $70 \mathrm{kDa}$ and contain a flavin cofactor. The sequences of LCAOs from Candida and Arabidopsis contain a CXXC heme-binding motif and the enzyme from Candida cloacae has been shown to contain a covalently bound heme cofactor $(32,33)$. The specific roles played by LCAO's two cofactors during catalysis have yet to be determined. The substrate scope of LCAO from Candida maltosa has been studied in detail and shown to be rather broad (30). Its best substrates are medium-chain 1-alkanols (1-octanol and 1-decanol), but its catalytic scope extends to the oxidation of medium- and long-chain 1-alkenols, alkanediols, $\omega$-hydroxyacids, aromatic primary alcohols and medium-chain 2-alkanols. The oxidation of 2-alkanols by LCAO proceeds enantioselectively, with the $(R)$-enantiomers being preferentially converted. 
LCAO from Aspergillus fumigatus has been combined with an $\omega$-transaminase to achieve the synthesis of primary amines 4 from a range of medium-chain 1-alkanols (Scheme 5) (34). Alkanols with a chain length of 4 to 11 carbon atoms could be converted, with $\mathrm{C}_{6}-\mathrm{C}_{8} 1$-alkanols giving the highest conversions.

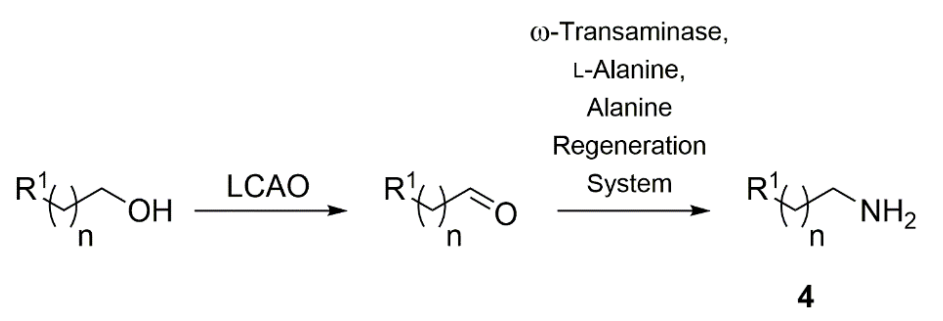

\begin{tabular}{|ccc|}
\hline $\mathbf{n}$ & $\mathbf{R}^{\mathbf{1}}$ & Conversion to 3 (\%) \\
\hline 3 & $\mathrm{H}$ & 20 \\
4 & $\mathrm{H}$ & 75 \\
5 & $\mathrm{H}$ & $>99$ \\
6 & $\mathrm{H}$ & $>99$ \\
7 & $\mathrm{H}$ & 82 \\
8 & $\mathrm{H}$ & 46 \\
9 & $\mathrm{H}$ & 16 \\
10 & $\mathrm{H}$ & 74 \\
5 & $\mathrm{Br}$ & 98 \\
5 & $\mathrm{Cl}$ & 96 \\
7 & $\mathrm{Cl}$ & 42 \\
5 & $\mathrm{C} \equiv \mathrm{N}$ & $>99$ \\
5 & $\mathrm{C} \equiv \mathrm{CH}$ & - \\
5 & $\mathrm{NH} H_{2}$ & - \\
5 & $\mathrm{OH}$ & - \\
5 & $\mathrm{SH}$ & \\
\hline
\end{tabular}

Scheme 5: Synthesis of primary amines (4) from 1-alkanols using long-chain alcohol oxidase and an $\omega$-transaminase (34).

Compounds with halogen, nitrile or alkyne substituents at the C-terminal end of the 1alkanol were converted, but compounds with an amino, hydroxyl or thiol substituent at this position were not. Secondary alcohols and benzylic or allylic alcohols were also not converted.

\section{Aryl alcohol oxidase}

Aryl alcohol oxidase (AAO, also known as veratryl alcohol oxidase or aromatic alcohol oxidase, EC 1.1.3.7) is a GMC-type oxidase primarily found in white-rot lignin-degrading fungi (35). AAOs are monomeric glycoproteins with molecular masses of 70-80 kDa and contain a non-covalently bound FAD cofactor. The most extensively studied AAO is the enzyme from Pleurotus eryngii (36). AAO oxidises a wide variety of primary $\alpha$-aryl alcohols to the corresponding aldehydes (Scheme 6$)(37,38)$. Substituents on the aromatic ring have a 
large effect on AAO activity, with electron-donating substituents (e.g. methoxy groups) leading to increased activity, but electron-withdrawing substituents (e.g. nitro groups) leading to diminished activity. para-Hydroxy substituted compounds are poorly converted by AAO. In addition to $\alpha$-aryl alcohols, primary $\gamma$-aryl alcohols can be oxidised, but only when an $\alpha$ - $\beta$ double bond is present [e.g. cinnamyl alcohol (3-phenylprop-2-en-1-ol)]. The aliphatic alcohol 2,4-hexadien-1-ol is also a good substrate, suggesting that AAO requires the presence of a conjugated double bond system, but not necessarily an aromatic ring, to enable catalysis. AAO also oxidises various $\alpha$-aryl aldehydes to carboxylic acids, particularly when electron withdrawing substituents (e.g. nitro, chloro or fluoro groups) are present on the aromatic ring (39). This reaction proceeds via the hydration of the aldehydes to their gem-diols, which are subsequently oxidised by the enzyme.

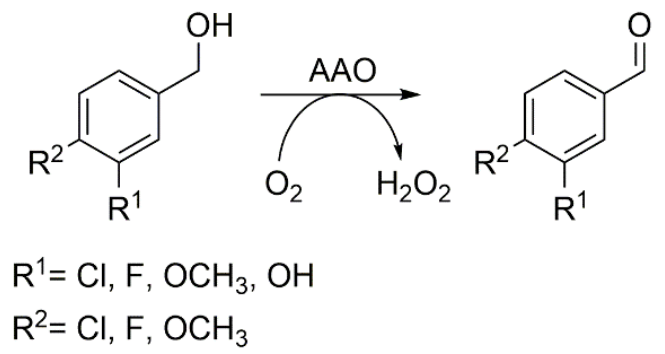

Scheme 6: Oxidation of $\alpha$-aryl alcohols catalysed by aryl alcohol oxidase (AAO).

Using deuterated 4-methoxybenzyl alcohol it was shown that the transfer of a hydride anion to the FAD cofactor during catalysis by AAO occurs enantioselectively, with the pro- $R$ hydrogen being preferentially removed (40). The enantioselectivity of AAO was confirmed by evaluating its reactivity with secondary alcohols. Despite being a poor substrate, 1-(4'methoxyphenyl)ethanol was resolved by $\mathrm{AAO}$, with the $R$-enantiomer remaining in an enantiomeric excess of $>98 \%$ after long-term conversion. The authors also identified an AAO mutant (F501A) that has heightened reactivity towards secondary alcohols and enhanced enantioselectivity compared to the wild-type enzyme. Further optimisation of the reactivity of AAO towards secondary alcohols may open the door to its use in the production of enantiomerically pure secondary $\alpha$-aryl alcohols. Although, P. eryngii AAO is poorly active with para-hydroxy substituted phenols, AAO from Bjerkandera adusta is able to oxidise these substrates (41).

\section{5-(Hydroxymethyl)furfural oxidase}

Another enzyme that catalyses the oxidation of $\alpha$-aryl alcohols is the GMC-type oxidase 5(hydroxymethyl)furfural oxidase (HMFO, EC 1.1.3.47). HMFO from Methylovorus sp. MP688 is a $70 \mathrm{kDa}$ protein that contains a non-covalently bound FAD cofactor. The enzyme is capable of oxidising 5-(hydroxymethyl)furfural (HMF) to 2,5-furandicarboxylic acid (FDCA) (42). HMF can be obtained from biomass by the conversion of sugars such as glucose and fructose and is a key building block for the synthesis of biobased chemicals (43). Its conversion to FDCA is of interest as this compound can function as a starting material for the synthesis of polyesters. HMFO catalyses all three two-electron oxidations that are required for this conversion (Scheme 7$)(42,44)$. As with AAO, the oxidation of the aldehydes to carboxylic acids proceeds via the oxidation of the gem-diol formed by hydration of the aldehydes. 
Dijkman et al. demonstrated that 95\% conversion of $4 \mathrm{mM} \mathrm{HMF}$ to FDCA could be achieved using HMFO, albeit with a relatively high enzyme concentration $(20 \mu \mathrm{M})$ and $24 \mathrm{~h}$ reaction time. Variants of the enzyme that displayed improved conversion of HMF to FDCA have also been described (45). The oxidation of HMF to FDCA by AAO has also been explored (46). However, efficient production of FDCA could not be achieved using only AAO, as it did not convert 2,5-formylfurancarboxylic acid (FFCA), the final intermediate in the reaction sequence, to FDCA. Conversion of FFCA formed from HMF by AAO to FDCA could be achieved by the subsequent addition of a peroxygenase. However, a reaction time of $120 \mathrm{~h}$ was required to achieve $91 \%$ conversion of $3 \mathrm{mM} \mathrm{HMF}$ to FDCA.

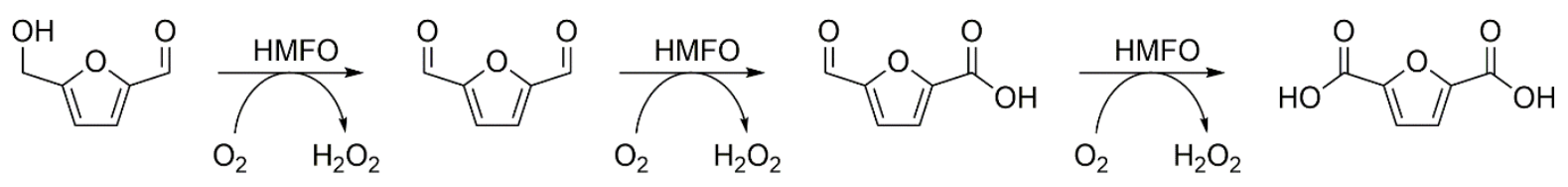

Scheme 7: Three-step oxidation of 5-(hydroxymethyl)furfural to 2,5-furandicarboxylic acid by 5(hydroxymethyl)furfural oxidase (HMFO).

In addition to the oxidation of HMF, HMFO catalyses the oxidation of various primary benzylic alcohols. As for AAO, the presence of a conjugated double bond system (not necessarily an aromatic ring) in a substrate is the minimum requirement to allow catalysis by HMFO, as it also oxidises cinnamyl alcohol and 2,4-hexadien-1-ol.

\section{Vanillyl alcohol oxidase}

The canonical member of the VAO/PCMH family is vanillyl alcohol oxidase from Penicillium simplicissimum (VAO, EC 1.1.3.38) (47). VAO is a $64 \mathrm{kDa}$ protein that is present in solution as an equilibrium of dimers and octamers. The octamer is the predominant form, but both forms are catalytically active (48). The enzyme contains an FAD cofactor, which is covalently bound to a histidine residue $(47,49)$. VAO catalyses the oxidation of various primary or secondary para-hydroxyl substituted benzylic alcohols, to the corresponding aldehydes or ketones (Scheme 8$)(47,50)$. Interestingly, the oxidation of secondary $\alpha$-aryl alcohols by VAO occurs enantioselectively, e.g. the reaction with 1-(4'-hydroxyphenyl)ethanol is selective towards the (S)-enantiomer, with an $E$-value of 57 (51). This raises the possibility that VAO could be used for the production of enantiomerically pure secondary $\alpha$-aryl alcohols by the resolution of racemic alcohols. The catalytic scope of VAO is not limited to the oxidation of alcohols: the enzyme also catalyses the deamination of benzylic amines, the demethylation of 4(methoxymethyl)phenol, the hydroxylation of 4-allylphenols, and the hydroxylation or dehydrogenation of 4-alkylphenols $(50,51)$.

In addition to the $P$. simplicissimum enzyme, an enzyme that displays VAO activity has been identified in the fungus Byssochlamys fulva V107 (52). This enzyme forms dimers with a subunit mass of approximately $58 \mathrm{kDa}$ and catalyses the oxidation of 4-hydroxybenzylic alcohols, 4-alkylphenols and 4-allyphenols, but not of 4-hydroxybenzylic amines or 4(methoxymethyl)phenol. Jin et al. identified a VAO homologue of bacterial origin in Rhodococcus jostii RHAI, which they named eugenol oxidase (EUGO) (53). EUGO is more efficient at catalysing the oxidation of vanillyl alcohol than $P$. simplicissimum VAO. However, 


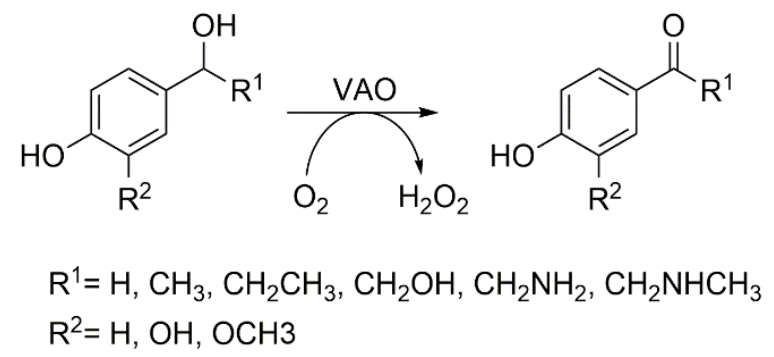

Scheme 8: Oxidation of 4-hydroxybenzylic alcohols catalysed by vanillyl alcohol oxidase (VAO).

4-alkyphenols and 4-(methoxymethyl)phenol are poor substrates for EUGO. The oxidation of the secondary alcohol 1-(4'-hydroxy-3'-methoxyphenyl)ethanol by EUGO was found to occur enantioselectively (54). Conversion of racemic 1-(4'-hydroxy-3'-methoxyphenyl)ethanol led to enrichment of the $(S)$-enantiomer, though the achieved $e e$ values were modest at $\leq 65 \%$.

\section{(S)-2-Hydroxy acid oxidases}

(S)-2-Hydroxy acid oxidases (HAO, EC 1.1.3.15) catalyse the oxidation of 2-hydroxy acids to 2-keto acids. The enzymes display enantioselectivity, preferentially converting the $(S)$ enantiomers of their substrates. Unlike most other flavin-dependent oxidases, HAOs contain FMN as a cofactor, rather than FAD. Three types of HAO can be defined based on their substrate specificity: glycolate oxidase, L-lactate oxidase and long-chain 2-hydroxy acid oxidase.

Glycolate oxidase (GlyO) is found in a variety of plants and mammals, with the most extensively studied variant being the enzyme from spinach (Spinacia oleracea), which is commercially available (55-57). GlyO's best substrate is glycolic acid, which is oxidised to glyoxylic acid (Scheme 9). Glyoxylic acid can be oxidised further to yield oxalic acid, though this process is less efficient than the oxidation of glycolic acid (58).

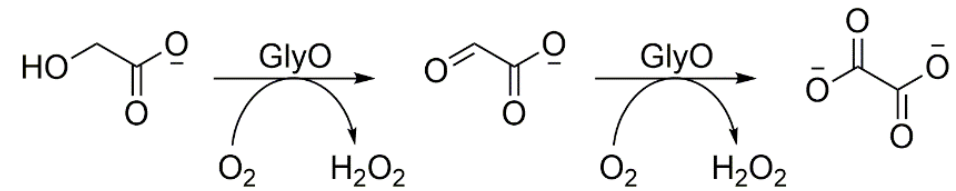

Scheme 9: Oxidation of glycolic acid catalysed by glycolate oxidase (GlyO).

GlyO also catalyses the enantioselective oxidation of (S)-2-hydroxy acids with longer chain lengths (up to 9 carbon atoms) (59). Adam et al. used spinach GlyO to produce various $(R)-2-$ hydroxy acids by the kinetic resolution of racemates, obtaining the $(R)$-enantiomers in excellent enantiomeric excesses at approximately 50\% conversion (59). This process was extended by the subsequent enantioselective reduction of the formed 2-keto acids using a lactate dehydrogenase, yielding the $(R)$-2-hydroxy acids $5 \mathbf{a}$ and $5 \mathbf{b}$ in yields of $>84 \%$ and enantiomeric excesses of $>93 \%$ (Scheme 10) (60). Permeabilised whole-cell preparations of $P$. pastoris containing overexpressed spinach GlyO and catalase have also been used as a biocatalyst for the production of various enantiopure (R)-2-hydroxy acids (61). 
L-lactate oxidase (LLO) is found in lactic acid bacteria, with well-known enzymes being those from Aerococcus viridans and Pediococcus sp., both of which are commercially available (62-64). LLO oxidises (S)-lactic acid to pyruvate. The enzyme is highly specific towards (S)-lactic acid, as it does not accept glycolic acid or 2-hydroxybutyric acid as a substrate (63). The oxidation of (S)-lactic acid by LLO has been combined with the non-enantioselective reduction of the formed pyruvate by sodium borohydrate to achieve the synthesis of $(R)$-lactic acid starting from racemic lactic acid, (S)-lactic acid or pyruvate (65).

Long-chain 2-hydroxy acid oxidases (LHAO) oxidise various 2-hydroxy acids with chain lengths of over three carbon atoms. Sources of LHAO include rat and pig kidney $(66,67)$. Good substrates of the enzyme include the $(S)$-enantiomers of aliphatic 2-hydroxy acids such as 2-hydroxybutyric acid and 2-hydroxyisocaproic acid, and aromatic 2-hydroxy acids, such as mandelic acid and phenyllactic acid (67-70).

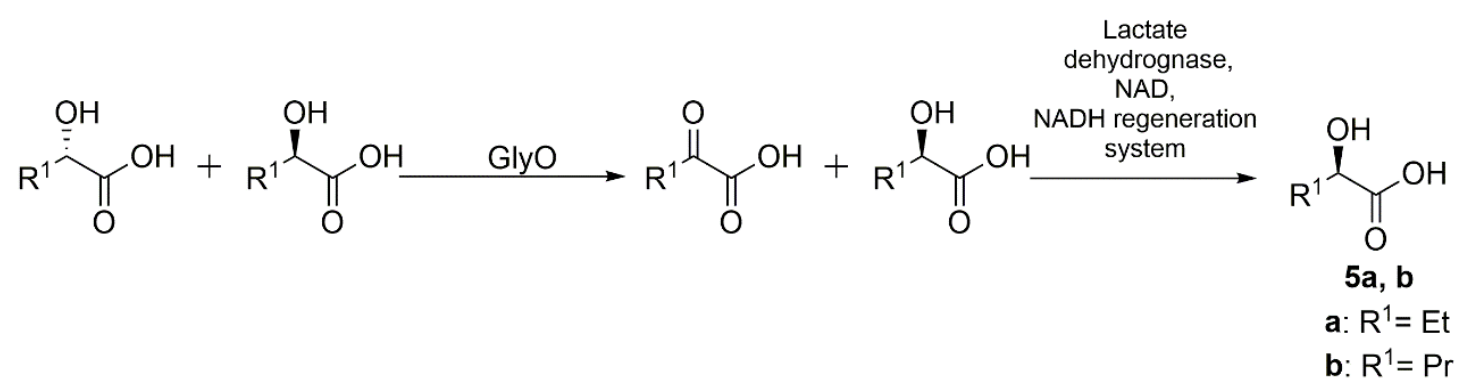

Scheme 10: Synthesis of (R)-2-hydroxy acids (5) by deracemisation using glycolate oxidase (GlyO) and lactate dehydrogenase (60).

\section{Cholesterol oxidase}

Cholesterol oxidase (ChoX, EC 1.1.3.6) is a bacterial flavoenzyme that catalyses the oxidation of the C3 hydroxyl group of cholesterol, yielding cholest-5-en-3-one. Most ChoXs also catalyse its subsequent isomerisation to cholest-4-en-3-one through the transfer of a proton from C4 to C6 via an active site base (Scheme 11) (71). Two different forms of ChoX exist: one which belongs to the GMC family, in which the FAD cofactor is non-covalently bound to the enzyme, and one which belongs to the $\mathrm{VAO} / \mathrm{PCMH}$ family, in which the FAD cofactor is covalently bound to the enzyme (71). Examples of both forms of the enzyme are commercially available, e.g. Streptomyces sp. ChoX for the GMC-type and Brevibacterium sp. ChoX for the VAO/PCMHtype. In addition to cholesterol, ChoX is active with various other sterols, such as pregnenolone and cholestanol $(71,72)$. Rhodococcus erythropolis ChoX can also catalyse the enantioselective oxidation of smaller cyclic unsaturated alcohols, such as 3-methylcyclohex2-en-1-ol (73).

ChoX can be a useful tool in the synthesis of rare steroids, which are of use as reference compounds for bioanalytical purposes. Alexander and Fisher reported the synthesis of $7 \alpha-$ hydroxycholest-4-en-3-one (8), an intermediate in the biosynthesis of bile acid, from $3 \beta$ hydroxycholest-5-en-7-one (6) (Scheme 12) (74). Three chemical steps were used to convert 6 to cholest-5-en-3 $\beta, 7 \alpha$-diol (7), which was converted to 8 using ChoX. 


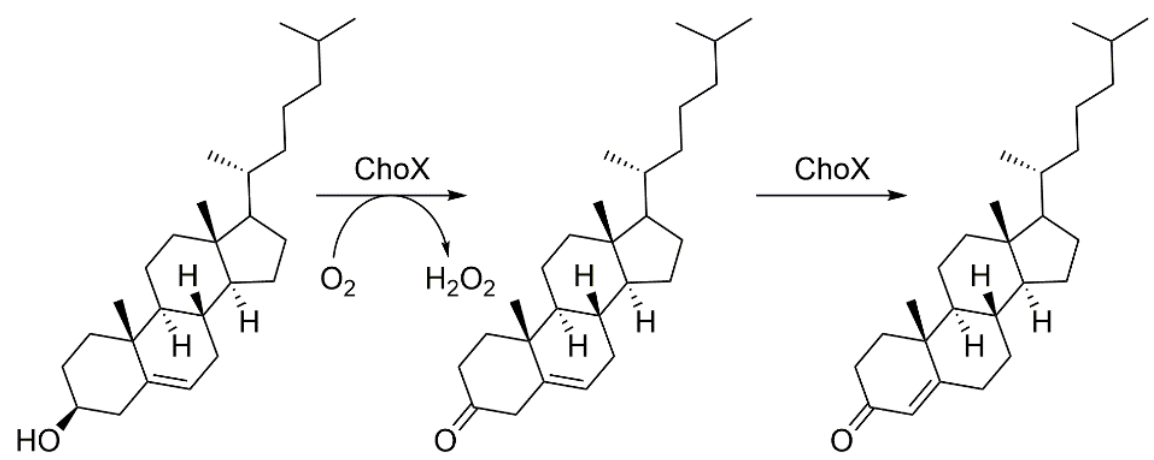

Scheme 11: Oxidation and isomerisation of cholesterol catalysed by cholesterol oxidase (ChoX).

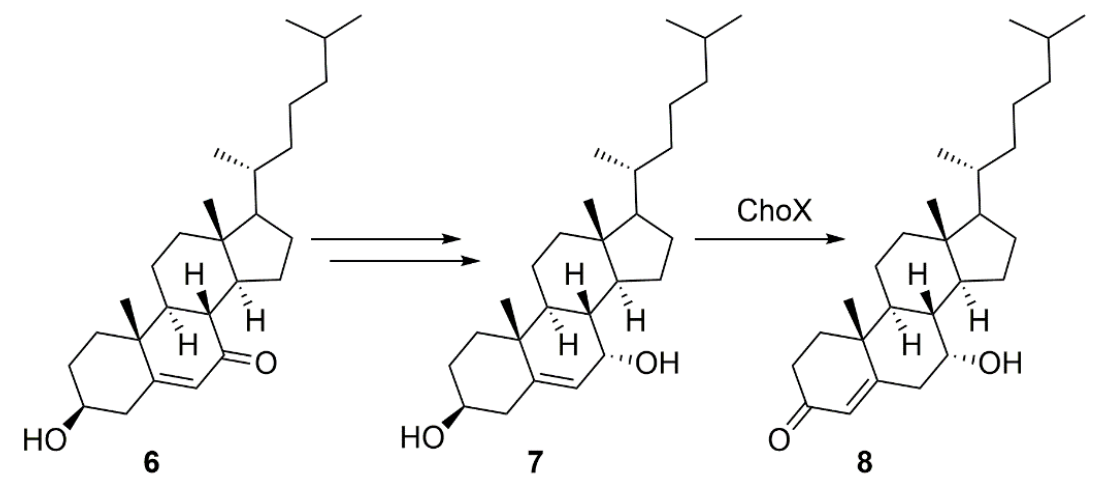

Scheme 12: Synthesis of 7 $\alpha$-hydroxycholest-4-en-3-one (8) using cholesterol oxidase (ChoX) (74).

ChoX has also been applied in the synthesis of $7 \beta$-hydroxytestosterone (11) starting from dehydroepiandrosterone (9) (Scheme 13) (75). A C 8 ester group was introduced at the C17 position of $\mathbf{9}$ to mimic the bulky side chain cholesterol has at this position. Subsequently, the hydroxyl group at C7 was introduced enantioselectively by chemical means, yielding compound 10, which served as a substrate for ChoX. After conversion of $\mathbf{1 0}$ by ChoX, the C17 acyl substituent was removed using porcine lipase, yielding $\mathbf{1 1}$ in an overall yield of $17 \%$.

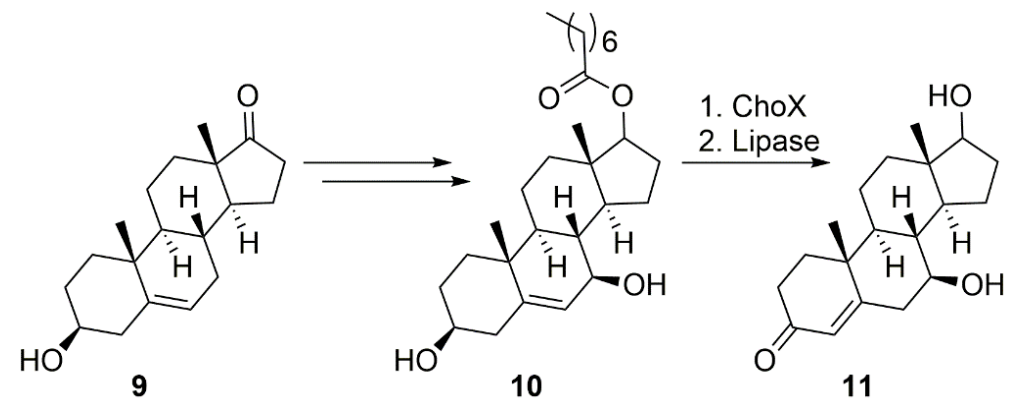

Scheme 13: Synthesis of 7 $\beta$-hydroxytestosterone (11) using cholesterol oxidase (ChoX) (75).

Guo et al. used ChoX in the synthesis of 5 $\beta$-pregn-7-en-3 $\alpha, 17 \alpha, 20$-triol (15) starting from 12 (Scheme 14) (76). 12 was converted to $\mathbf{1 3}$ by introduction of the $\Delta^{7}$ double bond by bromination-dehydrobromination, followed by removal of the acetate groups by solvolysis. Subsequently, 13 was oxidised and isomerised to 14 by ChoX. 14 was converted to 15 by hydrogenation over $\mathrm{Pd} / \mathrm{C}$, followed by reduction of the carbonyl groups using $\mathrm{LiAlH}_{4}$. 


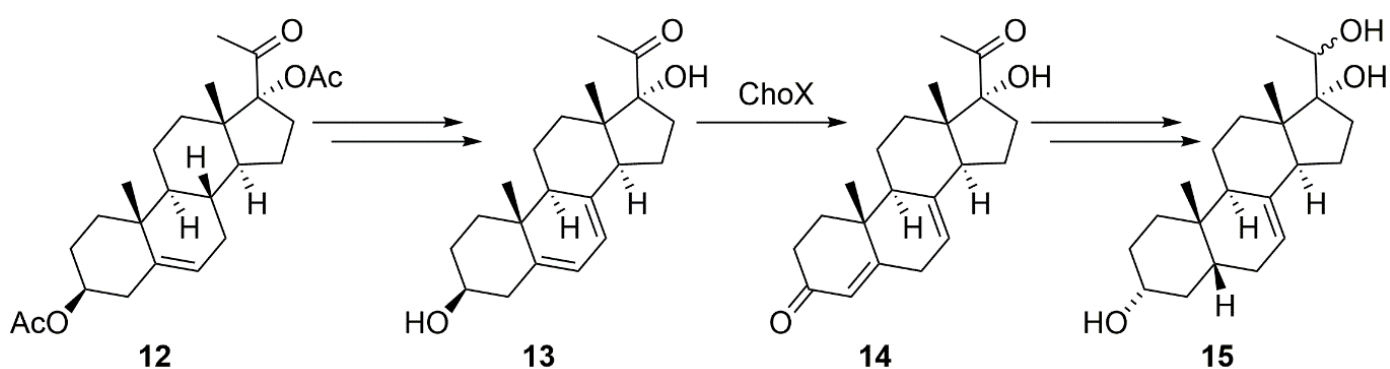

Scheme 14: Synthesis of 5 $\beta$-pregn-7-en-3 $\alpha, 17 \alpha, 20$-triol (15) involving cholesterol oxidase (ChoX) (76).

\section{Glycerol 3-phosphate oxidase}

Glycerol 3-phosphate oxidase (GPO, EC 1.1.3.21) is an FAD-dependent oxidase, which catalyses the oxidation of L-glycerol 3-phoshpate to dihydroxyacetone phosphate, which is a useful precursor for the synthesis of sugars by asymmetric aldol additions. GPOs from various bacterial sources are commercially available. Krämer and Steckhan used immobilised GPO in combination with D-fructose 1,6-bisphosphate aldolase to convert L-glycerol 3-phosphate and glyceraldehyde to D-fructose 1-phosphate and L-sorbose 1-phosphate, which were subsequently dephosphorylated using acid phosphatase to obtain the unmodified monosaccharides (77). GPO has also been applied in a one-pot multi-enzyme cascade reaction for the synthesis of the carbohydrate fragments 17a-c starting from glycerol and aldehydes 16a-c (Scheme 15) (78).
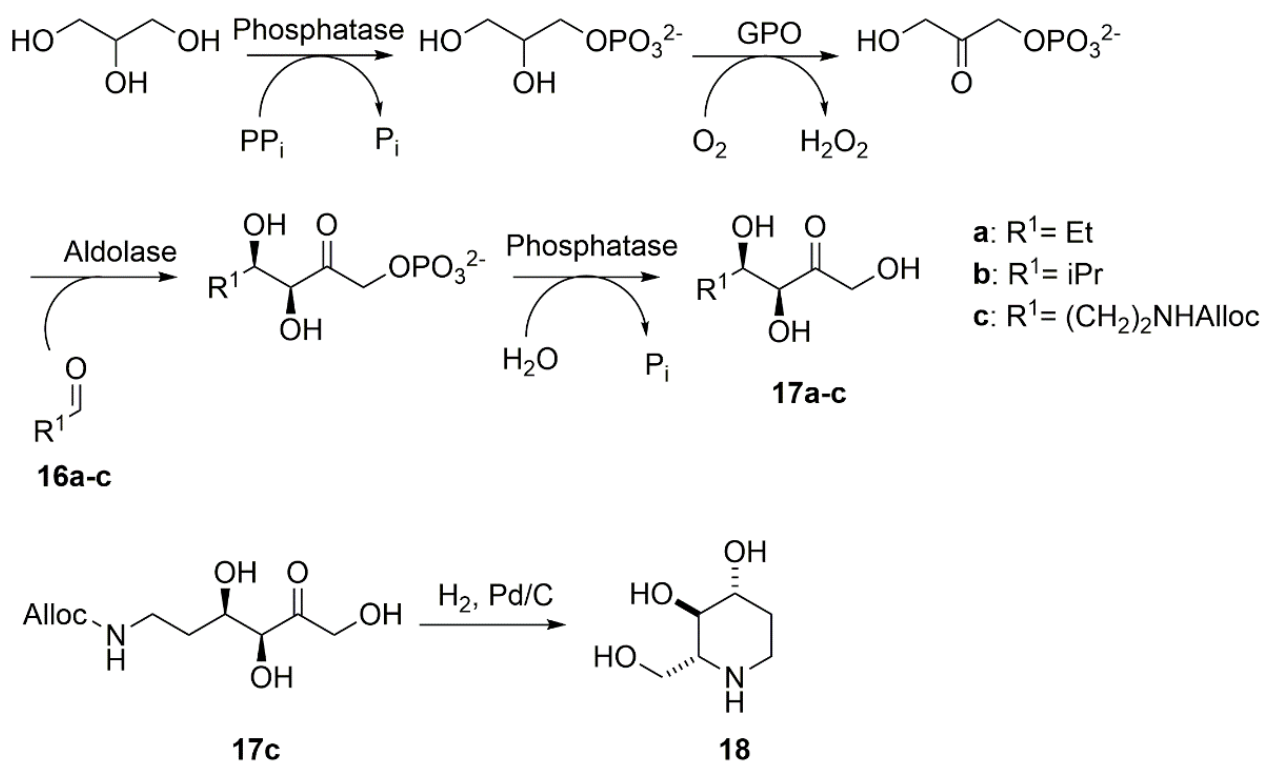

Scheme 15: Synthesis of carbohydrate fragments (17) from glycerol and aldehydes by a multi-enzyme cascade involving glycerol 3-phosphate oxidase (GPO) (78).

In this cascade, glycerol was first phosphorylated by an acid phosphatase, followed by the oxidation of the formed glycerol 3-phosphate to dihydroxyacetone phosphate by GPO. Subsequently, the dihydroxyacetone phosphate was stereoselectively coupled to the relevant aldehyde by an aldolase, followed by dephosphorylation of the product by acid phosphatase. The use of four aldolases with varying steroeselectivities enabled the synthesis of all four 
stereoisomers of $\mathbf{1 7} \mathbf{a}$, though in some cases the stereoselectivity of the coupling reaction was poor. When followed by reductive amination using $\mathrm{H}_{2}, \mathrm{Pd} / \mathrm{C}$, this enzymatic cascade could be used for the chemoenzymatic synthesis of the sugar D-fagomine (18) from glycerol and aldehyde 16c.

\section{Choline oxidase}

Choline oxidase (ChlO, EC 1.1.3.17) is a GMC-type oxidase that catalyses the oxidation of choline to the carboxylic acid betaine, via the aldehyde intermediate betaine aldehyde (Scheme 16). The most extensively studied ChlO is the enzyme from Arthrobacter globiformis, which is commercially available (79). A. globiformis $\mathrm{ChlO}$ is a homodimer composed of $60 \mathrm{kDa}$ subunits which contain a covalently bound FAD cofactor (80). The choline analogues $N, N-$ dimethylethanolamine, $\mathrm{N}$-methylethanolamine, triethanolamine, diethanolamine and 3,3dimethyl-1-butanol can also be oxidised by $\operatorname{ChlO}(79,81)$. Linear aliphatic alcohols and aldehydes are not oxidized by the enzyme.

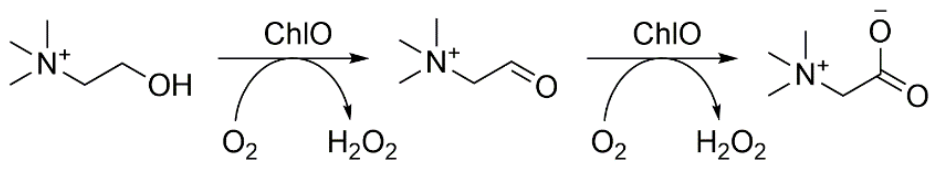

Scheme 16: Oxidation of choline catalysed by choline oxidase (ChlO).

\section{Glucose oxidase}

Glucose oxidase (GOX, EC 1.1.3.4) catalyses the oxidation of the C1 hydroxyl group of $\beta$ D-glucose, yielding D-glucono-1,5-lactone, which spontaneously hydrolyses to gluconic acid (Scheme 17). GOX from Aspergillus niger is a dimeric glycoprotein and belongs to the GMCtype oxidoreductase family. Each $75 \mathrm{kDa}$ subunit contains a non-covalently bound FAD cofactor. GOX has a rather narrow substrate range. $\alpha$-D-Glucose, pentoses, hexoses other than D-glucose and various deoxy analogues of $\beta$-D-glucose are poor substrates. An exception is 2deoxy-D-glucose which is a reasonably good substrate (82). This narrow substrate range limits the synthetic utility of GOX. Nevertheless, GOX has been applied as a biocatalyst for the production of gluconic acid (83), which is an important antioxidant in food and drinks, and also for the synthesis of D-glucosaminic acid, a chiral synthon for the asymmetric synthesis of amino acids (84). Although aldoses other than glucose are poor GOX substrates, Pezzotti and Therisod demonstrated that GOX can be used for the synthesis of aldonic acids from Dmannose, D-galactose and D-xylose (85).

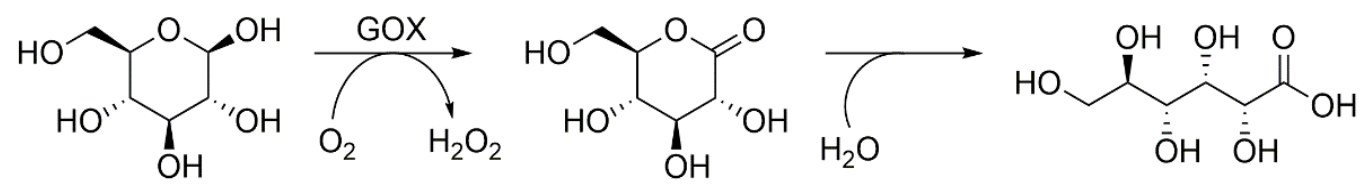

Scheme 17: Oxidation of $\beta$-D-glucose catalysed by glucose oxidase (GOX). 
Pyranose 2-oxidase (P2O, EC 1.1.3.10) is a GMC-type oxidase. The enzyme catalyses the oxidation of D-glucose and other aldopyranoses at the $\mathrm{C} 2$ position yielding the corresponding ketoaldoses. $\mathrm{P} 2 \mathrm{O}$ has been isolated from a number of wood-degrading white-rot basidiomycetes (86). The native enzyme is a homotetramer of $280 \mathrm{kDa}$, with each subunit containing one molecule of FAD, which is covalently linked to a histidine residue (87). P2O selectively oxidises aldopyranoses at the $\mathrm{C} 2$ position, but can also oxidise the $\mathrm{C} 3$ position if the C2 hydroxyl group is absent, as in the case of 2-deoxy-D-glucose. Unlike GOX, P2O can oxidise both the $\beta$ - and $\alpha$-anomers of D-glucose. P2O is also active with other aldopyranoses such as D-xylose, L-sorbose, D-allose and D-galactose, but D-glucose and its deoxy analogues are by far the best substrates $(88,89)$.

$\mathrm{P} 2 \mathrm{O}$ is a valuable enzyme for synthetic purposes (89). Oxidation of the C2 hydroxyl group of D-glucose yields 2-keto-D-glucose (D-glucosone, 19), which is an important intermediate for the synthesis of rare sugars, fine chemicals, and drugs (Scheme 18). Catalytic hydrogenation of D-glucosone yields the important sweetener D-fructose (20). This two-step chemoenzymatic synthesis is known as the 'Cetus process' (90). Enzymatic dehydratation of D-glucosone with pyranosone dehydratase results in the formation of the $\beta$-pyrone antibiotic cortalcerone (21) (91). P2O can also be used for the regioselective oxidation of D-galactose. The resulting D-galactosone (22) can be converted according to the Cetus procedure into the rare sugar D-tagatose (23) (92). The enzyme also catalyses the oxidation of 2-deoxy-D-glucose to 2-deoxy3-keto-D-glucose (24), which is subsequently oxidised by hydrogen peroxide (or oxygen) and decarboxylated to form a mixture of 1-deoxy-D-ribulose (25) and 1-deoxy-D-xylulose (26), a precursor in vitamin synthesis $(93,94)$. P2O oxidises the reducing sugar moiety of various 1,6disaccharides to their 2-ketoaldose forms, which can subsequently be converted to the ketose forms by an aldose reductase (95). The enzyme has also been applied in the synthesis of regioselectively functionalized dextran (a polysaccharide composed of glucose units linked primarily through $\alpha$-(1-6)-glycosidic bonds) (96). This was achieved by oxidizing sucrose at the $\mathrm{C} 2$ position of its glucose moiety and subsequently polymerizing the modified sucrose using the enzyme dextransucrase.

\section{Alditol oxidase}

Alditol oxidase (AldO, EC 1.1.3.41) is a biocatalytically interesting member of the VAO/PCMH family. The most extensively studied AldO is that from the bacterium Streptomyces coelicolor A3(2) (97). Similar enzymes have been identified in Streptomyces sp. H-7775 (known as sorbitol oxidase) and the thermophilic bacteria Streptomyces sp. IKD472 (known as xylitol oxidase) and Acidothermus cellulolyticus 11B (98-100). AldO from S. coelicolor is a monomeric protein with a molecular mass of $46 \mathrm{kDa}$. The enzyme contains an FAD cofactor, which is covalently bound to a histidine residue $(97,101)$. AldO catalyses the regioselective oxidation of the primary hydroxyl groups of various polyols (Scheme 19). The best substrates are alditols such as xylitol and D-sorbitol, which are oxidised to the corresponding D-aldoses (97). AldO oxidises various other polyols, with a strong preference for substrates with a hydroxyl group at the $\mathrm{C} 2$ position. In some cases, the final product is a carboxylic acid rather than an aldehyde. This has been shown to be the case for the oxidation of 1,2-pentanediol and 1-phenyl-1,2-ethanediol and is 
due to the oxidation of the gem-diol formed upon hydration of the aldehyde product (102). AldO displays selectivity regarding the configuration of the chiral centre at the $\mathrm{C} 2$ position of its 1,2-diol substrates. D-sorbitol is a significantly better substrate than its C2 epimer Dmannitol, due to the orientation of D-mannitol's C2 hydroxyl group leading to steric clashes with the active site residue His-343 $(97,101)$. The conversions of 1,2-pentanediol and 1-phenyl1,2-ethanediol to the corresponding aldehydes proceed enantioselectively. The $(R)$ enantiomers are preferentially converted with $E$-values of $>145$ and 74 respectively (102).

Hexose oxidase

Hexose oxidase (HOX, EC 1.1.3.5) is a carbohydrate oxidase which, like GOX, oxidises the C1 hydroxyl group of aldose substrates, yielding lactones which can hydrolyse to form aldonic acids. HOX was first isolated from the marine red alga Chondrus crispus (103). HOX consists<smiles>O=C1C=CCOC1(O)C(O)(O)O</smiles>

21

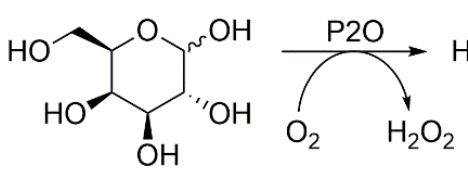<smiles>O=C1[C@@H](O)O[C@H](CO)[C@@H](O)[C@@H]1O</smiles><smiles>CCCCCC</smiles><smiles>OCC1(O)OC[C@@H](O)[C@H](O)[C@H]1O</smiles>

22 23<smiles>OC[C@H]1O[C@@H](O)C[C@@H](O)[C@@H]1O</smiles>

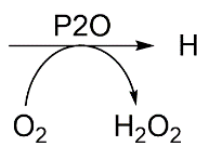<smiles>O=C1C[C@H](Cl)O[C@@H](CO)[C@@H]1O</smiles>

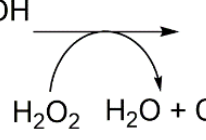<smiles>CC(=O)C[C@@H](O)CO</smiles>

24<smiles>CC(=O)[C@H](O)[C@@H](O)CO</smiles>

Scheme 18: Oxidations catalysed by pyranose-2-oxidase (P2O). From top to bottom: the oxidation of Dglucose to D-glucosone (19), which can subsequently be hydrogenated to D-fructose (20) or dehydrated to cortalcerone (21); the oxidation of D-galactose to D-galactosone (22), which can subsequently be hydrogenated to D-tagatose (23); the oxidation of 2-deoxy-D-glucose to 2-deoxy-3-keto-D-glucose (24), which is subsequently oxidatively decarboxylated to yield 1-deoxy-D-ribulose (25) and 1-deoxy-Dxylulose (26). 


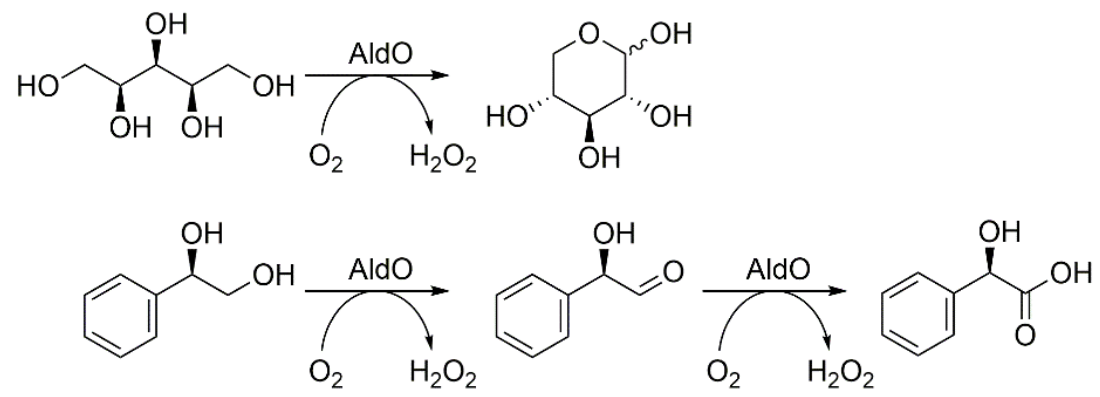

Scheme 19: Oxidations catalysed by alditol oxidase (AldO). Top: the oxidation of xylitol to D-xylose. Bottom: the oxidation of $(R)-1$-phenyl-1,2-ethanediol to $(R)$-2-hydroxy-2-phenylacetic acid. This reaction is enantioselective, as $(R)$-1-phenyl-1,2-ethanediol is more efficiently converted than the corresponding $(S)$-enantiomer.

of two subunits ( $37 \mathrm{kDa}$ and $25 \mathrm{kDa}$ ), which are formed upon proteolytic activation of a 62 $\mathrm{kDa}$ precursor protein, with two of each subunit combining to form the native enzyme (104, 105). HOX is a member of the VAO/PCMH family and contains a bicovalently bound FAD cofactor (106).

HOX has a far more relaxed substrate specificity than GOX, catalysing the oxidation of a variety of mono-, di-, tri-, and tetra-aldose sugars, including D-glucose, D-galactose, xylose, lactose, maltose, cellobiose, arabinose, maltotriose, and maltotetraose. The monosaccharides D-glucose and D-galactose are the substrates that are most efficiently converted (107). Due to its broad substrate specificity, HOX has great potential for the synthesis of lactones or aldonic acids from various sugars.

\section{Oligosaccharide oxidases}

A number of oxidases have been identified that, like GOX and HOX, catalyse the oxidation of the $\mathrm{C} 1$ hydroxyl group of aldose moieties, but which are primarily active with oligosaccharide substrates. These oligosaccharide oxidases all belong to the VAO/PCMH family. One of these enzymes is lactose oxidase (LAO) from Microdochium nivale. LAO is a $55 \mathrm{kDa}$ protein that contains an FAD cofactor, which is predicted to be bicovalently linked to the enzyme (108, 109). The enzyme is able to oxidise mono-, oligo- and polysaccharides, with the highest oxidation rates being observed with the glucooligosaccharides D-cellotriose and Dcellotetraose (108). A highly similar oxidase is glucooligosaccharide oxidase (GOOX) from Sarocladium strictum (formerly Acremonium strictum) (110). Mature GOOX has a molecular mass of $61 \mathrm{kDa}$ and contains a bicovalently bound FAD cofactor (111). GOOX oxidises the reducing end glycosyl residues of oligosaccharides linked by $\alpha$ - or $\beta-1,4$ bonds and the monosaccharide D-glucose $(110,112)$. Oligosaccharide oxidases that oxidise glucooligosaccharides have also been identified in sunflower (Helianthus annuus) and the fungus Paraconiothyrium sp. $(113,114)$.

Another related enzyme is chitooligosaccharide oxidase (ChitO) from Fusarium graminearum. ChitO also contains a bicovalently linked FAD cofactor and oxidises the $\mathrm{C} 1$ hydroxyl group of the reducing end of its oligosaccharide substrates (115). The main difference with other oligosaccharide oxidases is that ChitO preferentially oxidises chitooligosaccharides 
(oligosaccharides composed of $\mathrm{N}$-acetyl-D-glucosamine units). The catalytic efficiencies for the oxidation of chitooligosaccharides by ChitO are three orders of magnitude larger than those for the oxidation of their non $\mathrm{N}$-acetylated analogues (115). Xylitol oxidase (XylO) from Myceliophthora thermophila C1 is an oligossacharide oxidase that preferentially oxidises xylooligosaccharides, with xylobiose, xylotriose and xylotetraose being the substrates that are most efficiently converted (116).

\section{Non-flavin alcohol oxidases}

Although the majority of characterised alcohol oxidases are flavin-dependent enzymes, a small number of enzymes are known to use a different cofactor, such as copper or non-heme iron, to catalyse alcohol oxidation. These enzymes and their potential applications in organic synthesis are discussed below.

\section{Galactose oxidase}

Galactose oxidase (GAO, EC 1.1.3.9) is a copper-containing oxidase that catalyses the oxidation of a wide range of carbohydrates and primary alcohols to the corresponding aldehydes. The oxidation of hexose substrates, such as D-galactose, by GAO is highly regioselective, as only the hydroxyl group at the C6 position is oxidised (Scheme 20).

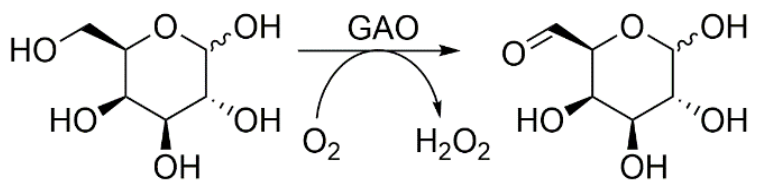

Scheme 20: Oxidation of D-galactose catalysed by galactose oxidase (GAO).

GAO from Fusarium sp. NRRL 2903 (previsouly known as Dactylium dendroides) is the most extensively characterised GAO and is commercially available (117). It is a monomeric protein with a molecular mass of $69 \mathrm{kDa}$ and contains a single copper ion as a cofactor (118). The 3D structure of GAO revealed that in the active site the mononuclear copper is coordinated by two tyrosine residues (Tyr-272 and Tyr-495), two histidine residues (His-496 and His-581) and one solvent molecule (119). The side chain of Tyr-272 is post-translationally modified to form a covalent thioether linkage with a cysteine residue (Cys-228). This promotes the formation of a Tyr-272 radical, which is present in the active form of the enzyme. The catalytic mechanism of GAO has been studied in detail $(117,120)$. Substrate binding induces a proton transfer from the alcohol substrate to Tyr-495, followed by hydrogen atom transfer from the substrate to the Tyr-272 radical. This results in the intermediate redox state of the enzyme [Cu(II), no radical present]. Subsequently, one-electron reduction of the copper cofactor and release of the aldehyde product brings the enzyme into the fully reduced state $[\mathrm{Cu}(\mathrm{I})$, no radical present]. The reduced enzyme can then be reoxidised by molecular oxygen.

GAO is active towards D-galactose and many derivatives thereof, polysaccharides containing a D-galactose moiety, dihydroxyacetone and benzyl alcohol derivatives (117). Besides being strictly regioselective, GAO is highly stereoselective. L-Galactose is not a substrate and the oxidation of glycerol results exclusively in the formation of (S)-glyceraldehyde. In the case of 
chiral alcohol substrates, only the enantiomer with a conformation resembling that of D-galactose is converted (121).

GAO has been employed in the regioselective modification of various carbohydrates at the C6 position. Leppänen et al. used GAO in the allylation of methyl $\alpha$-D-galactopyranoside (27) (Scheme 21) (122). Following the oxidation of 27 by GAO, the formed aldehydes were reacted with allyl halides in the presence of indium as a metal mediator, yielding 28a-c. This procedure has been extended to the allylation and propargylation of oligo- and polysaccharides (123).

Fuchs et al. used a similar procedure for the synthesis of homoallyl alcohols from benzyl alcohol and various derivatives thereof (124). Following oxidation of the alcohols by E. coli containing overexpressed GAO, the formed aldehydes were allylated by reacting them with allyl bromide or allylboronic acid pinacol ester.

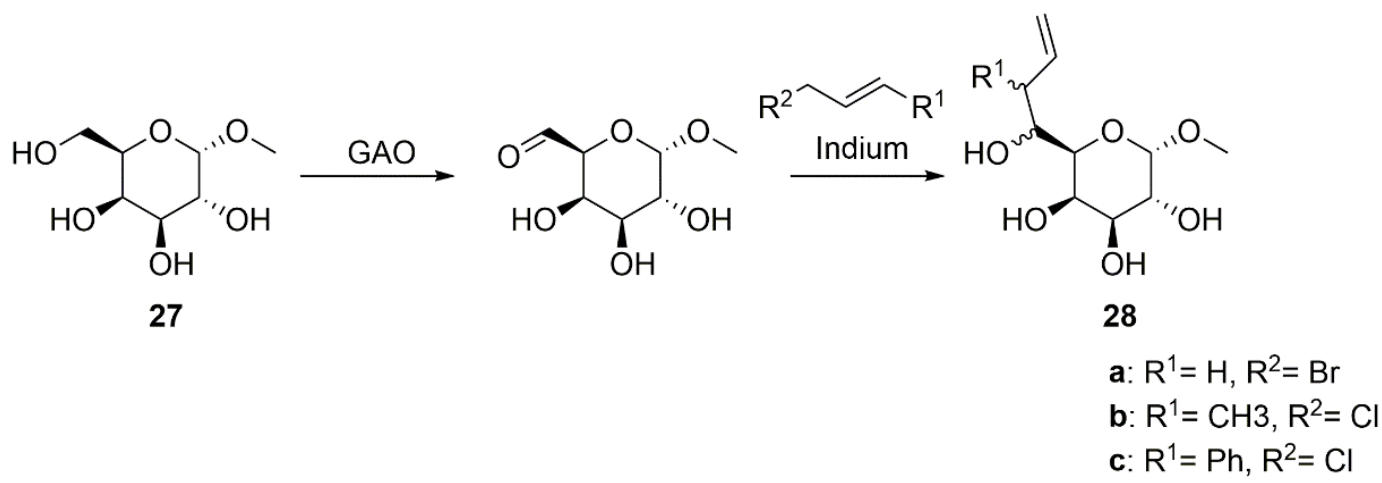

Scheme 21: Synthesis of homoallylic alcohol sugar derivatives (28) involving galactose oxidase (GAO) (122).

GAO has been used for the C6-biotinylation of the nucleotide sugars UDP-galacatose and UDP- $N$-acetlygalactosamine and the synthesis of various poly- $N$-acetyllactosamine derivatives (125-128). It has also been employed in a multi-enzyme cascade for the synthesis of the non-natural sugar L-fructose from the simple achiral building blocks glycerol and dihydroxyacetone phosphate (129). Here, glycerol is first stereosselectively converted to Lglyceraldehyde by GAO. Then, L-glyceraldehyde is coupled to dihydroxyacetone phosphate by a stereoselective aldol addition catalysed by an aldolase and the formed L-fructose 1phosphate is dephosphorylated by acid phosphatase, yielding L-fructose.

GAO has also been applied in combination with a transaminase in a one-pot cascade reaction for the synthesis of amines 29 from alcohols (Scheme 22) (130). Using two different $\omega$-transaminases, various aromatic alcohols could be aminated, though the efficiency of the conversion varied greatly depending on the nature of substituents on the aromatic ring. The procedure starting from cinnamyl alcohol extended by reductive amination of the formed amine 29a with 1-naphtaldehyde and $N$-methylation of the formed secondary amine, to yield the antifungal agent naftifine (30) in an overall yield of $51 \%$. 
GAO variants that display activity towards various aromatic secondary alcohols (1phenylethanol and derivatives thereof) have been created by directed evolution (131). These variants enantioselectively converted the $R$--enantiomer of 1-(3'-fluorophenyl)ethanol. Using one of the variants, racemic 1-(3'-fluorophenyl)ethanol was kinetically resolved, yielding the (S)-enantiomer in an enantiomeric excess of $99 \%$. Various other racemic secondary aromatic alcohols were also succesfully resolved in this manner. One of these variants was also used in combination with an aldehyde oxidase to achieve the conversion of 5(hydroxymethyl)furfural to 2,5-furandicarboxylic acid $(132,133)$.

\section{Mannitol oxidase}

Mannitol oxidase from the snail Helix aspersa (MannO, EC 1.1.3.40) is a non-flavin enzyme capable of oxidising sugar alcohols (134). The cofactor of this membrane protein has not been identified and the mechanism of substrate oxidation is unknown. Like AldO, MannO oxidises the $\mathrm{C} 1$ hydroxyl group of its substrates, with D-mannitol being converted to D-mannose (135).

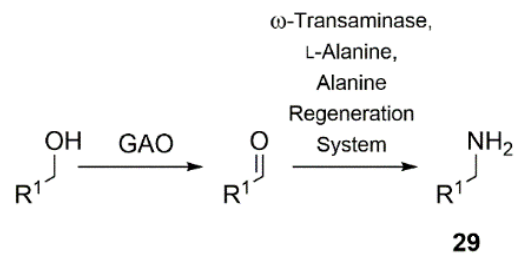

\begin{tabular}{|ccc|}
\hline $\mathbf{R}^{1}$ & Transaminase $^{*}$ & Conversion to 29 (\%) \\
\hline $\mathrm{Ph}$ & $\mathrm{Vf}$ & $>99$ \\
$2^{\prime}-\mathrm{ClC}_{6} \mathrm{H}_{4}$ & $\mathrm{Vf}$ & Trace \\
$2^{\prime}-\mathrm{MeOC}_{6} \mathrm{H}_{4}$ & $\mathrm{Vf}$ & 18 \\
$3^{\prime}-\mathrm{ClC}_{6} \mathrm{H}_{4}$ & $\mathrm{Vf}$ & 96 \\
$3^{\prime}-\mathrm{MeOC}_{6} \mathrm{H}_{4}$ & $\mathrm{Vf}$ & 81 \\
$3^{\prime}-\mathrm{MeC}_{6} \mathrm{H}_{4}$ & $\mathrm{Pd}$ & $>99$ \\
$4^{\prime}-\mathrm{ClC}_{6} \mathrm{H}_{4}$ & $\mathrm{Vf}$ & 44 \\
$4^{\prime}-\mathrm{MeOC}_{6} \mathrm{H}_{4}$ & $\mathrm{Pd}$ & 68 \\
$4^{\prime}-\mathrm{MeC}_{6} \mathrm{H}_{4}$ & $\mathrm{Pd}$ & 82 \\
$4^{\prime}-\mathrm{FC} 6 \mathrm{H}_{4}$ & $\mathrm{Pd}$ & 75 \\
$\mathrm{Piperonyl}$ & $\mathrm{Vf}$ & 35 \\
Piperonyl & $\mathrm{Pd}$ & 36 \\
3-Phenylallyl & $\mathrm{Vf}$ & 3 \\
3-Phenylallyl & $\mathrm{Pd}$ & 92 \\
\hline
\end{tabular}

${ }^{a} \mathrm{Vf}=$ Vibrio fluvialis $\omega$-transaminase; $\mathrm{Pd}=$ Paracoccus denitrificans $\omega$-transaminase

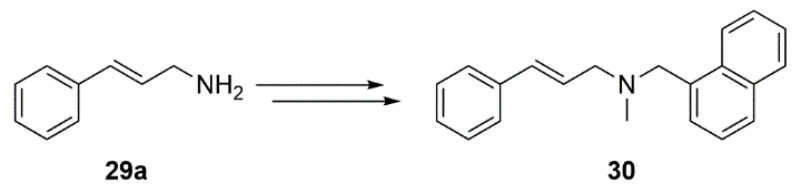

Scheme 22: Synthesis of aromatic amines (29) by a two-step transamination cascade involving galactose oxidase (GAO) (130). 
However, MannO's substrate specificity differs from AldO's, with D-arabinitol and Dmannitol being the enzyme's best substrates and xylitol being a poor substrate, suggesting the two enzymes have different preferences regarding the stereochemical configuration of their substrates (134).

\section{Secondary alcohol oxidase}

A few non-flavin oxidases have been identified that can catalyse the oxidation of aliphatic secondary alcohols to ketones. These oxidases are found in various Pseudomonas strains, where they are involved in the degradation of polyvinyl alcohol, and have been classified as secondary alcohol oxidase (SAO, EC 1.1.3.18) or polyvinyl alcohol oxidase (EC 1.1.3.30) (136). Relatively little is known about the biochemical properties and substrate oxidation mechanism of SAO. The SAOs that have been purified display similar catalytic properties and contain a single non-heme iron atom per protein molecule, but differ in properties such as molecular weight (ranging from 26 to $75 \mathrm{kDa}$ ), pI (ranging from 4.5 to 10) and localisation (most are secreted, but a single membrane-associated SAO has been described) (137-141). In addition to polyvinyl alcohol, SAO oxidises various medium-chain secondary alcohols, with substrates with chain lengths of six to eight carbon atoms being oxidised most efficiently (though the observed decrease in activity for alcohols with longer chain lengths may be due to their poor solubility in water). Cyclic alcohols (cyclohexanol) and aromatic secondary alcohols (1-phenylethanol) are also oxidised by SAO. Unfortunately, no information is available on whether these oxidations take place enantioselectively. Further investigations into the catalytic scope of these enzymes could pave the way for their use in enzymatic methods for the production of enantiomerically pure aliphatic secondary alcohols.

\section{Practical considerations}

\section{Hydrogen peroxide removal}

Although the use of molecular oxygen as a final electron acceptor is advantageous from the point of view of its cost and easy availability, the production of hydrogen peroxide as a byproduct can be problematic. The accumulation of hydrogen peroxide may lead to the inactivation of the biocatalyst and undesired side-reactions with products or substrates. The production of hydrogen peroxide is also undesirable from a waste management perspective. Thus, a method to convert hydrogen peroxide into chemically and environmentally benign compounds is required. The simplest and most commonly used method to dispose of hydrogen peroxide during enzymatic reactions is the addition of catalase to the reaction mixture. This commercially available enzyme catalyses the decomposition of hydrogen peroxide into water and oxygen. This has the added advantage of regenerating $50 \%$ of the oxygen consumed by the oxidase. Alternatively, cascade reactions may be designed where the hydrogen peroxide formed by an oxidase is used to enable a subsequent reaction by a hydrogen peroxide-dependent enzyme, such as a peroxidase or peroxygenase. For example, both GOX and MO have been used for the in situ generation of hydrogen peroxide to enable the synthesis of chiral sulfoxides from thioethers using a peroxidase from Coprinus cinereus (142-144). 


\section{Enzyme immobilisation}

One factor hampering the large-scale adoption of biocatalysis is the relative instability of enzymes, particularly at elevated temperatures and in organic solvents. The immobilisation of enzymes can increase enzyme stability, lengthening the lifetime of a biocatalyst. Immobilisation also greatly facilitates the separation of the catalyst from the reaction products in an active form and thus enables catalyst reuse. Various immobilisation techniques have been described including binding to solid supports, entrapment in polymer matrices or carrier-free methods such as the formation of cross-linked enzyme aggregates or crystals [reviewed in $(145,146)]$. The development of an effective immobilisation protocol is not trivial, but immobilisation of a number of the oxidases mentioned above, e.g. GOX (147), GlyO (148) and P2O (149), has already been investigated.

\section{Outlook and future perspectives}

The use of biocatalysts is a promising alternative to traditional methods of alcohol oxidation. As illustrated above, alcohol oxidases have a broad catalytic scope, being capable of oxidising a wide range of substrates with exquisite regio- and enantioselectivity. In future, this catalytic scope will increase further, as the number of characterised enzymes grows and our ability to modulate their reactivity by mutagenesis increases. The development and identification of more stable enzymes, together with further advances in techniques for their immobilisation, will contribute to the creation of robust biocatalysts suitable for repeated use in industrial processes. The low toxicity and biological origin of enzymes and the mild reaction conditions under which they are active, make biocatalytic processes attractive from the point of view of sustainability. Development of one-pot multi-step biocatalytic cascade reactions, will enable the design of efficient redox processes without the need for costly intermediate purification steps (150). Thus, alcohol oxidases have the potential to be valuable biocatalysts in organic synthesis, on both the laboratory- and industrial-scale.

\section{References}

1. Tojo, G., and Fernández, M. (2006) Oxidation of alcohols to aldehyes and ketones: A guide to current common practice, Springer (New York)

2. Parmeggiani, C., and Cardona, F. (2012) Transition metal based catalysts in the aerobic oxidation of alcohols. Green Chem. 14, 547-564

3. Wertz, S., and Studer, A. (2013) Nitroxide-catalyzed transition-metal-free aerobic oxidation processes. Green Chem. 15, 3116-3134

4. Kroutil, W., Mang, H., Edegger, K., and Faber, K. (2004) Biocatalytic oxidation of primary and secondary alcohols. Adv. Synth. Catal. 346, 125-142

5. Hollmann, F., Arends, I. W. C. E., Buehler, K., Schallmey, A., and Bühler, B. (2011) Enzymemediated oxidations for the chemist. Green Chem. 13, 226-265

6. Heuts, D. P. H. M., Scrutton, N. S., McIntire, W. S., and Fraaije, M. W. (2009) What's in a covalent bond?: On the role and formation of covalently bound flavin cofactors. FEBS J. 276, 3405-3427

7. Fraaije, M. W., and Mattevi, A. (2000) Flavoenzymes: Diverse catalysts with recurrent features. Trends Biochem. Sci. 25, 126-132

8. Wongnate, T., and Chaiyen, P. (2013) The substrate oxidation mechanism of pyranose 2-oxidase and other related enzymes in the glucose-methanol-choline superfamily. FEBS J. 280, 3009-3027

9. Fitzpatrick, P. F. (2007) Insights into the mechanisms of flavoprotein oxidases from kinetic 
isotope effects. J. Label. Compd. Radiopharm. 50, 1016-1025

10. Gadda, G. (2008) Hydride transfer made easy in the reaction of alcohol oxidation catalyzed by flavin-dependent oxidases. Biochemistry. 47, 13745-13753

11. Chaiyen, P., Fraaije, M. W., and Mattevi, A. (2012) The enigmatic reaction of flavins with oxygen. Trends Biochem. Sci. 37, 373-380

12. Gadda, G. (2012) Oxygen activation in flavoprotein oxidases: The importance of being positive. Biochemistry. 51, 2662-2669

13. Dijkman, W. P., de Gonzalo, G., Mattevi, A., and Fraaije, M. W. (2013) Flavoprotein oxidases: Classification and applications. Appl. Microbiol. Biotechnol. 97, 5177-5188

14. Kiess, M., Hecht, H.-J., and Kalisz, H. M. (1998) Glucose oxidase from Penicillium amagasakiense: Primary structure and comparison with other glucose-methanol-choline (GMC) oxidoreductases. Eur. J. Biochem. 252, 90-99

15. Fraaije, M. W., van Berkel, W. J. H., Benen, J. A. E., Visser, J., and Mattevi, A. (1998) A novel oxidoreductase family sharing a conserved FAD-binding domain. Trends Biochem. Sci. 23, 206207

16. Sahm, H., and Wagner, F. (1973) Microbial assimilation of methanol: The ethanol- and methanoloxidizing enzymes of the yeast Candida boidinii. Eur. J. Biochem. 36, 250-256

17. van Dijken, L. P., Otto, R., and Harder, W. (1976) Growth of Hansenula polymorpha in a methanollimited chemostat: Physiological responses due to the involvement of methanol oxidase as a key enzyme in methanol metabolism. Arch. Microbiol. 111, 137-144

18. Kato, N., Omori, Y., Tani, Y., and Ogata, K. (1976) Alcohol oxidases of Kloeckera sp. and Hansenula polymorpha: Catalytic properties and subunit structures. Eur. J. Biochem. 64, 341-350

19. Couderc, R., and Baratti, J. (1980) Oxidation of methanol by the yeast, Pichia pastoris: Purification and properties of the alcohol oxidase. Agric. Biol. Chem. 44, 2279-2289

20. Eriksson, K.-E., and Nishida, A. (1988) Methanol oxidase of Phanerochaete chrysosporium. In: Biomass Part B: Lignin, Pectin, and Chitin, Methods Enzymol. vol. 161, pp. 322-326

21. Isobe, K., Kato, A., Ogawa, J., Kataoka, M., Iwasaki, A., Hasegawa, J., and Shimizu, S. (2007) Characterization of alcohol oxidase from Aspergillus ochraceus AIU 031. J. Gen. Appl. Microbiol. 53, 177-183

22. Kellogg, R. M., Kruizinga, W., Bystrykh, L. V, Dijkhuizen, L., and Harder, W. (1992) Structural analysis of a stereochemical modification of flavin adenine dinucleotide in alcohol oxidase from methylotrophic yeasts. Tetrahedron. 48, 4147-4162

23. van Berkel, W. J. H., Eppink, M. H. M., and Schreuder, H. A. (1994) Crystal structure of $p$ hydroxybenzoate hydroxylase reconstituted with the modified FAD present in alcohol oxidase from methylotrophic yeasts: Evidence for an arabinoflavin. Protein Sci. 3, 2245-2253

24. Siebum, A., van Wijk, A., Schoevaart, R., and Kieboom, T. (2006) Galactose oxidase and alcohol oxidase: Scope and limitations for the enzymatic synthesis of aldehydes. J. Mol. Catal. B Enzym. 41, 141-145

25. Pérez-Sánchez, M., Müller, C. R., and Domínguez de María, P. (2013) Multistep oxidase-lyase reactions: Synthesis of optically active 2-hydroxyketones by using biobased aliphatic alcohols. ChemCatChem. 5, 2512-2516

26. Shanmuganathan, S., Natalia, D., Greiner, L., and Domínguez de Maria, P. (2012) Oxidationhydroxymethylation-reduction: A one-pot three-step biocatalytic synthesis of optically active $\alpha$ aryl vicinal diols. Green Chem. 14, 94-97

27. Schmidt, S., Pedroso de Almeida, T., Rother, D., and Hollmann, F. (2017) Towards environmentally acceptable synthesis of chiral $\alpha$-hydroxy ketones via oxidase-lyase cascades. Green Chem. 19, 1226-1229

28. Yamashita, N., Motoyoshi, T., and Nishimura, A. (1999) Purification and characterization of isoamyl alcohol oxidase ("Mureka"-forming enzyme). Biosci. Biotechnol. Biochem. 63, 1216-1222

29. Kemp, G. D., Dickinson, F. M., and Ratledge, C. (1988) Inducible long chain alcohol oxidase from alkane-grown Candida tropicalis. Appl. Microbiol. Biotechnol. 29, 370-374 
30. Mauersberger, S., Drechsler, H., Oehme, G., and Müller, H.-G. (1992) Substrate specificity and stereoselectivity of fatty alcohol oxidase from the yeast Candida maltosa. Appl. Microbiol. Biotechnol. 37, 66-73

31. Cheng, Q., Liu, H.-T., Bombelli, P., Smith, A., and Slabas, A. R. (2004) Functional identification of AtFao3, a membrane bound long chain alcohol oxidase in Arabidopsis thaliana. FEBS Lett. 574, $62-68$

32. Vanhanen, S., West, M., Kroon, J. T. M., Lindner, N., Casey, J., Cheng, Q., Elborough, K. M., and Slabas, A. R. (2000) A consensus sequence for long-chain fatty-acid alcohol oxidases from Candida identifies a family of genes involved in lipid $\omega$-oxidation in yeast with homologues in plants and bacteria. J. Biol. Chem. 275, 4445-4452

33. Cheng, Q., Sanglard, D., Vanhanen, S., Huan, T. L., Bombelli, P., Smith, A., and Slabas, A. R. (2005) Candida yeast long chain fatty alcohol oxidase is a $c$-type haemoprotein and plays an important role in long chain fatty acid metabolism. Biochim. Biophys. Acta - Mol. Cell Biol. Lipids. 1735, 192-203

34. Pickl, M., Fuchs, M., Glueck, S. M., and Faber, K. (2015) Amination of $\omega$-functionalized aliphatic primary alcohols by a biocatalytic oxidation-transamination cascade. ChemCatChem. 7, 31213124

35. Hernández-Ortega, A., Ferreira, P., and Martínez, A. T. (2012) Fungal aryl-alcohol oxidase: A peroxide-producing flavoenzyme involved in lignin degradation. Appl. Microbiol. Biotechnol. 93, 1395-1410

36. Guillén, F., Martinez, A. T., and Martínez, M. J. (1990) Production of hydrogen peroxide by arylalcohol oxidase from the ligninolytic fungus Pleurotus eryngii. Appl. Microbiol. Biotechnol. 32, 465469

37. Guillén, F., Martínez, A. T., and Martínez, M. J. (1992) Substrate specificity and properties of the aryl-alcohol oxidase from the ligninolytic fungus Pleurotus eryngii. Eur. J. Biochem. 209, 603-611

38. Ferreira, P., Medina, M., Guillén, F., Martínez, M. J., van Berkel, W. J. H., and Martínez, Á. T. (2005) Spectral and catalytic properties of aryl-alcohol oxidase, a fungal flavoenzyme acting on polyunsaturated alcohols. Biochem. J. 389, 731-738

39. Ferreira, P., Hernández-Ortega, A., Herguedas, B., Rencoret, J., Gutiérrez, A., Martínez, M. J., Jiménez-Barbero, J., Medina, M., and Martínez, Á. T. (2010) Kinetic and chemical characterization of aldehyde oxidation by fungal aryl-alcohol oxidase. Biochem. J. 425, 585-593

40. Hernández-Ortega, A., Ferreira, P., Merino, P., Medina, M., Guallar, V., and Martínez, A. T. (2012) Stereoselective hydride transfer by aryl-alcohol oxidase, a member of the GMC superfamily. ChemBioChem. 13, 427-435

41. Romero, E., Ferreira, P., Martínez, Á. T., and Martínez, M. J. (2009) New oxidase from Bjerkandera arthroconidial anamorph that oxidizes both phenolic and nonphenolic benzyl alcohols. Biochim. Biophys. Acta - Proteins Proteomics. 1794, 689-697

42. Dijkman, W. P., and Fraaije, M. W. (2014) Discovery and characterization of a 5hydroxymethylfurfural oxidase from Methylovorus sp. strain MP688. Appl. Environ. Microbiol. 80, 1082-1090

43. Rosatella, A. A., Simeonov, S. P., Frade, R. F. M., and Afonso, C. A. M. (2011) 5Hydroxymethylfurfural (HMF) as a building block platform: Biological properties, synthesis and synthetic applications. Green Chem. 13, 754-793

44. Dijkman, W. P., Groothuis, D. E., and Fraaije, M. W. (2014) Enzyme-catalyzed oxidation of 5hydroxymethylfurfural to furan-2,5-dicarboxylic acid. Angew. Chem. Int. Ed. 53, 6515-6518

45. Dijkman, W. P., Binda, C., Fraaije, M. W., and Mattevi, A. (2015) Structure-based enzyme tailoring of 5-hydroxymethylfurfural oxidase. ACS Catal. 5, 1833-1839

46. Carro, J., Ferreira, P., Rodríguez, L., Prieto, A., Serrano, A., Balcells, B., Ardá, A., JiménezBarbero, J., Gutiérrez, A., Ullrich, R., Hofrichter, M., and Martínez, A. T. (2015) 5hydroxymethylfurfural conversion by fungal aryl-alcohol oxidase and unspecific peroxygenase. FEBS J. 282, 3218-3229 
47. de Jong, E., van Berkel, W. J. H., van der Zwan, R. P., and de Bont, J. A. M. (1992) Purification and characterization of vanillyl-alcohol oxidase from Penicillium simplicissimum: A novel aromatic alcohol oxidase containing covalently bound FAD. Eur. J. Biochem. 208, 651-657

48. Fraaije, M. W., Mattevi, A., and van Berkel, W. J. H. (1997) Mercuration of vanillyl-alcohol oxidase from Penicillium simplicissimum generates inactive dimers. FEBS Lett. 402, 33-35

49. Mattevi, A., Fraaije, M. W., Mozzarelli, A., Olivi, L., Coda, A., and van Berkel, W. J. H. (1997) Crystal structures and inhibitor binding in the octameric flavoenzyme vanillyl-alcohol oxidase: The shape of the active-site cavity controls substrate specificity. Structure. 5, 907-920

50. Fraaije, M. W., Veeger, C., and van Berkel, W. J. H. (1995) Substrate specificity of flavindependent vanillyl-alcohol oxidase from Penicillium simplicissimum: Evidence for the production of 4-hydroxycinnamyl alcohols from 4-allylphenols. Eur. J. Biochem. 234, 271-277

51. van den Heuvel, R. H. H., Fraaije, M. W., Laane, C., and van Berkel, W. J. H. (1998) Regio- and stereospecific conversion of 4-alkylphenols by the covalent flavoprotein vanillyl-alcohol oxidase. J. Bacteriol. 180, 5646-5651

52. Furukawa, H., Wieser, M., Morita, H., Sugio, T., and Nagasawa, T. (1999) Purification and characterization of vanillyl-alcohol oxidase from Byssochlamys fulva V107. J. Biosci. Bioeng. 87, 285-290

53. Jin, J., Mazon, H., van den Heuvel, R. H. H., Janssen, D. B., and Fraaije, M. W. (2007) Discovery of a eugenol oxidase from Rhodococcus sp. strain RHA1. FEBS J. 274, 2311-2321

54. Nguyen, Q.-T., de Gonzalo, G., Binda, C., Rioz-Martínez, A., Mattevi, A., and Fraaije, M. W. (2016) Biocatalytic properties and structural analysis of eugenol oxidase from Rhodococcus jostii RHA1: A versatile oxidative biocatalyst. ChemBioChem. 17, 1359-1366

55. Zelitch, I., and Ochoa, S. (1953) Oxidation and reduction of glycolic and glyoxylic acids in plants. I. Glycolic acid oxidase. J. Biol. Chem. 201, 707-718

56. Frigerio, N. A., and Harbury, H. A. (1958) Preparation and some properties of crystalline glycolic acid oxidase of spinach. J. Biol. Chem. 231, 135-157

57. Stenberg, K., and Lindqvist, Y. (1996) High-level expression, purification, and crystallization of recombinant spinach glycolate oxidase in Escherichia coli. Protein Expr. Purif. 8, 295-298

58. Richardson, K. E., and Tolbert, N. E. (1961) Oxidation of glyoxylic acid to oxalic acid by glycolic acid oxidase. J. Biol. Chem. 236, 1280-1284

59. Adam, W., Lazarus, M., Boss, B., Saha-Möller, C. R., Humpf, H.-U., and Schreier, P. (1997) Enzymatic resolution of chiral 2-hydroxy carboxylic acids by enantioselective oxidation with molecular oxygen catalyzed by the glycolate oxidase from spinach (Spinacia oleracea). J. Org. Chem. 62, 7841-7843

60. Adam, W., Lazarus, M., Saha-Möller, C. R., and Schreier, P. (1998) Quantitative transformation of racemic 2-hydroxy acids into ( $R$-2-hydroxy acids by enantioselective oxidation with glycolate oxidase and subsequent reduction of 2-keto acids with D-lactate dehydrogenase. Tetrahedron: Asymmetry. 9, 351-355

61. Das, S., Glenn IV, J. H., and Subramanian, M. (2010) Enantioselective oxidation of 2-hydroxy carboxylic acids by glycolate oxidase and catalase coexpressed in methylotrophic Pichia pastoris. Biotechnol. Prog. 26, 607-615

62. Misaki, H., Horiuchi, Y., Matsuura, K., and Harada, S. (1980) Lactate oxidase process for the manufacture thereof and analytical method and kit for the use of the same, United States Patent. $4,237,222$

63. Duncan, J. D., Wallis, J. O., and Azari, M. R. (1989) Purification and properties of Aerococcus viridans lactate oxidase. Biochem. Biophys. Res. Commun. 164, 919-926

64. Maeda-Yorita, K., Aki, K., Sagai, H., Misaki, H., and Massey, V. (1995) L-Lactate oxidase and Llactate monooxygenase: Mechanistic variations on a common structural theme. Biochimie. 77, 631-642

65. Oikawa, T., Mukoyama, S., and Soda, K. (2001) Chemo-enzymatic D-enantiomerization of DLlactate. Biotechnol. Bioeng. 73, 80-82 
66. Blanchard, M., Green, D. E., Nocito-Carroll, V., and Ratner, S. (1946) L-Hydroxy acid oxidase. J. Biol. Chem. 163, 137-144

67. Robinson, J. C., Keay, L., Molinari, R., and Sizer, I. W. (1962) L- $\alpha$-Hydroxy acid oxidases of hog renal cortex. J. Biol. Chem. 237, 2001-2010

68. Nakano, M., and Danowski, T. S. (1966) Crystalline mammalian L-amino acid oxidase from rat kidney mitochondria. J. Biol. Chem. 241, 2075-2083

69. Domenech, C. E., Machado de Domenech, E. E., and Blanco, A. (1973) Molecular forms of L- $\alpha-$ hydroxy acid oxidase from rat kidney. Biochim. Biophys. Acta - Enzymol. 321, 54-63

70. Urban, P., Chirat, I., and Lederer, F. (1988) Rat kidney L-2-hydroxyacid oxidase: Structural and mechanistic comparison with flavocytochrome $b_{2}$ from baker's yeast, Biochemistry. 27, 7365-7371

71. Vrielink, A., and Ghisla, S. (2009) Cholesterol oxidase: Biochemistry and structural features. FEBS J. 276, 6826-6843

72. Pollegioni, L., Piubelli, L., and Molla, G. (2009) Cholesterol oxidase: Biotechnological applications. FEBS J. 276, 6857-6870

73. Dieth, S., Tritsch, D., and Biellmann, J.-F. (1995) Resolution of allylic alcohols by cholesterol oxidase isolated from Rhodococcus erythropolis. Tetrahedron Lett. 36, 2243-2246

74. Alexander, D. L., and Fisher, J. F. (1995) A convenient synthesis of 7 $\alpha$-hydroxycholest-4-en-3one by the hydroxypropyl- $\beta$-cyclodextrin-facilitated cholesterol oxidase oxidation of $3 \beta, 7 \alpha-$ cholest-5-ene-3,7-diol. Steroids. 60, 290-294

75. Labaree, D., Hoyte, R. M., and Hochberg, R. B. (1997) A direct stereoselective synthesis of 7 $\beta-$ hydroxytestosterone. Steroids. 62, 482-486

76. Guo, L.-W., Wilson, W. K., Pang, J., and Shackleton, C. H. L. (2003) Chemical synthesis of 7- and 8-dehydro derivatives of pregnane-3,17 $\alpha, 20$-triols, potential steroid metabolites in Smith-LemliOpitz syndrome. Steroids. 68, 31-42

77. Krämer, L., and Steckhan, E. (1997) Coimmobilization of L- $\alpha$-glycerophosphate oxidase with catalase and its application for the synthesis of dihydroxyacetone phosphate. Tetrahedron. 53, 14645-14650

78. Babich, L., van Hemert, L. J. C., Bury, A., Hartog, A. F., Falcicchio, P., van der Oost, J., van Herk, T., Wever, R., and Rutjes, F. P. J. T. (2011) Synthesis of non-natural carbohydrates from glycerol and aldehydes in a one-pot four-enzyme cascade reaction. Green Chem. 13, 2895-2900

79. Ikuta, S., Imamura, S., Misaki, H., and Horiuti, Y. (1977) Purification and characterization of choline oxidase from Arthrobacter globiformis. J. Biochem. 82, 1741-1749

80. Fan, F., Ghanem, M., and Gadda, G. (2004) Cloning, sequence analysis, and purification of choline oxidase from Arthrobacter globiformis: A bacterial enzyme involved in osmotic stress tolerance. Arch. Biochem. Biophys. 421, 149-158

81. Gadda, G., Powell, N. L. N., and Menon, P. (2004) The trimethylammonium headgroup of choline is a major determinant for substrate binding and specificity in choline oxidase. Arch. Biochem. Biophys. 430, 264-273

82. Leskovac, V., Trivić, S., Wohlfahrt, G., Kandrač, J., and Peričin, D. (2005) Glucose oxidase from Aspergillus niger: The mechanism of action with molecular oxygen, quinones, and one-electron acceptors. Int. J. Biochem. Cell Biol. 37, 731-750

83. Godjevargova, T., Dayal, R., and Turmanova, S. (2004) Gluconic acid production in bioreactor with immobilized glucose oxidase plus catalase on polymer membrane adjacent to anionexchange membrane. Macromol. Biosci. 4, 950-956

84. Pezzotti, F., Therisod, H., and Therisod, M. (2005) Enzymatic synthesis of D-glucosaminic acid from D-glucosamine. Carbohydr. Res. 340, 139-141

85. Pezzotti, F., and Therisod, M. (2006) Enzymatic synthesis of aldonic acids. Carbohydr. Res. 341, 2290-2292

86. Giffhorn, F. (2000) Fungal pyranose oxidases: Occurrence, properties and biotechnical applications in carbohydrate chemistry. Appl. Microbiol. Biotechnol. 54, 727-740

87. Halada, P., Leitner, C., Sedmera, P., Haltrich, D., and Volc, J. (2003) Identification of the covalent 
flavin adenine dinucleotide-binding region in pyranose 2-oxidase from Trametes multicolor. Anal. Biochem. 314, 235-242

88. Leitner, C., Volc, J., and Haltrich, D. (2001) Purification and characterization of pyranose oxidase from the white rot fungus Trametes multicolor. Appl. Environ. Microbiol. 67, 3636-3644

89. Giffhorn, F., Köpper, S., Huwig, A., and Freimund, S. (2000) Rare sugars and sugar-based synthons by chemo-enzymatic synthesis. Enzyme Microb. Technol. 27, 734-742

90. Geigert, J., Neidleman, S. L., and Hirano, D. S. (1983) Convenient, laboratory procedure for reducing D-glucosone to D-fructose. Carbohydr. Res. 113, 159-162

91. Koths, K., Halenbeck, R., and Moreland, M. (1992) Synthesis of the antibiotic cortalcerone from D-glucose using pyranose 2-oxidase and a novel fungal enzyme, aldos-2-ulose dehydratase. Carbohydr. Res. 232, 59-75

92. Freimund, S., Huwig, A., Giffhorn, F., and Köpper, S. (1996) Convenient chemo-enzymatic synthesis of D-tagatose. J. Carbohydr. Chem. 15, 115-120

93. Freimund, S., Huwig, A., Giffhorn, F., and Köpper, S. (1998) Rare keto-aldoses from enzymatic oxidation: Substrates and oxidation products of pyranose 2-oxidase. Chem. Eur. J. 4, 2442-2455

94. Blagg, B. S. J., and Poulter, C. D. (1999) Synthesis of 1-deoxy-D-xylulose and 1-deoxy-D-xylulose5-phosphate, J. Org. Chem. 64, 1508-1511

95. Leitner, C., Mayr, P., Riva, S., Volc, J., Kulbe, K. D., Nidetzky, B., and Haltrich, D. (2001) Enzymatic redox isomerization of 1,6-disaccharides by pyranose oxidase and NADHdependent aldose reductase. J. Mol. Catal. B Enzym. 11, 407-414

96. Seto, H., Kawakita, H., Ohto, K., Harada, H., and Inoue, K. (2008) Novel carbonyl-groupcontaining dextran synthesis by pyranose-2-oxidase and dextransucrase. Carbohydr. Res. 343, 2417-2421

97. Heuts, D. P. H. M., van Hellemond, E. W., Janssen, D. B., and Fraaije, M. W. (2007) Discovery, characterization, and kinetic analysis of an alditol oxidase from Streptomyces coelicolor. J. Biol. Chem. 282, 20283-20291

98. Hiraga, K., Kitazawa, M., Kaneko, N., and Odat, K. (1997) Isolation and some properties of sorbitol oxidase from Streptomyces sp. H-7775. Biosci. Biotechnol. Biochem. 61, 1699-1704

99. Yamashita, M., Omura, H., Okamoto, E., Furuya, Y., Yabuuchi, M., Fukahi, K., and Murooka, Y. (2000) Isolation, characterization, and molecular cloning of a thermostable xylitol oxidase from Streptomyces sp. IKD472. J. Biosci. Bioeng. 89, 350-360

100. Winter, R. T., Heuts, D. P. H. M., Rijpkema, E. M. A., van Bloois, E., Wijma, H. J., and Fraaije, M. W. (2012) Hot or not? Discovery and characterization of a thermostable alditol oxidase from Acidothermus cellulolyticus 11B. Appl. Microbiol. Biotechnol. 95, 389-403

101. Forneris, F., Heuts, D. P. H. M., Delvecchio, M., Rovida, S., Fraaije, M. W., and Mattevi, A. (2008) Structural analysis of the catalytic mechanism and stereoselectivity in Streptomyces coelicolor alditol oxidase. Biochemistry. 47, 978-985

102. van Hellemond, E. W., Vermote, L., Koolen, W., Sonke, T., Zandvoort, E., Heuts, D. P. H. M., Janssen, D. B., and Fraaije, M. W. (2009) Exploring the biocatalytic scope of alditol oxidase from Streptomyces coelicolor. Adv. Synth. Catal. 351, 1523-1530

103. Sullivan Jr., J. D., and Ikawa, M. (1973) Purification and characterization of hexose oxidase from the red alga Chondrus crispus. Biochim. Biophys. Acta - Enzymol. 309, 11-22

104. Hansen, O. C., and Stougaard, P. (1997) Hexose oxidase from the red alga Chondrus crispus. Purification, molecular cloning, and expression in Pichia pastoris. J. Biol. Chem. 272, 11581-11587

105. Groen, B. W., de Vries, S., and Duine, J. A. (1997) Characterization of hexose oxidase from the red seaweed Chondrus crispus. Eur. J. Biochem. 244, 858-861

106. Rand, T., Qvist, K. B., Walter, C. P., and Poulsen, C. H. (2006) Characterization of the flavin association in hexose oxidase from Chondrus crispus. FEBS J. 273, 2693-2703

107. Poulsen, C., and Høstrup, P. B. (1998) Purification and characterization of a hexose oxidase with excellent strengthening effects in bread. Cereal Chem. 75, 51-57

108. Xu, F., Golightly, E. J., Fuglsang, C. C., Schneider, P., Duke, K. R., Lam, L., Christensen, S., Brown, 
K. M., Jørgensen, C. T., and Brown, S. H. (2001) A novel carbohydrate:acceptor oxidoreductase from Microdochium nivale. Eur. J. Biochem. 268, 1136-1142

109. Leferink, N. G. H., Heuts, D. P. H. M., Fraaije, M. W., and van Berkel, W. J. H. (2008) The growing VAO flavoprotein family. Arch. Biochem. Biophys. 474, 292-301

110. Lin, S.-F., Yang, T.-Y., Inukai, T., Yamasaki, M., and Tsai, Y.-C. (1991) Purification and characterization of a novel glucooligosaccharide oxidase from Acremonium strictum T1. Biochim. Biophys. Acta - Protein Struct. Mol. Enzymol. 1118, 41-47

111. Huang, C.-H., Lai, W.-L., Lee, M.-H., Chen, C.-J., Vasella, A., Tsai, Y.-C., and Liaw, S.-H. (2005) Crystal structure of glucooligosaccharide oxidase from Acremonium strictum: A novel flavinylation of 6-S-cysteinyl, 8 $\alpha$-N1-histidyl FAD. J. Biol. Chem. 280, 38831-38838

112. Fan, Z., Oguntimein, G. B., and Reilly, P. J. (2000) Characterization of kinetics and thermostability of Acremonium strictum glucooligosaccharide oxidase. Biotechnol. Bioeng. 68, 231237

113. Custers, J. H. H. V., Harrison, S. J., Sela-Buurlage, M. B., van Deventer, E., Lageweg, W., Howe, P. W., van der Meijs, P. J., Ponstein, A. S., Simons, B. H., Melchers, L. S., and Stuiver, M. H. (2004) Isolation and characterisation of a class of carbohydrate oxidases from higher plants, with a role in active defence. Plant J. 39, 147-160

114. Kiryu, T., Nakano, H., Kiso, T., and Murakami, H. (2008) Purification and characterization of a carbohydrate:acceptor oxidoreductase from Paraconiothyrium sp. that produces lactobionic acid efficiently. Biosci. Biotechnol. Biochem. 72, 833-841

115. Heuts, D. P. H. M., Janssen, D. B., and Fraaije, M. W. (2007) Changing the substrate specificity of a chitooligosaccharide oxidase from Fusarium graminearum by model-inspired site-directed mutagenesis. FEBS Lett. 581, 4905-4909

116. Ferrari, A. R., Rozeboom, H. J., Dobruchowska, J. M., van Leeuwen, S. S., Vugts, A. S. C., Koetsier, M. J., Visser, J., and Fraaije, M. W. (2016) Discovery of a xylooligosaccharide oxidase from Myceliophthora thermophila C1. J. Biol. Chem. 291, 23709-23718

117. Whittaker, J. W. (2002) Galactose oxidase. Adv. Protein Chem. 60, 1-49

118. McPherson, M. J., Ogel, Z. B., Stevens, C., Yadav, K. D. S., Keen, J. N., and Knowles, P. F. (1992) Galactose oxidase of Dactylium dendroides: Gene cloning and sequence analysis. J. Biol. Chem. 267, 8146-8152

119. Ito, N., Phillips, S. E. V, Stevens, C., Ogel, Z. B., McPherson, M. J., Keen, J. N., Yadav, K. D. S., and Knowles, P. F. (1991) Novel thioether bond revealed by a $1.7 \AA$ crystal structure of galactose oxidase. Nature. 350, 87-90

120. Whittaker, J. W. (2005) The radical chemistry of galactose oxidase. Arch. Biochem. Biophys. 433, 227-239

121. Klibanov, A. M., Alberti, B. N., and Marletta, M. A. (1982) Stereospecific oxidation of aliphatic alcohols catalyzed by galactose oxidase. Biochem. Biophys. Res. Commun. 108, 804-808

122. Leppänen, A.-S., Niittymäki, O., Parikka, K., Tenkanen, M., Eklund, P., Sjöholm, R., and Willför, S. (2010) Metal-mediated allylation of enzymatically oxidized methyl $\alpha$-D-galactopyranoside. Carbohydr. Res. 345, 2610-2615

123. Leppänen, A.-S., Xu, C., Parikka, K., Eklund, P., Sjöholm, R., Brumer, H., Tenkanen, M., and Willför, S. (2014) Targeted allylation and propargylation of galactose-containing polysaccharides in water. Carbohydr. Polym. 100, 46-54

124. Fuchs, M., Schober, M., Pfeffer, J., Kroutil, W., Birner-Gruenberger, R., and Faber, K. (2011) Homoallylic alcohols via a chemo-enzymatic one-pot oxidation-allylation cascade. Adv. Synth. Catal. 353, 2354-2358

125. Bülter, T., Schumacher, T., Namdjou, D.-J., Gutiérrez Gallego, R., Clausen, H., and Elling, L. (2001) Chemoenzymatic synthesis of biotinylated nucleotide sugars as substrates for glycosyltransferases. ChemBioChem. 2, 884-894

126. Namdjou, D.-J., Sauerzapfe, B., Schmiedel, J., Dräger, G., Bernatchez, S., Wakarchuk, W. W., and Elling, L. (2007) Combination of UDP-Glc(NAc) 4'-epimerase and galactose oxidase in a one-pot 
synthesis of biotinylated nucleotide sugars. Adv. Synth. Catal. 349, 314-318

127. Kupper, C. E., Rosencrantz, R. R., Henßen, B., Pelantová, H., Thönes, S., Drozdová, A., Křen, V., and Elling, L. (2012) Chemo-enzymatic modification of poly- $N$-acetyllactosamine (LacNAc) oligomers and $N, N$-diacetyllactosamine (LacDiNAc) based on galactose oxidase treatment. Beilstein J. Org. Chem. 8, 712-725

128. Laaf, D., Steffens, H., Pelantová, H., Bojarová, P., Křen, V., and Elling, L. (2017) Chemoenzymatic synthesis of branched $N$-acetyllactosamine glycan oligomers for galectin-3 inhibition. Adv. Synth. Catal. 359, 4015-4024

129. Franke, D., Machajewski, T., Hsu, C.-C., and Wong, C.-H. (2003) One-pot synthesis of L-fructose using coupled multienzyme systems based on rhamnulose-1-phosphate aldolase. J. Org. Chem. 68, 6828-6831

130. Fuchs, M., Tauber, K., Sattler, J., Lechner, H., Pfeffer, J., Kroutil, W., and Faber, K. (2012) Amination of benzylic and cinnamic alcohols via a biocatalytic, aerobic, oxidationtransamination cascade. RSC Adv. 2, 6262-6265

131. Escalettes, F., and Turner, N. J. (2008) Directed evolution of galactose oxidase: Generation of enantioselective secondary alcohol oxidases. ChemBioChem. 9, 857-860

132. McKenna, S. M., Leimkühler, S., Herter, S., Turner, N. J., and Carnell, A. J. (2015) Enzyme cascade reactions: Synthesis of furandicarboxylic acid (FDCA) and carboxylic acids using oxidases in tandem. Green Chem. 17, 3271-3275

133. McKenna, S. M., Mines, P., Law, P., Kovacs-Schreiner, K., Birmingham, W. R., Turner, N. J., Leimkühler, S., and Carnell, A. J. (2017) The continuous oxidation of HMF to FDCA and the immobilisation and stabilisation of periplasmic aldehyde oxidase (PaoABC). Green Chem. 19, 4660-4665

134. Vorhaben, J. E., Smith Jr., D. D., and Campbell, J. W. (1986) Mannitol oxidase: Partial purification and characterization of the membrane-bound enzyme from the snail Helix aspersa. Int. J. Biochem. 18, 337-344

135. Vorhaben, J. E., Scott, J. F., and Campbell, J. W. (1980) D-Mannitol oxidation in the land snail, Helix aspersa. J. Biol. Chem. 255, 1950-1955

136. Kawai, F., and Hu, X. (2009) Biochemistry of microbial polyvinyl alcohol degradation. Appl. Microbiol. Biotechnol. 84, 227-237

137. Suzuki, T. (1976) Purification and some properties of polyvinyl alcohol-degrading enzyme produced by Pseudomonas O-3. Agric. Biol. Chem. 40, 497-504

138. Morita, M., and Watanabe, Y. (1977) A secondary alcohol oxidase: A component of a polyvinyl alcohol degrading enzyme preparation. Agric. Biol. Chem. 41, 1535-1537

139. Shimao, M., Tsuda, T., Takahashi, M., Kato, N., and Sakazawa, C. (1983) Purification of membrane-bound polyvinyl alcohol oxidase in Pseudomonas sp. VM15C. FEMS Microbiol. Lett. 20, 429-433

140. Sakai, K., Hamada, N., and Watanabe, Y. (1985) Purification and properties of secondary alcohol oxidase with an acidic isoelectric point. Agric. Biol. Chem. 49, 817-825

141. Kawagoshi, Y., and Fujita, M. (1997) Purification and properties of polyvinyl alcohol oxidase with broad substrate range obtained from Pseudomonas vesicularis var. povalolyticus PH. World J. Microbiol. Biotechnol. 13, 273-277

142. Okrasa, K., Guibé-Jampel, E., and Therisod, M. (2000) Tandem peroxidase-glucose oxidase catalysed enantioselective sulfoxidation of thioanisoles. J. Chem. Soc. Perkin Trans. 1. pp. 10771079

143. Pezzotti, F., Okrasa, K., and Therisod, M. (2005) Bienzymatic synthesis of chiral heteroarylmethyl-sulfoxides. Tetrahedron: Asymmetry. 16, 2681-2683

144. Pezzotti, F., and Therisod, M. (2007) Enantioselective oxidation of thioanisole with an alcohol oxidase/peroxidase bienzymatic system. Tetrahedron: Asymmetry. 18, 701-704

145. Garcia-Galan, C., Berenguer-Murcia, A., Fernandez-Lafuente, R., and Rodrigues, R. C. (2011) Potential of different enzyme immobilization strategies to improve enzyme performance. Adv. 
Synth. Catal. 353, 2885-2904

146. Hwang, E. T., and Gu, M. B. (2013) Enzyme stabilization by nano/microsized hybrid materials. Eng. Life Sci. 13, 49-61

147. Betancor, L., López-Gallego, F., Hidalgo, A., Alonso-Morales, N., Dellamora-Ortiz, G., Guisán, J. M., and Fernández-Lafuente, R. (2006) Preparation of a very stable immobilized biocatalyst of glucose oxidase from Aspergillus niger. J. Biotechnol. 121, 284-289

148. Seip, J. E., Fager, S. K., Gavagan, J. E., Anton, D. L., and Di Cosimo, R. (1994) Glyoxylic acid production using immobilized glycolate oxidase and catalase. Bioorganic Med. Chem. 2, 371-378

149. Olsson, L., Mandenius, C. F., Kubatova, E., and Volc, J. (1991) Immobilization of pyranose oxidase (Phanerochaete chrysosporium): Characterization of the enzymic properties. Enzyme Microb. Technol. 13, 755-759

150. Schrittwieser, J. H., Sattler, J., Resch, V., Mutti, F. G., and Kroutil, W. (2011) Recent biocatalytic oxidation-reduction cascades. Curr. Opin. Chem. Biol. 15, 249-256

\section{Abbreviations}

AAO: aryl alcohol oxidase, AldO: alditol oxidase, ChitO: chitooligosaccharide oxidase, ChlO: choline oxidase, ChoX: cholesterol oxidase, EUGO: eugenol oxidase, FAD: flavin adenine dinucleotide, FDCA: 2,5-furandicarboxylic acid, FFCA: 2,5-formylfurancarboxylic acid, FMN: flavin mononucleotide, GAO: galactose oxidase, GlyO: glycolate oxidase, GMC: glucose-methanol-choline, GOX: glucose oxidase, GOOX: glucooligosaccharide oxidase, GPO: glycerol 3-phosphate oxidase, (L)HAO: (long-chain) (S)-2hydroxy acid oxidase, $\operatorname{HMF}(\mathrm{O})$ : 5-(hydroxymethyl)furfural (oxidase), HOX: hexose oxidase, IAAO: isoamyl alcohol oxidase, LAO: lactose oxidase, LCAO: long-chain alcohol oxidase, LLO: L-lactate oxidase, MannO: mannitol oxidase, MO: methanol oxidase, $\mathrm{NAD}(\mathrm{P})$ : nicotinamide adenine dinucleotide (phosphate), P2O: pyranose-2-oxidase, SAO: secondary alcohol oxidase, VAO: vanillyl alcohol oxidase, $\mathrm{XylO}$ : xylooligosaccharide oxidase 




\title{
6
}

\section{A xylenol orange-based screening assay for the substrate specificity of flavin-dependent para-phenol oxidases}

\author{
Tom A. Ewing ${ }^{1}$, Aster van Noord ${ }^{1}$, Caroline E. Paul ${ }^{2}$ and Willem J. H. van Berkel ${ }^{1}$ \\ ${ }^{1}$ Laboratory of Biochemistry, Wageningen University \& Research, Wageningen, The Netherlands \\ ${ }^{2}$ Laboratory of Organic Chemistry, Wageningen University \& Research, Wageningen, \\ The Netherlands
}

Based on Molecules (2018), 23, 164

\section{Summary}

Vanillyl alcohol oxidase (VAO) and eugenol oxidase (EUGO) are flavin-dependent enzymes that catalyse the oxidation of para-substituted phenols. This makes them potentially interesting biocatalysts for the conversion of lignin-derived aromatic monomers to valueadded compounds. To facilitate their biocatalytic exploitation, it is important to develop methods by which variants of the enzymes can be rapidly screened for increased activity towards substrates of interest. Here, we present the development of a screening assay for the substrate specificity of para-phenol oxidases based on the detection of hydrogen peroxide using the ferric-xylenol orange complex method. The assay was used to screen the activity of VAO and EUGO towards a set of twenty-four potential substrates. This led to the identification of 4-cyclopentylphenol as a new substrate of VAO and EUGO and 4cyclohexylphenol as a new substrate of VAO. Screening of a small library of VAO and EUGO active-site variants for alterations in their substrate specificity led to the identification of a VAO variant (T457Q) with increased activity towards vanillyl alcohol and a EUGO variant (V436I) with increased activity towards chavicol and 4-cyclopentylphenol. This assay provides a quick and efficient method to screen the substrate specificity of para-phenol oxidases, facilitating the enzyme engineering of known para-phenol oxidases and the evaluation of the substrate specificity of novel para-phenol oxidases. 



\section{Introduction}

Lignin, one of the major constituents of lignocellulosic biomass, is a heterogenous aromatic polymer, formed from the monolignols para-coumaryl alcohol, coniferyl alcohol and sinapyl alcohol through a radical coupling process $(1,2)$. It is obtained as a by-product during the processing of plant biomass in biorefineries and as such represents an attractive renewable feedstock of aromatic compounds. In order to harness the full potential of lignin as a renewable chemical feedstock, it is desirable to develop novel methods to depolymerise it to monomeric aromatic compounds and subsequently convert these to value-added compounds. One way in which lignin-derived aromatic molecules can be converted to value-added compounds is through the action of oxidative enzymes. Two such enzymes are the flavindependent oxidases vanillyl alcohol oxidase (VAO, EC 1.1.3.38) from Penicillium simplicissimum and eugenol oxidase (EUGO) from Rhodococcus jostii RHA1 (3, 4), which both belong to auxiliary activity family 4 (AA4) of the carbohydrate active enzyme (CAZy) database (5).

$\mathrm{VAO}$ and EUGO are members of the VAO/PCMH flavoprotein family, which includes flavoenzymes characterised by the presence of a conserved FAD-binding domain $(6,7$, Chapter 2). VAO and EUGO both catalyse the two-electron oxidation of para-substituted phenols at the $\mathrm{C} \alpha$ position of their substituent (Scheme 1). Molecular oxygen acts as the electron acceptor for the reaction, being converted to hydrogen peroxide. Despite the fact that VAO and EUGO share significant sequence similarity (45\% identical) and very similar secondary and tertiary structures, they differ in terms of their oligomerisation state and substrate specificity $(8,9)$. VAO displays a broad substrate specificity, catalysing the oxidation of alcohols to aldehydes, the oxidative deamination of amines, the oxidative demethylation of ethers, the dehydrogenation or hydroxylation of alkyl groups and the hydroxylation of allyl groups $(10,11)$. EUGO displays a somewhat narrower substrate specificity. Although alcohols and 4-allylphenols are good substrates for the enzyme, 4-alkylphenols and ethers are hardly accepted (4). In terms of its oligomerisation state, VAO is predominantly octameric in solution, though active dimers may also be present under certain conditions $(3,12)$. In contrast, EUGO is exclusively dimeric in solution, with its structure strongly resembling that of a dimer of $\operatorname{VAO}(9)$.

A number of the reactions catalysed by VAO and EUGO are of interest for potential industrial applications. The oxidation of vanillyl alcohol (4-hydroxy-3-methoxybenzyl alcohol) or vanillyl amine [4-(aminomethyl)-2-methoxyphenol] yields vanillin (4-hydroxy-3methoxybenzaldehyde), the main flavour and fragrance compound in vanilla (14). VAO can also be used for the synthesis of chiral secondary alcohols, with the oxidation of short-chain linear 4-alkylphenols yielding the $(R)$-enantiomers of the corresponding alcohols in high enantiomeric excess (15). The hydroxylation of 4-allylphenols by VAO or EUGO has been employed in multi-enzyme cascades for the synthesis of the plant lignan pinoresinol and model lignin compounds (16-18). The hydroxylation of eugenol (4-allyl-2-methoxyphenol) by $\mathrm{VAO}$ has also garnered interest as the first step in fermentation processes for the conversion of eugenol to ferulic acid, which can be used as a precursor for vanillin (19-24). 


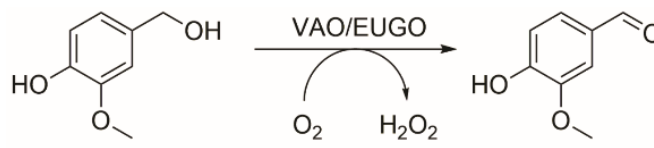

14

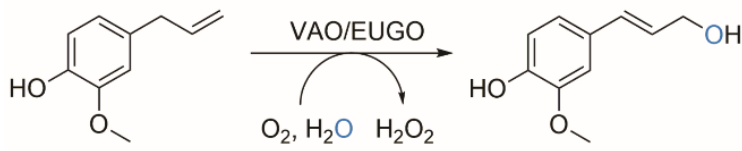

18

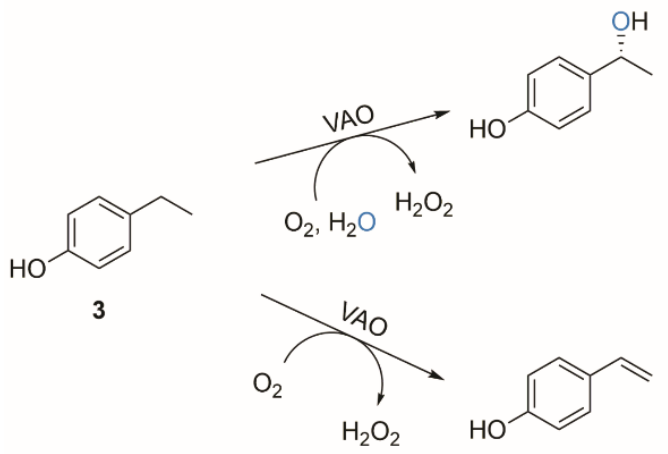

Scheme 1: Examples of reactions catalysed by VAO and/or EUGO. Both enzymes can catalyse the oxidation of vanillyl alcohol (14) to the aldehyde vanillin and of eugenol (18) to coniferyl alcohol. VAO converts 4-ethylphenol (3) to a mixture of products consisting of 4-vinylphenol and 1-(4'hydroxyphenyl)ethanol. The hydroxylation reaction occurs enantioselectively, predominantly yielding the $(R)$-enantiomer of the alcohol. In contrast, previous studies failed to reveal any activity towards 3 for EUGO (13).

Up to now, the molecular determinants of the differences in substrate specificity between VAO and EUGO are unclear. The differing oligomerisation states of the enzymes do not appear to be involved, as a VAO variant that exclusively forms dimers displayed similar catalytic properties to the wild-type enzyme (13). To improve our understanding of the determinants of the substrate specificity of these para-phenol oxidases and to facilitate efforts to modify their reactivity by enzyme engineering, it would be beneficial to be able to rapidly screen variants of VAO and EUGO for activity towards multiple substrates. To enable this, we here describe a method for the rapid screening of the substrate specificity of para-phenol oxidases using the ferric-xylenol orange complex method for the detection of hydrogen peroxide.

The ferric-xylenol orange complex method (xylenol orange assay) is a colourimetric method for the determination of the concentration of hydroperoxides. When an assay solution containing $\mathrm{Fe}^{2+}$ ions and xylenol orange is added to a sample containing a hydroperoxide under acidic conditions, the hydroperoxide will oxidise $\mathrm{Fe}^{2+}$ to $\mathrm{Fe}^{3+}$. The $\mathrm{Fe}^{3+}$ subsequently forms a complex with xylenol orange that can be quantified from its absorbance at $560 \mathrm{~nm}(25$, 26). The assay has been applied to measure the concentration of lipid or protein hydroperoxides in biological samples and as an activity assay for the lipid hydroperoxideforming enzyme lipoxygenase (27-29). Detection of hydrogen peroxide via the ferric-xylenol orange complex method can be used to detect the activity of oxidases and has been applied in a biosensor for lactose and in an in-gel screening assay for L-amino acid oxidase activity (30, $31)$. 
Methods used previously to determine the activity of VAO or EUGO typically relied on the measurement of the absorption of reaction products or the consumption of oxygen $(4,10)$. These assays are not suitable for high-throughput substrate specificity screening as they are either dependent on the identity of the phenolic reaction product (measuring absorption of the product) or cannot easily be performed for multiple samples simultaneously (measuring consumption of oxygen). In contrast, the xylenol orange assay does not depend on the identity of the phenolic reaction product and multiple samples can be evaluated simultaneously by performing the assay in a 96-wells plate. The xylenol orange assay is also better suited for our purpose than assays based on the detection of hydrogen peroxide via the formation of a coloured compound by peroxidases, as the phenolic substrates converted by VAO and EUGO are typically also substrates for peroxidases (32).

The method reported here consists of an efficient benchtop purification of His-tagged versions of VAO and EUGO, followed by a rapid screening of their activity towards a set of twentyfour potential substrates using the xylenol orange assay. After developing the procedure using the wild-type enzymes, we applied the assay to screen fourteen active-site variants for alterations in their substrate specificity. This led to the identification of new substrates of the wild-type enzymes and two variants that displayed increased activity towards at least one substrate.

\section{Results}

\section{Purification and characterisation of His-VAO and EUGO-His}

To enable screening of the substrate specificity of multiple VAO and EUGO variants, we first needed a method for the rapid and efficient purification of the enzymes. To this end, we expressed the enzymes from new expression vectors containing genes encoding His-tagged variants of the enzymes. VAO was expressed as an N-terminally His-tagged protein, His-VAO, and EUGO was expressed as a C-terminally His-tagged protein, EUGO-His. To evaluate whether these constructs can be used for our study, we expressed and purified the His-tagged wild-type enzymes and evaluated whether their catalytic properties are similar to those of the non-His-tagged proteins.

Both His-tagged enzymes were successfully expressed in E. coli and purified by a simple benchtop procedure consisting of a single affinity chromatography step using a Ni-NTA column followed by buffer exchange using a desalting column. The purified enzymes contained flavin, as determined from their characteristic flavin absorption spectra (Fig. S1+S2). Upon precipitation of the proteins using trichloroacetic acid, a yellow pellet and colourless supernatant were obtained, demonstrating that the flavin cofactor is covalently bound to the protein. To determine whether the presence of a His-tag on the protein affects catalysis, we determined the steady-state kinetic parameters for the oxidation of vanillyl alcohol by HisVAO and EUGO-His (Table 1). This revealed that the catalytic properties of the His-tagged enzymes are highly similar to those of the non-His-tagged enzymes, demonstrating that the introduction of the His-tag does not affect catalysis. Therefore, these His-tagged variants of $\mathrm{VAO}$ and EUGO provide a suitable experimental system to rapidly purify variants of the enzymes and study their catalytic properties. 
Table 1: Steady-state kinetic parameters for the oxidation of vanillyl alcohol by VAO, His-VAO, EUGO and EUGO-His in $50 \mathrm{mM}$ potassium phosphate buffer, $\mathrm{pH} 7.5$, at $25^{\circ} \mathrm{C}$.

\begin{tabular}{|llll|}
\hline Enzyme & $k_{\text {cat }}\left(\mathbf{s}^{-1}\right)$ & $K_{\mathrm{m}}(\boldsymbol{\mu M})$ & $k_{\text {cat }} / K_{\mathrm{m}}\left(\mathbf{s}^{-1} \mathbf{m M}^{-1}\right)$ \\
\hline VAO & $2.6^{\mathrm{a}}$ & $149^{\mathrm{a}}$ & 17 \\
His-VAO & $2.5 \pm 0.1$ & $129 \pm 15$ & 19 \\
EUGO & $7.1^{\mathrm{a}}$ & $79^{\mathrm{a}}$ & 90 \\
EUGO-His & $8.0 \pm 0.2$ & $47 \pm 5$ & 170 \\
\hline
\end{tabular}

${ }^{a}$ Data taken from (13).

Development of the xylenol orange assay using His-VAO and EUGO-His

To determine whether the xylenol orange assay can be used to accurately measure the activity of His-VAO and EUGO-His, we used it to follow the oxidation of vanillyl alcohol and eugenol by the enzymes in time. To this end, $2 \mathrm{mM}$ vanillyl alcohol or eugenol was allowed to react with EUGO-His or His-VAO for time periods ranging from one to twenty min, after which the amount of hydrogen peroxide formed was determined using the xylenol orange assay. In all cases, the measured hydrogen peroxide concentration was found to increase in time. The increase was linear for the first ten min of the reactions, with the exception of the oxidation of vanillyl alcohol by EUGO-His, where the increase was linear for the first $7.5 \mathrm{~min}$ of the reaction (Fig. 1).

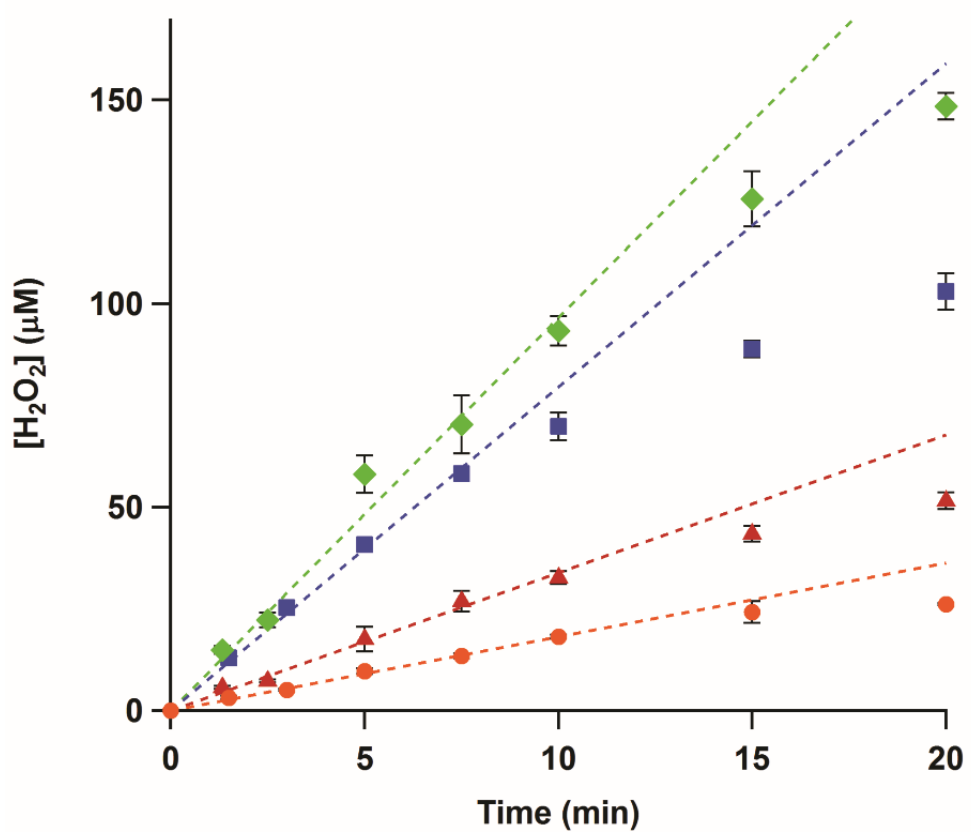

Figure 1: Conversion of eugenol or vanillyl alcohol by His-VAO or EUGO-His followed in time using the xylenol orange assay. His-VAO (orange circles for vanillyl alcohol, green diamonds for eugenol) or EUGO-His (blue squares for vanillyl alcohol, red triangles for eugenol) was incubated with $2 \mathrm{mM}$ substrate in $50 \mathrm{mM}$ potassium phosphate buffer, $\mathrm{pH} 7.5$, at room temperature $\left(19-20{ }^{\circ} \mathrm{C}\right)$. Reactions were allowed to proceed for the specified time, after which the amount of hydrogen peroxide formed was determined using the xylenol orange assay. Error bars represent the standard deviation of triplicate measurements. Dashed lines are fits to the linear range of the data that were used to determine reaction rates (see Table 2). 
Reaction rates were determined by fitting the linear range of the data. These rates (Table 2) were similar to the $k_{\text {cat }}$ values determined previously using other methods, as would be expected considering that our reactions were performed using saturating substrate concentrations. Thus, determination of the amount of hydrogen peroxide formed using the xylenol orange assay is a suitable way to determine the rates of reactions catalysed by HisVAO or EUGO-His.

Table 2: Reaction rates for the oxidation of $2 \mathrm{mM}$ vanillyl alcohol or eugenol by His-VAO or EUGOHis in $50 \mathrm{mM}$ potassium phosphate buffer, $\mathrm{pH} 7.5$, at room temperature $\left(19-20^{\circ} \mathrm{C}\right)$ as determined using the xylenol orange assay. The $k_{\text {cat }}$ values for the non-His-tagged enzymes measured previously using other methods are shown for comparison. These $k_{\text {cat }}$ values were measured under identical experimental conditions, with the exception of the temperature, which was $25^{\circ} \mathrm{C}$ instead of $19-20^{\circ} \mathrm{C}$.

\begin{tabular}{|c|c|c|c|c|}
\hline & \multicolumn{2}{|c|}{ (His)-VAO } & \multicolumn{2}{|c|}{ EUGO-(His) } \\
\hline & $\begin{array}{l}\text { Vanillyl } \\
\text { alcohol }\end{array}$ & Eugenol & $\begin{array}{l}\text { Vanillyl } \\
\text { alcohol }\end{array}$ & Eugenol \\
\hline$k_{\text {obs }} x y l e n o l$ orange assay & $1.5 \pm 0.1$ & $8.0 \pm 0.3$ & $6.6 \pm 0.1$ & $3.2 \pm 0.1$ \\
\hline$k_{\text {cat }}\left(\mathrm{s}^{-1}\right)$ & $2.6^{\mathrm{a}}$ & $11^{\mathrm{a}}$ & $7.1^{\mathrm{a}}$ & $2.9^{a}$ \\
\hline
\end{tabular}

a Data taken from (13).

Subsequently, we used the xylenol orange assay to evaluate the conversion of a set of twentyfour (potential) substrates by wild-type His-VAO and EUGO-His. The set of compounds contained various 4-(cyclo)alkylphenols, 4-hydroxybenzyl alcohols, 4-allylphenols and a 4hydroxybenzylic amine. In addition to these para-substituted phenols, 2-hydroxy- and 3hydroxybenzyl alcohol were included in the set of compounds in order to screen for variants that no longer have a strict specificity for para-substituted phenols. For a full list of the compounds and their structural formulae, see Table 3.

The rate of conversion of these compounds by His-VAO and EUGO-His at a substrate concentration of $2 \mathrm{mM}$ was determined by allowing the enzymes to react with the substrate for ten min and measuring the amount of hydrogen peroxide formed using the xylenol orange assay. Rates of the reactions of His-VAO and EUGO-His with the tested substrates, calculated by assuming that the formation of hydrogen peroxide in time is linear during the reaction period, are shown in Figure 2. For substrates where a $k_{\text {cat }}$ value has been determined for the non-His-tagged enzymes under similar experimental conditions, this value is shown for comparison. The reaction rates measured using the xylenol orange assay are generally in good agreement with previously obtained data regarding the substrate specificity of VAO and EUGO. For compounds for which the $k_{\text {cat }}$ had previously been determined and found to lie above the detection limit, the reaction rates measured with the xylenol orange assay typically lie close to the $k_{\text {cat }}$ values. This is to be expected, as the substrate concentration used for the xylenol orange assay $(2 \mathrm{mM})$ is at least ten times the $K_{\mathrm{m}}$ for all these compounds and as such the measurements were performed under saturating substrate concentrations. The only cases where no activity was observed with the xylenol orange assay despite the $k_{\text {cat }}$ lying above the detection limit were the oxidation of $4-n$-butylphenol (6) by His-VAO and 5 -indanol (20) by EUGO-His. The $k_{\text {cat }}$ for the oxidation of $4-n$-butylphenol by VAO $\left(1.2 \mathrm{~s}^{-1}\right)$ is only slightly higher than the detection limit of the assay $\left(0.83 \mathrm{~s}^{-1}\right)$ and therefore it is not too surprising that no activity was detected for its oxidation using the xylenol orange assay. However, the $k_{\text {cat }}$ for the 
Table 3. Compounds tested in the substrate specificity screening.

\begin{tabular}{|c|c|c|}
\hline Entry & $\begin{array}{l}\text { Compound name } \\
\text { (trivial name) }\end{array}$ & Structure \\
\hline 1 & $\begin{array}{l}\text { 4-methylphenol } \\
\text { (para-cresol) }\end{array}$ & \\
\hline 2 & $\begin{array}{l}\text { 2-methoxy-4-methylphenol } \\
\text { (para-creosol) }\end{array}$ & \\
\hline 3 & 4-ethylphenol & \\
\hline 4 & 4-n-propylphenol & \\
\hline 5 & 4-isopropyphenol & \\
\hline 6 & 4- $n$-butylphenol & \\
\hline 7 & 4-sec-butylphenol & \\
\hline 8 & 4-n-pentylphenol & \\
\hline 9 & 4- $n$-hexylphenol & \\
\hline 10 & 4-n-nonylphenol & \\
\hline 11 & 2-hydroxybenzyl alcohol & \\
\hline 12 & 3-hydroxybenzyl alcohol & \\
\hline 13 & 4-hydroxybenzyl alcohol & \\
\hline 14 & $\begin{array}{l}\text { 4-hydroxy-3-methoxybenzyl alcohol } \\
\text { (vanillyl alcohol) }\end{array}$ & \\
\hline 15 & 4-hydroxy-3,5-dimethoxybenzyl alcohol & \\
\hline 16 & 4-hydroxy-2-methoxybenzyl alcohol & \\
\hline 17 & $\begin{array}{l}\text { 4-allylphenol } \\
\text { (chavicol) }\end{array}$ & \\
\hline
\end{tabular}




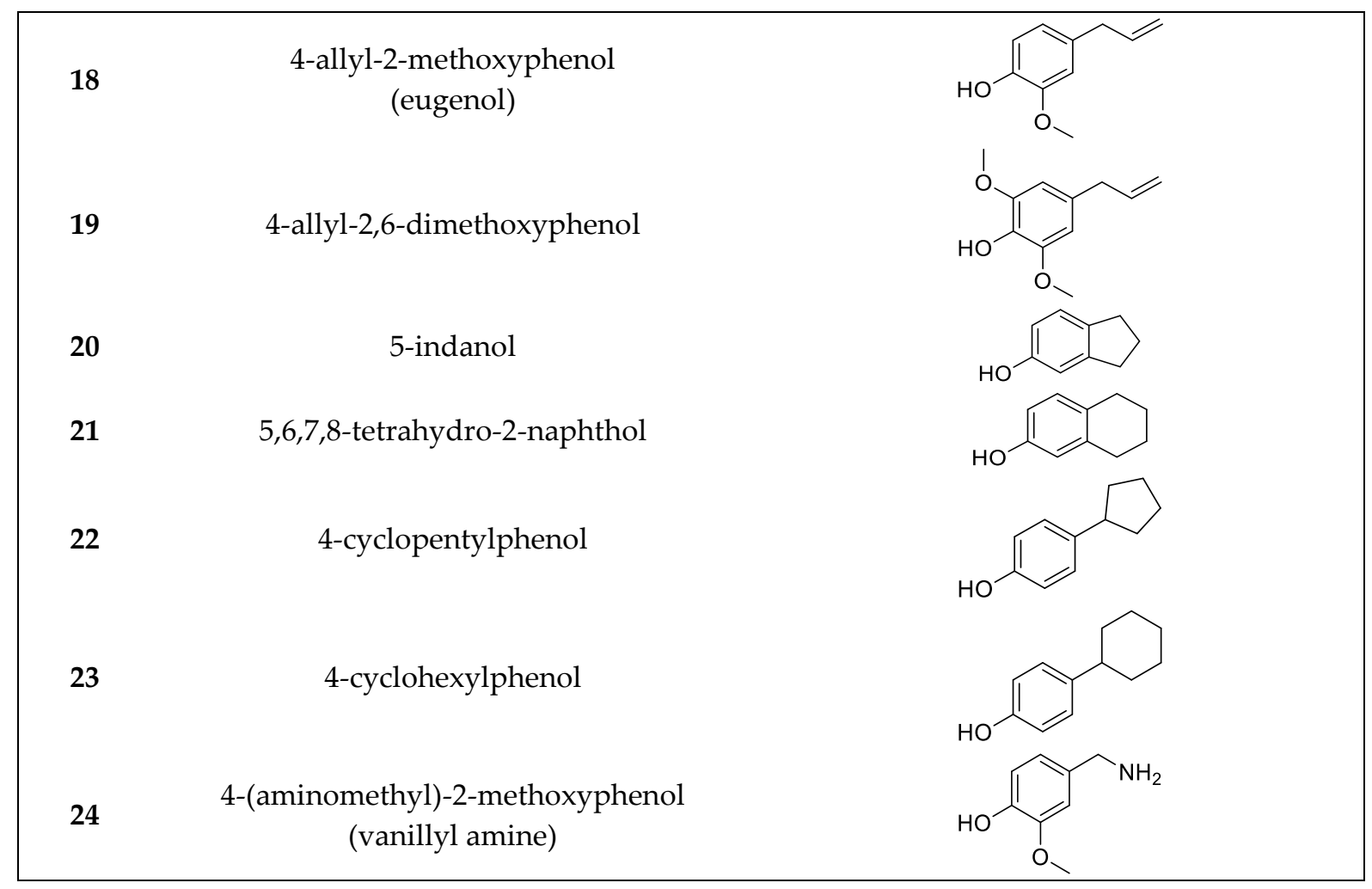

oxidation of 5-indanol by EUGO $\left(2.4 \mathrm{~s}^{-1}\right)$ lies well above the detection limit of the xylenol orange assay. To investigate this discrepancy further, the rate of oxidation of $2 \mathrm{mM} 5$-indanol by EUGO-His was determined by monitoring the consumption of oxygen during the reaction. The rate of oxygen consumption was found to be $0.064 \mathrm{~s}^{-1}$, significantly lower than the detection limit for the xylenol orange assay. This is in reasonable agreement with the rate of oxidation of $2 \mathrm{mM} 5$-indanol by EUGO determined by Nguyen et al. $\left(0.18 \mathrm{~s}^{-1}\right.$; the higher activity may be attributable to the addition of $10 \%$ DMSO as a cosolvent) (9). Therefore, at a substrate concentration of $2 \mathrm{mM}$, the rate of conversion of 5-indanol by EUGO-His is significantly lower than the detection limit of the xylenol orange assay. For all substrates where the previously determined $k_{\text {cat }}$ value is lower than the detection limit, no activity was observed using the xylenol orange assay.

Interestingly, our substrate specificity screening revealed that a number of compounds not previously described as substrates of VAO or EUGO are converted by the enzymes. Chavicol (17), which had previously been shown to be a substrate for VAO, was also converted by EUGO-His, though at a lower rate than by His-VAO. More surprisingly, both His-VAO and EUGO-His were active with 4-cyclopentylphenol (22), which had not previously been shown to be a substrate for either enzyme. Particularly with His-VAO, 4-cyclopentylphenol was quite efficiently converted, with the observed rate of $3.9 \mathrm{~s}^{-1}$ being higher than that observed for all other substrates except eugenol and chavicol. The ability of His-VAO and EUGO-His to convert 4-cyclopentylphenol was confirmed by allowing $1 \mu \mathrm{M}$ His-VAO or EUGO-His to react with $2 \mathrm{mM}$ 4-cyclopentylphenol at $25^{\circ} \mathrm{C}$ and analysing the reaction products by GC and GC/MS (Fig. S3+4). With both enzymes, the substrate was almost completely converted after $2 \mathrm{~h}(>99 \%$ conversion). The reaction yielded a single product, which was identified as 4-(1cyclopenten-1-yl)phenol by GC/MS and ${ }^{1} \mathrm{H}-\mathrm{NMR}$ (25, Scheme 2, Fig. S4+5). The rate of 


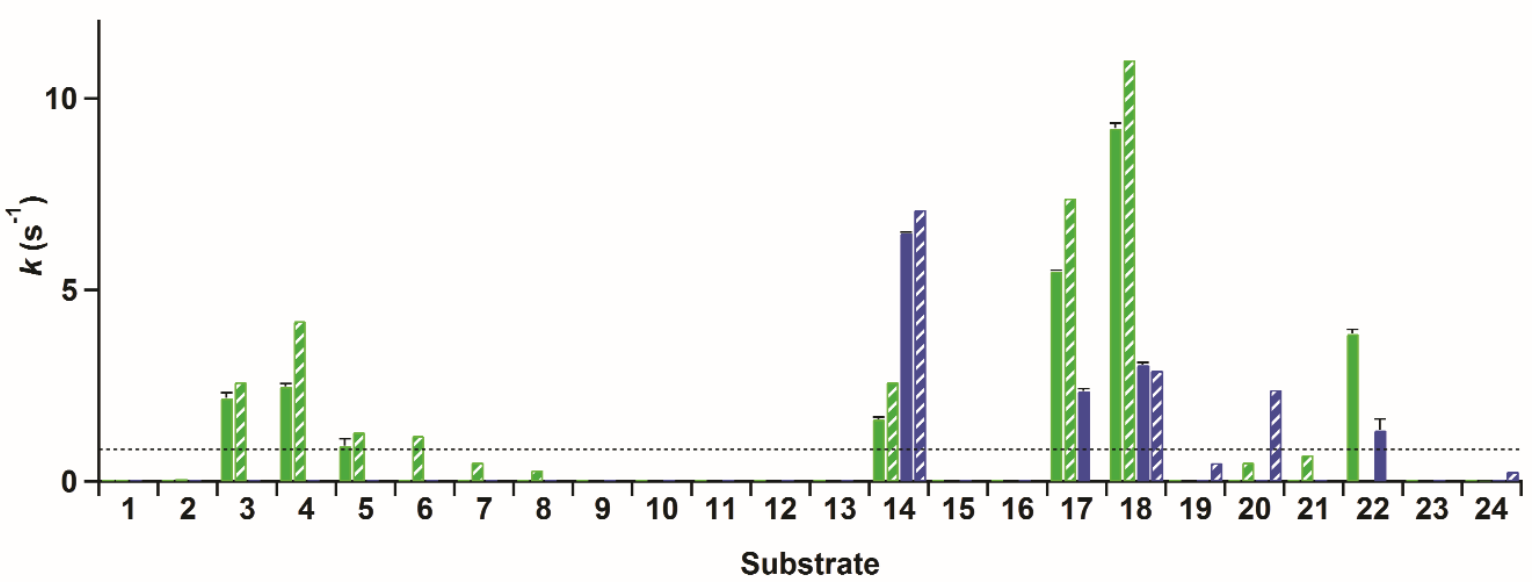

Figure 2: Rate of the reactions of His-VAO and EUGO-His with the compounds used in the substrate specificity screening in $50 \mathrm{mM}$ potassium phosphate buffer, $\mathrm{pH} 7.5$, at room temperature $\left(19-20^{\circ} \mathrm{C}\right)$ as determined using the xylenol orange assay. Solid bars give the reaction rates measured with the xylenol orange assay for His-VAO (green) and EUGO-His (blue). Error bars represent the standard deviation of duplicate measurements. Striped bars give $k_{\text {cat }}$ values measured previously by other methods with the non-His-tagged enzymes. These $k_{\text {cat }}$ values were measured under identical experimental conditions, with the exception of the temperature, which was $25^{\circ} \mathrm{C}$ instead of $19-20^{\circ} \mathrm{C}$. The striped horizontal line indicates the detection limit of the xylenol orange assay, which corresponds to a reaction rate of $0.83 \mathrm{~s}^{-1}$. The $k_{\text {cat }}$ values were from the following sources. VAO: compounds, 5-8, 20, 21 (11), 3, 14, 18 (13), 2, 24 (14), 1, 4 (33), 17 (34). EUGO: 20, 24 (4), 19 (9), 14, 18 (13). Numbering is according to Table 3.

oxidation of $2 \mathrm{mM}$ 4-cyclopentylphenol by His-VAO and EUGO-His was also determined by monitoring oxygen consumption during the reaction. This yielded rates of $3.7 \mathrm{~s}^{-1}$ for His-VAO and $1.7 \mathrm{~s}^{-1}$ for EUGO-His, in good agreement with the rates determined using the xylenol orange assay ( $3.9 \mathrm{~s}^{-1}$ and $1.3 \mathrm{~s}^{-1}$ for His-VAO and EUGO-His, respectively).

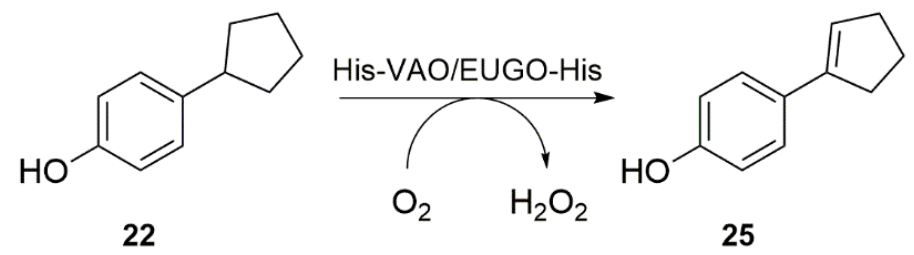

Scheme 2: Oxidation of 4-cyclopentylphenol (22) to 4-(1-cyclopenten-1-yl)phenol (25) by His-VAO or EUGO-His, as catalysed in $50 \mathrm{mM}$ potassium phosphate buffer, $\mathrm{pH} 7.5$, at $25^{\circ} \mathrm{C}$.

Taken together, these results demonstrate that the xylenol orange assay can be used to accurately measure the activity of His-VAO and EUGO-His towards a range of substrates and to identify hitherto unknown substrates of the enzymes. Therefore, it is a suitable assay for use in screening the substrate specificity of His-VAO and EUGO-His variants. Having established this, we set out to use the assay to screen a small library of His-VAO and EUGOHis variants for changes in their substrate specificity. 
To design the variants, we examined the active sites of the proteins to identify residues that differ between them and therefore may be responsible for the observed differences in substrate specificity. Both enzymes contain a solvent-inaccessible substrate binding pocket on the si-side of the FAD cofactor. This pocket is lined by 19 residues of which 12 are identical in both enzymes (Fig. 3). The conserved residues include all those known to be directly involved in catalysis, including Arg-504 (numbering of amino acids is as in non-His-tagged VAO), which is thought to stabilise the negative charge that develops at the $\mathrm{N} 1-\mathrm{C} 2=\mathrm{O} 2$ locus of FAD upon its reduction, Tyr-108 and Tyr-503, which stabilise the deprotonated form of phenolic substrates in the active site, Asp-170, which promotes flavin reduction by stabilising the reduced FAD cofactor through hydrogen bonding with the protonated N5 atom, and His-422, to which the FAD cofactor is covalently bound (8,34,35, Chapter 4$)$. The seven differing residues form a cluster on the side of the substrate opposite the flavin cofactor. Interestingly, the competitive inhibitor isoeugenol binds in a different orientation in each enzyme. In EUGO, it is flipped $180^{\circ}$ as compared to in VAO. This is likely due to the presence of Gly-392 in EUGO instead of the bulky Phe- 424 found at this position in VAO. Based on these observations, we hypothesised that differences in the cluster of differing residues may be responsible for the observed differences in substrate specificity between VAO and EUGO. To investigate this, we made seven His-VAO and seven EUGO-His variants, exchanging each of the differing residues for the amino acid that is found at this position in the other enzyme and set out to study their activity and substrate specificity using the xylenol orange assay (for a list of the residues and variants see Table 4, numbering as in the sequences of non-His-tagged VAO and EUGO).

\section{Substrate specificity screening of His-VAO and EUGO-His variants}

All fourteen variants were successfully expressed in E. coli and purified as described for the wild-type enzymes. A single variant, F424G His-VAO, did not contain any flavin after purification, as judged from the lack of a yellow colour and of the distinctive flavin absorption spectrum. Attempts to incorporate flavin into this variant by incubating it with FAD failed and it was not studied further. The other six His-VAO variants and all seven EUGO-His variants contained flavin as determined from their characteristic flavin absorption spectra (Fig. $\mathrm{S} 1+\mathrm{S} 2$ ). Upon precipitation of the proteins using trichloroacetic acid, a yellow pellet and colourless supernatant were obtained, demonstrating that the flavin cofactor is covalently bound to the protein. The absorption spectra of all seven EUGO-His variants had a similar shape to that of wild-type EUGO-His. A number of the His-VAO variants, most notably T457Q and C470L, displayed a somewhat altered flavin absorption spectrum, suggesting that in these variants the electronic environment of the flavin is slightly altered by the introduced mutations. The variants were screened for changes in their substrate specificity using the xylenol orange assay. The results of this screening assay are shown in Figure 4.

For His-VAO, all six remaining variants displayed activity with at least some of the tested compounds. Two of the variants, L316M and T459I, displayed substrate specificity profiles that were similar to that of the wild-type enzyme. The I468V and C470L variants displayed similar activity to the wild-type enzyme with the substrates vanillyl alcohol (14), chavicol (17) 
VAO

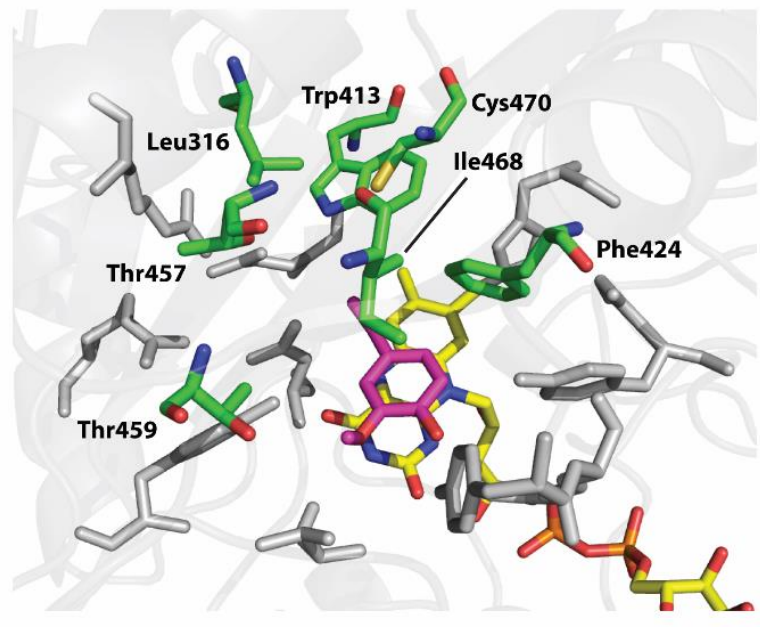

EUGO

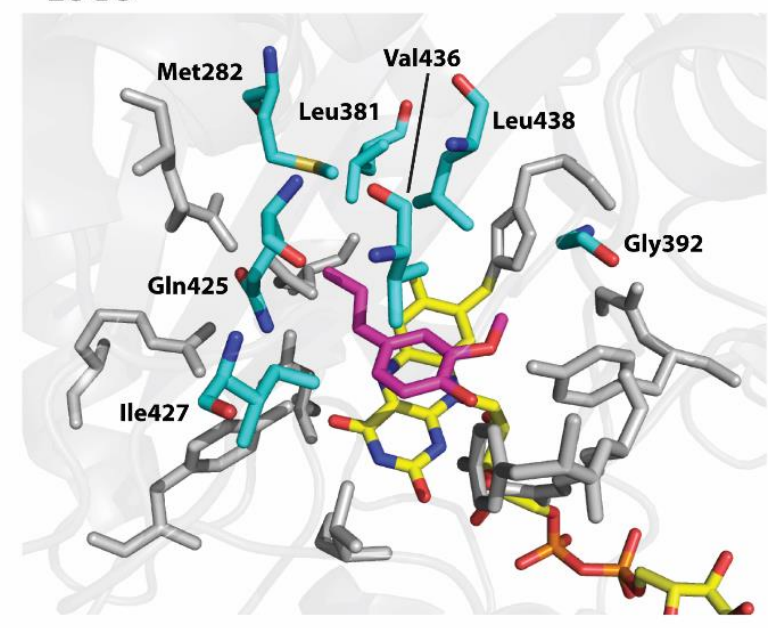

Figure 3: Structures of the substrate binding pockets of VAO and EUGO with the competitive inhibitor isoeugenol bound. Structures are shown in stick representation with residues that are identical in each protein shown in grey, residues that are different in each protein shown with green (VAO) or cyan (EUGO) carbon atoms, the FAD cofactor shown with yellow carbon atoms and isoeugenol shown with pink carbon atoms. Nitrogen atoms are shown in blue, oxygen atoms in red and phosphorus atoms in orange. The VAO and EUGO structures were downloaded from the PDB website (PDB IDs: 2VAO and 5FXD respectively). Figure was prepared using PyMOL (Schrödinger LLC, New York, NY, USA).

Table 4: List of residues forming part of the substrate binding pocket that are different in VAO and EUGO and of the His-VAO and EUGO-His variants that were made during this study. The residues are numbered according to their position in the non-His-tagged wild-type proteins.

\begin{tabular}{|ccccc|}
\hline Position & Residue in His-VAO & Residue in EUGO-His & $\begin{array}{c}\text { His-VAO } \\
\text { Variant }\end{array}$ & $\begin{array}{c}\text { EUGO-His } \\
\text { Variant }\end{array}$ \\
\hline 1 & Leu-316 & Met-282 & L316M & M282L \\
2 & Trp-413 & Leu-381 & W413L & L381W \\
3 & Phe-424 & Gly-392 & F424G & G392F \\
4 & Thr-457 & Gln-425 & T457Q & Q425T \\
5 & Thr-459 & Ile-427 & T459I & I427T \\
6 & Ile-468 & Val-436 & I468V & V436I \\
7 & Cys-470 & Leu-438 & C470L & L438C \\
\hline
\end{tabular}

and eugenol (18). However, these variants did not display any activity towards linear 4alkylphenols. The T457Q variant was the only variant to have significantly increased activity with vanillyl alcohol as a substrate, displaying a reaction rate that was approximately threefold higher than that of the wild-type enzyme. However, with all other substrates the T457Q variant displayed a decrease in activity. The only variant for which activity was detected with a compound for which no activity was detected for wild-type His-VAO was W413L. This variant displayed activity towards 4-cyclohexylphenol (23), a compound that so far had not been described as a substrate for VAO or EUGO. In addition to this novel reactivity, the W413L variant displayed activity towards 4-cyclopentylphenol (22), eugenol, chavicol and 4-isopropylphenol (5). No activity was detected with linear 4-alkylphenols or vanillyl alcohol. 


\section{His-VAO}
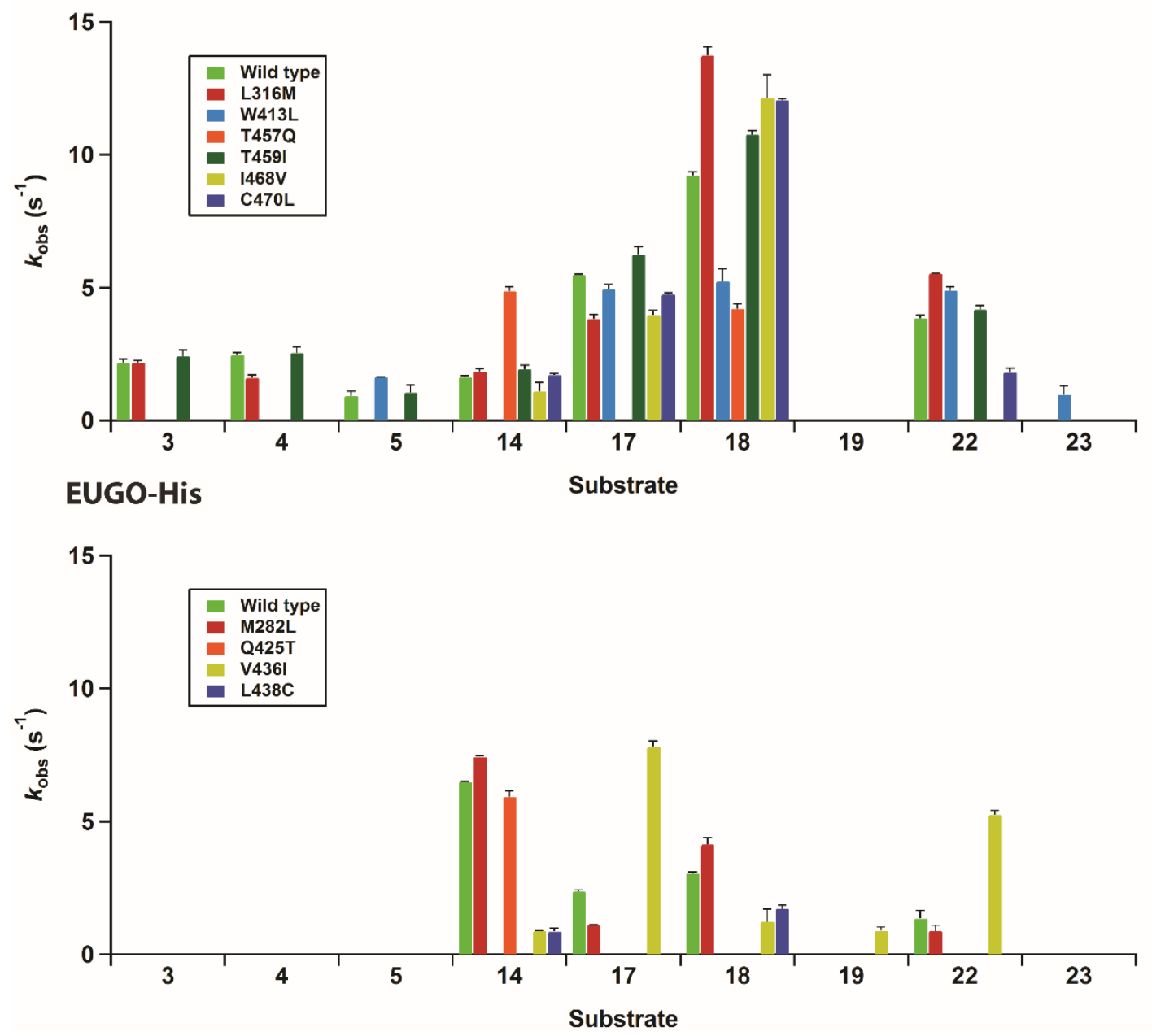

Figure 4: Rates of the reactions of His-VAO and EUGO-His variants with the compounds used in the substrate specificity screening in $50 \mathrm{mM}$ potassium phosphate buffer, $\mathrm{pH} 7.5$, at room temperature (19$20^{\circ} \mathrm{C}$ ) as determined using the xylenol orange assay. Error bars represent the standard deviations of duplicate measurements. For all compounds that are not included in the graphs, no variants gave a signal above the detection limit of the assay. The F424G His-VAO variant was not studied, as it did not contain any flavin cofactor. For the L381W, G392F and I427T EUGO-His variants, no response higher than the detection limit was measured for any compound.

To confirm that W413L His-VAO oxidises 4-cyclohexylphenol, $1 \mu \mathrm{M}$ enzyme was incubated with $2 \mathrm{mM} 4$-cyclohexylphenol for $4 \mathrm{~h}$ at $25^{\circ} \mathrm{C}$ and the reaction products were analysed by GC and GC/MS (Fig. S6+7). This revealed that 4-cyclohexylphenol is indeed converted by the enzyme yielding a single product, though the degree of conversion was only $27 \%$. GC/MS analysis revealed that the product displays a molecular ion at $m / z=174.1$, suggesting that it is formed by the dehydrogenation of the substrate. As VAO typically dehydrogenates the C $\alpha$ $\mathrm{C} \beta$ bond of alkyl groups, the product of 4-cyclohexylphenol oxidation is presumably 4-(1cyclohexen-1-yl)phenol. Similar experiments were performed using wild-type His-VAO and EUGO-His to evaluate whether they are also capable of converting 4-cyclohexylphenol. This revealed that after a $4 \mathrm{~h}$ incubation period, wild-type His-VAO had converted $51 \%$ of the substrate yielding the same product as observed with W413L His-VAO. In contrast, no 
conversion of 4-cyclohexylphenol by wild-type EUGO-His was observed under these reaction conditions.

The substrate specificity screening of the EUGO-His variants revealed that three of the seven variants, L381W, G392F and I427T, did not display measurable activity towards any of the tested compounds. One of the four remaining variants, M282L, displayed a substrate specificity profile that was similar to that of wild-type EUGO-His. Another variant, Q425T, displayed similar activity to the wild-type enzyme with vanillyl alcohol, but did not display measurable activity towards any other compounds. The L438C variant displayed lowered activity towards all substrates as compared to the wild-type enzyme. The V436I variant displayed lowered activity towards vanillyl alcohol and eugenol. However, this variant had significantly increased activity towards chavicol and 4-cyclopentylphenol and also displayed activity towards the dimethoxylated substrate 2,6-dimethoxy-4-allylphenol (19). This compound had previously been described to be a substrate for EUGO (9), but the $k_{\text {cat }}$ value for its oxidation $\left(0.49 \mathrm{~s}^{-1}\right)$ is lower than the detection limit of our assay, explaining the lack of activity detected for wild-type EUGO-His. No variants were identified that displayed activity towards compounds that had previously been identified as substrates for VAO, but not for EUGO, such as linear 4-alkylphenols.

In summary, the majority of the studied variants displayed similar or significantly reduced activity towards all tested compounds when compared to the corresponding wild-type enzyme. Two variants were identified that had significantly (more than twofold) increased activity towards one or more substrates.

\section{Discussion}

Here, we described the development of a method for the efficient screening of variants of VAO and EUGO for alterations in their substrate specificities. The method is based on a facile benchtop purification of His-tagged versions of the enzymes, followed by a substrate specificity screening using a xylenol orange assay. After demonstrating that this method allows accurate determination of reaction rates of the wild-type enzymes with a range of substrates, we used it to screen a library of fourteen His-VAO or EUGO-His variants towards a set of twenty-four (potential) substrates. The use of the xylenol orange assay, a spectrophotometric detection method for the oxidase product hydrogen peroxide, allows the simultaneous measurement of activity towards a range of substrates in a high-throughput fashion. This compares favourably to previous methods for measuring the activity of VAO and EUGO. These either relied on measuring the absorption of the product of the reaction, making the detection method dependent on the substrate used and on prior knowledge of the identity of the product, or on measuring the consumption of oxygen, which is not practical for high-throughput screening purposes. Although generic methods for the high-throughput screening of oxidase activity have been developed in the past, they typically rely on peroxidase-coupled activity assays, where a peroxidase uses the formed hydrogen peroxide to catalyse the production of a coloured product (36). This type of assay is not suitable for use with VAO or EUGO, as their phenolic substrates are typically also converted by peroxidases (32). 
Although the number of substrates and variants used in our screen is relatively small, the xylenol orange assay is highly scalable, particularly when robotic pipetting systems are available. In our case, the limiting factor for the number of variants that can be screened is the protein purification method, which relies on the use of gravity-flow Ni-NTA columns. However, we did demonstrate that this single purification step allowed us to obtain sufficiently pure His-VAO and EUGO-His variants. High-throughput methods for the production and purification of His-tagged proteins using E. coli expression systems have been described (37). Such a system could be used to achieve the purification of a large number of His-VAO or EUGO-His variants for use in the substrate specificity screening. Alternatively, cell free extracts of $E$. coli expressing the variants could be used for the assay. However, in our hands this gave poor results, most likely due to degradation of the formed hydrogen peroxide by catalase present in the E. coli extracts.

The substrate specificity profiling of wild-type His-VAO and EUGO-His led to the identification of 4-cyclopentylphenol as a substrate of the enzymes. Its conversion yielded 4(1-cyclopenten-1-yl)phenol as the sole product. This is in agreement with the previous finding that while VAO can catalyse both the hydroxylation and the dehydrogenation of 4alkylphenols, the dehydrogenation reaction is preferred when the alkyl side chain is relatively large (11). Our results also revealed that His-VAO and the W413L His-VAO variant catalyse the dehydrogenation of 4-cyclohexylphenol, presumably to 4-(1-cyclohexen-1-yl)phenol. However, this reaction was not catalysed by EUGO-His.

Although the substrate specificity profiling of fourteen His-VAO or EUGO-His variants did not lead us to a thorough understanding of the molecular determinants of the differing substrate specificities of the enzymes, it did give some interesting insights into the importance of certain non-catalytic active site residues for enzyme activity. One interesting finding was that the F424G His-VAO variant did not contain any FAD when purified from E. coli and attempts to incorporate FAD into the protein failed. Possibly, the extra flexibility conferred by the introduction of a glycine residue in this variant destabilises the structure of the active site, leading to impaired flavin binding. Three of the seven EUGO-His variants, L381W, G392F and I427T, did not display any activity in the substrate specificity profiling, despite containing covalently bound flavin. Presumably, the introduced mutations affect the structure of the active site in such a way that the substrate is no longer bound in an orientation that is productive for catalysis.

The I468V and C470L His-VAO variants also displayed interesting changes in substrate specificity, maintaining similar reaction rates to the wild-type enzyme with vanillyl alcohol, chavicol and eugenol, but displaying no activity towards linear 4-alkylphenols. Ile-468 and Cys-470 are positioned close together at the top of the substrate-binding pocket (Fig. 3). Possibly, the identity of these two residues is important for maintaining VAO's reactivity with linear 4-alkylphenols. As the ability to convert linear 4-alkylphenols is one of the main differences between VAO and EUGO in terms of substrate specificity, it would be interesting to investigate whether further mutagenesis at these positions could create a EUGO variant that is capable of catalysing this reaction. 
Only two variants that displayed significantly increased activity towards a compound for which activity was also detected with the wild-type enzymes were identified. T457Q His-VAO had a threefold increased reaction rate for the oxidation of vanillyl alcohol compared to wildtype His-VAO and I436V EUGO-His had three- and fourfold increased reaction rates for the oxidation of chavicol and 4-cyclopentylphenol respectively when compared to wild-type EUGO-His. It also displayed increased activity towards 4-allyl-2,6-dimethoxyphenol, though the magnitude of the increase could not be quantified as the reaction rate of wild-type EUGOHis with this compound is below the detection limit of the assay.

In summary, we developed an efficient method for screening variants of VAO and EUGO for changes in their substrate specificity consisting of the benchtop purification of His-tagged versions of the enzymes followed by substrate specificity screening using a xylenol orange assay. This method was employed for the substrate specificity profiling of the wild-type enzymes and of fourteen enzyme variants, leading to the identification of 4-cyclopentylphenol as a new substrate for both wild-type enzymes and of 4-cyclohexylphenol as a new substrate for wild-type His-VAO. Two variants, T457Q His-VAO and V436I EUGO-His were found to display significant increases in activity towards certain substrates. This method has the potential to be used to screen larger variant libraries in future, facilitating efforts to design VAO and EUGO variants for biocatalytic applications.

\section{Materials and methods}

\section{Materials}

4-Hydroxy-3,5-dimethoxybenzyl alcohol was from Alfa Aesar (Haverhill, MA, USA). 4-nButylphenol and 4-n-nonylphenol were from Lancaster Synthesis (Haverhill, MA, USA). Ferrous sulphate heptahydrate was from Merck (Burlington, MA, USA). 4-Allylphenol (chavicol) was from Quest International (Naarden, The Netherlands). 4-Allyl-2,6dimethoxyphenol, 4-allyl-2-methoxyphenol (eugenol), 4-(aminomethyl)-2-methoxyphenol hydrochloride (vanillyl amine), 4-sec-butylphenol, 4-cyclohexylphenol, 4-cyclopentylphenol, 4-ethylphenol, 4-n-hexylphenol, 2-hydroxybenzyl alcohol, 3-hydroxybenzyl alcohol, 4hydroxybenzyl alcohol, 4-hydroxy-2-methoxybenzyl alcohol, 4-hydroxy-3-methoxybenzyl alcohol (vanillyl alcohol), 5-indanol, 4-isopropylphenol, 2-methoxy-4-methylphenol (paracreosol), 4-methylphenol (para-cresol), 4- $n$-pentylphenol, 4- $n$-propylphenol, 5,6,7,8tetrahydronaphthol and xylenol orange tetrasodium salt were from Sigma-Aldrich (St. Louis, MO, USA). All other chemicals were from commercial sources and of the purest grade available. The pJ404-His-VAO and pBAD-EUGO-His plasmids were a kind gift from Prof. Dr. Marco Fraaije (University of Groningen).

\section{Site-directed mutagenesis}

Plasmids encoding the His-VAO and EUGO-His variants were created by linear wholeplasmid amplification from the pJ404-His-VAO and pBAD-EUGO-His plasmids respectively. The pJ404-His-VAO plasmid contains a version of the vaoA gene from $P$. simplicissimum that is codon-optimised for expression in E. coli behind the IPTG-inducible T5 promoter. The gene is extended with a sequence that encodes an N-terminal 6x-His-tag followed by a single 
glycine residue. The pBAD-EUGO-His plasmid contains the eugo gene from Rhodococcus jostii RHA1 with an extension that encodes the polypeptide sequence GKLGPEQKLISEEDLNSAVDHHHHHH at the C-terminus of the protein. The extension contains both a C-terminal 6x-His tag and a Myc-tag (EQKLISEEDL). This construct is placed behind the L-arabinose-inducible pBAD promoter. Constructs encoding variants of the enzymes were amplified from the corresponding plasmid using the mutagenic primers listed in Table 5. Following the amplification reaction, remaining template DNA was digested with DpnI and the mutated plasmids were transformed into DH5 $\alpha$ E. coli. Subsequently, plasmid DNA was isolated and the introduction of the correct mutations was confirmed by sequencing. The mutated plasmids were transformed into BL21 (for His-VAO) or TOP10 (for EUGO-His) E. coli for protein expression.

Table 5: List of primers used for site-directed mutagenesis in this study. Sites in italics indicate the position of the mutations introduced to achieve the amino acid substitution. Underlined sites indicate the position of mutations introduced to remove or introduce a restriction site to enable screening for the introduction of the correct mutations by restriction enzyme digestion (these are either the same mutations used for the amino acid substitution or silent mutations close to this site). The identity of the used restriction enzymes is given in the third column (+ indicates that a restriction site for this enzyme was introduced, - indicates that a restriction site for this enzyme was removed).

\begin{tabular}{|c|c|c|}
\hline Variant & Primer & $\begin{array}{l}\text { Restriction } \\
\quad \text { Site }\end{array}$ \\
\hline L316M His-VAO & ATCCGCCATATTCTGATGGATGCTGCAAGTGCTGGGTGACA & +PstI \\
\hline W413L His-VAO & 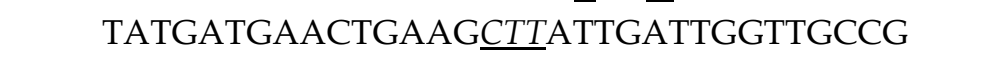 & +HindIII \\
\hline F424G His-VAO & $\begin{array}{c}\text { AATGGTGCCCACCTGGGTTTCAGCCCGATTGCGAAGGTGT } \\
\text { CTGGT }\end{array}$ & - PvuI \\
\hline T457Q His-VAO & $\begin{array}{c}\text { TGCCAGGAAGCAGGTCT } \underline{\text { AGACTTCATTGGTCAATTCACTG }} \\
\text { TTGGTATG }\end{array}$ & $+\mathrm{XbaI}$ \\
\hline T459I His-VAO & TTCATTGGTACGTTCATTGTTGGTATGCGTGAG & None \\
\hline I468V His-VAO & ATGCGTGAGATGCATCATGTTGTTTGCATCGTGTTT & + NsiI \\
\hline C470L His-VAO & ATGCGTGAGATGCATCATATTGTTTTAATCGTGTTTAACA & +NsiI \\
\hline M282L EUGO- & CTGCGCAACATCTTCTTGGACGCGGCCGCGGTG & None \\
\hline L381W EUGO- & CTGGACGAACTGCA $\underline{G T G G C T G G A C T G G G T G C C C ~}$ & + PstI \\
\hline G392F EUGO-His & AACGGCGGGCACATCTTTTTCTCÁACGGTGTCGGCGCCG & + AgeI \\
\hline Q425T EUGO- & AAGGACTACGCCGCGACATTCATCATCGGGCTC & None \\
\hline I427T EUGO-His & TACGCCGCGCAATTCACGATCGGGCTCCGCGAG & + PvuI \\
\hline V436I EUGO-His & CGCGAGATGCACCAC $\underline{A}$ T $\underline{A} T G C C T G T T C A T C T A C$ & + NdeI \\
\hline L438C EUGO-His & CTCCGCGAGATGCATCACGTGTGCTGCTTCATCTACGACA & $+\mathrm{NsiI}$ \\
\hline
\end{tabular}

Protein expression and purification

For the expression of His-VAO and its variants, BL21 E. coli containing the correct plasmid was grown in $100 \mathrm{~mL}$ LB medium (Duchefa Biochemie, Haarlem, The Netherlands) containing $100 \mu \mathrm{g} / \mathrm{mL}$ ampicillin at $37^{\circ} \mathrm{C}$ until the OD 600 was 0.6 . Subsequently, protein expression was induced by adding IPTG to a final concentration of $0.8 \mathrm{mM}$ and cells were grown overnight at $25{ }^{\circ} \mathrm{C}$. Next, cells were harvested by centrifugation $\left(4200 \mathrm{~g}, 15 \mathrm{~min}, 4{ }^{\circ} \mathrm{C}\right)$ and resuspended in $50 \mathrm{mM}$ potassium phosphate buffer, $\mathrm{pH} 7.5$, containing $500 \mathrm{mM} \mathrm{NaCl}, 5 \%$ glycerol (v/v), 
$0.5 \mathrm{mM} \mathrm{MgSO}_{4}$ and one cOmplete ${ }^{\mathrm{TM}}$ protease inhibitor pill (Roche, Basel, Switzerland) and 1 mg DnaseI (Roche) per $50 \mathrm{~mL}$. Cells were lysed by sonication using 6 cycles of 30 seconds at maximum power with an MSE sonication probe. Samples were cooled on ice during sonication. Following this, cell debris was removed by centrifugation $\left(39,000 \mathrm{~g}, 45 \mathrm{~min}, 4{ }^{\circ} \mathrm{C}\right)$ and the supernatant was loaded onto a gravity flow column containing $2 \mathrm{~mL}$ HisPur Ni-NTA resin (Thermo Scientific, Waltham, MA, USA) equilibrated in $50 \mathrm{mM}$ potassium phosphate buffer, $\mathrm{pH} 7.5$, containing $500 \mathrm{mM} \mathrm{NaCl}$ and $5 \%$ glycerol. The column was washed with this buffer until all unbound proteins had eluted as judged from the absorption of the flowthrough at $280 \mathrm{~nm}$. Subsequently, His-VAO was eluted using $50 \mathrm{mM}$ potassium phosphate buffer, pH 7.5, containing $500 \mathrm{mM} \mathrm{NaCl}, 5 \%$ glycerol (v/v) and $200 \mathrm{mM}$ imidazole. Fractions containing His-VAO were pooled and transferred to $50 \mathrm{mM}$ potassium phosphate buffer, $\mathrm{pH}$ 7.5, containing $150 \mathrm{mM} \mathrm{NaCl}$ and $10 \%$ glycerol by passing them over an Econo-Pac 10DG desalting column (Bio-Rad, Hercules, CA, USA) equilibrated in this buffer. This procedure typically yielded $1-3 \mathrm{mg}$ protein from $100 \mathrm{~mL}$ E. coli culture.

After purification of F424G His-VAO, the protein did not contain any flavin cofactor. In an attempt to obtain flavin-containing protein, the purified F424G His-VAO variant was incubated with $1 \mathrm{mM}$ FAD in $50 \mathrm{mM}$ potassium phosphate buffer, $\mathrm{pH} 7.5$, containing $75 \mathrm{mM}$ $\mathrm{NaCl}$ and $5 \%$ glycerol at room temperature for $4 \mathrm{~h}$. Subsequently, free FAD was removed by passing the mixture over an Econo-Pac 10DG desalting column (Bio-Rad) equilibrated in 50 $\mathrm{mM}$ potassium phosphate buffer, $\mathrm{pH} 7.5$, containing $150 \mathrm{mM} \mathrm{NaCl}$ and $10 \%$ glycerol. Fractions containing the protein were collected and analysed for flavin content by measuring their absorption spectra. This revealed that the protein did not contain any FAD. No further attempts were made to incorporate FAD into the F424G His-VAO variant.

For the expression of EUGO-His and its variants, TOP10 E. coli containing the correct plasmid was grown in $100 \mathrm{~mL}$ TB medium (Difco, Becton Dickinson, Franklin Lake, NJ, USA) containing $100 \mu \mathrm{g} / \mathrm{mL}$ ampicillin at $37{ }^{\circ} \mathrm{C}$ until the OD 600 was 0.6. Subsequently, protein expression was induced by adding L-arabinose to a final concentration of $0.02 \%(\mathrm{w} / \mathrm{v})$ and cells were grown overnight at $30^{\circ} \mathrm{C}$. Next, cells were harvested by centrifugation $(4200 \mathrm{~g}, 15$ min, $4{ }^{\circ} \mathrm{C}$ ) and resuspended in $50 \mathrm{mM}$ potassium phosphate buffer, $\mathrm{pH} 7.5$, containing $20 \mathrm{mM}$ imidazole, $0.5 \mathrm{mM} \mathrm{MgSO}_{4}$ and one cOmplete ${ }^{\mathrm{TM}}$ protease inhibitor pill (Roche) and $1 \mathrm{mg}$ DnaseI (Roche) per $50 \mathrm{~mL}$. Cells were lysed by sonication using 6 cycles of 30 seconds at maximum power with an MSE sonication probe. Samples were cooled on ice during sonication. Following this, cell debris was removed by centrifugation $\left(39,000 \mathrm{~g}, 45 \mathrm{~min}, 4{ }^{\circ} \mathrm{C}\right)$ and the supernatant was loaded onto a gravity flow column containing $2 \mathrm{~mL}$ HisPur Ni-NTA resin (Thermo Scientific) equilibrated in $50 \mathrm{mM}$ potassium phosphate buffer, $\mathrm{pH}$ 7.5, containing $20 \mathrm{mM}$ imidazole. The column was washed with this buffer until all unbound proteins had eluted as judged from the absorption of the flow-through at $280 \mathrm{~nm}$. Subsequently, EUGO-His was eluted using $50 \mathrm{mM}$ potassium phosphate buffer, $\mathrm{pH}$ 7.5, containing $500 \mathrm{mM}$ imidazole. Fractions containing EUGO-His were pooled and transferred into $50 \mathrm{mM}$ potassium phosphate buffer, $\mathrm{pH} 7.5$, by passing them over an Econo-Pac 10DG desalting column (Bio-Rad) equilibrated in this buffer. This procedure typically yielded 5-10 $\mathrm{mg}$ protein from $100 \mathrm{~mL}$. coli culture. 


\section{Analytical methods}

All experiments were performed in $50 \mathrm{mM}$ potassium phosphate buffer, $\mathrm{pH} 7.5$, unless indicated otherwise. Absorption spectra were recorded on a Hewlett Packard 8453 photodiode array spectrophotometer (Agilent Technologies, Santa Clara, CA, USA). Concentrations of His-VAO and its variants were determined using the extinction coefficient of non-His-tagged VAO at $439 \mathrm{~nm}\left[\varepsilon=12,500 \mathrm{M}^{-1} \mathrm{~cm}^{-1}(3)\right]$ and concentrations of EUGO-His and its variants were determined using the extinction coefficient of non-His-tagged EUGO at $441 \mathrm{~nm}\left[\varepsilon=14,200 \mathrm{M}^{-1} \mathrm{~cm}^{-1}\right.$ (4)]. For trichloroacetic acid precipitations, $10 \mu \mathrm{L} \mathrm{50 \% (w/v)}$ trichloroacetic acid solution was added to $90 \mu \mathrm{L} 11 \mu \mathrm{M}$ enzyme solution in $50 \mathrm{mM}$ potassium phosphate buffer, $\mathrm{pH} 7.5$, (EUGO-His and variants) or $50 \mathrm{mM}$ potassium phosphate buffer, $\mathrm{pH} 7.5$, containing $150 \mathrm{mM} \mathrm{NaCl}$ and $10 \%$ glycerol (His-VAO and variants) to yield final concentrations of $5 \%(\mathrm{w} / \mathrm{v})$ trichloroacetic acid and $10 \mu \mathrm{M}$ enzyme. Mixtures were incubated on ice for $30 \mathrm{~min}$, after which the precipitate was pelleted by centrifugation $(21,000 \mathrm{~g}, 15 \mathrm{~min}$, $4{ }^{\circ} \mathrm{C}$ ). The presence of flavin in the pellet or supernatant was judged from their colour and fluorescence upon irradiating them with UV light. Steady-state kinetic parameters for the oxidation of vanillyl alcohol to vanillin by His-VAO and EUGO-His were determined by following the absorption of the product at $340 \mathrm{~nm}\left(\varepsilon=14,000 \mathrm{M}^{-1} \mathrm{~cm}^{-1}\right)$ and fitting the obtained reaction rates to the Michaelis-Menten equation using Igor Pro v. 6.10A (Wavemetrics, Lake Oswego, OR, USA). Oxygen consumption measurements were performed using a Hansatech Oxytherm system (Hansatech Instruments, King's Lynn, UK).

\section{Enzymatic reactions for the xylenol orange assay}

Enzymatic reactions were performed in 96-wells plates. Substrate solution (180 $\mu \mathrm{L})$ was added to $20 \mu \mathrm{L}$ enzyme solution to give a reaction mixture containing $20 \mathrm{nM}$ enzyme and $2 \mathrm{mM}$ substrate in $50 \mathrm{mM}$ potassium phosphate buffer, $\mathrm{pH}$ 7.5. For measurements where the oxidation of eugenol or vanillyl alcohol was followed in time, the reaction mixtures were incubated for the desired time (ranging from 1-20 min) at room temperature (19-20 ${ }^{\circ} \mathrm{C}$ ), after which $20 \mu \mathrm{L}$ of the reaction mixture was removed and the hydrogen peroxide concentration was determined using the xylenol orange assay as described below. Reaction rates were determined by fitting a curve to the linear range of the data using Igor Pro. For substrate specificity screening, the reaction mixtures were incubated for $10 \mathrm{~min}$ at room temperature $\left(19-20^{\circ} \mathrm{C}\right)$, after which a $20 \mu \mathrm{L}$ sample was taken and the hydrogen peroxide concentration was determined using the xylenol orange assay as described below. Three of the tested compounds, 4- $n$-nonylphenol, 4-hydroxybenzyl alcohol and 4-cyclohexylphenol, were poorly soluble in water at the used concentration and therefore were added to the reaction mixtures as (partial) suspensions. Reaction rates were estimated by assuming that there is a linear increase in hydrogen peroxide concentration during the reaction time.

\section{Xylenol orange assay}

$20 \mu \mathrm{L}$ of the sample to be analysed was added to $180 \mu \mathrm{L}$ xylenol orange assay mix in a 96wells plate. This yielded a final assay mixture containing $100 \mu \mathrm{M}$ xylenol orange, $250 \mu \mathrm{M}$ $\mathrm{FeSO}_{4}, 25 \mathrm{mM} \mathrm{H}_{2} \mathrm{SO}_{4}$ and the tenfold diluted analyte solution. This mixture was incubated in the dark at room temperature $\left(19-20^{\circ} \mathrm{C}\right)$ for $30 \mathrm{~min}$, after which the absorption at $560 \mathrm{~nm}$ was 
measured using an xMark microplate spectrophotometer (Bio-Rad). A calibration curve of hydrogen peroxide solutions with known concentrations in the range 10-200 $\mu \mathrm{M}$ (concentration prior to addition to the xylenol orange assay mixture) was used to convert the measured absorbance values to the hydrogen peroxide concentration in the analyte solution. To ensure that the measured hydrogen peroxide concentrations reflect actual hydrogen peroxide formed during the enzymatic reaction, a detection limit of $10 \mu \mathrm{M}$ hydrogen peroxide, which corresponds to the lowest point of the calibration curve, was used. All samples where the measured hydrogen peroxide concentration was lower than this were deemed to have had no significant formation of hydrogen peroxide during the reaction period.

Conversion of 4-cyclopentylphenol and 4-cyclohexylphenol and identification of the reaction products

For GC and GC/MS analysis, reaction mixtures $(100 \mu \mathrm{L})$ containing $2 \mathrm{mM}$ substrate and $1 \mu \mathrm{M}$ enzyme in $50 \mathrm{mM}$ potassium phosphate buffer, $\mathrm{pH} 7.5$, (EUGO-His) or $50 \mathrm{mM}$ potassium phosphate buffer, $\mathrm{pH} 7.5$, containing $30 \mathrm{mM} \mathrm{NaCl}$ and $2 \%$ (w/v) glycerol (His-VAO), were incubated for $2 \mathrm{~h}$ (4-cyclopentylphenol) or $4 \mathrm{~h}$ (4-cyclohexylphenol) at $25^{\circ} \mathrm{C}$ under shaking (500 rpm). Subsequently, the reactions were stopped by extracting the reaction mixtures twice with $100 \mu \mathrm{L}$ EtOAc. The EtOAc layers were combined and dried over $\mathrm{Na}_{2} \mathrm{SO}_{4}$. Reaction products were analysed by GC and GC/MS. GC was performed on an Agilent 6890 GC system equipped with an Agilent 7673 injector using a $60 \mathrm{~m} \times 0.25 \mathrm{~mm} \times 0.25 \mu \mathrm{m}$ DB-1 column (Agilent Technologies). The injector temperature was $220^{\circ} \mathrm{C}$ and the FID temperature was $250^{\circ} \mathrm{C}$. The split ratio was 10:1. Samples from reactions with 4-cyclopentylphenol were run at a flow rate of $1.7 \mathrm{~mL} / \mathrm{min}$ using the following temperature programme: $100{ }^{\circ} \mathrm{C}$ for $5 \mathrm{~min}$, followed by a temperature gradient to $230{ }^{\circ} \mathrm{C}$ with an increment of $10{ }^{\circ} \mathrm{C} / \mathrm{min}$, followed by 3 $\min$ at $230^{\circ} \mathrm{C}$. Samples from reactions with 4-cyclohexylphenol were run at a flow rate of 1.7 $\mathrm{mL} / \mathrm{min}$ using the following temperature programme: $100{ }^{\circ} \mathrm{C}$ for $4 \mathrm{~min}$, followed by a temperature gradient to $160^{\circ} \mathrm{C}$ with an increment of $10{ }^{\circ} \mathrm{C} / \mathrm{min}$, followed by $10 \mathrm{~min}$ at $160{ }^{\circ} \mathrm{C}$, followed by a temperature gradient to $230{ }^{\circ} \mathrm{C}$ with an increment of $10{ }^{\circ} \mathrm{C} / \mathrm{min}$, followed by 3 min at $230{ }^{\circ} \mathrm{C}$. Reported conversion percentages are average values from duplicate experiments. GC/MS was performed on an Agilent 6890 GC system equipped with an Agilent 7975C MS detector and an Agilent 7683B injector using a $30 \mathrm{~m} \times 0.25 \mathrm{~mm} \times 0.25 \mu \mathrm{m} \mathrm{HP}-5 \mathrm{MS}$ column (Agilent Technologies). The injector temperature was $275^{\circ} \mathrm{C}$. The split ratio was 100:1. Samples were run at a flow rate of $1.1 \mathrm{~mL} / \mathrm{min}$ using the following programme: $50{ }^{\circ} \mathrm{C}$ for 3 min, followed by a temperature gradient to $100{ }^{\circ} \mathrm{C}$ with an increment of $5{ }^{\circ} \mathrm{C} / \mathrm{min}$, followed by a temperature gradient to $250^{\circ} \mathrm{C}$ with an increment of $10{ }^{\circ} \mathrm{C} / \mathrm{min}$, followed by $3 \mathrm{~min}$ at $250{ }^{\circ} \mathrm{C}$.

For ${ }^{1} \mathrm{H}-\mathrm{NMR}$ analysis, a reaction mixture $(25 \mathrm{~mL})$ containing $2.5 \mathrm{mM} 4$-cyclopentylphenol and $0.5 \mu \mathrm{M}$ EUGO-His was incubated in $50 \mathrm{mM}$ potassium phosphate buffer, $\mathrm{pH}, 7.5$, at $25^{\circ} \mathrm{C}$ for $16 \mathrm{~h}$ under shaking $(500 \mathrm{rpm})$. Following this, reaction products were extracted twice with 25 mL EtOAc. The EtOAc layers were combined and dried over $\mathrm{Na}_{2} \mathrm{SO}_{4}$. Subsequently, EtOAc was removed by evaporation and the remaining solid was dissolved in $\mathrm{CDCl}_{3} .{ }^{1} \mathrm{H}-\mathrm{NMR}$ spectra were recorded on an Avance III $400 \mathrm{MHz}$ NMR spectrometer (Bruker, Billerica, MA, USA) at $400 \mathrm{MHz}$. This revealed that the obtained product was a mixture of $40 \% 4$ cyclopentylphenol and 60\% 4-(1-cyclopenten-1-yl)phenol. 
4-cyclopentylphenol. ${ }^{1} \mathrm{H}-\mathrm{NMR}\left(400 \mathrm{MHz}, \mathrm{CDCl}_{3}\right) \delta$ (ppm): $7.12(\mathrm{ap} \mathrm{d}, J=8.5 \mathrm{~Hz}, 2 \mathrm{H}), 6.77$ (ap d, $J=8.5 \mathrm{~Hz}, 2 \mathrm{H}), 4.66(\mathrm{~s}, 1 \mathrm{H}), 2.93(\mathrm{~m}, 1 \mathrm{H}), 2.14-1.96(\mathrm{~m}, 2 \mathrm{H}), 1.87-1.72(\mathrm{~m}, 2 \mathrm{H}), 1.75-1.59(\mathrm{~m}$, $2 \mathrm{H}), 1.61-1.46(\mathrm{~m}, 2 \mathrm{H})$.

4-(1-cyclopenten-1-yl)phenol. ${ }^{1} \mathrm{H}-\mathrm{NMR}\left(400 \mathrm{MHz}, \mathrm{CDCl}_{3}\right) \delta(\mathrm{ppm}): 7.33(\mathrm{ap} \mathrm{d}, J=8.6 \mathrm{~Hz}, 2 \mathrm{H})$, 6.78 (ap d, $J=8.9 \mathrm{~Hz}, 2 \mathrm{H}), 6.04(\mathrm{~m}, 1 \mathrm{H}), 4.80$ (br s, 1H), 2.73-2.56 (m, 2H), 2.56-2.45 (m, 2H), $2.09-1.96(\mathrm{~m}, 2 \mathrm{H})$.

\section{Acknowledgments}

We thank Sigrid Berglund for technical assistance and Prof. Dr. Marco Fraaije and Mohamed Habib (both from University of Groningen, The Netherlands) for providing the pJ404-HisVAO and pBAD-EUGO-His plasmids and expression and purification protocols for His-VAO and EUGO-His. This research was supported by the European Union through the INDOX project (FP7-KBBE-2013-7-613549).

\section{References}

1. Ragauskas, A. J., Beckham, G. T., Biddy, M. J., Chandra, R., Chen, F., Davis, M. F., Davison, B. H., Dixon, R. A., Gilna, P., Keller, M., Langan, P., Naskar, A. K., Saddler, J. N., Tschaplinski, T. J., Tuskan, G. A., and Wyman, C. E. (2014) Lignin valorization: Improving lignin processing in the biorefinery. Science. 344, 1246843

2. Rinaldi, R., Jastrzebski, R., Clough, M. T., Ralph, J., Kennema, M., Bruijnincx, P. C. A., and Weckhuysen, B. M. (2016) Paving the way for lignin valorisation: Recent advances in bioengineering, biorefining and catalysis. Angew. Chem. Int. Ed. 55, 8164-8215

3. de Jong, E., van Berkel, W. J. H., van der Zwan, R. P., and de Bont, J. A. M. (1992) Purification and characterization of vanillyl-alcohol oxidase from Penicillium simplicissimum: A novel aromatic alcohol oxidase containing covalently bound FAD. Eur. J. Biochem. 208, 651-657

4. Jin, J., Mazon, H., van den Heuvel, R. H. H., Janssen, D. B., and Fraaije, M. W. (2007) Discovery of a eugenol oxidase from Rhodococcus sp. strain RHA1. FEBS J. 274, 2311-2321

5. Levasseur, A., Drula, E., Lombard, V., Coutinho, P. M., and Henrissat, B. (2013) Expansion of the enzymatic repertoire of the CAZy database to integrate auxiliary redox enzymes. Biotechnol. Biofuels. 6, 41

6. Fraaije, M. W., van Berkel, W. J. H., Benen, J. A. E., Visser, J., and Mattevi, A. (1998) A novel oxidoreductase family sharing a conserved FAD-binding domain. Trends Biochem. Sci. 23, 206207

7. Ewing, T. A., Fraaije, M. W., Mattevi, A., and van Berkel, W. J. H. (2017) The VAO/PCMH flavoprotein family. Arch. Biochem. Biophys. 632, 104-117

8. Mattevi, A., Fraaije, M. W., Mozzarelli, A., Olivi, L., Coda, A., and van Berkel, W. J. H. (1997) Crystal structures and inhibitor binding in the octameric flavoenzyme vanillyl-alcohol oxidase: The shape of the active-site cavity controls substrate specificity. Structure. 5, 907-920

9. Nguyen, Q.-T., de Gonzalo, G., Binda, C., Rioz-Martínez, A., Mattevi, A., and Fraaije, M. W. (2016) Biocatalytic properties and structural analysis of eugenol oxidase from Rhodococcus jostii RHA1: A versatile oxidative biocatalyst. ChemBioChem. 17, 1359-1366

10. Fraaije, M. W., Veeger, C., and van Berkel, W. J. H. (1995) Substrate specificity of flavindependent vanillyl-alcohol oxidase from Penicillium simplicissimum: Evidence for the production of 4-hydroxycinnamyl alcohols from 4-allylphenols. Eur. J. Biochem. 234, 271-277

11. van den Heuvel, R. H. H., Fraaije, M. W., Laane, C., and van Berkel, W. J. H. (1998) Regio- and stereospecific conversion of 4-alkylphenols by the covalent flavoprotein vanillyl-alcohol oxidase. J. Bacteriol. 180, 5646-5651 
12. Fraaije, M. W., Mattevi, A., and van Berkel, W. J. H. (1997) Mercuration of vanillyl-alcohol oxidase from Penicillium simplicissimum generates inactive dimers. FEBS Lett. 402, 33-35

13. Ewing, T. A., Gygli, G., and van Berkel, W. J. H. (2016) A single loop is essential for the octamerization of vanillyl alcohol oxidase. FEBS J. 283, 2546-2559

14. van den Heuvel, R. H. H., Fraaije, M. W., Laane, C., and van Berkel, W. J. H. (2001) Enzymatic synthesis of vanillin. J. Agric. Food Chem. 49, 2954-2958

15. Drijfhout, F. P., Fraaije, M. W., Jongejan, H., van Berkel, W. J. H., and Franssen, M. C. R. (1998) Enantioselective hydroxylation of 4-alkylphenols by vanillyl alcohol oxidase. Biotechnol. Bioeng. 59, 171-177

16. Ricklefs, E., Girhard, M., Koschorreck, K., Smit, M. S., and Urlacher, V. B. (2015) Two-step onepot synthesis of pinoresinol from eugenol in an enzymatic cascade. Chem CatChem. 7, 1857-1864

17. Ricklefs, E., Girhard, M., and Urlacher, V. B. (2016) Three-steps in one-pot: Whole-cell biocatalytic synthesis of enantiopure (+)- and (-)-pinoresinol via kinetic resolution. Microb. Cell Fact. 15, 78

18. Habib, M. H. M., Deuss, P. J., Lončar, N., Trajkovic, M., and Fraaije, M. W. (2017) A biocatalytic one-pot approach for the preparation of lignin oligomers using an oxidase/peroxidase cascade enzyme system. Adv. Synth. Catal. 359, 3354-3361

19. Overhage, J., Steinbüchel, A., and Priefert, H. (2003) Highly efficient biotransformation of eugenol to ferulic acid and further conversion to vanillin in recombinant strains of Escherichia coli. Appl. Environ. Microbiol. 69, 6569-6576

20. Overhage, J., Steinbüchel, A., and Priefert, H. (2006) Harnessing eugenol as a substrate for production of aromatic compounds with recombinant strains of Amycolatopsis sp. HR167. J. Biotechnol. 125, 369-376

21. Plaggenborg, R., Overhage, J., Loos, A., Archer, J. A. C., Lessard, P., Sinskey, A. J., Steinbüchel, A., and Priefert, H. (2006) Potential of Rhodococcus strains for biotechnological vanillin production from ferulic acid and eugenol. Appl. Microbiol. Biotechnol. 72, 745-755

22. Zucca, J., Lambert, F., Mane, J., Ness, F., and Aigle, M. (2013) System for producing aromatic molecules by bioconversion, United States Patent. 8,404,472

23. Lambert, F., Zucca, J., Ness, F., and Aigle, M. (2014) Production of ferulic acid and coniferyl alcohol by conversion of eugenol using a recombinant strain of Saccharomyces cerevisiae. Flavour Fragr. J. 29, 14-21

24. Zhou, R., and Xiaodan, Y. (2015) Methods of making vanillin via the microbial fermentation of ferulic acid from eugenol using a plant dehydrogenase. Patent application. WO/2015/066722

25. Gupta, B. L. (1973) Microdetermination techniques for $\mathrm{H}_{2} \mathrm{O}_{2}$ in irradiated solutions. Microchem. J. 18, 363-374

26. Gay, C., Collins, J., and Gebicki, J. M. (1999) Hydroperoxide assay with the ferric-xylenol orange complex. Anal. Biochem. 273, 149-155

27. Nourooz-Zadeh, J., Tajaddini-Sarmadi, J., and Wolff, S. P. (1994) Measurement of plasma hydroperoxide concentrations by the ferrous oxidation-xylenol orange assay in conjunction with triphenylphosphine. Anal. Biochem. 220, 403-409

28. Waslidge, N. B., and Hayes, D. J. (1995) A colorimetric method for the determination of lipoxygenase activity suitable for use in a high throughput assay format. Anal. Biochem. 231, 354358

29. Gay, C. A., and Gebicki, J. M. (2003) Measurement of protein and lipid hydroperoxides in biological systems by the ferric-xylenol orange method. Anal. Biochem. 315, 29-35

30. Yu, Z., Wang, J., Zhou, N., Zhao, C., and Qiu, J. (2013) A highly sensitive method for quantitative determination of L-amino acid oxidase activity based on the visualization of ferric-xylenol orange formation. PLoS One. 8, e82483

31. Jasti, L. S., Dola, S. R., Fadnavis, N. W., Addepally, U., Daniels, S., and Ponrathnam, S. (2014) Co-immobilized glucose oxidase and $\beta$-galactosidase on bovine serum albumin coated allyl glycidyl ether (AGE)-ethylene glycol dimethacrylate (EGDM) copolymer as a biosensor for 
lactose determination in milk. Enzyme Microb. Technol. 64-65, 67-73

32. BRENDA enzyme database EC 1.11.1.7: peroxidases (https://www.brendaenzymes.org/enzyme.php?ecno=1.11.1.7), Accessed on 11 December 2017

33. Fraaije, M. W., van den Heuvel, R. H. H., Roelofs, J. C. A. A., and van Berkel, W. J. H. (1998) Kinetic mechanism of vanillyl-alcohol oxidase with short-chain 4-alkylphenols. Eur. J. Biochem. 253, 712-719

34. Ewing, T. A., Nguyen, Q.-T., Allan, R. C., Gygli, G., Romero, E., Binda, C., Fraaije, M. W., Mattevi, A., and van Berkel, W. J. H. (2017) Two tyrosine residues, Tyr-108 and Tyr-503, are responsible for the deprotonation of phenolic substrates in vanillyl-alcohol oxidase. J. Biol. Chem. 292, 1466814679

35. van den Heuvel, R. H. H., Fraaije, M. W., Mattevi, A., and van Berkel, W. J. H. (2000) Asp-170 is crucial for the redox properties of vanillyl-alcohol oxidase. J. Biol. Chem. 275, 14799-14808

36. Holt, A., and Palcic, M. M. (2006) A peroxidase-coupled continuous absorbance plate-reader assay for flavin monoamine oxidases, copper-containing amine oxidases and related enzymes. Nat. Protoc. 1, 2498-2505

37. Scheich, C., Sievert, V., and Büssow, K. (2003) An automated method for high-throughput protein purification applied to a comparison of His-tag and GST-tag affinity chromatography. BMC Biotechnol. 3, 12

\section{Abbreviations}

EUGO(-His): (His-tagged) eugenol oxidase, FAD: flavin adenine dinucleotide, (His-)VAO: (His-tagged) vanillyl alcohol oxidase 


\section{Supplementary Figures}
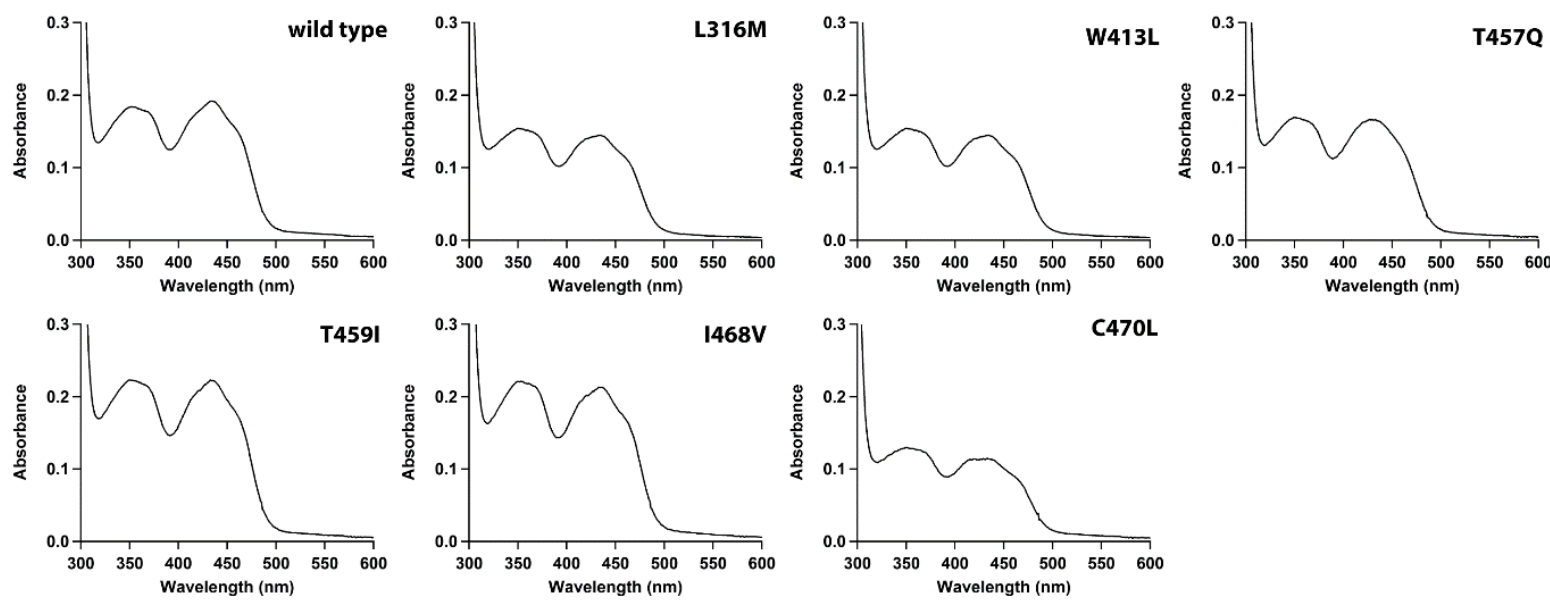

Figure S1: Absorption spectra of His-VAO variants recorded in $50 \mathrm{mM}$ potassium phosphate buffer, $\mathrm{pH} 7.5$, at $25^{\circ} \mathrm{C}$.
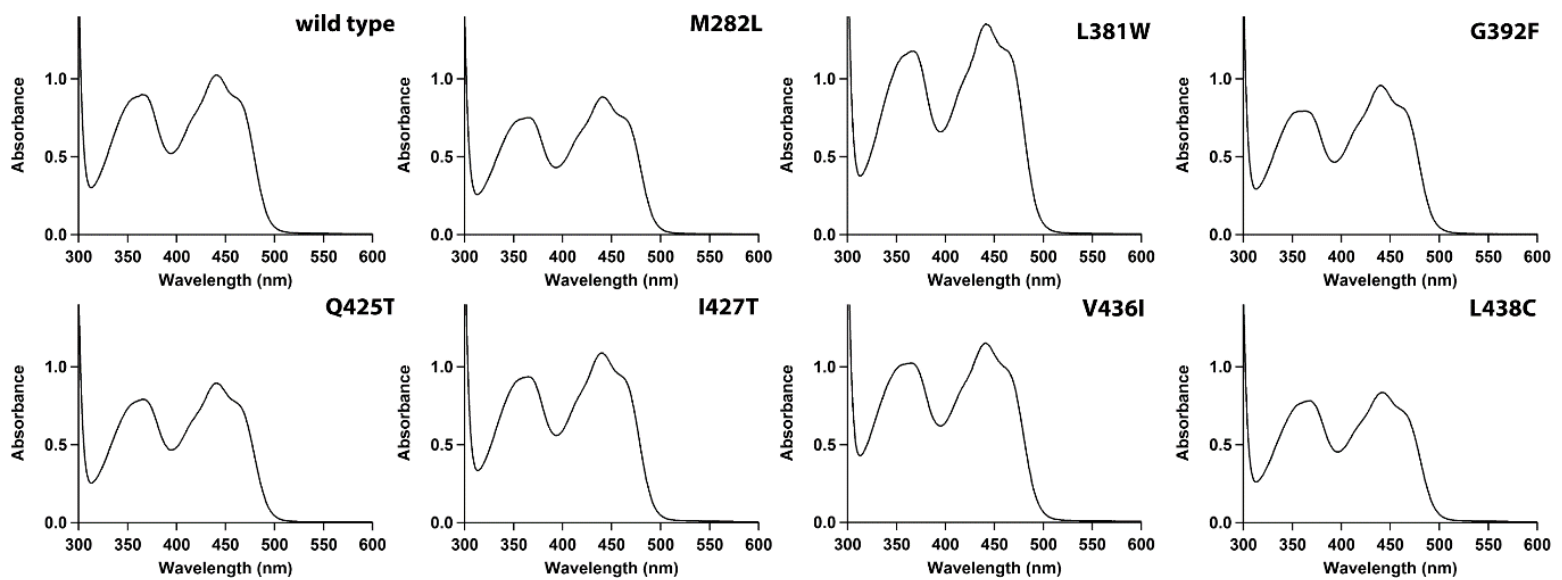

Figure S2: Absorption spectra of EUGO-His variants recorded in $50 \mathrm{mM}$ potassium phosphate buffer, $\mathrm{pH} 7.5$, at $25^{\circ} \mathrm{C}$. 

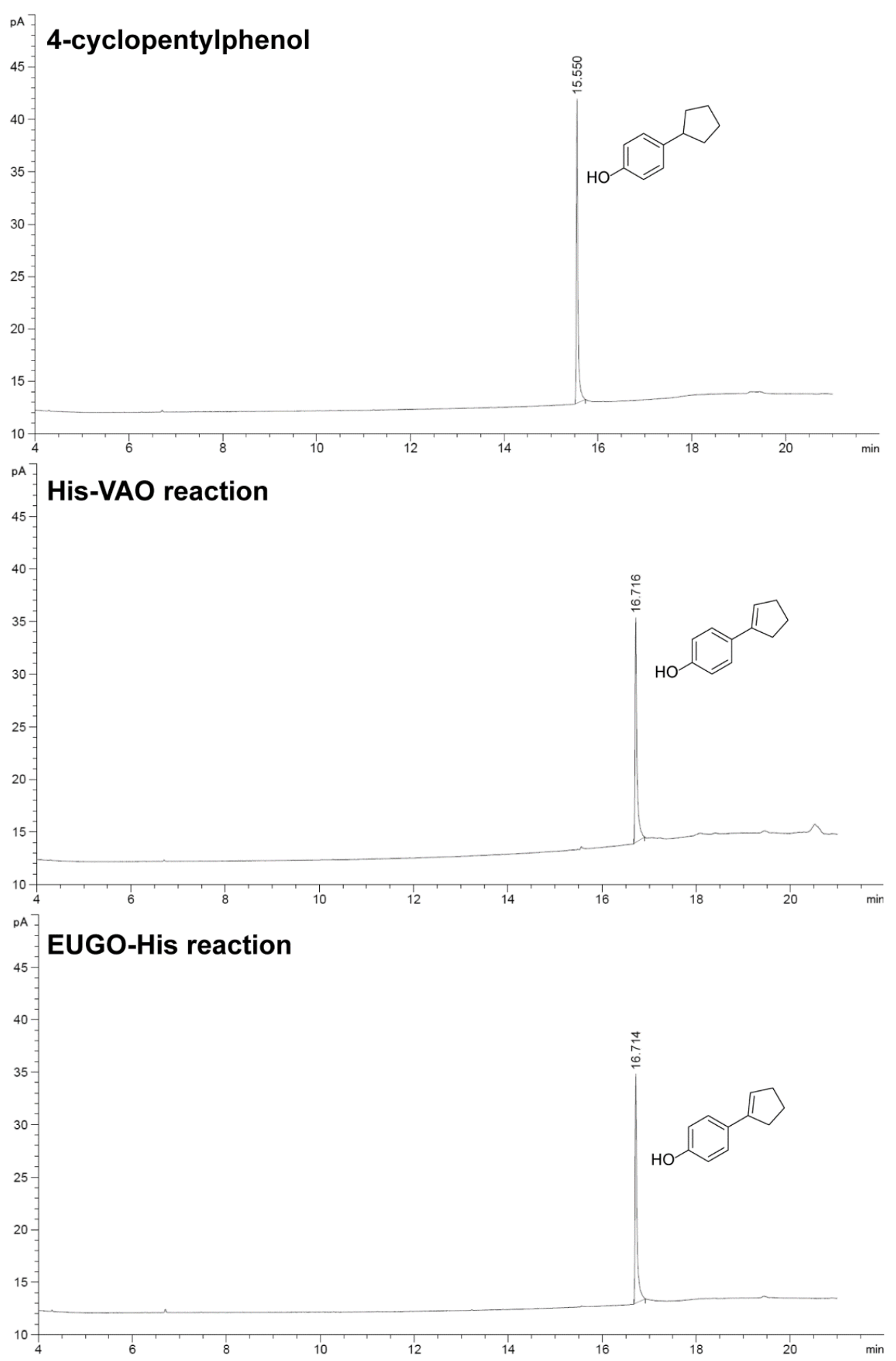

Figure S3: GC chromatograms of the reaction products of the conversion of 4-cyclopentylphenol by His-VAO or EUGO-His. $2 \mathrm{mM}$ 4-cyclopentylphenol and $1 \mu \mathrm{M}$ enzyme were incubated for $2 \mathrm{~h}$ at $25^{\circ} \mathrm{C}$ in $50 \mathrm{mM}$ potassium phosphate buffer, $\mathrm{pH}$ 7.5, (EUGO-His) or $50 \mathrm{mM}$ potassium phosphate buffer, $\mathrm{pH} 7.5$, containing $30 \mathrm{mM} \mathrm{NaCl}$ and $2 \%$ (w/v) glycerol (His-VAO). Subsequently, the reaction products were extracted and analysed by GC. For both reactions, a single product, 4-(1-cyclopenten-1yl)phenol, was formed at $>99 \%$ conversion. Retention times: 4-cyclopentylphenol: $15.6 \mathrm{~min}, 4-(1-$ cyclopenten-1-yl)phenol: $16.7 \mathrm{~min}$. 

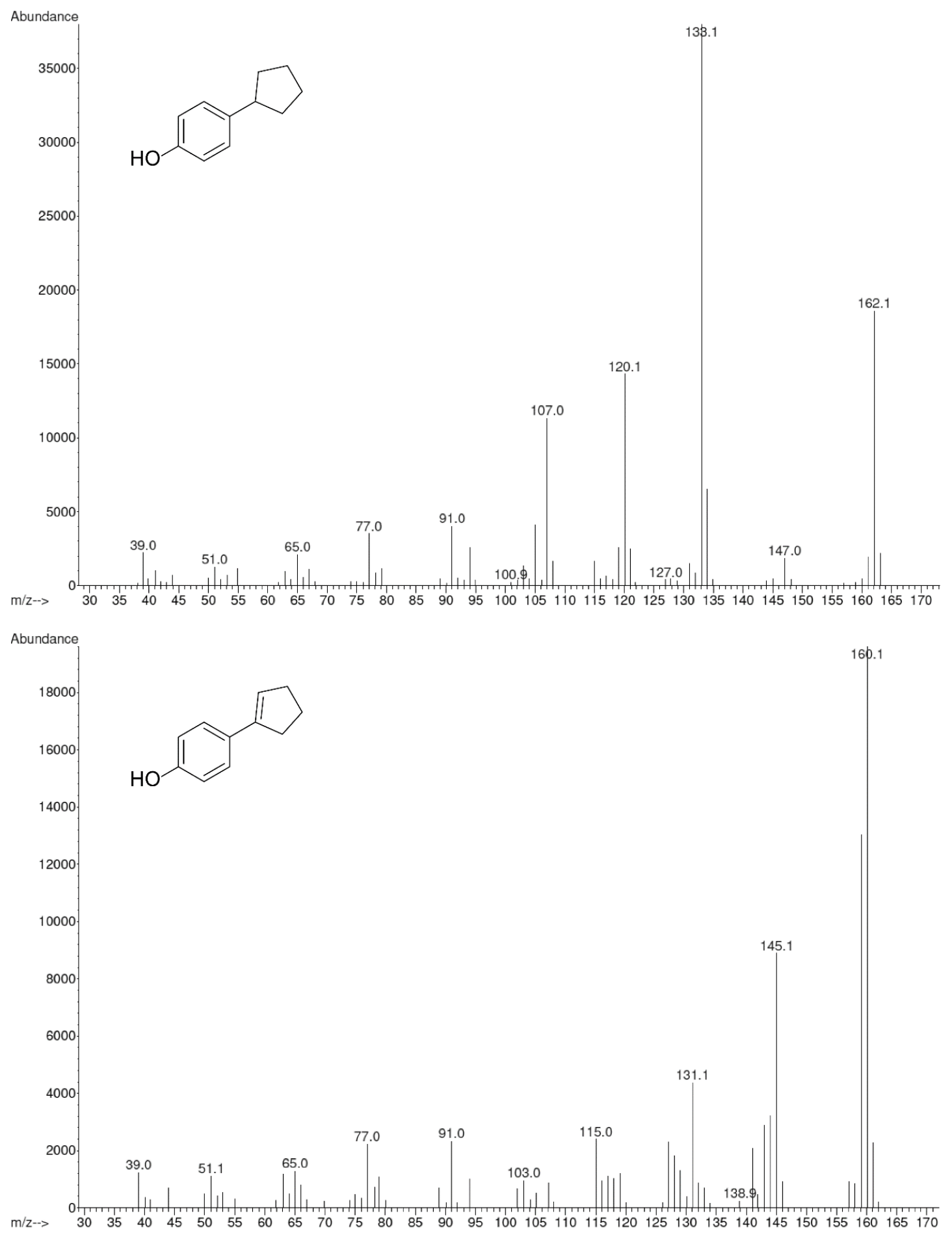

Figure S4: Mass spectra of 4-cyclopentylphenol (top) and 4-(1-cyclopenten-1-yl)phenol formed from its conversion by His-VAO (bottom) as obtained by GC/MS analysis. $2 \mathrm{mM} 4$-cyclopentylphenol and 1 $\mu \mathrm{M}$ enzyme were incubated for $2 \mathrm{~h}$ at $25^{\circ} \mathrm{C}$ in $50 \mathrm{mM}$ potassium phosphate buffer, $\mathrm{pH} 7.5$, containing $30 \mathrm{mM} \mathrm{NaCl}$ and $2 \%(\mathrm{w} / \mathrm{v})$ glycerol. Subsequently, the reaction products were extracted and analysed by GC/MS. A single product peak was observed, the mass spectrum of the corresponding compound is shown in the bottom panel. The product formed by the conversion of 4-cyclopentylphenol by EUGO-His displayed an identical mass spectrum. A 4-cyclopentylphenol standard also displayed a single peak on the chromatogram, the mass spectrum of the corresponding compound is shown in the top panel. 


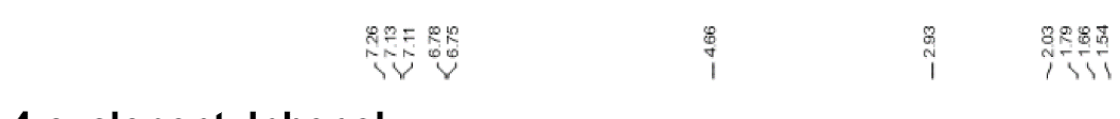

\section{4-cyclopentylphenol}<smiles>Oc1ccc(C2CCCC2)cc1</smiles>
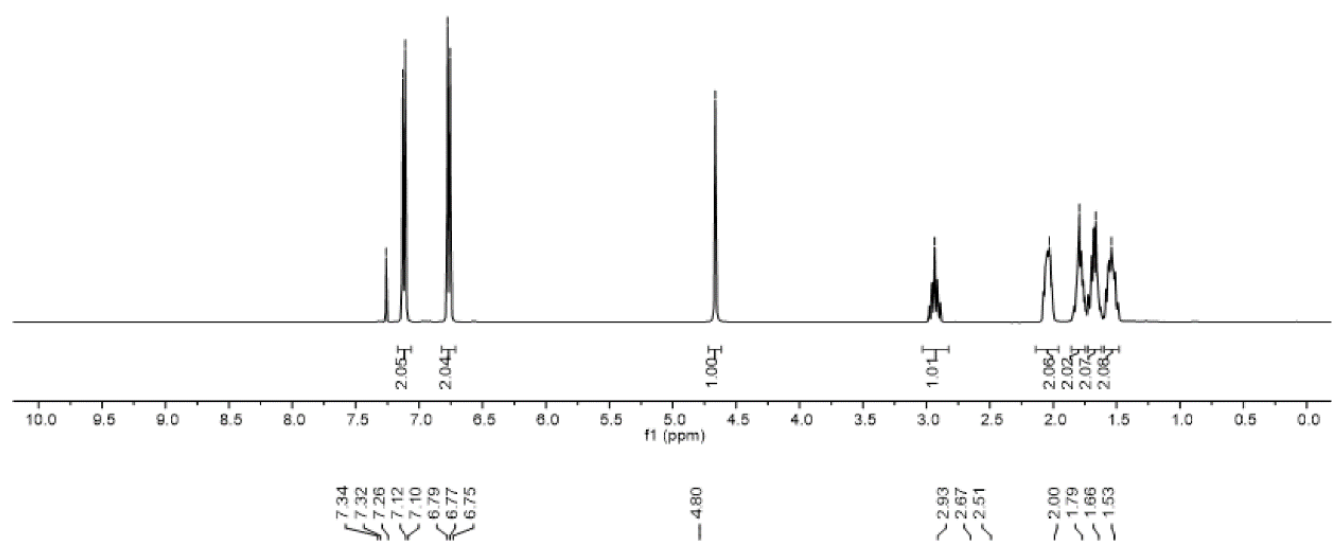

\section{EUGO-His reaction}<smiles>Oc1ccc(C2=CCCC2)cc1</smiles>

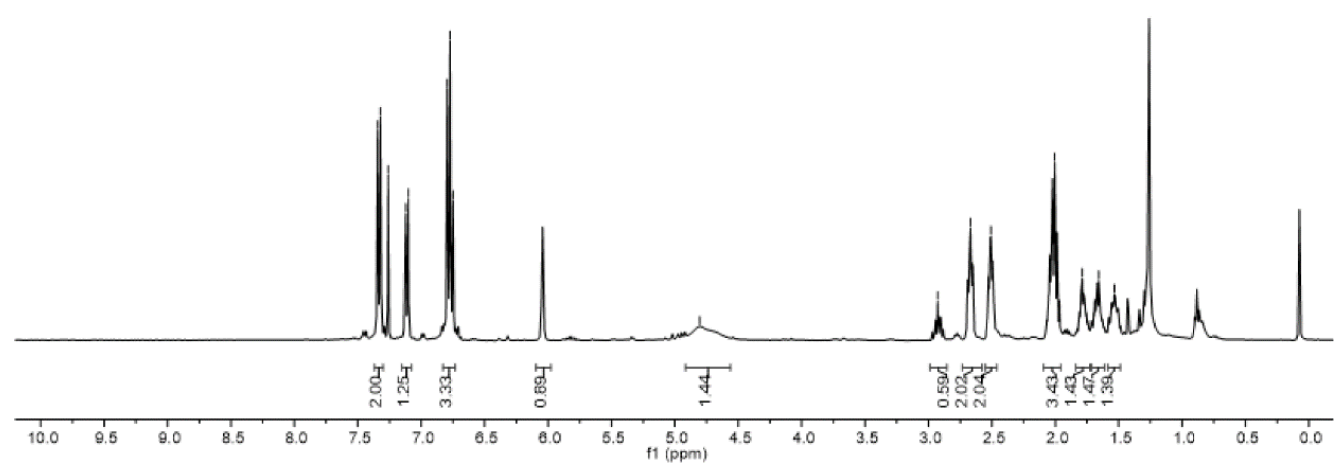

Figure S5: ${ }^{1} \mathrm{H}-\mathrm{NMR}$ spectra of 4-cyclopentylphenol and the products of the conversion of 4cyclopentylphenol by EUGO-His. $2.5 \mathrm{mM}$ 4-cyclopentylphenol and $0.5 \mu \mathrm{M}$ EUGO-His were incubated in $50 \mathrm{mM}$ potassium phosphate buffer, $\mathrm{pH} 7.5$, at $25^{\circ} \mathrm{C}$ for $16 \mathrm{~h}$. Subsequently, reaction products were extracted and analysed by ${ }^{1} \mathrm{H}-\mathrm{NMR}$. This revealed that the product is a mixture of 4cyclopentylphenol (40\%) and 4-(1-cyclopenten-1-yl)phenol (60\%). Peaks below $1.5 \mathrm{ppm}$ are due to impurities in the $\mathrm{CDCl}_{3}$. 4-cyclopentylphenol: ${ }^{1} \mathrm{H}-\mathrm{NMR}\left(400 \mathrm{MHz}, \mathrm{CDCl}_{3}\right) \delta(\mathrm{ppm}): 7.12(\mathrm{ap} \mathrm{d}, J=8.5 \mathrm{~Hz}$, $2 \mathrm{H}), 6.77(\mathrm{ap} \mathrm{d}, J=8.5 \mathrm{~Hz}, 2 \mathrm{H}), 4.66(\mathrm{~s}, 1 \mathrm{H}), 2.93(\mathrm{~m}, 1 \mathrm{H}), 2.14-1.96(\mathrm{~m}, 2 \mathrm{H}), 1.87-1.72(\mathrm{~m}, 2 \mathrm{H}), 1.75-$ $1.59(\mathrm{~m}, 2 \mathrm{H}), 1.61-1.46(\mathrm{~m}, 2 \mathrm{H}) .4$-(1-cyclopenten-1-yl)phenol: ${ }^{1} \mathrm{H}-\mathrm{NMR}\left(400 \mathrm{MHz}, \mathrm{CDCl}_{3}\right) \delta(\mathrm{ppm}): 7.33$ $($ ap d, $J=8.6 \mathrm{~Hz}, 2 \mathrm{H}), 6.78($ ap d, $J=8.9 \mathrm{~Hz}, 2 \mathrm{H}), 6.04(\mathrm{~m}, 1 \mathrm{H}), 4.80(\mathrm{br} \mathrm{s}, 1 \mathrm{H}), 2.73-2.56(\mathrm{~m}, 2 \mathrm{H}), 2.56-$ $2.45(\mathrm{~m}, 2 \mathrm{H}), 2.09-1.96(\mathrm{~m}, 2 \mathrm{H})$. 

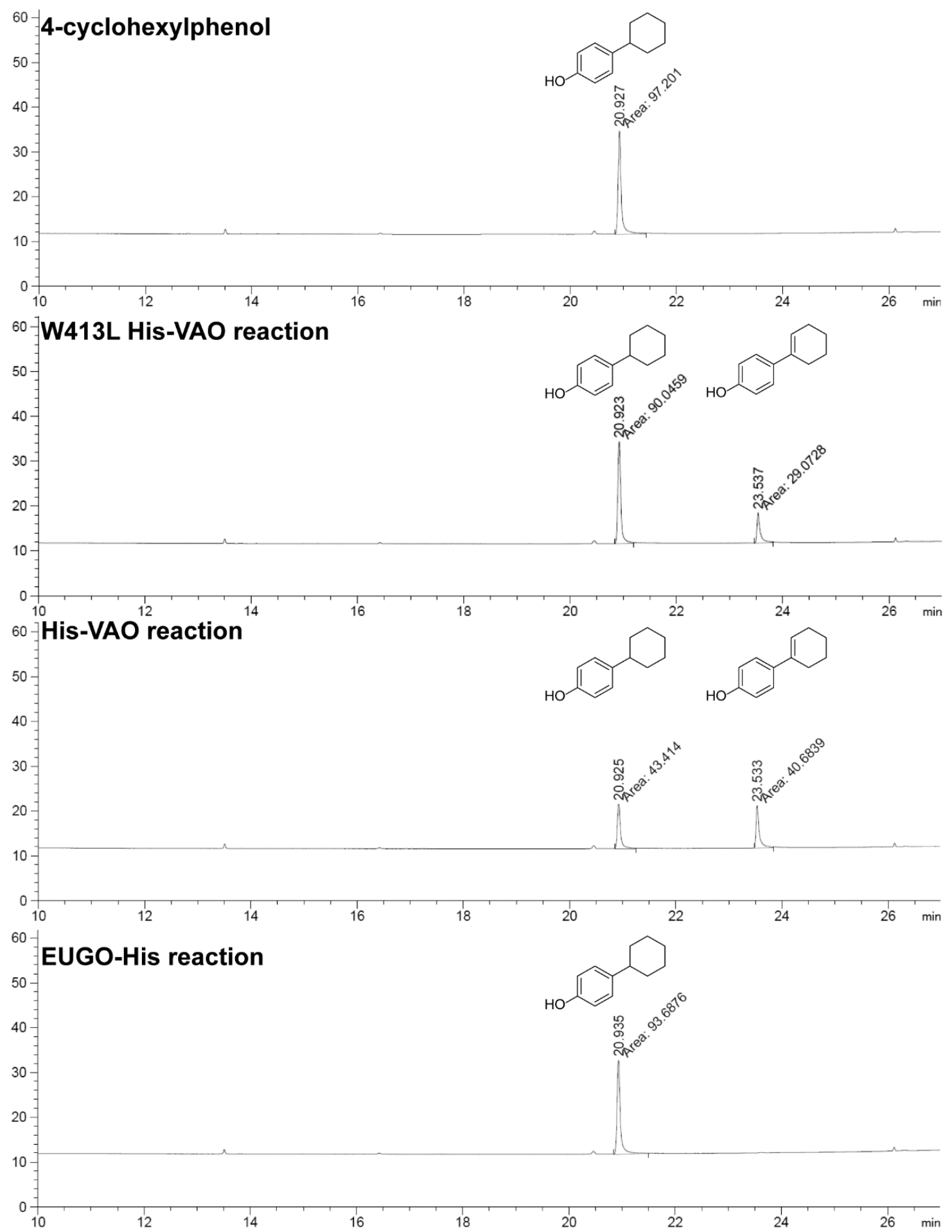

Figure S6: GC chromatograms of the reaction products of the conversion of 4-cyclohexylphenol by W413L His-VAO, His-VAO or EUGO-His. $2 \mathrm{mM}$ 4-cyclohexylphenol and $1 \mu \mathrm{M}$ enzyme were incubated for $4 \mathrm{~h}$ at $25^{\circ} \mathrm{C}$ in $50 \mathrm{mM}$ potassium phosphate buffer, pH 7.5, (EUGO-His) or $50 \mathrm{mM}$ potassium phosphate buffer, $\mathrm{pH} 7.5$, containing $30 \mathrm{mM} \mathrm{NaCl}$ and $2 \%(\mathrm{w} / \mathrm{v})$ glycerol (His-VAO and W413L His-VAO). Subsequently, the reaction products were extracted and analysed by GC. With W413L His-VAO and His-VAO, a single product, 4-(1-cyclohexen-1-yl)phenol, was formed at 27\% and $51 \%$ conversion respectively (average of two reactions). With EUGO-His, no conversion of the substrate was observed. Retention times: 4-cyclohexylphenol: $20.9 \mathrm{~min}$, 4-(1-cyclohexen-1-yl)phenol: $23.5 \mathrm{~min}$. 


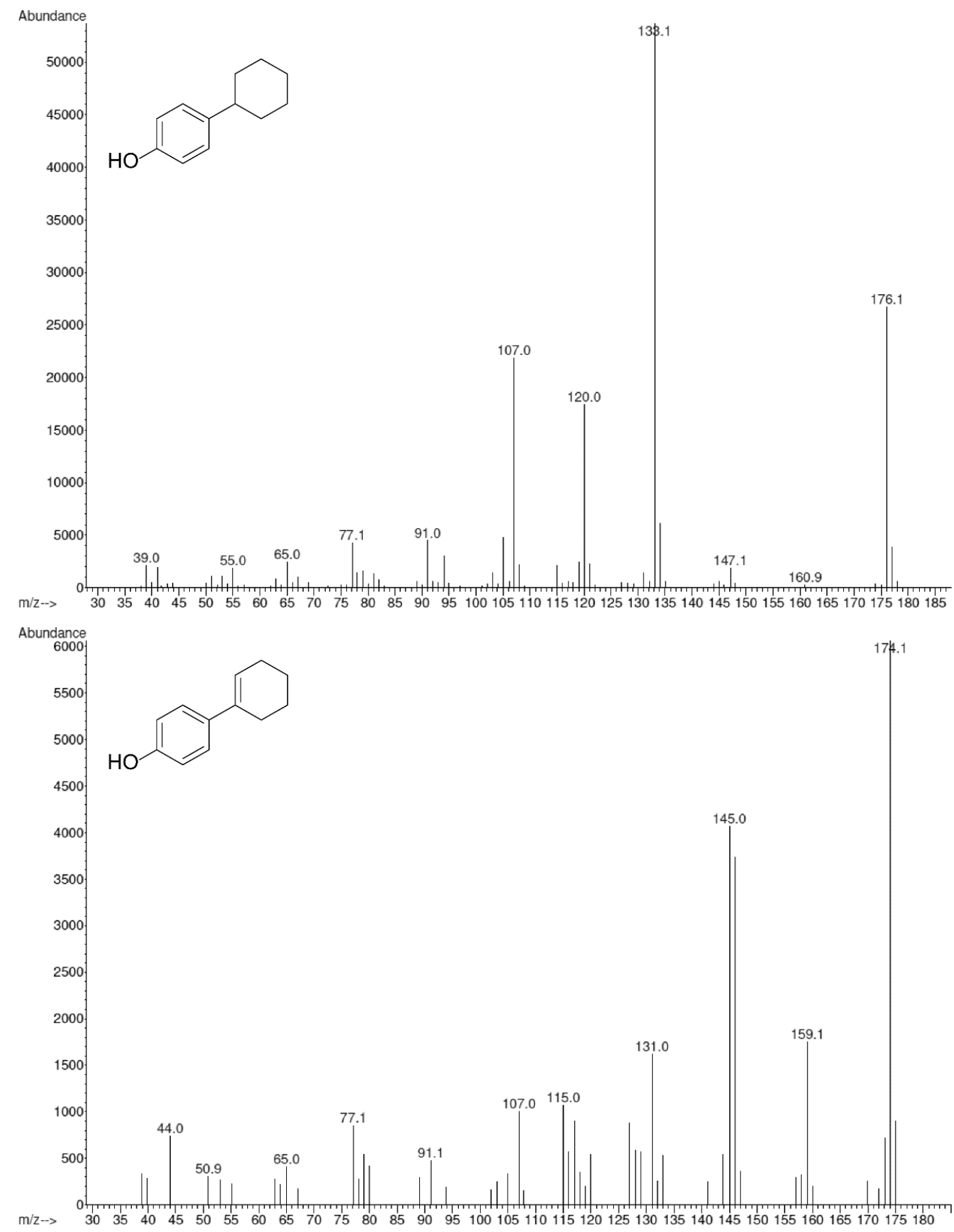

Figure S7: Mass spectra of 4-cyclohexylphenol (top) and 4-(1-cyclohexen-1-yl)phenol formed from its conversion by W413L His-VAO (bottom) as obtained by GC/MS analysis. $2 \mathrm{mM}$ 4-cyclohexylphenol and $1 \mu \mathrm{M}$ enzyme were incubated for $4 \mathrm{~h}$ at $25^{\circ} \mathrm{C}$ in $50 \mathrm{mM}$ potassium phosphate buffer, $\mathrm{pH}$ 7.5, containing $30 \mathrm{mM} \mathrm{NaCl}$ and $2 \%(\mathrm{w} / \mathrm{v})$ glycerol. Subsequently, the reaction products were extracted and analysed by GC/MS. Two peaks were observed, one corresponding to unconverted substrate and a single product peak, the mass spectrum of the product is shown in the bottom panel. The product formed by the conversion of 4-cyclohexylphenol by wild-type His-VAO displayed an identical mass spectrum. A 4-cyclohexylphenol standard also displayed a single peak on the chromatogram, the mass spectrum of the corresponding compound is shown in the top panel. 



\title{
Multigram scale enzymatic synthesis of (R)-1-(4'-hydroxyphenyl)ethanol using vanillyl alcohol oxidase
}

\author{
Tom A. Ewing ${ }^{1}$, Jasmin Kühn², Silvia Segarra ${ }^{3}$, Marta Tortajada ${ }^{3}$, Ralf Zuhse ${ }^{2}$ \\ and Willem J. H. van Berkel ${ }^{1}$ \\ ${ }^{1}$ Laboratory of Biochemistry, Wageningen University \& Research, Wageningen, The Netherlands \\ ${ }^{2}$ Chiracon GmbH, Luckenwalde, Germany \\ ${ }^{3}$ Biopolis S. L., Paterna, Spain
}

Based on Advanced Synthesis and Catalysis (2018), https://doi.org/10.1002/adsc.201800197

\section{Summary}

The enantioselective oxyfunctionalisation of $\mathrm{C}-\mathrm{H}$ bonds is a highly interesting reaction, as it provides access to chiral alcohols that are important pharmaceutical building blocks. However, it is hard to achieve using traditional methods. One way in which it can be achieved is through the action of oxidative enzymes. Although many reports of the oxyfunctionalisation capabilities of enzymes at an analytical scale have been published, reports on the use of enzymes to achieve oxyfunctionalisation on a synthetically relevant scale are fewer. Here, we describe the scale-up of the conversion of 4-ethylphenol to $(R)-1-\left(4^{\prime}-\right.$ hydroxyphenyl)ethanol using the flavin-dependent enzyme vanillyl alcohol oxidase. The process was optimised by testing different reaction media and substrate and enzyme concentrations and by performing it under an oxygen atmosphere. Under optimised reaction conditions, $4.1 \mathrm{~g}(R)-1-(4$ '-hydroxyphenyl)ethanol at 97\% ee was obtained from $10 \mathrm{~g}$ 4ethylphenol (isolated yield $36 \%$ ). These results highlight some of the challenges that can be encountered during scale-up of an enzymatic oxyfunctionalisation process to a synthetically relevant scale and will be of use for the development of enzymatic processes for the synthesis of industrially relevant compounds. 



\section{Introduction}

The selective oxyfunctionalisation of $\mathrm{C}-\mathrm{H}$ bonds under mild reaction conditions is of great interest to synthetic organic chemists. In recent years, much effort has been put into developing metal-based catalysts that can introduce oxygen atoms using environmentally benign oxidants such as molecular oxygen $(1,2)$. An attractive alternative to traditional chemical catalysts is the use of enzymes capable of introducing oxygen atoms, which often display exquisite regio- and enantioselectivity and are active under mild reaction conditions. Well-known classes of oxygenating enzymes include heme-dependent cytochrome P450 enzymes and peroxygenases, non-heme iron oxygenases and flavin-dependent oxygenases (3-7). Some of these enzymes have been utilised to obtain valuable products from preparativescale transformations (8-12). For many others, however, our knowledge of their oxyfunctionalisation capabilities is limited to the analytical scale. In order to stimulate the use of oxidoreductases in industrial chemical synthesis, it is crucial to demonstrate that they can be used to obtain compounds on a synthetically relevant scale (13).

Chiral alcohols, which are often important intermediates in the synthesis of active pharmaceutical ingredients, present an interesting target for these preparative-scale transformations (14). One class of alcohols that are important pharmaceutical building blocks are chiral $\alpha$-aryl alcohols $(15,16)$. A number of biocatalytic approaches for their synthesis have been described, including enantioselective reduction of a prochiral ketone, kinetic resolution or deracemisation of racemic alcohols and the enantioselective hydration of vinylphenol derivatives (17-22). Another potential enzymatic synthesis route for chiral $\alpha$-aryl alcohols is the direct oxyfunctionalisation of alkyl-substituted aromatic molecules. An enzyme that can be used for this purpose is vanillyl alcohol oxidase from Penicillium simplicissimum (VAO, EC 1.1.3.38), which is capable of catalysing the enantioselective hydroxylation of 4-alkylphenols. Here, we describe the development of the multigram scale enzymatic synthesis of $(R)-1-\left(4^{\prime}-\right.$ hydroxyphenyl)ethanol using VAO.

VAO catalyses the oxidation of a wide range of para-substituted phenols at their C $\alpha$ atom using molecular oxygen as an electron acceptor (23-25). VAO displays a broad substrate scope, catalysing the oxidation of benzylic alcohols or amines, the oxidative demethylation of 4(methoxymethyl)phenol and the hydroxylation of 4-allylphenols. In addition, VAO oxidises phenols bearing unfunctionalised alkyl chains as the para-substituent. This leads to mixtures of hydroxylated and dehydrogenated products, with the ratio between the two being determined by the size of the alkyl chain $(25,26$, Chapter 6$)$ and the composition of the reaction medium (27). The hydroxylation of short chain 4-alkylphenols proceeds with high regio- and enantioselectivity. The conversion of 4-ethylphenol, 4-propylphenol or 2-methoxy4-propylphenol by VAO yields the corresponding 1-(4'-hydroxyphenyl)alcohols as the sole hydroxylation product, in addition to more minor amounts of vinylic by-products from dehydrogenation of the substrate and ketone by-products from further oxidation of the formed alcohols (Scheme 1) (28). The hydroxylation reaction is highly enantioselective, with the $(R)$-enantiomers of the alcohol product being formed at $94 \%$ ee in all cases.

The catalytic mechanism of VAO provides an explanation for this regio- and enantioselectivity (29-31, Chapter 4). Binding of the phenolate form of the substrate to the active site is followed 
by hydride transfer from the $\mathrm{C} \alpha$ atom of the substrate to the N5 atom of the FAD cofactor (Scheme 2). This yields a planar para-quinone methide intermediate, which is subsequently hydrated in the enzyme's active site. The enantioselectivity of the hydration is determined by the presence of an active site base (Asp-170) that activates water for attack at one side of the planar para-quinone methide. Accumulation of the $(R)$-enantiomer is further enhanced by the fact that the (S)-enantiomer of 1-(4'-hydroxyphenyl)ethanol is more easily oxidised further to 4-hydroxyacetophenone than the $(R)$-enantiomer is (25). A VAO variant (D170S/T457E) where the active site base was moved to the other side of the substrate displayed reversed enantioselectivity, converting 4-ethylphenol to (S)-1-(4'- hydroxyphenyl)ethanol at $80 \%$ ee (32). Its ability to catalyse regio- and enantioselective hydroxylations using only molecular oxygen as a cosubstrate makes VAO an interesting candidate for biocatalytic applications.

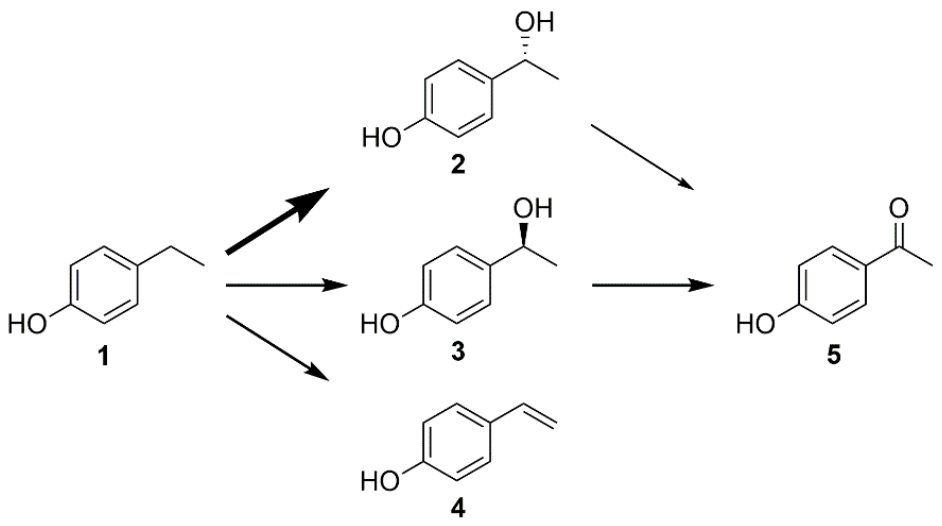

Scheme 1: Conversion of 4-ethylphenol (1) by VAO. Thickness of the arrows gives a qualitative indication of the relative rates at which the depicted reactions occur. VAO readily converts 4ethylphenol to $(R)-1-\left(4^{\prime}\right.$-hydroxyphenyl)ethanol (2), with (S)-1-(4'-hydroxyphenyl)ethanol (3) and 4vinylphenol (4) being formed as by-products. The formed alcohol can subsequently be oxidised further to 4-hydroxyacetophenone (5), with the (S)-enantiomer being more readily converted than the $(R)$ enantiomer. Scheme adapted from (25).

The synthetic utility of $\mathrm{VAO}$ at small scale has been demonstrated previously. The conversion of 4-ethylphenol and 2-methoxy-4-propylphenol was performed at $100 \mathrm{mg}$ scale, yielding 33.2 $\mathrm{mg}(28 \%)$ and $52 \mathrm{mg}(45 \%)$ respectively of the corresponding purified alcohols (28). To evaluate the potential of VAO to be used as a biocatalyst for the synthesis of chiral alcohols on a more synthetically relevant scale, we scaled up the conversion of 4-ethylphenol to the 10 g scale.

\section{Results}

\section{Optimisation of Reaction Conditions}

As a first step in the scale-up process, we optimised the reaction conditions for the conversion of 4-ethylphenol (1) at an analytical scale by testing the effect of the addition of organic solvents to the reaction medium. To this end, the conversion of $5 \mathrm{mg} \mathbf{1}$ was performed in 84 $\mathrm{mM}$ potassium phosphate buffer, $\mathrm{pH} 7.5$, in the presence of $10 \%$ acetone or acetonitrile. The composition of reaction mixtures after the reaction as determined by gas chromatography is given in Table 1. Using acetone as a cosolvent gave the best results, with complete conversion 


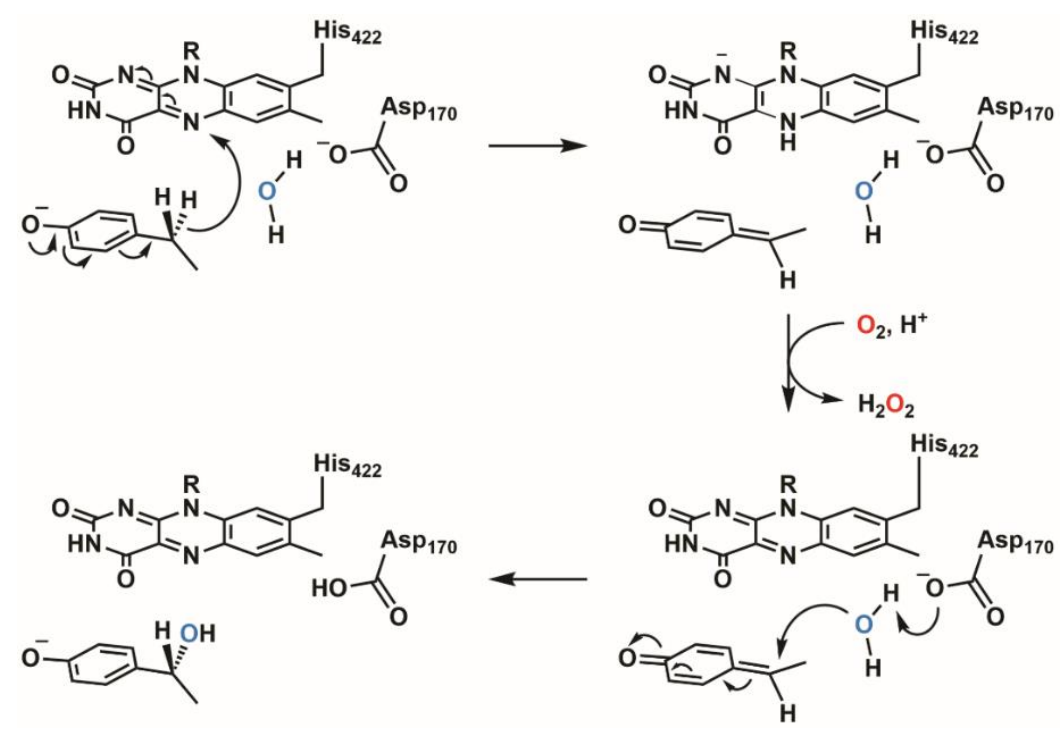

Scheme 2: Mechanism of the enantioselective hydroxylation of 4-ethylphenol by VAO. First, 4ethylphenol binds in the enzyme's active site in its phenolate form. Following this, a hydride is transferred from the $\mathrm{C} \alpha$ atom of its ethyl group to the N5 atom of FAD, yielding a para-quinone methide intermediate and reduced FAD. Subsequently, a water molecule is activated by Asp-170 and attacks the $\mathrm{C} \alpha$ atom of the para-quinone methide intermediate, yielding the alcohol product. Stopped-flow kinetics analysis has demonstrated that this occurs after (or possibly concomitant with) the reoxidation of FAD by molecular oxygen (30). The position of Asp-170 relative to the planar para-quinone methide intermediate explains the preferential formation of the $(R)$-enantiomer of 1-(4'-hydroxyphenyl)ethanol.

of 1 and $83 \%$ of the formed product being (R)-1-(4'-hydroxyphenyl)ethanol (2) after $4 \mathrm{~h}$. The formation of 2 is underestimated, as part of the 1-(4'-hydroxyphenyl)ethanol undergoes elimination of water during the gas chromatographic analysis, yielding 4-vinylphenol (4) [as confirmed by GC analysis of racemic pure 1-(4'-hydroxyphenyl)ethanol]. Further scale-up was performed using the reaction medium containing $10 \%$ acetone.

Table 1: Products obtained from the conversion of 4-ethylphenol (1) by VAO in the presence of organic solvents. $5 \mathrm{mg} 1$ (at a concentration of $2.9 \mathrm{mM}$ ) and $0.4 \mathrm{U}$ VAO were incubated in $84 \mathrm{mM}$ potassium phosphate buffer, $\mathrm{pH} 7.5$, at $30{ }^{\circ} \mathrm{C}$ in the presence of $10 \%$ acetone or $10 \%$ acetonitrile. After the incubation period, reaction products were extracted and analysed by GC. Compounds are numbered as in Scheme 1. GC chromatograms are shown in Fig. S1. The given numbers are the amount of a compound as a percentage of the total present upon analysis.

\begin{tabular}{|ccccccc|}
\hline Reaction conditions & $\mathbf{1}(\mathbf{\%})$ & $\mathbf{2}(\mathbf{\%})$ & $\mathbf{3}(\mathbf{\%})$ & $\mathbf{2 - e e}(\mathbf{\%})$ & $\mathbf{4}(\mathbf{\%})$ & $\mathbf{5}(\mathbf{\%})$ \\
\hline $10 \%$ acetone, $4 \mathrm{~h}$ & - & 83 & 0.7 & 98 & 14 & 3.0 \\
& & & & & & \\
$10 \%$ acetonitrile, $22 \mathrm{~h}$ & 5.9 & 76 & 2.5 & 94 & 14 & 1.3 \\
\hline
\end{tabular}

Scaling up the reaction to $100 \mathrm{mg}$ scale with increased substrate concentrations revealed that almost complete conversion (97\%) could be achieved using a substrate concentration of 23 $\mathrm{mM}$ and $2 \mathrm{U} \mathrm{VAO}$ (Table 2, reaction a). Increasing the substrate concentration further to 45 $\mathrm{mM}$ led to lower conversion (87\%) even though the same enzyme to substrate ratio was used 
(Table 2, reaction b). Therefore, $23 \mathrm{mM}$ substrate with $0.02 \mathrm{U} \mathrm{VAO} / \mathrm{mg}$ substrate was chosen as the conditions for scale-up.

We subsequently tested whether we could eliminate the need for protein purification by performing the reaction using a cell-free extract of Escherichia coli expressing an N-terminally His-tagged variant of VAO. The plasmid encoding for this His-tagged VAO, pJ404-His-VAO, contains a version of the gene encoding for VAO that is codon-optimised for expression in $E$. coli. This construct allows VAO to be expressed at higher levels than the previously used plasmid, pBC11 (33), which contains the vaoA gene from $P$. simplicissimum. Using this cell-free extract, the conversion of $100 \mathrm{mg} \mathbf{1}$ gave a similar result to that using the purified enzyme (Table 2, reaction c). Apparently, the use of E. coli cell-free extract did not lead to any undesired side reactions taking place. Therefore, the cell-free extract was used for further scale-up reactions.

Scale-up to $10 \mathrm{~g}$ Scale

Having established the optimised reaction conditions for the conversion of $\mathbf{1}$ to $\mathbf{2}$, we next set out to scale up the reaction to a more synthetically relevant scale of $10 \mathrm{~g}$ of substrate. First, the reaction was scaled up to $1 \mathrm{~g}$ of substrate using the same reaction conditions as for $100 \mathrm{mg}$. This led to almost complete conversion (93\%) of $\mathbf{1}$ and to similar formation of $\mathbf{2}$ as was obtained at $100 \mathrm{mg}$ scale (Table 2, reaction d). Analysis of the reaction products by ${ }^{1} \mathrm{H}-\mathrm{NMR}$ allowed quantification of the products without being hindered by the decomposition of 1-(4'hydroxyphenyl)ethanol to 4 observed during GC analysis, revealing that the enzymatic reaction yields less than $3 \%$ of 4 as a by-product (Table 2, reaction d). To obtain pure 2 , it was purified from the crude reaction mixture by recrystallisation, yielding $244.3 \mathrm{mg}$ (21\%) pure 2 at $99 \%$ ee (Fig. S3+S4).

Subsequently, the reaction was scaled up to our final target scale of approximately $10 \mathrm{~g}$ of starting material. However, using the same reaction conditions with $13.4 \mathrm{~g} 1$ led to lower conversion of the substrate [63\% (Table 2, reaction e)]. A possible explanation is that the larger reaction volume required for this conversion led to oxygen depletion becoming limiting for the reaction. Therefore, we performed the reaction with $10 \mathrm{~g} 1$ under identical conditions, but with the reaction vessel under oxygen atmosphere for the first $9 \mathrm{~h}$ of the reaction. Under these conditions, the substrate was almost completely converted after $24 \mathrm{~h}$ [94\% (Table 2, reaction f)]. After recrystallisation, pure 2 was obtained in 36\% isolated yield and 97\% ee (Fig. S3+S4).

\section{Discussion}

Here, we described the scale-up of the synthesis of (R)-1-(4'-hydroxyphenyl)ethanol starting from 4-ethylphenol using VAO. Optimisation of reaction conditions at analytical scale revealed that the reaction proceeds efficiently in the presence of $10 \%$ acetone. The addition of acetone likely improves the solubility of the aromatic substrate and products. In addition, the solubility of oxygen in acetone is increased as compared to in water $[277 \mathrm{mg} / \mathrm{L}$ in acetone at $25^{\circ} \mathrm{C}$ and $44 \mathrm{mg} / \mathrm{L}$ in water at $20^{\circ} \mathrm{C}$ at 1 bar pure oxygen atmosphere (34)], preventing oxygen depletion from becoming limiting for the reaction. 
Table 2: Products obtained from the conversion of 4-ethylphenol (1) by VAO. 1 and VAO or His-VAO cell-free extract were incubated in $88 \mathrm{mM}$ potassium phosphate buffer, $\mathrm{pH} 7.5$, containing $10 \%$ acetone at $30{ }^{\circ} \mathrm{C}$ for $24 \mathrm{~h}$. Subsequently, reaction products were extracted and analysed by GC or ${ }^{1} \mathrm{H}-\mathrm{NMR}$. Compounds are numbered as in Scheme 1. GC chromatograms are shown in Fig. S2, ${ }^{1} \mathrm{H}-\mathrm{NMR}$ spectra in Fig. S4. The given numbers are the amount of a compound as a percentage of the total present upon analysis. CFE: cell-free extract.

\begin{tabular}{|c|c|c|c|c|c|c|c|c|c|c|}
\hline \multicolumn{2}{|c|}{ Reaction } & \multirow{2}{*}{$\begin{array}{c}\text { Substrate } \\
100 \mathrm{mg} \\
23 \mathrm{mM}\end{array}$} & \multirow{2}{*}{$\begin{array}{l}\text { Enzyme } \\
2 \mathrm{UVAO}\end{array}$} & \multirow{2}{*}{\begin{tabular}{c|}
1 \\
$(\%)$ \\
3.1
\end{tabular}} & \multirow{2}{*}{\begin{tabular}{c|}
2 \\
$(\%)$ \\
83
\end{tabular}} & \multirow{2}{*}{$\begin{array}{c}3(\%) \\
4.0\end{array}$} & \multirow{2}{*}{$2+3(\%)$} & \multirow{2}{*}{$\begin{array}{c}\text { 2-еe (\%) } \\
91\end{array}$} & \multirow{2}{*}{$\begin{array}{c}4(\%) \\
8.3\end{array}$} & \multirow{2}{*}{$\begin{array}{r}5(\%) \\
1.5\end{array}$} \\
\hline $\mathrm{a}$ & GC & & & & & & & & & \\
\hline$b$ & GC & $\begin{array}{l}100 \mathrm{mg} \\
45 \mathrm{mM}\end{array}$ & $2 \mathrm{U}$ VAO & 13 & 58 & 4.1 & & 87 & 24 & 1.0 \\
\hline c & GC & $\begin{array}{l}100 \mathrm{mg} \\
23 \mathrm{mM}\end{array}$ & $\begin{array}{c}2 \mathrm{U} \\
\mathrm{His}-\mathrm{VAO} \\
\mathrm{CFE}\end{array}$ & 0.8 & 74 & 2.1 & & 95 & 23 & 0.9 \\
\hline $\mathrm{d}$ & GC & $\begin{array}{c}1.0 \mathrm{~g} \\
23 \mathrm{mM}\end{array}$ & $\begin{array}{c}20 \mathrm{U} \\
\text { His-VAO } \\
\text { CFE }\end{array}$ & 6.5 & 83 & 1.3 & & 97 & 8.6 & 0.8 \\
\hline & $\begin{array}{c}{ }^{1} \mathrm{H}- \\
\mathrm{NMR}\end{array}$ & & & 7.5 & & & 88 & & 2.6 & 1.8 \\
\hline $\mathrm{e}$ & GC & $\begin{array}{c}13.4 \mathrm{~g} \\
23 \mathrm{mM}\end{array}$ & $\begin{array}{c}268 \mathrm{U} \\
\text { His-VAO } \\
\text { CFE }\end{array}$ & 37 & 51 & 1.9 & & 93 & 9.2 & 1.1 \\
\hline $\mathrm{f}$ [a] & GC & $\begin{array}{c}10.0 \mathrm{~g} \\
23 \mathrm{mM}\end{array}$ & $\begin{array}{c}202 \mathrm{U} \\
\text { His-VAO } \\
\text { CFE }\end{array}$ & 6.0 & 82 & 4.1 & & 90 & 6.5 & 1.0 \\
\hline & $\begin{array}{c}{ }^{1} \mathrm{H}- \\
\text { NMR }\end{array}$ & & & 6.3 & & & 89 & & 2.7 & 1.8 \\
\hline
\end{tabular}

[a] Reaction vessel was kept under pure $\mathrm{O}_{2}$ atmosphere for the first $9 \mathrm{~h}$

Tests at the $100 \mathrm{mg}$ scale revealed that the substrate concentration could be increased to 23 $\mathrm{mM}$, while still maintaining almost full conversion of the substrate. However, upon further increasing the substrate concentration to $45 \mathrm{mM}$, lower conversions were obtained. This may be due to the limited solubility of the aromatic substrate. Cell-free extract of E. coli was successfully substituted for the purified enzyme, eliminating the cost associated with protein purification.

The reaction was successfully scaled up from the $100 \mathrm{mg}$ to the $1 \mathrm{~g}$ scale using essentially the same reaction conditions. However, upon further scale-up to approximately $10 \mathrm{~g}$ of substrate, 
lower conversions were observed due to oxygen depletion becoming limiting for the reaction. This was solved by performing the reaction under an oxygen atmosphere. This observation that maintaining sufficient oxygen levels is crucial to allow the reaction to proceed is in agreement with previous studies regarding biotransformations using monooxygenasecontaining whole-cell biocatalysts, where oxygen depletion was found to be limiting for the reaction under certain conditions $(8,35,36)$. As the use of an oxygen atmosphere may be undesired when performing chemical reactions at an industrial scale, due to it posing an explosion hazard, alternative ways in which to obtain sufficient oxygenation of the reaction medium such as the use of bubble aeration should be considered in future.

One aspect of the process described here that can still be improved, is the fact that despite obtaining $82 \%$ formation of 2 in the largest scale reaction, the isolated yield of $36 \%$ is relatively low. This low yield is attributable to significant losses of material during both the extraction of the product from the reaction mixture $(6.51 \mathrm{~g}$ crude product obtained from $10 \mathrm{~g}$ starting material) and during recrystallisation. The loss of material during the extraction procedure may be attributable to the relatively high polarity of the product making its extraction into organic solvents inefficient. This may be solved in future by testing other solvents for extraction or by performing more rounds of extraction. From the GC data, it can be calculated that the crude compound obtained after extraction contains $5.34 \mathrm{~g} \mathrm{2}$, which corresponds to a crude yield of $47 \%$. A further $25 \%$ of the product was lost during its purification by recrystallisation. Although this loss is significant, recrystallisation is likely the most suitable method to increase the $e e$ of the product by removing the $(S)-1-\left(4^{\prime}\right.$-hydroxyphenyl)ethanol formed as a by-product.

In short, we described the scale-up of the synthesis of $(R)-1-\left(4^{\prime}\right.$-hydroxyphenyl)ethanol by the enantioselective hydroxylation of 4-ethylphenol using VAO. Under optimised reaction conditions, $94 \%$ conversion of $10 \mathrm{~g}$ 4-ethylphenol was obtained after $24 \mathrm{~h}$. Purification of the product yielded $(R)-1-(4$-hydroxyphenyl)ethanol in 36\% isolated yield and $97 \%$ ee. These results highlight some of the issues that may be encountered during scale-up of an enzymatic hydroxylation reaction from analytical scale to a synthetically relevant scale and may be of use in facilitating such scale-up for the synthesis of chiral pharmaceutical intermediates in an industrial setting.

\section{Materials and methods}

\section{Materials}

4-Ethylphenol and 4-hydroxyacetophenone were from abcr (Karlsruhe, Germany). An analytical standard for 1-(4'-hydroxyphenyl)ethanol was synthesised as a racemic mixture from 4-hydroxyacetophenone:

A solution of 4-hydroxyacetophenone $(3.4 \mathrm{~g}, 25 \mathrm{mmol})$ in $\mathrm{MeOH}(50 \mathrm{~mL})$ was stirred at 15 $20^{\circ} \mathrm{C}$ and a solution of $\mathrm{NaBH}_{4}(0.57 \mathrm{~g}, 15 \mathrm{mmol})$ in $0.2 \mathrm{M}$ aqueous $\mathrm{NaOH}(10 \mathrm{~mL})$ was added. Subsequently, the reaction mixture was stirred at room temperature for $20 \mathrm{~h}$. After this, $\mathrm{MeOH}$ was removed by evaporation and water $(10 \mathrm{~mL})$ was added to the residue. The mixture was extracted with $\mathrm{CHCl}_{3} /$ isopropanol (3/1) and the organic layer was dried over $\mathrm{Na}_{2} \mathrm{SO}_{4}$ and the 
solvent was removed by evaporation. The residue ( $2.2 \mathrm{~g}$, yellow solid) was chromatographed on silica gel with dichloromethane/ $\mathrm{MeOH}$ (50/1), yielding pure (R,S)-1-(4'hydroxyphenyl)ethanol (1.45 g, white solid, 42\%). ${ }^{1} \mathrm{H}-\mathrm{NMR}$ (d6-DMSO, 400MHz): $\delta$ (ppm): $9.10(\mathrm{~s}, 1 \mathrm{H}), 7.12(\mathrm{dt}, J=8.02 \mathrm{~Hz}, 2 \mathrm{H}), 6.68(\mathrm{dt}, J=8.69 \mathrm{~Hz}, 2 \mathrm{H}), 4.85(\mathrm{~d}, J=4.29 \mathrm{~Hz}, 1 \mathrm{H}), 4.63-$ $4.57(\mathrm{~m}, 1 \mathrm{H}), 1.27(\mathrm{~d}, J=6.44 \mathrm{~Hz}, 3 \mathrm{H})$.

The elution times of the 1-(4'-hydroxyphenyl)ethanol enantiomers upon gas chromatography analysis were assigned by comparison with 1-(4'-hydroxyphenyl)ethanol formed by conversion of 1 by VAO [primarily $(R)$-enantiomer].

All other chemicals were from commercial sources and of the purest grade available.

\section{Expression and purification of $V A O$}

For small-scale tests with purified enzyme, VAO was expressed and purified as described previously (37, Chapter 3). For larger-scale reactions using cell-free extracts, N-terminally Histagged VAO was expressed from the pJ404-His-VAO plasmid, which contains a version of the vaoA gene from $P$. simplicissimum that is codon-optimised for expression in E. coli behind an IPTG-inducible T5 promoter. BL21 E. coli cells containing this plasmid were grown at $37^{\circ} \mathrm{C}$ in LB medium containing $100 \mu \mathrm{g} / \mathrm{mL}$ ampicillin until the OD 600 of the cultures was 0.6. Subsequently, IPTG was added to a concentration of $0.8 \mathrm{mM}$ and the cells were grown overnight at $25{ }^{\circ} \mathrm{C}$. Following this, cells were harvested by centrifugation $(4,000 \mathrm{~g}, 10 \mathrm{~min}$, $10{ }^{\circ} \mathrm{C}$ ), resuspended in $50 \mathrm{mM}$ potassium phosphate buffer, $\mathrm{pH} 7.0$, containing $0.5 \mathrm{mM}$ dithiothreitol, $0.5 \mathrm{mM} \mathrm{MgSO}_{4}$ and one cOmplete ${ }^{\mathrm{TM}}$ protease inhibitor pill (Roche, Basel, Switserland) and $1 \mathrm{mg}$ DnaseI (Roche) per $50 \mathrm{~mL}$, and lysed by passing them through a precooled SLM Aminco French Pressure Cell three times. Subsequently, cell debris was removed by centrifugation $\left(39,000 \mathrm{~g}, 45 \mathrm{~min}, 4^{\circ} \mathrm{C}\right)$ and the obtained soluble fractions were used for the reactions.

VAO activity was determined by monitoring the conversion of vanillyl alcohol (4-hydroxy-3methoxybenzyl alcohol) to vanillin (4-hydroxy-3-methoxybenzaldehyde) at $25^{\circ} \mathrm{C}$ in $50 \mathrm{mM}$ potassium phosphate buffer, $\mathrm{pH} 7.5$, by measuring the absorption of the product at $340 \mathrm{~nm}(\varepsilon$ $=14,000 \mathrm{M}^{-1} \mathrm{~cm}^{-1}$ ) using a Hewlett Packard 8453 diode array spectrophotometer (Agilent Technologies, Santa Clara, CA, USA).

\section{Gas chromatography}

Gas chromatography experiments were performed on a GC-2010 plus (Shimadzu, Kyoto, Japan) with an FID detector $\left(260^{\circ} \mathrm{C}\right)$ and a Hydrodex $\beta$-6TBDM column (25 m x $2.45 \mathrm{~mm}$, Machery-Nagel, Düren, Germany). Hydrogen was used as a carrier gas at a flow rate of 1.5 $\mathrm{mL} / \mathrm{min}$ and the injector temperature was $80^{\circ} \mathrm{C}$. Runs were performed using a $20 \mathrm{~min}$ gradient from 80 to $220^{\circ} \mathrm{C}$ followed by $10 \mathrm{~min}$ at $220^{\circ} \mathrm{C}$. 


\section{Analytical-scale conversions of 4-ethylphenol}

$1(5 \mathrm{mg}, 41 \mu \mathrm{mol}), \mathrm{VAO}(0.4 \mathrm{U}, 0.8 \mathrm{~mL}$ of a $0.5 \mathrm{U} / \mathrm{mL}$ solution in $50 \mathrm{mM}$ potassium phosphate buffer, $\mathrm{pH} 7.5)$ the specified organic solvent $(1.4 \mathrm{~mL})$ and $100 \mathrm{mM}$ potassium phosphate buffer, $\mathrm{pH} 7.5,(11.8 \mathrm{~mL})$ were stirred at $30^{\circ} \mathrm{C}$. After the reaction, the solution was concentrated under reduced pressure. The residue was extracted with EtOAc $(4 \times 5 \mathrm{~mL})$. The organic layer was dried over $\mathrm{Na}_{2} \mathrm{SO}_{4}$ and filtered, and the solvent was removed by evaporation. The residue was dissolved in $\mathrm{MeOH}(1 \mathrm{~mL})$ and analysed by GC.

Scale-up to the $100 \mathrm{mg}$ scale was performed using the same procedure, but with the volume of the reaction mixture adapted to achieve the desired substrate concentration and using $2 \mathrm{U}$ VAO (or His-VAO cell-free extract).

Synthesis of (R)-1-(4'-hydroxyphenyl)ethanol at $1 \mathrm{~g}$ scale

$1(1 \mathrm{~g}, 8.2 \mathrm{mmol})$ was dissolved in acetone $(34.5 \mathrm{~mL}) .100 \mathrm{mM}$ potassium phosphate buffer, $\mathrm{pH} 7.5,(296 \mathrm{~mL})$ and His-VAO cell-free extract $(20 \mathrm{U}, 14.3 \mathrm{~mL}$ of a $1.4 \mathrm{U} / \mathrm{mL}$ solution) were added to the solution. The solution was stirred with a magnetic stirrer. The reaction solution was stirred for $24 \mathrm{~h}$ at $30^{\circ} \mathrm{C}$ (water bath) under air atmosphere. After $24 \mathrm{~h}$, the solution was concentrated under reduced pressure at $50{ }^{\circ} \mathrm{C}$ (water bath). During concentration, a slimy white solid was formed. The suspension was filtered and the aqueous phase was extracted with EtOAc. The organic phase was washed with brine, dried over $\mathrm{Na}_{2} \mathrm{SO}_{4}$ and concentrated under reduced pressure to give a crude compound (502 $\mathrm{mg}$, green beige solid). This compound was analysed by GC and ${ }^{1} \mathrm{H}-\mathrm{NMR}$ to evaluate the success of the conversion. Subsequently, the crude product was purified by recrystallisation. EtOAc $(3 \mathrm{~mL})$ was added to the crude product and the suspension was heated to $80^{\circ} \mathrm{C}$ (oil bath) until all solid was dissolved. Subsequently, the solution was cooled to room temperature, during which a solid crystallised. The solid was filtered and washed with a hexane/EtOAc mixture (1/1). The solid was dried under reduced pressure at $50{ }^{\circ} \mathrm{C}$ (water bath) to give the product ( $244.3 \mathrm{mg}$, white solid, 22\%).

Synthesis of (R)-1-(4'-hydroxyphenyl)ethanol at $10 \mathrm{~g}$ scale

$1(10 \mathrm{~g}, 82 \mathrm{mmol})$ was dissolved in acetone $(345 \mathrm{~mL}) .100 \mathrm{mM}$ potassium phosphate buffer, pH 7.5, (3075 mL) and His-VAO cell-free extract $(202 \mathrm{U}, 31 \mathrm{~mL}$ of a $6.5 \mathrm{U} / \mathrm{mL}$ solution) were added to the solution. The solution was stirred with an overhead stirrer $(230 \mathrm{rpm})$. The reaction vessel was evacuated and then brought under $\mathrm{O}_{2}$ atmosphere. The solution was stirred for $9 \mathrm{~h}$ under $\mathrm{O}_{2}$ atmosphere and, subsequently, $15 \mathrm{~h}$ under air atmosphere at $30{ }^{\circ} \mathrm{C}$ (water bath). After $24 \mathrm{~h}$, the solution was concentrated under reduced pressure at $50^{\circ} \mathrm{C}$ (water bath). During concentration, a slimy white solid was formed. The suspension was filtered and the aqueous phase was extracted with EtOAc. The organic phase was washed with brine, dried over $\mathrm{Na}_{2} \mathrm{SO}_{4}$ and concentrated under reduced pressure to give a crude compound (6.51 $\mathrm{g}$, green beige solid). This compound was analysed by GC and ${ }^{1} \mathrm{H}-\mathrm{NMR}$ to evaluate the success of the conversion. Subsequently, the crude product was purified by recrystallisation. EtOAc $(38 \mathrm{~mL})$ was added to the crude compound and the suspension was heated to $90^{\circ} \mathrm{C}$ (oil bath) until all solid was dissolved. Subsequently, the solution was cooled 
to room temperature, during which a solid crystallised. The solid was filtered and washed with a hexane/EtOAc mixture (1/1). The solid was dried under reduced pressure at $50{ }^{\circ} \mathrm{C}$ (water bath) to give the product (4.10 g, white solid, 36\%).

\section{Acknowledgements}

We would like to thank Prof. Dr. Marco Fraaije (University of Groningen, The Netherlands) for supplying the pJ404-His-VAO plasmid and Dr. Caroline Paul (Wageningen University \& Research) for critically reading the manuscript. This work was funded by the European Union under the INDOX project (FP7-KBBE-2013-7-613549).

\section{References}

1. Enthaler, S., and Company, A. (2011) Palladium-catalysed hydroxylation and alkoxylation. Chem. Soc. Rev. 40, 4912-4924

2. Lindhorst, A. C., Haslinger, S., and Kühn, F. E. (2015) Molecular iron complexes as catalysts for selective C-H bond oxygenation reactions. Chem. Commun. 51, 17193-17212

3. Kovaleva, E. G., and Lipscomb, J. D. (2008) Versatility of biological non-heme Fe(II) centers in oxygen activation reactions. Nat. Chem. Biol. 4, 186-193

4. Leisch, H., Morley, K., and Lau, P. C. K. (2011) Baeyer-Villiger monooxygenases: More than just green chemistry. Chem. Rev. 111, 4165-4222

5. Hofrichter, M., and Ullrich, R. (2014) Oxidations catalyzed by fungal peroxygenases. Curr. Opin. Chem. Biol. 19, 116-25

6. Girvan, H. M., and Munro, A. W. (2016) Applications of microbial cytochrome P450 enzymes in biotechnology and synthetic biology. Curr. Opin. Chem. Biol. 31, 136-145

7. Romero, E., Gómez Castellanos, J. R., Gadda, G., Fraaije, M. W., and Mattevi, A. (2018) Same substrate, many reactions: Oxygen activation in flavoenzymes. Chem. Rev. 118, 1742-1769

8. Bühler, B., Bollhalder, I., Hauer, B., Witholt, B., and Schmid, A. (2003) Chemical biotechnology for the specific oxyfunctionalization of hydrocarbons on a technical scale. Biotechnol. Bioeng. 82, 833-842

9. Baldwin, C. V. F., Wohlgemuth, R., and Woodley, J. M. (2008) The first 200-L scale asymmetric Baeyer-Villiger oxidation using a whole-cell biocatalyst. Org. Process Res. Dev. 12, 660-665

10. Bernhardt, R., and Urlacher, V. B. (2014) Cytochromes P450 as promising catalysts for biotechnological application: Chances and limitations. Appl. Microbiol. Biotechnol. 98, 6185-6203

11. Holtmann, D., Fraaije, M. W., Arends, I. W. C. E., Opperman, D. J., and Hollmann, F. (2014) The taming of oxygen: Biocatalytic oxyfunctionalisations. Chem. Commun. 50, 13180-13200

12. Fernández-Fueyo, E., Ni, Y., Gomez Baraibar, A., Alcalde, M., van Langen, L. M., and Hollmann, F. (2016) Towards preparative peroxygenase-catalyzed oxyfunctionalization reactions in organic media. J. Mol. Catal. B Enzym. 134, 347-352

13. Martínez, A. T., Ruiz-Dueñas, F. J., Camarero, S., Serrano, A., Linde, D., Lund, H., Vind, J., Tovborg, M., Herold-Majumdar, O. M., Hofrichter, M., Liers, C., Ullrich, R., Scheibner, K., Sannia, G., Piscitelli, A., Pezzella, C., Sener, M. E., Kılıç, S., van Berkel, W. J. H., Guallar, V., Lucas, M. F., Zuhse, R., Ludwig, R., Hollmann, F., Fernández-Fueyo, E., Record, E., Faulds, C. B., Tortajada, M., Winckelmann, I., Rasmussen, J.-A., Gelo-Pujic, M., Gutiérrez, A., del Río, J. C., Rencoret, J., and Alcalde, M. (2017) Oxidoreductases on their way to industrial biotransformations. Biotechnol. Adv. 35, 815-831

14. Zheng, G.-W., and Xu, J.-H. (2011) New opportunities for biocatalysis: Driving the synthesis of chiral chemicals. Curr. Opin. Biotechnol. 22, 784-792

15. Karl, U., and Simon, A. (2009) BASF's ChiPros ${ }^{\circledR}$ chiral building blocks: The cornerstones of your API syntheses! Chim. Oggi. 27, 66-69 
16. Patel, R. N. (2013) Biocatalytic synthesis of chiral alcohols and amino acids for development of pharmaceuticals. Biomolecules. 3, 741-777

17. Stampfer, W., Kosjek, B., Faber, K., and Kroutil, W. (2003) Biocatalytic asymmetric hydrogen transfer employing Rhodococcus ruber DSM 44541. J. Org. Chem. 68, 402-406

18. Neupert, A., Ress, T., Wittmann, J., Hummel, W., and Gröger, H. (2010) Enantioselective biocatalytic reduction of non-protected hydroxyacetophenones. Z. Naturforsch. B. 65, 337-340

19. Silva, C. R., Souza, J. C., Araújo, L. S., Kagohara, E., Garcia, T. P., Pelizzari, V. H., and Andrade, L. H. (2012) Exploiting the enzymatic machinery of Arthrobacter atrocyaneus for oxidative kinetic resolution of secondary alcohols. J. Mol. Catal. B Enzym. 83, 23-28

20. Wuensch, C., Gross, J., Steinkellner, G., Gruber, K., Glueck, S. M., and Faber, K. (2013) Asymmetric enzymatic hydration of hydroxystyrene derivatives. Angew. Chem. Int. Ed. 52, 22932297

21. Nasário, F. D., Cazetta, T., Moran, P. J. S., and Rodrigues, J. A. R. (2016) Deracemization of 1phenylethanol via tandem biocatalytic oxidation and reduction. Tetrahedron: Asymmetry. 27, 404-409

22. Payer, S. E., Pollak, H., Glueck, S. M., and Faber, K. (2018) A rational active-site redesign converts a decarboxylase into a $\mathrm{C}=\mathrm{C}$ hydratase: "Tethered acetate" supports enantioselective hydration of 4-hydroxystyrenes. ACS Catal. 8, 2438-2442

23. de Jong, E., van Berkel, W. J. H., van der Zwan, R. P., and de Bont, J. A. M. (1992) Purification and characterization of vanillyl-alcohol oxidase from Penicillium simplicissimum: A novel aromatic alcohol oxidase containing covalently bound FAD. Eur. J. Biochem. 208, 651-657

24. Fraaije, M. W., Veeger, C., and van Berkel, W. J. H. (1995) Substrate specificity of flavindependent vanillyl-alcohol oxidase from Penicillium simplicissimum: Evidence for the production of 4-hydroxycinnamyl alcohols from 4-allylphenols. Eur. J. Biochem. 234, 271-277

25. van den Heuvel, R. H. H., Fraaije, M. W., Laane, C., and van Berkel, W. J. H. (1998) Regio- and stereospecific conversion of 4-alkylphenols by the covalent flavoprotein vanillyl-alcohol oxidase. J. Bacteriol. 180, 5646-5651

26. Ewing, T. A., van Noord, A., Paul, C. E., and van Berkel, W. J. H. (2018) A xylenol orange-based screening assay for the substrate specificity of flavin-dependent para-phenol oxidases. Molecules. 23, 164

27. van den Heuvel, R. H. H., Partridge, J., Laane, C., Halling, P. J., and van Berkel, W. J. H. (2001) Tuning of the product spectrum of vanillyl-alcohol oxidase by medium engineering. FEBS Lett. 503, 213-216

28. Drijfhout, F. P., Fraaije, M. W., Jongejan, H., van Berkel, W. J. H., and Franssen, M. C. R. (1998) Enantioselective hydroxylation of 4-alkylphenols by vanillyl alcohol oxidase. Biotechnol. Bioeng. 59, 171-177

29. Fraaije, M. W., and van Berkel, W. J. H. (1997) Catalytic mechanism of the oxidative demethylation of 4-(methoxymethyl)phenol by vanillyl-alcohol oxidase: Evidence for formation of a p-quinone methide intermediate. J. Biol. Chem. 272, 18111-18116

30. Fraaije, M. W., van den Heuvel, R. H. H., Roelofs, J. C. A. A., and van Berkel, W. J. H. (1998) Kinetic mechanism of vanillyl-alcohol oxidase with short-chain 4-alkylphenols. Eur. J. Biochem. 253, 712-719

31. Ewing, T. A., Nguyen, Q.-T., Allan, R. C., Gygli, G., Romero, E., Binda, C., Fraaije, M. W., Mattevi, A., and van Berkel, W. J. H. (2017) Two tyrosine residues, Tyr-108 and Tyr-503, are responsible for the deprotonation of phenolic substrates in vanillyl-alcohol oxidase. J. Biol. Chem. 292, 1466814679

32. van den Heuvel, R. H. H., Fraaije, M. W., Ferrer, M., Mattevi, A., and van Berkel, W. J. H. (2000) Inversion of stereospecificity of vanillyl-alcohol oxidase. Proc. Natl. Acad. Sci. U. S. A. 97, 94559460

33. van den Heuvel, R. H. H., Fraaije, M. W., Mattevi, A., and van Berkel, W. J. H. (2000) Asp-170 is crucial for the redox properties of vanillyl-alcohol oxidase. J. Biol. Chem. 275, 14799-14808 
34. Kaltofen, R. K. (1998) Tabellenbuch Chemie, Verlag Harri Deutsch (Frankfurt am Main)

35. Baldwin, C. V. F., and Woodley, J. M. (2006) On oxygen limitation in a whole cell biocatalytic Baeyer-Villiger oxidation process. Biotechnol. Bioeng. 95, 362-369

36. Hilker, I., Baldwin, C., Alphand, V., Furstoss, R., Woodley, J., and Wohlgemuth, R. (2006) On the influence of oxygen and cell concentration in an SFPR whole cell biocatalytic Baeyer-Villiger oxidation process. Biotechnol. Bioeng. 93, 1138-1144

37. Ewing, T. A., Gygli, G., and van Berkel, W. J. H. (2016) A single loop is essential for the octamerization of vanillyl alcohol oxidase. FEBS J. 283, 2546-2559

\section{Abbreviations}

FAD: flavin adenine dinucleotide, (His)-VAO: (His-tagged)-vanillyl alcohol oxidase 


\section{Supplementary Figures}

$10 \%$ acetone, $4 \mathrm{~h}$

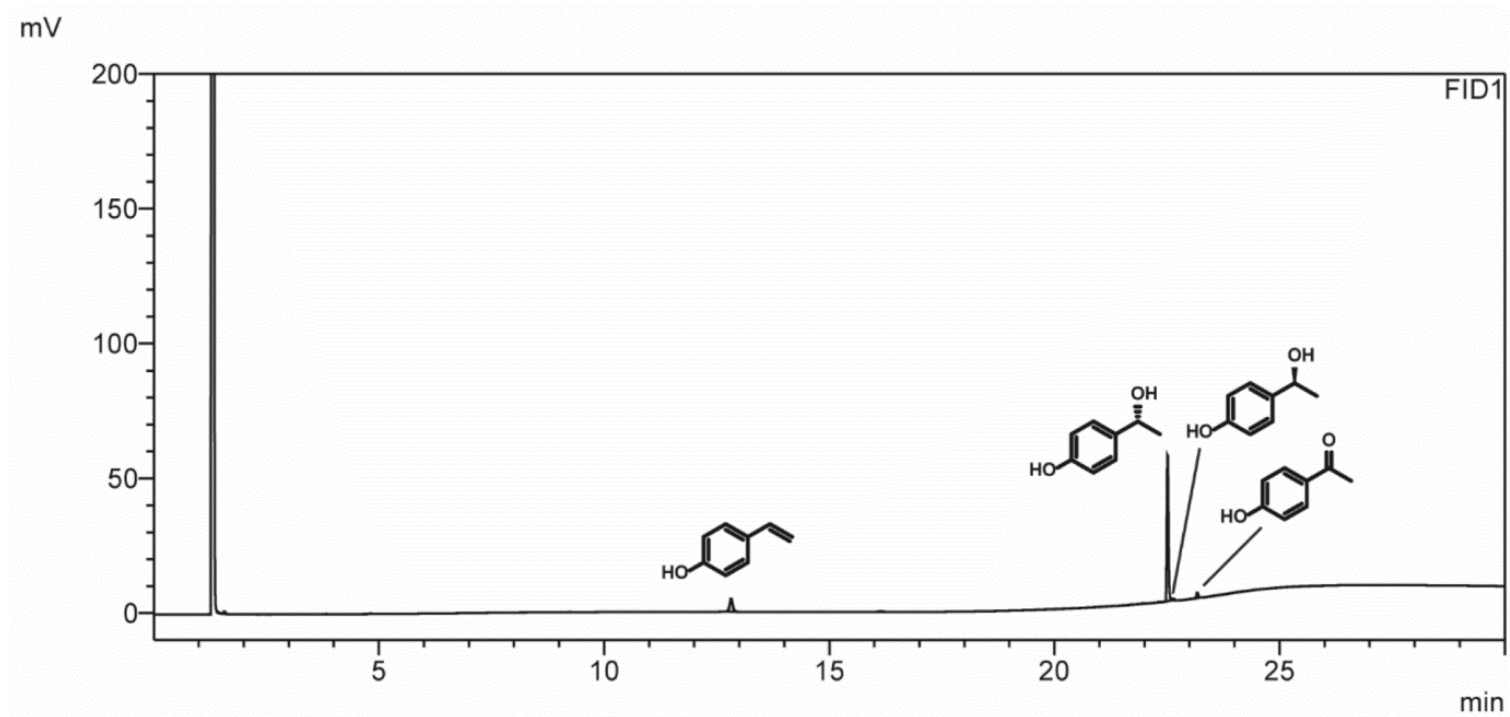

$10 \%$ acetonitrile, $22 \mathrm{~h}$

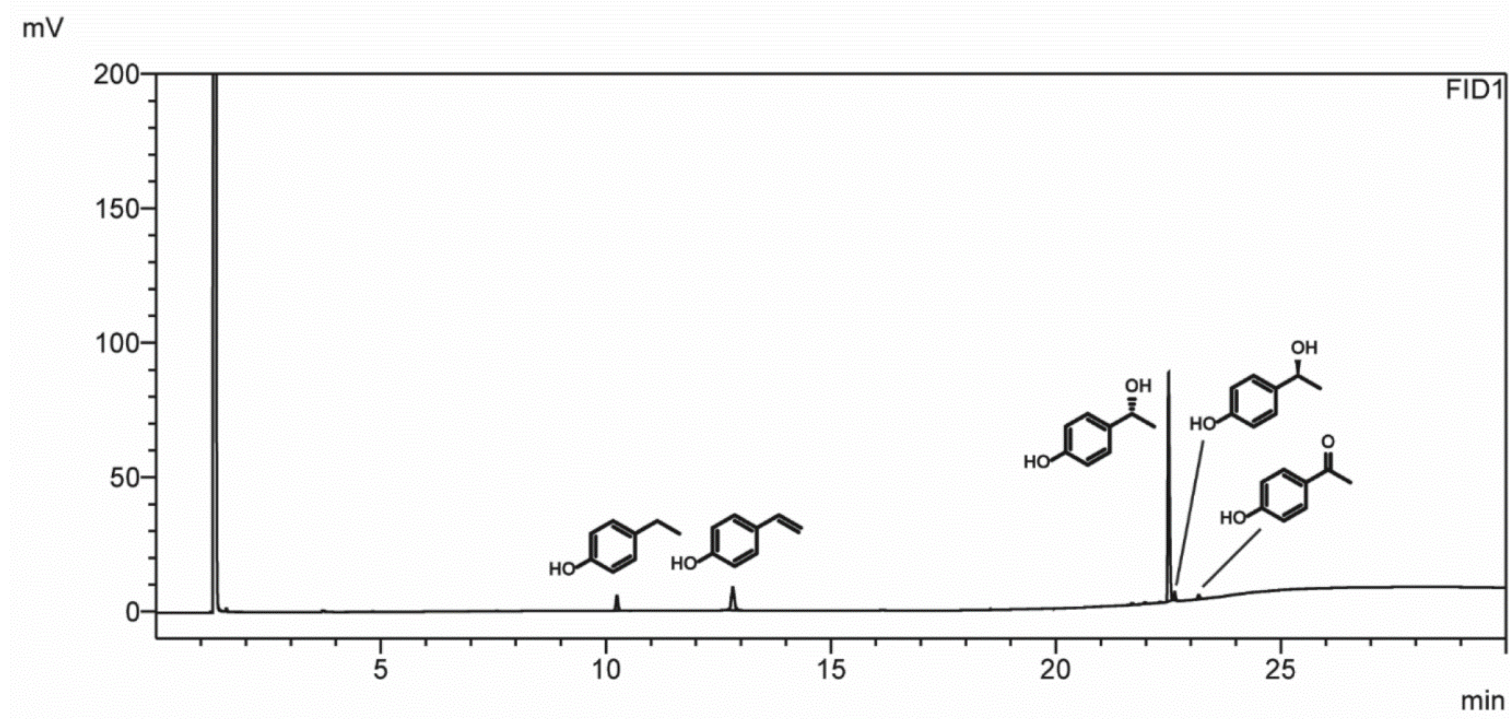

Figure S1: GC chromatograms showing the products obtained from the conversion of 4-ethylphenol by VAO. Reaction mixtures containing $5 \mathrm{mg}$ 4-ethylphenol (at a concentration of $2.9 \mathrm{mM}$ ) and $0.4 \mathrm{U}$ $\mathrm{VAO}$ in $84 \mathrm{mM}$ potassium phosphate buffer, $\mathrm{pH} 7.5$, containing $10 \%$ acetone or $10 \%$ acetonitrile were incubated at $30{ }^{\circ} \mathrm{C}$ for the specified reaction times. Subsequently, the reaction products were extracted and analysed by GC. Retention times: 4-ethylphenol (1): $10.2 \mathrm{~min}$, 4-vinylphenol (4): $12.8 \mathrm{~min},(R)-1-$ (4'-hydroxyphenyl)ethanol (2): $22.5 \mathrm{~min},(S)-1-\left(4^{\prime}\right.$-hydroxyphenyl)ethanol (3): $22.6 \mathrm{~min}, 4$ hydroxyacetophenone (5): $23.2 \mathrm{~min}$. 
Reaction a: $100 \mathrm{mg}, 23 \mathrm{mM}$ 4-ethylphenol, 2 U VAO

$\mathrm{mV}$

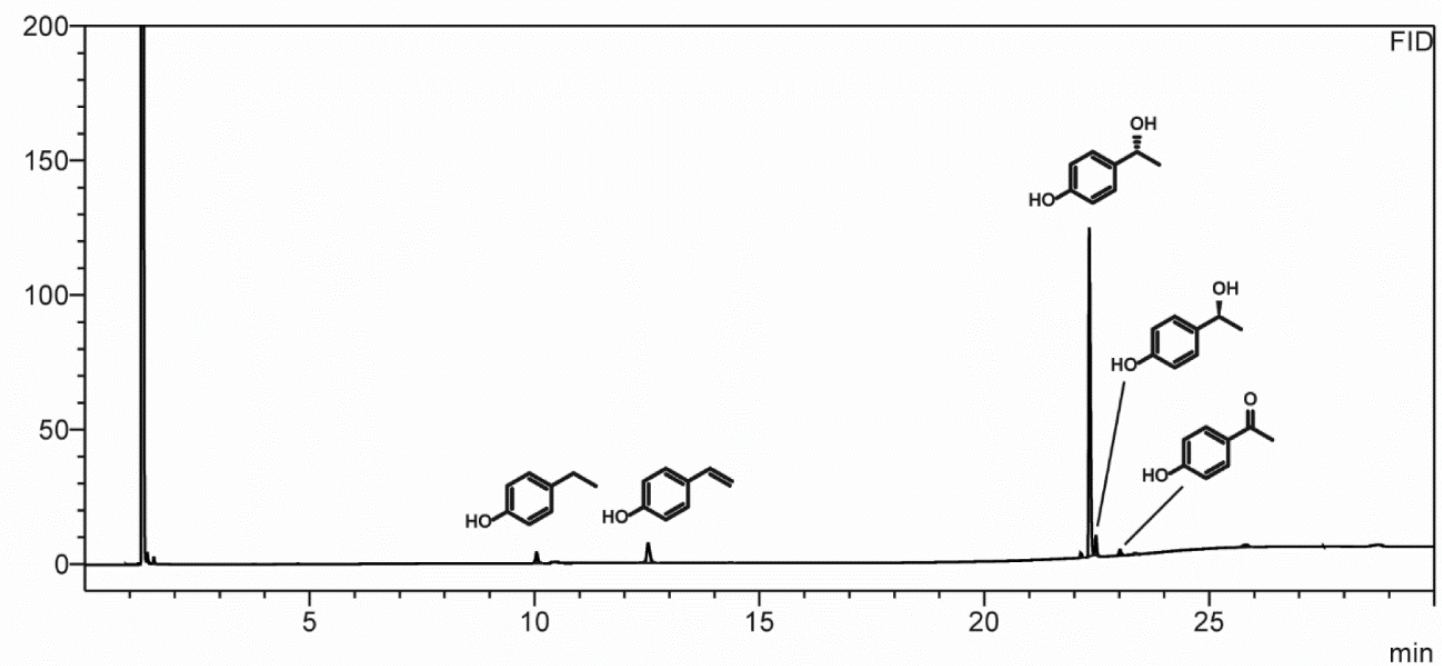

Reaction b: 100 mg, 45 mM 4-ethylphenol, 2 U VAO

$\mathrm{mV}$

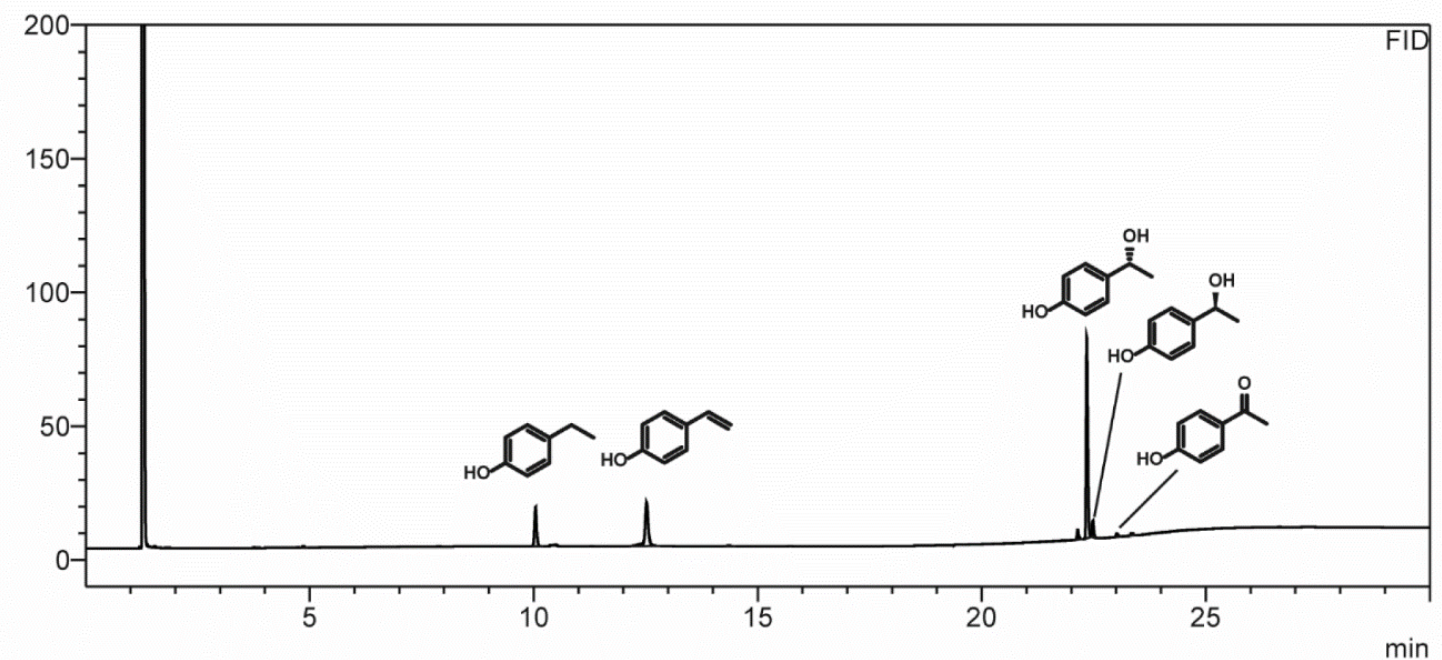

Reaction c: $100 \mathrm{mg}, 23 \mathrm{mM}$ 4-ethylphenol, $2 \mathrm{U}$ His-VAO cell-free extract $\mathrm{mV}$

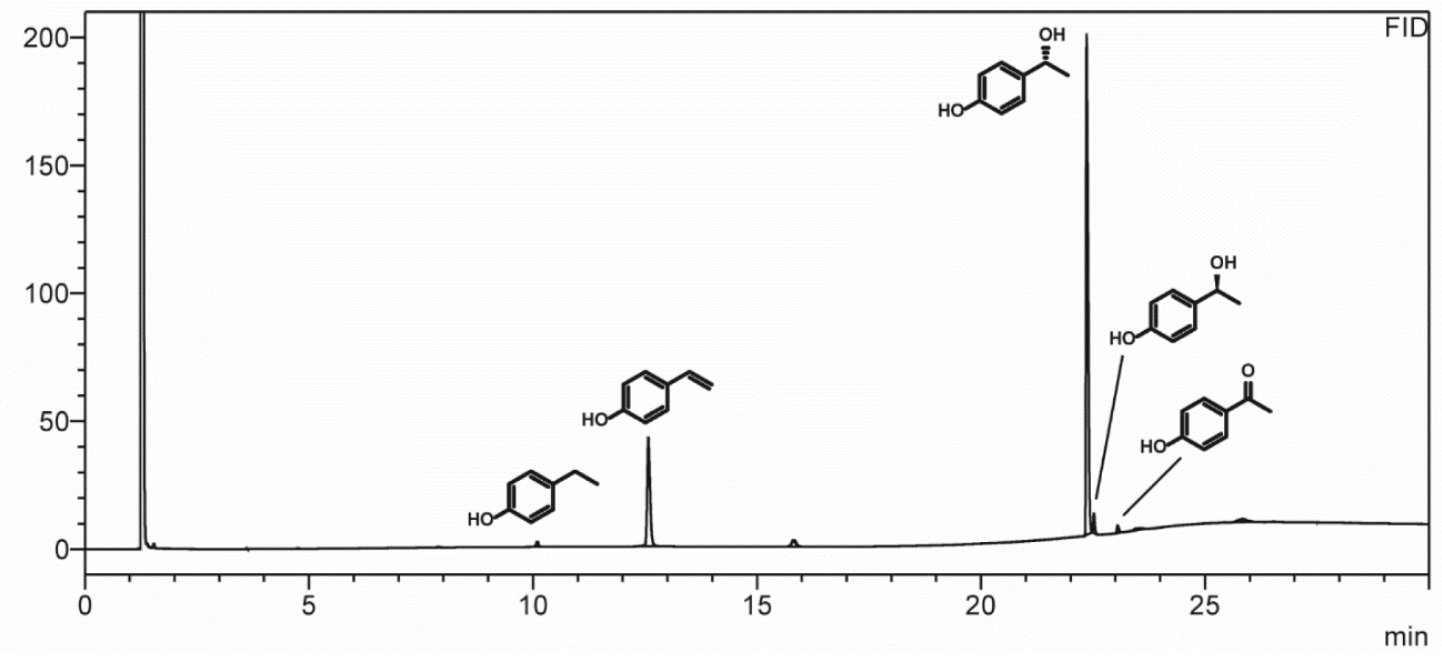


Reaction d: 1.0 g, 23 mM 4-ethylphenol, 20 U His-VAO cell-free extract $\mathrm{mV}$

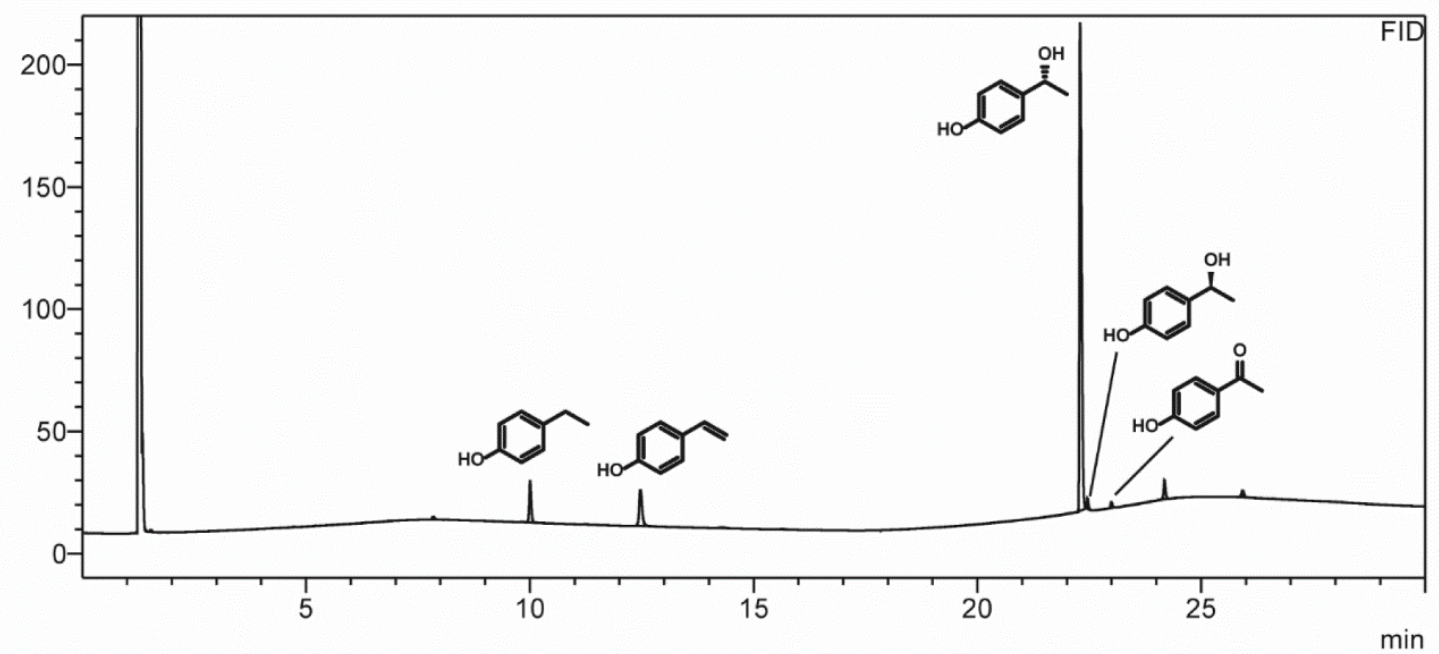

Reaction e: 13.4 g, 23 mM 4-ethylphenol, 268 U His-VAO cell-free extract $\mathrm{mV}$

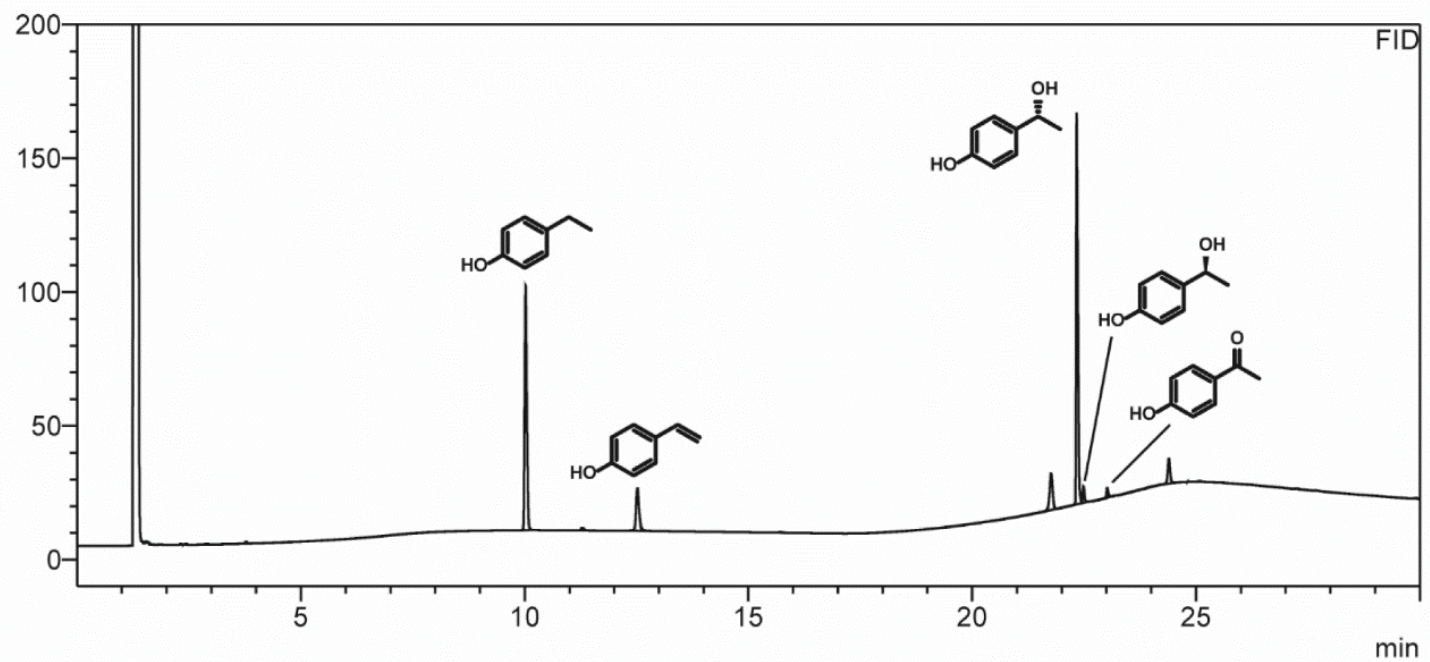

Reaction f: $10.0 \mathrm{~g}, 23 \mathrm{mM}$ 4-ethylphenol, $202 \mathrm{U}$ His-VAO cell-free extract $\mathrm{O}_{2}$ atmosphere for first $9 \mathrm{~h}$

$\mathrm{mV}$

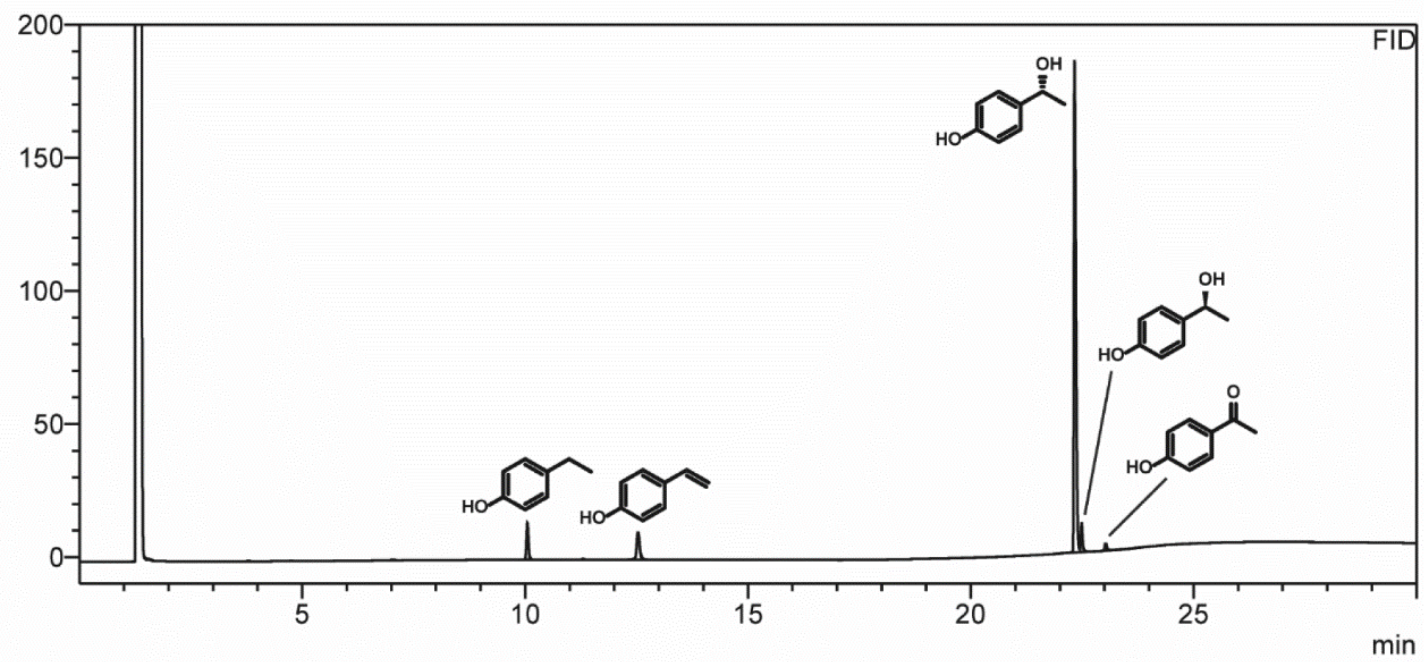


Figure S2: GC chromatograms showing the products obtained from the conversion of 4-ethylphenol by VAO. Reaction mixtures containing 4-ethylphenol and VAO or His-VAO cell-free extract were incubated in $88 \mathrm{mM}$ potassium phosphate buffer, $\mathrm{pH}$ 7.5, containing 10\% acetone for 24 hours at $30{ }^{\circ} \mathrm{C}$. Subsequently, the reaction products were extracted and analysed by GC. Reactions are labelled as in Table 2. Retention times: 4-ethylphenol (1): $10.0 \mathrm{~min}$, 4-vinylphenol (4): $12.5 \mathrm{~min},(R)-1-\left(4^{\prime}-\right.$ hydroxyphenyl)ethanol (2): $22.3 \mathrm{~min},(S)-1-\left(4^{\prime}\right.$-hydroxyphenyl)ethanol (3): $22.5 \mathrm{~min}, 4$ hydroxyacetophenone (5): $23.0 \mathrm{~min}$.

\section{Reaction d, after recrystallisation}

$\mathrm{mV}$

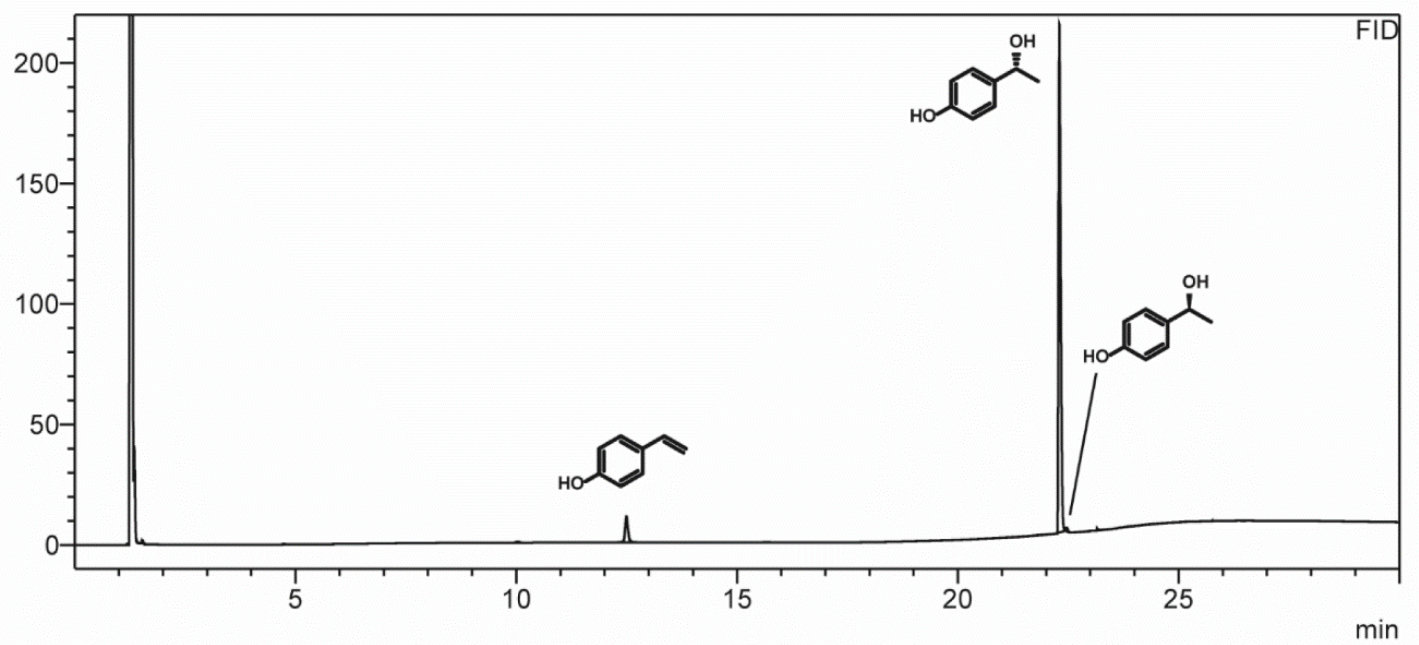

\section{Reaction $\mathrm{f}$, after recrystallisation}

$\mathrm{mV}$

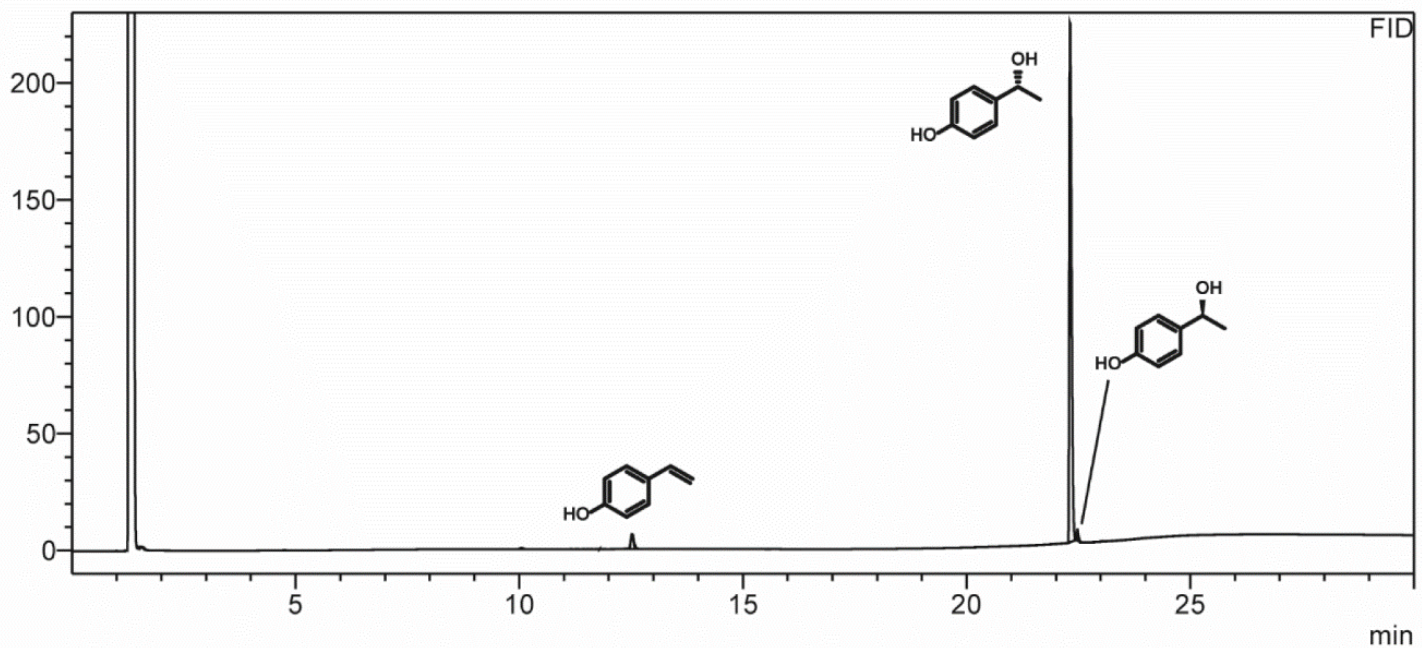

Figure S3: GC chromatograms of purified $(R)-1-\left(4^{\prime}\right.$-hydroxyphenyl)ethanol. Pure $(R)-1-\left(4^{\prime}-\right.$ hydroxyphenyl)ethanol was obtained from crude product mixtures from the conversion of 4ethylphenol by His-VAO cell-free extract by recrystallisation and analysed by GC. The 4 -vinylphenol observed in the GC chromatograms is formed by the decomposition of 1-(4'-hydroxyphenyl)ethanol during analysis, as demonstrated by the absence of any signal corresponding to 4-vinylphenol in the ${ }^{1} \mathrm{H}-\mathrm{NMR}$ spectrum of the product and the presence of 4-vinylphenol upon GC analysis of pure racemic 1-(4'-hydroxyphenyl)ethanol. Reactions are labelled as in Table 2. Retention times: 4vinylphenol (4): $12.5 \mathrm{~min},(R)-1-\left(4^{\prime}\right.$-hydroxyphenyl)ethanol (2): $22.3 \mathrm{~min},(S)-1-\left(4^{\prime}-\right.$ hydroxyphenyl)ethanol (3): $22.5 \mathrm{~min}$. 
Racemic 1-(4'-hydroxyphenyl)ethanol

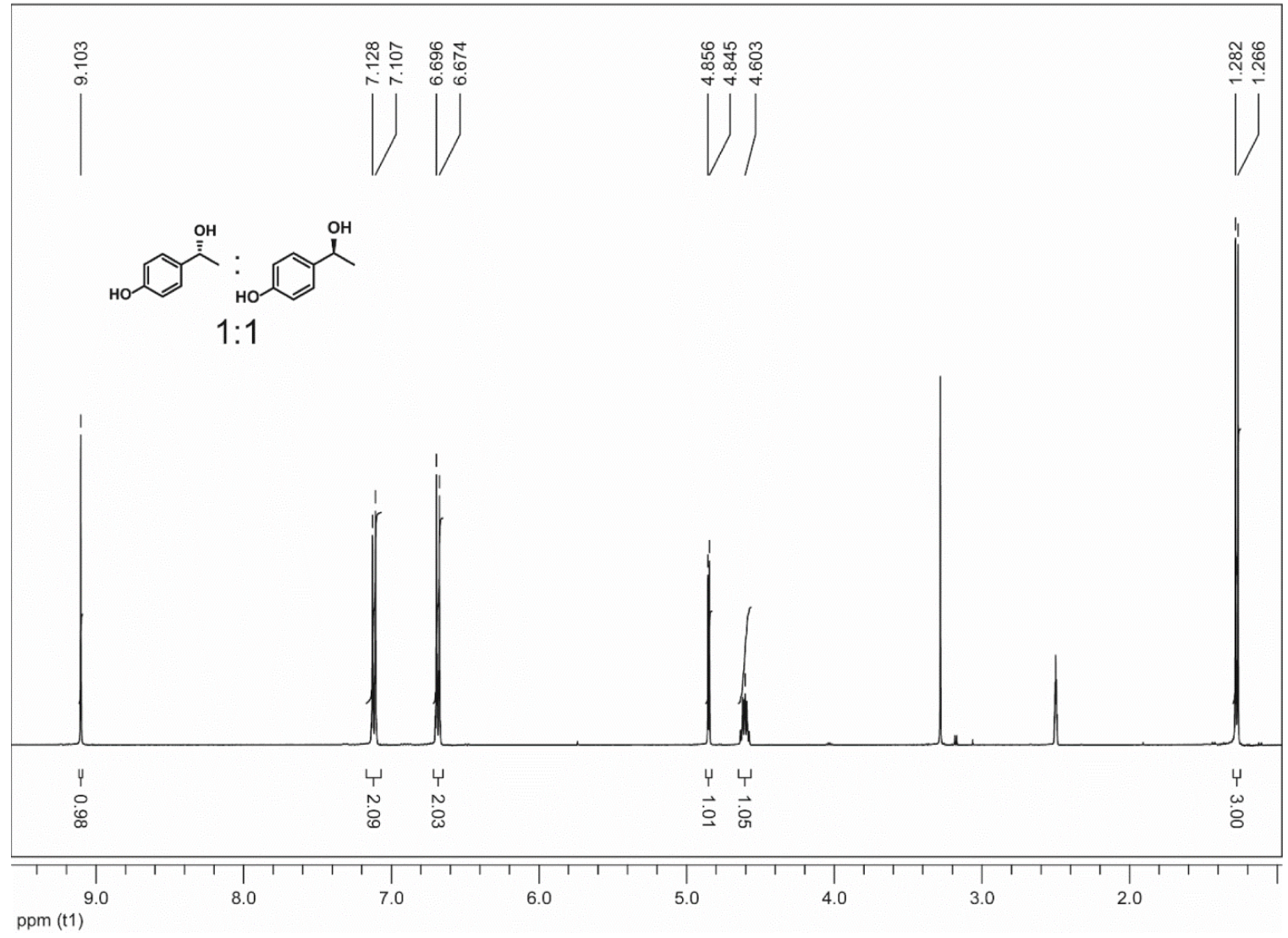

Reaction d, crude product

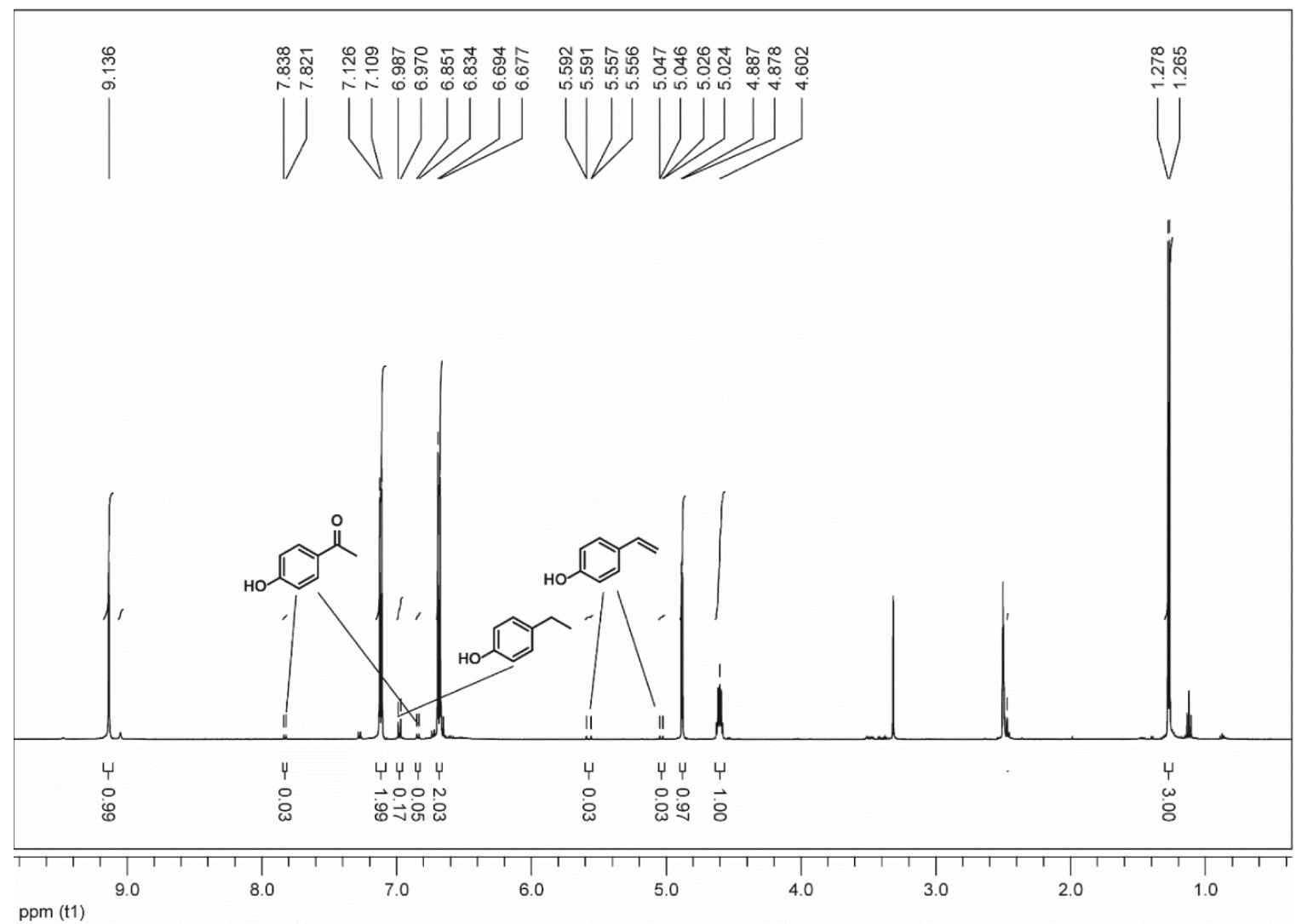




\section{Reaction d, pure product}

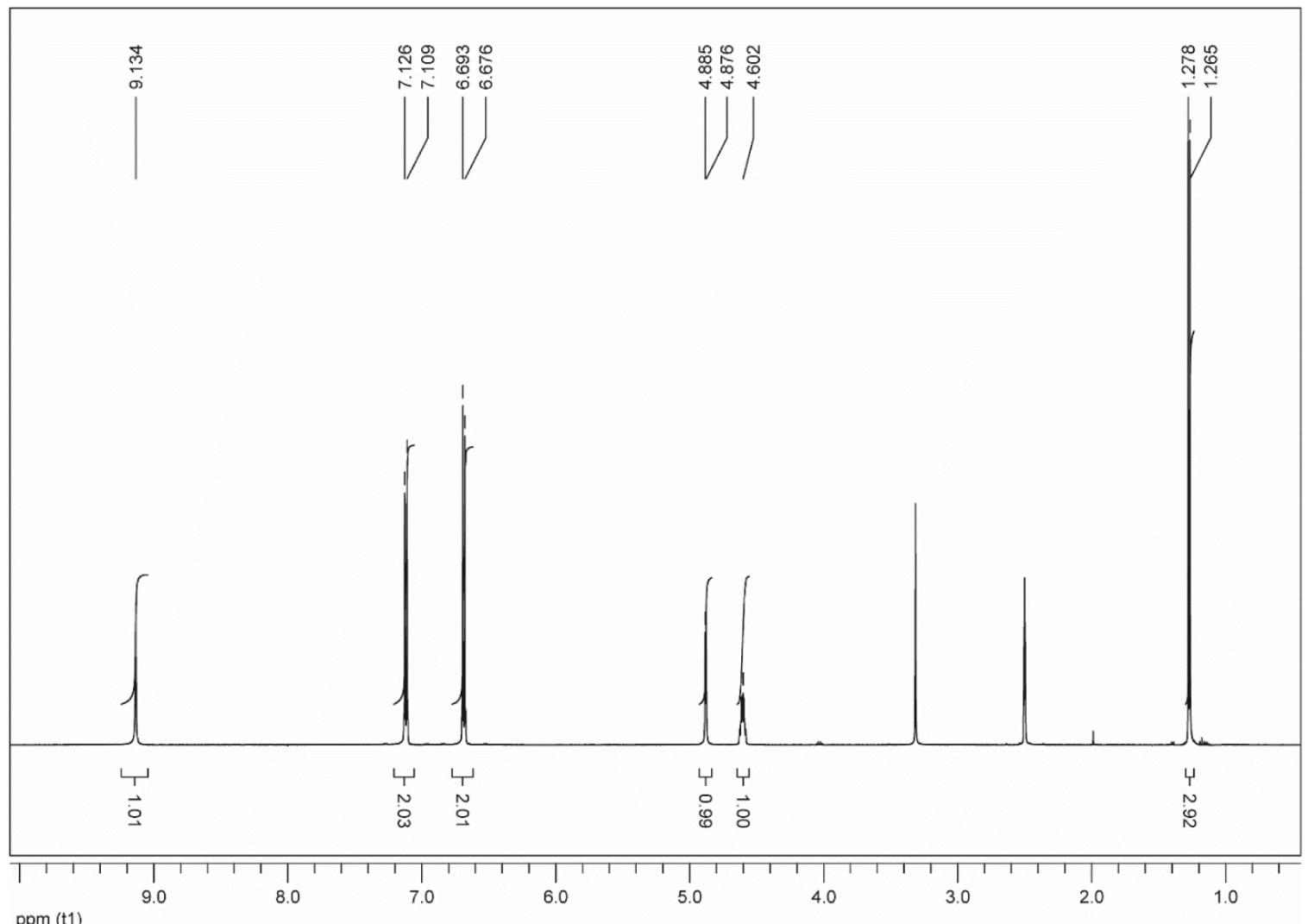

Reaction $f$, crude product

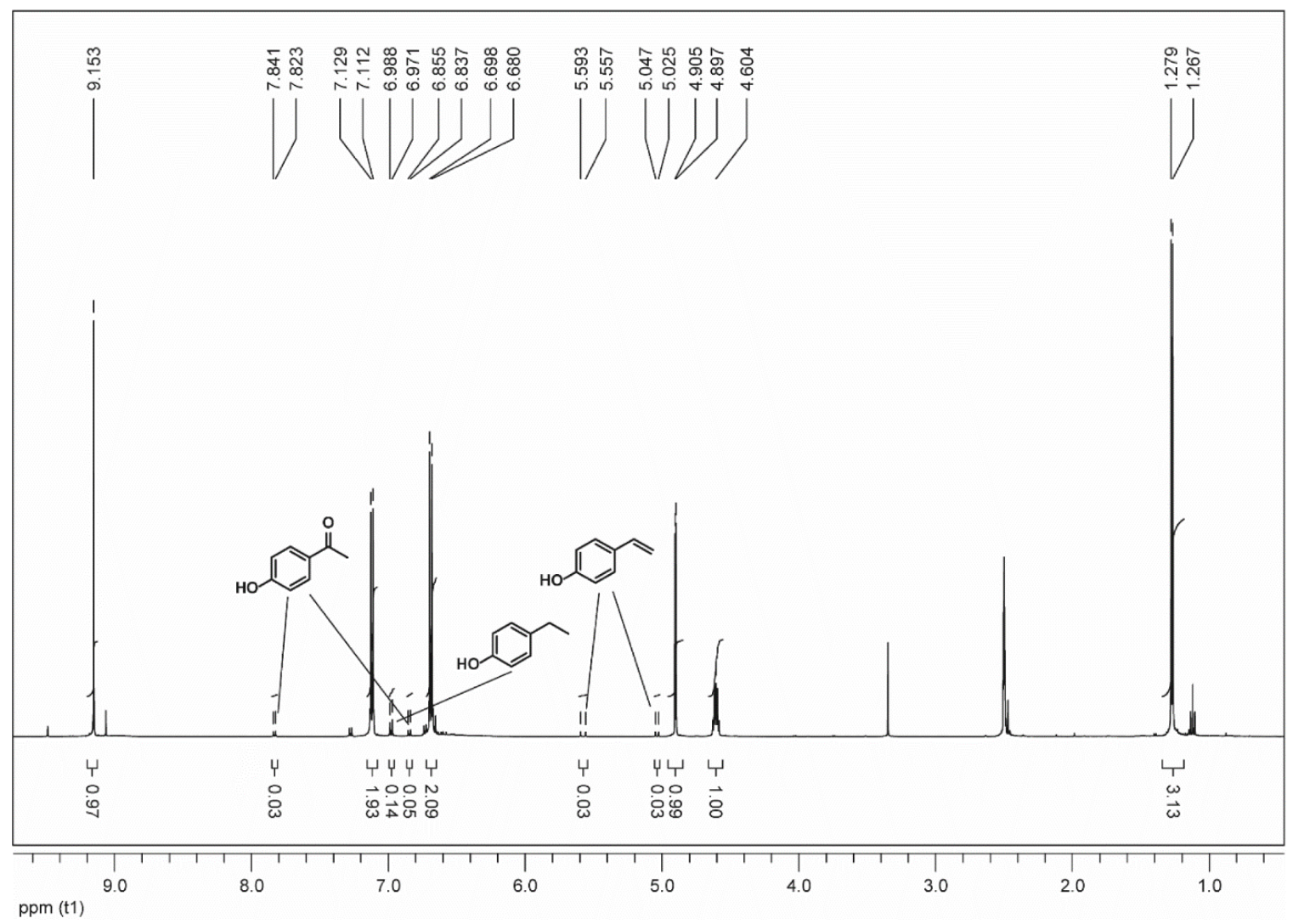




\section{Reaction f, pure product}

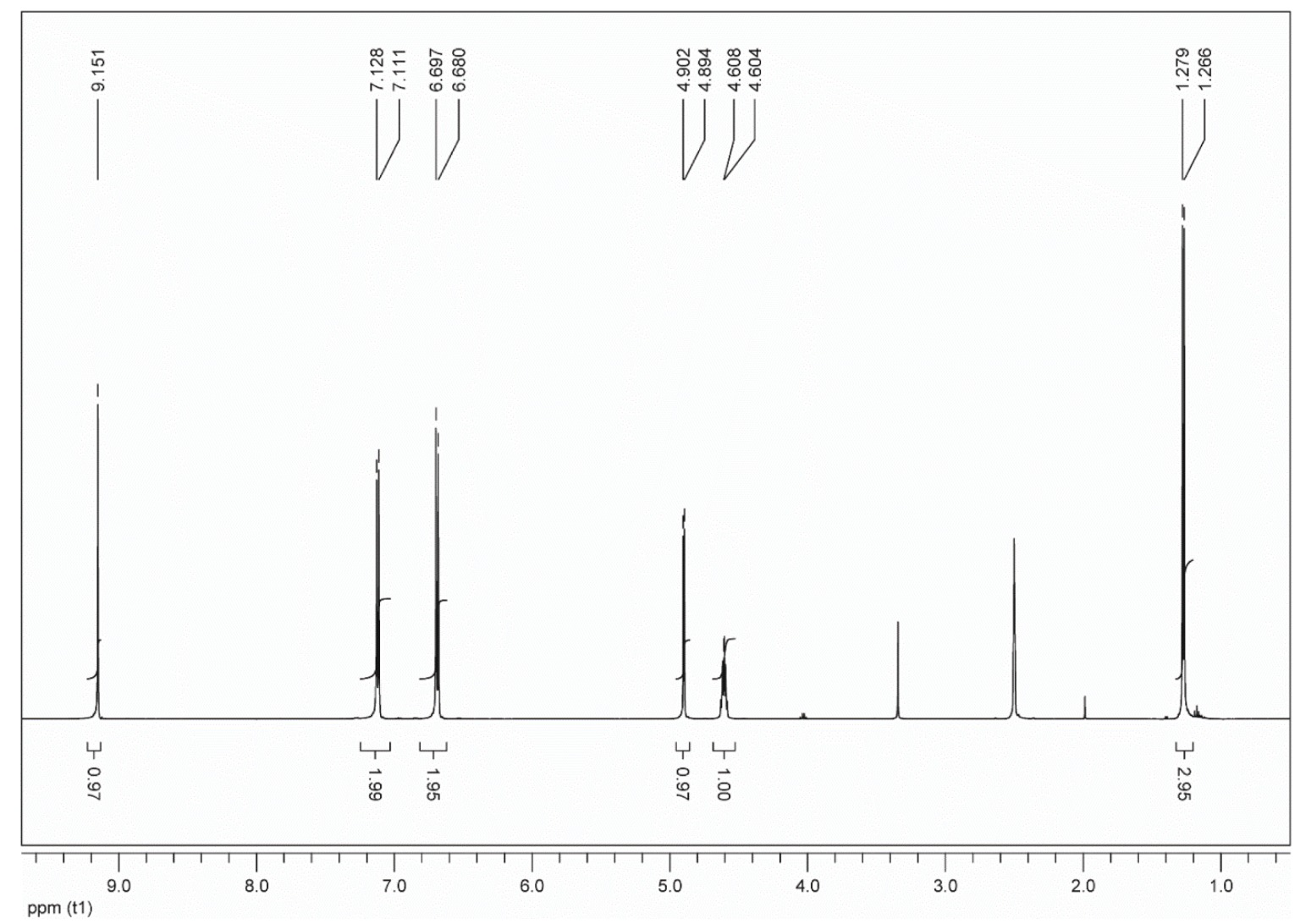

Figure S4: ${ }^{1} \mathrm{H}-\mathrm{NMR}$ spectra of the reaction products of the conversion of 4 -ethylphenol by His-VAO cell-free extract recorded in d6-DMSO. Spectra of the crude product mixtures obtained after (multi)gram scale conversion of 4-ethylphenol by His-VAO cell-free extract and of pure (R)-1-(4'hydroxyphenyl)ethanol obtained from the crude product mixtures by recrystallisation are shown. The spectrum of a racemic 1-(4'-hydroxyphenyl)ethanol standard is shown for comparison. Peaks at 2.5 and $3.3 \mathrm{ppm}$ are from the solvent. 1-(4'-hydroxyphenyl)ethanol (2+3): $\delta(\mathrm{ppm}): 9.10(\mathrm{~s}, 1 \mathrm{H}), 7.12(\mathrm{dt}, J=$ $8.02 \mathrm{~Hz}, 2 \mathrm{H}), 6.68(\mathrm{dt}, J=8.69 \mathrm{~Hz}, 2 \mathrm{H}), 4.85(\mathrm{~d}, J=4.29 \mathrm{~Hz}, 1 \mathrm{H}), 4.63-4.57(\mathrm{~m}, 1 \mathrm{H}), 1.27(\mathrm{~d}, J=6.44 \mathrm{~Hz}$, $3 \mathrm{H})$. Impurities in the crude product were identified and quantified using the following diagnostic peaks: 4-ethylphenol (1): $6.98 \mathrm{ppm}(\mathrm{m}, 2 \mathrm{H})$, 4-vinylphenol (4): 5.55-5.60 ppm (m, 1H), 5.02-5.05 ppm (m, $1 \mathrm{H})$, 4-hydroxyacetophenone (5): $7.83 \mathrm{ppm}(\mathrm{m}, 2 \mathrm{H}), 6.84 \mathrm{ppm}(\mathrm{m}, 2 \mathrm{H})$. 




\title{
The oxidation of thiols by flavoprotein oxidases:
} A biocatalytic route to reactive thiocarbonyls

\author{
Tom A. Ewing ${ }^{1^{*}}$, Willem P. Dijkman ${ }^{2 *}$, Jacques M. Vervoort ${ }^{1}$, Marco W. Fraaije ${ }^{2}$ \\ and Willem J. H. van Berkel ${ }^{1}$ \\ ${ }^{1}$ Laboratory of Biochemistry, Wageningen University \& Research, Wageningen, The Netherlands \\ ${ }^{2}$ Molecular Enzymology Group, University of Groningen, Groningen, The Netherlands \\ *Contributed equally
}

Based on Angewandte Chemie International Edition (2014), 53, 13206-13209

\begin{abstract}
Summary
Flavoprotein oxidases are a diverse class of biocatalysts, most of which catalyse the oxidation of $\mathrm{C}-\mathrm{O}, \mathrm{C}-\mathrm{N}$ or $\mathrm{C}-\mathrm{C}$ bonds. Flavoprotein oxidases that are known to catalyse the oxidation of C-S bonds are rare. Here, we report that various flavoprotein oxidases previously thought to solely act on alcohols also catalyse the oxidation of thiols to thiocarbonyls. These results highlight the versatility of enzymatic catalysis and provide a potential biocatalytic route to reactive thiocarbonyl compounds, which have a variety of applications in synthetic organic chemistry.
\end{abstract}





\section{Introduction}

Flavoprotein oxidases constitute a diverse class of oxidoreductases that contain a flavin cofactor in the form of flavin adenine dinucleotide (FAD) or flavin mononucleotide (FMN). They catalyse the oxidation of a wide range of substrates, with molecular oxygen functioning as the final oxidant. Their catalytic cycle consists of two half-reactions. During the reductive half-reaction, two electrons are transferred from the substrate to the flavin cofactor, a process that is generally believed to occur via the direct transfer of a hydride to the flavin's N5 atom $(1,2)$. In the oxidative half-reaction, the flavin cofactor is reoxidised by molecular oxygen, which is reduced to hydrogen peroxide. Unlike many other oxidative enzymes, e.g. dehydrogenases, oxidases do not require stoichiometric amounts of expensive organic cofactors, making them interesting candidates for biocatalytic applications. Most of the characterized flavoprotein oxidases catalyse the oxidation of $\mathrm{C}-\mathrm{O}, \mathrm{C}-\mathrm{N}$ or $\mathrm{C}-\mathrm{C}$ bonds to the corresponding double bonds (3). A small number of flavoprotein oxidases are known to oxidise sulfur-containing compounds. Prenylcysteine lyase (EC 1.8.3.5) and farnesylcysteine lyase (EC 1.8.3.6) catalyse the oxidative cleavage of thioethers into aldehydes and thiols, while sulfhydryl oxidases (EC 1.8.3.2 and 1.8.3.3) catalyse the formation of disulfide bonds from thiols. It has been reported that the flavin-dependent alcohol oxidases glucose oxidase from Aspergillus niger (GOX, EC 1.1.3.4) and methanol oxidase from Hanselula polymorpha (MO, or alcohol oxidase, EC 1.1.3.13) can catalyse the oxidation of thiols as well as of alcohols $(4,5)$. However, the reaction was not studied in detail. Here, we describe the finding that the flavindependent alcohol oxidases alditol oxidase from Streptomyces coelicolor (AldO, EC 1.1.3.41) and 5-(hydroxymethyl)furfural oxidase from Methylovorus sp. strain MP688 (HMFO, EC 1.1.3.47) catalyse the oxidation of thiols that are structurally similar to their alcohol substrates, yielding the corresponding thiocarbonyls.

\section{Results}

AldO is a flavin-dependent oxidase that catalyses the regioselective oxidation of the primary hydroxyl groups of a broad range of polyols, with the best substrates being alditols such as xylitol, D-sorbitol and L-threitol $(6,7)$. The oxidation of polyols by AldO occurs stereoselectively. For example, D-sorbitol is converted significantly more efficiently than its $\mathrm{C} 2$ epimer D-mannitol. AldO belongs to the VAO/PCMH family of structurally homologous flavoproteins. These flavoproteins contain a conserved FAD-binding domain as well as a variable substrate-binding domain, which determines their substrate specificity $(8$, Chapter 2). The enzyme contains an FAD cofactor which is covalently linked to a histidine residue (9). To investigate whether AldO reacts with thiols, the enzyme was first expressed and purified as a fusion protein with an $\mathrm{N}$-terminal maltose-binding protein tag. This fusion protein (mAldO) displays highly similar catalytic properties to the wild type enzyme (6). Oxygen consumption measurements showed that m-AldO catalyses the oxidation of DL-dithiothreitol (DL-DTT), which is structurally identical to the known AldO substrate threitol, except for the presence of thiol rather than hydroxyl groups at the terminal carbon atoms. Steady-state kinetic analysis revealed that $\mathrm{m}$-AldO oxidises racemic DTT with $k_{\text {cat }}=0.79 \mathrm{~s}^{-1}$ and $K_{\mathrm{m}}=3.7$ $\mathrm{mM}$ (Table 1). Enantiopure L-DTT was converted with similar efficiency. m-AldO also oxidises dithioerythritol, but the reaction with this epimer of DTT is less efficient, primarily due to a higher $K_{\mathrm{m}}$. The tested thiols are remarkably good substrates for m-AldO. The catalytic 
efficiency for the oxidation of L-DTT is very similar to that for the oxidation of its alcohol analogue L-threitol. In fact, of all known m-AldO substrates, only xylitol and D-sorbitol display higher catalytic efficiencies than L-DTT $(6,7)$.

HPLC analysis revealed that three main products are formed during the oxidation of DL-DTT by m-AldO (Fig. S1). Addition of the hydrogen peroxide scavenger sodium pyruvate to the reaction mixture demonstrated that two of the products are formed by side reactions involving hydrogen peroxide. The third compound, which is the product of the enzymatic reaction, was collected and identified by ${ }^{1} \mathrm{H}-\mathrm{NMR}$ as the dithiohemiacetal 2 [Scheme 1 (Lenantiomers shown), Fig. S2]. Formation of this product presumably occurs via the thioaldehyde intermediate 1, which is formed upon oxidation of DL-DTT by m-AldO and subsequently undergoes an intramolecular cyclisation to yield $\mathbf{2}$. This dithiohemiacetal is rather sensitive to oxidation, being entirely oxidised to the corresponding sulfenic acid after 6 days of storage at $-20{ }^{\circ} \mathrm{C}$ dissolved in acetonitrile. By comparison to a reference compound, one of the two side products was identified as the disulfide trans-4,5-dihydroxy-1,2-dithiane, which was shown to be formed upon reaction of DL-DTT with hydrogen peroxide. The second unidentified compound is likely the disulfide formed after reaction of two molecules of 2 upon its oxidation by hydrogen peroxide. Alternatively, it may be the mixed disulfide formed between one molecule of 2 and one molecule of DTT.

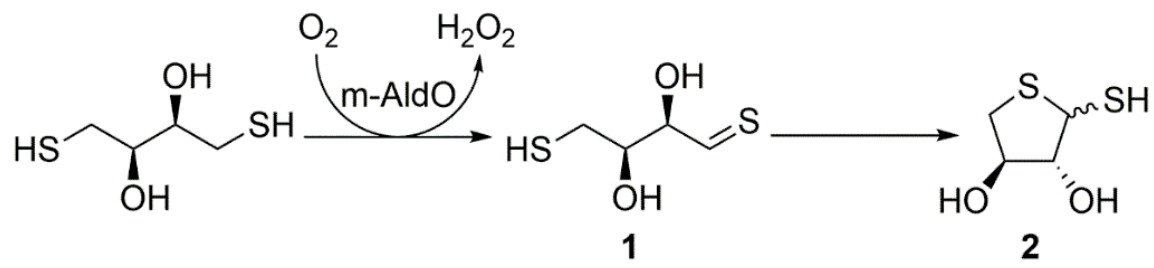

Scheme 1: The oxidation of L-dithiothreitol catalysed by m-AldO.

To further investigate the reaction of m-AldO with L-DTT, changes in the spectral properties of the enzyme's flavin cofactor during the reductive half-reaction were followed using a stopped flow-apparatus. Upon mixing m-AldO and L-DTT under anaerobic conditions, a rapid increase in absorbance was observed at $530 \mathrm{~nm}$ (Fig. 1A). This increase in absorbance at high wavelengths is indicative of the formation of a charge transfer complex between the thiolate anion form of L-DTT and the FAD cofactor. Such flavin-thiol charge transfer complexes have been observed in various members of the sulfhydryl oxidase family, though in these cases it is due to an interaction with an active site cysteine residue $(10,11)$. Fitting the data to a single exponential function yielded a rate of $555 \mathrm{~s}^{-1}$ for the formation of the charge transfer complex at an L-DTT concentration of $25 \mathrm{mM}$. Subsequently, the absorbance at 530 $\mathrm{nm}$ decays concomitantly with the absorbance at $450 \mathrm{~nm}$, suggesting the charge transfer interaction is broken by transfer of electrons from L-DTT to the flavin cofactor (Fig. 1B). The decay of the absorbance at $530 \mathrm{~nm}$ was biphasic, with $k_{\mathrm{obs} 1}=2.6 \mathrm{~s}^{-1}$ and $k_{\mathrm{obs} 2}=0.84 \mathrm{~s}^{-1}$. At an LDTT concentration of $25 \mathrm{mM}$ the decay in absorbance at $450 \mathrm{~nm}$ could only be satisfactorily fit using a triple exponential function. Two of the phases had similar rate constants to those observed for the decay of the absorbance at $530 \mathrm{~nm}, k_{\mathrm{obs} 1}=2.8 \mathrm{~s}^{-1}$ and $k_{\mathrm{obs} 2}=0.83 \mathrm{~s}^{-1}$. The third phase was too slow to be catalytically relevant $\left(k_{\mathrm{obs} 3}=0.089 \mathrm{~s}^{-1}\right)$ and its contribution to the drop in absorbance was very minor. Of the two catalytically relevant phases, the slower phase had the largest contribution to the decrease in absorbance. The rate of this phase displayed a 
hyperbolic dependence on substrate concentration, approaching a value of $1.13 \mathrm{~s}^{-1}$ at nonlimiting substrate concentrations. This suggests this phase represents irreversible reduction of the flavin cofactor. As the rate constant for reduction is close to $k_{\text {cat, }}$ the electron transfer from L-DTT to the flavin is the rate-limiting step in catalysis. The faster phase was poorly defined, likely due to its rate constant lying in the same range as the slower phase and its contribution to the drop in absorbance being more minor, and did not display any distinguishable dependence on substrate concentration. This phase may represent spectral changes associated with another step in catalysis, e.g. product release. Alternatively, the enzyme may exist in two distinct conformations, in which the reduction proceeds at different rates. Monitoring spectral changes using a photodiode array detector would help clarify what process is responsible for this phase. However, the high-intensity illumination required for this led to rapid photoreduction of the FAD cofactor. Enzyme-monitored turnover experiments under aerobic conditions revealed that during steady-state catalysis $\mathrm{m}$-AldO is predominantly present as the charge transfer complex (Fig. 1C).
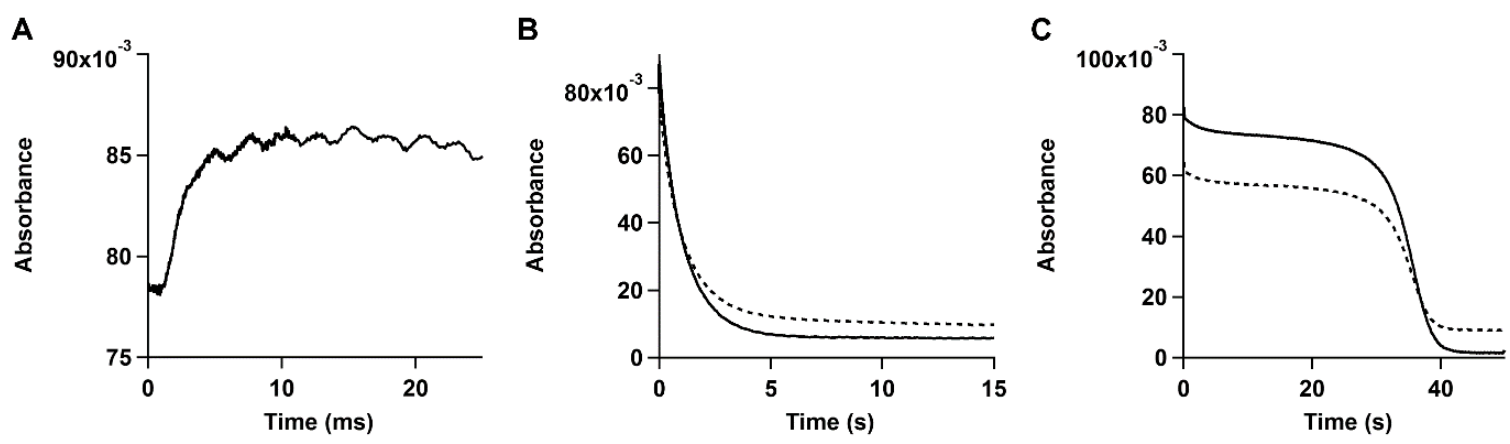

Figure 1: Spectral changes observed during the reaction of m-AldO with L-DTT as measured using a stopped-flow apparatus. Traces show the absorbance at $530 \mathrm{~nm}$ (solid lines) and $450 \mathrm{~nm}$ (dashed lines). Traces were obtained after mixing $10 \mu \mathrm{M}$ m-AldO with $25 \mathrm{mM}$ L-DTT under anaerobic (A+B) or aerobic conditions (C). Measurements were performed at least in triplicate, representative traces are shown.

The VAO/PCMH family, to which AldO belongs, displays a remarkably broad range of reactivities. The oxidation of alcohols and amines, hydroxylation of alkyl groups, C-C bond formation and the demethylation of ethers have all been described (12-15). The results presented above show that the oxidation of thiols can be added to this list. To determine whether the oxidation of thiols is also performed by more strict alcohol oxidases from the glucose-methanol-choline (GMC) family, we investigated the recently discovered 5(hydroxymethyl)furfural oxidase (HMFO) from Methylovorus sp. strain MP688 (16). HMFO catalyses the oxidation of primary alcohols. Its substrate range includes phenylic, furanic and linear unsaturated alcohols. In addition, HMFO is active towards several aldehydes. However, this activity is restricted to aldehydes which are spontaneously hydrated to the gem-diol form when dissolved in water (17).

We tested HMFO for activity towards phenylmethanethiol, which is the thiol analogue of its known substrate benzyl alcohol, and the related (4'-nitrophenyl)methanethiol. Interestingly, both thiols were readily oxidised by HMFO. The steady-state kinetic parameters of HMFO for these two aromatic thiol substrates are shown in Table 1 . The $k_{\text {cat }}$ values for both thiols are 
slightly lower than those for their alcohol counterparts (Table 1). Also, the $K_{\mathrm{m}}$ values for both thiols are significantly higher than for the alcohols. Taken together, the catalytic efficiency of HMFO is higher for alcohols than for thiols. Still, both tested thiols can be regarded as good substrates with $k_{\text {cat }}$ values of $>2 \mathrm{~s}^{-1}$ and $K_{\mathrm{m}}$ values in the millimolar range.

Table 1: Steady-state kinetic parameters for the oxidation of thiols and their alcohol analogues by flavoprotein oxidases. Steady state kinetic parameters were determined at $25{ }^{\circ} \mathrm{C}$ in the following buffers: $\mathrm{m}$-AldO: $20 \mathrm{mM}$ potassium phosphate buffer, $\mathrm{pH}$ 7.2; HMFO: $50 \mathrm{mM}$ potassium phosphate buffer, pH 8.0; GOX: 50 mM citrate buffer, $\mathrm{pH}$ 5.5.

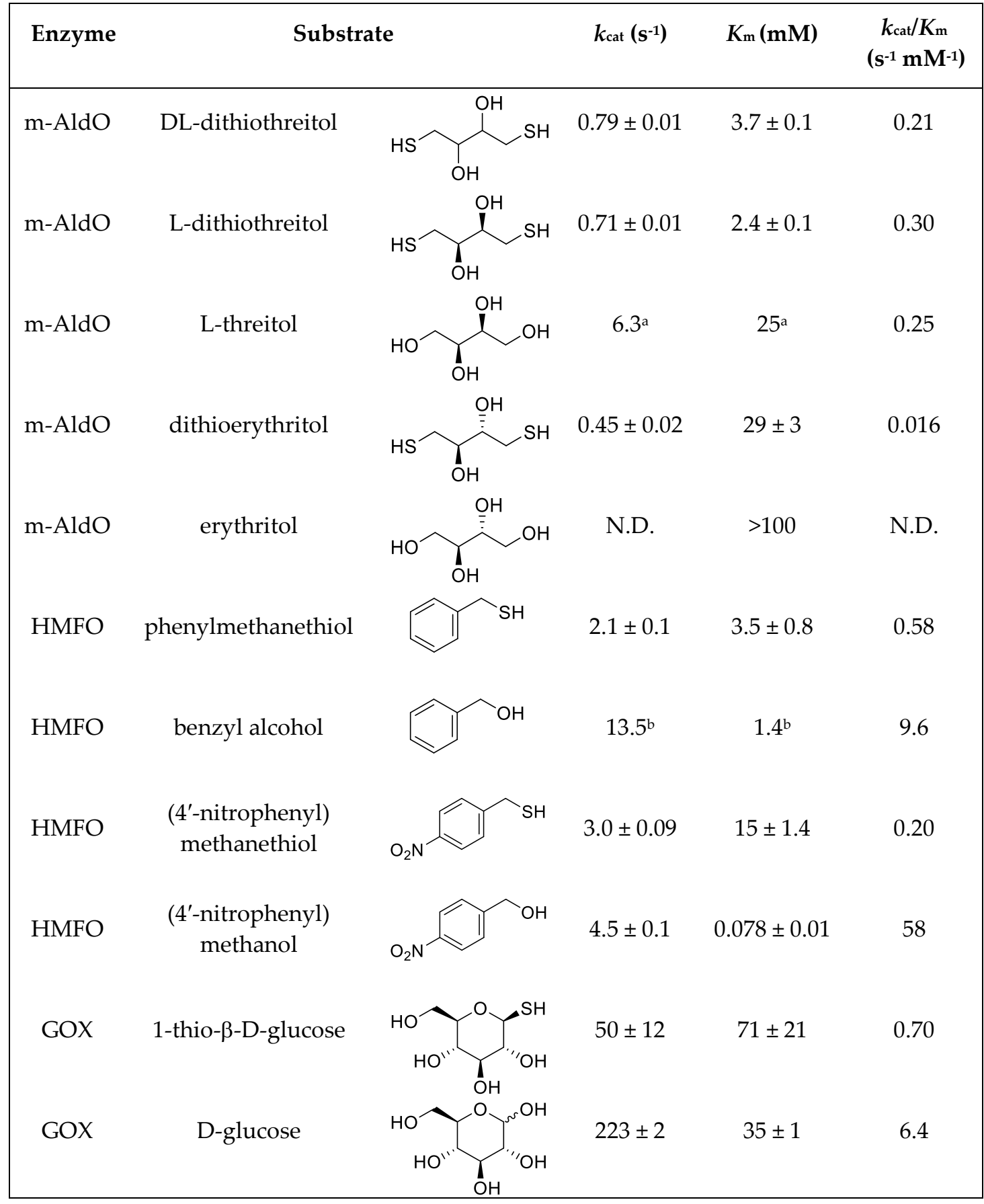

a Data taken from (6).

b Data taken from (16). 
The products formed upon oxidation of the two aromatic thiol substrates by HMFO were identified using GC/MS and LC/MS. At $\mathrm{pH}$ 7.0, phenylmethanethiol is oxidised to the corresponding aromatic thioaldehyde, benzothialdehyde (3) (Scheme 2, Fig. S3+S4). No formation of 1,2-dibenzyldisulfane was observed, demonstrating that HMFO does not catalyse the formation of disulfide bonds. At $\mathrm{pH} 8.0$, two products are formed. While after 10 minutes only benzothialdehyde was formed, after 60 minutes benzaldehyde (4) was detected as well. This suggests that the thioaldehyde is slowly hydrated, yielding the aldehyde as the final product. This is analogous to the reaction performed by amine oxidases, where the oxidation of amines to imines is followed by their reaction with water, yielding aldehydes or ketones (18). To confirm the involvement of water, the reaction at $\mathrm{pH} 8.0$ was performed in the presence of ${ }^{18} \mathrm{O}$-labelled water. In the presence of $\mathrm{H}_{2}{ }^{18} \mathrm{O}$, benzaldehyde with $\mathrm{m} / z=108$ was observed, whereas in unlabelled water only $m / z=106$ was detected (Fig. S4). This confirms that, at $\mathrm{pH}$ 8.0, water performs a nucleophilic attack on the initially formed thioaldehyde, yielding benzaldehyde as the final product. Using (4'-nitrophenyl)-methanethiol as a substrate, the reaction at $\mathrm{pH} 8.0$ again yielded the corresponding aldehyde. Because this aldehyde is easily hydrated to the gem-diol (19), it was oxidised further by HMFO, yielding 4nitrobenzoic acid as the final product. In addition to these main products, several minor peaks were detected by GC and LC analysis, probably due to the reactive nature of the formed thioaldehyde.

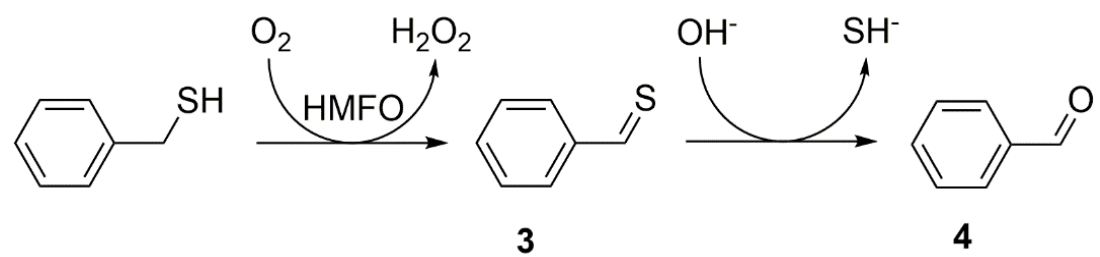

Scheme 2: The oxidation of phenylmethanethiol to benzothialdehyde (3) and benzaldehyde (4) catalysed by HMFO.

We also investigated a number of commercially available oxidases for their reactivity towards thiols. GOX is a fungal glycoprotein, which also belongs to the GMC family and is widely applied as an oxidative biocatalyst. Like HMFO, GOX contains a non-covalently bound FAD cofactor. GOX regioselectively oxidizes $\beta$-D-glucose to 1,5-D-gluconolactone, which spontaneously hydrolyses to gluconic acid (20). Glucose oxidase (GOX) from Aspergillus niger has been reported to display activity towards 1-thio- $\beta$-D-glucose (5). Oxygen consumption measurements showed that this enzyme indeed oxidises 1-thio- $\beta$-D-glucose with $k_{\mathrm{cat}}=50 \mathrm{~s}^{-1}$, $K_{\mathrm{m}}=71 \mathrm{mM}$, and with substrate inhibition occurring with a $K_{\mathrm{i}}$ of $42 \pm 13 \mathrm{mM}$ (Table 1). However, the oxidation of 1 -thio- $\beta$-D-glucose by GOX is less efficient than that of its alcohol analogue D-glucose, which occurs with a higher $k_{\mathrm{cat}}\left(223 \mathrm{~s}^{-1}\right)$ and lower $K_{\mathrm{m}}(35 \mathrm{mM})$ (Table 1$)$. Methanol oxidase (MO) is another fungal GMC-type oxidase. MO contains a non-covalently bound FAD cofactor and catalyses the oxidation of linear unbranched primary alcohols of up to five carbon atoms in length (21). Oxygen consumption measurements showed that $\mathrm{MO}$ from Candida boidinii is able to oxidise ethanethiol, displaying a specific activity of $0.32 \pm 0.03$ $\mathrm{U} / \mathrm{mg}$ at a substrate concentration of $20 \mathrm{mM}$, as compared to a specific activity of $0.51 \pm 0.02$ $\mathrm{U} / \mathrm{mg}$ for the oxidation of $20 \mathrm{mM}$ ethanol. 


\section{Discussion}

In summary, all tested flavin-dependent alcohol oxidases are capable of catalysing the oxidation of thiols, yielding thiocarbonyls. Though various free flavins are also known to react with some thiols, these reactions solely yield disulfides as products, suggesting they are mechanistically distinct from the flavoenzyme-catalysed reactions described here $(22,23)$. In the reaction of $\mathrm{m}$-AldO with L-DTT, the thiol substrate binds to the enzyme as a thiolate anion, leading to a strong charge transfer interaction with the oxidised flavin cofactor. Binding of the substrate in its thiolate form is likely stimulated by two positively charged residues, Arg-322 and Lys-375, that are in close proximity to the terminal hydroxyl group of the substrate in the crystal structure of AldO with xylitol bound (9). Subsequently, the flavin is slowly reduced by the transfer of two electrons from the substrate, yielding the thiocarbonyl product. The electron transfer probably occurs through the direct transfer of a hydride from the $\mathrm{C} \alpha$ atom of the substrate to the N5 atom of the flavin cofactor, as is believed to be the case for the oxidation of alcohols and amines by flavoprotein oxidases $(1,2)$. In the case of the oxidation of DTT by $\mathrm{m}$-AldO, the formed thioaldehyde rearranges intramolecularly to yield dithiohemiacetal 2. For the aromatic thiols oxidised by HMFO, such a rearrangement is not possible because of the absence of a second thiol group and in the case of phenylmethanethiol the formed thioaldehyde 3 is stable enough to be detected. Because HMFO has a broad substrate scope, this biocatalyst can be used to generate a large variety of thioaldehydes. The fusion protein m-AldO also has a broad substrate scope and could potentially be used to generate more stable thioaldehydes in cases where no second thiol group is present to enable intramolecular cyclisation. Because the ability to oxidise thiols appears to be a general property of flavin-dependent oxidases, many other enzymes may be used for similar purposes. Thus, our results not only highlight the promiscuity and versatility of enzymatic catalysis, but also provide a potential biocatalytic route to reactive thiocarbonyl compounds, which have a variety of applications in synthetic organic chemistry (24).

\section{Materials and methods}

\section{Materials}

DL-dithiothreitol was from Serva (Heidelberg, Germany). L-dithiothreitol, dithioerythritol, erythritol, trans-4,5-dihydroxy-1,2-dithiane, 1-thio- $\beta$-D-glucose, phenylmethanethiol, (4'nitrophenyl)methanethiol, benzyl alcohol, (4'-nitrophenyl)methanol, methanol oxidase (alcohol oxidase) from Candida boidinii and ethanethiol were from Sigma-Aldrich (St. Louis, MO, USA). Glucose oxidase from Aspergillus niger was from Boehringer Mannheim (Mannheim, Germany). D-glucose was from Merck (Kenilworth, NJ, USA). Ethanol was from VWR Chemicals (Radnor, PA, USA). The purity of thiol substrates was checked by NMR spectroscopy. No evidence for the presence of alcohol impurities was found.

\section{Protein expression and purification}

m-AldO was expressed from the pBAD-m-AldO plasmid (6) which contains the m-AldO construct behind the L-arabinose-inducible pBAD promoter. TOP10 E. coli containing the pBAD-m-AldO plasmid was grown at $37^{\circ} \mathrm{C}$ in Terrific Broth medium (Difco, Becton Dickinson, Franklin Lake, NJ, USA) supplemented with $100 \mu \mathrm{g} / \mathrm{mL}$ ampicillin until the OD 600 of the culture was 0.8. Subsequently, the cells were incubated on ice for $15 \mathrm{~min}$, protein 
expression was induced by adding L-arabinose at $0.02 \%(\mathrm{w} / \mathrm{v})$ and the cells were grown at $17^{\circ} \mathrm{C}$ for $40 \mathrm{~h}$. After this period, the cells were transferred to $20 \mathrm{mM}$ potassium phosphate buffer, $\mathrm{pH} 7.2$, supplemented with $4 \mathrm{mM} \mathrm{MgCl}_{2}$ and two cOmplete ${ }^{\mathrm{TM}}$ protease inhibitor pills (Roche, Basel, Switzerland) and $1 \mathrm{mg}$ DnaseI (Roche) per $50 \mathrm{~mL}$ and lysed by passing them through a pre-cooled SLM Aminco French pressure cell three times. The soluble fraction obtained after centrifugation $\left(39,000 \mathrm{~g}, 45 \mathrm{~min}, 4^{\circ} \mathrm{C}\right)$ was loaded onto an amylose resin column (New England Biolabs, Ipswich, MA, USA). Non-binding proteins were removed by washing with $20 \mathrm{mM}$ potassium phosphate buffer, $\mathrm{pH}$ 7.2, after which bound proteins were eluted with $20 \mathrm{mM}$ potassium phosphate buffer, $\mathrm{pH}$ 7.2, supplemented with $10 \mathrm{mM}$ maltose. Subsequently, m-AldO was purified further using a SourceQ anion exchange column. After loading, bound proteins were eluted using a linear 40 min gradient from 0 to $0.5 \mathrm{M} \mathrm{KCl}$ in 20 $\mathrm{mM}$ potassium phosphate buffer, $\mathrm{pH}$ 7.2. HMFO was purified as a His6-SUMO-HMFO fusion protein as described elsewhere (16).

Determination of steady-state kinetic parameters

Concentrations of $\mathrm{m}-\mathrm{AldO}, \mathrm{GOX}$ and $\mathrm{HMFO}$ solutions were determined using previously published molar absorption coefficients $\left[\varepsilon_{452}=12,500 \mathrm{M}^{-1} \mathrm{~cm}^{-1}\right.$ for m-AldO (6), $\varepsilon_{450}=14,100 \mathrm{M}^{-}$ ${ }^{1} \mathrm{~cm}^{-1}$ for GOX (25) and $\varepsilon_{456}=10,700 \mathrm{M}^{-1} \mathrm{~cm}^{-1}$ for $\left.\mathrm{HMFO}(17)\right]$. The concentration of MO solutions was determined using a BCA assay kit (Thermo Scientific, Waltham, MA, USA). Initial rates of the enzymatic reactions were determined by monitoring oxygen consumption using a Hansatech Oxytherm system (Hansatech Instruments) for m-AldO, GOX and MO and REDFLASH sensor spots and a Firesting $\mathrm{O}_{2}$ detector and light source (Pyroscience, Aachen, Germany) for HMFO. m-AldO activity was measured in $20 \mathrm{mM}$ potassium phosphate buffer, $\mathrm{pH}$ 7.2, HMFO activity in $50 \mathrm{mM}$ potassium phosphate buffer, $\mathrm{pH}$ 8.0, GOX activity in $50 \mathrm{mM}$ citrate buffer, $\mathrm{pH} 5.5$, and $\mathrm{MO}$ activity in $100 \mathrm{mM}$ potassium phosphate buffer, $\mathrm{pH}$ 7.5. All measurements were performed at $25^{\circ} \mathrm{C}$. Steady-state kinetic parameters were determined by fitting the measured initial rates to the Michaelis-Menten equation using Igor Pro v. 6.10A (Wavemetrics, Lake Oswego, OR, USA). In cases where substrate inhibition was observed, the initial rates were fit to a modified version of the Michaelis-Menten equation that includes a term to take substrate inhibition into account:

$$
k_{o b s}=\frac{k_{c a t} *[S]}{K_{m}+[S]+\frac{[S]^{2}}{K_{i}}}
$$

Where $k_{\mathrm{obs}}$ is the measured initial rate, $k_{\mathrm{cat}}$ is the turnover number, $K_{\mathrm{m}}$ is the Michaelis constant, $K_{\mathrm{i}}$ is the inhibition constant for substrate inhibition and [S] is the substrate concentration.

\section{Identification of reaction products}

To analyse the products of the oxidation of DL-DTT by m-AldO, reaction mixtures containing $10 \mu \mathrm{M}$ m-AldO and $25 \mathrm{mM}$ DL-DTT in $20 \mathrm{mM}$ potassium phosphate buffer, $\mathrm{pH}$ 7.2, were incubated under shaking $(350 \mathrm{rpm})$ at $25{ }^{\circ} \mathrm{C}$, in the presence or absence of $50 \mathrm{mM}$ sodium pyruvate. After 3 or $6 \mathrm{~h}$, the enzyme was removed by ultrafiltration and $0.5 \%(\mathrm{v} / \mathrm{v})$ trifluoroacetic acid was added to the flow-through. HPLC analysis of the products was performed using a system consisting of an Alltima C18 column $(150 \times 4.6 \mathrm{~mm}$, Hichrom, Reading, UK), a Waters 600E pump system and a Waters 996 photodiode array detector (Waters, Milford, MA, USA). The reaction products were eluted using a 15 min linear gradient from 0 to $15 \%(\mathrm{v} / \mathrm{v})$ acetonitrile in water, with both eluents containing $0.1 \%(\mathrm{v} / \mathrm{v})$ 
trifluoroacetic acid, and detected by monitoring the absorbance at $210 \mathrm{~nm}$. To identify the product of the enzymatic reaction, the corresponding peak was collected and the product was extracted twice with 1 volume of EtOAc. Subsequently, the solvent was removed by evaporation under nitrogen flow and the residue was dissolved in deuterated acetonitrile. NMR-spectra were recorded on a $600 \mathrm{MHz}$ Avance $3 \mathrm{NMR}$-spectrometer (Bruker, Billerica, MA, USA). To test whether free FAD is capable of catalysing the conversion of DL-DTT under similar conditions, $10 \mu \mathrm{M}$ FAD and $25 \mathrm{mM}$ DL-DTT were incubated in $20 \mathrm{mM}$ potassium phosphate buffer, $\mathrm{pH} 7.2$, for $6 \mathrm{~h}$ at $25{ }^{\circ} \mathrm{C}$ under shaking (350 rpm). Subsequently, reaction products were analysed by HPLC as described above. No conversion of DL-DTT was observed under these reaction conditions.

Phenylmethanethiol $(10 \mathrm{mM})$ was oxidised by $5 \mu \mathrm{M}$ HMFO in $50 \mathrm{mM}$ Tris/ $\mathrm{HCl}$ buffer, $\mathrm{pH} 7.0$ or 8.0 , or $50 \mathrm{mM}$ potassium phosphate buffer, $\mathrm{pH}$ 6.0, containing $100 \mathrm{U} / \mathrm{mL}$ catalase (SigmaAldrich) to remove hydrogen peroxide. The reactions were performed at $25{ }^{\circ} \mathrm{C}$ under shaking (1000 rpm). Reactions were stopped after 10,60 or $300 \mathrm{~min}$ by extracting with $125 \mu \mathrm{L}$ to 250 $\mu \mathrm{L} \mathrm{CHCl}_{3}$. Control reactions with free riboflavin or FAD $(5 \mu \mathrm{M})$ were performed at $\mathrm{pH}$ 7.0. The reaction products were analysed on a HP6890 GC-system with an HP 5973 Mass selective detector (Agilent Technologies, Santa Clara, CA, USA) using an HP1 column (25 m x $0.25 \mathrm{~mm}$ $x 0.25 \mu \mathrm{m}$, Agilent). After a pre-run of $5 \mathrm{~min}$ at $60^{\circ} \mathrm{C}$, the temperature was increased to $230{ }^{\circ} \mathrm{C}$ at $10{ }^{\circ} \mathrm{C} / \mathrm{min}$ followed by a post-run of $5 \mathrm{~min}$ at $300{ }^{\circ} \mathrm{C}$. The split ratio was set to $50: 1$ and the detector temperature to $325^{\circ} \mathrm{C}$.

For the oxidation of (4'-nitrophenyl)methanethiol $(20 \mathrm{mM}), 5 \mu \mathrm{M}$ HMFO was used in $50 \mathrm{mM}$ ammonium bicarbonate buffer, $\mathrm{pH}$ 8.0. After 0.5 or $5 \mathrm{~h}$, the reactions were stopped by heating the sample for $5 \mathrm{~min}$ at $70{ }^{\circ} \mathrm{C}$, and removing denatured protein by centrifugation. Reaction products were analyzed with an Alltima HP C18 column, $3 \mu \mathrm{m}$ (Hichrom) on a Surveyor LC (Thermo Finnigan, San Jose, CA, USA), using a gradient of acetonitrile (B) in $50 \mathrm{mM}$ ammonium bicarbonate buffer, $\mathrm{pH} 8.0$ (A). After 2 min of $100 \% \mathrm{~A}, \mathrm{~B}$ was increased from $0 \%$ to $80 \%$ in $30 \mathrm{~min}$ and kept constant at $80 \%$ for $5 \mathrm{~min}$. Subsequently, B was decreased from $80 \%$ to $0 \%$ in $1 \mathrm{~min}$, followed by $100 \% \mathrm{~A}$ for the last $10 \mathrm{~min}$ of the run. Detection was performed using a diode array detector between 200 and $600 \mathrm{~nm}$. Mass detection was performed on a LCQ Fleet MS (Thermo) using APCI ionisation of the sample.

To check for side products formed by hydrogen peroxide during catalysis, phenylmethanethiol and (4'-nitrophenyl)methanethiol (both $10 \mathrm{mM}$ ) were incubated with 10 $\mathrm{mM}$ hydrogen peroxide and products were analysed as described.

\section{$\mathrm{H}_{2}{ }^{18} \mathrm{O}$ incorporation}

To investigate the role of water in the formation of benzaldehyde from benzothialdehyde, 5 $\mu \mathrm{M}$ HMFO was used to oxidise $10 \mathrm{mM}$ phenylmethanethiol in the presence of $30 \% \mathrm{H}_{2}{ }^{18} \mathrm{O}$ and $100 \mathrm{U} / \mathrm{mL}$ catalase in $50 \mathrm{mM}$ Tris/ $\mathrm{HCl}$ buffer, $\mathrm{pH}$ 8.0. After $2 \mathrm{~h}\left(25^{\circ} \mathrm{C}, 1000 \mathrm{rpm}\right)$ the products were analysed as described above.

\section{Stopped-flow kinetics}

Changes in the spectral properties of m-AldO's FAD cofactor during its reaction with L-DTT were analysed using an SX20 stopped-flow apparatus (Applied Photophysics, Leatherhead, UK). All experiments were performed at $25^{\circ} \mathrm{C}$ in $20 \mathrm{mM}$ potassium phosphate buffer, $\mathrm{pH}$ 7.2. $\mathrm{m}$-AldO $(10 \mu \mathrm{M})$ was mixed with various concentrations of L-DTT and the absorbance was 
monitored at selected wavelengths using a photomultiplier detector. For experiments where anaerobic conditions were required, the enzyme and substrate solutions contained $10 \mathrm{mM}$ putrescine. Prior to mixing, the solutions were flushed with nitrogen and subsequently putrescine oxidase was added to a concentration of $10 \mu \mathrm{g} / \mathrm{ml}$ to scavenge residual oxygen. Observed rate constants were obtained by fitting the measured traces to single, double or triple exponential functions as appropriate.

\section{References}

1. Fitzpatrick, P. F. (2007) Insights into the mechanisms of flavoprotein oxidases from kinetic isotope effects. J. Label. Compd. Radiopharm. 50, 1016-1025

2. Gadda, G. (2008) Hydride transfer made easy in the reaction of alcohol oxidation catalyzed by flavin-dependent oxidases. Biochemistry. 47, 13745-13753

3. Dijkman, W. P., de Gonzalo, G., Mattevi, A., and Fraaije, M. W. (2013) Flavoprotein oxidases: Classification and applications. Appl. Microbiol. Biotechnol. 97, 5177-5188

4. Suylen, G. M. H., Large, P. J., van Dijken, J. P., and Kuenen, J. G. (1987) Methyl mercaptan oxidase, a key enzyme in the metabolism of methylated sulphur compounds by Hyphomicvobium EG. J. Gen. Microbiol. 133, 2989-2997

5. Saa, L., and Pavlov, V. (2012) Enzymatic growth of quantum dots: Applications to probe glucose oxidase and horseradish peroxidase and sense glucose. Small. 8, 3449-3455

6. Heuts, D. P. H. M., van Hellemond, E. W., Janssen, D. B., and Fraaije, M. W. (2007) Discovery, characterization, and kinetic analysis of an alditol oxidase from Streptomyces coelicolor. J. Biol. Chem. 282, 20283-20291

7. van Hellemond, E. W., Vermote, L., Koolen, W., Sonke, T., Zandvoort, E., Heuts, D. P. H. M., Janssen, D. B., and Fraaije, M. W. (2009) Exploring the biocatalytic scope of alditol oxidase from Streptomyces coelicolor. Adv. Synth. Catal. 351, 1523-1530

8. Fraaije, M. W., van Berkel, W. J. H., Benen, J. A. E., Visser, J., and Mattevi, A. (1998) A novel oxidoreductase family sharing a conserved FAD-binding domain. Trends Biochem. Sci. 4, 206207

9. Forneris, F., Heuts, D. P. H. M., Delvecchio, M., Rovida, S., Fraaije, M. W., and Mattevi, A. (2008) Structural analysis of the catalytic mechanism and stereoselectivity in Streptomyces coelicolor alditol oxidase. Biochemistry. 47, 978-985

10. Hoober, K. L., and Thorpe, C. (1999) Egg white sulfhydryl oxidase: Kinetic mechanism of the catalysis of disulfide bond formation. Biochemistry. 38, 3211-3217

11. Schaefer-Ramadan, S., Gannon, S. A., and Thorpe, C. (2013) Human augmenter of liver regeneration: Probing the catalytic mechanism of a flavin-dependent sulfhydryl oxidase. Biochemistry. 52, 8323-8332

12. Fraaije, M. W., Veeger, C., and van Berkel, W. J. H. (1995) Substrate specificity of flavindependent vanillyl-alcohol oxidase from Penicillium simplicissimum: Evidence for the production of 4-hydroxycinnamyl alcohols from 4-allylphenols. Eur. J. Biochem. 234, 271-277

13. Fraaije, M. W., and van Berkel, W. J. H. (1997) Catalytic mechanism of the oxidative demethylation of 4-(methoxymethyl)phenol by vanillyl-alcohol oxidase: Evidence for formation of a $p$-quinone methide intermediate. J. Biol. Chem. 272, 18111-18116

14. Drijfhout, F. P., Fraaije, M. W., Jongejan, H., van Berkel, W. J. H., and Franssen, M. C. R. (1998) Enantioselective hydroxylation of 4-alkylphenols by vanillyl alcohol oxidase. Biotechnol. Bioeng. 59, 171-177

15. Kutchan, T. M., and Dittrich, H. (1995) Characterization and mechanism of the berberine bridge enzyme, a covalently flavinylated oxidase of benzophenanthridine alkaloid biosynthesis in plants. J. Biol. Chem. 270, 24475-24481

16. Dijkman, W. P., and Fraaije, M. W. (2014) Discovery and characterisation of a 5- 
(hydroxymethyl)furfural oxidase from Methylovorus sp. Strain MP688. Appl. Environ. Microbiol. 80, 1082-1090

17. Dijkman, W. P., Groothuis, D. E., and Fraaije, M. W. (2014) Enzyme-catalyzed oxidation of 5hydroxymethylfurfural to furan-2,5-dicarboxylic acid. Angew. Chem. Int. Ed. 53, 6515-6518

18. Turner, N. J. (2011) Enantioselective oxidation of C-O and C-N bonds using oxidases. Chem. Rev. 111, 4073-4087

19. Ferreira, P., Hernández-Ortega, A., Herguedas, B., Rencoret, J., Gutiérrez, A., Martínez, M. J., Jiménez-Barbero, J., Medina, M., and Martínez, Á. T. (2010) Kinetic and chemical characterization of aldehyde oxidation by fungal aryl-alcohol oxidase. Biochem. J. 425, 585-593

20. Leskovac, V., Trivić, S., Wohlfahrt, G., Kandrač, J., and Peričin, D. (2005) Glucose oxidase from Aspergillus niger: The mechanism of action with molecular oxygen, quinones, and one-electron acceptors. Int. J. Biochem. Cell Biol. 37, 731-750

21. Sahm, H., and Wagner, F. (1973) Microbial assimilation of methanol: The ethanol- and methanoloxidizing enzymes of the yeast Candida boidinii. Eur. J. Biochem. 36, 250-256

22. Gibian, M. J., Elliott, D. L., Kelly, C., Borge, B., and Kupecz, K. (1972) Studies of reactions between flavins and quinones, mercaptans, and enolates. Z. Naturforsch. B. 27, 1016-1020

23. Loechler, E. L., and Hollocher, T. C. (1980) Reduction of flavins by thiols. 1: Reaction mechanism from the kinetics of the attack and breakdown steps. J. Am. Chem. Soc. 102, 7312-7321

24. Metzner, P. (1999) Thiocarbonyl compounds as specific tools for organic synthesis. In: Organosulfur Chemistry I (Page, P. C. B. ed.), Top. Curr. Chem. vol. 204, pp. 127-181

25. Swoboda, B. E. P., and Massey, V. (1965) Purification and properties of the glucose oxidase from Aspergillus niger. J. Biol. Chem. 240, 2209-2215

\section{Abbreviations}

(m-)AldO: (maltose binding protein-)alditol oxidase, DTT: dithiothreitol, FAD: flavin adenine dinucleotide, FMN: flavin mononucleotide, GMC: glucose-methanol-choline, GOX: glucose oxidase, HMFO: 5-(hydroxymethyl)furfural oxidase, MO: methanol oxidase 


\section{Supplementary Figures}

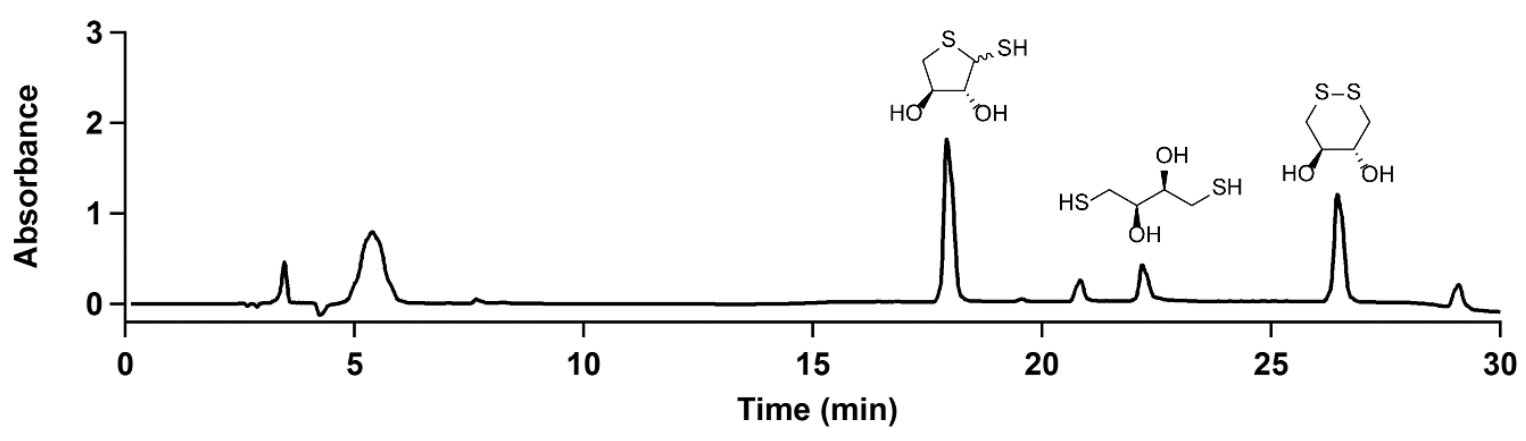

Figure S1: HPLC analysis of the products formed upon oxidation of DL-dithiothreitol catalysed by alditol oxidase. $10 \mu \mathrm{M} \mathrm{m}$-AldO and $25 \mathrm{mM}$ DL-DTT were incubated in $20 \mathrm{mM}$ potassium phosphate buffer, $\mathrm{pH} 7.2$, at $25^{\circ} \mathrm{C}$ in the presence of $50 \mathrm{mM}$ sodium pyruvate. After $6 \mathrm{~h}$, the enzyme was removed by ultrafiltration and products were analysed by HPLC. Retention times: dithiohemiacetal 2 : $17.9 \mathrm{~min}$, unidentified compound 1: $20.9 \mathrm{~min}$, DL-DTT: $22.2 \mathrm{~min}$, trans-4,5-dihydroxy-1,2-dithiane: 26.4 min, unidentified compound 2: $29.1 \mathrm{~min}$. The broad peak with a retentiom time of 5-6 min is pyruvate. trans-4,5-dihydroxy-1,2-dithiane and unidentified compound 2 are formed by sidereactions involving hydrogen peroxide, as they were far more abundant in the absence of the $\mathrm{H}_{2} \mathrm{O}_{2}$ scavenger pyruvate. Unidentified compound 1 was present in similar minor amounts regardless of the presence of pyruvate. Possibly, it reflects the formation of a sulfenic acid by the oxidation of $\mathbf{2}$ by molecular oxygen. The structures used to label the peaks show the L-enantiomer of DTT and the dithiohemiacetal formed from its oxidation. 
A

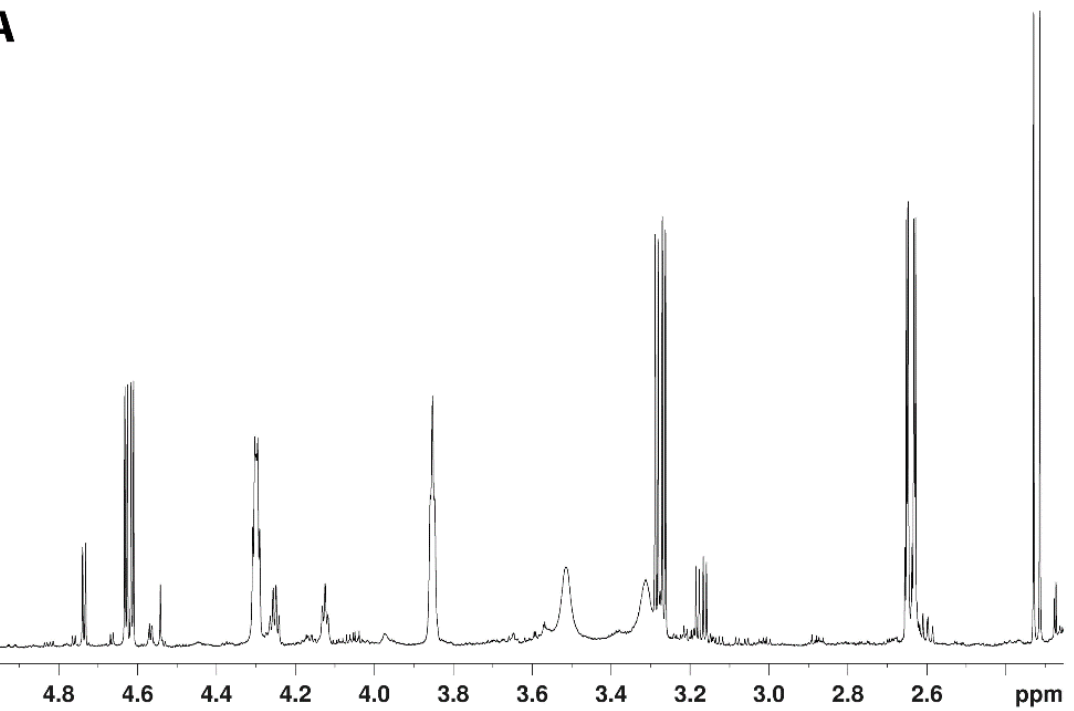

B

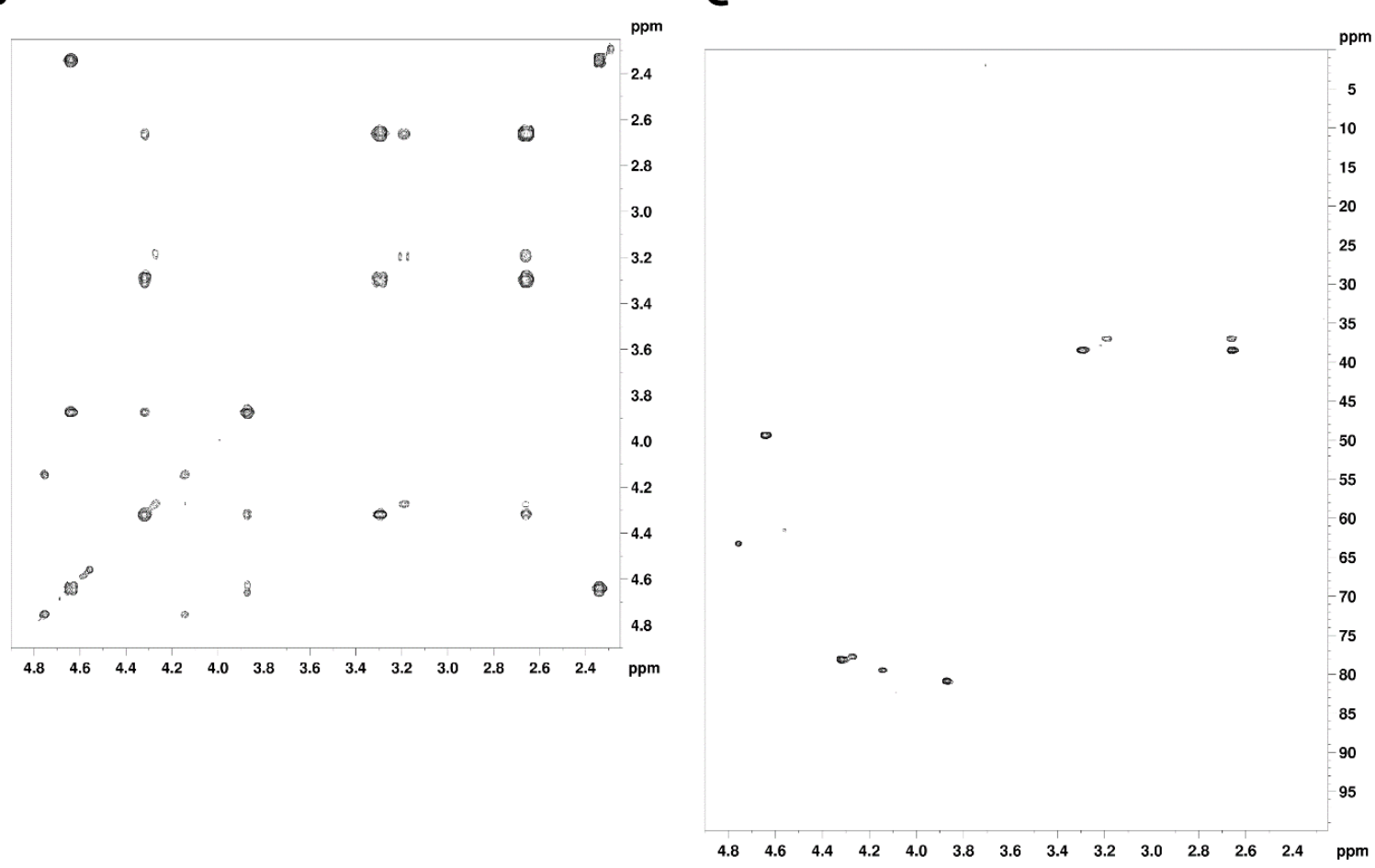

Figure S2: NMR spectra of dithiohemiacetal 2 formed by the oxidation of DL-DTT by m-AldO. (A) 1-D ${ }^{1} \mathrm{H}-\mathrm{NMR}$, (B) ${ }^{1} \mathrm{H}-{ }^{-1} \mathrm{H}-\mathrm{COSY}(\mathrm{C}){ }^{1} \mathrm{H}-{ }^{13} \mathrm{C}$ HSQC. $10 \mu \mathrm{M}$ m-AldO and $25 \mathrm{mM}$ DL-DTT were incubated in 20 $\mathrm{mM}$ potassium phosphate buffer, $\mathrm{pH} 7.2$, at $25^{\circ} \mathrm{C}$ in the presence of $50 \mathrm{mM}$ sodium pyruvate.

Compound 2 was purified from the reaction mixture using HPLC and analysed by NMR. During the measurement time, compound 2 was partially oxidised to the corresponding sulfenic acid. After 6 days of storage at $-20{ }^{\circ} \mathrm{C}$ dissolved in acetonitrile, 2 was completely converted to this sulfenic acid. 2: ${ }^{1} \mathrm{H}-\mathrm{NMR}\left(600 \mathrm{MHz}, \mathrm{CD}_{3} \mathrm{CN}\right) \delta(\mathrm{ppm}): 4.64 \mathrm{ppm}(\mathrm{dd}, J=9.3,3.8 \mathrm{~Hz}, 1 \mathrm{H}), 4.32(\mathrm{~m}, 1 \mathrm{H}), 3.87 \mathrm{ppm}(\mathrm{m}$, $1 \mathrm{H}), 3.30 \mathrm{ppm}(\mathrm{dd}, J=11.0,4.8 \mathrm{~Hz}, 1 \mathrm{H}), 2.66 \mathrm{ppm}(\mathrm{dd}, J=11.0,2.5 \mathrm{~Hz}, 1 \mathrm{H}), 2.34 \mathrm{ppm}(\mathrm{d}, J=9.3 \mathrm{~Hz}$, $1 \mathrm{H}) .{ }^{1} \mathrm{H}-{ }^{13} \mathrm{C}-\mathrm{HSQC}\left(600 \mathrm{MHz}, \mathrm{CD}_{3} \mathrm{CN}\right):{ }^{13} \mathrm{C}, \delta(\mathrm{ppm}): 80.8,78.0,49.3,38.4$. Sulfenic acid oxidation product: ${ }^{1} \mathrm{H}-\mathrm{NMR}\left(600 \mathrm{MHz}, \mathrm{CD}_{3} \mathrm{CN}\right) \delta(\mathrm{ppm}): 4.76(\mathrm{~d}, J=4.42 \mathrm{~Hz}, 1 \mathrm{H}), 4.27(\mathrm{~m}, 1 \mathrm{H}), 4.14(\mathrm{~m}, 1 \mathrm{H}), 3.58$ $(\mathrm{d}, J=4.7 \mathrm{~Hz}, 1 \mathrm{H}), 3.41(\mathrm{~d}, J=4.3 \mathrm{~Hz}, 1 \mathrm{H}), 3.19(\mathrm{dd}, J=10.7,4.8 \mathrm{~Hz}, 1 \mathrm{H}), 2.66(\mathrm{dd} J=10.8,3.8 \mathrm{~Hz}, 1 \mathrm{H})$. ${ }^{1} \mathrm{H}-{ }^{13} \mathrm{C}-\mathrm{HSQC}\left(600 \mathrm{MHz}, \mathrm{CD}_{3} \mathrm{CN}\right){ }^{13} \mathrm{C}, \delta$ (ppm): 79.4, 77.6, 63.2, 37.0. 
A

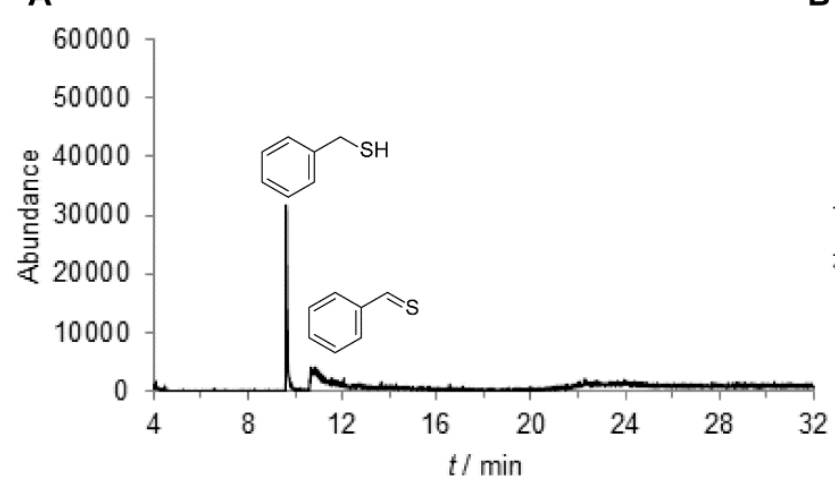

B

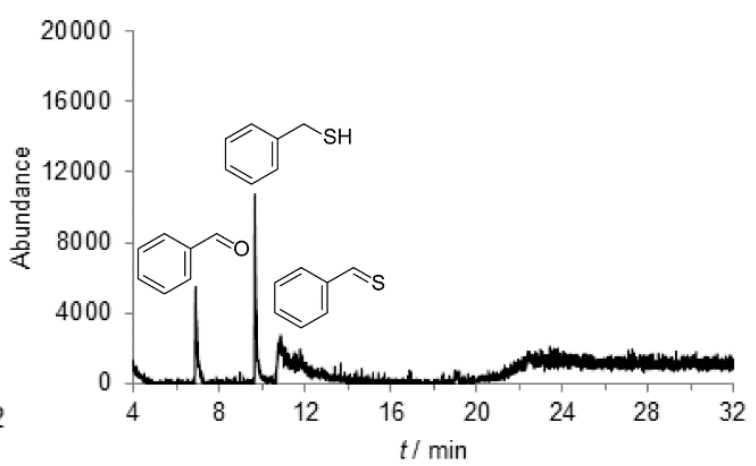

Figure S3: GC/MS analysis of the oxidation of phenylmethanethiol by HMFO. $10 \mathrm{mM}$ phenylmethanethiol and $5 \mu \mathrm{M}$ HMFO were incubated for $5 \mathrm{~h}$ in $50 \mathrm{mM}$ Tris/ $\mathrm{HCl}$ buffer, $\mathrm{pH} 7.0$ or 8.0, at $25{ }^{\circ} \mathrm{C}$. Following this, reaction products were extracted and analysed by GC/MS. (A) At pH 7.0, phenylmethanethiol is partially converted to benzothialdehyde. (B) The same reaction at $\mathrm{pH} 8.0$ yields benzaldehyde as an additional product. Retention times: benzaldehyde: $6.9 \mathrm{~min}$, phenylmethanethiol: $9.6 \mathrm{~min}$, benzothialdehyde: $10.8 \mathrm{~min}$.

A

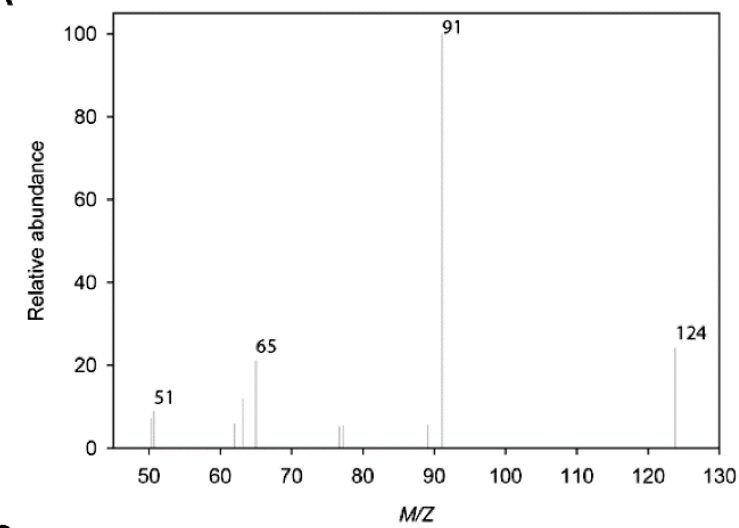

C

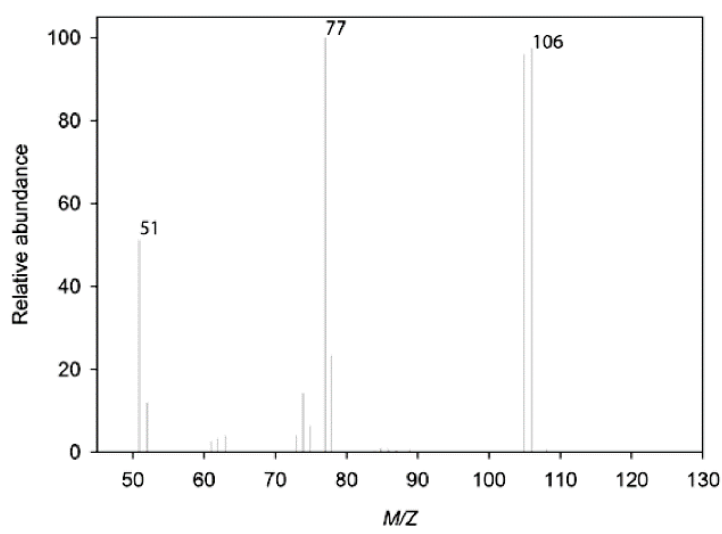

B

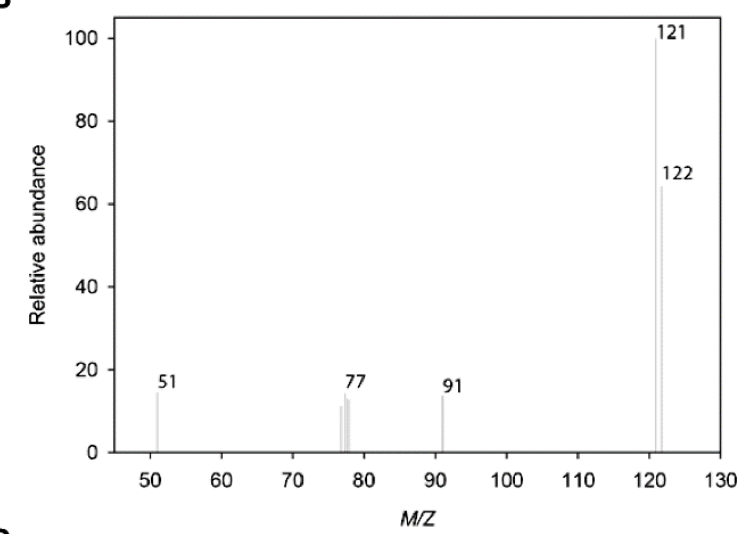

D

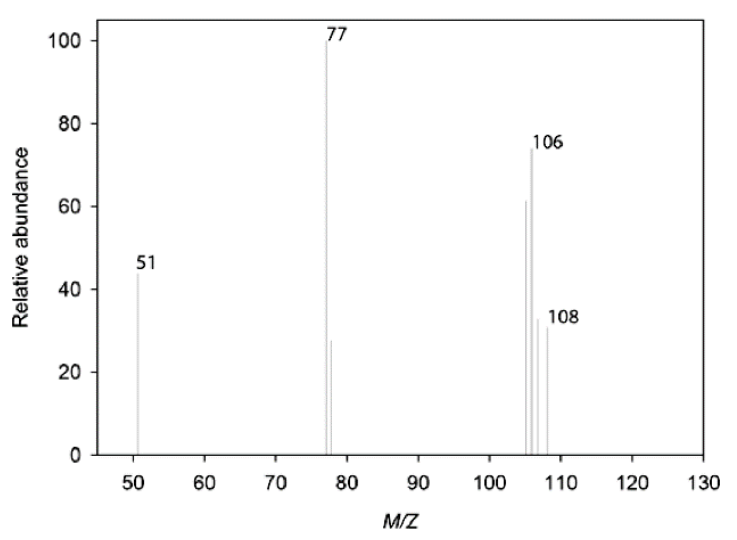

Figure S4: Mass spectra of the products of the oxidation of phenylmethanethiol by HMFO. $10 \mathrm{mM}$ phenylmethanethiol and $5 \mu \mathrm{M}$ HMFO were incubated for $5 \mathrm{~h}$ in $50 \mathrm{mM}$ Tris/HCl buffer, $\mathrm{pH} 7.0$ or 8.0, at $25{ }^{\circ} \mathrm{C}$. Following this, reaction products were extracted and analysed by GC/MS (see also Fig. S3). (A) Phenylmethanethiol $\left(M_{w}=124 u\right)$. (B) Benzothialdehyde $\left(M_{w}=122 u\right)$. (C) Benzaldehyde $\left(M_{w}=106 u\right.$, only observed when the reaction was performed at $\mathrm{pH}$ 8.0). (D) When the reaction is performed at $\mathrm{pH}$ 8.0 in the presence of $30 \% \mathrm{H}_{2}{ }^{18} \mathrm{O}$, benzaldehyde with ${ }^{18} \mathrm{O}$ incorporated $\left(M_{\mathrm{w}}=108 \mathrm{u}\right)$ is observed. 

9

Discussion 

Historically, the industrial application of enzymes has been dominated by the use of hydrolases such as glycosidases, proteases and lipases (1). In recent years, however, the potential of oxidoreductases for industrial applications has been increasingly recognised (2). Oxidoreductases that are of interest in this context include copper-containing enzymes, such as laccases and lytic polysaccharide monooxygenases, heme-containing enzymes such as peroxygenases and peroxidases and flavin-dependent enzymes such as GMC-type and $\mathrm{VAO} / \mathrm{PCMH}$-type oxidoreductases (3). In order to facilitate the adoption of these enzymes by industry, it is of importance to develop enzymes with improved properties for potential industrial applications. This can be achieved by identifying and characterising novel enzymes in sequenced genomes or modifying existing enzymes by protein engineering. Efforts to modify existing enzymes are greatly facilitated by recent advances in the development of random mutagenesis methods (such as directed evolution) and in the computational modelling of enzymatic reactions, which enables protein engineering by rational design methods $(4,5)$. These efforts can also be supported by fundamental enzymological studies, as a better understanding of the mechanisms by which enzymes achieve catalysis will facilitate their redesign. In addition to modifying the enzymes themselves, it is also necessary to define which factors are of importance in designing an efficient biocatalytic process and what impediments may be encountered when scaling up such a process from the laboratory to the industrial scale. In the research presented in this thesis, a number of these aspects have been explored.

Fundamental enzymological studies provided interesting new insights into the molecular mechanisms of oligomerisation (Chapter 3) and substrate activation (Chapter 4) in 4-phenol oxidising (4PO) flavoproteins. To facilitate the biocatalytic application of these enzymes, the development of an assay to efficiently screen variants of vanillyl alcohol oxidase (VAO) and eugenol oxidase (EUGO) for changes in their substrate specificities (Chapter 6) and the scaleup of the synthesis of the chiral secondary alcohol (R)-1-(4'-hydroxyphenyl)ethanol using VAO (Chapter 7) were also described. Below, these results are discussed in the context of other recent developments in our understanding of the occurrence, function and mechanisms of 4PO subfamily members. Remaining questions and challenges that are yet to be overcome in enabling the biocatalytic application of these enzymes are also discussed. In addition, the potential applications of the oxidation of thiols to thiocarbonyls by flavin-dependent oxidases, as described in Chapter 8, are discussed briefly.

\section{Occurrence and physiological function of 4PO oxidases}

\section{Physiological function of $4 P O$ oxidases}

Although the 4PO oxidases VAO and EUGO have been extensively studied in terms of their in vitro biochemical properties, their exact function in vivo remains somewhat elusive. The ascomycetous fungus Penicillium simplicissimum and the actinobacterium Rhodococcus jostii RHA1 both grow in environments rich in plant biomass and it has been reported that Rhodococcus jostii RHA1 possesses the ability to degrade lignin $(6,7)$. As VAO and EUGO catalyse the oxidation of monomeric phenolic compounds, it seems likely that they are involved in the catabolism of lignin-derived aromatic molecules. For VAO, it has been 
proposed that 4 -(methoxymethyl)phenol is the physiological substrate, as it is the only substrate that has been shown to induce expression of the enzyme (Scheme 1) (8). In vivo, the 4-hydroxybenzaldehyde formed by its oxidation by $\mathrm{VAO}$ is oxidised further to 4hydroxybenzoate. This is then hydroxylated on the aromatic ring to yield 3,4dihydroxybenzoate (protocatechuate), which can be broken down further through the $\beta$ ketoadipate pathway (9). As the oxidation of various other substrates by VAO also yields 4hydroxybenzaldehyde [or derivatives thereof $(10,11)$ ] it is plausible that VAO could be a multifunctional enzyme, catalysing the conversion of various lignin-derived compounds to aldehydes that are subsequently further broken down via the same pathway as observed for 4-(methoxymethyl)phenol. Although the physiological role of EUGO has not been studied, it seems likely that it performs a similar function.

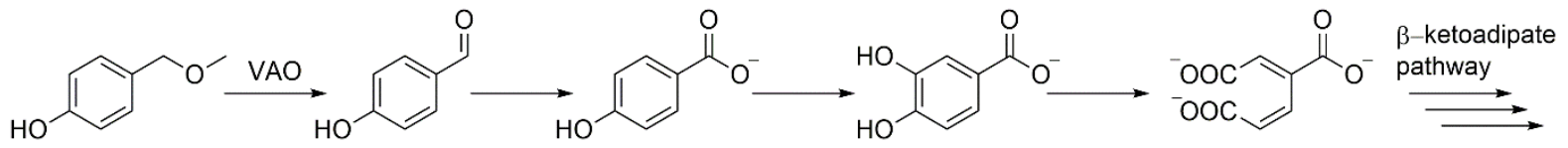

Scheme 1: Pathway for the breakdown of 4-(methoxymethyl)phenol by Penicillium simplicissimum. In the first step, 4-(methoxymethyl)phenol is converted to 4-hydroxybenzaldehyde by VAO. Next, the aldehyde is converted to 4-hydroxybenzoate, which is then hydroxylated to yield 3,4dihydroxybenzoate (protocatechuate). This compound is further broken down in the $\beta$-ketoadipate pathway, the first step of which is its oxidative intradiol ring cleavage to yield $\beta$-carboxymuconate. Other compounds that are converted to an aldehyde by VAO (e.g. vanillyl alcohol) could be degraded in a similar manner. Scheme adapted from (8).

A recent survey of the occurrence of genes encoding putative VAO-like oxidases in sequenced fungal genomes showed that they are widespread within the fungal kingdom (12). No clear correlation was observed between the habitat or lifestyle of the fungi and the presence of genes encoding VAO-like oxidases. However, many of the species in which VAO-like oxidase genes were identified are either plant pathogens or wood-decay fungi, consistent with a lifestyle where lignin-derived compounds would be present in their environment. Characterisation of the proteins encoded by some of these genes or evaluation of the effect of their deletion on the physiology of the host organism may be of use for the further elucidation of the physiological function of this class of enzymes.

\section{Oligomerisation state of $4 P O$ oxidases}

One of the most striking differences between VAO and EUGO lies in their oligomerisation state, with VAO predominantly forming octamers that can be described as tetramers of stable dimers and EUGO being exclusively dimeric $(13,14)$. In Chapter 3, the molecular determinant of this difference in oligomerisation state was found to be a loop that is located at the dimerdimer interface of a VAO octamer. Deletion of this loop led to the formation of a VAO variant that is exclusively dimeric in solution. This variant displayed similar catalytic properties to the wild-type enzyme, suggesting that the function of octamerisation is not to modulate the reactivity of the enzyme. Therefore, it seems likely that octamerisation is important for VAO's function in vivo, e.g. by promoting interactions with partner proteins or by simply enabling the clustering of a large number of VAO dimers in a small volume. Achieving a better 
understanding of the role played by VAO-like oxidases in fungal physiology may be of help in understanding the function of the octamerisation of VAO.

Recently, an attempt was made to characterise five novel fungal VAO-like oxidases after expressing them in Komagataella phaffii (formerly Pichia pastoris) (15). Unfortunately, the enzymes could not be characterised in terms of their activity, as expression in K. phaffii led to the isolation of proteins containing a remarkably stable anionic flavin semiquinone. However, size exclusion analysis revealed that all five of the enzymes displayed an octameric quaternary structure, despite two of them not containing a dimer-dimer interacting loop. This suggests that VAO-like proteins can also achieve octamer formation through other mechanisms. Interestingly, a multiple sequence alignment revealed that the two proteins that contain no dimer-dimer interacting loop have an N-terminal extension that is not found in the other proteins. As the crystal structure of $P$. simplicissimum $\mathrm{VAO}$ reveals that the $\mathrm{N}$-terminus of the protein is located close to the dimer-dimer interface (16), it is possible that this N-terminal extension might take over the role of the dimer-dimer interacting loop in enabling the octamerisation of these proteins. Even more surprisingly, a bacterial VAO-like protein from Cycloclasticus sp. was also reported to display an octameric quaternary structure, despite not containing a dimer-dimer interacting loop or an N-terminal extension (15). More extensive characterisation of this protein is required to determine what factors are involved in enabling its octamerisation.

\section{Catalytic mechanism of $4 \mathrm{PO}$ subfamily members}

\section{Mechanism of substrate activation}

The results presented in Chapter 4 led to a new model for the catalytic mechanism for the conversion of vanillyl alcohol (4-hydroxy-3-methoxybenzyl alcohol) and chavicol (4allylphenol) by VAO. According to this model, substrates can bind to the active site with their phenolic hydroxyl group either in its deprotonated or protonated form. When the substrate binds in its deprotonated form, it simply reacts with the FAD cofactor via hydride transfer to give the para-quinone methide intermediate. When the substrate binds in its protonated form, it must first be actively deprotonated by the enzyme to enable hydride transfer to occur. This occurs more slowly than the reaction of the deprotonated substrate with the enzyme and therefore, two distinct rates for the reduction of the flavin cofactor by its substrates are observed. Two tyrosine residues, Tyr-108 and Tyr-503, are responsible for stabilising the deprotonated form of the substrate in the active site, thus achieving preferential binding of the deprotonated substrate. When the substrate binds in its protonated form, one of the two tyrosine residues likely acts as a base, removing the proton and activating the substrate for reaction with FAD.

A recent computational study provided insights into how substrates migrate from the bulk solution into VAO's active site. This indicated that substrates migrate through a channel found at the monomer-monomer interface of a VAO dimer before entering the active site through a gate formed by His-466 and Tyr-503 (17). These simulations also suggested that the substrate (in this case para-creosol) cannot migrate into the active site in its deprotonated form 
due to a high energy barrier encountered when the substrate is roughly $15 \AA$ from the FAD cofactor. Therefore, it seems likely that the substrate becomes deprotonated only upon passing this position in the enzyme. Thus, after entering the active site, the $p K_{a}$ of the substrate drops due to the presence of Tyr-108 and Tyr-503, leading to an equilibrium of deprotonated and protonated substrate being bound in the active site, with the deprotonated form predominating. After this, the deprotonated form of the substrate can rapidly react with the FAD cofactor. The reaction with the protonated form is slower, this may be due to it binding in a slightly different, non-optimal orientation within the active site.

\section{Oxygen reactivity}

Another interesting aspect of the $4 \mathrm{PO}$ subfamily members that has not yet been explored is why some of the enzymes are oxidases capable of using molecular oxygen as an electron acceptor (EUGO and VAO), while others are dehydrogenase that rely on the presence of alternative electron acceptors for their activity [para-cresol methylhydroxylase (PCMH)]. Inspection of the crystal structure of VAO revealed that there is a chloride anion bound in a pocket located on the re-side of the FAD cofactor (16). A similar pocket has been described as being the site at which oxygen reacts with FAD in the VAO/PCMH family members alditol oxidase and berberine bridge enzyme and chloride has previously been found to bind to the oxygen binding site of flavin-dependent oxidases such as glucose oxidase and monomeric sarcosine oxidase (18-20). Therefore, it seems likely that this pocket may be the site of the reaction with oxygen in VAO. Interestingly, inspection of the crystal structures of EUGO and PCMH revealed that this pocket is also present in EUGO, but that in PCMH a proline residue (Pro-155) extends into the pocket, sterically blocking it (Fig. 1) (21, 22). This proline residue is conserved in all close homologues of PCMH identified in the bioinformatics analysis presented in Chapter 3. A leucine residue is found at the corresponding position in VAO and EUGO and a leucine or isoleucine residue is found at this position in all of the close homologues of these oxidases identified in Chapter 3. In contrast to the situation in PCMH, where the side chain of Pro-155 extends into the pocket found in VAO and EUGO, the side chains of Leu-171 in VAO and Leu-152 in EUGO point away from the pocket, thus potentially providing space for oxygen to bind to the enzyme and react with FAD.

To test whether this structural difference is responsible for the differences in oxygen reactivity between these enzymes, a EUGO variant was created where Leu-152 was replaced by the proline found at this position in PCMH: L152P EUGO. Based on its absorption and circular dichroism spectra and the yellow pellet obtained upon precipitation of the protein using trichloroacetic acid, the purified L152P variant is correctly folded and contains covalently bound FAD. Preliminary studies of the reactivity of this variant using stopped-flow spectroscopy revealed that upon anaerobically mixing L152P EUGO and $2 \mathrm{mM}$ vanillyl alcohol the enzyme is completely reduced by the substrate, albeit significantly more slowly than the wild-type enzyme is (Fig. 2A). Upon mixing reduced enzyme with buffer containing atmospheric oxygen levels, however, full reoxidation of the enzyme was not achieved, even after a reaction time of $60 \mathrm{~s}$ (Fig. 2B). This suggests that the putative oxygen binding pocket is indeed the site of the reaction of oxygen with FAD in EUGO. More detailed studies on this 

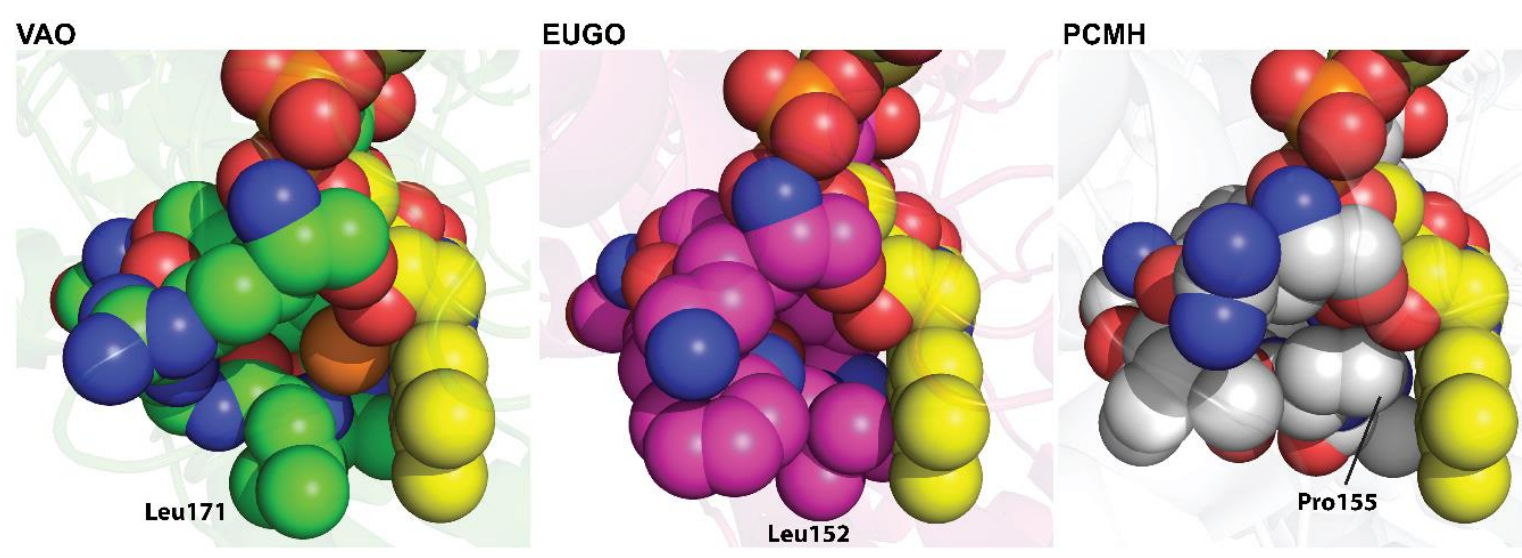

Figure 1: Space-filling model of the structures of the putative oxygen binding pocket of VAO and EUGO and the corresponding region in PCMH. VAO is shown with green carbon atoms, EUGO with pink carbon atoms and PCMH with grey carbon atoms. The FAD cofactors are shown with yellow carbon atoms. Nitrogen atoms are shown in blue and oxygen atoms in red. The chloride anion bound to the putative oxygen binding pocket of VAO is shown in orange. The crystal structures of VAO, EUGO and PCMH were downloaded from the PDB website (PDB IDs: 1VAO, 5FXD and 1DIQ respectively). Figure was prepared using PyMOL (Schrödinger LLC, New York, NY, USA).

variant, including a comprehensive stopped-flow kinetics analysis, are required to fully understand the effect of the introduced mutation on the oxygen reactivity of EUGO. Determination of the crystal structure of the variant, to demonstrate that the introduced proline residue indeed blocks the putative oxygen binding pocket, would also be of use. Further mutagenesis at this position could be used to study the effect that changing the environment of the oxygen binding pocket has on the reaction of EUGO with oxygen. It would also be highly interesting to evaluate whether the flavoprotein subunit of PCMH can be turned into an oxidase by replacing Pro-155 by other amino acids.

\section{Mechanism of reaction with 4-alkylphenols}

Another intriguing open question regards the mechanism of the reaction of VAO and EUGO with 4-alkylphenols. It has been shown previously that, despite readily converting 4ethylphenol and 4-propylphenol, VAO does not efficiently convert 4-methylphenol (paracresol) due to the formation of a covalent adduct between this substrate and the enzyme's FAD cofactor $(16,23)$. A recent computational study, where the reaction mechanism of VAO with 3-methoxy-4-methylphenol (para-creosol) and vanillyl alcohol was studied by QM modelling, revealed a potential explanation for the fact that this covalent adduct is exclusively formed with 4-methylphenols (12). After hydride transfer from para-creosol to FAD yields the para-quinone methide intermediate and reduced FAD, the active site base Asp-170 adopts a conformation that allows it to deprotonate the N5 atom of the reduced FAD. The deprotonated FAD subsequently attacks the $\mathrm{C} \alpha$ of the para-quinone methide, leading to formation of the adduct. Adduct formation is a reversible process. After it breaks down, the formed paraquinone methide can react with water, explaining the slow formation of hydroxylated product observed with para-creosol. In the case of vanillyl alcohol, the more bulky side chain of the substrate prevents Asp-170 from adopting the conformation in which it can deprotonate the $\mathrm{N} 5$ of reduced FAD, thus preventing adduct formation. 
A

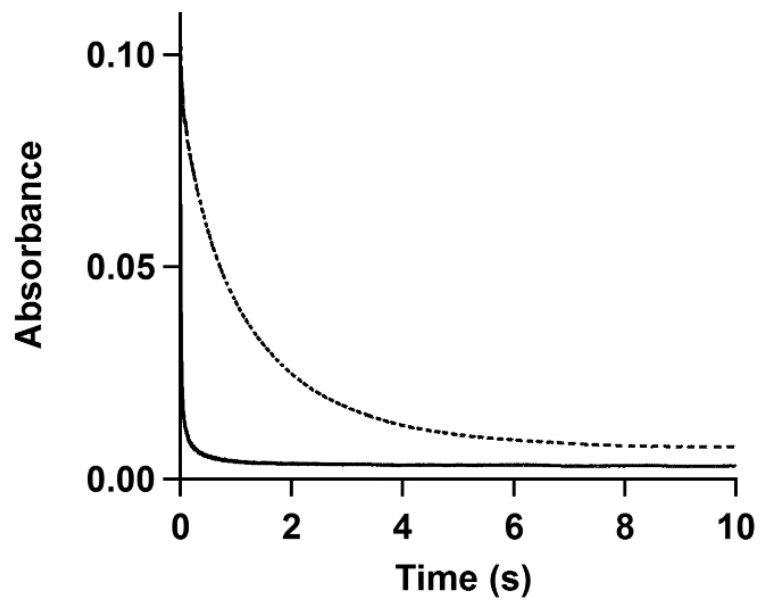

B

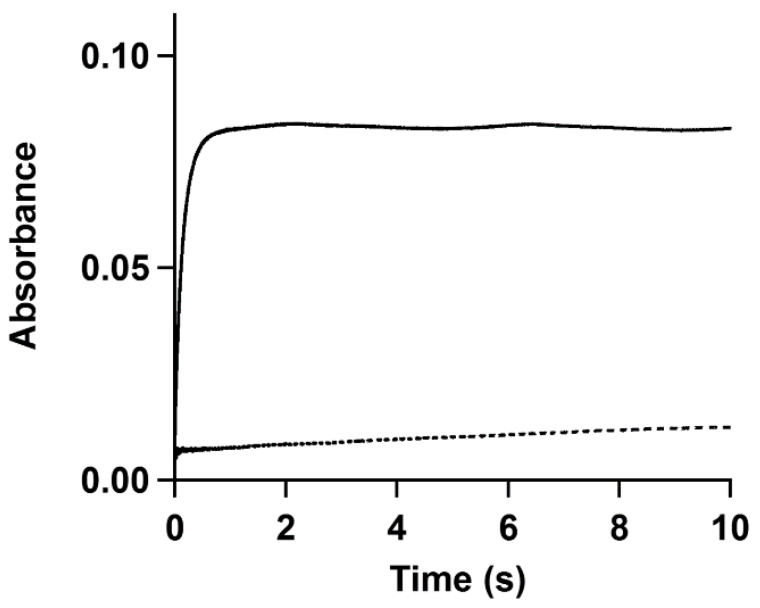

Figure 2: Traces of the absorption of the FAD cofactor of wild-type EUGO (solid lines) and L152P EUGO (dashed lines) at $441 \mathrm{~nm}$ during their reductive (A) and oxidative (B) half-reactions. (A) Traces of the absorbance at $441 \mathrm{~nm}$ after mixing $10 \mu \mathrm{M}$ oxidised enzyme with $2 \mathrm{mM}$ vanillyl alcohol in $50 \mathrm{mM}$ potassium phosphate buffer, $\mathrm{pH} 7.5$, under anaerobic conditions at $25^{\circ} \mathrm{C}$. (B) Traces of the absorbance at $441 \mathrm{~nm}$ after mixing $10 \mu \mathrm{M}$ reduced enzyme (reduced by mixing with 1.4 equivalents of vanillyl alcohol under anaerobic conditions) with $127 \mu \mathrm{M}$ oxygen in $50 \mathrm{mM}$ potassium phosphate buffer, $\mathrm{pH}$ 7.5 , at $25^{\circ} \mathrm{C}$. Even upon longer reaction times (maximum $60 \mathrm{~s}$ ) full reoxidation of the FAD cofactor of L152P EUGO was not achieved.

When VAO is incubated with 4-ethylphenol or 4-propylphenol under anaerobic conditions, a covalent adduct similar to that observed with para-cresol is formed (23). However, the rate of formation of this species observed in stopped-flow experiments under anaerobic conditions is too slow to be catalytically relevant and the substrates are readily converted by the enzyme. This implies that in the reaction of VAO with 4-ethylphenol or 4-propylphenol, after formation of the para-quinone methide intermediate, the enzyme's FAD cofactor is rapidly reoxidised by its reaction with molecular oxygen and that this stimulates the breakdown of the para-quinone methide intermediate by its reaction with water or intramolecular rearrangement. Thus, the outcome of the reaction of a 4-alkylphenol with VAO is a combination of the rate at which a covalent adduct is formed between the para-quinone methide intermediate and FAD (and the rate of the reverse reaction) and the rate at which the para-quinone methide intermediate breaks down either by reacting with water or rearranging intramolecularly. The rate of adduct formation may be affected by the identity of the substrate, with more bulky substrates impairing adduct formation by preventing the deprotonation of the N5 of FAD by Asp-170. For many substrates, the breakdown of the para-quinone methide intermediate is connected to the reoxidation of FAD by molecular oxygen $(23,24)$. In addition, the rate of this reoxidation may be affected by the presence of a para-quinone methide intermediate in the active site, as has been observed with 4-(methoxymethyl)phenol (24). This illustrates that in order to gain a comprehensive understanding of the factors governing the reactivity of 4 -alkyphenols with $4 \mathrm{PO}$ oxidases it is not sufficient to understand how the identity of the substrate effects processes occurring during the reductive half-reaction. The effect of the presence of a particular para-quinone methide intermediate in the active site on the reaction of the enzyme with molecular oxygen must also be taken into account. 
In this context, it is interesting to study why $\mathrm{VAO}$ readily converts 4-alkylphenols such as 4ethylphenol, whereas EUGO does not. A potential explanation could be that, in EUGO, 4ethylphenol more easily forms an adduct with the enzyme's FAD cofactor than in VAO. It would be of interest to study the reaction of EUGO with 4-ethylphenol in more detail, e.g. by studying whether the enzyme is reduced by 4-ethylphenol and whether the spectral properties of the enzyme upon reduction are consistent with the formation of an FAD-4ethylphenol adduct. If evidence for the formation of an adduct is obtained, it would be of interest to obtain its crystal structure. Finally, QM modelling of the reaction of VAO and EUGO with 4-ethylphenol may give us a more comprehensive understanding of why VAO can convert this compound, but EUGO cannot.

\section{Towards the biocatalytic application of $4 \mathrm{PO}$ subfamily members}

The $4 \mathrm{PO}$ subfamily members have been shown to be of use for a number of biocatalytic applications such as the synthesis of vanillin or model lignin compounds (25-34). They may also be of interest for the synthesis of chiral $\alpha$-aryl alcohols and in Chapter 7 we demonstrated that VAO can be used to obtain such a compound on a multigram scale. Further research into this subfamily of enzymes could increase their potential for industrial applications.

\section{Expanding the catalytic scope of the $4 P O$ subfamily}

First of all, it would be interesting to further explore the natural diversity of $4 \mathrm{PO}$ enzymes by characterising potential family members identified by mining genome databases. This might lead to the identification of enzymes with desired properties from the perspective of industrial applications such as higher reaction rates with interesting substrates, the ability to convert substrates that are not accepted by the currently-known enzymes or heightened thermostability or tolerance to organic solvents. Such improved properties could also be achieved by the modification of existing enzymes, either by rational design or directed evolution. All such attempts will be facilitated by the high-throughput substrate specificity screening assay presented in Chapter 6.

One interesting target for such mutagenesis efforts would be to obtain VAO variants with heightened reactivity towards 4-methylphenols, which would enable the design of an efficient enzymatic process for the synthesis of vanillin from para-creosol. It would also be of interest to obtain a EUGO variant that is able to catalyse the oxidation of 4-alkylphenols. This might be facilitated by further QM computational studies that could provide a more comprehensive picture of the factors governing the formation of adducts between the FAD cofactor of VAO or EUGO and 4-alkylphenols.

Another interesting question is whether the catalytic scope of $4 \mathrm{PO}$ subfamily members can be extended beyond the strict specificity for para-substituted phenols. In light of the results presented in Chapter 8, one might hypothesise that compounds with activating groups other than a hydroxyl group on the aromatic ring might also be accepted as substrates by $4 \mathrm{PO}$ subfamily members. For example, substrates with a thiol or amino group at this position might be converted by the enzymes, with catalysis proceeding via a thioquinone methide or quinone methide imine respectively. In this light, the ability of VAO and EUGO to catalyse 
the conversion of 4-ethylthiophenol and 4-aminobenzyl alcohol was evaluated using oxygen consumption measurements. No significant oxygen consumption was observed using substrate concentrations of $200 \mu \mathrm{M}$ and enzyme concentrations of $460 \mathrm{nM}$ and $630 \mathrm{nM}$ for

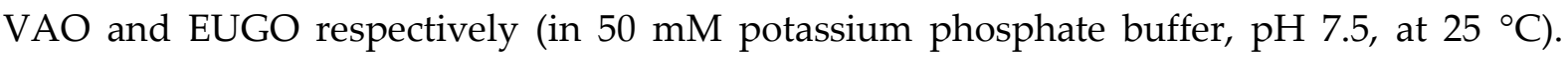
Nevertheless, it might be interesting to perform long-term incubations of these substrates with the enzymes to evaluate whether their conversion can be achieved.

Another interesting question is whether our current understanding of the $4 \mathrm{PO}$ subfamily members could allow us to design an oxidase that specifically catalyses the oxidation of the $\mathrm{C} \alpha$ atom of ortho-substituted phenols. This reaction could proceed via a mechanism similar to that observed in $4 \mathrm{PO}$ subfamily members, involving an ortho-quinone methide intermediate. However, modifying an existing $4 \mathrm{PO}$ subfamily member so as to achieve this reaction may be extremely challenging, as it would entail completely remodelling the enzyme's active site so as to move the phenolate binding pocket to a different position. However, combining the mechanistic understanding gathered from previous mutagenesis studies (35, Chapter 4) with computational studies that allow us to understand the structure of the transition state during catalysis by $4 \mathrm{PO}$ subfamily members (12), may allow the construction of an ortho-phenol oxidase using de novo enzyme design methods (36). Achieving this goal would demonstrate that we truly understand all factors that are required to enable catalysis in $4 \mathrm{PO}$ subfamily members.

\section{Development of biocatalytic processes involving $4 P O$ enzymes}

The characterisation of novel $4 \mathrm{PO}$ subfamily members and the redesign of existing ones by mutagenesis will lead to the identification of enzymes that (more) efficiently catalyse reactions that are of interest for biocatalytic applications. In order for these enzymes to be successfully applied, efforts must also be made to understand what factors are of importance in designing large-scale biocatalytic processes involving them.

The main limiting factor identified during the scale-up of the synthesis of $(R)-1-\left(4^{\prime}-\right.$ hydroxyphenyl)ethanol from 4-ethylphenol presented in Chapter 7 was oxygen depletion, which became the limiting factor for the conversion achieved at the largest scale tested. This is likely due to the large reaction volume leading to the rate of oxygen transfer into the reaction medium becoming insufficient. This was solved by performing the reaction under an oxygen atmosphere. However, this is not desirable for industrial applications, as the use of pure oxygen presents an explosion hazard. Use of reactor set-ups that increase the rate of transfer of oxygen into the reaction medium, e.g. by bubble aeration (37), is a possible solution. Another option is the addition of higher amounts of organic solvents in which the solubility of oxygen is higher than in water to the reaction medium. The addition of higher amounts of organic solvents could also increase the substrate loadings that can be used, further facilitating the design of a cost-effective biocatalytic process.

Another factor that was found to be important in the research presented in Chapter 7 is to minimise the amount of by-products formed during a reaction. During the VAO-mediated synthesis of (R)-1-(4'-hydroxyphenyl)ethanol, a significant amount of the desired product (at 
least $20 \%$ ) was lost during its purification by recrystallisation. Although alternative purification methods such as column chromatography may be used to purify the product with lower losses, they are less attractive for use in industrial processes. The creation of VAO variants that lead to less (or ideally no) by-product formation could be a useful solution to this issue.

\section{Potential applications of thiol oxidation by flavoprotein oxidases}

Chapter 8 presents the finding that flavin-dependent alcohol oxidases are capable of oxidising thiol analogues of their alcohol substrates to the corresponding thiocarbonyl compounds (Scheme 2). This was found to hold true for all tested oxidases, suggesting it is a general property of this class of enzymes. The oxidation of thiols by 5-(hydroxymethyl)furfural oxidase has recently been applied for the synthesis of enantiomerically pure secondary thiols by the kinetic resolution of racemates (38). In addition, the thiocarbonyl compounds formed upon oxidation of thiols are of interest for a number of applications. For example, they can be applied as dienophiles in Diels-Alder type cycloadditions for the synthesis of sulphurcontaining heterocycles $(39,40)$. Nucleophilic addition to thiocarbonyls is also of interest as the similar electronegativity of sulphur and carbon allows addition to the sulphur atom in some cases, enabling C-S bond formation $(39,41)$. Considering the fact that all tested flavoprotein oxidases can convert thiols as well as alcohols, it would be interesting to study whether their reactivity extends to the oxidation of other functional groups as well. For example, it would be interesting to test whether they are capable of oxidising selenols, which display chemical properties that are quite similar to those of thiols (42).

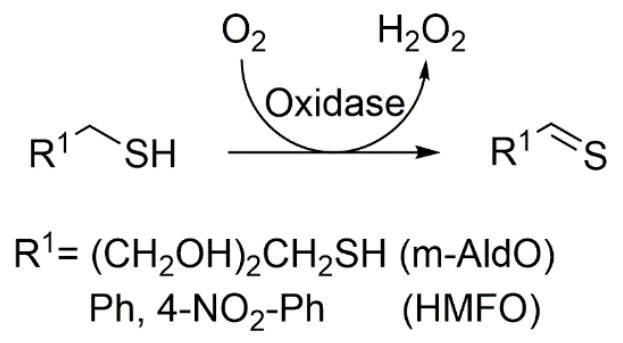

Scheme 2: Oxidation of thiols to thiocarbonyls by alditol oxidase (m-AldO) and 5-(hydroxymethyl)furfural oxidase (HMFO).

\section{Summarising remarks}

The research presented in this thesis provides advances in our understanding of the molecular mechanisms governing oligomerisation and catalysis in $4 \mathrm{PO}$ subfamily members. In addition, it contains the description of the development of a high-throughput screening assay for the activity of $4 \mathrm{PO}$ subfamily oxidases and the scale-up of the synthesis of a chiral secondary alcohol using VAO. Intriguing open questions still exist regarding the exact physiological function of $4 \mathrm{PO}$ subfamily members and certain aspects of their catalytic mechanisms such as the molecular mechanisms by which their reactivity with oxygen is governed and why they differ in terms of their reactivity with 4-alkylphenols. A comprehensive understanding of the factors governing catalysis in these enzymes will enable us to modify them so as to have properties that are desirable from the point of view of biocatalytic applications. 
Unconventional avenues of exploration, such as reactions that fall outside the known catalytic scope of the enzymes, may yield unexpected results with interesting potential applications. It will be fascinating to see what surprising advances the future will bring regarding $4 \mathrm{PO}$ subfamily members, the VAO/PCMH flavoprotein family and the biocatalytic application of enzymes in general.

\section{Acknowledgements}

I thank Eva Ramos Becares and Tom Arfman for their work on the oxygen reactivity of EUGO and Prof. Dr. Andrea Mattevi (University of Pavia) for his input regarding this topic.

\section{References}

1. Pellis, A., Cantone, S., Ebert, C., and Gardossi, L. (2018) Evolving biocatalysis to meet bioeconomy challenges and opportunities. Nat. Biotechnol. 40, 154-169

2. Gygli, G., and van Berkel, W. J. H. (2015) Oxizymes for biotechnology. Curr. Biotechnol. 4, 100110

3. Martínez, A. T., Ruiz-Dueñas, F. J., Camarero, S., Serrano, A., Linde, D., Lund, H., Vind, J., Tovborg, M., Herold-Majumdar, O. M., Hofrichter, M., Liers, C., Ullrich, R., Scheibner, K., Sannia, G., Piscitelli, A., Pezzella, C., Sener, M. E., Kılıç, S., van Berkel, W. J. H., Guallar, V., Lucas, M. F., Zuhse, R., Ludwig, R., Hollmann, F., Fernández-Fueyo, E., Record, E., Faulds, C. B., Tortajada, M., Winckelmann, I., Rasmussen, J.-A., Gelo-Pujic, M., Gutiérrez, A., del Río, J. C., Rencoret, J., and Alcalde, M. (2017) Oxidoreductases on their way to industrial biotransformations. Biotechnol. Adv. 35, 815-831

4. Paladino, A., Marchetti, F., Rinaldi, S., and Colombo, G. (2017) Protein design: From computer models to artificial intelligence. Wiley Interdiscip. Rev. Comput. Mol. Sci. 7, e1318

5. Bunzel, H. A., Garrabou, X., Pott, M., and Hilvert, D. (2018) Speeding up enzyme discovery and engineering with ultrahigh-throughput methods. Curr. Opin. Struct. Biol. 48, 149-156

6. de Jong, E., Beuling, E. E., van der Zwan, R. P., and de Bont, J. A. M. (1990) Degradation of veratryl alcohol by Penicillium simplicissimum. Appl. Microbiol. Biotechnol. 34, 420-425

7. Ahmad, M., Taylor, C. R., Pink, D., Burton, K., Eastwood, D., Bending, G. D., and Bugg, T. D. H. (2010) Development of novel assays for lignin degradation: Comparative analysis of bacterial and fungal lignin degraders. Mol. Biosyst. 6, 815-821

8. Fraaije, M. W., Pikkemaat, M., and van Berkel, W. J. H. (1997) Enigmatic gratuitous induction of the covalent flavoprotein vanillyl-alcohol oxidase in Penicillium simplicissimum. 63, 435-439

9. Wells Jr., T., and Ragauskas, A. J. (2012) Biotechnological opportunities with the $\beta$-ketoadipate pathway. Trends Biotechnol. 30, 627-637

10. de Jong, E., van Berkel, W. J. H., van der Zwan, R. P., and de Bont, J. A. M. (1992) Purification and characterization of vanillyl-alcohol oxidase from Penicillium simplicissimum: A novel aromatic alcohol oxidase containing covalently bound FAD. Eur. J. Biochem. 208, 651-657

11. Fraaije, M. W., Veeger, C., and van Berkel, W. J. H. (1995) Substrate specificity of flavindependent vanillyl-alcohol oxidase from Penicillium simplicissimum: Evidence for the production of 4-hydroxycinnamyl alcohols from 4-allylphenols. Eur. J. Biochem. 234, 271-277

12. Gygli, G. (2018) A kaleidoscopic look at vanillyl alcohol oxidase. Ph.D. thesis, ISBN 978-94-6343741-7

13. Fraaije, M. W., Mattevi, A., and van Berkel, W. J. H. (1997) Mercuration of vanillyl-alcohol oxidase from Penicillium simplicissimum generates inactive dimers. FEBS Lett. 402, 33-35

14. Jin, J., Mazon, H., van den Heuvel, R. H. H., Janssen, D. B., and Fraaije, M. W. (2007) Discovery of a eugenol oxidase from Rhodococcus sp. strain RHA1. FEBS J. 274, 2311-2321

15. Gygli, G., and van Berkel, W. J. H. (2017) Vanillyl alcohol oxidases produced in Komagataella phaffii contain a highly stable noncovalently bound anionic FAD semiquinone. Biocatalysis. 3, 
$17-26$

16. Mattevi, A., Fraaije, M. W., Mozzarelli, A., Olivi, L., Coda, A., and van Berkel, W. J. H. (1997) Crystal structures and inhibitor binding in the octameric flavoenzyme vanillyl-alcohol oxidase: The shape of the active-site cavity controls substrate specificity. Structure. 5, 907-920

17. Gygli, G., Lucas, M. F., Guallar, V., and van Berkel, W. J. H. (2017) The ins and outs of vanillyl alcohol oxidase: Identification of ligand migration paths. PLoS Comput. Biol. 13, e1005787

18. Baron, R., Riley, C., Chenprakhon, P., Thotsaporn, K., Winter, R. T., Alfieri, A., Forneris, F., van Berkel, W. J. H., Chaiyen, P., Fraaije, M. W., Mattevi, A., and McCammon, J. A. (2009) Multiple pathways guide oxygen diffusion into flavoenzyme active sites. Proc. Natl. Acad. Sci. U. S. A. 106, 10603-10608

19. Kommoju, P.-R., Chen, Z.-W., Bruckner, R. C., Mathews, F. S., and Jorns, M. S. (2011) Probing oxygen activation sites in two flavoprotein oxidases using chloride as an oxygen surrogate. Biochemistry. 50, 5521-5534

20. Zafred, D., Steiner, B., Teufelberger, A. R., Hromic, A., Karplus, P. A., Schofield, C. J., Wallner, S., and Macheroux, P. (2015) Rationally engineered flavin-dependent oxidase reveals steric control of dioxygen reduction. FEBS J. 282, 3060-3074

21. Nguyen, Q.-T., de Gonzalo, G., Binda, C., Rioz-Martínez, A., Mattevi, A., and Fraaije, M. W. (2016) Biocatalytic properties and structural analysis of eugenol oxidase from Rhodococcus jostii RHA1: A versatile oxidative biocatalyst. ChemBioChem. 17, 1359-1366

22. Cunane, L. M., Chen, Z.-W., Shamala, N., Mathews, F. S., Cronin, C. N., and McIntire, W. S. (2000) Structures of the flavocytochrome $p$-cresol methylhydroxylase and its enzyme-substrate complex: Gated substrate entry and proton relays support the proposed catalytic mechanism. J. Mol. Biol. 295, 357-374

23. Fraaije, M. W., van den Heuvel, R. H. H., Roelofs, J. C. A. A., and van Berkel, W. J. H. (1998) Kinetic mechanism of vanillyl-alcohol oxidase with short-chain 4-alkylphenols. Eur. J. Biochem. 253, 712-719

24. Fraaije, M. W., and van Berkel, W. J. H. (1997) Catalytic mechanism of the oxidative demethylation of 4-(methoxymethyl)phenol by vanillyl-alcohol oxidase: Evidence for formation of a $p$-quinone methide intermediate. J. Biol. Chem. 272, 18111-18116

25. van den Heuvel, R. H. H., Fraaije, M. W., Laane, C., and van Berkel, W. J. H. (2001) Enzymatic synthesis of vanillin. J. Agric. Food Chem. 49, 2954-2958

26. Overhage, J., Steinbüchel, A., and Priefert, H. (2003) Highly efficient biotransformation of eugenol to ferulic acid and further conversion to vanillin in recombinant strains of Escherichia coli. Appl. Environ. Microbiol. 69, 6569-6576

27. Plaggenborg, R., Overhage, J., Loos, A., Archer, J. A. C., Lessard, P., Sinskey, A. J., Steinbüchel, A., and Priefert, H. (2006) Potential of Rhodococcus strains for biotechnological vanillin production from ferulic acid and eugenol. Appl. Microbiol. Biotechnol. 72, 745-755

28. Overhage, J., Steinbüchel, A., and Priefert, H. (2006) Harnessing eugenol as a substrate for production of aromatic compounds with recombinant strains of Amycolatopsis sp. HR167. J. Biotechnol. 125, 369-376

29. Zucca, J., Lambert, F., Mane, J., Ness, F., and Aigle, M. (2013) System for producing aromatic molecules by bioconversion. United States Patent. 8,404,472

30. Lambert, F., Zucca, J., Ness, F., and Aigle, M. (2014) Production of ferulic acid and coniferyl alcohol by conversion of eugenol using a recombinant strain of Saccharomyces cerevisiae. Flavour Fragr. J. 29, 14-21

31. Zhou, R., and Xiaodan, Y. (2015) Methods of making vanillin via the microbial fermentation of ferulic acid from eugenol using a plant dehydrogenase. Patent application. WO/2015/066722

32. Ricklefs, E., Girhard, M., Koschorreck, K., Smit, M. S., and Urlacher, V. B. (2015) Two-step onepot synthesis of pinoresinol from eugenol in an enzymatic cascade. ChemCatChem. 7, 1857-1864

33. Ricklefs, E., Girhard, M., and Urlacher, V. B. (2016) Three-steps in one-pot: Whole-cell biocatalytic synthesis of enantiopure (+)- and (-)-pinoresinol via kinetic resolution. Microb. Cell 
Fact. 15, 78

34. Habib, M. H. M., Deuss, P. J., Lončar, N., Trajkovic, M., and Fraaije, M. W. (2017) A biocatalytic one-pot approach for the preparation of lignin oligomers using an oxidase/peroxidase cascade enzyme system. Adv. Synth. Catal. 359, 3354-3361

35. van den Heuvel, R. H. H., Fraaije, M. W., Mattevi, A., and van Berkel, W. J. H. (2000) Asp-170 is crucial for the redox properties of vanillyl-alcohol oxidase. J. Biol. Chem. 275, 14799-14808

36. Zanghellini, A. (2014) De novo computational enzyme design. Curr. Opin. Biotechnol. 29, 132-138

37. Li, X., Li, P., Zu, L., and Yang, C. (2016) Gas-liquid mass transfer characteristics with microbubble aeration - I: Standard stirred tank. Chem. Eng. Technol. 39, 945-952

38. Pickl, M., Swoboda, A., Romero, E., Winkler, C. K., Binda, C., Mattevi, A., Faber, K., and Fraaije, M. W. (2018) Kinetic resolution of sec-thiols by enantioselective oxidation with rationally engineered 5-(hydroxymethyl)furfural oxidase. Angew. Chem. Int. Ed. 57, 2864-2868

39. Metzner, P. (1999) Thiocarbonyl compounds as specific tools for organic synthesis. In: Organosulfur Chemistry I (Page, P. C. B., ed.), Top. Curr. Chem. vol. 204, pp. 127-181

40. Jiang, H., Cruz, D. C., Li, Y., Lauridsen, V. H., and Jørgensen, K. A. (2013) Asymmetric organocatalytic thio-diels-alder reactions via trienamine catalysis. J. Am. Chem. Soc. 135, 52005207

41. Bailey, W. F., Bartelson, A. L., and Wiberg, K. B. (2012) Contrasting reactions of ketones and thioketones with alkyllithiums: A coordinated experimental and computational investigation. J. Am. Chem. Soc. 134, 3199-3207

42. Wessjohann, L. A., Schneider, A., Abbas, M., and Brandt, W. (2007) Selenium in chemistry and biochemistry in comparison to sulfur. Biol. Chem. 388, 997-1006

\section{Abbreviations}

4PO: 4-phenol oxidising, EUGO: eugenol oxidase, FAD: flavin adenine dinucleotide, PCMH: para-cresol methylhydroxylase, VAO: vanillyl alcohol oxidase 


Acknowledgements 



\section{Acknowledgements}

After slightly more than four years of work, it is a great feeling to have finally completed my $\mathrm{PhD}$ thesis. It is now time to look back and reflect on a very fulfilling and educational experience and of course to thank all those who contributed to it.

First of all my promotor, Willem van Berkel. I still remember the first time we met. As a prospective MSc thesis student, I spent an hour or so in your office being introduced to the world of flavoenzymes. I recall being particularly impressed by a beautiful protein structure that was hanging on your wall and the world record for "weighing molecules" that was associated with it. Little did I realise at the time, that I would devote four years of my life to studying it. I feel privileged to have had you as a supervisor during my $\mathrm{PhD}$, your constant support and infectious enthusiasm for all things yellow were of immeasurable importance in allowing me to successfully complete it. Thank you!

I would also like to extend my special thanks to Adrie Westphal. After Willem had infected me with "yellow fever", you were tasked with teaching me all the laboratory skills required in order to be a flavoenzymologist. Thank you for all the guidance during my entire time at Biochemistry!

I would also like to thank my other colleagues in the "protein group" at Biochemistry. Gudrun, I really enjoyed having a partner-in-VAO-crime during my PhD. Thank you for being my paranymph and good luck in your future career! Mieke, we shared an office for many years, during which you ensured I always had someone to chat with. Thanks for agreeing to be my paranymph! Joseline, thanks for ensuring that I was provided with sufficient amounts of vitamin $\mathrm{C}$ during my $\mathrm{PhD}$. Without all the grapefruits that magically appeared on my desk, I surely wouldn't have made it to where I am today. Antsje, you were always a beacon of calm in our otherwise chatty group. Thanks for organising many nice gaming events. Caroline, it was great to have someone with your level of expertise regarding biocatalysis around during the last period of my PhD. Thanks for all the help with the analytical chemistry! Jan-Willem and Carlo, thanks for attending our group meetings and for your useful and insightful comments and suggestions.

During my PhD, I had the pleasure of supervising a number of BSc and MSc students. Aster, Eva, Ilmi, Jacob-Paul, Mark, Rianne, Robert, Sigrid and Tom A, each of you contributed, directly or indirectly, to the work presented in this thesis. Thanks for all the hard work, I wish you all the best for the future!

I would also like to thank Jacques Vervoort for his help with the analytical chemistry during the thiol oxidation project, it was greatly appreciated.

To all my other colleagues at the Laboratory of Biochemistry, thanks for making our department a pleasant place to work. I very much enjoyed all the borrels, dinners, pub quizzes, lunchtime runs, movie nights and the PhD trip I shared with you. 
Thanks also, to my scientific collaborators outside of Wageningen. First, to Andrea Mattevi, who was kind enough to host me for six weeks in his laboratory at the University of Pavia. I really enjoyed my stay in Italy, both in the professional and personal sense. Thank you Andrea, for providing me with this opportunity. Thai, I would also like to thank you for your help during this period. I really learned a lot about protein crystallography from you, good luck back in Vietnam!

I would also like to thank Marco Fraaije for hosting me on multiple occasions to perform stopped-flow experiments at the University of Groningen. I really enjoyed performing these experiments and also benefitted from your extensive knowledge and experience regarding $\mathrm{VAO}$ and other flavoenzymes. Elvira, thank you for supervising me in the lab during these trips, you truly are a stopped-flow expert and I learned a great deal from you. Willem, thank you for your collaboration during the "smelly" project.

Thanks also, to all those I collaborated with during the INDOX project. In particular to Jasmin and Ralf from Chiracon and Silvia and Marta from Biopolis, for the pleasant collaboration during the "scaling up" project.

I would also like to thank some people from my personal life for providing support and enjoyment outside of work.

Jimmy en Ruben, door alle bestelavonden en concert- en festivalbezoeken bleef ik tijdens mijn PhD een "happy camper". Rik, jij was er wat minder vaak bij, maar was wel altijd mooi. Met name de reis naar Oeganda was een groot hoogtepunt (sorry Jimmy!).

Job, jij stond altijd klaar om gebroederlijk op de bank, glas whisky of jenever in de ene hand, $\mathrm{X}$-box controller in de andere, de straten van Wageningen vrij te houden van buitenaardse indringers. Success met je promotieonderzoek!

To all my friends at NVLTB and Walhalla, in particular the members of the active tennis group (Bram, Chidu, Jaap, Jort and Viet) and Marion, thanks for providing some relaxation on the tennis courts. I look forward to seeing you there soon!

To my siblings, Robert and Catriona. 2018 will be a unique year for the Ewing family, with all three of us getting a degree. I look forward to coming to your graduation ceremonies, I am proud of you!

To Mum and Dad. The foundations of this $\mathrm{PhD}$ thesis were laid in my early childhood, when you always encouraged me to follow my natural inclination to learn about and explore the world around me. It must have been frustrating at times to have such a scientifically inclined child (But whyyyy?), but you always encouraged me to pursue my interests and I am grateful for that. Thanks for all the support!

Dearest $\mathrm{Lu}$, you were a great support to me during the last period of my PhD. I look forward to seeing you finishing up your own studies in the coming months and to continuing our life in Wageningen together! 我爱你! 


About the author 



\section{About the author}

Thomas (Tom) Alasdair Ewing was born in Farnborough, United Kingdom, on the $29^{\text {th }}$ of August 1990. After moving to the Netherlands with his parents in 1993, he grew up in Bergschenhoek, a small town close to Rotterdam. After completing secondary school, he studied chemistry at Utrecht University and graduated cum laude in 2013. During the final stages of his MSc programme, during which he specialised in Molecular and Cellular Life Sciences, he performed an internship in the research group led by Prof. Dr. Willem van Berkel at the Laboratory of Biochemistry of Wageningen University

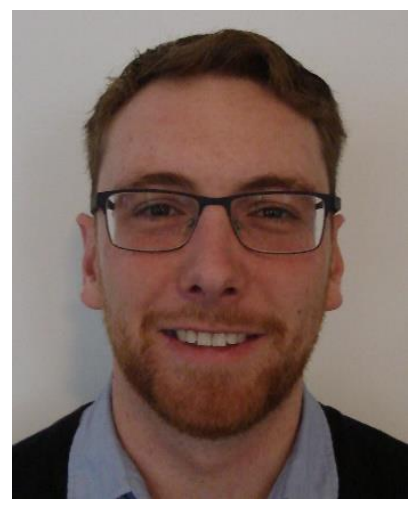
\& Research. In January 2014, he started his PhD studies in the same group, working on the flavin-dependent oxidase vanillyl alcohol oxidase (VAO) as part of the EU-financed INDOX consortium. During his PhD studies, Tom spent six weeks in the research group of Prof. Dr. Andrea Mattevi at the University of Pavia, Italy, where he learned how to perform protein Xray crystallography. He also visited the research group of Prof. Dr. Marco Fraaije at the University of Groningen, where he performed stopped-flow kinetics experiments. He will now continue his career in the field of intellectual property law, working at the Servicedesk Intellectual Property of Wageningen University \& Research. 


\section{List of publications}

Ewing, T. A., Kühn, J., Segarra, S., Tortajada, M., Zuhse, R., and van Berkel, W. J. H. (2018) Multigram scale enzymatic synthesis of (R)-1-(4'-hydroxyphenyl)ethanol using vanillyl alcohol oxidase. Advanced Synthesis and Catalysis, https://doi.org/10.1002/adsc.201800197

Ewing, T. A., van Noord, A., Paul, C. E., and van Berkel, W. J. H. (2018) A xylenol orangebased screening assay for the substrate specificity of flavin-dependent para-phenol oxidases. Molecules, 23, 164

Ewing, T. A., Fraaije, M. W., Mattevi, A., and van Berkel, W. J. H. (2017) The VAO/PCMH flavoprotein family. Archives of Biochemistry and Biophysics, 632, 104-117

Ewing, T. A., Nguyen, Q.-T., Allan, R. C., Gygli, G., Romero, E., Binda, C., Fraaije, M. W., Mattevi, A., and van Berkel, W. J. H. (2017) Two tyrosine residues, Tyr-108 and Tyr-503, are responsible for the deprotonation of phenolic substrates in vanillyl-alcohol oxidase. Journal of Biological Chemistry, 292, 14668-14679

Ewing, T. A., Gygli, G., and van Berkel, W. J. H. (2016) A single loop is essential for the octamerization of vanillyl alcohol oxidase. FEBS Journal, 283, 2546-2559

Ewing, T. A., Fraaije, M. W., and van Berkel, W. J. H. (2015) Oxidations using alcohol oxidases. In: Science of Synthesis: Biocatalysis in Organic Synthesis 3 (Faber, K., Fessner, W.-D., and Turner, N. J. eds.), pp. 157-185

Ewing, T. A.*, Dijkman, W. P. , Vervoort, J. M., Fraaije, M. W., and van Berkel, W. J. H. (2014) The oxidation of thiols by flavoprotein oxidases: A biocatalytic route to reactive thiocarbonyls. Angewandte Chemie International Edition, 53, 13206-13209

*Equal contribution 


\section{Overview of completed training activities}

\section{Discipline specific activities}

\section{Courses}

Applied Biocatalysis

Wageningen 2014

Food and Biorefinery Enzymology

Wageningen 2017

Conferences and meetings

6 INDOX project meetings ${ }^{1}$

Various

2014-2016

4 NWO CHAINS meetings ${ }^{1,2}$

Veldhoven

2014-2017

$7^{\text {th }}$ European meeting on OxiZymes ${ }^{1}$

Vienna

2014

$18^{\text {th }}$ International Symposium on Flavins and Flavoproteins ${ }^{2}$

Cha-Am

2014

$12^{\text {th }}$ International Symposium on Biocatalysis and Biotransformations ${ }^{2}$

Vienna

2015

$8^{\text {th }}$ European meeting on OxiZymes ${ }^{1}$

Wageningen

2016

$19^{\text {th }}$ International Symposium on Flavins and Flavoproteins ${ }^{2}$

Groningen

2017

\section{$\underline{\text { General courses }}$}

VLAG PhD week

Project and Time Management

Techniques for Writing and Presenting a Scientific Paper

Scientific Publishing

Career Perspectives

\section{Optional activities}

Preparing PhD research proposal

Biochemistry group meetings and seminars

PhD trip Biochemistry

${ }^{1}$ Oral presentation, ${ }^{2}$ Poster presentation
Baarlo

2014

Wageningen

2016

Wageningen

2016

Wageningen

2017

Wageningen

2017

Wageningen

2014

Wageningen

2014-2017

Barcelona

2017 
The research described in this thesis was performed at the Laboratory of Biochemistry, Wageningen University \& Research, and was financially supported by the European Union through the INDOX project (FP7-KBBE-2013-7-613549).

Financial support from the Laboratory of Biochemistry, Wageningen University \& Research, for printing this thesis is gratefully acknowledged.

Cover design and layout by Tom A. Ewing Printed by Digiforce || ProefschriftMaken, Vianen, the Netherlands

Tom A. Ewing, July 2018 